O) $m$ $\infty$ $\infty$

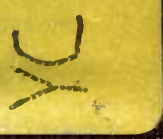




\section{LIBRARY}

OF THE

UNIVERSITY OF CALIFORNIA.

Received

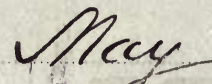

viurssion No. $6 \mathrm{rg}$ gy 2 . Class No.

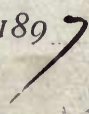

\section{BTOLOCY \\ IIERARY}

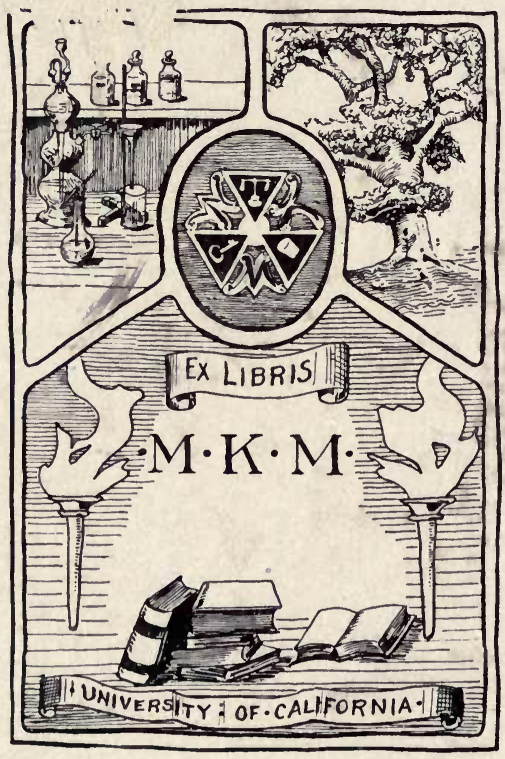




\title{
A TEXT-BOOK \\ oF \\ PHYSIOLOGICAL CHEMISTRY.
}

BY

\section{OLOF HAMMARSTEN,}

PROFESSOR OF MEDICAL AND PHYSIOLOGICAL CHEMISTRY IN THE UNIVERSITY OF UPSALA.

\author{
Authorized Cranslation \\ FROM THE SECOND SWEDISH EDITION AND FROM THE \\ AUTHOR'S ENLARGED AND REVISED \\ GERMAN EDITION \\ $B Y$
}

JOHN A. MANDEL,

ASSISTANT TO THE CHAIR OF CHEMISTRY, ETC., IN THE BELLEVUE HOSPITAL MEDICAL COLLEGE AND IN THE COLLEGE OF THE CITY OF NEW YORK.

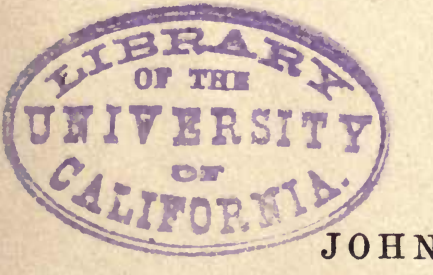

FIRST EDITION. FIRST THOUSAND.

NEW YORK :

53 East Tenth Street.

1893. 


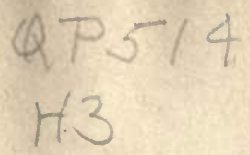

BIOLOGY

LISRARY

Copyright, 1893,

BY

JOHN A. MANDEL.

65872

ROBRRT DRUMMOND,

Electrotyper,

414 \& 146 Pearl Street, New York. 
THIS TRANSLATION

Is RESPECTFULLY DEDICATED TO

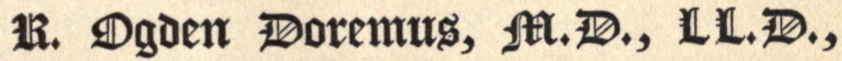

WHO GAVE THE IMPETUS TO THE STPUDY OF PHYSIOLOGICAL CHEMISTRY BY FOUNDING THE FIRST CHEMICAL LABORATORY IN CONNECTION WITH A MEDICAL COLLEGE IN THIS COUNTRY, BY THE TRANSLATOR. 



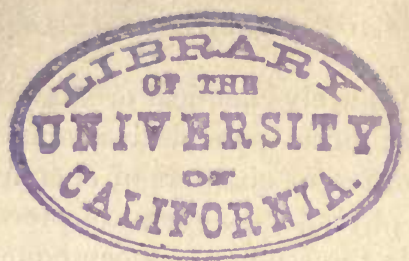

PREFACE TO THE GERMAN EDITION.

AFTER the appearance of the first Swedish edition of this textbook, I was asked by several co-laborers abroad to provide a German translation, which was at that time impossible, for several reasons. But I found it very difficult to decline a similar proposal which I received from many colleagues after the second edition appeared.

I yielded, therefore, to their expressed wishes ; but I found after a time that it was impossible to obtain a translator in this special province of science, notwithstanding the unwearied exertions of my publisher. Nothing remained for me but to undertake the translation myself; hence I ask the reader's indulgence for possible idiomatic or orthographic errors.

Specialists will at once perceive that the book before us is not a complete or detailed text-book. My intention was merely to supply students and physicians with a condensed and as far as possible objective representation of the principal results of physiologicochemical research and also with the principal features of physiologico-chemical methods of work. It seems to me that I have followed a common, practical, even if not strictly correct usage in allowing space in this book to the more important pathologicochemical facts, although I have given the book the title Textbook of Physiological Chemistry.

The arrangement of subject-matter, which deviates considerably from that generally followed in text-books, was caused by the manner in which physiological chemistry is studied in Sweden. Here physiologico- and pathologico-chemical laboratory practice is obligatory for all students of medicine. In the arrangement of such practical work I continually kept in view that it should not 
consist of isolated, purely chemical or analytico-chemical problems, but that always, as far as possible, it should go hand in hand with the study of the different chapters of chemical physiology.

The study of physiologico-chemical processes within the animal body must precede the study of its component parts, its fluids and tissues; and this latter study, according to my experience, will then only inspire true interest if the study of the physiological significance of those component parts be closely pursued in connection with that of the transformations which take place in these fluids and tissues.

In view of this arrangement of subject-matter, and in order to render my book of greater interest and utility to those who do not wish to take cognizance of its analytico-chemical part, I have distinguished the latter by different setting of the type. With the exception of urinary analysis, which practically is of particular importance and which has been treated somewhat elaborately, this part in general depicts only the main points in the methods of preparation and of analytical methods. The instructor who superintends the laboratory practice and who chooses the problems for work has ample opportunity to give the beginner the necessary advanced directions, and for the more experienced student, as well as for the specialist, the excellent works of HopPe-SEyLer, Neubauer-HuPPERT, and others render more explicit directions superfluous.

Upsatia, October, 1890.

Olof Hammarsten. 


\section{TRANSLATOR'S PREFACE.}

KNowing the demands of the medical student and practising physician for a more extended knowledge of physiological chemistry, and at the same time knowing the lack of literature on this subject in the English language, I have been led to make a translation of this most admirable work. The subject of physiological chemistry is being more and more advanced in this country, until it will soon become an obligatory study in our medical schools, and the enlargement of the literature on the subject will greatly help its progress.

It will be seen at a glance that the work is well suited as a laboratory book, for it contains the best methods for the preparation, detection, and quantitative estimation of most of the substances found in the organism and its excretions and secretions. At the author's request I have made no additions or changes whatsoever in the manuscript, and it may appear that some of the methods described, especially on the urine, are too lengthy and troublesome for the practising physician; still the quick or clinical methods are well described in smaller hand-books on the subject. In the work of translation I have adhered as closely as possible to the author's enlarged German edition and also the original Swedish edition, and therefore the literary errors will perhaps be pardoned.

I must here express my appreciation to Mon. A. Bodrgougnon who has kindly gone carefully over the manuscript and read the proof-sheets.

New York, October, 1893.

J. A. Mandel.

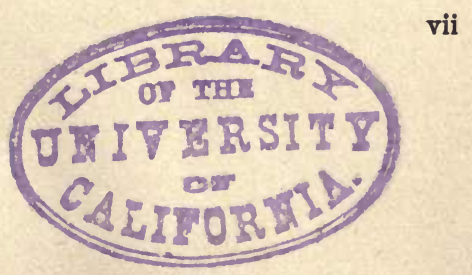





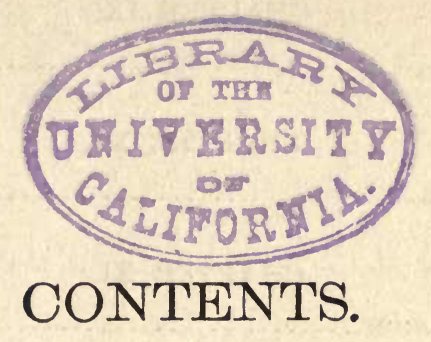

CHAPTER I.

INTRODUCTION

CHAPTER II.

The Protein Substances.

CHAPTER III.

The Amrmal Cell.

CHAPTER IV.

The BLood.

CHAPTER V.

Chyle, Lymph, Transudations and Exudations.................. 117

CHAPTER VI.

The Liver..................................... 135

CHAPTER VII.

Digestion....................................... 167

CHAPTER VIII.

Tissues of the Connective Substance.................... 233 
CHAPTER IX.

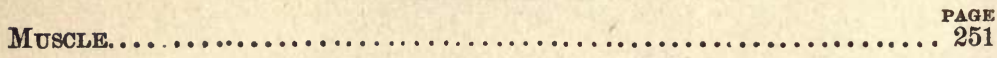

CHAPTER $\mathbf{X}$.

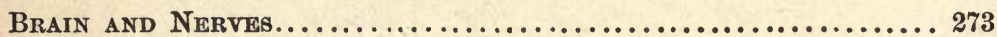

CHAPTER XI.

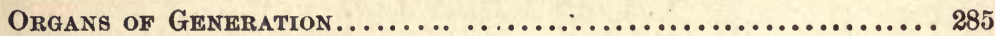

CHAPTER XII.

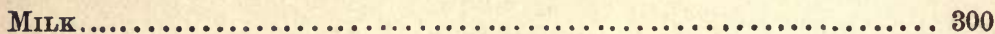

CHAPTER XIII.

The Skin and its Secretions................................ 32

CHAPTER XIV.

The Urine..................................... 330

CHAPTER XV.

The Exchange of Material......................... 435 


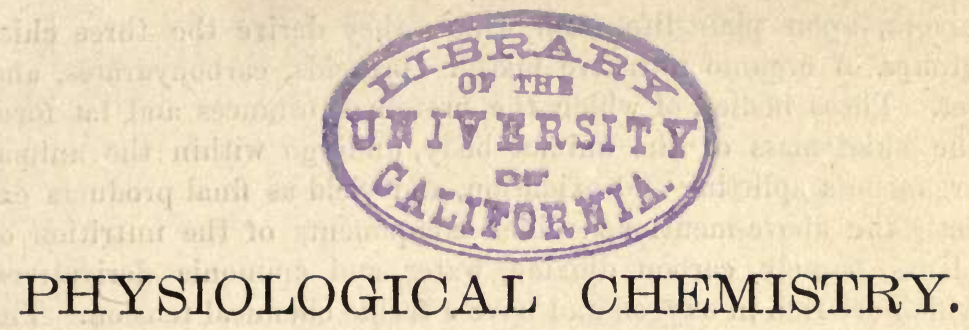

CHAPTER I.

INTRODUCTION.

IT follows from the law of the conservation of force and matter that living beings, plants and animals, can neither produce new matter nor new force. They are only called upon to appropriate and assimilate already existing material and to transform it into new forms of force.

Out of a few relatively simple combinations, especially carbon dioxide and water, together with ammonium compounds or nitrates, and a few mineral substances, which serve as its food, the plant builds up the extremely complicated constituents of its organism, albumins, carbohydrates, fats, resins, organic acids, etc. The chemical work which is performed in the plant must therefore, in the majority of cases, consist in syntheses; but besides these, processes of reduction take place to a great extent. The vis viva of the sunlight induces the green parts of the plant to split off oxygen from the carbon dioxide and water, and therefore the chief constituents of the plant contain less oxygen than the material serving as food. The vis viva of the sun, which produces this splitting, is not lost; it is only transformed into another form of force, into the potential energy or chemical tension of the free oxygen on the one side and the combinations less oxygenated produced by the synthesis, on the other side.

These conditions are not the same in animals. They are dependent either directly, as the herbivora, or indirectly, as the car- 
nivora, upon plant-life, from which they derive the three chief groups of organic nutritive matter-proteids, carbohydrates, and fat. These bodies, of which the protein substances and fat form the chief mass of the animal body, undergo within the animal organism a splitting and oxidation, and yield as final products exactly the above-mentioned chief components of the nutrition of plants, namely, carbon dioxide, water, and ammonia derivatives, which are rich in oxygen and have a feeble chemical tension. The chemical tension, which is partly combined with the free oxygen and partly stored up in the above-mentioned more complex chemical compounds, is transformed into vis viva, heat, and mechanical work. While in the plant reduction processes and syntheses, which are active in the conversion of living force into potential energy or chemical tension, are the prevailing forces, we find in the animal body the reverse of this, namely, splitting and oxidation processes, which convert chemical tension into living force (vis viva).

This difference between animals and plants must not be overrated, nor must we consider that there exists a sharp boundary-line between the two. This is not the case. There are not only lower plants, free from chlorophyll, which in regard to chemical processes represent intermediate steps between higher plants and animals, but the difference existing between the higher plants and animals is more of a quantitative than a qualitative kind. Plants require oxygen as peremptorily as do animals. Like the animal, the plant also, in the dark and by means of those parts which are free from chlorophyll, takes up oxygen and eliminates carbon dioxide, while in the light the oxidation processes going on in the green parts are overshadowed or hidden beneath the more intense reduction processes. Like the animal the vegetable ferments transform chemical tension into living energy and heat; and even in a few of the higher plants-as the aroidece when bearing fruit-a considerable development of heat has been observed. The reverse is found in the animal organism, for, besides oxidation and splitting, reduction processes and syntheses also take place. The contrast which seemingly exists between animals and plants consists merely in that in the animal organism the processes of oxidation and splitting are prevalent, while in the plant those of reduction and synthesis have mostly been observed. 
WöHLER in 1824 furnished the first example of sYNTHETICAL processes within the animal organism. He showed that when benzoic acid is introduced into the stomach it reappears as hippuric acid in the urine, after its coupling with glycocoll (amido-acetic acid). Since the discovery of this synthesis, which may be expressed by the following equation,

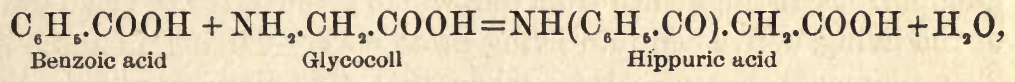

and which is ordinarily considered as a type of an entire series of syntheses occurring in the body where water is eliminated, the number of known syntheses in the animal kingdom has increased considerably. Many of these syntheses have also been artificially produced outside of the organism, and numerous examples of animal syntheses of which the history is absolutely clear will be found in the following pages. Besides these well-studied syntheses, there occur in the animal body also similar processes unquestionably of the greatest importance to animal life, but of which we know nothing with positiveness. We enumerate as examples of this kind of synthesis the new formation of the red blood-corpuscles (the hæmoglobin), the formation of the different albumins from the peptones, the formation of fat from carbohydrates, and others.

The chemical processes in the animal body we have mentioned above as consisting chiefly of oxidation and splitting processes. The oxygen of inhaled air, as also that of the blood, is now called neutral, molecular oxygen, and the old assumption that ozone occurs in the organism has now been discarded for several reasons. There are few substances which can be introduced from the outside, such as aldehydic compounds and certain alcohols, for example benzylalcohol (SCHMIEDEBERG), which can be oxidized within the animal organism by the neutral oxygen; while, on the contrary, albumin and fat, which form the chief part of the organic constituents of the animal body, are almost indifferent to neutral oxygen. The question arises, how then is the oxidation of these and other bodies possible in the animal organism?

Formerly the view was generally accepted that ANIMAL OxIDATION took place in the animal fluids, while to-day we are of the 
opinion that it is connected with the form-elements and the tissues. The question how this oxidation in the form-elements pro. ceeds and how it is induced cannot be answered with certainty.

In conformity with the views of PFLÜGER and others, it is often asserted that the albumin outside of the organism, and also that which circulates in the blood and fluids, is to be regarded as "dead albumin," as distinguished from that which is converted by the work of the living, active cell into living protoplasm-" living albumin." The statement has also been made that this living protoplasm albumin is differentiated from the "dead albumin" by a greater mobility of the atoms within the molecule, and it may be characterized by a greater inclination towards intramolecular changes of position of these atoms. The reason for this greater inner movement PFLÜGER ascribes to the presence of certain groups, such as cyanogen, while LoEw attributes it to the presence of aldehydic groups in the albumen molecule.

In these differences between ordinary albumins and living protoplasm albumin PFLÜGER sees the reason for the animal oxidation processes which show certain similarity to the oxidation of phosphorus in air containing oxygen. In the last-mentioned process the phosphorus is not only itself oxidized, but, as it splits the oxygen molecule and sets free oxygen atoms (active oxygen), it may also have at the same time an indirect or secondary oxidizing action upon other bodies present. In an analogous way the living protoplasm albumin, which is not, like the dead albumin, indifferent to neutral oxygen, can disintegrate the oxygen molecule, thus becoming itself oxidized, and at the same time the setting free of oxygen atoms may cause a secondary oxidation of other less oxidizable substances.

Another very widely-diffused view exists in regard to the origin of the activity of the oxygen, so that by the decomposition processes in the tissues reducing substances are formed which split the neutral oxygen molecule, uniting with one oxygen atom and setting the other free.

The formation of reducing substances by fermentation and putrefaction is generally known. The butyric fermentation of sugar in which hydrogen is set free $-\mathrm{C}_{6} \mathrm{H}_{12} \mathrm{O}_{6}=\mathrm{C}_{4} \mathrm{H}_{8} \mathrm{O}_{2}+2 \mathrm{CO}_{2}$ $+2\left(\mathrm{H}_{2}\right)$-is an example of this kind. Another example is the 
appearance of nitrates in consequence of an oxidation of nitrogen in cases of putrefaction, which process is ordinarily explained by the statement that, in putrefaction, reducing, easily-oxidizable bodies are formed which split oxygen molecules, liberating oxygen atoms which afterward oxidize the nitrogen. It is assumed also that the cells of the animal tissues and organs, like these lower organisms, which cause fermentation and putrefaction, undergo such splitting processes in which easily-oxidizable substances, perhaps also hydrogen in statu nascendi (HOPPE-SEYLER), are produced. The observations of EHRLICH, that certain blue coloring mattersalizarin blue and indophenol blue-are decolorized by the tissues of the living animal and become blue again on exposure to air, seem also to be a proof of the occurrence of easily-oxidizable combinations in the tissues. A further proof of this is found in the observations of C. Ludwig and Alex. Schmidt that in the blood of asphyxiated animals, as well as in the absence of oxygen, an accumulation of reducing, easily-oxidizing substances takes place.

In accordance with what has been above stated, we may assume that the oxidation in the animal body takes place in the following manner: The forces peculiar to protoplasm, unknown to us, but acting similarly to heat, increase the intramolecular movements of the atoms in such a. way that a loosening or splitting of the molecule occurs and an aggregation of the oxygen is made possible ("primary oxidation," NASSE). The new products formed in this manner may perhaps in part be in direct combination with neutral oxygen ("direct oxidation," NASSE) and gradually burned within the body, but they must probably first undergo a further splitting, and then succumb to consecutive oxidation, until, after repeated splitting and oxidation, the final products of the exchange of material are formed. Finally, the easily-oxidizable products of decomposition, when they split the oxygen molecule and only combine with one of the oxygen atoms, may act on difficultlyoxidizable substances in an indirect or, as NASsE has called it, a "secondary oxidation" by the setting free of the second atom.

Thus the oxidation within the animal body is caused by the action of forces acting similarly to heat, which loosens or splits the molecules; and since this oxidation has long been known as combustion, this view is easily reconcilable with the mode of action

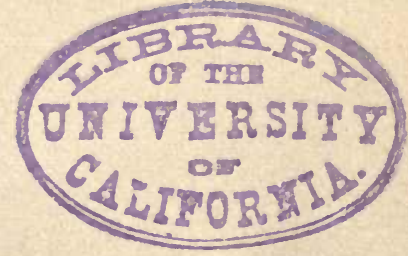


just described. In combustion in the ordinary sense, as, for example, the burning of wood or oil, we must not forget that the substances themselves do not combine with oxygen. It is only after the action of heat has decomposed these bodies to a certain degree that the oxidation of the products of such decomposition takes place and is accompanied by the phenomenon of light.

The numerous intermediary products of decomposition which we observe in the animal body teach us that the oxidations and splittings of the components of the body do not take place at once and suddenly, but only very gradually, step by step, until the final products of exchange are reached.

A very instructive example, of such a gradual decomposition outside of the organism has been shown by DRECHSEL in his investigation on the electrolysis of phenol by an alternating current. By experiments with alternating electric currents we obtain, of course, in the watery solution of the substance, at each electrode alternately, oxygen and hydrogen in great rapidity. Therefore oxidations and reductions must take place alternately, and we obtain syntheses as well as splittings with oxidations.

If phenol in watery solution is treated with such an alternating current, we produce, by the combined action of reduction and oxidation processes, where all double linking in the benzol ring is broken by the aggregation of hydrogen atoms with simultaneous solution, and followed by an oxidation with the elimination of hydrogen atoms, a new body, hydro-phenoketon, $\mathrm{C}_{6} \mathrm{H}_{10} \mathrm{O}$, or<smiles>COC1CCCC1C</smiles>

From the hydro-phenoketon a compound of the

fatty series is produced by the fixation of $\mathrm{O}+2 \mathrm{H}$ accompanied with the splitting of the benzol ring, namely, normal caproic acid, $\mathrm{C}_{6} \mathrm{H}_{12} \mathrm{O}_{2}$, or $\mathrm{H}_{2}^{\mathrm{H}} \mathrm{C} \mathrm{CH}_{2}^{\mathrm{COOH}}$. By further electrolysis of the caproic acid, with the removal of carbon as carbon dioxide and of 
hydrogen as water, a series of acids with decreasing amounts of carbon are obtained, and in this way we may, by properly directed combination of reductions and oxidations, pass from a body of the aromatic series to a body of the fatty series, and then to substances in which the amount of carbon decreases, until the final products of animal exchange are reached.

That reduction processes occur in the organism has already been stated, and in the following pages special examples of these are given. As Drechsel has also found that the same electro-syntheses (of urea and phenol-sulphuric acid) are produced by the continuous as well as by the alternating current, and since the occurrence of galvanic currents in the body has been positively shown, Drechsel concludes that not only do syntheses, but also the combustion of foods and constituents of the tissues, take place in the animal body in consequence of a quick succession of reductions and oxidations.

Another attempt to explain the animal oxidations has been made by M. Traube. Traube has brought forward powerful arguments against the view that an activity of the oxygen is caused by reducible substances, and he seems to believe that within the organism so-called oxygen transmitters occur which act similarly to nitric oxide in the manufacture of sulphuric acid where oxidation is the result of the absorption and liberation of oxygen by other substances which are themselves not directly oxidized by neutral oxygen. The presence of such bodies in the animal organism has not thus far been proved.

An important source of the vis viva developed in the body is to be sought for in the oxidation derived from oxygen of strong potential energy, but also in the splitting processes ; where more complicated chemical compounds are reduced to simpler ones, and when, therefore, the atoms change from a movable equilibrium to a stabler one and stronger chemical affinities are satisfied, the chemical potential energy is transformed into vis viva (or living energy). The best-known example of such a splitting process outside of the animal organism is the ordinary alcoholic fermentation of sugar, $\mathrm{C}_{6} \mathrm{H}_{12} \mathrm{O}_{6}=2 \mathrm{CO}_{2}+2 \mathrm{C}_{2} \mathrm{H}_{6} \mathrm{O}$, in which process heat is set free. The animal body may also have a source of vis viva in the splitting processes which are not dependent on the presence of free oxygen. 
The processes taking place in the living muscle yields an example of this kind. A removed muscle, which gives no oxygen when in a vacuum, may, as Hermans has shown, work, at least for a time, in an atmosphere devoid of oxygen, and give off carbon dioxide at the same time.

We call processes of splitting which are accompanied by a decomposition of water and then a taking up of its constituents $\left(\mathrm{H}_{3}\right.$ and 0) hydrolytic splittings. These splittings, which play an important rôle within the animal body, and which are most frequently met with in the process of digestion, are, for example, the transformation of starch into sugar and the splitting of neutral fats into the corresponding fatty acid and glycerin.

$$
\left.\underset{\text { Tristearin. }}{\mathrm{C}_{3} \mathrm{H}_{6}\left(\mathrm{C}_{18} \mathrm{H}_{35} \mathrm{O}_{2}\right)_{3}}+3 \mathrm{H}_{2} \mathrm{O}=\underset{\text { Glycerin. }}{\mathrm{C}_{3} \mathrm{H}_{6}(\mathrm{OH})_{3}}+\underset{\text { Stearic acid. }}{3\left(\mathrm{C}_{18} \mathrm{H}_{38} \mathrm{O}_{2}\right.}\right) \text {. }
$$

As a rule the hydrolytic splitting processes as they occur in the animal body may be performed outside of it by means of higher temperatures with or without the simultaneous action of acids or alkalies. Considering the two above-mentioned examples, we know that starch is converted into sugar when it is boiled with dilute acids, and also that the fats are split into fatty acids and glycerin on heating them with caustic alkalies or by the action of superheated steam. The heat of the chemical reagents which are used for the performance of these reactions would cause immediate death if applied to the living system. Consequently the animal organism must have other means at its disposal which will act similarly, but in such a manner that they may work without endangering the life or normal constitution of the tissues. Such means have been recognized in the so-called formless ferments or enzymes.

Alcoholic fermentation, as well as other processes of fermentation and putrefaction, is dependent upon the presence of living organisms, ferment fungi and splitting fungi of different kinds; and according to the researches of PASTEUR, these processes are to be considered as phases of life of these organisms. The name organized ferments or ferments has been given to such micro-organisms of which ordinary yeast is an example. However, the same name has also been given to certain bodies or mixtures of bodies of unknown 
organic origin which are products of the chemical work within the cell, and which, after they are separated from the cell, are capable in the smallest quantities of causing a decomposition or splitting in very considerable quantities of other substances without entering into combination with the decomposed body or with any of its products of splitting or decomposition. Such ferments are, for example, the diastase of malt and the ferments secreted by the different glands participating in the process of digestion. These non-organized or formless ferments are generally called, according to KüHNE, enzymes.

A ferment in a more restricted sense is therefore a living being, while an enzyme is a product of chemical processes in the cell, a product which has an individuality even without the cell, and which may be active when separated from the cell. The splitting of glucose or invert-sugar into carbon dioxide and alcohol by fermentation is a fermentative process closely connected with the life of the yeast. The inversion of cane-sugar is, on the contrary, an enzymotic process caused by one of the bodies or mixture of bodies formed by the living ferment, which can be severed from this ferment, and still remains active even after the death of the latter. Consequently ferments and enzymes are capable of manifesting a different behavior towards certain chemical reagents. Thus there exist a number of substances, among which we may mention arsenious acid, phenol, salicylic acid, boracic acid, chloroform, ether, and others, which in certain concentration kill ferments, but which do not noticeably impair the action of the enzymes.

It is doubtful, indeed highly improbable, whether it has been possible up to the present time to isolate any enzyme in a pure state. Therefore the nature of the enzymes and their elementary composition is unknown. Such as have been obtained thus far appear to be nitrogenized and to be similar in some degree to albuminous bodies. They may be extracted from the tissues by means of water or glycerin, especially by the latter, which forms very stable solutions and which, consequently, serves as a means of extraction of the enzymes. The enzymes, generally speaking, do not appear to be diffusible, they decompose hydrogen peroxide and are precipitated with other substances when these are in a finely-divided state. This property has also often been taken advantage of in the prepa- 
ration of pure enzymes. The continued heating of their solutions above $+80^{\circ} \mathrm{C}$. generally destroys most of the enzymes. In the dry state, however, certain enzymes may be heated to $100^{\circ}$ or indeed to $150^{\circ}-160^{\circ} \mathrm{C}$. without losing their power. The enzymes are precipitated from their solutions by alcohol.

We have no characteristic reactions for the enzymes in general, and each enzyme is characterized by its specific action and by the conditions under which it develops. But it must be stated that, however the different enzymes may vary in action, they all seem to have this in common, that by their presence an impulse is given to split more complicated combinations into simpler ones, whereby the atoms arrange themselves from an unstable equilibrium into a more stable one, chemical tension is transformed into living force, and new products are formed with lower heat of combustion than the original substance. The presence of water seems to be a necessary factor in the perfection of such decompositions, and the chemical process seems to consist in the taking up of the elements of water. The manner in which these enzymes work is still enveloped in darkness, but their action may be considered as very closely related to the so-called catalytic or contact action.

As above stated, the enzymes are of great importance for the chemical processes going on in the digestive tract, but we have to add that the results of their action is greatly complicated by processes of putrefaction which take place in the intestines at the same time, and which are caused by micro-organisms. Micro-organisms are physiological constituents of the contents of the intestinal canal, and it is therefore to be supposed that also lower organisms or their germs are to be encountered in the animal tissues and fluids generally, under normal conditions. The question is still unsettled as to how far this is the case. But as yet no positive or decisive proof for the justification of such a statement has been furnished. The lower organisms, on the contrary, when they enter into the animal fluids or tissues and develop and increase, are of the greatest pathological importance, and modern bacteriology, founded by KocH and PAsteur, in relation to the doctrine of infectious diseases, gives efficient testimony to these facts.

Putrefaction caused within the animal fluids and tissues by the lower organisms may produce, among others, combinations of a 
basic nature. Such bodies were first found by Selmi in human cadavers, and called by him cadaver alkaloids or ptomaines. These ptomaines, which have been studied by a great number of investigators, especially by Selmi, Brieger, and GaUtier, must be considered as products of chemical processes caused by putrefaction microbes. Some of these ptomaines are exceedingly poisonous, and consequently BRIEGER has called them toxines.

The formation of such poisonous products in the decompositions caused by putrefactive microbes makes it probable that the lower organisms acting in infectious diseases also produce poisonous substances which may cause by their action the symptoms or complieations of the disease. BRIEGER, who has become prominent by his study of this subject, has been able to isolate from typhus cultures a substance called typhotoxin which has a poisonous action on animals; and he has also prepared another substance, tetanin, from the amputated arm of a patient with tetanus, animals inoculated with which die exhibiting symptoms of developed tetanus. Admitting that the results obtained thus far upon this subject are not very numerous, we cannot refrain from stating that the facts already found open a promising field for further labor and research.

As above stated, the chemical processes in animals and plants do not stand in opposition to each other; they offer differences indeed, but still they are of the same kind from a qualitative standpoint.

PFLÜGER says that there exists a blood-relationship between all living cells of the animal and vegetable kingdoms, and that they originate from the same root; and if the organisms consisting of one cell can decompose protein substances in such a manner as to produce poisonous substances, why should not the animal body, which is only a collection of cells, be able to produce under physiological conditions similar poisonous substances? The poisonous secretions of toads, serpents, and numerous other animals prove, in fact, that the animal body has this power. Frequent efforts have been made of late to prove that the human organism under physiological conditions produces poisonous substances. In the human saliva (GAUTIER and others), and especially in the urine (Pouchet, Bouchard, and others), but also in the expired air (BROWN-SEQUARD and D'ARSONVAL), poisonous organic substances have been proved to exist. The correctness of many of these statements is contradicted 
by other investigators, and the interesting question as to the poison. ous nature of the human physiological secretions and excretions seens to require further proof.

The so-called teucomaines command a special interest. Those substances of basic nature which are incessantly and regularly produced as products of the decomposition of the protein substances of the organism, and which therefore are to be considered as products of the physiological exchange of substance, have been called leucomaines by GAUTIER in contradistinction to the ptomaines produced by micro-organisms. These substances, with the exception of a few which were known in animal extractives, were first found by GAUTIER in animal tissues and muscles, and among these he finds some which are poisonous in small doses. The leucomaines of late are considered of special importance as originators of disease. It has been contended that when these bodies accumulate, on account of an incomplete excretion or oxidation in the system, an autointoxication may be produced (BovcHaRD). Though this view is not by any means based upon generally-recognized facts, still it offers an interesting starting-point for physiological and pathological chemical research. 


\section{CHAPTER II.}

\section{THE PROTEIN SUBSTANCES.}

THE chief mass of the organic constituents of animal tissues consists of amorphous, nitrogenized, complex bodies of high molecular weight. These bodies, which are either albuminous in a special sense or bodies nearly related thereto, take first rank among the organic constituents of the animal body on account of their great abundance. For this reason they are classed together in a special group which has received the name protein group (from $\pi \rho \omega \tau \epsilon v i c$, I am the first or take the first place). The bodies belonging to these several groups are called protein substances, although in a few cases the albuminous bodies in a special sense are designated by the same name.

The several protein substances contain carbon, hydrogen, nitrogen, and oxygen. They generally contain also sulphur, a few phosphorus, and a few also iron. Copper has been found in some few cases. On heating the protein substances they gradually decompose, producing inflammable gases, ammoniacal compounds, carbon dioxide, water, nitrogenized bases, as well as many other bodies, and at the same time they emit a strong odor of burnt horn or wool. More highly heated they leave a porous, shining mass of carbon, and when this is thoroughly burnt an ash is obtained consisting chiefly of calcium and magnesium phosphates. The question whether the mineral bodies left by burning exist as impurities or whether they are constituents of the protein molecule has not been decided.

It is at present impossible to decide on a classification of the protein substances based upon their properties, reactions, and constitution, as well as upon their solubilities and precipitations, corresponding to the demands of science. The best classification is perhaps the following systematic summary of the better known 
and studied animal protein substances, due mainly to HoPPsSEYLER and Drechsel.

\section{Albuminous Bodies.}

Albumins..... $\left\{\begin{array}{l}\text { Serum albumin, } \\ \text { Ovalbumin, } \\ \text { Lactalbumin. }\end{array}\right.$
Globulins...... $\left\{\begin{array}{l}\text { Serum globulin, } \\ \text { Fibrinogen, } \\ \text { Myosin, } \\ \text { Musculin, } \\ \text { Vitellin (?). }\end{array}\right.$

Nucleoalbumins $\left\{\begin{array}{l}\text { Casein, } \\ \text { Ovovitellin (?), } \\ \text { Pyin, and others. }\end{array}\right.$

Albuminates.... $\left\{\begin{array}{l}\text { Acid albuminate, } \\ \text { Alkali albuminate. }\end{array}\right.$

Albumoses and Peptones.

Coagulated...... $\left\{\begin{array}{l}\text { Fibrin, } \\ \text { Albumin coagulated by heat, and others. }\end{array}\right.$

\section{Proteids.}

Mucine....... $\left\{\begin{array}{l}\text { Pure Mucin, } \\ \text { Mucoide or Mucinoide. }\end{array}\right.$

(Hyalogen.)

Hæmoglobin.

\section{.II. Albumoides or Albuminoides.}

Keratine.

Elastin.

Collagen.

(Amyloid.)

(Fibroin, Sericin, Cornein, Spongin, Conchiolin, Byssus, and others.)

To this summary must be added that we often find in the investigations of animal fluids and tissues protein substances which 
do not coincide with the above scheme or do so only with difficulty. At the same time it must be remarked that bodies will be found which seem to rank between the different groups.

\section{Native Albumins.}

The albuminous bodies are never-failing constituents of the animal and regetable organisms. They are especially found in the animal body, where they form the solid constituents of the muscles, glands, and the blood serum, and they are so generally distributed that there are only a few animal secretions and excretions, such as the tears, perspiration, and perhaps urine, in which they are entirely absent or only occur as traces.

All albuminous bodies contain carbon, hydrogen, nitrogen, oxygen, and sulphur; a few contain also phosphorus. Iron is generally found in traces in their ash, and it seems to be a regular constituent of a certain group of the albuminous bodies, namely, the nucleo-albumin group. The composition of the different albuminous bodies deviates a little, but the variations are within relatively close limits. For the better studied animal albuminous bodies the following conposition of the ash-free substance has been given:

$$
\begin{aligned}
& \text { C. . . . } 50.6-54.5 \text { per cent. } \\
& \mathrm{H} \text {. . . . . } 6.5-7.3 \text { " } \\
& \mathrm{N} \cdot . \cdot \cdot 15.0-17.6 \text {. } 6 \\
& \mathrm{~S} \cdot . \cdot . \cdot 0.8 \rightarrow 2.2 \text {. } 0 \\
& \mathrm{P} \text {. . . . } 0.42-0.85 \text { " } \\
& \text { O . . . . } 21.50-23.50 \text { “ }
\end{aligned}
$$

A part of the nitrogen of the albumin molecule is loosely combined and splits off easily as ammonia by the action of alkalies (NASSE). Sulphur shows the same property in nearly all albuminous bodies (Fleitmann, Danilewsky, KrüGer). A part of the sulphur separates as potassium or sodium sulphide on boiling with caustic potash or soda, and may be detected by lead acetate. What remains can only be detected after fusing with nitre and sodium carbonate and testing for sulphates. The albumin molecule therefore contains at least 2 atoms of sulphur. The molecular 
weight of the albumins has not been determined, therefore it is impossible to give formulæ. For the alkali albuminate, in whose formation from native albumins a part of the nitrogen and the loosely-bound sulphur is split off, LIEBERKüHN has given the formula $\mathrm{C}_{72} \mathrm{H}_{112} \mathrm{~N}_{18} \mathrm{SO}_{22}$.

The constitution of the albuminous bodies, notwithstanding numerous investigations, is still unknown. By heating albumin with barium hydrate and water in sealed tubes at $150^{\circ}-200^{\circ} \mathrm{C}$. for several days, ScHÜTZENBERGER obtained a number of products among which were ammonia, carbon dioxide, oxalic acid, acetic acid, and, as chief product, a mixture of amido-acids. This mixture contained, besides a little tyrosin and a few other bodies, chiefly acids of the series $\mathrm{C}_{n} \mathrm{H}_{2 n+1} \mathrm{NO}_{2}$ (leucines) and $\mathrm{C}_{n} \mathrm{H}_{2 n-1} \mathrm{NO}_{2}$ (leuceines). The sulphur of the albumins yields sulphites. The three bodies, carbon dioxide, oxalic acid, and ammonia, are formed in the same relative proportion as in the decomposition of urea and oxamid; therefore SCH ÜTZENBERGER suggests that perhaps albumin may be considered as a very complex ureid or oxamid. Such a conclusion cannot be derived from the above decomposition processes for several reasons, and the attempts to prepare urea directly by splitting albumin by means of trypsin, or by oxidation, have given negative, or at least not positive, results.

On fusing albumin with caustic alkali, ammonia and other volatile products are generated; among these leucin, from which volatile fatty acids, such as acetic acid, valerianic acid, and also butyric acid, are formed; also tyrosin, from which phenol, indol, and skatol are produced. The majority of these products are found as a result of putrefaction (see Chap. VII). On boiling with mineral acids, or still better by boiling with hydrochloric acid and zinc chloride (HLASIWETZ and HABERMANN), the albumins yields amido-acids, such as leucin, aspartic acid, glutamic acid, and tyrosin (and from vegetable albumin SCHULZE and BARBIERI obtained $\alpha$-phenylamido-propionic acid), also sulphuretted hydrogen, ammonia, and nitrogenized bases (DRECHSEL). As an essential difference between the action of acids and alkalies (barium hydrate) on albumins, DRECHSEL suggests that by the action of acids carbon dioxide, oxalic and acetic acids are not produced.

By the putrefaction of albumins, as well as by decomposition 
by means of acids or alkalies, and also by certain enzymes, among other products amido-acids are produced, and these have a certain significance for the probable formation of the albumins. It is more than likely that in the synthesis of albumin in the plant from the ammonia or the nitric acid of the soil, amido-acids or acid amids, among which asparagin plays an important rôle, are produced; and from these the albuminous bodies are derived by the influence of glucose or other non-nitrogenized combinations.

By the oxidation of albumins in acid solutions, volatile fatty acids, their aldehydes, nitriles, ketones, also benzoic acid are obtained, also hydrocyanic acid by oxidizing with potassium dichromate and acid. Nitric acid gives various nitro-products, such as xanthoproteic acid (VAN DEE PANTs), trinitroalbumin (LOEW) or oxynitroalbumin, nitrobenzoic acid, and others. With aqua regia fumaric acid, oxalic acid, chlorazol, and other bodies are produced. By the action of bromine under stronger pressure a large number of derivatives are obtained, such as bromanil and tribromacetic acid, bromoform, leucin, leucinimid, oxalic acid, tribromamido-benzoic acid, peptone, and bodies similar to humus.

By the dry distillation of albumins we obtain a large number of decomposition products of a disagreeable burnt odor, and a porous glistening mass of carbon containing nitrogen is left as a residue. The products of distillation are partly an alkaline liquid which contains ammonium carbonate and acetate, ammonium sulphide, ammonium cyanide, an inflammable oil and other bodies, and a brown oil which contains hydrocarbons, nitrogenized bases belonging to the aniline and pyridine series, and a number of unknown substances.

It is impossible here to discuss all the products obtained by the action of different reagents on the albumins, but from the abovedescribed bodies from the decomposition of albumin it is clear that the products belong in part to the fatty and in part to the aromatic series. Even though the constitution of the albumins has as yet not been successfully demonstrated, it seems to be a fact from the above that in the albumin molecule we have, besides the atomic arrangement belonging to the fatty series, at least one aromatic group present.

By the oxidation of albumin by means of potassium permanganate, MALY obtained an acid, the oxyprotosulphonic acid, C 51.21; H $6.89 ; \mathrm{N} 14.59$; S $1.77 ; 025.54$, which is not a product of splitting, but an oxidation product in which the group $\mathrm{SH}$ is changed into $\mathrm{SO}_{2} . \mathrm{OH}$. This acid does not give the proper color reaction with MrLLoN's reagent (see below), nor does it yield the ordinary aromatic splitting products of the albumins. Still the aromatic group is not absent, but it seems to be in another binding from that in ordinary albumin. On oxidizing with potassium dichromate and acid this group appears as benzoic acid, and on fusing with alkali, benzol is given off.

The animal albuminous bodies are odorless and tasteless, ordinarily amorphous. The crystalloid (DOTTERPL ̈̈TTCHEN) occurring 
in the eggs of certain fishes and amphibians does not consist of pure albumin, but of an albumin containing large amounts of lecithin which seems to be combined with mineral substances. Crystalline combinations of albumin with mineral substances have been prepared from seeds of various plants, and lately crystallized animal albumin in combination with salts has been prepared (HofMeIster). In the dry condition the albuminious bodies appear as a white powder, or when in thin layers as yellowish, hard, transparent plates. A few are soluble in water, others only soluble in salty or faintly alkaline, or acid solutions while others are insoluble in these solvents. All albuminous bodies when burnt leave an ash, and it is therefore questionable whether there exists an albuminous body which is soluble in water without the aid of mineral substances. Nevertheless it has not been thus far successfully proved that a native albuminous body can be prepared perfectly free from mineral substances without changing its constitution or its properties. The albuminous bodies are in most cases strong colloids. They diffuse, if at all, only very slightly through animal membranes or parchment-paper, and the albumins have generally a very high osmotic equivalent. All albuminous bodies are optically active and turn the ray of polarized light to the left.

On heating a solution of albumin to the temperatures depending on the albumin present, and with the proper reactions and in favorable external conditions, - as, for example, in the presence of neutral salts,-most albuminous bodies separate in the solid state as a crude or "coagulated" albumin. The different temperatures at which the various albuminous bodies coagulate in neutral, salty solutions give in many cases a good means for detecting and separating these bodies.

The general reactions for the albuminous bodies are numerous, but only the most important will be given here. To facilitate the study of these they have been divided into the two following groups.

\section{A. Precipitation Reactions of the Albuminous Bodies.}

1. Coagulation Test. An alkaline albumin solution does not coagulate on boiling, a neutral solution only partly and incompletely, and the reaction must therefore be acid for coagulation. 'The 
neutral liquid is first boiled and then the proper amount of acid added carefully. A flocculent precipitate is formed, and if properly done the filtrate should be water-clear. If dilute acetic acid be used for this test, the liquid must first be boiled and then 1,2, or 3 drops of acid added, depending on the amount of albumin present, and boiled before the addition of each drop. If dilute nitric acid be used, then to 10-15 c.c. of the previously-boiled liquid 15-20 drops of the acid must be added. If too little nitric acid be added a soluble combination of the acid and albumin is formed which is precipitated by more acid. An albumin solution containing a small amount of salts must first be treated with about $1 \% \mathrm{NaCl}$, since the heating test may fail, especially on using acetic acid, in the presence of only a slight amount of albumin. 2. Behavior towards Mineral Acids at Ordinary Temperatures. The albumins are precipitated by the three ordinary mineral acids and by metaphosphoric acid, but not by orthophosphoric acid. If nitric acid be placed in a reagent glass and the albumin solution be allowed to flow gently thereon, a white, opaque ring of precipitated albumin will form where the two liquids meet (HELLER's albumin test). 3. Precipitation by Metallic Salts. Copper sulphate, neutral and basic lead acetate (in small amounts), mercuric chloride, and other salts precipitate albumin. On this is based the use of albumins as antidotes in poisoning by metallic salts. 4. Precipitation by Ferro- or Ferricyanide of Potassium in Acetic Acid Solution. In these tests the relative quantities of reagent, albumin, or acid do not interfere with the delicacy of the test. 5. Precipitation by Neutral Salts, such as $\mathrm{Na}_{2} \mathrm{SO}_{4}$ or $\mathrm{NaCl}$, when added to saturation to the liquid acidified with acetic acid or hydrochloric acid. 6. Precipitation by Alcohol. The solution must not be alkaline, but must be either neutral or faintly acid. It must, at the same time, contain a sufficient quantity of neutral salts. \% Precipitation by Tannic Acid in acetic-acid solutions. The absence of neutral salts or the presence of free mineral acids may not cause the precipitate to appear, but after the addition of a sufficient quantity of sodium acetate the precipitate will in both cases appear. 8. Precipitation by Phospho-tungstic or Phospho-molybdic Acids in the presence of free mineral acids. Potassium-mercuric iodide and potassium-bismuth iodide precipitate albumin solutions acidified 
with hydrochloric acid. 9. Precipitation by Picric Acid in solutions acidified by organic acids.

\section{B. Color Reactions for Albuminous Bodies.}

1. Millon's reaction. ${ }^{1}$ A solution of mercurie nitrate in nitric acid containing some nitrous acid gives a precipitate in albumin solutions which at the ordinary temperature is slowly, but at the boiling-point more quickly, colored red; and the solution may also be colored a feeble or bright red, depending on the amount of albumin. Solid albuminous bodies, when treated by this reagent, give the same coloration. This reaction, which depends on the presence of the aromatic group in the albumin, is also given by tyrosin and other benzol derivatives, with a hydroxyl group in the benzol nucleus. 2. Xanthoproteic reaction. With strong nitric acid the albuminous bodies give, on heating to boiling, yellow flakes or a yellow solution. After saturating with ammonia or alkalies the color becomes orangeyellow. 3. Adamkiewicz' reaction. If a little albumin is added to a mixture of 1 vol. concentrated sulphuric acid and 2 vols. glacial acetic acid a reddish-violet color is obtained slowly at ordinary temperatures, but more quickly on heating. Gelatine does not give this reaction. 4. Biuret test. If an albumin solution be first treated with caustic potash or soda and then a dilute copper sulphate solution be added drop by drop, first a reddish, then a reddish-violet, and, lastly, a violet-blue color is obtained. 5. Albumin is soluble on heating with concentrated hydrochloric acid, producing a violet color, and when the albumin is first boiled with alcohol and then washed with ether (LIEBERMaNN) it gives a beautiful blue solution. 6. With concentrated sulphuric acid and sugar (in small quantities) the albuminous bodies give a beautiful red coloration. These color reactions apply to all albuminous bodies.

The delicacy of the same albumin reagent differs for the differ-

${ }_{1}^{1}$ The reagent is obtained in the following way : $1 \mathrm{pt}$. mercury is dissolved in 2 pts. of nitric acid (of sp. gr. 1.42), first when cold and later by warming. After complete solution of the mercury, add 1 volume of the solution to 2 volumes of water. Allow this to stand a few hours and decant the super natant liquid. 
ent albuminous bodies, and it is, on this account, impossible to give the degree of delicacy for each reaction for all albuminous bodies. Of the precipitation reactions HeLLer's test (if we eliminate the peptones and certain albumoses) is recommended in the first place for its delicacy, though it is not the most delicate reaction, and because it can be performed so easily. Among the precipitation reactions, that with basic lead acetate (when carefully and exactly executed) and the reactions $6,7,8$, and 9 are the most delicate. The color reactions 1 to 4 show a great delicacy in the order in which they are given.

No albumin reaction is in itself characteristic, and, therefore, in testing for albumin one reaction is not sufficient, but a number of precipitation and color reactions must be employed.

For the quantitative estimation of coagulable albumin the determination by boiling with acetic acid can be performed with advantage since, by operating carefully, it gives exact results. The precipitation by means of alcohol in the liquid which has first been neutralized may also be used for this purpose. The alcohol must be added so that the liquid contains 70 to 80 vols. per cent. In both cases small amounts of albumin may remain in the filtrate. This last may be determined by concentrating the filtrate sufficiently (in the alcohol method all the alcohol must be expelled), and removing any separated fat by shaking with ether, and then precipitating with tannic acid, with the addition of $\mathrm{NaCl}$ if necessary. Approximately $63 \%$ of the tannic acid precipitate washed with cold water and dried may be considered as albumen. The precipitation by means of copper sulphate may also be employed as a quantitative method, while the Biuret reaction may be used for the quantitative calorimetric estimation of peptones and albumoses. The quantitative estimation of albumin by means of the polariscope is not applicable in all cases and does not give sufficiently exact results.

The separation of albumins from a solution may, in most cases, be performed by boiling with acetic acid. Small amounts of albumin which remain in the filtrates may be separated by boiling with freshly-precipitated lead carbonate (HOFMEISTER) or with ferric acetate (HOPPE-SEYLER), as described in Chapter XIV on the urine. If the liquid cannot be boiled, the albumin may be precipitated by neutral salts and acid, or by the very careful addition of lead acetate, or by the addition of alcohol. If the liquid contains substances which are precipitated by alcohol, such as glycogen, then the albumin may be separated by the alternate addition of potassium-mercuric iodide and hydrochloric acid (BRÜCKE). 


\section{Synopsis of the Most Important Properties of the Different Chief Groups of Albuminous Bodies.}

Albumins. These bodies are insoluble in water and are not precipitated by the addition of a little acid or alkali. They are precipitated by the addition of large quantities of mineral acids or metallic salts. Their solution in water coagulates on boiling in the presence of neutral salts, but a weak saline solution does not. If $\mathrm{NaCl}$ or $\mathrm{MgSO}_{4}$ is added to saturation to a neutral solution in water at a normal temperature or at $+30^{\circ} \mathrm{C}$. no precipitate is formed; but if acetic acid is added to this saturated solution the albumin readily separates. When ammonium sulphate is added in substance to saturation to an albumin solution a complete precipitation occurs at ordinary temperature. Of all the albuminous bodies the albumins are the richest in sulphur, containing from 1.6 to $2.2 \%$.

Globulins. These albuminous bodies are insoluble in water, but dissolve in dilute neutral salt solutions. The globulins are precipitated unchanged from these solutions by sufficient dilution with water; and on heating they coagulate. The globulins dissolve in water on the addition of very little acid or alkali, and on neutralizing the solvent they reprecipitate.

The solution in a minimum amount of alkali is precipitated by carbon dioxide, but the precipitate may be redissolved by an excess of the precipitant. The neutral solutions of the globulins containing salts are partly or completely precipitated on saturation with $\mathrm{NaCl}$ or $\mathrm{MgSO}_{4}$ in substance at normal temperatures. The globulins contain an average amount of sulphur, not below $1 \%$.

A sharp line between the globulins on one side and the artificial albuminates on the other can hardly be drawn. The albuminates are, indeed, as a rule insoluble in dilute common-salt solutions; but an albuminate may be prepared by the action of strong alkali which is soluble in common-salt solutions immediately after precipitation. We also have globuiins which are insoluble in $\mathrm{NaCl}$ after having been in contact with water for some time.

Nucleoalbumins. These bodies are found widely diffused in both the animal and regetable kingdoms. They form one of the chief constituents of protoplasm, while the albumins and in part also the globulins are special constituents of the animal juices. 
The nucleoalbumins are found in organs abounding in cells, but they also occur in secretions and sometimes in other fluids in apparent solution as destroyed and altered protoplasm. The nucleoalbumins behave like rather strong acids; they are nearly insoluble in water, but dissolve easily with the aid of a little alkali. Such a solution, neutral or, indeed, a faintly acid one, does not coagulate on boiling. The nucleoalbumins resemble the globulins and the albuminates in solubility and precipitation properties, but differ from them in being hardly soluble in neutral salts. The most important difference between the nucleoalbumins, the globulins, and the albuminates is that the nucleoalbumins contain phosphorus, and by the action of pepsin hydrochloric acid on nucleoalbumins a phosphorized product, nuclein, is split off which, according to LIEBERMANN, is a combination of albumin with metaphosphoric acid. The nucleoalbumins seem habitually to contain less sulphur than the bodies of the preceding groups. Some iron is found as a constant constituent.

Alkali and Acid Albuminates. By the action of alkalies all native albuminous bodies are converted, with the elimination of nitrogen or by the action of stronger alkali with the emission of sulphur, into a new modification, called alkali albuminate, whose specific rotation is increased at the same time. If caustic alkali in substance or in strong solution be allowed to act on a concentrated albumin solution, such as blood-serum or egg-albumin, the alkali albuminate may be obtained as a solid which dissolves in water on heating forming a jelly, and which is called "LIEBERKÜHN's solid alkali albuminate." By the action of dilute caustic alkali solutions on dilute albumin solutions we have alkali albuminates, formed slowly at the ordinary temperature, but more rapidly on heating. These solutions may be modified by the source of the albumin acted upon, and also by the extent of the action of the alkali, but it is still always the same reaction.

If albumin is dissolved in an excess of concentrated hydrochloric acid, or if we digest an albumin solution acidified with 1-2 p. m. hydrochloric acid in the warmth, or digest the albumin alone with pepsin hydrochloric acid, we obtain a new modification of albumin which indeed may show somewhat varying properties, but has also certain reactions in common with ordinary albumin. 
These modifications, which may be obtained in a solid gelatinous condition by sufficient concentration, are called acid albuminates or acid albumin, sometimes also syntonin, though we prefer to call that acid albuminate syntonin which is obtained by extracting muscles with hydrochloric acid of $1 \mathrm{p} . \mathrm{m}$.

The alkali and acid albuminates have the following reactions in common: They are nearly insoluble in water and dilute commonsalt solutions (see previous page), but they dissolve easily in water after the addition of a very small quantity of acid or alkali. Such a solution or one nearly neutral does not coagulate on boiling, but is precipitated at the normal temperature on neutralizing the solvent by an alkali or an acid. A solution of an alkali or acid albuminate in acid is easily precipitated on saturating with $\mathrm{NaCl}$, but a solution in alkali is precipitated with difficulty or not at all, according to the amount of alkali it contains. The nearly neutral solutions are precipitated by mineral acids in excess, also by many metallic salts.

Notwithstanding this agreement in the reactions, the acid and alkali albuminates are essentially different, and by dissolving an alkali albuminate in some acid no acid albuminate solution is obtained, nor is an alkali albuminate formed on dissolving an acid albuminate in water by the aid of a little alkali. The alkali albuminates are relatively strong acids. They may be dissolved in water with the addition of $\mathrm{CaCO}_{3}$, with the elimination of $\mathrm{CO}_{2}$, which does not occur with typical acid albuminates, and they show in opposition to the acid albuminates also other variations which stand in connection with their strongly-marked acid nature. Dilute solutions of alkalies act more energetically on albumin than do acids of the same concentration. In the first case a part of the nitrogen, and often also the sulphur, is split off, and from this property we may obtain an alkali albuminate by the action of an alkali upon an acid albuminate; but we cannot obtain an acid albuminate by the reverse reaction. (K. MörNER.)

The preparation of the albuminates has been given above. By the action of alkalies or acids upon an albumin solution the corresponding albuminate may be precipitated by neutralizing with acid or alkali. The washed precipitate is dissolved in water by the aid of a little alkali or acid, and again precipitated by neutralizing the 
solvent. If this precipitate which has been washed in water is treated with alcohol and ether, the albuminate will be obtained in a pure form.

Albumoses and Peptones. Peptones are designated as the final products of the decomposition of albuminous bodies by means of proteolytic enzymes, in so far as these final products are still true albuininous bodies; while we designate as albumoses or propeptones the intermediate products produced in the peptonization of albumins in so far as they are substances not similar to albuminates.

Albumoses and peptones may also be produced by the hydrolytic decomposition of the albumins with acids or alkalies, also by the putrefaction of the same. They may also be formed in very small quantities as by-products in the investigations of animal fluids and tissues, and the question to what extent these exist preformed under physiological conditions requires very careful investigation.

Between the peptones which represent the last splitting products and those albumoses which stand closest to the original albumin we have undoubtedly a series of intermediate products. Under such circumstances it is a difficult problem to try to draw a sharp line between the peptone and the albumose group, and it is just as difficult to define our conception of peptones and albumoses in an exact and satisfactory manner.

The albumoses have been considered as those albuminous bodies whose solutions do not coagulate on boiling and which, to distinguish them from peptones, were characterized chiefly by the following properties. The watery solutions are precipitated at the ordinary temperature by nitric acid as well as by acetic acid and potassium ferrocyanide, and this precipitate has the peculiarity of disappearing on heating and reappearing on cooling. If a solution of albumoses is saturated with $\mathrm{NaCl}$ in substance, the albumoses are partly precipitated in neutral solutions, but on the addition of acid saturated with the salt they completely precipitate. This precipitate, which dissolves on warming, is a combination of albumose with the acid.

We formerly designated as peptone that albuminous body which was readily soluble in water and which did not coagulate by heat, whose solutions were precipitated neither by nitric acid, nor by 
acetic acid and potassium ferrocyanide, nor by neutral salts and acid.

The reactions and properties which the albumoses and peptones had in common were formerly considered as the following: They give all the color reactions of the albumins, but with the biuret test they give a more beautiful red color than the ordinary albumin. They are precipitated by ammoniacal lead acetate, by mercuric chloride, alcohol, tannic, phospho-tungstic, phospho-molybdic acids, potassium-mercuric iodide and hydrochloric acid, and lastly by picric acid. The albumoses and peptones have also a greater diffusive power than native albuminous bodies, and the diffusive power is greater the nearer the questionable substance stands to the final product, the so-called pure peptone.

These old views have undergone an essential change in the last few years. After Hexnsius' observation that ammonium sulphate was a general precipitant for albumin, also peptone in the old sense, KüHNE and his pupils proposed this salt as a means of separating albumoses and peptones. Those products of digestion which separate on saturating their solution with ammonium sulphate are considered by KüHNE and indeed by most of the modern investigators as albumoses, while those which remain in solution are called peptones or pure peptone. This pure peptone is formed in relatively large amounts in the pancreatic digestion, while in the pepsin digestion it is only small in quantity unless after prolonged digestion.

According to SchÜTZENBERGER and KüHNE the albumins yield two chief groups of new albuminous bodies when decomposed by dilute acids or with proteolytic enzymes; of these the anti group shows a greater resistance to further action of the acid and enzyme than the other, namely, the hemi group. Corresponding to these views KÜHNE divides the albumoses into two chief groups, the antialbumoses and hemialbumoses, and the peptones into two chief groups, the antipeptones and the hemipeptones. In the pepsin digestion we obtain, besides different albumoses, a mixture of antiand hemipeptone, which mixture KüHNE called amphopeptone. In the digestion with trypsin (the proteolytic enzyme of the pancreas) the hemipeptone is further split into leucin, tyrosin, and other substances, while the antipeptone remains unchanged. Only 
by the sufficiently energetic action of trypsin is one peptone at last obtained, the so-called antipeptone.

K $\ddot{H N E}$ and his pupils, who have conducted these complete investigations on the albumoses and peptones, classify the different kinds of albumoses according to their different solubilities and precipitation powers. In the pepsin digestion of fibrin they obtained the following albumoses: 1. Dysalbumose, which is insoluble in water and dilute salt solutions. 2. Heteroalbumose, insoluble in water but soluble in salt solution. 3. Protalbumose, soluble in salt solution and water. These three albumoses are precipitated by $\mathrm{NaCl}$ in a neutral solution, while 4, the Deuteroalbumose, which is soluble in salt solution or water, is precipitated (partly) on saturating with $\mathrm{NaCl}$, but only after the addition of an acid. This pre. cipitate is a combination of albumose and acid (HERTH).

HERTH claims that the relative proportion of acid or alkaki, salt, water, or albumose in a solution essentially changes the solubility and precipitation power of the same. He also claims that the occurrence of several different kinds of albumoses cannot be demonstrated, because with one and the same albumose, the above conditions being changed, its solubilities and precipitating powers are changed. HAMBURger found the same to be true from his investigations.

The albumoses obtained from different albuminous bodies do not seem to be identical. The globulinalbumoses of Kt'HNE and CHITtENDEN are called globuloses, the vitellin albumoses of NEUMEIsTER vitelloses, those of casein caseoses (CHITTENDEN), those of myosin myosinoses (KüHNE and CHITTENDEN), and so on. The different kinds of albumoses are distinguished as proto,hetero-, and deutero-caseoses, etc.

NEUMEISTER designates as atmidalbumose that body which is obtained by the action of superheated steam on fibrin. At the same time he also obtained a substance called atmidalbumin which stands between the albuminates and the albumoses.

Of the soluble albumoses NeUMEIsTer designates protoalbumose and heteroalbumose as primary albumoses, while the deuteroalbumose, which is nearly related to the peptones, he calls secondary albumose. As essential difference between the primary and secondary albumoses he suggests the following: The secondary albumoses are not precipitated by nitric acid in liquids free from salt, nor by copper sulphate solution (2 parts in 100), nor by $\mathrm{NaCl}$ in substance in a neutral liquid. Pure true peptones are not precipi. 
tated either by picric acid or by potassium-mercuric iodide and acid. The primary albumoses are completely precipitated by phospho-molybdic or phospho-tungstic acid, while the secondary are not quite completely precipitated, and the true peptones very incompletely. The peptones are also precipitated by mercuric chloride in neutral solutions, and also by tannic acid in liquids containing acetic acid. This precipitate may be dissolved in an excess of tannic acid (SEBELIEN).

The study of the albumoses and peptones, as above indicated, has undergone in the last few years an essential transformation. It may still be doubtful whether the behavior of a single salt, the ammonium sulphate, yields sufficient basis for the characterization of two groups of albuminous bodies, the albumoses and peptones; and this question is warranted since, according to NEUMEISTER, we have a deuteroalbumose (found in the pepsin digestion) which is not completely precipitated by ammonium sulphate. It seems that the transformation of albumins into peptones takes place through a number of intermediate steps such as starch undergoes in passing from dextrine into glucose. A complete separation of these several intermediate products, as well as their purification, is an extremely difficult task.

What relationship do the albumoses and peptones bear to the albumin from which they are formed? HERTH has found that fibrin albumose and fibrin have a similar constitution. KüHNE and Chitttenden, as also Chittenden and his pupils, have analyzed the different albumoses from fibrin, globulin, egg-albumin, myosin, and casein, and found in a few albumoses an increase and in others a decrease in the amount of carbon, nitrogen, and sulphur as compared with the mother-albumin. From the results of their analyses it has been found that, with the probable exception of the peptone standing closest to the albumoses, the difference in the constitution of the original albumins and the corresponding albumose is sometimes in one direction and sometimes in another, and is unessential.

According to the analyses of peptones (in the old sense) made by Maly, Herth, and Henninger, they seem to have the same constitution as the albumin. According to the analyses by KüHNE and ChitTenden of "true" fibrin peptone, part amphopeptone 
and part antipeptone prepared by pancreas infusion, this peptone was found to contain about the same amount of hydrogen and the same or a greater amount of nitrogen, but less carbon than the albumoses. In his investigations on casein CHITTENDEN found, on the other hand, that in antipeptone the amount of carbon was higher than in certain caseoses. As the preparation of true peptones in a pure condition is accompanied with great difficulty, and as the peptones (in the modern sense) analyzed have not always behaved as true peptones towards the peptone reagents as described by NeumeIster, it is most difficult to draw any positive conclusion from these analyses. It seems, nevertheless, that generally the so-called true peptones are perhaps somewhat poorer in carbon than the corresponding albumins.

The elementary analyses made up to the present time have not given us a positive answer in regard to the relationship existing between the albumins on one side and the albumoses and peptones on the other. The view that the peptone formation is a hydrolytic splitting is accepted by HoPPE-SEYLER, KüHNE, HENNINGER, and indeed by recent investigators. In support of this view we have the observations of HENNINGER and HoFMEISTER, according to which peptones are converted into an albumin similar to albuminates by the action of acetic acid anhydride, or by heating so that water is expelled. According to other investigators, as MALY, Herth, LoEw, and others, the formation of peptone is a depolymerization of the albumins. A third view is that albumins and peptones are isomeric bodies; while a fourth view (GRIESSMAYER) claims that the albumins consist of micell groups which on peptonization are first converted into micells and then further into a molecule. Though an ordinary albumin solution contains micells or micell bonds, so also a peptone solution contains an albumin molecule.

The preparation of different albumoses in a complete pure form is very troublesome and accompanied with a great many difficulties. For this reason there will be given here only the general methods by which the different albumose precipitates are obtained. If we proceed from a solution of fibrin in pepsin hydrochloric acid, we first neutralize, heat to boiling, filter, concentrate the filtrate, and saturate it with common salt in substance. The precipitate is filtered, 
washed with a saturated salt solution, and then treated with a 10 per cent $\mathrm{NaCl}$ solution; what remains is called dysalbumose. The filtered solution is repeatedly and completely dialyzed. A part separates which is heteroalbumin, while the protalbumose remains dissolved in the liquid. The above-mentioned filtrate which has had the primary albumoses removed and saturated with common salt is treated with acetic acid which has previously been saturated with salt. The precipitate consists of deuteroalbumose. The resulting filtrate, from which a new precipitate separates on saturation with ammonium sulphate, contains the remainder of the deuteroalbumose and a little peptone.

For the preparation of true peptone we may use a prolonged pepsin digestion, but much quicker results are obtained by the use of the trypsin digestion. The neutralized liquid is heated to boiling, filtered; sufficiently concentrated, and saturated while boiling hot with ammonium sulphate. The albumoses which separate are filtered. If any peptone is present in the cold filtrate, a beautiful biuret reaction is obtained by the addition of very strong caustic soda solution and a little copper sulphate solution, though when the products of pepsin digestion are employed this may depend on non-precipitated deuteroalbumose. The greater part of the ammonium sulphate may be removed from the filtrate by evaporation and crystallization or by partial precipitation by alcohol. What now remains is separated by the addition of barium hydrate, and lastly by barium carbonate with heat. 'The filtrate is concentrated and precipitated by alcohol. In regard to the more detailed accounts of the proposed methods for the preparation of pure peptone and the different albumoses the reader is referred to the works of Kühne, Chittenden, and Neumeister.

The methods for the detection of albumoses and peptones in fluids and tissue are not very accurate, and therefore, as they cannot be given without criticism which does not enter into the scope and plan of this work, they will be omitted.

Coagulated Albuminous Bodies. Albumin may be converted into the coagulated condition by different means: by heating (see page 18), by the action of alcohol, especially in the presence of neutral salts, and in certain cases, as in the conversion of fibrinogen into fibrin (Chapter IV), by the action of an enzyme. The nature of the processes which take place during coagulation is unknown. The coagulated albuminous bodies are insoluble in water, in neutral salt solutions, and in dilute acids or alkalies, at normal temperature. They are dissolved and converted into albuminates by the action of less dilute acids or alkalies, especially on heating. 


\section{Proteid.}

With this name, as suggested by HoPPE-SEYLER, we designate a class of bodies which are more complex than the albuminous bodies and which yield as nearest splitting products albuminous bodies on one side and non-protein bodies, such as coloring matters and carbohydrates, on the other.

The most important substances belonging to this group are the blood-coloring matter, hcomoglotin, which will be thoroughly treated in a following chapter (Chapter IV), and mucin substances or allied bodies.

Mucin Substances. We designate as mucin colloid substances whose solutions are mucilaginous and thready, and which when treated with acetic acid give a precipitate insoluble in an excess of acid, and on boiling with dilute mineral acids yield a substance capable of reducing copper oxyhydrate. This last-mentioned fact, which was first observed by EICHWALD, differentiates mucin from other bodies which have long been mistaken for it and.which have similar physical properties. On the other hand, bodies whose physical properties differ from it but which give a reducible substance on boiling with dilute mineral acids have also been designated as mucin.

The different bodies characterized as mucin substances correspond, first, either to true mucin or, second, to mucoid or mucinoid.

All mucin substances contain carbon, hydrogen, nitrogen, sulphur, and oxygen. Compared with albuminous bodies they contain less nitrogen and, as a rule, less carbon. As close decomposition products they yield albuminous bodies on one side and carbohydrates or acids related thereto on the other. On boiling with dilute mineral acids they all give a reducible substance.

The true mucins are characterized by their natural solution, or one prepared by a trace of alkali, being mucilaginous, thread-like, and giving a precipitate with acetic acid which is insoluble in excess of acid. The mucoids do not show these physical properties and have other solubilities and precipitative properties. As we have intermediate steps between different albuminous bodies, so also we have such between true mucins and mucoids, and a sharp line between these two groups cannot be drawn. 
True mucins are secreted by the larger mucous glands, by certain mucous membranes, also by the skin of snails and other animals. True mucin also occurs in the connective tissue and navel-cord. Sometimes, as in snails and in the membrane of the frog-egg (GIACOSA), a mother-substance of mucin, a mucinogen, has been found, which may be converted into mucin by alkalies. Mucoid substances are found in cartilage, certain cysts, etc. As the mucin question has been very little studied, it is at the present time impossible to give any positive statements in regard to the occurrence of mucins and mucoids, especially as without doubt in many cases non-mucinous substances have been described as mucins. So much is sure, that mucins or nearly-related bodies occur widely diffused in the organism of certain tissues. From their decomposition products we derive a great deal of knowledge in regard to the formation and splitting of carbohydrates or kindred bodies (glycuronic acid) from other complex atoms.

True Mucin. Thus far we have been able to obtain only a few mucins in a pure and unchanged condition due to the reagents used. The elementary analyses of these mucins have given the following results:

\section{$\begin{array}{llllllllll}\mathrm{C} & \mathrm{H} & \mathrm{N} & \mathrm{S} & \mathrm{O}\end{array}$}

$\begin{array}{llllllll}\text { Mucin from snail......... } & 50 & 32 & 6.84 & 13.65 & 1.75 & 27.44 & \text { (Hammarsten.) }\end{array}$

$\begin{array}{lllllll}\text { Mucin from nerve........ } & 48.30 & 6.44 & 11.75 & 0.81 & 32.70 & \text { (LoEBisch.) }\end{array}$

$\begin{array}{lllllll}\text { Mucin from sub-maxillaris } & 48.84 & 6.80 & 12.32 & 0.84 & 31.20 & \text { (Hammarsten.) }\end{array}$

The mucin of the snail-skin, which stands closest to keratin, contains more sulphur than the other mucins. The sulphur is moreover, at least in certain mucins, part in loose and part in strong chemical union.

By the action of superheated steam on mucin a carbohydrate, animal gum (LANDWEHR), is split off. On boiling mucin with dilute mineral acids, acid albuminate and bodies similar to albumose or peptone are obtained, besides a reducing substance which has not been closely studied. By the action of stronger acids we obtain among other bodies leucin, tyrosin, and lævulinic acid (LANDWEHR). Certain mucins, as the submaxillaris mucin, are easily changed by very dilute alkalies, as lime-water, while others, such as nerve-mucin, are not affected (LOEBISCH). If a strong 
caustic alkali solution, as a 5 per cent $\mathrm{KOH}$ solution, is allowed to act on sub-maxillaris mucin, we obtain alkali albuminate, a body similar to albumose and peptone, and one or more substances of an acid reaction and with strong reducing powers.

In one or the other respect the different mucins act somewhat differently. For example, the snail and nerve mucins are insoluble in dilute hydrochloric acid of 1-2 p. m., while the mucin of the submaxillary gland and the navel cord are soluble. Nerve-mucin becomes flaky with acetic acid, while the other mucins are precipitated in more or less fibrous, tough masses. Still all the mucins have certain reactions in common.

In the dry state mucin forms a white or yellowish-gray powder. When moist it forms, on the contrary, flakes or yellowish-white tough lumps or masses. The mucins are acid in reaction. They give the color reactions of the albuminous bodies. They are not soluble in water, but may give a neutral solution with water and the smallest quantity of alkali. Such a solution does not coagulate on boiling, while acetic acid gives at the normal temperature a precipitate which is insoluble in an excess of the precipitant. If $5-10 \% \mathrm{NaCl}$ be added to a mucin solution, this can now be carefully acidified with acetic acid without giving a precipitate. Such acidified solutions are copiously precipitated by tannic acid; with potassium ferrocyanide they give no precipitate, but on sufficient concentration they become thick or viscous. A neutral solution of mucinalkali is precipitated by alcohol in the presence of neutral salts; they also give precipitates with several metallic salts. If mucin is heated on the water-bath with dilute hydrochloric acid of about 2\%, the liquid gradually becomes a yellowish or dark brown and reduces copper oxhydrate from alkaline solutions.

The mucin most easily obtained in large quantities is the submaxillary mucin, which may be prepared in the following way: The filtered watery extract of the gland, as colorless as possible, is treated with $25 \%$ hydrochloric acid, so that the liquid contains $1.5 \mathrm{p} . \mathrm{m}$. HCl. On the addition of the acid the mucin is immediately precipitated, but dissolves on stirring. If this acid liquid is immediately diluted with $2-3$ vols. of water, the mucin separates and may be purified by redissolving in $1-5 \mathrm{p} . \mathrm{m}$. acid and diluting with water and washing therewith. The mucin of the navel-cord 
may be prepared in the same way. ${ }^{1}$ The nerve mucin is prepared from nerves which have first been freed from albumin by commonsalt solution and water. They are extracted with lime-water, the filtrate is precipitated with acetic acid, and the precipitate purified by redissolving in dilute alkali or lime-water, precipitating with acid, and washing with water (ROLLETT, LOEBISCH). Lastly, the mucin is treated with alcohol.

2. Mucoids or Mucinoids. To this group belong pseudomucin, which occurs in ovarial liquids, colloid, which is probably related thereto, and chondromucoid, which occurs in cartilage. These bodies will be treated of later in their respective chapters.

Hyalogen. Under this name KRUKENBERG has designated a number of differing protein bodies, which are characterized by the following: By the action of alkalies they change, with the splitting off of sulphur and nitrogen, into a soluble nitrogenized product called by him hyaline and which yields a pure carbohydrate by further decomposition. Within this group the most widely differing substances may find place, as for instance true mucin and mucoid, the so-called mucin of the holothuria, neossin of the edible bird'snest, the glycoproteid of the vineyard snail, the onuphin and spirographin, and other substances from the lower animals. It is of very little value to collect into one group all these differing substances, which have very little in common, until we have learned with some degree of certainty the nature of the reducible substances and other products obtained from them.

\section{Albumoids or Albuminoids.}

Under this name we collect into a special group all those protein bodies which cannot be placed in either of the other two groups, although they differ essentially among themselves and from a chemical standpoint do not show any prevailing difference from the ordinary albuminous bodies. The most important and abundant of the bodies belonging to this group are important constituents of the animal skeleton or the animal structure. They occur as a rule in an insoluble state in the organism, and they are marked in most cases by a great resistance to reagents which dissolve albumins or to chemical reagents in general.

The Keratin Group. Keratin is the chief constituent of the horny structure, of the epidermis, of hair, wool, of the nails, hoofs, horns, feathers, of tortoise-shcll, etc., etc. Keratin is also found as neurokeratin (KüHNE) in the brain and nerves. The shell-membrane of the hen's egg seems also to contain keratin.

1 The author has not been able to obtain this pure, so the analysis has not been given in the previous table of the mucins. 
It seems that there exist more than one keratin, and these varieties form a special group of bodies. This fact, together with the difficulty in isolating the keratin from the tissues in a pure condition without a partial decomposition, is sufficient explanation for the variation in the elementary constitution given below. As examples the analyses of a few tissues rich in keratin and of keratin itself are given as follows:

\begin{tabular}{|c|c|c|c|c|c|c|}
\hline & C & $\mathbf{H}$ & $\mathbf{N}$ & $\mathbf{S}$ & O & \\
\hline Human hair.... & 50.65 & 6.36 & 17.14 & 5.00 & 20.85 & (v. LAAR.) \\
\hline Nail ............. & 51.00 & 6.94 & 17.51 & 2.80 & 21.75 & (MULDER.) \\
\hline Neurokeratin... & $56.11-58.45$ & $7.26-8.02$ & $11.46-14.32$ & $1.63-2.24$ & $\ldots$ & (KUBNE.) \\
\hline Horn (average). & 50.86 & 6.94 & ... & 3.30 & $\ldots$ & (HoRBACZEWSK \\
\hline Tortoise-shell... & 54.89 & 6.56 & 16.77 & 2.22 & 19.56 & (MULDER.) \\
\hline Shell-membrane & 49.78 & 6.64 & 16.43 & 4.25 & 20.90 & (LINDVALL.) \\
\hline
\end{tabular}

Sulphur is at least in part in loose combination, and it is partly removed by the action of alkalies (as sulphides), or indeed by boiling with water (Chevreul). Combs of lead after long usage become black, and this is due to the action of the sulphur of the hair. On heating keratin with water in sealed tubes at a temperature of $150^{\circ}$ to $200^{\circ} \mathrm{C}$. it dissolves, with the elimination of sulphuretted hydrogen, forming a non-gelatinizing liquid which contains albumose (called keratinose by KRUKENBERG) and peptone (?). The keratin may be dissolved by alkalies, especially on heating, forming, besides alkali sulphides, albumoses and peptones (?). That the keratin in the organism is formed from the albumin is not to be denied. Drechsel believes that in keratin a part of the oxygen of the albumins is exchanged for sulphur, and a part of the ieucin or any other amido-acid is exchanged for tyrosin. The products of decomposition of keratin and of albumin are similar, except that the former gives proportionally a greater amount of tyrosin (3-5\%).

Keratin is amorphous or takes the form of the tissues from which it was prepared. On heating it decomposes and generates an odor of burnt horn. It is insoluble in water, alcohol, or ether. On heating with water to $150^{\circ}-200^{\circ} \mathrm{C}$. it dissolves. It also dissolves gradually in caustic alkalies, especially on heating. It is not dissolved by artificial gastric juice or by trypsin solutions. Keratin gives the xanthoproteic acid reaction, as well as the reac- 
tions with Millon's reagent, even though they are not always typical.

In the preparation of keratin a finely-divided horny structure is treated first with boiling water, then consecutively with diluted acid, pepsin-hydrochloric acid, and alkaline trypsin solution, and, lastly, with water, alcohol, and ether.

Elastin occurs in the connective tissue of higher animals, sometimes in so large quantities that it forms a special tissue. It occurs most abundantly in the cervical ligament (ligamentum nuchæ). It seems that there is more than one kind of elastin.

Elastin, according to the general view, is free from sulphur. According to the investigations of CHITTENDEN and HART, it is a question whether or not elastin contains sulphur which is removed by the action of the alkali in its preparation. The most trustworthy analyses of elastin from the cervical ligament have given the following results:

$\begin{array}{ccccl}\text { C } & \text { H } & \text { N } & \text { O } & \\ 54.32 & 6.99 & 16.75 & 21.94 & \text { (HoRBACZEWSKI.) } \\ 54.24 & 7.27 & 16.70 & 21.79 & \text { (CHITTENDEN and HART.) }\end{array}$

As splitting products we find leucin, tyrosin (in small quantity), glycocoll, amido-valerianic acid, ammonia, and others. No indol or phenol is obtained on putrefaction. On heating with water in closed vessels, on boiling with dilute acids, or by the action of a proteolytic enzym, the elastin dissolves and splits into two chief products, called by HoRBACZEWSKI hemielastin and elastinpeptone. According to CHITTENDEN and HART, these products correspond to two albumoses designated by them protoelastose and deuteroelastose. The first is soluble in cold water and separates on heating, and its solution is precipitated by mineral acid as well as by acetic acid and potassium ferrocyanide. The watery solution of the other does not become cloudy on heating, and is not precipitated by the above-mentioned reagents.

Pure dry elastin is a yellowish-white powder; in the moist state it appears like yellowish-white threads or membranes. It is insoluble in water, alcohol, or ether, and shows a resistance against the action of chemical reagents. It is not dissolved by strong caustic 
alkalies at the ordinary temperature, and only slowly at the boiling temperature. It is very slowly attacked by cold concentrated sulphuric acid, and it is relatively easily dissolved on warming with strong nitric acid. It gives MILlon's reaction.

On account of its great resistance to chemical reagents, elastin may be prepared (best from the ligamentum nuchæ) in the following way: First boil with water, then with $1 \%$ caustic potash, then again with water, and lastly with acetic acid. The residue is treated with cold 5\% hydrochloric acid for twenty-four hours, carefully washed with water, boiled again with water, and then treated with alcohol and ether.

Collagen, or glue-forming substance, occurs very extensively in the animal kingdom, especially in the vertebrates, seldom in the invertebrates. Collagen is the chief constituent of the fibres of the connective tissue and (as ossein) of the organic substances of the bony structure. It also occurs in the cartilaginous tissues as chief constituent, but it is here mixed with another substance which was formerly called chondrigen. Collagen from different tissues has not quite the same composition, and probably there are several varieties of collagen.

By continuously boiling with water (more easily in the presence of a little acid) collagen is converted into gelatine. HoFMeIsTER found that gelatine, on being heated to $130^{\circ} \mathrm{C}$., is transformed into collagen; and this last may be considered as the anhydride of gelatine. Collagen and gelatine have the following composition:

$\begin{array}{lccccl} & \text { C } & \text { H } & \text { N } & \text { S+O } & \\ \text { Collagen ................ } & 50.75 & 6.47 & 17.86 & 24.92 & \text { (HoFMEIstER.) } \\ \text { Gelatine (from hartshorn). } & 49.31 & 6.55 & 18.37 & 25.77 & \text { (MULDER.) } \\ \text { Gelatine (from bones)...... } & 50.00 & 6.50 & 17.50 & 26.00 & \text { (FREMY.) }\end{array}$

The gelatine contains about $0.6 \%$ sulphur, which probably belongs to the gelatine and hardly exists there as an impurity from the albumin.

The investigations in regard to the decomposition products of collagen have been made on gelatine. Gelatine yields, under similar conditions as albuminous bodies, amido-acids, but no tyrosin. It yields a large amount of glycocoll, to which, on this account, the name of glue-sugar has been given. On putrefaction gelatine gives 
neither tyrosin nor indol, in which it deviates from e albumins. Still the aromatic group is not absent in gelatine, and it acts like the oxidized albumin, the oxyprotsulphonic acid giving benzoic acid (MALY). On treating gelatine with hydrochloric acid and alcohol and then acting on this with a nitrite, BUCHNER and CuRTIUs obtained an ester of a diazo-fatty acid, probably diazo-oxyacrylic acid ester, and it is therefore also possible that the nucleus of the gelatine is formed of amido-acrolein.

Collagen is insoluble in water, salt solutions, dilute acids, and alkalies, but it swells up in dilute acids. By continuous boiling with water it is converted into gelatine. It is dissolved by the gastric juice and also by the pancreatic juice (trypsin solution) when it has previously been treated with acid or heated with water above $+70^{\circ} \mathrm{C}$. By the action of ferrous sulphate, corrosive sublimate, or tannic acid, collagen shrinks. Collagen treated by these bodies does not putrefy, and the tannic acid is therefore of great importance in the preparation of leather.

Gelatine or glue is colorless, amorphous, and transparent in thin layers. It swells in cold water without dissolving. It dissolves in warm water, forming a sticky liquid, which solidifies on cooling when sufficiently concentrated. The solution is lævogyrate; $\alpha \mathrm{j}$ at $+30^{\circ} \mathrm{C} .=-130^{\circ}$. Acetic acid and alkalies diminish the specific rotary power. Gelatine solutions on boiling are not precipitated either by mineral acids, acetic acid, alum, lead acetate, or mineral salts in general. A gelatine solution acidified with acetic acid may be precipitated by potassium ferrocyanide on carefully adding the reagent, but on the addition of too much potassium ferrocyanide the liquid remains clear. Gelatine solutions are precipitated by tannic acid in the presence of salt; by acetic acid and common salt in substance; mercuric chloride in the presence of $\mathrm{HCl}$ and $\mathrm{NaCl}$; phosphomolybdic acid in the presence of acid; and lastly by alcohol, especially when neutral salts are present. Gelatine solutions do not diffuse. Gelatine gives the biuret reaction, but not ADAMKIEWICZ's. It gives Millon's reaction and the xanthoproteic acid reaction so faintly that it probably occurs from an impurity consisting of albumin. By continuous boiling with water,-especially in the presence of dilute acid,-also by digesting with gastric 
juice or trypsin solution, gelatine loses the property of gelatinizing and is transformed into gelatine-peptone.

According to HoFMEISTER it splits into two substances, semiglutin and hemicollin. The former is insoluble in alcohol of $70-80 \%$, and is precipitated by platinum chloride. The latter, which is not precipitated by platinum chloride, dissolves in alcohol.

Collagen may be obtained from bones by extracting with hydrochloric acid (which dissolves the earthy matters) and then carefully removing the acid with water. It may be obtained from tendons treated with lime-water (which dissolves the albumin and mucin), and then thoroughly washing with water. Gelatine is obtained by boiling collagen with water. The finest commercial gelatine contains a little albumin, which may be removed by allowing the finelydivided gelatine to swell in cold water and extracting thoroughly with large quantities of fresh water. In regard to the preparation of gelatine from cartilage see Chapter VIII.

Chondrin is only a mixture of glue with the specific constituents of cartilage and their transformation products. Spongin forms the great mass of the ordinary sponge. It gives no gelatine, and on boiling with acids it yields leucin and glycocoll, but no tyrosin. Conchiolin is found in the shells of mussels and snails, and also in the egg-shells of these animals. It yields leucin but no tyrosin. Byssus contains a substance, closely related to conchiolin, which is soluble with difficulty. Cornein forms the axial system of the Antipathes and Gorgonia. It gives leucin and a crystallizable substance, cornicrystallin (KRU. KENBERG). Fibroin and Sericin are the two chief constituents of raw silk. By the action of superheated water the sericin dissolves and gelatinizes on cooling (silk gelatine), while the more difficultly soluble fibroin remains undissolved in the shape of the original fibre. On boiling with acid the fibroin yields alanin (WEYL), glycocoll, and a great deal (5-8\%) of tyrosin. Fibroin is dissolved in cold concentrated hydrochloric acid with the expulsion of $1 \%$ nitrogen as ammonia, and it is converted into another, nearly-related substance called sericoin (WEYL). Sericin yields no glycocoll but leucin and a crystallizable substance called serin. The composition of the above-nientioned bodies is as follows:

$\begin{array}{llllll}C & \mathrm{H} & \mathrm{N} & \mathrm{S} & \mathrm{O}\end{array}$

\begin{tabular}{|c|c|c|c|c|c|}
\hline Conchiolin (from snail-eggs) & 50.92 & 6.88 & 17.86 & 0.31 & 24.34 (KRUKENBERG) \\
\hline Spongin................. & 46.50 & 6.30 & 16.20 & 0.5 & 27.50 (CROOCKEWITT.) \\
\hline & 48.75 & 6.35 & 16.40 & & .. (Posselt.) \\
\hline Cornein. & 48.96 & 5.90 & 1681 & .... & 28.33 (KRUKENBERG.) \\
\hline Fibroin.. & 48.23 & 6.27 & 18.31 & .... & 27.19 (CRAMER.) \\
\hline Sericin $\ldots \ldots \ldots \ldots \ldots \ldots$ & 44.32 & 6.18 & 18.30 & .... & 30.20 (Cramer.) \\
\hline
\end{tabular}

Amyloid, so called by VIRchow, is a protein substance appearing under pathological conditions in the internal organs, such as the spleen, liver, and kidneys, as infiltrations; and in serous membranes as granules with concentric layers. It probably also occurs as a constituent of a few prostate calculi. Amyloid has not been 
obtained pure, therefore its composition cannot be given with certainty. Friedreich and KeKulé found C $53.6 ; \mathrm{H} 7.0 ; \mathrm{N} 15.0$; and $\mathrm{S}+024.4 \%$. KüHNE and RUDNEFF found $1.3 \%$ sulphur. Amyloid is not related to the carbohydrates, and on boiling with acids it gives neither glucose nor any other reducing substance. On the contrary, it yields leucin and tyrosin.

It is insoluble in water, alcohol, ether, dilute hydrochloric acid, and acetic acid. It is dissolved in concentrated hydrochloric acid or caustic alkali, and is converted into acid or alkali albuminates according to the agents employed. According to KostJurin, amyloid is dissolved by the gastric juice, which is the reverse of older theories. Amyloid gives the xanthoproteic acid reaction and the reactions of MILLON and ADAMKIEWICZ. Its most important property is its behavior with certain coloring matters. It is colored reddish brown or a dingy violet by iodine; a violet or blue by iodine and sulphuric acid; red by methylaniline iodide, especially on the addition of acetic acid; and red by aniline green.

Amyloid is prepared by extracting the tissue with cold and then boiling water, afterwards with alcohol and ether. After boiling with alcohol containing hydrochloric acid and digesting with gastric juice, that which is insoluble is considered as amyloid. As the amyloid may be dissolved by the gastric juice, the utility of this method seems doubtful. 


\section{CHAPTER III.}

THE ANIMAL CELL.

THE cell is the unit of the manifold, variable forms of the organism; it forms the simplest physiological apparatus, and as such is the seat of chemical processes. It is generally admitted that all chemical processes of importance do not take place in the animal fluids, but proceed in the cells, which may be considered as the chemical laboratory of the organism. It is also principally the cells which, through their greater or less activity, regulate or govern the range of the chemical processes and also the intensity of the total exchange of material.

It is natural that the chemical investigation of the animal cell should in most cases coincide with the study of those tissues of which it forms the chief constituent. Only in a few cases can the cells be directly, by relatively simple manipulations, isolated in a rather pure state from the tissues, as, for example, in the investigation of pus or of tissue very rich in cells. But even in these cases the chemical investigation may not lead to any positive results in regard to the constituents of the uninjured living cells. By the process of chemical transformation new substances may be formed at the death of the cell, and at the same time physiological constituents of the cell may be destroyed or transported into the surrounding menstruum and therefore escape investigation. For this and other reasons we possess only a very limited knowledge of the constituents and the constitution of the cell, especially of the living one.

While young cells of different origin in the early period of their existence may show a certain similarity in regard to their form and chemical constitution, they may, on further development, not only take the most varied forms, but may also offer from a chemical standpoint the greatest diversity. As a description of the constituents and the constitution of the different cells occurring in the animal organism is nearly equivalent to a demonstration of the chemical relations of most animal tissues, and as this exposition 
will be found in their respective chapters, we will here only discuss the chemical constituents of the young cells or the cells in general.

We must first differentiate between the protoplasm and the nucleus.

The Protoplasm of the generative cell consists during life of a semi-solid body contractile under certain conditions, very rich in water, and the mass of which consists mainly of albuminous bodies. If the cell be deprived of the physiological conditions of life, or if exposed to destructive exterior influences, such as the action of high temperatures, of chemical agents, or indeed of distilled water, the protoplasm dies. The albuminous bodies which it contains coagulate at least partially, and other chemical changes are found to take place. The alkaline reaction of the living cell may be converted into an acid by the appearance of paralactic acid, and the carbohydrate, the glycogen, which habitually occurs in the young generative cell may after its death be quickly changed and consumed.

The albuminous bodies of the protoplasm consist, according to the general view, chiefly of globulins, but albumins are also found. The occurrence of globulins in the animal as well as in the regetable cell has been specially shown by HOPPE-SEYLER, and according to this investigator two globulin substances, vitellin and myosin, occur in all protoplasm. HALLIBURToN has lately closely studied the albuminous bodies of the lymphatic cells, and found two globulins besides an albumin probably identical with serum albumin (see Chapter IV). Of the globulins, one which occurs only in small quantities coagulates at the temperature of $48-50^{\circ} \mathrm{C}$., while the other, which occurs in abundance, is coagulated like serum globulin by a $5 \%$ solution of $\mathrm{MgSO}_{4}$ at $75^{\circ} \mathrm{C}$. The widespread occurrence of globulins and also of albumins in the protoplasm of the animal cell has been unquestionably demonstrated, but these two groups of albuminous bodies do not, at least in many cases, form the chief mass of the protoplasm. The protoplasm seems to consist in great part of very complex protein substances, the proteids on one side and the nucleoalbumins on the other. Of all these the nucleoalbumins appear to be regular constituents of the protoplasm, and they do not only appear in the pus cells or in the cells of the lymphatic glands (HALLIBURTON), but also in almost all varieties of glandular cells. The chief mass of the albumins found 
in the protoplasm appear to be phosphorized, a condition which is of importance as showing the genetic connection between the cell nucleus rich in phosphorus and the protoplasm.

The extent to which the proteids occur in the young generative cells has not been sufficiently investigated; nevertheless these substances occur habitually and in significant amounts in certain epithelium and glandular cells. Difficultly soluble protein substances are also found in dead cells or in organs rich in cells which act like coagulated albuminous bodies when treated with the ordinary reagents.

In cases in which the protoplasm is surrounded by an outer, condensed layer or a cell membrane, this envelope seems to consist of albumoid substances. In a few cases-and these, according to DoNDERs, answer for the primary animal cell membrane-these substances seem to be closely related to elastin; in other cases, on the contrary, they seem rather to belong to the keratin group. The chemical processes by which these albumoid substances are formed from the albuminous bodies or proteids of the protoplasm are unknown.

The occurrence of phosphorized organic combinations in all protoplasm is without doubt of the greatest importance for the functional task, as also for the development of the cell. Of the phosphorized combinations in the cell there are at least two chief groups. To one belongs lecithin, and to the other nuclein, the latter occurring partly in the nucleoalbumin, and forming a part of the chief constituents of the cell nucleus.

Lecithin. This body is, according to the investigations of Strecker, Hundeshagen, and Gilson, an ether-like combination of glycerophosphoric acid substituted by fatty acid radicals, with a base, cholin. Therefore there may be different lecithins according to the fatty acid contained in the lecithin molecule. One of these-distearyllecithin - has been closely studied by HoPPESEYLER and DiACONOW:

$$
\mathrm{C}_{44} \mathrm{H}_{90} \mathrm{NPO}_{9}=\mathrm{HO} \cdot\left(\mathrm{CH}_{8}\right)_{3} \mathrm{~N}_{2} \mathrm{C}_{2} \mathrm{H}_{4} \cdot \mathrm{O}(\mathrm{OH}) \mathrm{PO} \cdot 0 . \mathrm{C}_{8} \mathrm{H}_{8}:\left(\mathrm{C}_{18} \mathrm{H}_{36} \mathrm{O}_{2}\right)_{3} \text {. }
$$

In agreement with this, if lecithin be boiled with baryta-water it yields fatty acids, glycerophosphoric acid, and cholin. It is only slowly decomposed by dilute acids. Besides small quantities of 
glycerophosphoric acid (perhaps also distearylglycerophosphoric acid) we have large quantities of free phosphoric acid split off.

Glycerophosphoric aCID (HO) ${ }_{2}$ PO. $0 . \mathrm{C}_{3} \mathrm{H}_{6}(\mathrm{OH})_{2}$ is a bibasic acid, which probably only occurs in the animal fluids and tissues as splitting product of lecithin. The cHoLIN, which seems to be identical with the bases SINKALIN (in mustard-seed) and AMANITIN (in agaricus muscarius), has the formula $\mathrm{HO} \cdot \mathrm{N}\left(\mathrm{CH}_{3}\right)_{3} \cdot \mathrm{C}_{2} \mathrm{H}_{4} \cdot \mathrm{OH}$ and is therefore considered as trimethylethoxylium hydrate. Cholin according to BRIEGER is not identical with the base, NEURIN, prepared by LIEBREICH as a decomposition product from the brain, which is considered as trimethylvinylium hydrate, $\mathrm{HO} \cdot \mathrm{N}\left(\mathrm{CH}_{3}\right)_{3} \cdot \mathrm{O}_{2} \mathrm{H}_{3}$. The combination of cholin with hydrochloric acid gives with platinum chloride a crystalline double combination which is easily soluble in water, insoluble in alcohol and ether, and which crystallizes in sixsided orange-colored plates. This combination is used in detecting this base.

Lecithin occurs, as HOPPE-SEYLER has especially shown, widely diffused in the vegetable and animal kingdoms. According to this investigator, it occurs also in many cases in combination with other bodies, such as albuminous bodies, hæmoglobin, and others. Lecithin, according to HOPPE-SEYLER, is found in nearly all animal and vegetable cells thus far studied, and also in nearly all fluids. It is specially abundant in the brain, nerves, fish-eggs, yolk of the egg, electrical organs of the gymnotus electricus, semen and pus, and also in the muscles and blood-corpuscles, blood-plasma, lymph, milk, and bile, as well as in other animal juices and liquids. Lecithin is also found in pathological tissues or liquids.

Lecithin may be obtained in grains or warty masses composed of small crystalline plates by strongly cooling its solution in strong alcohol. In the dry state it has a waxy appearance, is mouldable and soluble in alcohol, especially on heating (to $40^{\circ}-50^{\circ}$ C.); it is less soluble in ether. It is dissolved also by chloroform, carbon disulphide, benzol, and fatty oils. It swells in water to a pasty mass which shows under the microscope slimy, oily drops and threads, so-called myelin forms (see Chap. X). On warming this swollen mass or the concentrated alcoholic solution, decomposition takes place with the production of a brown color. On allowing the solution or the swollen mass to stand, decomposition 
takes place and the reaction becomes acid. In putrefying lecithin yields glycerophosphoric acid and cholin; the latter further decomposes with the formation of methylamin, ammonia, carbon dioxide, and marsh-gas (HASEBROEK). If dry lecithin be heated it decomposes, takes fire and burns, leaving a phosphorized coke. On fusing with caustic alkali and saltpetre it yields alkali phosphates. Lecithin is easily carried down during the precipitation of other compounds such as the albuminous bodies, and may therefore very greatly change the solubilities of the latter.

Lecithin combines with acids and bases. The combination with hydrochloric acid gives with platinum chloride a double salt which is insoluble in alcohol, soluble in ether, and which contains $10.2 \%$ platinum.

It may be prepared tolerably pure from the yolk of the hen's egg by the following methods, as suggested by HoPPE-SEYLER and DIACONow: The yolk, deprived of albumin, is extracted with cold ether until all the yellow color is removed. 'Then the residue is extracted with water at $50-60^{\circ} \mathrm{C}$. After the evaporation of the alcoholic extract at 50-60 $\mathrm{C}$., the syrupy matter is treated with ether and the insoluble residue dissolved in as little alcohol as possible. On cooling this filtered alcoholic solution to $-5^{\circ}$ to $-20^{\circ}$ C. the lecithin gradually separates in small grains. According to Gilson, a new portion of lecithin may be obtained from the ether used in extracting the yolk by dissolving the residue after the evaporation of the ether in petroleum ether and then shaking this solution with alcohol. The petroleum ether takes the fat, while the lecithin remains dissolved in the alcohol and may be obtained therefrom rather easily by using the proper precautions.

The detection and the quantitative estimation of lecithin in animal fluids or tissues is based on the solubility of the lecithin (at $50-60^{\circ} \mathrm{C}$.) in alcohol-ether, by which the phosphoric acid or glycerophosphoric acid salts which may be present at the same time are not dissolved. The alcohol-ether extract is evaporated, the residue dried and burnt with soda and saltpetre. Phosphoric acid is formed from the lecithin, and it can be detected and quantitatively estimated. The distearyllecithin yields $8.798 \% \mathrm{P}_{2} \mathrm{O}_{5}$. This method is, however, not exactly correct, for it is possible that other phosphorized organic combinations, such as jecorin (see Chapter VI), may have passed into the alcohol-ether extract. For the detection of lecithin, boiling with baryta-water and the preparation of the double platinum salt of cholin is sufficient.

The study of the second phosphorized constituents of the cell, the nuclein, is generally pursued with the study of the cell nucleus. 
The Cell Nucleus, as far as investigated, contains nuclein as chief constituent.

Nucleins. By this name Hoppe-Seyler and Miescher designated the chief constituent of the nucleus of the pus cell first isolated by them. Since that time, as by continuous investigations the same body is found very widely diffused in the animal and vegetable kingdoms, especially in organs rich in cells, we now designate as nuclein a number of phosphorized bodies which are partly obtained as splitting products of the nucleoalbumins, and partly the chief constituents of the cell nucleus.

According to HoPPE-SEYLER, these bodies may be divided into three groups. The first, to which belong the nuclein of yeast; pus, nucleated red blood-corpuscles, and probably the cell nucleus in general, on boiling with acids yields as splitting products albuminous bodies, xanthin bodies, and phosphoric acid. To the second group, which yields as splitting products albumin and phosphoric acid, belong the nuclein of the yolk of the egg and casein-in other words, from the nucleo-albumins in general, and to the third group, which gives as splitting products only phosphoric acid and hypoxanthin, belongs only the nuclein of the sperm of the salmon. LieberMANN has split off metaphosphoric acid from the nuclein of yeast, and he has also found that the metaphosphoric acid gives a combination with albumin which acts like a nuclein of the second group. PoHL has also come to the same result in so far as he has been able to prepare a combination of metaphosphoric acid with serum albumin and albumose which is similar to nuclein. LIEBERMANN therefore considers nuclein as a combination between albumin and metaphosphoric acid. The xanthin bodies, which, according to KosseL, are decomposition products of the nucleins, according to LIEBERMANN probably come from admixture.

That we find different constitutions for nucleins of different origin is not remarkable. A variation of $3.2-9.6 \%$ in the amount of phosphorus has been found in different nucleins. Under such conditions, and as the nuclein question is at present doubtful, it is hardly of any use to give the results of the elementary analyses of the different nucleins.

The nucleins are colorless, amorphous, insoluble, or only slightly soluble in water. They are insoluble in alcohol and ether. Thev 
are more or less easily dissolved by alkalies; in dilute mineral acids they are insoluble or dissolve with difficulty. The nucleins are not dissolved by pepsin-hydrochloric acid, or only slightly by its continuous action. The nucleins containing albumin answer to the biuret test and MiLLoN's reaction. With dilute mineral acids at ordinary temperature they give off (at least for nuclein from yeast or yolk of egg) metaphosphoric acid. On boiling with caustic alkali they decompose and alkali phosphates are formed. On burning they leave an acid-reacting, difficultly-burnt coke which contains metaphosphoric acid. On fusing with saltpetre and soda they give alkali phosphates.

To prepare nucleins from nucleoalbumins casein is the best material to employ. This is first dissolved in water containing about 2 p.m HCl, the filtered solution treated with pepsin and digested at the temperature of the body. After a little time a precipitate consisting of nuclein appears, which is purified by repeated solution in water with the aid of the smallest quantity of alkali, and by reprecipitating with acid, washing with water, and extracting with alcohol and ether. From cells or tissues first remove the chief mass of the albumins by the artificial digestion with pepsin-hydrochloric acid, digest the residue with very dilute ammonia, filter, and precipitate with hydrochloric acid. This precipitate is now digested with artificial gastric juice and treated as above described. In detecting nuclein the same method is used, and the last product is fused with soda and saltpetre, and phosphoric acid tested for in the melted mass. Naturally the phosphate lecithin (and jecorin) must first be removed by acid, alcohol, and ether respectively. No exact methods are known for the quantitative estimation of the nucleins in organs and tissues.

Among the decomposition products of the nucleins the socalled xanthin bodies are especially of great interest. Although Lieberman,, in opposition to the views of KosseL, considers these bodies not as real decomposition products of nuclein, but only as admixtures, yet until this question is settled more definitely, and since the xanthin bodies stand in close relationship to the cell nucleus, it is perhaps most proper to speak of these bodies in connection with the cell nucleus and the nucleins.

Xanthin Bodies. With this name we designate a group of bodies consisting of carbon, hydrogen, nitrogen, and in most cases 
also of oxygen, which, by their constitution, show a relationship not only among themselves, but also with uric acid. These bodies are xanthin, hypoxanthin, guanin, adenin, heteroxanthin, paraxanthin, and carnin. The bodies THEOBROMIN and THEOPHYLLIN (both dimethyl xanthin) and CAFFEIN (trimethyl xanthin) occurring in the vegetable kingdom also belong to this group. The relation of these bodies to one another is shown in the following list:

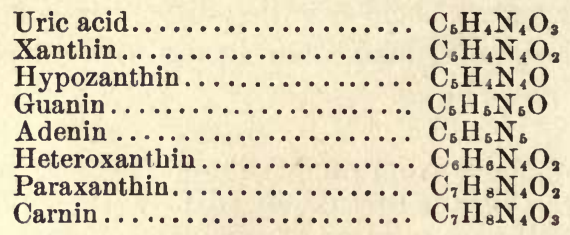

Guanin may be converted into xanthin, and adenin into hypoxanthin, by nitrous acid, also by putrefaction. Carnin is converted into hydrobromic-acid hypoxanthin by bromine-water. Adenin, as is shown by its formula, is a polymeric substance of hydrocyanic acid, and on its decomposition with alkali yields alkali cyanides. The relationship of these bodies to cyanogen is also shown by GAUTIER, who prepared xanthin synthetically from hydrocyanic acid.

The significance of the xanthin bodies as decomposition products of the cell nucleus and of nuclein was first pointed out by Kossel, who discovered the two bodies adenin and theophyllin, and his researches have greatly contributed towards the knowledge of xanthin products. - In those tissues in which, as in the glands, the cells have kept their original state, the xanthin bodies are not found free, but in combination with other atomic groups (nucleins). In such tissue, on the contrary, as in muscles, which are poor in cell nuclei, the xanthin bodies are found in the free state. If the xanthin bodies, as suggested by KosseL, stand in close relationship to the cell nucleus, it is easy to understand why the mass of these bodies is so greatly increased when large quantities of nucleated cells appear in such places as were before relatively poorly endowed. As an example of this we have in leucæmia blood extremely rich in leucocytes. In such blood KosSEL found 1.04 
p. m. xanthin bodies, against only traces in the normal blood. That the xanthin bodies are also intermediate steps in the formation of urea or uric acid in the animal organism, as will be shown later (see Chapter XIV), is probable.

Those xanthin bodies which have thus far been obtained in the decomposition of the nucleins, or generally from cells, or from tissue rich in cell nuclei, and on this account will be described here, are xanthin, hypoxanthin, guanin, and adenin, all of which are found in the vegetable kingdom (Schulze and BosshaRd, Kossel, BAGinsky). The carnin, which has only been found in meat extracts (WEIDEL) and in the flesh of certain fishes, as also in the bodies paraxanthin and heteroxanthin (SALOMON), which are only found in urine, will be described in their proper chapters, namely, IX and XIV.

$$
\text { Xanthin, } \mathrm{C}_{6} \mathrm{H}_{4} \mathrm{~N}_{4} \mathrm{O}_{2}=\begin{aligned}
& \text { NH.CH : } \mathrm{C} \cdot \mathrm{NH} \\
& \dot{\mathrm{CO}} \mathrm{O} . \mathrm{NH} \text {. } \dot{\mathrm{C}: \mathrm{N}}
\end{aligned}>\mathrm{CO} \text { (E. Fischer), is }
$$

found in the muscles, liver, spleen, pancreas, kidneys, testicles, carp-sperm, thymus, and brain. It occurs in the smallest amounts as a physiological constituent of urine, and it has been found rarely as a urinary sediment or calculus. It was first observed in such a stone by MARCET. Xanthin is found in larger amounts in a few varieties of guano (Jarvis guano).

Xanthin is amorphous, or forms granular masses of crystals. It is very slightly soluble in water, in $14,151-14,600$ parts at $+16^{\circ} \mathrm{C}$, and in $1300-1500$ parts at $100^{\circ} \mathrm{C}$. (ALMÉN). It is insoluble in alcohol or ether, but is dissolved by alkalies or acids. With hydrochloric acid it gives a crystalline, difficultly-soluble combination. Xanthin dissolved in ammonia gives with silver nitrate an insoluble, gelatinous precipitate of xanthin silver. This precipitate is dissolved by nitric acid, and by this method an easilysoluble double combination is formed. A watery xanthin solution is precipitated on boiling with copper acetate. At ordinary temperatures xanthin is precipitated by mercuric chloride and by ammoniacal lead acetate.

When evaporated to dryness in a porcelain dish with nitric acid xanthin gives a yellow residue, which turns, on the addition of caustic soda, first red, and, after heating, purple red. If we add some chloride of lime to some caustic soda in a porcelain dish 
and add the xanthin to this mixture, at first a dark green and then quickly a brownish halo forms around the xanthin grains and then disappears (HOPPE-SEYLER). If xanthin be warmed in a small vessel on the water-bath with chlorine-water and a trace of nitric acid and evaporated to dryness, when the residue is exposed under a bell-jar to the vapors of ammonia a red or purple-violet color is produced (WEIDEI's reaction). It is still a question whether entirely pure xanthin will give this reaction.

Hypoxanthin or SARKIN, $\mathrm{O}_{6} \mathrm{H}_{4} \mathrm{~N}_{4} \mathrm{O}$. This body is found in the tissues containing xanthin. It is especially abundant in the sperm of salmon and carp. It occurs also in the marrow. In normal urine it appears in very small quantities, and it seems also to be found in milk. It is found in rather significant quantities in the blood and urine in leucocythæmia.

Hypoxanthin forms very small colorless crystalline needles. It is more soluble than xanthin. It dissolves in 300 parts cold and 78 parts boiling water. It is nearly insoluble in alcohol, but it is dissolved by alkalies and acids. The combination with hydrochloric acid crystallizes and is more soluble than the corresponding xanthin combination. It acts in ammoniacal solution like xanthin with silver nitrate. The silver combination of hypoxanthin dissolves with difficulty in boiling nitric acid of $1.1 \mathrm{sp}$. gr., and on cooling the double combination separates as crystalline needles. Treated like xanthin with nitric acid, hypoxanthin gives a nearly colorless residuum which does not become red by heating with alkali. Hypoxanthin does not give WeIDEL's reaction. After the action of hydrochloric acid and zinc a hypoxanthin solution becomes first ruby-red and then brownish red in color on the addition of an excess of alkali (KosseL).

Guanin, $\mathrm{C}_{5} \mathrm{H}_{5} \mathrm{~N}_{6} \mathrm{O}=\begin{array}{r}\mathrm{NH} . \mathrm{CH}: \dot{\mathrm{C} . \mathrm{NH}} \\ \mathrm{NH}: \dot{\mathrm{C}} \cdot \mathrm{NH} \cdot \dot{\mathrm{C}: \mathrm{N}}>\mathrm{CO} . \quad \text { Guanin is }\end{array}$ found in organs rich in cells, such as the liver, spleen, pancreas, testicles, and in salmon-sperm. It is further found in the muscles (in very small amounts), in the scales and in the air-bladder of certain fishes as iridescent crystals of guanin lime; in the retina epithelium of fishes, in guano, and in the excrement of spiders it is found as chief constituent. Under pathological conditions it has been found in 


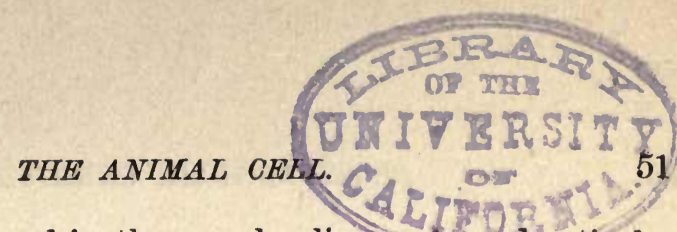

leucocythæmic blood, and in the muscles, ligaments, and articulations of pigs with guanin gout.

Guanin is a colorless, ordinarily amorphous powder which may be obtained as small crystals by allowing its solution in concentrated ammonia to spontaneously evaporate. It is nearly insoluble in water, alcohol, and ether. It is easily dissolved by mineral acids and alkalies, but it dissolves with great difficulty in ammonia. The silver combination dissolves with difficulty in boiling nitric acid, and on cooling the double combination crystallizes. Guanin acts like xanthin in the nitric-acid test, but gives with alkalies on heating a stronger bluish-violet color. A warm solution of guanin hydrochloride gives with a cold saturated solution of picric acid a yellow precipitate consisting of silky needles (CAPRANICA). With a concentrated solution of potassium chromate a guanin solution gives a crystalline, orange-red precipitate, and with a concentrated solution of potassium ferricyanide a yellowish brown, crystalline precipitate (CAPRANICA).

Adenin, $\mathrm{C}_{6} \mathrm{H}_{6} \mathrm{~N}_{5}$, was first found by Kossel in the pancreas gland. It occurs in greatest quantities in the sperm of the carp and in the thymus (Kossel and SchMidLer). It also occurs in the liver, spleen, lymphatic glands, and kidneys (not in muscles). It has been observed in leucocythæmic urine.

Adenin crystallizes in long needles. It dissolves in cold water with difficulty (1086 parts), but easily in warm. Pure adenin dissolves slightly in boiling alcohol, in cold not at all; impure adenin is, however, dissolved by cold alcohol. It is insoluble in ether. Adenin is easily dissolved by acids and alkalies. In dilute ammonia it dissolves with more difficulty than hypoxanthin, but more easily than guanin. The silver combination dissolves with difficulty in boiling nitric acid and crystallizes on cooling. The nitric-acid test and WeIDEL's reaction act the same as with hypoxanthin. The same is true for its behavior with hydrochloric acid and zinc with addition of alkali.

The principle for the preparation, detection, and the quantitative estimation of the four above-described xanthin bodies is, according to Kossel and Kossel and Schindler, as follows: The finely-divided organ or tissue is boiled for three or four hours with sulphuric acid of about $5 \mathrm{p} . \mathrm{m}$. The filtered liquid is freed from albumin by lead 
acetate, and the new filtrate is treated with sulphuretted hydrogen to remove the lead, again filtered, concentrated, and, after adding an excess of ammonia, precipitated with silver nitrate. The silver combination (with the addition of some urea to prevent nitrification) is dissolved in not too large a quantity of boiling nitric acid of sp. gr. 1.1, and this solution filtered boiling hot. On cooling the silver xanthin remains in the solution, while the double combination of guanin, hypoxanthin, and adenin crystallizes. The xanthin silver may be removed from the filtrate by the addition of ammonia, and the xanthin set free by means of sulphuretted hydrogen. The three above-mentioned silver nitrate combinations are decomposed in water with ammonium sulphide and heat; the silver sulphide is filtered, the filtrate concentrated, saturated with ammonia, and digested on the water-bath. The guanin remains undissolved, while the other two bases pass into solution. A part of the guanin is still retained by the silver sulphide, and may be liberated by boiling it with dilute hydrochloric acid and then saturating the filtrate with ammonia. When the above filtrate, containing the adenin and hypoxanthin, which has been, if necessary, freed from ammonia by evaporation, is allowed to cool, the adenin separates, while the hypoxanthin remains in solution. The chief points in the above method are used for the quantitative estimation of the xanthin bodies. If the solution of adenin and hypoxanthin is evaporated to dryness, the residue weighed, and the amount of nitrogen determined, from this determination and from the amount of nitrogen in hypoxanthin $(41.17 \%)$ and in adenin $(51.8 \%$ ) the quantity of each of these bodies may be calculated.

In the generative animal cells, and especially in those which develop embryonic tissue, Cl. Bernard and Hensen have discovered a carbohydrate, the glycogen. According to HopPESEYLER it seems to be a never-failing constituent of the cells as soon as they show embryonic movements, and he found this carbohydrate in the white blood-corpuscles but not in the developed motionless pus-corpuscles. The relationship which exists between the consumption of glycogen and muscular work (see Chapter IX) leads us to suppose that such a consumption takes place in the movements of the animal protoplasm. On the other side the widely-diffused occurrence of glycogen in embryonic tissues, as also its occurrence in pathological swellings and in abundant cellformations, seems of the greatest importance in the formation and development of the cell.

In grown animals the glycogen is found in the muscles and 
certain other organs, foremost of these being the liver; it will therefore be more thoroughly described in connection with this organ (Chapter VI).

Another body or more correctly a group of bodies which occur very widely diffused in the animal and vegetable kingdoms and habitually in the cells are the cholesterines, whose best-known representative is the ordinary cholesterin, specially known as the chief constituent of certain biliary calculi and occurring in large quantities in the brain and nerves. It is hardly to be admitted that this body has any direct importance in the life and the development of the cell. It is more probable, as HopPE-SEYLER suggests, that cholesterin is a splitting product appearing in the general lifeprocesses of the cells. Also according to HoPPE-SEYLER the fat, which does not constantly appear in the cell, has nothing to do with the general process of life.

Mineral bodies are also never-failing constituents of the cell. These minerals are potassium, sodium, calcium, magnesium, iron, phosphoric acid, and chlorine. In regard to the alkalies we find in general in the animal organism that the sodium combinations are more abundant in the fluids, the potassium combinations occur chiefly in the form-constituents and in the protoplasm. Corresponding to this the cell contains potassium, chiefly as phosphate, while the sodium and chlorine combinations occur less abundantly. According to the ordinary views the potassium combinations, especially the potassium phosphate, are of the greatest importance for the life and development of the cell, even though we do not know the nature of the importance. At least we must not overlook the fact that a part of the phosphoric acid which is obtained from the cell or tissue rich in cells may originate from the nuclein and lecithin in the process of ashing. Also the iron, which often occurs in the ash as ferric phosphate, seems, at least in part, to be formed from the nucleo-albumin. The habitual occurrence of earthy phosphates in all cells and tissues, together with the difficulty or almost impossibility of separating these bodies from the protein substances without decomposition, leads us to suppose that these mineral bodies are indeed, though their rôle is still unknown, of the greatest importance for the life of the cells and the chemical processes which accompany their evolution. 


\section{CHAPTER IV.}

THE BLOOD.

THE blood is to be considered from a certain standpoint as a fluid tissue, and it consists of a transparent liquid, the bloodplasma, in which an immense number of solid particles, the red and colorless blood-corpuscles (and the blood-tablets) are suspended.

Outside of the organism the blood, as is well known, coagulates more or less quickly; but this coagulation is accomplished generally in a few minutes after leaving the body. All varieties of blood do not coagulate with the same degree of rapidity. Some coagulate more quickly, others more slowly. Among the varieties of blood thus far investigated the blood of the horse coxgulates most slowly. The coagulation may be more or less retarded by quickly cooling; and if we allow equine blood to flow directly from the vein into a glass cylinder which is not too wide and which has been cooled, and let it stand at $0^{\circ} \mathrm{C}$., the blood may be kept fluid for several days. An upper, amber-yellow layer of plasma gradually separates from a lower, red layer composed of blood-corpuscles with only a little plasma. Between these we observe a whitish-gray layer, which consists of white blood-corpuscles.

The plasma thus obtained and filtered is a clear amber-yellow alkaline liquid which remains fluid for some time when kept at $0^{\circ}$ C., but soon coagulates at the ordinary temperature.

The coagulation of the blood may be prevented in other ways. After the injection of peptone or, more correctly, albumose solutions into the blood (in the living dog), the blood does not coagulate on leaving the veins (FANo, SchmidT-MÜLHeim). The plasma obtained from such blood by means of centrifugal force is called "peptone-plasma." The coagulation of the blood of warm- 
blooded animals is prevented by the injection of an effusion of the mouth of the officinal leech into the blood-current (HAYCRAFT). If the blood-circulation of a dog is cut off between the liver and intestines and the blood allowed to flow only through the head and the viscera of the thoracic cavity, the coagulation of the blood is destroyed (PAwLow, BoHR). If we allow the blood to flow directly, while we stir it, into a neutral salt solution-best a saturated magnesium sulphate solution (1 vol. salt solution and 3 vols. blood)-we obtain a mixture of blood and salt which remains uncoagulated for several days. The blood-corpuscles which, because of their adhesiveness and elasticity, would otherwise pass easily through the pores of the filter-paper are made solid and stiff by the salt, so that they may be easily filtered. The plasma thus obtained, which does not coagulate spontaneously, is called "saltplasma."

On coagulation there separates in the previously fluid blood an insoluble or a very difficultly-soluble albuminous substance, fibrin. When this separation takes place without stirring the blood coagulates to a solid mass which, when carefully severed from the sides of the vessel, contracts, and a clear, generally yellow-colored liquid, the blood-serum, exudes. The solid coagulum which incloses the blood-corpuscles is called the blood-clot (placenta sanguinis). If the blood is beaten during coagulation, the fibrin separates in elastic threads or fibrous masses, and the defibrinated blood which separates is sometimes called cruor, ${ }^{1}$ and consists of blood-corpuscles and blood-serum.

The defibrinated blood consists of blood-corpuscles and serum, while the uncoagulated blood consists of blood-corpuscles and blood-plasma. The essential chemical difference between bloodserum and blood-plasma is that the blood-serum does not contain the mother-substance of fibrin, the fibrinogen, which exists in the blood-plasma, and the serum is proportionally richer in another body, the fibrin ferment (see page 58).

1 The name cruor is used in different senses. We sometimes understand thereby only the blood when coagulated to a red solid mass, in other cases the blood-clot after the separation of the serum, and lastly the sediment consisting of red blood-corpuscles which is obtained from defibrinated blood by means of centrifugal force or by letting it stand. 


\section{Blood-plasma and Blood-serum.}

\section{The Blood-plasma.}

In the coagulation of the blood a chemical transformation takes place in the plasma. A part of the albumins separates as insoluble fibrin. The albuminous bodies of the plasma must therefore be first described. They are fibrinogen, serum globulin, and serum albumin.

Fibrinogen occurs in blood-plasma, chyle, lymph, and in certain transudations and exudations. ${ }^{1}$

It has the general properties of the globulins, but differs from other globulins as follows: In a moist condition it forms white flakes which are soluble in dilute common salt solutions, and which easily conglomerate into tough, elastic masses or lumps. The solution in $\mathrm{NaCl}$ of $5-10 \%$ coagulates on heating to $+52^{\circ}$ to $55^{\circ} \mathrm{C}$., and the faintly alkaline or nearly neutral weak salt solution coagulates at $+56^{\circ} \mathrm{C}$., or at exactly the same temperature at which the blood-plasma coagulates. Fibrinogen solutions are precipitated by an equal volume of a saturated common salt solution, and are completely precipitated by adding an excess of $\mathrm{NaCl}$ in substance (thus differing from serum globulin). It differs from myosin of the muscles, which coagulates at about the same temperature, and from other albuminous bodies, in the property of being converted into fibrin under certain conditions. Fibrinogen has a strong decomposing action on hydrogen peroxide.

The fibrinogen may be easily separated from the salt-plasma by precipitation with an equal volume of a saturated NaCl solution. For further purification the precipitate is pressed, redissolved in an $8 \%$ salt solution, the filtrate precipitated by a saturated salt solution as above, and after precipitating in this way three times, the precipitate at last obtained is pressed between filter-paper and finely divided in water. The fibrinogen dissolves with the aid of the small amount of $\mathrm{NaCl}$ contained in itself, and the solution may be made salt-free by dialysis with very faintly alkaline water. From transudations we ordinarily obtain a fibrinogen which is strongly

1 The question as to the occurrence of other fibrinogens (WooldRIDGE) will be spoken of in connection with the complete discussion of the coagulation of the blood. (See further on.) 
contaminated with lecithin and which can hardly be purified without decomposing. The method for the detection and quantitative estimation of fibrinogen in a liquid is based on its property of yielding fibrin on the addition of a little blood, of serum, or of fibrin ferment.

The fibrinogen stands in close relation to its transformationproduct, the fibrin.

Fibrin is the name of that albuminous body which separates on the so-called spontaneous coagulation of blood, lymph, and transudations, as also in the coagulation of a fibrinogen solution after the addition of serum or fibrin ferment (see below).

If the blood is beaten during coagulation, the fibrin separates in elastic fibrous masses. The fibrin of the blood-clot may be beaten to small, less elastic, and not particularly fibrous lumps. The typical, fibrous, and elastic white fibrin, after washing, stands in regard to its solubility close to the coagulable albuminous bodies. It is insoluble in water, alcohol, or ether. It expands in hydrochloric acid of 1 p. m., as also in caustic potash or soda of 1 p. m., to a gelatinous mass, which dissolves at the ordinary temperature only after several days, but at the temperature of the body it dissolves more readily but still slowly. The fibrin expands in a 5$10 \%$ solution of common salt or saltpetre, but only dissolves in the presence of contaminating enzymes or by putrefaction. Fibrin decomposes hydrogen peroxide, but this property is destroyed by heating or by the action of alcohol.

What has been said of the solubility of fibrin relates only to the typical fibrin obtained from the arterial blood of mammalia or man by whipping and washing first with water and with common-salt solution, and then with water again. The blood of various kinds of animals yields fibrin with somewhat different properties and of varying purity, and likewise blood from different parts of the body may yield fibrim with unlike solubilities (DENIS).

The fibrin obtained by beating the blood and purified as above described is always contaminated by enclosed blood-corpuscles or remains thereof, and also by lymphoid cells. It can only be obtained pure from filtered plasma or filtered transudations. For the pure preparation, as well as for the quantitative estimation of fibrin, the spontaneously coagulating liquid is at once, or the non-spontaneously coagulating liquid only after the addition of blood-serum or fibrin 
ferment, thoroughly beaten with a glass rod or whale-bone, and the separated coagulum is washed first in.water, and then with a 5\% common-salt solution, and again with water, and lastly extracted with alcohol and ether.

A pure fibrinogen solution may be kept at the ordinary temperature until putrefaction begins without showing a trace of fibrin coagulation. But if to this solution we add a water-washed fibrin clot or a little blood-serum, it immediately coagulates and may yield perfectly typical fibrin. The transformation of the fibrogen into fibrin requires the presence of another body contained in the blood-clot and in the serum. This body, whose importance in the coagulation of fibrin was first observed by BUCHANAN, was later rediscovered by ALEXANDER SCHMIDT, and designated "fibrin ferment." The nature of this enzymotic body has not been ascertained. According to the investigations of GAMGEE, LEA, and Green and Halliburton, the "fibrin ferment" seems to be a substance of the nature of the globulins. According to HalliBURTON, it is a body derived from the lymphoid cells, a special globulin, " cell-globulin," which differs from serum globulin partly by fibrino-plastic properties and partly by having another temperature of coagulation $\left(+60^{\circ} \mathrm{C}\right.$., or somewhat higher in a solution containing $10 \% \mathrm{NaCl}$ ). The so-called fibrin ferment corresponds to the enzymes in that only the very smallest amounts of it are required for action, and further that on heating the solution it becomes inactive.

The isolation of the fibrin ferment has been tried in several ways. Ordinarily it may be prepared by the following method proposed by ALEX. SchMIDT : Precipitate the serum or defibrinated blood with 15-20 vols. of alcohol and allow it to stand a few months. The precipitate is then filtered and dried over sulphuric acid. The ferment may be extracted from the dried powder by means of water.

If a fibrinogen solution containing salt, as above described, is treated with a solution of "fibrin ferment," it coagulates at the ordinary temperature more or less quickly and yields a typical fibrin. Besides the fibrin ferment the presence of neutral salts is necessary, for without them AlEx. SCHMidT has shown the coagulation of fibrin does not take place. The amount of fibrin obtained 
on coagulation is always smaller than the amount of fibrinogen from which the fibrin is derived, and we always find a small amount of globulin substance in the solution. It is therefore not improbable that the coagulation of fibrin, in accordance with the views of DeNrs, is a splitting process in which the soluble fibrinogen is split into an insoluble albuminous body, the fibrin, which forms the chief mass, and a soluble globulin substance, which is only formed in small amounts. The globulin substance, which is called "fibrin globulin" by the author, coagulates at $+64^{\circ} \mathrm{C}$. and has the following composition : C 52.70\% ; H 6.98\% ; N 16.06\%.

The coagulation of the blood consists chiefly in the conversion of the fibrinogen of the plasma into fibrin. The coagulation of the blood is a much more complicated process than the coagulation of a fibrinogen solution, inasmuch as the first involves other important questions, as, for instance, the reason for the blood remaining fluid in the body, the origin of the fibrin ferment, and the importance of the form-elements in the coagulation. A fuller discussion of the various hypotheses and theories concerning the coagulation of the blood must therefore be given later.

Serum Globulin, also called paraglobulin (KüHNE), fibrinoplastic substance (Alex. Schmidt), serum casein (PANUM), fibrine soluble (DENIs), occurs in the plasma, serum, lymph, transudations and exudations, in the white and red corpuscles, and probably in many animal tissues and form-elements, though in small quantities. It is also found in the urine in many diseases.

Serum globulin has the general properties of the globulins. In a moist condition it forms a snow-white flaky mass neither tough nor elastic. The essential differences between serum globulin and fibrinogen are the following: Serum-globulin solutions are only incompletely precipitated by adding $\mathrm{NaCl}$ to saturation, and not precipitated at all by an equal volume of a saturated common-salt solution. The coagulation temperature is, with $5-10 \% \mathrm{NaCl}$ in solution, $+75^{\circ} \mathrm{C}$. It is completely precipitated by $\mathrm{MgSO}_{4}$ in substance added to saturation, as also by an equal volume of a saturated solution of ammonium sulphate. The specific rotary power, according to FRÉDERICQ, for serum globulin (from-ox blood) solutions containing salt is $\alpha(\mathrm{D})=-47.8^{\circ}$. 
Serum globulin may be easily separated as a fine flocculent precipitate from blood-serum by neutralizing or making faintly acid with acetic acid and then diluting with 10-20 vols. of water. For further purification this precipitate is dissolved in dilute commonsalt solution, or in water by the aid of the smallest possible amount of alkali, and then reprecipitated by diluting with water or by the addition of a little acetic acid. The serum globulin may also be separated from the serum by means of magnesium or ammonium sulphate; in these cases it is difficult to completely remove the salt by dialysis. The serum globulin from blood-serum is always contaminated by lecithin and the so-called fibrin ferment. A serum globulin free from fibrin ferment may be prepared from fermentfree transudations, as sometimes from hydrocele fluids, and this shows that the serum globulin and the fibrin ferment are different bodies. For the detection and the quantitative estimation of serum globulin we may use the precipitation by magnesium sulphate added to saturation (AUTHOR), or by an equal volume of a saturated neutral ammonium sulphate solution (HOFMEISTER and KAUDER and $\mathrm{POHL}$ ). In the quantitative estimation the precipitate is collected on a weighed filter, washed with the salt solution employed, dried with the filter at about $115^{\circ} \mathrm{C}$., then washed with boilinghot water, so as to completely remove the salt, extracted with alcohol and ether, dried, weighed and burnt to determine the ash.

Serum Albumin is found in large quantities in blood-serum, blood-plasma, lymph, transudations, and exudations. Probably it also occurs in other animal liquids and tissues. The albumin which passes into the urine under pathological conditions consists largely of serum albumin.

In the dry state serum albumin forms a transparent, gummy; brittle, hygroscopic mass, or a white powder which may be heated to $100^{\circ} \mathrm{C}$. without decomposing. Its solution in water gives the ordinary reactions for albumin; the specific rotary power for serum albumin free from paraglobulin, obtained from human transudations, is, according to STARK, $\alpha(\mathrm{D})=-62.6^{\circ}$ to $-64.6^{\circ}$. The coagulation temperature of a serum-albumin solution is $+70^{\circ}$ to $+75^{\circ} \mathrm{C}$., according to most authorities, but this varies to a great extent with a varying concentration and amount of salt. A 1-2\% albumin solution may, in the presence of very little $\mathrm{NaCl}$, coagulate at $+50^{\circ} \mathrm{C}$. or below; in the presence of $5 \% \mathrm{NaCl}$ it coagulates 
at $+75^{\circ}$ to $+90^{\circ} \mathrm{C}$. By the careful addition of acid the coagulation temperature may be lowered; by the addition of alkali it may be raised. In blood-serum from certain animals and in human transudations HALLIBURTON found the coagulation to take place on heating to the following temperatures: $+70^{\circ}$ to $73^{\circ} \mathrm{C}$; $777^{\circ}$ to $78^{\circ} \mathrm{C}$; and $82^{\circ}$ to $85^{\circ} \mathrm{C}$. He therefore considers the serum albumin a mixture of three albumins, $\alpha$, $\beta$, and $\gamma$, which coagulate at the three points mentioned. In cold-blooded animals he found only the albumin $\alpha$.

The serum albumin differs from the albumin of the white of the hen's egg in the following particulars: it is more lævogyrate; the precipitate formed by hydrochloric acid easily dissolves in an excess of the acid; it is much less insoluble in alcohol; and lastly it acts differently inside of the organism. If egg-albumin is introduced into the blood system it passes into the urine, while the serum albumin does not. A solution of serum albumin positively free from mineral bodies has never yet been prepared. A solution as poor as possible in salts does not coagulate either on boiling or on the addition of alcohol. After the addition of a little common salt it coagulates in both cases.

In preparing serum albumin, first remove the globulins by saturating with magnesium sulphate at about $+30^{\circ} \mathrm{C}$., and filter at the same temperature. The cooled filtrate is separated from the crystallized salt and is treated with acetic acid of about $1 \%$ The precipitate formed is filtered, pressed, dissolved in water with the addition of alkali to neutral reaction, and the solution freed from salt by dialysis. The serum albumin may also be separated from the filtrate saturated with magnesium sulphate by adding sodium sulphate to saturation at about $+40^{\circ} \mathrm{C}$. The pressed precipitate is also in this case dissolved. in water and the solution freed from salt by dialysis. The albumin may be obtained in a solid form from the dialyzed solution either by evaporating the solution to dryness at gentle heat or by precipitating with alcohol, which must be removed quickly. In the detection and quantitative estimation of serum albumin, the filtrate from the globulins which have been removed by magnesium sulphate is heated to boiling, after the addition of a little acetic acid if necessary. The simplest way is to consider the difference between the total albumins and the globulins as serum albumin. 
Summary of the elementary composition of the above mentioned and described albuminous bodies:

\begin{tabular}{|c|c|c|c|c|c|c|}
\hline Fibrinogen...... & $\begin{array}{c}\text { C } \\
52.93\end{array}$ & $\underset{6.90}{H}$ & $\underset{16.66}{\mathrm{~N}}$ & $\begin{array}{c}\mathrm{S} \\
1.25\end{array}$ & $\begin{array}{c}0 \\
22.26\end{array}$ & IMARSTEN.) \\
\hline & 52.68 & 6.83 & 1691 & 1.10 & 22.48 & “ \\
\hline obulin. & 52.70 & 698 & 16.06 & & & “. \\
\hline ulin. & & 7.0 & 15. & 1.1 & 23.24 & “ \\
\hline rum albumin (1) & 53.06 & 6.8 & 16.04 & 1.80 & 22.26 & “ \\
\hline rum albumin & & 6.65 & 15.88 & 2.25 & 22.95 & ، \\
\hline
\end{tabular}

The serum albumin (2) came from a human exudation, and the other bodies from the blood of a horse. 'The tibrin was prepared from a filtered commonsalt plasma.

\section{The Blood-serum.}

As above stated, the blood-serum is the clear liquid which is pressed out by the contraction of the blood-clot. It differs chiefly from the plasma in the absence of fibrinogen and the presence of a little fibrin globulin and an abundance of fibrin ferment. Considered qualitatively the blood-serum contains the same chief constituents as the blood-plasma.

If undiluted serum be sufficiently acidified with acetic acid, a precipitate is obtained consisting of partly unchanged serum globulin, fibrin globulin, lecithin, and, in some cases, coloring matters (bile coloring matters in the serum of the horse). By the same process WooLDRIDGE precipitated from the blood-serum of the sheep and $\operatorname{dog}$ a substance which is closely related to fibrinogen and was called by him "serum fibrinogen."

Blood-serum is a sticky liquid which is more alkaline than the plasma. The specific gravity in man is $1.02 \%$ to 1.032 , average 1.028 The color is strongly or faintly yellow; in human blood-serum it is pale yellow with a shade towards green, and in horses it is often amber-yellow. The serum is ordinarily clear; after a meal it may be opalescent, cloudy, or milky-white, according to the amount of fat contained in the food.

Besides the above-mentioned bodies, the following constituents are found in the blood-plasma or blood-serum:

Fat occurs from 1-r p. m. in fasting animals. After partaking of food the amount is increased, and if the food is rich in fat as much as 12.5 p. m. has been found in the blood of dogs (RöHRIG). We also find soaps (HopPe-SEYLER), cholesterin, and lecithin.

Glucose seems to be a physiological constituent of the plasma. According to the investigations of ABELES, EwALD, KüLZ, and SEEGEN, the sugar found in the plasma is glucose. OTto found in the plasma, besides glucose, another reducing, non-fermentable substance. The amount of glucose in the blood is about 1-1.5 p. m. 
OTro found in human blood $1.18 \mathrm{p} . \mathrm{m}$. glucose and $0.29 \mathrm{p}$. m. of the other reducing substance. The amount of glucose in the blood seems to be almost independent of the food; nevertheless after feeding with large quantities of glucose and dextrin BLEILE observed a significant increase of glucose. If the amount is more than 3 p. m., according to CL. BERNARD, the glucose passes into the urine, producing glycosuria. The different amounts of glucose in the blood from different vessels and under various conditions will be fully discussed later.

Among the bodies which are found in the blood and without doubt met with in smaller or greater amounts in the plasma are to be mentioned urea, uric acid (found in human blood by ABELES), creatin, carbamic acid, paralactic acid, and hippuric acid. Under pathological conditions the following have been found: hypoxanthin, leucin, tyrosin, and biliary constituents.

The coloring malters of the blood-serum are very little known. In equine blood-serum biliary coloring matters, bilirubin, besides other coloring matters, occur. The yellow coloring matter of the serum seems to belong to the group of luteins, which are often called lipochromes or fat-coloring matters. From ox-serum KRUKENBERG was able to isolate with amyl alcohol a so-called lipochrome whose solution shows two absorption-bands, of which one encloses the line $F$ and the other lies between $F$ and $G$. Halliburton found in the blood of birds and amphibia a yellow coloring matter which only showed one absorption-band.

The mineral bodies in serum and plasma are qualitatively, but not quantitatively, the same. A part of the calcium, magnesium, and phosphoric acid is removed on the coagulation of the fibrin. By means of dialysis, the presence of sodium chloride, which forms the chief mass or $60-70 \%$ of the total mineral bodies, also lime salts, sodium carbonate, besides traces of sulphuric and phosphoric acids and potassium, may be shown in the serum. Traces of silicic acid, fluorine, copper, iron, manganese, and ammonia are claimed to have been found in the serum. As in most animal fluids, the chlorine and sodium are in the blood-serum in excess of the phosphoric acid and potassium (the occurrence of which in the serum is even doubted). The acids found in the ash are not sufficient to saturate the bases found, a condition which shows that a part of the bases is combined with organic substances, perhaps albumin. 
The gases of the blood-serum, which consist chiefly of carbon dioxide with only a little nitrogen and oxygen, will be described when treating of the gases of the blood.

Because of the difficulty of obtaining plasma only a few analyses have been made. As an example the results of the analyses of the blood-plasma of the horse will be given below. The analysis No. 1 was made by HopPe-SEYler. No. 2 is the average of the results of three analyses made by the AUTHOR. The figures are given in 1000 parts of the plasma.

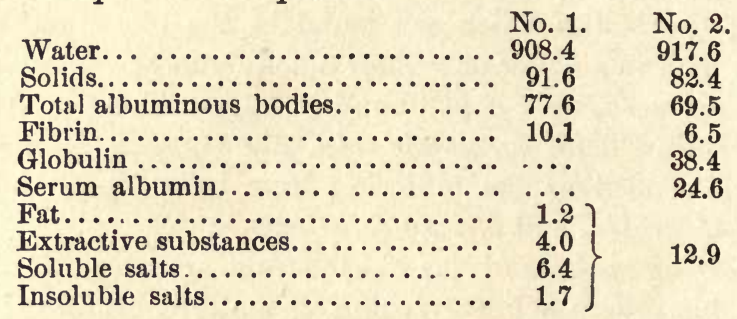

As an example of the constitution of the blood-serum with special regard to the relationship of the different albuminous bodies to each other, the following analyses are given. The results are in 1000 parts.

\begin{tabular}{|c|c|c|c|c|c|c|c|}
\hline $\begin{array}{l}\text { Serum } \\
\text { from }\end{array}$ & Solids. & $\begin{array}{c}\text { Total } \\
\text { Albumin- } \\
\text { ous } \\
\text { Bodies. }\end{array}$ & $\begin{array}{c}\text { Serum } \\
\text { Globulin }\end{array}$ & $\begin{array}{l}\text { Serum } \\
\text { Albumin. }\end{array}$ & $\begin{array}{l}\text { Lecithin, } \\
\text { Fat, } \\
\text { Salts, etc. }\end{array}$ & 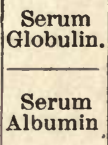 & Authority. \\
\hline Man.... & 92.07 & 76.20 & 31.04 & 45.16 & 15.88 & $\frac{1}{1.5}$ & HAMMARSTEN \\
\hline Horse.. & 85.97 & 72.57 & 45.65 & 26.92 & 13.40 & $\frac{1}{0.591}$ & "“ \\
\hline $\mathrm{Ox} \ldots .$. & 89.65 & 74.99 & 41.69 & 33.30 & 14.66 & $\frac{1}{0.842}$ & “ \\
\hline Dog.... & $\ldots \ldots$ & 58.20 & 20.50 & 37.70 & $\ldots$. & $\frac{1}{1.8}$ & SERToLI \\
\hline Hen.... & 54.00 & 39.49 & 7.84 & 31.65 & 14.51 & $\frac{1}{4.03}$ & HAMMARSTEN \\
\hline Frog... & $\cdots \cdots$ & 25.40 & 21.80 & 3.60 & $\cdots \cdots$ & $\frac{1}{0.165}$ & HALLIBURTON \\
\hline Eel..... & $\cdots \cdots$ & 67.30 & 52.80 & 14.50 & $\cdots \cdots$ & $\frac{1}{0.275}$ & 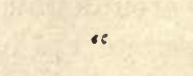 \\
\hline
\end{tabular}


According to HALLIBURTON, the amount of the albumins in comparison with the globulins in cold-blooded animals is not only proportionally smaller, but the total amount of albuminous bodies is smaller than in the warm-blooded animals.

By a comparative investigation of serum and plasma from the same individual, we find more serum globulin in the one than in the other. The reason for this may lie partly in the fact that in the coagulation of fibrin from the fibrinogen some fibrin globulin is formed which in the quantitative estimation is precipitated with the serum globulin, and partly because the white corpuscles yield serum globulin in the fibrin coagulation (Alex. Schmidt).

The quantity of mineral bodies in the serum has been determined by many investigators.

The conclusion drawn from the analyses is that there exists a rather close correspondence between human and animal blood-serum, and it is therefore sufficient to give here the analysis of C. ScHMIDT of (1) human blood, and of (2) pig- and (3) ox-blood by Bunge. As in the calcination of lecithin and albumins incorrect results are obtained for the phosphoric and sulphuric acids, these result will not be given below. All figures correspond to 1000 parts of serum.

$\begin{array}{lcccc} & & 1 & 2 & 3 \\ \mathrm{~K}_{2} \mathrm{O} \ldots \ldots \ldots \ldots \ldots \ldots \ldots & 0.392 & 0.273 & 0.254 \\ \mathrm{Na}_{2} \mathrm{O} \ldots \ldots \ldots \ldots \ldots \ldots \ldots & 4.462 & 4.272 & 4.351 \\ \mathrm{Cl} \ldots \ldots \ldots \ldots \ldots \ldots \ldots & 3.612 & 3.611 & 3.717 \\ \mathrm{CaO} \ldots \ldots \ldots \ldots \ldots \ldots \ldots & 0.163 & 0.136 & 0.126 \\ \mathrm{MgO} \ldots \ldots \ldots \ldots \ldots \ldots & 0.101 & 0.038 & 0.045\end{array}$

The amount of $\mathrm{NaCl}$ is $6-7 \mathrm{p} . \mathrm{m}$., and it is remarkable that this amount of $\mathrm{NaCl}$ remains almost constant, so that with food containing an excess of $\mathrm{NaCl}$ it is quickly eliminated by the urine, and with food poor in chlorides the amount in the blood first decreases, but increases after taking chlorides from the tissues. The secretion of chlorides by the urine is thereby diminished.

The amount of phosphoric acid, calculated as $\mathrm{Na}_{2} \mathrm{HPO}_{4}$, in the serum freed from lecithin has been determined as $0.02-0.09 \mathrm{p} . \mathrm{m}$. by Sertoli and Mroczkowski in different varieties of serum. The small amount of iron sometimes found in the serum probably originates from a contamination with the blood-coloring matters. 


\section{The Form-elements of the Blood.}

\section{The Red Blood-corpuscles.}

The blood-corpuscles are round, biconcave disks without membrane and nucleus in man and mammalia (with the exception of the llama, the camel, and their congeners). In the latter animals, as also in birds, amphibia, and fishes (with the exception of the cyclostoma), the corpuscles have in general a nucleus, are biconvex and more or less elliptical. The size varies in different animals. In man they have an average diameter of 7 to $8 \mu(\mu=0.001 \mathrm{~m} . \mathrm{m}$. $)$ and a maximum thickness of $1.9 \mu$. Their specific gravity is 1.088 to 1.089 (C. SchMIDT) or 1.105 (WELCKER). They are heavier than the blood-plasma or serum, and therefore sink in these liquids. In the discharged blood they may lie sometimes with their flat surfaces together, forming a cylinder like a roll of coin. The reason for this is unknown, but as it may be observed in defibrinated blood it seems probable that the formation of fibrin has nothing to do with it. Seen with the microscope, each blood-corpuscle has a pale yellow color, and only in moderately thick layers is the color somewhat reddish.

The number of red blood-corpuscles is different in the blood of various animals. In the blood of man there are generally 5 million red corpuscles in $1 \mathrm{c.mm}$., and in woman 4 to 4.5 million.

On diluting the blood with water and alternately freezing and thawing it, as also on shaking it with ether, or by the action of chloroform or bile, a remarkable change takes place. The bloodcoloring matters, which are hardly free in the blood-corpuscles, but rather, according to the view of HOPPE-SEYLER, are combined with some other substance, perhaps lecithin, are by this means set free from these combinations and pass into solution, while the remainder of each blood-corpuscle forms a swollen mass. By the action of carbon dioxide, by the careful addition of acids, acid salts, tincture of iodine, or certain other bodies, this residue, rich in albumin, condenses and in many cases the form of the blood-corpuscles may be again obtained. This residue has been called the stroma of the red blood-corpuscles. 
To isolate the stromata of the blood-corpuscles they are washed first by diluting the blood with 10-20 vols. of a 1-2\% common-salt solution and then separating the mixture by centrifugal force or by allowing it to stand at a low temperature. 'This is repeated a few times until the blood-corpuscles are freed from serum. These purified blood-corpuscles are, according to WooLDRIDGE, mixed with 5-6 vols. of water and then a little ether is added until complete solution is obtained. The leucocytes gradually settle to the bottom, a movement which may be accelerated by centrifugal force, and the liquid which separates therefrom is very carefully treated with a $1 \%$ solution of KHSO, until it is about as dense as the original blood. The separated stromata is collected on a filter and quickly washed.

WOOLDRIDGE found as constituents of the stroma lecithin, cholesterin, and a globulin which, according to HALLIBURTON, is the above-mentioned (page 58) fibrinoplastic acting cell-globulin. Nucleo-albumin and albumoses could not be detected (HaLLIBURTON). The nucleated red blood-corpuscles of the bird contain, according to PLOS'z and HOPPE-SEYLER, nuclein and an albuminous body which swells to a slimy mass in a $10 \%$ common-salt solution and which seems to be closely related to the hyaline substance (hyaline substance of RovIDA) occurring in the lymph-cells. The red blood-corpuscles without any nucleus are, as a rule, very poor in albumin but are rich in hæmoglobin; the nucleated corpuscles are richer in albumin and poorer in hæmoglobin.

A gelatinous, albuminous, fibrin-like body may be obtained from the red blood-corpuscles. This fibrin-like mass has been observed on freezing and then thawing the sediment of the blood-corpuscles, or on discharging the spark from a large Leyden jar through the blood, or on dissolving the blood-corpuscles of one kind of animal in the serum of another (LANDoIs, stroma fibrin). In none of these cases has it been shown that we have to deal with a fibrin formation at the expense of the stroma. It seems only to have been shown that the red blood-corpuscles of frog's blood contain fibrinogen (Alex. Schmidt and Semmer).

The mineral bodies of the red corpuscles are chiefly potassium, phosphoric acid, and chlorine; in the red corpuscles of man, dog, and the ox sodium has also been found. 
The most important constituent of the blood-corpuscles from a physiological standpoint is the red coloring matter.

\section{Blood-coloring Matters.}

According to HOPPE-SEYLER the coloring matter of the red blood-corpuscles is not in a free state but combined with some other substance. The crystalline coloring matter, the hæmoglobin or oxyhæmoglobin, which may be isolated from the blood, is considered, according to HOPPE-SEYLER, as a splitting product of this combination, and it acts in many ways like the questionable combination itself. This combination is insoluble in water and uncrystallizable. It strongly decomposes hydrogen peroxide without being oxidized itself; it shows a greater resistance to certain chemical reagents (as potassium ferricyanide) than the free coloring matter, and lastly it gives off its loosely-combined oxygen much more easily in vacuum than the free coloring matter. To distinguish between the splitting products, the hæmoglobin and the oxyhæmoglobin, we may call the combination of the blood-coloring matter of the venous blood-corpuscles phlebin, and that of the arterial arterin (HoPPE-SEYLER). Since the above-mentioned combination of the blood-coloring matters with other bodies, for example (if they really do exist) with lecithin, have not been closely studied, the following statements will only apply to the free coloring matter, the hæmoglobin.

The color of the blood depends in part on haemoglobin and in part on a molecular combination of this with oxygen, the oxyhomoglobin. We find in blood after suffocation almost exclusively hæmoglobin, in arterial blood disproportionately large amounts of oxyhæmoglobin, and in venous blood a mixture of both. Blood-coloring matters are found also in striated as well as in certain smooth muscles, and lastly in solution in different invertebrata. The quantity of hæmoglobin in human blood may indeed be somewhat variable under different circumstances, but amounts averaging about $14 \%$ or 8.5 grammes have been determined for each kilo of the weight of the body.

The hæmoglobin belongs to the group of proteids, and yields as splitting products, besides very small amounts of volatile fatty acids 
and other bodies, chiefly albumin (96\%), and a coloring matter, hoemochromogen (4\%), containing iron, which in the presence of oxygen is easily oxidized into homatin.

The hæmoglobin prepared from different kinds of blood has not exactly the same constitution, which seems to indicate the presence of different hæmoglobins. The analyses of different investigators of the hæmoglobin from the same kind of blood do not always agree with one another, which probably depends upon the somewhat various methods of preparation. The following analyses are given as examples of the constitution of different hæmoglobins:

\begin{tabular}{|c|c|c|c|c|c|c|c|}
\hline $\begin{array}{l}\text { Hæmoglobi } \\
\text { from the }\end{array}$ & in $\mathrm{C}$ & H & $\mathrm{N}$ & $\mathbf{S}$ & $\mathrm{Fe}$ & 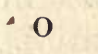 & $\mathrm{P}_{2} \mathrm{O}_{6}$ \\
\hline $\operatorname{Dog} \ldots .$. & 53.85 & 7.32 & 16.17 & 0.390 & 0.430 & 21.84 & .... (HOPPE-SEYLER. \\
\hline & & & & & & & JAOUET) \\
\hline Horse & & 6.97 & & & & 19. & KOSSEL.) \\
\hline & & 6. & & & $0.2^{2}-x-1$ & 23.43 & . (ZINOFFSKY.) \\
\hline$\ldots$ & & & & & 0.400 & 19.5 & . (HÜFNER.) \\
\hline Pig...... & & 7.38 & 16.23 & 0.660 & 0.430 & 21.360 & (тто.) \\
\hline & & 7.38 & 17. & 0.479 & 0.399 & 19. & (HÜFNER.) \\
\hline & & & & & 0.480 & & . . (HOPPE-SEYLER.) \\
\hline & & 7.39 & 16. & & 0.5 & & “ \\
\hline & & 7. & & & & & $0.77 \quad$ " \\
\hline & & 7.19 & 16.45 & 0.857 & 0.335 & 22.500 & 0.197 (JACQUET.) \\
\hline
\end{tabular}

The question whether the amount of phosphorus in the hæmoglobin from birds exists as a contamination or as a constituent has not been decided. In the hæmoglobin from the horse (ZINOFFSKY), the pig, and the ox (HÜFNER) we have 1 atom of iron to 2 atoms of sulphur, while in the hæmoglobin from the dog (JACQUET) the relation is 1 to 3. From the data of the elementary analysis, as also from the amount of loosely-combined oxygen, HÜFNER has calculated the molecular weight of dog-hæmoglobin as 14,129 and the formula $\mathrm{C}_{686} \mathrm{H}_{1025} \mathrm{~N}_{164} \mathrm{FeS}_{3} \mathrm{O}_{181}$. The molecular weight is therefore very high. The hæmoglobin from various kinds of blood not only show a diverse constitution, but also a different solubility and crystalline form, and a varying quantity of water of crystallization, from which we infer that there are several kinds of hæmoglobin.

0xyhæmoglobin, which has also been called HжMatogLobULiN or HAMATOCR YSTALLIN, is a molecular combination of hæmoglobin and oxygen. For each molecule of hæmoglobin 1 molecule of oxygen exists; and the amount of loosely-combined oxygen which is united to $1 \mathrm{grm}$. oxyhæmoglobin (of the dog) has been determined by 
HÜFNER as 1.582 c. c. (at $0^{\circ} \mathrm{C}$. and $760 \mathrm{~m} \cdot \mathrm{m} . \mathrm{Hg}$ ). The ability of hæmoglobin to take up oxygen seems to be a function of the iron it contains, and when this is calculated as about $0.33-0.40 \%$, then 1 atom of iron in the hæmoglobin corresponds to about 2 atoms $=1$ molecule of oxygen. The combination of hæmoglobin with oxygen is, as has been stated, loose and dissociatable, and the quantity of oxygen taken up by a hæmoglobin solution depends upon the pressure of the oxygen at that temperature. If this latter be decreased by means of a vacuum, especially on gently heating or by passing some indifferent gas through the solution, all of the oxygen may be expelled from an oxyhæmoglobin solution so that only hæmoglobin remains. The reverse of this is true of a hæmoglobin solution which by its remarkable attraction for oxygen may be converted into oxyhæmoglobin. Oxyhæmoglobin is generally considered as a weak acid.

Oxyhæmoglobin has been obtained in crystals from several varieties of blood. These crystals, first observed by REICHERT and FUNKE, are blood-red, transparent, silky, and may be 2-3 m.m. long. The oxyhæmoglobin from squirrel's blood crystallizes in sixsided plates of the hexagonal system; the other varieties of blood yield needles, prisms, tetrahedra, or plates which belong to the rhombic system. The quantity of water of crystallization varies. between 3-10\% for the different oxyhæmoglobins. When completely dried at a low temperature over sulphuric acid the crystals may be heated to $110^{\circ}-115^{\circ} \mathrm{C}$. without decomposing. At higher temperatures, somewhat above $160^{\circ} \mathrm{C}$., they decompose, giving an odor of burnt horn, and leave, after complete combustion, an ash consisting of oxide of iron. The oxyhæmoglobin crystals from difficultly-crystallizable kinds of blood, for example from such as ox's, human, and pig's blood, are easily soluble in water. The oxyhæmoglobin from easily-crystallizable blood, as from that of the horse, dog, squirrel, and guinea-pig, are soluble with difficulty in the order above given. The oxyhæmoglobin dissolves more easily in a very dilute solution of alkali carbonate than in pure water, and this solution may be kept. The presence of a little too much alkali causes the oxyhæmoglobin to quickly decompose. The crystals are insoluble without decolorization in absolute alcohol. According to NENCKI, it is converted 
into an isomeric or polymeric modification, called by him parahcemoglobin. Oxyhæmoglobin is insoluble in ether, chloroform, benzol, and carbon disulphide.

A solution of oxyhæmoglobin in water is not precipitated by many metallic salts, but is precipitated by sugar of lead. On heating the watery solution it decomposes at $60^{\circ}$ to $70^{\circ} \mathrm{C}$., and it splits off albumin and hæmatin. It is also decomposed by acids, alkalies, and many metallic salts. It gives the ordinary reactions for albumin with the albumin reagents which first decompose the oxyhæmoglobin with the splitting off of albumin.

The oxyhæmoglobin may, when it is gradually oxidized, act as an "ozone exciter" by the disjoining of neutral oxygen, making the oxygen active (PFLÜGER). It may also have another relation to ozone, since it has the property of an "ozone transmitter" in that it causes the reaction of certain reagents (turpentine) containing ozone upon ozone reagents such as tincture of guaiacum (ScHöNBEIN, His). According to KowalewsKy, it is not ozone but an oxidation-product of turpentine that we have to deal with; but this question requires further proof.

A sufficiently dilute solution of oxyhæmoglobin or arterial blood shows a spectrum with two absorption-bands between the FRAUNHOFER lines $D$ and $E$. The one band $\alpha$, which is narrower but darker and sharper, lies on the line $D$; the other, broader, less defined and less dark band $\beta$, lies at $E$. These bands can be detected in a.layer of $1 \mathrm{~cm}$. of a $0.1 \mathrm{p}$. m. solution of oxyhæmoglobin. In a still weaker dilution the band $\beta$ first disappears. By increased concentration of the solution the two bands become broader, the space between them smaller or entirely obliterated, and at the same time the blue and violet part of the spectrum is darkened. The oxyhæmoglobin may be differentiated from other coloring matters having a similar absorption-spectrum by its behavior towards reducing substances. (See below.)

A great many methods have been proposed for the preparation of oxyhæmoglobin crystals, but in their chief features they all agree with the following method as suggested by HOPPE-SEYLER : The washed blood-corpuscles (best those from the dog or the horse) are stirred with 2 vols. water and then shaken with ether. After decanting the ether and allowing the ether which is retained by 
the blood solution to evaporate in an open dish in the air, cool the filtered blood solution to $0^{\circ} \mathrm{C}$., add while stirring $\frac{1}{4}$ vol. of alcohol also cooled, and allow to stand a few days at $-5^{\circ}$ to $-10^{\circ} \mathrm{C}$. The crystals which separate may be repeatedly recrystallized by dissolving in water of about $35^{\circ} \mathrm{C}$, cooling and adding cooled alcohol as above. Lastly, they are washed with cooled water containing alcohol ( $\frac{1}{4}$ vol. alcohol) and dried in vacuum at $0^{\circ} \mathrm{C}$. or a lower temperature. According to GscheidLen's investigations, oxyhæmoglobin crystals may be obtained from difficulty crystallizable varieties of blood by allowing the blood first to putrify slightly in sealed tubes. After shaking with air by which the blood is again arterialized, proceed as above.

For the preparation of oxyhæmoglobin crystals in small quantities from blood easily crystallized, it is often sufficient to stir a drop of blood with a little water on a microscope slide and allow the mixture to evaporate so that the drop is surrounded by a dried ring. After covering with a thin glass, the crystals gradually appear radiating from the ring. These crystals are formed in a surer manner if the blood is first mixed with some water in a test-tube and shaken with ether and a drop of the lower deep-colored liquid treated as above on the slide.

Hæmoglobin, also called Reduced Hæmoglobin or Purple CrUorin (STOKES), occurs only in very small quantities in arterial blood, in larger quantities in venous blood, and is nearly the only blood-coloring matter after suffocation.

Hæmoglobin is much more soluble than the oxyhæmoglobin, and it can therefore only be obtained as crystals with difficulty. These crystals are as a rule isomorphous to the corresponding oxyhæmoglobin crystals, but are darker, having a shade towards the blue or purple, and are decidedly more pleochromatic. Its solutions in water are darker and more violet or purplish than solutions of oxyhæmoglobin of the same concentration. They absorb the blue and the violet rays of the spectrum in a less marked degree, but strongly absorb the rays lying between $C$ and $D$. In proper dilution the solution shows a spectrum with one broad, not sharply-defined band between $D$ and $E$. This band does not lie in the middle between $D$ and $E$, but is towards the red end of the spectrum, a little over the line $D$. A hæmoglobin solution actively absorbs oxygen from the air and is converted into an oxyhæmoglobin solution.

A solution of oxyhæmoglobin may be easily converted into a hæmoglobin solution by means of a vacuum, by passing an indiffer- 
ent gas through, or by the addition of a reducing substance, as, for example, an ammoniacal ferrotartrate solution (STOKES' reductionliquid). If an oxyhæmoglobin solution or arterial blood is kept in a sealed tube, we observe a gradual reduction of the oxyhæmoglobin into hæmoglobin. If the solution has a proper concentration, a crystallization of hæmoglobin may occur in the tube at lower temperatures (HÜFNER).

Carbon Monoxide Hæmoglobin is the molecular combination between $1 \mathrm{~mol}$. hæmoglobin and $1 \mathrm{~mol}$. CO. This combination is stronger than the oxygen combination of hæmoglobin. The oxygen is for this reason easily driven off by carbon monoxide, and this explains the poisonous action of carbon monoxide, which kills by the expulsion of the oxygen of the blood.

Carbon monoxide hæmoglobin is formed by saturating blood or a hæmoglobin solution with carbon monoxide, and may be obtained as crystals by the same means as oxyhæmoglobin. These crystals are isomorphous to the oxyhæmoglobin crystals, but are less soluble, more constant, and their bluish-red color is more marked. For the detection of carbon monoxide hæmoglobin its absorption spectrum is of the greatest importance. This spectrum shows two bands which are very similar to those of oxyhæmoglobin, but they occur more towards the violet part of the spectrum. These bands do not change noticeably on the addition of reducing substances; this constitutes an important difference between carbon monoxide and oxyhæmoglobin. If the blood contains oxyhæmoglobin and carbon monoxide hæmoglobin at the same time, we will obtain on the addition of a reducing substance (ammoniacal ferrotartrate solution) a mixed spectrum originating from the hæmoglobin and carbon monoxide hæmoglobin.

A great many reactions have been suggested for the testing of carbon-monoxide hæmoglobin in medico-legal cases. A simple and at the same time a good one is HoPPE-SEYLER's soda test. The blood is treated with double its volume of caustic-soda solution of $1.3 \mathrm{sp}$. gr., by which ordinary blood is converted into a dingy brownish mass, which when spread out on porcelain is brown with a shade of green. Carbon-monoxide blood gives under the same conditions a red mass, which if spread out on porcelain shows a beautiful red color. Several modifications of this test have been proposed.

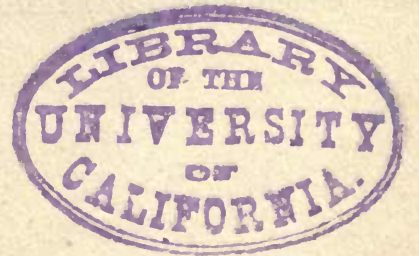


Nitric-oxide Hæmoglobin is also a crystalline molecular combination which is even stronger than the carbon-monoxide hæmoglobin. Its solution shows two absorption-bands which are paler and less sharp than the carbon-monoxide hæmoglobin bands, and they disappear on the addition of reducing bodies.

Hæmoglobin forms also a molecular combination with acetylene. It is also claimed that it forms a combination with hydrocyanic acid.

Carbon - dioxide Hæmoglobin. Hæmoglobin forms a molecular combination with carbon dioxide whose spectrum is similar to that of hæmoglobin (ToRUP). By the action of carbon dioxide on hæmoglobin part of the latter is decomposed with the separation of albumin (BoHR, ToRUP), and the combination seems to be rather carbon-dioxide hæmochromogen (see below : Hæmochromogen).

Methæmoglobin. This name has been given to a coloring matter which is easily obtained from the oxyhæmoglobin as a transformation product and which has been correspondingly found in transudations and cystin fluids containing blood, in urine, in hæmaturia or hæmoglobinuria, also in urine and blood on poisoning with potassium chlorate, amyl nitrite or alkali nitrite, and many other bodies.

Methæmoglobin does not contain any oxygen in molecular or dissociable combination, but still the oxygen seems to be of importance in the formation of methæmoglobin. If arterial blood be sealed up in a tube, it gradually consumes its oxygen and becomes venous, and by this absorption of oxygen a little methæmoglobin is formed. The same occurs on the addition of a small quantity of acid to the blood. By the spontaneous decomposition of blood some methæmoglobin is formed, and by the action of ozone, potassium permanganate, potassium ferricyanide, and certain other bodies on the blood an abundant formation of methæmoglobin takes place.

According to a few investigators, among others SoRBY and JäDERHOLM, the methæmoglobin contains more oxygen than oxyhæmoglobin, while according to others, among whom may be mentioned HoPPE-SEYler, it contains less. HüFNER and OTTO claim that it contains just as much oxygen, but that it is more strongly combined. J̈̈DERHOLM and SAARBACH claim that a 
methæmoglobin is first converted into an oxyhæmoglobin and then into a hæmoglobin solution by reducing substances, while HoPPESEYLER claims that it is converted directly into a hæmoglobin solution.

Methæmoglobin has the same constitution as oxyhæmoglobin (HÜFNER and OTTO). It was first shown by them that it crystallizes in brownish-red needles, prisms, or six-sided plates. It dissolves easily in water ; the solution has a brown, color and becomes a beautiful red on the addition of alkali. The solution of the pure substance is not precipitated by lead acetate alone, but by lead acetate and ammonia. The absorption-spectrum of a watery or acidified solution of methæmoglobin is very similar to that of hæmatin in acid solution, but is easily distinguished from the latter since, on the addition of a little alkali and a reducing substance, the former passes over to the spectrum of reduced hæmoglobin, while a hæmatin solution under the same conditions gives the spectrum of an alkaline hæmochromogen solution (see below). Methæmoglobin in alkaline solution shows two absorptionbands which are like the two oxyhæmoglobin bands, but they differ from these in that the band $\beta$ is stronger than $\alpha$. By the side of the band $\alpha$ and united with it by a shadow lies a third, fainter band between $C$ and $D$, near to $D$.

Crystallized methæmoglobin may be easily obtained by treating a concentrated solution of oxyhæmoglobin with a sufficient quantity of concentrated potassium ferricyanide solution to give the mixture a porter-brown color. After cooling to $0^{\circ} \mathrm{C}$. add $\frac{1}{4}$ vol. cooled alcohol and allow the mixture to stand a few days in the cold. The crystals may be easily purified by recrystallizing from water by the addition of alcohol.

Carbon monoxide methæmoglobin has been prepared by WEYL and $\nabla$. ANSEP by the action of potassium permanganate on carbon monoxide hæmoglobin. Sulphur methæmoglobin is the same given by HOPPE-SEYLER to that coloring matter which is formed by the action of sulphuretted hydrogen on oxyhæmoglobin. The solution has a greenish-red, dirty color and shows an absorption-band in the red. This coloring matter is claimed to be the greenish color seen on the surface of putrefying flesh.

Decomposition products of the blood coloring matters. By its decomposition hæmoglobin yields, as above stated, albumin and a ferruginous coloring matter as chief products. If the decomposition takes place in the absence of oxygen, a coloring matter 
is obtained which is called by HOPPE-SEYLER hoemochromogen, by other investigators (STOKES) reduced homatin. In the presence of oxygen, hæmochromogen is quickly oxidized to hæmatin, and we obtain in this case as a colored decomposition product another coloring matter, homatin. As hæmochromogen is easily converted by oxygen into hæmatin, so this latter may be reconverted into hæmochromogen by reducing substances.

Hæmochromogen was discovered by HOPPE-SEYLER to whom of all investigators we are more indebted for our knowledge in regard to the blood-coloring matters and their decomposition products. $\mathrm{He}$ has also lately been able to obtain this coloring matter as crystals. Hæmochromogen is, according to HoPPE-SEYLER, the colored atomic group of hæmoglobin and its combination with gases, and this atomic group is combined with albumin in the coloring matter. The characteristic absorption of light depends on the hæmochromogen, and it is also this atomic group which binds in the oxyhæmoglobin $1 \mathrm{~mol}$. oxygen and in the carbon monoxide hæmoglobin 1 mol. carbon monoxide with 1 atom iron. HoppeSEYLER has observed a combination between hæmochromogen and carbon monoxide, and this combination shows the spectral appearance of carbon monoxide hæmoglobin.

An alkaline hæmochromogen solution has a beautiful red color. It shows two absorption-bands, first described by STokes, of which the one is darker and lies between $D$ and $E$, and the other, broader but not so dark, covers the lines $E$ and $b$. In acid solution hæmochromogen shows four bands, which according to J̈̈DERHOLM depend on a mixture of hæmochromogen and hæmatoporphyrin (see below), this last formed by a partial decomposition resulting from the action of the acid.

Hæmochromogen may be obtained as crystals by the action of caustic soda on hæmoglobin at $100^{\circ} \mathrm{C}$. in the absence of oxygen (HOPPE-SEYLER). By the decomposition of hæmoglobin by acids (of course in the absence of air) we obtain hæmochromogen contaminated with a little hæmatoporphyrin. An alkaline hæmochromogen solution is easily obtained by the action of a reducing substance (STOKES' reduction liquid) in an alkaline hæmatin solution.

Hæmatin, also called 0xyhæmatin, is sometimes found in old transudations. It is formed by the action of gastric or pancreatic 
juices on oxyhæmoglobin, and is therefore also found in the fæces after hemorrhage in the intestinal canal, and also after a meat diet and food rich in blood. It is stated that hæmatin may occur in urine after poisoning with arseniuretted hydrogen. As shown above, the hæmatin is formed by the decomposition of oxyhæmoglobin, or at least of hæmoglobin, in the presence of oxygen.

The constitution of hæmatin may, according to HOPPE-SEYLER, be expressed by the formula $\mathrm{C}_{34} \mathrm{H}_{35} \mathrm{~N}_{4} \mathrm{FeO}_{5}$. According to NENCKI and SIEBER it has the formula $\mathrm{C}_{32} \mathrm{H}_{52} \mathrm{~N}_{4} \mathrm{FeO}_{4}$, and they claim that hæmatin consists of a body not yet isolated, hæmin, $\mathrm{C}_{32} \mathrm{H}_{30} \mathrm{~N}_{4} \mathrm{FeO}_{3}$, with $1 \mathrm{~mol} . \mathrm{H}_{2} \mathrm{O}$.

Hæmatin is amorphous, dark brown or bluish black. It may be heated to $180^{\circ} \mathrm{C}$. without decomposition; on burning it leaves a residue consisting of iron oxide. It is insoluble in water, dilute acids, alcohol, ether, and chloroform, but it dissolves slightly in warm glacial acetic acid. Hæmatin dissolves in acidified alcohol or ether. It easily dissolves in alkalies, even when very dilute. The alkaline solutions are dichroitic; in thick layers they appear red by reflected light, and in thin layers greenish. The alkaline solutions are precipitated by lime- and baryta-water, as also by solutions of neutral salts of the alkaline earths. The acid solutions are always brown.

An acid hæmatin solution absorbs the red part of the spectrum less and the violet part more. The solution shows a rather sharplydefined band between $C$ and $D$ whose position may change with the variety of acid used as a solvent. Between $D$ and $F$ a second, much broader, less sharply-defined band occurs which by proper dilution of the liquid is converted into two bands. The one between $b$ and $F$, lying near $F$, is darker and broader, the other, between $D$ and $E$, lying near $E$, is lighter and narrower. Also by proper dilution a fourth very faint band is observed between $D$ and $E$ lying near $D$. Hæmatin may thus in acid solution show four absorption-bands; ordinarily one sees distinctly only the bands between $C$ and $D$ and the broad, dark band-or the two bands-between $D$ and $F$. In alkaline solution the hæmatin shows a broad absorption-band, which lies in greatest part between $C$ and $D$, but reaches a little over the line $D$ towards the right in the space between $D$ and $E$.

Hæmatin is dissolved by concentrated sulphuric acid in the 
presence of air, forming a purple-red liquid. The iron is here split off and the new coloring matter, called homatoporphyrin by HOPPE-SEYLER, is iron-free. The hæmatin yields with concentrated sulphuric acid, in the absence of air, a second iron-free coloring matter called hoematolin (HOPPE-SEYLER). Hæmatoporphyrin may also be prepared by the action of glacial acetic acid saturated with hydrobromic acid on hæmin crystals (NENCKI and SIEBER). This coloring matter is, according to NENCKI and Sieber, an isomer of the bile-coloring matter bilirubin, and the formula is, according to them, $\mathrm{C}_{16} \mathrm{H}_{18} \mathrm{~N}_{2} \mathrm{O}_{3}$. The formation of hæmatoporphyrin from hæmatin occurs according to the following equation:

$$
\mathrm{C}_{32} \mathrm{H}_{32} \mathrm{~N}_{4} \mathrm{O}_{4} \mathrm{Fe}+2 \mathrm{H}_{2} \mathrm{O}-\mathrm{Fe}=2\left(\mathrm{C}_{16} \mathrm{H}_{18} \mathrm{~N}_{2} \mathrm{O}_{3}\right) \text {. }
$$

The combinations of hæmatoporphyrin with $\mathrm{Na}$ and with $\mathrm{HCl}$ have been obtained as crystals by NENCKI and Sieber. The hæmatoporphyrin prepared by them does not seem to be identical with that prepared by HOPPE-SEYLER even though they have the same spectrum. A dilute solution with alkali carbonate shows a spectrum with four absorption-bands, namely, a band between $C$ and $D$; a second, broader, surrounding $D$ and with its broadest part between $D$ and $E$; a third, lighter and narrower, between $D$ and $E$; and lastly a fourth, broad and dark band between $b$ and $F$. By the action of reducing agents on hæmatoporphyrin a coloring matter has been obtained which stands closely related to urobilin (Hoppe-Seyler, NeNCKI and Sieber). Hæmatoporphyrin occurs also in the animal kingdom preformed (MACMUNN).

Hæmin, Hamin Crystals, or Teichmann's Crystals. Hæmin, according to HOPPE-SEYLER, is a combination between hæmatin and hydrochloric acid, having the formula $\mathrm{C}_{34} \mathrm{H}_{35} \mathrm{~N}_{4} \mathrm{FeO}_{5} \cdot \mathrm{HCl}$. NeNCKI and Sieber designate as hæmin, on the contrary (see page r7), a body not yet isolated, of the formula $\mathrm{C}_{32} \mathrm{H}_{30} \mathrm{~N}_{4} \mathrm{FeO}_{3}$, which may be considered as hæmatin $-\mathrm{H}_{2} \mathrm{O}$ or $\mathrm{C}_{32} \mathrm{H}_{32} \mathrm{~N}_{4} \mathrm{FeO}_{4}-\mathrm{H}_{2} \mathrm{O}$. The hæmin crystals are, according to the latest views, a combination of this substance, hæmin, and $\mathrm{HCl}$, according to the formula $\mathrm{C}_{32} \mathrm{H}_{30} \mathrm{~N}_{4} \mathrm{FeO}_{3}$. $\mathrm{HCl}$.

According to the same experimenters, the hæmin crystals are a double combination with the solvent, amyl alcohol or acetic acid, which is used in their preparation; while HOPPE-SEYLER claims that the solvent is only held me- 
chanically by the crystals. The formula of the hæmin crystals prepared by means of amyl alcohol is, according to Nencki and Sreber,

$\left(\mathrm{C}_{32} \mathrm{H}_{30} \mathrm{~N}_{4} \mathrm{FeO}_{3} . \mathrm{HCl}\right)_{4} . \mathrm{C}_{5} \mathrm{H}_{12} \mathrm{O}$.

Hæmin crystals form in large masses a bluish-black powder, but are so small individually that they can only be seen by the microscope. They consist of dark-brown or nearly brownish-black, long, rhombic, or spool-like crystals, isolated, or grouped as crosses, rosettes, or starry forms. They are insoluble in water, dilute acids at the normal temperature, alcohol, ether, and chloroform. They are slightly dissolved by glacial acetic acid and warmth. They dissolve in acidified alcohol, as also in dilute caustic or carbonated alkalies; and in the last case they form, besides alkali chlorides, soluble hæmatin alkali, from which the hæmatin may be precipitated by an acid.

The preparation of hæmin crystals is always the starting-point for the preparation of hæmatin. According to HOPPE-SEYLER, shake the blood-corpuscles which have been washed with commonsalt solution (see page 66) with water and ether, then filter the solution of blood-coloring matters, concentrate strongly, mix with 10-20 vols. glacial acetic acid, and heat for 1-2 hours on the water-bath. After diluting with several volumes of water, allow the liquid to stand a few days. The crystals which separate are then washed with water, boiled with acetic acid, and then washed again with water, alcohol, and ether. NENCKI and SIEBER coagulate the sediment of the blood-corpuscles by alkali, allow the coagulum to dry incompletely in the air, rub it fine, and then boil it with amyl alcohol after the addition of a little hydrochloric acid. The crystals which separate from the filtrate after cooling are washed with water, alcohol, and ether. If hæmin crystals be dissolved in dilute caustic alkali, hæmatin may be precipitated from the solution by the addition of acid; and from this hæmatin pure hæmin crystals may be prepared by heating with glacial acetic acid and a little common salt.

In preparing hæmin crystals in small amounts proceed in the following manner: The blood is dried after the addition of a small quantity of common salt, or the dried blood may be rubbed with a trace of common salt. The dry powder is placed on a microscopeslide, moistened with glacial acetic acid, and then covered with the cover-glass. Add, by means of a glass rod, more glacial acetic acid by applying the drop at the edge of the cover-glass, until the space between the slide and the cover-glass is full. Now warm over a very small flame, with the precaution that the acetic acid does not boil 
and pass with the powder from under the cover-glass. If no crystals appear after the first warming and cooling, warm again, and if necessary add some more acetic acid. After cooling, if the experiment has been properly performed, a number of dark-brown or nearly black hæmin crystals of varying forms will be seen.

Hæmatoidin, thus called by VIRchow, is a coloring matter which crystallizes in orange-colored rhombic plates, and which occurs in old blood extravasations, and whose origin from the blood-coloring matters seems to be established (LANGHANs, CORDUA, QUINCKE, and others). A solution of hæmatoidin shows no absorption-bands, but only a strong absorption of the violet to the green (EWALD). According to most observers, hæmatoidin is identical with the bile-coloring matter bilirubin. It is not identical with the crystallizable lutein from the corpora lutea of the ovaries of the cow (PICcolo and LIEBen, KüHNE and EWALD).

In the detection of the above-described blood-coloring matters the spectroscope is the only entirely trustworthy means of investigation. If it is only necessary to detect blood in general and not to determine definitely whether the coloring matter is hæmoglobin, methæmoglobin, or hæmatin, then the presence of hæmin crystals is an absolute positive proof. The reader is referred to more extended text-books for exacter methods for the detection of blood in chemico-legal cases, and it is perhaps sufficient to give here the chief points of the investigation.

If spots on clothes, linen, wood, etc., are to be tested for the presence of blood, it is best, when possible, to scratch or shave off as much as possible, rub with common salt, and from this prepare the hæmin crystals. On obtaining positive results the presence of blood is not to be doubted. If you do not obtain sufficient material by the above means, then soak the spot with a few drops of water in a watch-crystal. If a colored solution is thus obtained, then remove the fibres, wood-shavings, and the like as far as possible, and dry all the solution in a watch-glass. The dried residue may be partly used for the spectroscope test directly, and part may be employed in the preparation of the hæmin crystals. It also serves to detect hæmochromogen in alkaline solution after previous treatment with alkali and the addition of reducing substances.

If a colorless solution is obtained after soaking with water, or the spots are on rusty iron, then digest with a little dilute alkali $(5 \mathrm{p} . \mathrm{m}$.). In the presence of blood the solution gives, after neutralization with hydrochloric acid and drying, a residue which may 
give the hæmin crystals with glacial acetic acid. Another part of the alkaline solution shows, after the addition of STOKE's reduction liquid, the absorption-bands of hæmochromogen in alkaline solution.

The methods proposed for the quantitative estimation of the blood-coloring matters are partly chemical and partly physical.

Among the chemical methods to be mentioned is the ashing of the blood and the determination of the amount of iron contained therein, from which the amount of hæmoglobin may be calculated. Another method consists in first saturating the blood completely with oxygen. Now pump out thoroughly this oxygen, and calculate from the amount of oxygen the amount of hæmoglobin present (GRÉHANT and QUINQUAUD). None of these methods is reliable.

The physical methods consist either in a colorimetric or a spectroscopic investigation.

The principle of HOPPE-SEYLER's colorimetric method is that a measured quantity of blood is diluted with an exactly measured quantity of water until the diluted blood solution has the same color as a pure oxyhæmoglobin solution of a known strength. The amount of coloring matter present in the undiluted blood may be easily calculated from the degree of dilution. In the colorimetric testing we use a glass vessel with parallel sides containing a layer of liquid $1 \mathrm{~cm}$. thick (hæmatinometer of HoPPE-SEYLER). The method is good, and the inconvenience that the normal solution of oxyhæmoglobin does not keep for any length of time without decomposing may be prevented by preserving the solution in sealed tubes. The oxyhæmoglobin is gradually reduced to a hæmoglobin solution which may be kept for years, and when required for use it is converted into an oxyhæmoglobin solution by aerating. A few observers (RAJEWSKY, LEsser, Mallassez) have tried to replace the oxyhæmoglobin solution by a solution of picrocarmin.

The quantitative estimation of the blood-coloring matters by means of the spectroscope may be done in different ways, but at the present time the spectrophotometric method is chiefly used, and this seems to be the most reliable. 'This method is based on the fact that the extinction coefficient of a colored liquid for a certain region of the spectrum is directly proportional to the concentration, so that $C: E=C_{1}: E_{1}$, when $C$ and $C_{1}$ represent the different concentrations and $E$ and $E_{1}$ the corresponding coefficient of extinction. From the equation $\frac{C^{\prime}}{E}=\frac{C_{1}}{E_{1}}$ it follows that for one and the same coloring matter this relation, which is called the absorption ratio, must be constant. If the absorption ratio is represented by $A$, the determined extinction coefficient by $E$, and the concen- 
tration (the amount of coloring matter in grams in 1 c. c.) by $C$, then $C=A . E$.

Different apparatus have been constructed (VIERoRDT and HÜFNER) for the determination of the extinction coefficient which is equal to the negative logarithm of those rays of light which remain after the passage of the light through a layer $1 \mathrm{~cm}$. thick of an absorbing liquia. In regard to these apparatus the reader is referred to other text-books.

As control the extinction coefficients are determined in two different regions of the spectrum, namely, $D 32 E-D 54 E$ and $D 63 E-D 84 E$. The constants or the absorption ratio for these two regions of the spectrum are designated by Htrner by $A$ and $A^{\prime}$. Before the determination the blood must be diluted with water, and if the proportion of dilution of the blood be represented by $V$, then the concentration or the amount of coloring matter in 100 parts of the undiluted blood is

$$
\begin{aligned}
& C=100 \cdot \underset{V}{V} \cdot A \cdot A \\
& C=100 \cdot \underset{A^{\prime}}{E} \cdot E^{\prime} \text {, and }
\end{aligned}
$$

The absorption ratio or the constants in the two above-mentioned regions of the spectrum have been determined for oxyhæmoglobin, hæmoglobin, carbon monoxide hæmoglobin, and methæmoglobin.

The figures for the above coloring matters obtained from canine blood are as follows:

$$
\begin{aligned}
& \text { Oxyhæmoglobin ............. } A_{o}=0.001330 \text { and } A_{o}{ }^{\prime}=0.001000 \\
& \text { Hæmoglobin................ } A_{r}=0.001091 \text { " } A_{r}^{\prime}=0.001351 \\
& \text { Carbon monoxide hæmoglobin } A_{c}=0.001130 \text { " } A_{c} \text { ' }=0.001000 \\
& \text { Methæmoglobin............. } A_{m}=0.003696 \text { " } A_{m}^{\prime}=0.002798
\end{aligned}
$$

The quantity of each coloring matter may be determined in a mixture of two blood-coloring matters by this method, which is of special importance in the determination of the quantity of oxyhæmoglobin and hæmoglobin present in blood at the same time. If we represent by $E$ and $E$ ' the extinction coefficients of the mixture in the above-mentioned regions of the spectrum, by $A_{o}$ and $A_{o}^{\prime}$ and $A_{r}$ and $A_{r}^{\prime}$ the constants for oxyhæmoglobin and reduced hæmoglobin, and by $V$ the degree of dilution of the blood, then the percentage of oxyhæmoglobin $H_{o}$ and of (reduced) hæmoglobin $H_{r}$ is

$$
H_{o}=100 \cdot V \cdot \frac{A_{o} A_{o}^{\prime}\left(E A_{r}-E^{\prime} A_{r}^{\prime}\right)}{A_{o}^{\prime} A_{r}-A_{o} A_{r^{\prime}}}
$$

and

$$
H_{r}=100 . V \cdot \frac{A_{r} A_{r}^{\prime}\left(E A_{o}^{\prime}-E A_{o}\right)}{A_{o}^{\prime} A_{r}-A_{o} A_{r}^{\prime}}
$$

Among the many apparatus constructed for clinical purposes for the quantitative estimation of hæmoglobin the hæmometer of FLEIsCHL is to be preferred. The determination by this apparatus is made by comparing the color of the blood diluted with water with the color of a wedge-shaped movable prism of red glass. If the blood shows the same color as the glass prism, then the amount of hæmoglobin in the blood may be directly read from the scale. 
The amount of hæmoglobin is expressed as percentage of the physiological amount of hæmoglobin.

Many other coloring matters are found besides the often-occurring hæmoglobin in the blood of invertebra. In a few arachnidæ, crustacea, gasteropodæ, and cephalopodæ a body analogous to hæmoglobin containing copper, hamocyanin, has been found. By the taking up of loosely-bound oxygen this body is converted into blue oxylicemocyanin (P. Bert, Frederice, KrUkenberg, MACMUNN), and by the escape of the oxygen becomes colorless again. A coloring matter called chlorocruorin by LANKESTER is found in certain clıætopodæ. Homerythrin, so called by KRUKENBERG but first observed by SchwalBe, is a red coloring matter from a few gephyrea. Besides hæmocyanin we find in the blood of certain crustacea the red coloring matter tetronerythrin (HALLIBURTON), which is also widely spread in the animal kingdom. Echinochrom, so named by MACMUNN, is a brown coloring matter occurring in the perivisceral tluid of a variety of echinoderms.

The quantitative constitution of the red blood-corpuscles is difficult to determine and we have hardly any sufficiently trustworthy analyses of them. The amount of water varies in different varieties of blood between $570-630 \mathrm{p}$. m., with a proportional amount, $430-3 \%$ p. m., of solids. In the blood of mammalia the chief mass (about nine tenths) of the dried substance consists of hæmoglobin.

According to the analyses of HOPPE-SEYLER and his pupils, the red corpuscles contain in 1000 parts of the dried substance:

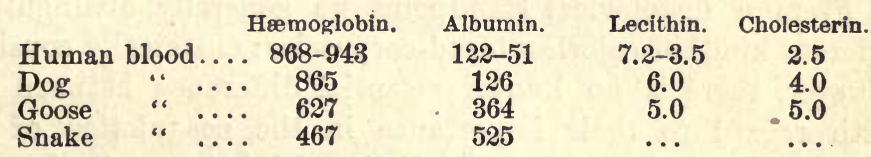

Of special interest is the varying proportion of the hæmoglobin to the albumin in the nucleated and in the non-nucleated blood-corpuscles. These last are much richer in hæmoglobin and poorer in albumin than the others. The amount of mineral bodies, as far as they have been determined, in the moist corpuscles is 4.8$8.9 \mathrm{p} . \mathrm{m}$. The chief mass consists of potassium, phosphoric acid, and chlorine. The blood-corpuscles of ox-blood contain, according to BUNGE, more sodium and chlorine than phosphoric acid and potassium. The blood-corpuscles of the pig and horse contain no sodium (BUNGE). Human-blood corpuscles contain, according to WANACH, about five times as much potassium as sodium, on an average $3.99 \mathrm{p} . \mathrm{m}$. potassium and $0.75 \mathrm{p} . \mathrm{m}$. sodium. 


\section{The Colorless Blood-corpuscles and the Blood-tablets.}

The Colorless Blood-corpuscles, also called Leucocytes or Lymphoid Cells, which occur in the blood in varying forms and sizes, form in a state of rest spherical lumps of a sticky, highly refractive power, capable of motion, non-membranous protoplasm, which show 1-4 nuclei on the addition of water or acetic acid. In human and mammalial blood they are larger than the red bloodcorpuscles. They have also a lower specific gravity than the red corpuscles, move in the circulating blood nearer to the walls of the vessel, and have also a slower motion.

The number of colorless blood-corpuscles varies not only in the different blood-vessels, but also under different physiological conditions. As an average we have only 1 colorless corpuscle for 350500 red corpuscles. According to the investigations of Alex. Schmidt and his pupils, the leucocytes are destroyed in great part -about $71 \%$ in the blood of the horse, according to HEYL-on the discharge of the blood before and during coagulation, so that discharged blood is much poorer in leucocytes than the circulating blood. The correctness of this statement has been denied by other investigators.

From a histological standpoint we generally distinguish the different kinds of colorless blood-corpuscles; chemically considered, however, there is no known essential difference between them. With regard to their importance in the coagulation of fibrin Alex. Schmidt and his pupils distinguish between the leucocytes which are destroyed by the coagulation and those which are not. The last mentioned are colored quickly by carmine and give with alkalies or common-salt solutions a slimy mass; the first do not show such behavior.

The protoplasm of the leucocytes has during life amoboid movements which partly make possible the wandering of the cells and partly the taking up of smaller grains or foreign bodies within the same. On these grounds the occurrence of myosin in them has been admitted even without any special proof thereof. Alex. ScHMidt found serum globulin in equine-blood leucocytes which had been washed with ice-cold water. There are also at least certain leucocytes which yield a slimy mass when treated 
with alkalies or $\mathrm{NaCl}$ solutions, which seem to be identical with the so-called hyaline substance of Rovida found in the pus-cells. On digesting the leucocytes with water a solution of a proteid body is obtained which can be precipitated by acetic acid and which is not soluble in an excess of the acid (ScHMidt and RAUSCHENBACH, WOOLDRIDGE). This proteid substance, designated "lymphfibrinogen " by WOOLDRIDGE, when obtained from certain leucocytes (but not from others) has a powerful action on the coagulation of fibrin. An important constituent of the colorless corpuscles is, according to ALEX. SCHMIDT, the fibrin ferment or perhaps more correctly a mother-substance of the same, a zymogen. In the destruction of the colorless corpuscles the fibrin ferment is set free and serum globulin also passes into the plasma at the same time. HaLLibuRtor has isolated two globulins and one albumin (see page 42) from the leucocytes of the lymphatic glands, besides RovIDA's hyaline substance, and albumoses and peptones as post-mortem products.

Among the other constituents of the pale corpuscles we must mention glycogen, which occurs in the living but not in the dead cell, lecithin, protagon (?), and cholesterin. The nucleus consists without doubt of nuclein. The mineral constituents are probably the same as in the pus-cells (see Chapter V).

The above statements in regard to the constituents of the leucocytes cover not only the pale blood-corpuscles, but also the leucocytes of the lymphatic glands. The leucocytes of the blood are considered as cells pierced from the outside, and it is probably the correct view because we know of no specific chemical properties or constituents in the leucocytes of the blood which differ from those of other leucocytes.

The blood-tablets (BIzzozero's), whose nature and physiological importance have been much questioned, are pale, colorless, gummy disks, round or more oval in shape and generally with a diameter two or three times smaller than the red blood-corpuscles. Their number, according to FUSARI, in healthy persons is $180,000-250,000$ in 1 c.mm. Certain investigators, for example Bizzozero, SCHIMMELBUSCH, and LAKER, claim that the blood-tablets occur preformed in the circulating blood, while others, for instance LöWIT and WooldrIDGE, deny this. Other investigators (HLAVA) 
claim that they may, at least in part, be formed from the colorless blood-corpuscles, while LöwIT claims that they are formed by the withdrawal of globulin substance from the white blood-corpuscles. BIzzozero and several other investigators consider the blood-tablets as the starting-point for the coagulation of the blood, while ALEX. ScHMidT's pupils deny this. LöwIT claims that the blood-tablets are formed from globulin substance, and has therefore given them the name globulin tablets. The relationship of these blood-tablets to the fibrin coagulation will be spoken of shortly.

\section{The Blood as a Mixture of Plasma and Blood- corpuscles.}

The blood in itself is a thick, sticky, lighter or darker red opaque liquid having a salty taste and even in thin layers a faint odor differing in different kinds of animals. On the addition of sulphuric acid to the blood the odor is more pronounced. In adult human beings the specific gravity averages 1.055 , ranging between 1.045 and 1.075. According to ScHERRENzISs the fotal blood has a lower specific gravity than the blood of grown persons. LLOYD E. JoNES found that the specific gravity is highest at birth and lowest in children when about two years old and in pregnant women.

The reaction of the blood is alkaline. The amount of alkali, calculated as $\mathrm{Na}_{2} \mathrm{CO}_{3}$, is in the dog about 2 (ZUNTZ), in rabbits about 2.5 (LASSAR), and in man $3.38-3.90$ p. m. (v. JAKSCH). The alkaline reaction diminishes outside of the body, and indeed the more quickly the greater the original alkalinity of the blood. This depends on the formation of acid in the blood, in which the red blood-corpuscles seem to take part in some way or another. After excessive muscular activity the alkalinity is diminished on account of the formation of acid in the muscles, and it is also decreased after the continuous use of acids (LASSAR).

The color of the blood is red-light scarlet-red in the arteries and dark bluish-red in the veins. Blood free from oxygen is dichroitic, dark red by reflected light, and green by transmitted light. The blood-coloring matters occur in the blood-corpuscles. For this reason blond is opaque in thin layers and acts as a "deckfarbe." If the hæmoglobin is removed from the stroma or dissolved from 
the blood-liquid by any of the above-mentioned means (see page 66) the blood becomes transparent and acts then like a "lacfarbe." Less light is now reflected from its interior, and this latter blood is therefore darker in thicker layers. On the addition of salt solutions to the blood-corpuscles they shrink and more light is reflected and the color appears lighter. A great abundance of red corpuscles makes the blood darker, while by diluting with serum or by a greater abundance of white corpuscles the blood becomes lighter in appearance. The different colors of arterial and of venous blood depend on the varying quantity of gas contained in these two varieties of blood, or better on the different amounts of oxyhæmoglobin and hæmoglobin they contain. The reason for the different colors of these two varieties of blood has been attributed in part to the unequal forms of the blood-corpuscles. In the arterial blood the blood-corpuscles are considered as more biconcave and therefore reflect the light more than in the venous blood (HARLESS). These statements do not coincide with later investigations.

The most striking property of blood consists in its coagulating within a shorter or longer time, but as a rule very shortly after leaving the vein. Different kinds of blood coagulate with varying rapidity; in human blood the first marked sign of coagulation is seen in 2-3 minutes, and within 7-8 minutes the blood is thoroughly converted into a gelatinous mass. If the blood is allowed to coagulate slowly, the red corpuscles have time to settle more or less before the coagulation, and the blood-clot then shows an upper, large, yellowish-gray or reddish-gray layer consisting of fibrin enclosing chiefly colorless corpuscles. This layer has been called crusta inflammatoria or phlogistica, because it has been especially observed in inflammatory processes, and is considered one of the characteristics of them. This crusta or "buffy coat" is not characteristic of any special disease, and it occurs chiefly when the blood coagulates slowly or when the blood-corpuscles settle more quickly than usual. A buffy coat has been observed often in the slow coagulation of equine blood. The blood from the capillaries is not supposed to have the power of coagulating.

Coagulation is retarded by cooling, by diminishing the oxygen and increasing the amount of carbon dioxide, which is the reason that venous blood and to a much higher degree blood after asphyx- 
iation coagulates more slowly than arterial blood. The coagulation may be retarded or prevented by the addition of acids, alkalies, or ammonia, even in small quantities; by concentrated solutions of neutral salts or alkalies and alkaline earths; also by egg-albumin, solutions of sugar or gum, glycerin, or much water; also by receiving the blood in oil. The coagulation may be prevented by the injection of an albumose solution or by an infusion of the leech into the circulating blood, but this infusion of the leech acts in the same way on blood just expelled. The coagulation may be facilitated by raising the temperature; by contact with foreign bodies, to which the blood adheres; by stirring or beating it; by admission of air; by diluting with very small amounts of water; by the addition of platinum-black or finely-powdered carbon; by the addition of lac-colored blood, which does not act by the presence of dissolved blood-coloring matters, but by the stromata of the blood-corpuscles (WooldrIDGE, NUCK), and also by the addition of the leucocytes from the lymphatic glands, or a watery saline extract of the lymphatic glands, testicles, or thymus. The active constituent of such a watery extract is, according to WooLDRIDGE, an albuminous body containing lecithin, which he calls tissue fibrinogen.

An important question to answer is why the blood remains fluid in the circulation while it quickly coagulates when it leaves the circulation.

When the blood leaves the vein it comes under new, abnormal conditions. It cools off, it comes in contact with the air, its motion stops, and it is deprived of the influence of the living walls of the ressels. That the cooling is not the reason of the coagulation is proved by the fact that cooling is a good means of retarding coagulation. That the contact with air is not essential is shown by the fact that when blood is collected over mercury, so that it cannot absorb or expel any gas, it likewise coagulates. That the cessation of the motion does not cause the coagulation follows, since blood collected over mercury coagulates whether it is shaken or not, and further from the fact that motion, such as beating the blood, facilitates the coagulation.

The reason why blood coagulates on leaving the body is therefore to be sought for in the influence which the walls of the living 
and entire blood-vessels exert upon it. These views are derived from the observations of many investigators. From the observations of Hewsor, Lister, and Frederice it is known that when a vein full of blood is tied at the two ends and removed from the body, the blood may remain fluid for a long time. BRÜCKE allowed the heart removed from a tortoise to beat at $0^{\circ} \mathrm{C}$., and found that the blood remained uncoagulated for some days. The blood from another heart quickly coagulated when collected over mercury. In a dead heart, as also in a dead blood-ressel, the blood soon coagulates, and also when the walls of the vessel are changed by pathological processes.

What then is the influence which the walls of the vessels exert on the liquidity of the circulating blood? FREUND has found that the blood remains fluid when collected by means of a greased canula under oil or in a vessel smeared with vaseline. If the blood collected in a greased vessel be beaten with a glass rod previously oiled, it does not coagulate, but it quickly coagulates on beating it with an unoiled glass rod or when it is poured into a vessel not greased. The non-coagulability of blood collected under oil has been confirmed later by HaYcraft and CarLier. Freund found on further investigating that the evaporation of the upper layers of blood or their contamination with small quantities of dust causes a coagulation even in a vessel treated with vaseline. According to FREUND, it is this adhesion between the blood or between its form-elements and a foreign substance-and the diseased walls of the vessels also act as such-that gives the impulse towards coagulation, while the lack of adhesion prevents the blood from coagulating. This adhesion of the form-elements of the blood to certain foreign substances seems to induce changes which apparently stand in a certain relationship to the coagulation of the blood.

That view of the coagulation of the blood which, with a few modifications, is accepted by most investigators is the theory proposed by Alexander Schmidt and the Dorpat School. According to Alex. Schmidt, who of all investigators has done most to elucidate this subject, an abundant destruction of the colorless bloodcorpuscles takes place in coagulation, and from this not only the fibrin ferment results, but also serum globulin and fibrinogen, which 
last, however, was previously present in the plasma. Under the action of the fibrin ferment the serum globulin and fibrinogen unite forming fibrin. In the coagulation a reciprocal action takes place between the protoplasm of the leucocytes and the plasma, so that the plasma quickly destroys the leucocytes and is itself destroyed by the setting free of fibrin ferment with the separation of fibrin (Schmidt and RAUSChenbach). Such an exchange action not only takes place between the blood-plasma and the leucocytes, but also between blood-plasma and animal protoplasm in general-indeed, even between vegetable protoplasm and blood-plasma (SсHмIDT and Grohmans). While the blood-serum in general acts as a conservator on the cells, the blood-plasma on the contrary has a destructive action, and this action develops the fibrin ferment. This lastmentioned is chiefly a decomposition product of the cells (SснміDт, Rauschenbach; Foa and Pellacani), and it may therefore be called "protozym" (RAUSCHENBACH). A destruction of the leucocytes may also occur in the blood under physiological conditions, and therefore traces of fibrin ferment are habitually formed in the blood. Within certain limits the organism may be guarded from a dangerous increase in these processes. According to Schmidt and Groth, and SCHMIDT and KRÜGER, the injection of leucocytes into the circulating blood may produce an intravascular coagulation, but the correctness of this statement is denied by WOOLDRIDGE. According to him, the pure, washed leucocytes are inactive and the action observed by ScHMIDT and his pupils is caused by contamination with "lymphfibrinogen." The lymphfibrinogen is a representative of an entire group of protein substances which are precipitated by acetic acid, contain lecithin; which occur in many organs and tissues but which have not been closely studied and which WOOLDRIDGE has named "tissue fibrinogens."

According to Alex. SchMidt, the coagulation of the blood is an enzymotic process, produced by the fibrin ferment, in which two protein substances, the serum globulin and the fibrinogen, form the material substrata of the fibrin. There is no basis for the assumption that the serum globulin in the newly-formed fibrin exists otherwise than as a mechanical contamination. It is true indeed that the strongly-contaminated serum globulin prepared from serum containing enzymes accelerates coagulation, and the amount 
of separated fibrin under the circumstances may be increased, but pure serum globulin prepared from transudation free from enzymes is inactive. The same action on the amount of fibrin separated which the impure serum globulin shows also occurs by the action of the substance precipitated by acetic acid from a watery extract of the leucocytes of the lymphatic glands. This substance, which is neither identical with serum globulin nor contaminated by it, may, according to SchMIDT and RAUSCHENBACH, increase the amount of fibrin in filtered plasma about 25\%. Finally, a fibrinogen solution free from serum globulin, and a fibrin ferment solution also free from serum globulin, may yield a typical fibrin (AUTHоR). It is therefore hardly possible to accept this part of ScHмIDT's theory. It is much more probable that the impure serum globulin furthers the separation of the fibrin indirectly, in the same way as $\mathrm{CaCl}_{2}$ (AUTHOR) and the lime salts in general (GREEN, KRÜGRR). It is a fact that a typical fibrin is formed from fibrinogen alone in the presence of fibrin ferment and mineral bodies (alkali chlorides and lime salts).

In regard to the importance of the colorless blood-corpuscles in the coagulation of the blood opinions are somewhat diverse. According to Brzzozero and others, it is not the colorless bloodcorpuscles but the blood-tablets which represent the starting-point for the formation of fibrin, a view against which weighty objections have been urged by LöwIT and others. WOoLDRIDGE also considers the colorless blood-corpuscles as only of secondary importance. As found by him, a peptone-plasma which has been freed from all form-constituents by centrifugal force yields large quantities of fibrin when it is not separated from a substance which precipitates by cooling and which, on microscopical examination, is very similar to Bizzozero's blood-tablets. Nevertheless, as Löwir has found that homogeneous drops may exude from the white blood-corpuscles before the coagulation which take a form similar to tablets, it is probable that the substance observed by WooLDRIDGE which, on cooling, separates as a formation similar to tablets originates from the colorless blood-corpuscles. That a coagulation without a destruction of the colorless bloodcorpuscles may take place has been clearly demonstrated by Löwit, who, however, does not dispute the importance of the 
colorless blood-corpuscles in the coagulation. On the contrary, he has observed that in crab's blood a so-called "plasmoschise," which is the exit of constituents of the cells in the plasma, takes place before the coagulation, and this process, he claims, stands in close relationship to the coagulation of the blood. If we do not give too much weight to the destruction of the leucocytes,-and we admit as most essential this part of ScHMIDT's theory that the impulse to the coagulation comes from the colorless blood-corpuscles, and that the constituents of the same, passing into the plasma, take part in the coagulation,- - then ScHMIDT's theory is not disproved by the investigations of the last years, but it is even supported by them.

In opposition to the view of Alex. Schmidt, who considers the fibrin coagulation as an enzymotic process, WooLDRIDGE is of the opinion that the fibrin ferment is not the cause of the coagulation, but is a product of the chemical processes taking place during coagulation. WOOLDRIDGE claims, on the contrary, that lecithin and proteid substances containing lecithin are of the greatest importance in the coagulation. This product is obtained by cooling the peptone-plasma which has been centrifugated, and the substance which separates has been called by WOOLDRIDGE $A$-fibrinogen. The plasma, according to WOoLDRIDGE, contains in itself all qualities necessary to produce a coagulation, and the form-elements are only of a secondary importance. Peptone-plasma which has been centrifugated and which is entirely free from formelements, but contains the $A$-fibrinogen, coagulates on diluting with water, by the passage of carbon dioxide through the liquid, or after the addition of a little acetic acid, and the fibrin ferment is thereby formed. WOoLDRIDGE designates as $C$-fibrinogen the ordinary fibrinogen isolated by the method suggested on page 56. This fibrinogen occurs indeed in transudations, but it only occurs in the peptone-plasma in very small quantities. A third fibrinogen occurs in the greatest amounts in the peptone-plasma, and this is the mother-substance of the $C$-fibrinogen, and called $B$-fibrinogen by WooLDRIDGE. The $B$-fibrinogen is converted into fibrin by lecithin and leucocytes from the lymphatic glands, but not by fibrin ferment or blood-serum. After the previous action of serum or fibrin ferment the $B$-fibrinogen yields fibrin on diluting with water. The one most essential for the fibrin coagulation is, ac- 
cording to WooLDRIDGE, a reciprocal action between $A$ - and $B$ fibrinogen. An exchange of lecithin from the $A$-fibrinogen to the $B$-fibrinogen takes place.

HALLIBURTON has opposed weighty arguments to this theory. It is also difficult to find in WooLDRIDGE's discussion conclusive proofs for the above views, and the experiments by which they are supported are interpreted with difficulty. The entire theory is chiefly based on experiments with "peptone-plasma;" but such plasma acts differently in certain respects than ordinary plasma. It should be especially mentioned that, while ordinary plasma and an ordinary fibrinogen solution act similarly on heating, the peptone-plasma shows quite different behavior. The peptone-plasma when diluted with water, or on passing $\mathrm{CO}_{2}$ through it, or on the addition of a little acid, also acts, when the so-called $A$-fibrinogen has not been removed, like a plasma in which the ordinary fibrinogen ( $C$-fibrinogen) has been partly converted into an intermediate step between fibrinogen and fibrin. Until the differences existing between ordinary plasma and peptone-plasma shall have been more thoroughly tested and generally accepted it will be difficult to give an unambiguous interpretation of observations made on peptoneplasma.

FrEUND seeks the reason for the coagulation in a separation of calcium phosphate whereby a part of the previously-dissolved albuminous bodies becomes insoluble as fibrin. By the adhesion existing before the coagulation the alkali phosphate of the formelements passes over to the plasma richer in lime salts and forms calcium phosphate. If the amount of this last is so large in the plasma or any other coagulable liquid that it cannot be kept completely in solution, then, according to Freund's views, the separation of the excess is the cause of a part of the albuminous bodies becoming insoluble, or, in other words, is the cause of the coagulation. It is a well-known fact that fibrin and fibrinogen yield an ash containing calcium phosphate, and that lime salts facilitate coagulation or cause a coagulation in liquids poor in ferment; and it is also well known that rennet ferment cannot cause a coagulation in a casein solution if there is a lack of lime salts. There is no question about the lime salts being of great importance in the fibrin coagulation; but that a separation of calcium phosphate is 
the cause of coagulation has been just as little proved as the assertion that the coagulation of milk in the preparation of cheese is produced by the calcium phosphate becoming insoluble without the action of the rennet ferment on the casein. The untenability of FreUND's theory has been shown by LATSOHENBERGER.

According to Dogiel and Holzman, the coagulation of the fibrin is an oxidation of the fibrinogen. The relation of oxygen to the coagulation is not quite clear; a certain influence, however, on the coagulation cannot be denied. But as the coagulation may take place also in the absence of free oxygen, the above statement seems not to be well grounded.

The very dark and complicated process of the intravascular coagulation and the relationship of the so-called tissue fibrinogen (WooldRIDGE) to the same have been so insufficiently studied that we cannot enter here into a discussion of this interesting question.

\section{The Gases of the Blood.}

Since the pioneer investigations of MAGNUS and LoTHAR MEYER the gases of the blood have been subjects for repeated, careful investigations by prominent experimenters, among whom we must mention first C. LUDwig and his pupils and E. PfLüger and his school. By these investigations not only has science been enriched by a mass of facts, but also the methods themselves have been made more perfect and accurate. In regard to these methods, as also in regard to the laws of the absorption of gases by liquids, dissociation, and other questions belonging here, the reader is referred to complete text-books on physiology, on physics, and on gasometric analysis.

The gases occurring in blood under physiological conditions are oxygen, carbon dioxide, and nitrogen. The last-mentioned gas is only found in very small amounts, on an average of $1.8 \mathrm{vol}$. per cent. The amount is here, as in all following experiments, calculated for $0^{\circ} \mathrm{C}$. and $760 \mathrm{~mm}$. pressure. The nitrogen seems to be simply absorbed into the blood, at least in great part. It appears to play no part in the processes of life, and its amount varies but slightly in the blood of different blood-vessels.

The oxygen and carbon dioxide behave otherwise, as their 
amounts have significant variations, not only in the blood from different hlood-vessels, but also because many conditions, such as a difference in the circulation, a different temperature, rest and activity, cause a change. The arterial and venous blood show the greatest difference in regard to the gases they contain.

The amount of oxygen in the arterial blood of dogs is on an average 22 vols. per cent (PFLÜGER). In human blood SETSCHENow found about the same amount, namely, 21.6 vols. per cent. Lower figures have been found for rabbit's or bird's blood, respectively 13.2\% and 10-15\% (WALTER, JOLYET). Venous blood has very variable amounts of oxygen. LuDwiG and Sczelkow found 6.8\% oxygen in the venous blood of quiet muscles and a still smaller amount in the venous blood of active muscles. Oxygen is entirely absent from blood after asphyxiation, or only occurs as traces. The venous blood of the glands seems, on the contrary, during secretion to be richer in oxygen than ordinary venous blood. By summarizing a great number of analyses by different experimenters, ZUNTZ has calculated that the venous blood of the right side of the heart contains on an average $\% .15 \%$ less oxygen than the arterial blood.

The amount of carbon dioxide in the arterial blood of dogs is 30 to 40 vols. per cent (Ludwig, Setschenow, Pflüger, P. Bert, and others), most generally about $40 \%$. SETSCHENow found 40.3 vols. per cent in human arterial blood. The amount of carbon dioxide in renous blood varies still more (LUDwIG, PFLüGER and their pupils, P. Bert, Mathreu and Urbain, and others). According to the calculations of $\mathrm{ZUNTZ}$ the venous blood of the right side of the heart contains about $8.2 \%$ more carbon dioxide than the arterial. The average amount may be put down as 48 vols. per cent. HoLMGREN found indeed in blood after asphyxiation 69.21 vols. per cent carbon dioxide.

Oxygen is absorbed only to a small extent by the plasma or serum, in which PFLÜGER found but $0.26 \%$. The greater part or nearly all of the oxygen is loosely combined with the hæmoglobin in oxyhæmoglobin. The quantity of oxygen which is contained in the blood of the dog corresponds closely to the quantity which we would expect from the activity of the hæmoglobin to combine with oxygen, and also the quantity of hæmoglobin in canine blood. It is difficult to ascertain how far the circulating 
arterial blood is saturated with oxygen, as immediately after bleeding a loss of oxygen always takes place. Still it seems to be unquestionable that it is not quite completely saturated with oxygen in life. Arterial canine blood, according to PFLÜGER, is saturated. to $\frac{9}{10}$ with oxygen, according to HÜFNER to $\frac{14}{15}$.

The question whether ozone occurs in the blood is to be answered decidedly in the negative. It is not only impossible to detect ozone in the blood, but the possibility of the occurrence of ozone in the fluids and tissues is even a priori to be denied. Ozone acts as nascent oxygen; and as easily-oxidized substances occur in the organism which combine with nascent oxygen, ozone, if such a formation should take place at all, would be destroyed instantly. But such a formation of ozone in the animal body cannot be admitted. Ozone may indeed be formed by slow oxidation, since the nascent oxygen formed in consequence combines with neutral oxygen forming ozone; but in the animal organism the nascent oxygen must be bound by the oxidized substances before it can form ozone.

It was formerly believed that the hæmoglobin acted as an "ozone-exciter," possessing the property of converting the inactive oxygen of the air into ozone. The red blood-corpuscles can by themselves also give a blue color with tincture of guaiacum, which is markedly seen when this tincture is dried on blotting-paper and a drop of blood previously diluted with 5-10 vols. water is added. According to PFLÜGER, we are here dealing with a decomposition and gradual oxidation of hæmoglobin, in which processes the neutral oxygen is split, setting free oxygen atoms.

The carbon dioxide of the blood occurs in part, and indeed, according to the investigations of Alex. SchMidT and L. FredericQ, generally $\frac{1}{3}$ in the blood-corpuscles, and it also occurs in part, and in fact the greatest part, in the plasma and serum respectively. Of the carbon dioxide in the form-elements a small part, according to Setschenow, occurs in the colorless corpuscles (probably combined with the globulin alkali), while the chief mass exists in the red blood-corpuscles.

The carbon dioxide of the red corpuscles is loosely combined, and the constituent uniting with the $\mathrm{CO}_{2}$ of the same seems to be the alkali combined with phosphoric acid, oxyhæmoglobin or hæmoglobin and globulin on one side and the hæmoglobin itself on the other. 
That in the red corpuscles alkali phosphate occurs in such quantities that it may be of importance in the combination with carbon dioxide is not to be doubted, and we must admit that from the diphosphate, by a greater partial pressure of the carbon dioxide, monophosphate and alkali carbonate are formed, while by a lower partial pressure of the carbon dioxide the mass action of the phosphoric acid comes again into play, so that with the carbon dioxide becoming free, a re-formation of alkali diphosphate takes place. It is generally admitted that the blood-coloring matters, especially the oxyhæmoglobin, which can expel carbon dioxide from sodium carbonates in vacuo (PREYER), act like an acid; and as the globulin also acts like an acid (see below), this body may also occur in the bloodcorpuscles as alkali combination. The alkali of the blood-corpuscles must therefore, according to the law of the action of the mass between the carbon dioxide, phosphoric acid, and the others, be considered as active acid constituents of the blood-corpuscles, and among these especially the blood-coloring matters, as the globulin can hardly be of importance because of its small quantity. By greater mass-action or greater partial pressure of the carbon dioxide, bicarbonate must be formed at the expense of the diphosphates and the other alkali combinations, while at a diminished partial pressure of the same gas, with the escape of carbon dioxide, the alkali diphosphate and the other alkali combinations must be re-formed at the cost of the bicarbonate.

Hæmoglobin must nevertheless, as the investigations of SETSCHENOW and ZUNTZ and especially those of BoHR and TORUP have shown, be able to hold the carbon dioxide loosely combined even in the absence of alkali. BOHR has also found that the dissociation curve of the carbon-dioxide hæmoglobin corresponds essentially to the curve of the absorption of carbon-dioxide, from which ground he and ToRUP consider the hæmoglobin itself as of importance in the binding of the carbon dioxide of the blood and not its alkali combinations. In regard to this question the conditions are not quite clear. If carbon dioxide is allowed to act on hæmoglobin, it unites (BoHR, ToRUP) with the colored atomic group of the hæmoglobin, splitting off albumin, and from this hæmoglobin, so decomposed, oxyhæmoglobin cannot be formed by the action of oxygen. According to BoHr, for each gramme of 
hæmoglobin at $+18.4^{\circ} \mathrm{C}$. and a pressure of $30 \mathrm{~mm} .2 .4 \mathrm{c} . \mathrm{cm}$. carbon dioxide are combined; and since in the arterial blood nearly all the hæmoglobin exists as oxyhæmoglobin, it is difficult to obtain, at least from the arterial blood, an appreciable fraction of the carbon dioxide as carbon-dioxide hæmoglobin. It cannot be denied that a not insignificant part of the carbon dioxide of the blood is held by the red corpuscles in loose combination; but how this combination takes place requires further investigation before it can be answered.

The chief part of the carbon dioxide of the blood is found in the blood-plasma or the blood-serum, which follows from the fact that the serum is richer in carbon dioxide than the corresponding blood itself. By experiments with the air-pump on bloodserum it has been found that the chief part of the carbon dioxide contained in the serum is given off in a vacuum, while a smaller part can only be pumped out after the addition of an acid. The red corpuscles also act as an acid, and therefore in blood all the carbon dioxide is expelled in vacuo. A part of the carbon dioxide is firmly chemically combined in the serum.

Absorption experiments with blood-serum have shown us further that the carbon dioxide which can be pumped out is in great part loosely chemically combined, and from this loose combination of the carbon dioxide it necessarily follows that the serum must also contain simply-absorbed carbon dioxide. For the form of binding of the carbon dioxide contained in the serum or the plasma we find the three following possibilities: 1 . A part of the carbon dioxide is simply absorbed; 2. Another part is loosely chemically combined; and 3. A third part is in firm chemical combination.

The quantity of simply-absorbed carbon dioxide has not been exactly determined. Setschenow considers the quantity in dogserum to be about $\frac{1}{10}$ of the total quantity of carbon dioxide. According to the tension of the carbon dioxide in the blood and its absorption coefficient, the quantity seems to be still smaller.

The quantity of firmly chemically combined carbon dioxide in the blood-serum is the same as the quantity of simple alkali carbonate in the serum. This quantity is not known, and it cannot be determined either by the alkalinity found by titration, nor can it be calculated from the excess of alkali found in the ash, because the 
alkali is not only combined with carbon dioxide but also with other bodies, especially with albumin. The quantity of firmly chemically combined carbon dioxide cannot be ascertained after pumping out in vacuo without the addition of acid, because to all appearances certain active constituents of the serum, as acids, expel carbon dioxide from the simple carbonate. The quantity of carbon dioxide not expelled from dog-serum by vacuum alone without the addition of acid amounts to 4.9 to 9.3 vols. per cent, according to the determinations of PFLÜGER.

From the occurrence of simple alkali carbonates in the bloodserum it naturally follows that a part of the loosely-combined carbon dioxide of the serum which can be pumped out must occur as bicarbonate. The occurrence of this combination in the bloodserum has been directly shown. In experiments with the pump, as well as in absorption experiments, the serum behaves in other ways as a solution of bicarbonate, or carbonate of a corresponding concentration; and the behavior of the loosely-combined carbon dioxide in the serum can be explained only by the occurrence of bicarbonate in the serum. By means of vacuum the serum always allows much more than one half of the carbon dioxido to be expelled, and it follows from this that in the pumping out not only may a dissociation of the bicarbonate take place, but also a conversion of the double sodium carbonate into a simple salt. As we know of no other carbon-dioxide combination besides the bicarbonate in the serum from which the carbon dioxide can be set free by simple dissociation in vacuo, we are obliged to assume that the serum must contain other faint acids in addition to the carbon dioxide, which contend with it for the alkalies and which expel the carbon dioxide from simple carbonates in vacuo. The carbon dioxide which is expelled by means of the pump and which, without regard to the simple absorbed quantity, is generally designated as "loosely chemically combined carbon dioxide," is thus only obtained in part in dissociable loose combination; in part it originates from the simple carbonates, from which it is expelled in vacuo by other faint acids.

These faint acids are thought to be in part phosphoric acid and in part globulin. The importance of the alkali phosphates for the carbon dioxide combination (see page 97 ) has been shown by the 
investigations of FERNET; but the quantity of these salts in the serum is, at least in certain kinds of blood, for example in oxserum, so small that it can hardly be of importance. In regard to the globulins SETSCHENow is of the opinion that they do not act as acids themselves, but form a combination with carbon dioxide, producing carboglobulinic acid which unites with the alkali. According to SerTour, whose views have lately found a supporter in TondP, the globulins themselves are the acids combined with the alkali of the blood-serum. In both cases the globulin would form, directly or indirectly, that chief constituent of the plasma or of the blood-serum which, according to the law of the action of masses, contends with the carbon dioxide for the alkalies. By a greater partial pressure of the carbon dioxide the latter deprives the globulin alkali of a part of its alkali, and bicarbonate is formed; by low partial pressure the carbon dioxide escapes, and the bicarbonate is taken up by the globulin alkali.

In the above statements it has been emphasized that the oxygen in the blood occurs in a dissociable combination with the hæmoglobin, and for the formation of this combination, the oxyhæmoglobin, a certain partial pressure of the oxygen is necessary at any temperature. Also the carbon dioxide of the blood, that which is contained in the blood-corpuscles as well as that in the plasma, occurs mostly in combinations which are dependent to a great extent upon the partial pressure of the carbon dioxide. For the study of the exchange of gases between the blood and the alveolar air on one side, and the blood and the tissues on the other, special regard must be paid to the question as to how far this exchange of gases is the result of the law of diffusion and how far other forces take part in it; also the tension of the oxygen and the carbon dioxide is of the greatest importance.

The law of the dissociation of oxyhæmoglobin has been studied by many investigators. Of the greatest physiological interest are those investigations which relate to the dissociation at the temperature of the body. In regard to these, many investigators (P. BERT, Herter, and Hufner) have found, partly by experiments on the living animal and partly by experiments with blood or pure hæmoglobin solutions, that the tension of the oxygen of the blood at the temperature of the body corresponds to an oxygen partial pressure 
of about $75-80 \mathrm{~mm}$. mercury. BoHR has obtained a somewhat different result from his investigations. He experimented on dogs which were injected with leech infusion or peptone solution so as to prevent coagulation of the blood, and he allowed the blood to circulate through an apparatus which was inserted between the central and peripheral end of the cut carotid, or between the central end of the carotid and the central end of the cut jugular vein, and in this way the exchange of gases between the blood and a gas mixture of known constitution could be determined. As measure of the tension of the oxygen in the arterial blood he obtained a disproportionate high value or an average pressure of $136.5 \mathrm{~mm}$. mercury. By a simultaneous determination of the oxygen tension in the blood and in the expired air of the same animals he found in several cases a higher value for the former than for the latter. While according to P. BERT's investigations the taking up of oxygen from the air of the lungs by the blood may be explained by the high oxygen partial pressure in the air of the lungs, this is not perceptible according to the experiments of BoHr, in which the tension of the oxygen in the blood is greater than in the expired air, and also for evident reasons it is still greater than in the alveolar air. Bонк is also of the opinion that the generally-accepted diffusion theory does not give sufficient explanation for the taking up of oxygen from the air, and that we must also admit that the lungtissue itself plays an active part in the taking up of oxygen.

As the chief quantity of the oxygen in the blood simply absorbed, corresponding to the pressure, is contained as a loose chemical combination, it is to be supposed that the amount of oxygen in the blood, at least within certain limits, is independent of the amount of oxygen in the air.

That the raising of the oxygen pressure, even to a pressure of one atmosphere, has no essential influence on the amount of oxygen taken up, and on the carbon dioxide eliminated, has been known for a long time (Lavoisier, Reanault, and Reiset). Further experiments in this direction have been made by PaUL Bert. He found that in pure oxygen at a pressure of 3 atmospheres, or in ordinary air at a pressure of 15 atmospheres, animals quickly died with convulsions. Before and during the spasms a lowering of temperature took place, and the consumption of oxygen, as well as 
the elimination of carbon dioxide and the combustion of the sugar of the blood, was lowered. By raising the oxygen pressure of the air to 3 atmospheres the amount of oxygen contained in the blood is somewhat increased. It seems that the amount of oxygen which is here taken up corresponds to that quantity which is simply absorbed by the blood at that pressure.

It is also of special interest to know to what extent the partial pressure of the oxygen of the air can be lowered without causing any injurious action or danger to life. A great many observations have been made on this subject, partly on man (P. BERT, Sivel and Crocé-Spinelli, Leblanc, and others) and partly on animals (by W. Müller, Hoppe-Seyler, Stroganow, Bert, Friedlander and Herter, Fränkel and Geppert). From these observations it seems that the partial pressure of the oxygen in the atmosphere may sink to one half without causing a disturbance. The respiration is hindered by an oxygen tension of $7-8 \%$ of an atmosphere, and at a still lower tension a lowering of the temperature is observed, lassitude, inability of muscular movement, and insensibility. At an oxygen tension which corresponds to about $3-3.5 \%$ of an atmosphere, death occurs.

In regard to the amount of oxygen in the blood at lower airpressures, the observations of FRÄNKEL and GEPPERT on dogs must be mentioned. At an air-pressure of $410 \mathrm{~mm}$. mercury the amount of oxygen in the arterial blood was normal; at an air-pressure of $3 \% 8-365 \mathrm{~mm}$. it was a little diminished, and only at a lowering of the pressure to $300 \mathrm{~mm}$. was a marked decrease of the oxygen observed.

The tension of the carbon dioxide in the blood has been determined in different ways by PFLÜGER and his pupils, WOLFBERG, Strassburg, and Nussbaum. According to the density method, the blood is allowed to flow directly from the artery or vein through a glass tube which contains a gas mixture of a known constitution. If the tension of the carbon dioxide in the blood is greater than the gas mixture, then the blood gives up carbon dioxide; while in the reverse case it takes up carbon dioxide from the gas mixture. The analysis of the gas mixture after passing the blood through it will also decide if the tension of the carbon dioxide in the blood is greater or less than in the gas mixture; and by a sufficiently great number of determinations, especially when: 
the amount of carbon dioxide of the gas mixture corresponds as nearly as possible in the beginning to the probable tension of this gas in the blood, we may learn the tension of the carbon dioxide in the blood.

According to this method, the carbon-dioxide tension of the arterial blood is on an average 2.8\% of an atmosphere, corresponding to a pressure of $21 \mathrm{~mm}$. mercury (STRASSBURG). In the blood from the pulmonary alveoli NussBaUM found a carbon-dioxide tenbion of 3.81\% of an atmosphere, corresponding to a pressure of 28.95 $\mathrm{mm}$. mercury. Strassbura, who experimented on tracheotomized dogs, in which the ventilation of the lungs was less active and therefore the carbon dioxide was removed from the blood with less readiness, found in the venous blood of the heart a carbon-dioxide tension of 5.4\% of an atmosphere, corresponding to a partial pressure of $41.01 \mathrm{~mm}$. mercury.

Another method is the catheterization of a flap of the lungs. By the introduction of a catheter of a special construction into a branch of a bronchia the corresponding flap of the lung may be hermetically sealed, while in the other flaps of the same lung and in the other lung the ventilation remains unhindered, so that no stowing of carbon dioxide takes place in the blood. When the cutting off lasts so long that a complete equalization between the gases of the blood and the cut-off air of the lungs is assumed, a sample of this air of the lungs is removed by means of the catheter and analyzed. In the air thus obtained from the lungs Nussbaum and Wolfberg found an average of $3.6 \% \mathrm{CO}_{2}$. Nussbaum has also determined the carbon-dioxide tension in the blood of the pulmonary alveoli in a case simultaneous with the catheterization of the lungs. He found nearly identical results, namely, a carbondioxide tension of $3.84 \%$ and $3.81 \%$ of an atmosphere.

While according to the just-mentioned determinations the carbon-dioxide pressure in the venous blood amounts to about $30 \mathrm{~mm}$. mercury and in the arterial to about $20 \mathrm{~mm}$., ВонR has, on the contrary, according to the above-described methods (page 101), found strikingly lower figures -2 to $3 \mathrm{~mm}$., and even lower than $1 \mathrm{~mm}$.

The quantity of carbon dioxide in the expired air amounts to about 2.8\% (WOLFBERG, BоHR). The air of the alveolus of the lungs is naturally richer in carbon dioxide, but the amount is not 
exactly known. If we start from the figures for the carbon-dioxide tension found by PFLUGER and his pupils, and when we recall, further, that Nussbaum found in the cut-off air of the lungs-which is more likely to be richer than poorer in carbon dioxide than the normal alveolar air of the lungs - the same carbon-dioxide tension as in the venous blood of the heart, then these observations easily agree with the view that elimination of the carbon dioxide from the blood in the lungs simply follows the laws of diffusion. According to the experiments of $\mathrm{BOHR}$, in which the blood and the expired air were investigated at the same time, and in which he found the carbon-dioxide tension in the blood significantly lower than the expired air-and also lower than the alveolar air,such an assumption as the above is impossible. BOHR also wishes to give by his experiments a proof of the view suggested long ago by LuDWIG's school, namely, that the lungs play a specific secretory rôle in the elimination of carbon dioxide. The necessity of further investigations for the explanation of the reason for these very deviating results obtained by different investigators is apparent.

As the carbon dioxide is always in part combined chemically, and as this part increases with the amount of alkali in the blood, it is evident that the amount of carbon dioxide and the carbondioxide tension must not always be parallel. 'That this is a fact has been shown by GAULE in experimenting on suffocated dogs. If the amount of alkali in the blood be dimpinished, then, naturally, the amount of carbon dioxide will be decreased. Such a behavior is found in poisoning by mineral acids. WALTER found only $2-3$ vols. per cent carbon dioxide in the blood of a rabbit which had had hydrochloric acid introduced into the stomach. In the comatose state of diabetes melitus the alkali of the blood seems to be saturated in great part by an acid combination ( $\beta$ oxybutyric acid) (Stadelmann, Minkowsky), and correspondingly Minkowsky found in the blood in comatose diabetes only 3.3 vols. per cent carbon dioxide.

In regard to the carbon-dioxide tension in the tissue, we must assume a priori that it is higher than in the blood. This is found to be true. Strassburg found in the urine of dogs and in the bile a carbon-dioxide tension of $9 \%$ and $\%$ of an atmosphere, respect- 
ively. The same experimenter has, further, injected atmospheric air into a loosened intestinal knot of a living dog, and analyzed the air taken out after some time. He found a carbon-dioxide tension of $7.7 \%$ of an atmosphere. The carbon-dioxide tension in the tissue is strikingly greater than in venous blood, even if we use as basis for our calculation the figures found by PFLÜGER and his pupils, as opposed to BоHR's results of relatively high values, for the carbondioxide tension. It is not disputed that the carbon dioxide simply passes from the tissue to the blood according to the laws of diffusion.

\section{The Quantitative Constitution of the Blood.}

The quantitative analysis of blood cannot be of value for the blood as an entirety. We must ascertain on one side the relationship of the plasma and blood-corpuscles to each other, and on the other side the constitution of each of these two chief constituents. The difficulties which stand in the way of such a task, especially in regard to the living, non-coagulated blood, have not been removed. Since the constitution of the blood may differ not only in different vascular regions, but also in the same region under different circumstances, which renders also number of blood analyses necessary, it can hardly appear remarkable that our knowledge of the con stitution of the blood is still relatively limited.

If any substance is found in the blood which belongs exclusively to the plasma and does not occur in the blood-corpuscles, then the amount of plasma contained in the blood may be calculated if we determine the amount of this substance in 100 parts of the plasma and serum, respectively, on one side and in 100 parts of the blood on the other. If we represent the amount of this substance in the plasma by $p$ and in the blood by $b$, then the amount $x$ of the plasma in 100 parts of blood is $x=\frac{100 . b}{p}$.

Such a substance, which occurs only in the plasma, is fibrin according to HOPPE-SEYLER, sodium according to BUNGE (in certain kinds of blood), and sugar according to OTro. The experimenters just named have tried to determine the amount of the plasma and the blood-corpuscles, respectively, in different kinds of blood, starting from the above-mentioned substances. 
Another method, suggested by HOPPE-SEYLER, is to determine the total amount of hæmoglobin and albumin in a portion of blood, and on the other hand the amount of hæmoglobin and albumin in the blood-corpuscles of an equal portion of the same blood which have been sufficiently washed with common-salt solution by centrifugal force. The figures obtained as a difference between these two determinations correspond to the amount of albumin which was contained in the serum of the first portion of blood. If we now determine the albumin in a special portion of serum of the same blood, then the amount of serum in the blood is easily determined. The usefulness of this method has been confirmed by BUNGE by the control experiments with the sodium determinations. If the amount of serum and blood-corpuscles in the blood is known, and we then determine the amount of the different blood-constituents in the blood-serum on one side and of the total blood on the other, the distribution of these different blood-constituents in the two chief components of the blood, plasma and blood-corpuscles may be ascertained. According to the just-mentioned procedure, the following analyses of pig's blood and ox's blood have been made by BUNGE. Analyses of human blood have been made for some time by C. ScHMIDT according to another method, which perhaps have given rather too high results for the weight of the bloodcorpuscles. All figures represent parts in 1000 parts of blood.

\begin{tabular}{|c|c|c|c|c|c|c|c|c|}
\hline & \multirow{2}{*}{\multicolumn{2}{|c|}{ Pig's Blood. }} & \multirow{2}{*}{\multicolumn{2}{|c|}{ Ox's Blood. }} & \multicolumn{4}{|c|}{ Human Blood. } \\
\hline & & & & & \multicolumn{2}{|c|}{ Man's. } & \multicolumn{2}{|c|}{ Woman's. } \\
\hline & $\begin{array}{l}\text { Blood- } \\
\text { cor- } \\
\text { puscles } \\
436.8\end{array}$ & $\begin{array}{c}\text { Serum } \\
\mathbf{5 6 3 . 2}\end{array}$ & $\begin{array}{c}\text { Blood- } \\
\text { cor- } \\
\text { puscles } \\
318.7\end{array}$ & \begin{tabular}{|c|} 
Serum \\
681.3
\end{tabular} & $\begin{array}{c}\text { Blood- } \\
\text { cor- } \\
\text { puscles } \\
513.02\end{array}$ & $\begin{array}{c}\text { Serum } \\
486.98\end{array}$ & $\begin{array}{l}\text { Blood- } \\
\text { cor- } \\
\text { puscles } \\
396.24\end{array}$ & $\begin{array}{l}\text { Serum } \\
603.76\end{array}$ \\
\hline Water. & 276.100 & 517.900 & 191.200 & 622.200 & 349.690 & 439.020 & 272.560 & 551.990 \\
\hline Solids........ & 160.700 & 45.300 & 127.500 & 59.100 & 163.330 & 47.690 & 123.680 & 51.70 \\
\hline $\left.\begin{array}{l}\text { Hæmoglobin and } \\
\text { Albumin }\end{array}\right\} . .$. & 151.600 & 38.100 & 123.600 & 49.900 & 159.590 & 43.820 & 120.130 & 46.700 \\
\hline Remaining org. bodies. & 5.200 & 2.800 & 2.400 & 3.800 & & & & \\
\hline Inorganic bodies........ & $\begin{array}{l}3.900 \\
2.421\end{array}$ & 4.300 & 1.500 & 5.400 & $\begin{array}{l}3.740 \\
1.586\end{array}$ & $\begin{array}{l}4.140 \\
0.153\end{array}$ & $\begin{array}{l}3.550 \\
1.412\end{array}$ & $\begin{array}{l}5.070 \\
0.200\end{array}$ \\
\hline $\begin{array}{l}\mathrm{K}_{2} \mathrm{O} \\
\mathrm{N}_{2}{ }_{2} \mathrm{O} \ldots \ldots \ldots \ldots \ldots \ldots \ldots \ldots\end{array}$ & ........ & $\begin{array}{l}0.154 \\
2.406\end{array}$ & $\begin{array}{l}0.238 \\
0.667\end{array}$ & $\begin{array}{l}0.173 \\
2.964\end{array}$ & $\begin{array}{l}1.580 \\
0.241\end{array}$ & 1.661 & 0.648 & 1.916 \\
\hline 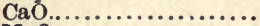 & $\ldots \ldots$ & 0.072 & & 0.070 & $\ldots .$. & ......... & ......... & $\ldots \ldots$ \\
\hline $\mathrm{MgO} \ldots \ldots \ldots \ldots \ldots$ & 0.069 & 0.021 & 0.005 & 0.031 & (n....... & $\ldots \ldots$ & $\cdots \cdots$ & $\ldots \ldots$ \\
\hline $\begin{array}{c}\mathrm{Fe}_{2} \mathrm{O}_{3} \ldots \ldots \ldots \ldots \ldots \ldots \\
\mathrm{Cl}\end{array}$ & $0 . \dot{657}$ & $\begin{array}{l}0.006 \\
2.034\end{array}$ & $\dddot{0} \dddot{5} 2 \mathrm{i}$ & $\begin{array}{l}0.007 \\
2.532\end{array}$ & 0.898 & $\cdots 1.723$ & $\ddot{0} 362$ & $\dddot{0.144}$ \\
\hline $\mathrm{P}_{2} \mathrm{O}_{5} \ldots \ldots \ldots \ldots \ldots \ldots$ & 0.903 & 0.106 & 0.224 & 0.181 & 0.695 & 0.071 & 0.643 & $2.20 \%$ \\
\hline
\end{tabular}

Hoppe-Seyler, Sacharjin, and Otto found 584.9-693.5 p. m. plasma and 415.1-306.5 p. m. blood-corpuscles in horse's blood. BUNGE found, on the contrary, in an analysis 468.5 p. m. serum and 531.5 p. m. blood-corpuscles-more blood-corpuscles, therefore, 
than serum. For human blood ARronet has found 478.8 p. m. blood-corpuscles and 521.2 p. m. serum (in defibrinated blood) as an average of nine determinations.

The relative amount of blood-corpuscles and plasma therefore varies. In human blood the plasma is about 50\% of the weight of the blood; but in other cases it seems generally to be somewhat greater. In a fow cases it may indeed be $\frac{2}{3}$ of the weight of the blood. Water occurs in the greatest amount in the plasma or serum, which latter ordinarily contains at least $\frac{9}{10}$ water, while the bloodcorpuscles contains only a little more than $\frac{1}{2}$ or about $\frac{2}{3}$ water. Iron probably occurs only in the blood-corpuscles. Chlorine and codium prevail generally in the plasma, potassium and phosphoric acid in the blood-corpuscles. In a few varieties of blood (pig's and horse's blood) the sodium is found exclusively in the plasma or serum, the potassium prevailing in the blood-corpuscles (BUNGE). In dog's and ox's blood the blood-corpuscles are, however, richer in sodium than in potassium (BUNGE). In man the potassium is contained in the largest quantities in the blood-corpuscles and only in very small quantities in the plasma (C. ScHмIDT, WANACH). The alkaline earths occur chiefly in the plasma. Manganese has also been found in the blood $(0.06 \mathrm{p} . \mathrm{m}$. according to BURIN DE BUISSON), as well as traces of lithium, copper, lead, and silver. The blood as a whole contains in ordinary cases $7 \% 0-820$ p. m. water, with $180-230$ p. m. solids; of these $173-220$ p. m. are organic and $6-10 \mathrm{p} . \mathrm{m}$. inorganic. The organic consist, deducting 6-12 p. m. extractive bodies, of albumin and hæmoglobin. The amount of these last-mentioned bodies in the blood is about 130-150 p. m.

The amount of sugar in the blood is on an average 1-1.5 p. m. The quantity of urea, which amounts to $0.2-0.9 \mathrm{p}$. m., is greater after partaking of food than during fasting (GRÉHANT and QUINQUAUD). The quantity of uric acid may be $0.1 \mathrm{p}$. m. in bird's blood (v. SCHRÖDER), and the quantity of lactic acid may reach $0.71 \mathrm{p}$. m. in human venous blood (BERLINERBLAU). 


\section{The Constitution of the Blood in different Vascular Regions and under different Physiological Conditions.}

Arterial and Venous Blood. The most striking difference between these two kinds of blood is the variation in color caused by their containing different amounts of gas and different amounts of oxyhæmoglobin and hæmoglobin. The arterial blood is light red; the venous blood is dark red, dichroitic, greenish by transmitted light through thin layers. The arterial coagulates more quickly than the venous blood. The latter, on account of the transudation which takes place in the capillaries, is somewhat poorer in water but richer in blood-corpuscles and hæmoglobin than the arterial blood (Heidenhain, Nasse, Otto). The quantity of sugar is somewhat greater in the arterial blood than in the venous (ОтTо).

Blood from the Portal Vein and the Hepatic Vein. The blood of the hepatic vein is poorer in ordinary red blood-corpuscles but richer in colorless and so-called young red blood-corpuscles. A few investigators have decided from this that a formation of red blood-corpuscles takes place in the liver, while others claim that a destruction takes place.

In consideration of the relationship to the simultaneous formation of small quantities of bile and lymph in the unit of time of the large quantities of blood circulating throuhg the liver we can hardly expect to detect a positive difference in the constitution of the blood of the hepatic vein by chemical analysis. The statements in regard to such a difference are in fact contradictory. For example, Drospoff has found more hæmoglobin in the hepatic than in the portal veins, while Отто found less. In regard to the different amounts of sugar in these two kinds of blood we have also much contradiction. According to a few experimenters, as OTTO and above all others SEEGEN, the blood of the hepatic vein is richer in sugar, which corresponds with the older statements of Cladde Bernard. Such a difference may, however, be caused by the operative interference in the collection of the blood from the hepatic vein (ABELES), and as a rule investigators nowadays do not seem to agree with BERNARD's view. During the digestion of food rich in carbohydrates the blood of the portal vein may not only be richer in glucose but also contain other carbohydrates (v. MERING. 
OTTo). The amount of urea in the blood from the hepatic vein is greater than in other blood (GRÉHANT and QUiNQUAUD).

Blood of the Splenic Vein is decidedly richer in leucocytes than the blood from the splenic artery. The red blood-corpuscles of the blood from the splenic vein are smaller than the ordinary, less flattened, and show a greater resistance to water. The blood from the splenic vein is also claimed to be richer in water, fibrin, and albumin than the ordinary venous blood (BÉCLARD). According to v. MIDDENDORFF, it is richer in hæmoglobin than arterial blood. It coagulates more slowly.

The Blood from the Veins of the Glands. The blood circulates with greater rapidity through a gland during activity (secretion) than when at rest, and the outflowing venous blood has therefore during activity a lighter red color and a greater amount of oxygen. Because of the secretion the venous blood also becomes somewhat poorer in water and richer in solids.

The blood from the Muscular Veins shows an opposite behavior, for during activity it is darker and more venous in its properties because of the increased absorption of oxygen by the muscles or still greater production of carbon dioxide than when at rest.

Menstrual Blood has, according to an old statement, not the power of coagulating. This statement is nevertheless false, and the apparent uncoagulability depends in part on the womb and the vagina retaining the blood-clot, so that only fluid cruor is at times eliminated, and in part on a contamination with vaginal mucus which disturbs the coagulation.

The Blood of the Two Sexes. Woman's blood coagulates somewhat more quickly, has a lower specific gravity, a greater amount of water, and a smaller quantity of solids than the blood of man. The amount of blood-corpuscles and hæmoglobin is somewhat smaller in woman's blood. The amount of hæmoglobin is, according to ОтTо, $146 \mathrm{p}$. m. for man's blood and $133 \mathrm{p}$. m. for woman's.

During pregnancy NASSE has observed a decrease in the specific gravity, with an increase in the amount of water towards the end of the eighth month. From then the specific gravity increases, and at delivery it is normal again. The amount of fibrin is somewhat increased (BECQUEREL and RoDIER, NASSE). The number of 
blood-corpuscles seems to decrease. In regard to the amount of hæmoglobin the statements are somewhat contradictory.

The Blood at Different Periods of Life. Fœtal blood is strikingly poorer in blood-corpuscles and hæmoglobin than the blood of the adult. The fotal blood at the moment of birth has, according to ScherRenziss, a lower specific gravity, a markedly lower amount of hærnoglobin, and a little less fibrin, but a greater amount of mineral bodies, especially proportionally more sodium (but less potassium) than the blood of adults. A few hours after birth the blood of the child has the same quantity of hæmoglobin as the blood of the mother (CoHNstein, Zuntz, OtTo). The amount of hæmoglobin and of blood-corpuscles then quickly increases; still they do not both increase at the same rate, as the amount of hæmoglobin increases much faster. Two to three days after birth the hæmoglobin reaches a maximum (20-21\%), which is greater than at any other period of life. 'This is the cause of the great abundance of solids in the blood of new-born infants as observed by Denis, Panum, and other investigators. The quantity of hæmoglobin and blood-corpuscles sinks gradually from this first maximum to a minimum of about 11\% hæmoglobin, which minimum appears in human beings between the fourth and eighth year. The quantity of hæmoglobin then increases again until about the twentieth year, when a second maximum of $13.7-15 \%$ is reached. The hæmoglcbin remains at this point only towards the forty-fifth year, and then gradually and slowly decreases (LEICHTENSTERN, Отто). According to older statements, the blood at old age is poorer in blood-corpuscles and albuminous bodies but richer in water and salts.

The Influence of Food on the Blood. In complete starvation a decrease in the amount of solid blood constituents is found to take place (PANUM and others). The amount of hæmoglobin is a little increased (SuввотіN, Oтто), and also the number of red blood-corpuscles is greater (WorM Müller, BuntzeN), which probably depends on the fact that the blood-corpuscles are not so quickly transformed as the serum. As after-effect the inanition causes an anæmic condition (Worm Müller, OtTo, BuntzeN).

After a rich meal the relative number of blood-corpuscles, especially after secretion of digestive juices or absorption of 
nutritive liquids, may be increased or diminished (Buntzen, LEICHTENSTERN). The number of colorless blood-corpuscles may be increased to such an extent, after a diet rich in albumin, that a true digestion leucocytose appears (HoFMEISTER and PoHL). After a diet rich in fat the plasma becomes, even after a short time, more or less milky-white, like an emulsion. The constitution of the food acts essentially on the amount of hæmoglobin in the blood. The blood of herbivora is generally poorer in hæmoglobin than that from carnivora, and SuBBotin has observed in dogs after a partial feeding with food rich in carbohydrates that the amount of hæmoglobin sank from the physiological average of $13 \% .5 \mathrm{p} . \mathrm{m}$. to 103.2-93.7 p. m. According to Leichtenstern, a gradual increase in the amount of hæmoglobin is found to take place in the blood of human beings on enriching the food, and according to the same investigator the blood of lean persons is generally somewhat richer in hæmoglobin than blood from fat ones of the same age. The addition of iron salts to the food greatly influences the number of blood-corpuscles and also the amount of hæmoglobin they contain, so that, according to NASSE, the iron, especially in combination with fat, is active. According to the investigations of HAYEM and Mallassez, the iron preparations increase the amount of hæmoglobin contained in the blood in anæmia to a higher degree than the number of blood-corpuscles.

The Constitution of the Blood under Physiological Conditions may be changed either by the appearance of a foreign substance or by the quantities of any one or more of the blood constituents being abnormally increased or diminished. Changes of this last kind occur frequently.

An increase in the number of red corpuscles, a true "PLETHORA POLYCYTH EMICA," takes place after transfusion of blood of the same species of animal. According to the observations of PANUM and WORM MÜLLER, the blood-liquid is quickly eliminated and transformed in this case,- the water being eliminated principally by the kidneys, and the albumin burned into urea, etc.,--while the blood-corpuscles are preserved longer and cause a "POLYCYTH мIA." A relative increase in the number of red corpuscles is found after abundant transudations from the blood, as in cholera and heart-failure, with considerable accumulation. 
A decrease in the number of red corpuscles occurs in anæmia from different causes. Each hemorrhage causes an acute anæmia or oligæmia. Even during the bleeding the remaining blood becomes richer in water by diminished secretion and excretion, as also by an abundant absorption of parenchymous fluid somewhat poorer in albumin and strikingly poorer in red blood-corpuscles. The oligæmia passes soon into a hydræmia. The amount of albumin then gradually increases again; but the re-formation of the red blood-corpuscles is slower, and after the hydræmia follows also an oligocythæmia. After a little time the number of blood-corpuscles rises to normal; but the re-formation of hæmoglobin does not keep pace with the re-formation of the corpuscles, and a chlorotic condition may appear (LAache, Buntzen, OtTo). A considerable decrease in the number of red corpuscles occurs also in chronic anæmia and chlorosis; still in such cases an essential decrease in the amount of hæmoglobin occurs without an essential decrease in the number of blood-corpuscles. The decrease in the amount of hæmoglobin is more characteristic of chlorosis than a decrease in the number of red corpuscles.

A very considerable decrease in the number of red corpuscles (from 300,000 to 400,000 in $1 \mathrm{c.} \mathrm{mm}$.) and diminishing in the amount of hæmoglobin (from $\frac{1}{8}$ to $\frac{1}{10}$ ) occurs in malignant anæmia (HAYEM, LÉPINe, LAACHe, and others). On the contrary, the individual red corpuscles are larger and richer in hæmoglobin than they ordinarily are, and the number stands in an inverse relationship to the amount of hæmoglobin (HAYEM).

The Constitution of the Red Corpuscles. Irrespective of the changes in the amount of hæmoglobin, as just mentioned, the constitution of the blood-corpuscles may be changed in other ways. By abundant transudation, as in cholera, the blood-corpuscles may give up water, potassium, and phosphoric acid to the concentrated plasma and become correspondingly richer in organic substances (C. Schмidт). By a few other transudations processes, as in dysentery and dropsy with albuminuria, a considerable amount of albumin passes from the blood, the plasma becomes richer in water, and the blood-corpuscles may take up water and so become poorer in organic substance (C. SснмIDT).

The number of colorless blood-corpuscles is found to be in- 
creased by suppuration in puerperal fever, pyæmia, and many other diseases, but especially in leucæmia, which disease is characterized by a great abundance of leucocytes in the blood. The number of leucocytes is not only absolutely increased in this disease, but also in proportion to the number of red blood-corpuscles, which is considerably diminished in leucæmia. The blood from a leucæmic patient has a lower specific gravity than the ordinary (1.035-1.040) and a lighter color, as if it were mixed with pus. The reaction after death is often acid, probably due to a decomposition of the considerably-increased lecithin. In leucæmic blood, volatile fatty acids, lactic acid, glycero-phosphoric acid, large amounts of xanthin bodies (SALOMON, KOSSEL) and the so-called CHarcot's crystals (see Chapter XI) have been found.

The quantity of water in the blood is increased by general dropsy, with or without kidney disease, by the different forms of anæmia, by scurvy, and by febrile diseases. The amount of water is diminished by abundant transudations, by powerful laxatives, by diarrhœa, and especially by cholera.

The amount of albumins in the blood may be relatively increased (HYPERALBUMINOSE) in cholera and after the action of laxatives. A decrease in the amount of albumin (HYPALBUMINOSE) occurs after direct loss of albumin from the blood, as in bleeding albuminuria, dysentery, abundant formation of pus, etc., etc. The amount of fibrin is increased (HYPERINOSE) in inflammatory diseases, pneumonia, acute muscular rheumatism, and erysipelas, in which the blood yields a "CRUSTA PHLOGISTICA" because it coagulates more slowly. The statements in regard to the occurrence of a hyperinose in scurvy and hydræmia seems to require further investigation. A decrease in the amount of fibrin (HYPINOSE) has been observed in malaria, pyæmia, and malignant anæmia. These statements also require further corroboration.

The amount of fat in the blood (LIP \&MIA) increases, irrespective of the increase after a diet rich in fat, in drunkards, in corpulent individuals, after fracture of the bones, and also in diabetes. In the last-mentioned case the increase in fat depends, according to PAVy and Hoppe-SeYler, upon defective digestion.

An increase in the amount of fat in the blood has also been observed in diseases of the liver, Bright's disease, tuberculosis, mala- 
ria, and cholera. v. JAKSCH has observed volatile fatty acids in the blood (LIPACID ÆMIA) in febrile diseases and sometimes in diabetes.

The amount of salts in the blood is increased in dropsy, dysentery, and in cholera immediately after the first violent attack, but diminishes later after the attack in cholera, in scurvy, and in inflammatory diseases. The decrease of alkali salts, especially common salt, is only trifling, but in pneumonia the salt disappears almost entirely from the urine. A decrease in the alkalinity of the blood has been observed in many cases, as in fevers, uræmia, carbon-monoxide poisoning, diseases of the liver, leucæmia, malignant anæmia, and diabetes. The above-mentioned (page 104) decrease in the alkalinity of the blood in diabetes mellitus is of special interest.

The quantity of glucose is increased in diabetes (mellitæmia). HopPe-SEYLER found in one case $9 \mathrm{p} . \mathrm{m}$. glucose in the blood. According to ClaUde BeRNaRd, when the quantity of glucose in the blood amounts to $3 \mathrm{p}$. m. it passes into the urine. The quantity of urea is augmented in fevers, also in increased exchange of albumin, and by an increased formation of urea caused thereby. A further increase in the amount of urea in the blood occurs in a retarded micturition, as in cholera as well as in cholera infantum (K. MörNER), and in affections of the kidneys and the urinary passages. After a ligature of the ureters or after extirpation of the kidneys of animals an accumulation of urea takes place in the blood. In uræmia, ammonia may occur in the blood, which originates from a decomposition of the urea. Uric acid is found increased in the blood in gout (GARROD, SALOMON); oxalic acid was also found in the blood in the same disease by GaRRoD.

Among the foreign bodies which are found in the blood the following must be mentioned here: BILIARY ACIDS and BILIARY COLORING MATTERS (which latter may occur under physiological conditions in a few varieties of blood) in iceterus; LEUCIN and TYROSIN in acute atrophy of the liver; ACETON specially in fevers (v. JAKSCH). In melanæmia, especially after continuous malarial fever, black, less often light brown or yellowish, grains of pigment occur in the blood, which, according to the generally-received opinion, come into the blood from the spleen. After poisoning with potassium chlo- 
rate, metahæmoglobin is observed in human and in canine blood (MARCHAND and KAHN); but, on the contrary, no formation of metahæmoglobin takes place in the blood of rabbits (STOKVIS and KIMMYSER). A formation of metahæmoglobin may be caused at the expense of the hæmoglobin by the inhalation of amyl nitrite, as also by the action of a number of other medicinal bodies (HAYEM and others).

The quantity of blood is indeed somewhat variable in different species of animals and in different conditions of the body; in general we consider the entire quantity of blood in adults as about $\frac{1}{13}-\frac{1}{14}$ of the weight of the body, and in new-born infants about $\frac{1}{19}$. Fat individuals are relatively poorer in blood than lean ones. During inanition the quantity of blood decreases less quickly than the weight of the body (PANUM), and it may therefore be also proportionally greater in starving individuals than in well-fed ones.

By careful bleeding the quantity of blood may be considerably diminished without any dangerous symptoms. The loss of blood to $\frac{1}{4}$ of the normal quantity has as sequence no durable sinking of the blood-pressure in the arteries; while the smaller arteries accommodate themselves to the small quantities of blood by contracting (WorM MüLLER). A loss of blood to $\frac{1}{3}$ of the quantity reduces the blood-pressure considerably, and a loss of $\frac{1}{2}$ of the blood in adults is dangerous to life. The faster the bleeding the more dangerous it is. New-born infants are very sensitive to loss of blood, and likewise fat, old, and weak persons cannot stand much loss of blood. Women can stand loss of blood better than men.

The quantity of blood may be considerably increased by the injection of blood from the same species of animal (PANUM, LANDoIs, WorM Müller, PoNfick). According to WorM Müller, the normal quantity of blood may indeed be increased to $83 \%$ without producing any abnormal conditions or continuing high blood-pressure. An increase of the quantity of blood to $150 \%$ may be directly dangerous to life (WorM MüLLER). If the quantity of blood of an animal is increased by transfusion with blood of the same kind of animal, an abundant formation of lymph takes place. The water in excess is eliminated by the urine; and as the albumin of the blood serum is quickly decomposed, while the red blood-corpuscles are 
destroyed much more slowly ('TschirJew, Forster, Panum, Worm MÜLLER), a polycythæmia is gradually produced.

If blood of another kind is transfused, then under certain conditions, according to the quantity of blood introduced, more or less menacing symptoms appear. These appear, for instance, when the blood-corpuscles of the receiver are dissolved easily by the serum of the introduced blood, as, for example, the blood-corpuscles of rabbits on transfusion with a different kind of blood, or the reverse, when the blood-corpuscles of the transfused blood are dissolved by the blood of the receiver; for instance, when the blood of a dog is transfused with rabbit's or lamb's blood, or the blood of a man with lamb's blood (LANDoIs). Before dissolving, the blood-corpuscles may unite in tough agglomerated heaps, which clog up the smaller vessels (LANDOIS). On the other hand, the stromata of the dissolved blood-corpuscles may also give rise to an extensive intravascular coagulation causing death.

The transfusion should therefore when possible be made with the blood of the same kind of animal, and for the resuscitating action of the blood it is immaterial whether or not it contains the fibrin or the mother-substance of the same. The action of transfused blood depends on its blood-corpuscles, and therefore defibrinated blood acts just like non-defibrinated (PANUM, LANDOIS).

The quantity of blood in the different organs depends essentially on the activity of the same. During work the exchange of material in an organ is more active than when at rest, and the increased exchange of material is combined with a more abundant flow of blood. Although the total quantity of blood in the body remains constant, the distribution of the blood in the various organs may be different at different times. As a rule, the quantity of blood in an organ can be an approximate measure of the more or less active exchange of material going on in the same, and from this point of view the distribution of the blood in the different organs and groups of organs is of interest. According to RANKE, to whom we are especially indebted for our knowledge of the relationship of the activity of the organs to the quantity of blood contained therein, of the total quantity of blood (in the rabbit) about $\frac{1}{4}$ comes to the muscles in rest, $\frac{1}{4}$ to the heart and the large blood-vessels, $\frac{1}{4}$ to the liver, and $\frac{1}{4}$ to the other organs. 


\section{CHAPTER V.}

\section{CHYLE, LYMPH, TRANSUDATIONS AND EXUDATIONS.}

\section{Chyle and Lymph.}

From the close relationship which exists between blood and lymph, and the dependence which the formation of lymph has upon the blood-circulation and the blood-pressure, it is to be expected that a close correspondence in the chemical constitution between bloodplasma and lymph should exist. The lymph is generally considered as transudated plasma. Qualitatively the lymph contains the same substances as the plasma. The essential difference is of a quantitative nature and consists in that the lymph is poorer in albumin. No essential chemical difference has been found between the lymph and the chyle of starving animals. After the assimilation of fatty food the chyle differs from the lymph in its wealth of minutelydivided fat-globules which give it a milky appearance; hence the old name "milk-juice."

Chyle and lymph, as well as the plasma, contain serum albumin, serum globulin, fibrinogen, and fibrin ferment. The two last-mentioned bodies occur only in very small amounts; therefore the chyle and lymph coagulate slowly (but spontaneously) and yield but little fibrin. Like other liquids poor in fibrin ferment, chyle and lymph do not at once coagulate completely, but repeated coagulations take place.

The extractive bodies seem to be the same as in plasma. Glucose is found in about the same quantity as in the blood-serum (v. Mering), but in larger quantities than in the blood (PoiseuILle and Lefort, Ginsberg); this depends on the fact that the blood-corpuscles contain no glucose. The amount of urea has been 
determined by WURTZ as $0.12-0.28 \mathrm{p} . \mathrm{m}$. The mineral bodies appear to be the same as in plasma.

As form-elements leucocytes and red blood-corpuscles are common to both chyle and lymph. When it has not left the connivent valves chyle contains very few leucocytes, but in the vessels moving on the peritoneal side of the intestines it is richer in leucocytes. The greatest quantity of leucocytes is found in the chyle between the great mesenteric gland and the cisterna chyli. The chyle is poorer in leucocytes in the thoracic duct, probably becanse a mixing takes place here with lymph, from other parts of the body that is poorer in form-constituents.

The red blood-corpuscles occur in the chyle and lymph in very small quantities. In these liquids, which seem to be free from oxygen, the blood-corpuscles are darker-colored, and only after they have come in contact with the air do they have the light-red color of oxyhæmoglobin and give the surface of the fibrin clot a beautiful light-red appearance. It has been suggested that this red color originates from the transition forms between red and white blood-corpuscles, in which blood-coloring matters are first formed by the action of the oxygen.

The chyle of starving animals has the appearance of lymph. After partaking of fat or food rich in fat it is milky, and this is partly due to the presence of large fat-globules, as in milk, or partly, and indeed chiefly, the finely-divided fat. The nature of the fats occurring in the chyle depends on the variety of fat in the food. The disproportionally greater part consists of neutral fats, and even after feeding with abundant amounts of free fatty acids MUNK and LEBEDEFF found in the chyle chiefly neutral fats with a small quantity of fatty acids or soaps.

The gases of the chyle have not been studied, and it seems that the gases of an entirely normal human lymph have not thus far been investigated. The gases from dog-lymph contain only traces of oxygen and consist of 37.4-53.1\% $\mathrm{CO}_{2}$ and $1.6 \% \mathrm{~N}$ (AUTHOR) calculated at $0^{\circ} \mathrm{C}$. and $760 \mathrm{~mm}$. mercury. The chief mass of the carbon dioxide of the lymph seems to be firmly chemically combined. Comparative analyses of blood and lymph have shown that the lymph contains more carbon dioxide than arterial but less than venous blood. The tension of the carbon dioxide of lymph is, 
aecording to PfLUtger and Strassburg, smaller than in venous but greater than in arterial blood.

The quantitative constitution of the chyle must naturally be very variable. The analyses thus far made refer only to that mixture of chyle and lymph which is obtained from the thoracic duct. The specific gravity varies between $1.00 \%$ and 1.043. As example of the constitution of human chyle we will here give two analyses. The first is by OwEN.REES, of the chyle of an executed person, and the second by HoPPE-SEYLER, of the chyle in a case of rupture of the thoracic duct. In the latter case the fibrin had previously separated. The results are in 1000 parts.

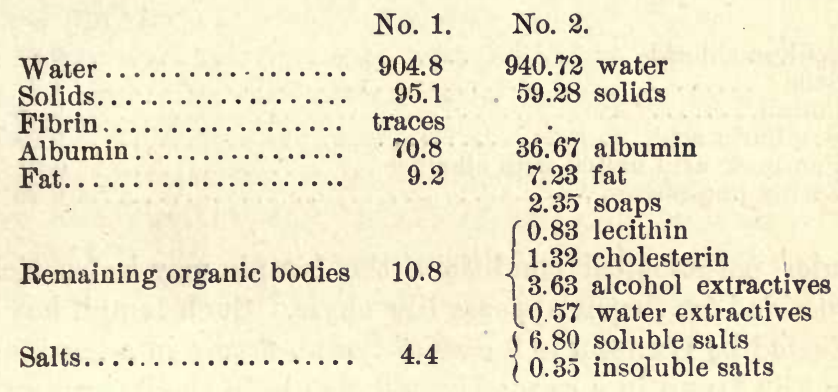

The quantity of fat is very variable and may be considerably increased by partaking food rich in fats.

A great many analyses of chyle from animals have been made, and they chiefly show the fact that the chyle is a liquid with a very changeable composition which stands closely related to bloodplasma, but with the chief difference that it contains more fat and less solids. The reader is referred to special works for these analyses, as, for example, to v. GorUP-BeSANEz's "Lehrbuch der physiologischen Chemie," 4th edition.

The composition of the lymph is also very changeable, and its specific gravity shows about the same variation as the chyle. In the following analyses, 1 and 2, made by Gubler and Quevenne, are the results obtained from lymph from the upper part of the thigh of a woman aged 39 ; and 3, made by v. ScHERER, is an analysis of lymph from the sac-like dilated vessels of the spermatic cord. No.

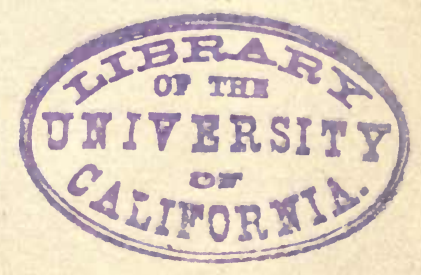


4 was made by C. ScHMidT, the data being obtained from lymph from the neck of a colt. The results are in parts per 1000.

\begin{tabular}{|c|c|c|c|c|}
\hline & 1 & 2 & 3 & \\
\hline Water. . & 939.9 & 934.8 & 957.6 & 955 \\
\hline Solids....... & 60.1 & 65.2 & 42.4 & 44 \\
\hline Fibrin ............... & 0.5 & 0.6 & 0.4 & 2 \\
\hline 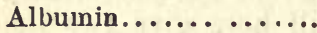 & 42.7 & 42.8 & 34.7 & \\
\hline t, cholesterin, lecithin & 3.8 & 9.2 & $\ldots$. & 34 \\
\hline Extractive bodies...... & 5.7 & 4.4 & & \\
\hline alts $\ldots \ldots \ldots \ldots \ldots \ldots$ & 7.3 & 8.2 & 7.3 & \\
\hline
\end{tabular}

The mixture of salts found by C. Schmidt in the lymph of the horse has the following composition, calculated in parts per 1000 parts of the lymph:

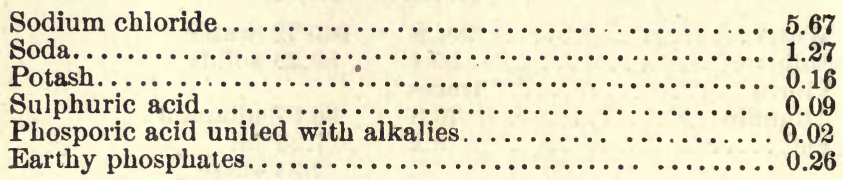

Under pathological conditions the lymph may be so rich in finely-divided fat that it appears like chyle. Such lymph has been investigated by HeNSEN in a case of lymph fistula in a ten-year-old boy, and by LANG in a case of lymph fistula in the left upper part of the thigh of a girl of seventeen. The lymph investigated by HeNSEN contained as an average of nineteen analyses $19 \mathrm{p}$. m. of fat and $0.6 \mathrm{p}$. m. of cholesterin, while that investigated by LANG contained $24.8 \mathrm{p}$. m. of fat.

The quantity of chyle and lymph must naturally change considerably, therefore, for this and other reasons, the calculations of the quantity in 24 hours are not to be depended upon. The food plays a very important rôle in the quantity of chyle and lymph. NASSE has observed in dogs that the formation of lymph is $36 \%$ more after feeding with meat than after feeding with potatoes, and about 54\% more than after 24 hours' deprivation of food.

The amount of lymph is increased by the following influences, namely, by increasing the total quantity of blood, as by transfusion of blood (WORM MÜLLER), raising the blood-pressure (LUDWIG and ToMsA), increased influx of the arterial blood (LUDwIG, RAGOwICZ, Giandzzi), and, above all, by preventing the discharge of the blood 
by tying the veins (BIDDER, EmminghaUs, Weiss). The quantity of lymph is also increased by strong active or passive muscular movements (LESSER). On poisoning with curara the secretion of lymph is increased (Puschutin, Lesser), and the solids of the lymph are also increased at the same time.

\section{Transudations and Exudations.}

The serous membranes are normally kept moistened by liquids whose quantity is only sufficient in a few instances, as in the pericardial cavity and the arachnoid membrane, for a complete chemical analysis to be made of them. Under diseased conditions an abundant transudation may take place from the blood into the serous cavities, into the subcutaneous tissues, or under the epidermis; and in this way pathological transudations are formed. Such true transudations, which are similar to lymph, are generally poor in form-elements and leucocytes, and yield only very little or almost no fibrin, while the inflammatory transudations, the so-called exudations, are generally rich in leucocytes and yield proportionally more fibrin. As a rule, the richer a transudation is in leucocytes the closer it stands to pus, while when it has a diminished quantity of leucocytes it is more nearly like real transudations or lymph.

It is ordinarily accepted that filtration is of the greatest importance in the formation of transudations and exudations. The facts coincide with this view, namely, that all these fluids contain the salts and extractive bodies occurring in the blood-plasma in about the same quautity as the blood-plasma, while the amount of albumin is habitually smaller. While the different fluids belonging to this group have about the same quantities of salts and extractive bodies, they differ from each other chiefly in containing differing quantities of albumin and form-elements, as well as varying quantities of transformation and decomposition products of these latter-changed blood-coloring matters, cholesterin, etc., etc. The largest quantity of albumin habitually occurs in inflammatory processes with changed permeability of the walls of the vessels. The condition of the capillaries in the different vascular regions affects the amount of albumin. For example, the 
amount of albumin in the PERICARDIAL, PLEURAL, and PERITONEAL FLUIDS is considerably greater than in those fluids which are found in the ARACHNOID MEMBRANE, in the SUB-CUTANEOUS TISSUES, or in the AqUEOUS HUMOR. The condition of the blood also greatly affects the transudations, for in hydræmia the amount of albumin in the transudation is very small. With the increase of the age of a transudation, of a hydrocele fluid for instance, the quantity of albumin is increased, probably by resorption of water, and indeed exceptional cases may occur in which the amount of albumin, without any previous bleeding, is greater than in the blood-serum. It is natural to suppose that the state of the circulation and the pressure must have an influence on the quantity and composition of the transudation even though their action is little known. By increasing the vein-pressure SENATOR caused an increase in the quantity of transudation and the amount of albumin contained therein, while the amount of salts was not essentially changed. Of the variation in the amount of albumin by simple arterial hyperæmia nothing is positively known.

The gases of the transudations consist of carbon dioxide besides small amounts of nitrogen and traces of oxygen. The tension of the carbon dioxide is greater in the transudations than in the blood (EWALD). On mixing with pus the amount of carbon dioxide is decreased.

The extractives are, as above stated, the same as in the bloodplasma; but sometimes extractive bodies occur, such as allantoin in dropsical fluids (Moscatelli), which have not been detected in the blood. Urea seems to occur in very variable amounts. Glucose, or at least a substance which reduces copper oxide in alkaline liquids, occurs in most transudations. Succinic acid has been found in a few cases in hydrocele fluids, while in other cases it is entirely absent. Leucin and tyrosin have been found in transudations from diseased livers and in pus-like transudations which have decomposed. Among other extractives found in transudations we must mention uric acid, allantoin, xanthin, creatin, inosit, and pyrocatechin.

As above stated, irrespective of the varying number of formelements contained in , the different transudations, the quantity of albumin is the most characteristic chemical distinction in their 
composition; therefore a quantitative analysis is of little importance except in determining the quantity of albumin.

Pericardial Fluid. The quantity of this fluid is also, under certain physiological conditions, so large that a sufficient quantity for chemical investigation was obtained from a person who had been executed. This fluid is lemon-yellow in color, somewhat sticky, and yields more fibrin than other transudations $(6-8 \mathrm{p} . \mathrm{m}$.). The amount of solids, according to the analyses performed by v. GoRUPBesanez, Wachsmuth, and Hoppe-SeYler, is 37.5-44.9 p. m., and the amount of albumin is $22.8-24.7 \mathrm{p}$. $\mathrm{m}$. In a case of chylopericardium, which was probabiy due to the rupture of a chylus vessel or caused by a capillary exudation of chyle because of stowing, HASEBROEK found in 1000 parts of the analyzed fluid 103.61 parts solids, $73 . \% 9$ albuminous bodies, $10.7 \%$ fat, 3.34 cholesterin, 1.77 lecithin, and 9.34 salts.

The pleural fluid occurs under physiological conditions in such small quantities that a chemical analysis of the same cannot be made. Under pathological conditions this fluid may show very variable properties. In a few cases it is nearly serous, in others again sero-fibrinous, and in others similar to pus. There is a corresponding variation in the specific gravity and the properties in general. If a pus-like exudation is kept closed for a long time in the pleural cavity, a more or less complete maceration and solution of the pus-corpuscles is found to take place. The ejected yellowishbrown or greenish fluid may then be as rich in solids as the bloodserum; and an abundant flocculent precipitate of a neucleo-albumin (the pyin of early writers) may be obtained on the addition of acetic acid. This precipitate is soluble with difficulty by adding an excess of acetic acid.

According to MÉHU, who has investigated a great number of pleural fluids, the specific gravity is generally higher than 1.020 in acute pleurisy, the amount of solids is $6.5 \mathrm{p} . \mathrm{m}$., and the quantity of fibrin not higher than $1.2 \mathrm{p} . \mathrm{m}$. In chronic pleurisy with gathering of pus the specific gravity is higher than 1.018 and may rise to 1.024 (according to the observations of the author it may rise indeed to 1.030). The quantity of solids may in these cases be $60-70 \mathrm{p} . \mathrm{m}$. or even more-90-100 p. m. (AUTHOR). Fibrin is absent. In disturbed circulation, as in cirrhosis of the liver or in heart troubles, 
the specific gravity is usually lower than 1.015 and the quantity of solids averages $20-30$ p. m.

The quantity of peritoneal fluid is very small under physiological conditions. The investigations refer only to the fluid under diseased conditions (dropsical or ascites fluid.) The color, transparency, and consistency of these may vary greatly.

In cachectic conditions the fluid is nearly colorless, milky-opalescent, watery, does not coagulate spontaneously, is of a very low specific gravity, 1.005-1.015, and nearly free from form-constituents. In carcinomatous peritonitis it may have a cloudy, dirty-gray appearance due to its richness in form-elements of various kinds. The specific gravity is then higher, the quantity of solids greater, and it often coagulates spontaneously. In inflammatory processes it is straw - or lemon-yellow in color, somewhat cloudy or reddish, due to leucocytes and red blood-corpuscles, and from great richness in leucocytes it may appear more like pus. It coagulates spontaneously, may be relatively richer in solids, and may have a specific gravity of 1.030 or more. By rupture of a chylous vessel the dropsical fluid may be rich in very finely-emulsified fat (CHYLOUS ASCITES). In such cases $3.86-10.30 \mathrm{p}$. m. fat has been found in the dropsical fluid (Goinochet, HaY). By admixture of this fluid with the fluid from an ovarian cyst it may sometimes contain pseudomucin (see Chapter XI). We also have cases in which the ascitical fluid contains mucoids which may be precipitated by alcohol after removal of the albumins by coagulation at boiling temperature. Such substances, which yield a reducible substance on boiling with acids, have been found by the author in tuberculous peritonitis and in cirrhosis hepatis syphilitica.

In order to show the amount of albumin in ascitic fluids we give below the results of RUNEBERG's analyses. They are in parts per 1000 parts of the fluid.

\begin{tabular}{|c|c|c|c|c|c|}
\hline & & & Max. & Min. & Average. \\
\hline Ascit & fluid in & 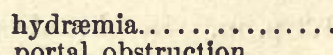 & & 0.2 & 2.1 \\
\hline 6 & “ & se & 23.0 & 8.4 & 16.7 \\
\hline "6 & “، & ca & 54.2 & 27.0 & 35.1 \\
\hline
\end{tabular}

Urea has also been found in ascitical fluids, sometimes only as traces, sometimes in larger quantities (4 p. m. in albuminuria), also uric acid, allantoin in cirrhosis of the liver (MOSCATELLI), xanthin, creatin, cholesterin, and glucose. 
Hydrocele and Spermatocele Fluids. These fluids differ from each other in various ways. The hydrocele fluids are generally colored light or darker yellow, sometimes brownish with a shade of green. They have a relatively higher specific gravity, 1.016-1.026, with a variable but generally higher amount of solids, an average of $60 \mathrm{p} . \mathrm{m}$. They sometimes coagulate spontaneously, sometimes only after the addition of fibrin ferment or blood. They contain as form-elements chiefly leucocytes. Sometimes they contain smaller or larger amounts of cholesterin crystals.

The spermatocele fluids, on the contrary, are as a rule colorless, thin, cloudy like water mixed with milk. They sometimes have an acid reaction. They have a lower specific gravity, 1.006 to 1.010 , a lower amount of solids-an average of about $13 \mathrm{p}$. m.,-and do not coagulate either spontaneously or after the addition of blood. They are, as a rule, poor in albumin and contain spermatozoa, cell-detritus, and fat-globules as form-constituents. To show the unequal composition of these two kinds of fluids we will give the average results (calculated in parts per 1000 parts of the fluid) of 17 analyses of hydrocele fluids and 4 of spermatocele fluids made by the author:

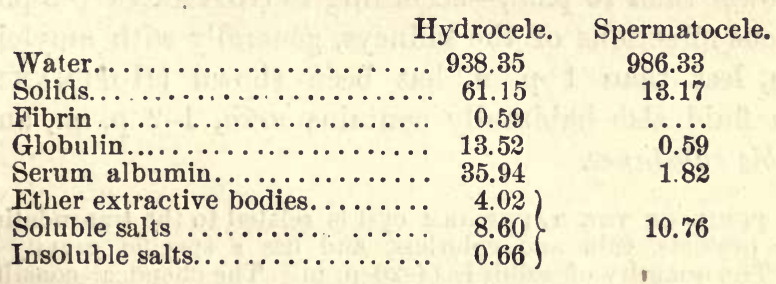

In the hydrocele fluids traces of uree and a reducing substance have been found, and in a few cases also succinic acid and inosit.

Cerebrospinal Fluid. This fluid, which in certain respects is more of a secretion than a transudation (C. ScHMidt, HALLIBURToN), is thin, water-clear, and has a low specific gravity (1.005). It is very poor in solids, $10-15 \mathrm{p} . \mathrm{m}$., and ordinarily contains about $10 \mathrm{p}$. m. albumin. This albumin is generally a mixture of globulin and albumose; occasionally some peptone occurs, and more rarely, in special cases, serum albumin appears (HALLIBURTON). An optically inactive, non-fermentable, reducible substance, seemingly pyrocatechin (HALLIBURTON), has been observed in this fluid. 
The older statement that the cerebro-spinal fluid differs from the transudations in a greater wealth of potassium salts has not been confirmed by recent investigations.

Aqueous Humor. This fluid is clear, alkaline, and has a specific gravity of 1.003-1.009. The amount of solids is on an average 13 p. m. and the amount of proteids only $0.8-1.2 \mathrm{p} . \mathrm{m}$. The proteids consists of about equal parts serum globulin and globulin (КАНN). According to GRUENHAGEN, it contains paralactic acid, another dextro-gyrate substance, and a reducible body which is not similar to glucose or dextrine.

Blister-fluid. The content of blisters caused by burns, and of vesicator blisters and the blisters of the pemphigus chronicus, is generally a fluid rich in solids and albumin (40-65 p. m.). 'This is especially true of the contents of vesicatory blisters, which also contain a substance that reduces copper oxide. The fluid of the pemphigus is slimy and alkaline in reaction.

The fluid of subcutaneous œdema. This is, as a rule, very poor in solids, purely serous, does not contain fibrinogen, and has a specific gravity of 1.005 to 1.010 . The quantity of proteids is in most cases lower than 10 p.m.,-according to HoFFMANN 1-8 p.m.,- -and in serious affections of the kidneys, generally with amyloid degeneration, less than 1 p. m. has been shown (Hoffmass). The œdema fluid also habitually contains urea, 1-2 p. m., and also a reducible substance.

The FLUID OF THE TAPEWORM cyst is related to the transudations. It is poor in proteids, thin and colorless, and has a specific gravity of 1.0051.015. The quantity of solids is $14-20 \mathrm{p} . \mathrm{m}$. The chemical constituents are glucose (2.5 p. m.), inosit, -traces of urea, creatin, succinic acid, and salts (8.3$9.7 \mathrm{p}$. m.). Albumin is only found in traces, and then only after an inflammatory irritation. In the last-mentioned case $7 \mathrm{p} . \mathrm{m}$. albumin has been found in the fluid.

The Synovial Fluid and Fluid in Synovial Cavities around Joints, etc. The synovia is hardly a transudation, but it is often treated as an appendix to the transudations.

The synovia is an alkaline, sticky, fibrous, yellowish fluid which is cloudy, from the presence of cell-nuclei and remains of destroyed cells, but is sometimes clear. It contains also, besides albumin and salts, a mucin-like nucleoalbumin. The presence of pure mucin has not been shown. The composition of synovia is 
not constant, but varies in rest and in motion. In the lastmentioned case the quantity of fluid is less, but the amount of the mucin-like body, albumin, and of the extractive bodies is greater, while the quantity of salts is diminished. This may be seen from the following analyses by Frerichs. The figures represent parts per 1000.

\begin{tabular}{|c|c|c|}
\hline & $\begin{array}{l}\text { I. Synovia from } \\
\text { a stall-fed Ox. }\end{array}$ & $\begin{array}{l}\text { II. Synovia from } \\
\text { a Field-fed Ox. }\end{array}$ \\
\hline Water. & .. 969.9 & 948.5 \\
\hline Solids............. & 30.1 & 51.5 \\
\hline Mucin-like body $\ldots \ldots \ldots \ldots \ldots \ldots \ldots$ & 2.4 & 5.6 \\
\hline Albumin and extractives............. & 15.7 & 35.1 \\
\hline Fat.................... & 0.6 & 0.7 \\
\hline (................. & 11.3 & 9.9 \\
\hline
\end{tabular}

The synovia of new-born babes corresponds to that of resting animals. The fluid of the brusæ mucosæ, as also the fluid in the synovial cavities around joints, etc., is similar to synovia from a qualitative standpoint.

\section{The Pus.}

Pus is a yellowish-gray or yellowish-green, creamy mass of a faint odor and an unsavory, sweetish taste. It consists of a fluid, the pus-serum, in which solid particles, the pus-cells, swim. The number of these cells varies so considerably that the pus may at one time be thin and at another time so thick that it scarcely contains a drop of serum. The specific gravity, therefore, may also greatly vary, namely, between 1.020 and 1.040 , but ordinarily it is 1.031-1.033. The reaction of fresh pus is generally alkaline, but it may become neutral or acid from a decomposition in which fatty acids, glycero-phosphoric acid, and also lactic acid are formed. It may become strongly alkaline when putrefaction occurs with the formation of ammonia.

In the chemical investigation of pus the pus-serum and the pus-corpuscles must be studied separately.

Pus-serum. Pus does not coagulate spontaneouslý nor after the addition of defibrinated blood. The fluid in which the pus-corpuscles are suspended is not to be compared with the plasma, but rather with the serum. The pus-serum is pale yellow, yellowish green, or brownish yellow, and has an alkaline reaction. It contains, for the most part, the same constituents as the blood- 
serum; but sometimes besides these-when, for instance, the pus has remained in the body for a long time,-it contains a nucleoalbumin which is precipitated by acetic acid and soluble with great difficulty in an excess of the acid (pyin of the older authors). This nucleoalbumin seems to be formed from the hyaline substance of the pus-cells by maceration. The pus-serum contains, moreover, at least in many cases, no fibrin ferment. According to the analyses of HOPPE-SEYLER, the pus-serum contains in 1000 parts:

I.

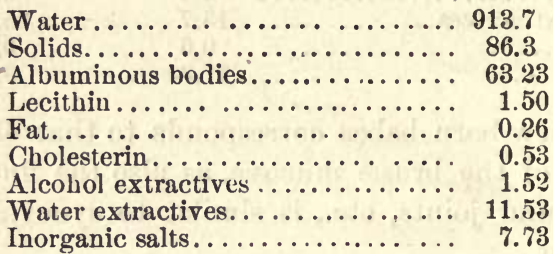

II.

905.65

94.35

77.21

056

0.29

0.87

0.73

6.92

7.77

The ash of pus-serum has the following composition, calculated to 1000 parts of the serum :

I.

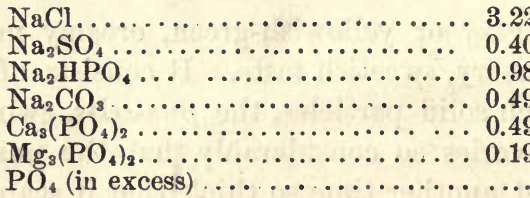

II.

5.39

0.31

0.46

1.13

0.31

0.12

.05

The pus-corpuscles are generally thought to consist in great part of emigrated colorless blood-corpuscles (emigration hypothesis), and their chemical properties have therefore been given above. We consider the molecular grains, fat-globules, and red blood-corpuscles rather as casual form-elements.

The pus-cells may be separated from the serum by centrifugal force or by decantation directly or after dilution with a solution of sodium sulphate in water ( 1 vol. saturated sodium-sulphate solution and 9 vols. water), and then washed by this same solution in the same manner as the blood-corpuscles.

The chief constituents of the pus-corpuscles are albuminous bodies of which the largest proportion seems to be a nucleoalbumious substance which is insoluble in water and which expands into a tough, slimy mass when treated with a $10 \%$ common-salt solution. This protein substance, which is soluble in alkali but quickly 
changed thereby, is called Rovidas's hyaline substance, and the property of the pus of being converted into a slime-like mass by a solution of common salt depends on this substance. Besides this substance we find in the pus-cells also an albuminous body which coagulates at $48-49^{\circ}$ C., as well as serum-globulin (?), serumalbumin, a substance similar to coagulated albumin (MIESCHER), and lastly peptone (HoFMEISTER).

We also find in the protoplasm of the pus-cells, besides the proteids, lecithin, cholesterin, xanthin bodies, fat, soaps, and cerebrin (see Chapter X). HOPPE-SEYLER claims that glycogen appears only in the living, contractile white blood-cells and not in the dead pus-corpuscles. Salomon has nevertheless found glycogen in pus. The cell-nucleus contains nuclein and some lecithin.

The mineral constituents of the pus-corpuscles are potassium, sodium, calcium, magnesium, and iron. A part of the alkalies is found as chlorides, and the remainder, as well as the other bases, exists as phosphates.

The quantitative composition of the pus-cells from the analyses of HopPE-SEYLER is as follows, in parts per 1000 of the dried substance:

I.

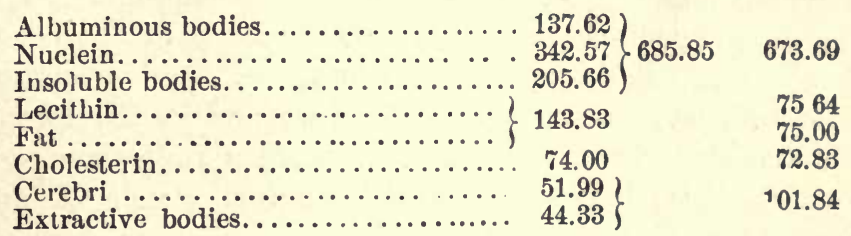

MINERAL SUBSTANCES.

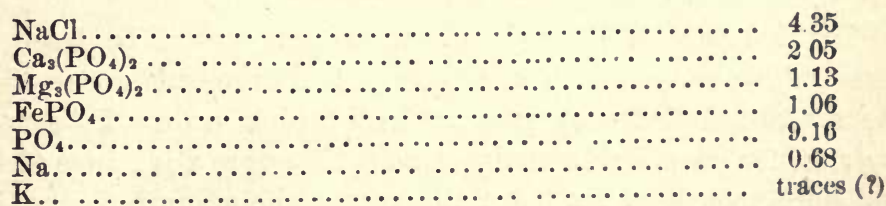

Miescher has obtained other results for the alkali combinations, namely : potassium phosphate 12 , sodium phosphate 6.1. earthy phosphate and iron phosphate 4.2 , sodium chloride 1.4, and phosphoric acid combined with organic substances 3.14-2.03 p. m.

In pus from congested abscesses which have stagnated for some time we find peptone, leucin and tyrosin, free fatty acids and 
volatile fatty acids, such as formic acid, butyric acid, valerianic acid. We also sometimes find chondrin (?) and glutin (?), urea, glucose (in diabetes), biliary coloring matters, and bile acids (in catarrhal icterus).

As more specific but not constant constituents of the pus we must mention the following : pyin, which seems to be a nucleoalbumin precipitable by acetic acid, and also pyinic acid and chlorrhodinic acid, which have been so little studied that they cannot be more fully treated here.

In many cases a blue, more rarely a green color, has been observed in the pus. This depends on the presence of a variety of vibrios (LüCKE) from which Fordos and LücKE have isolated a crystallizable coloring matter partly blue and partly yellow, pyocyanin and pyoxanthose.

\section{Appendix.}

\section{Lymphatic Glands, Spleen, etc.}

The Lymphatic Glands. According to Foster and LaNKESTER and HaLLiburton, we find in the cells of the lymphatic glands the four albuminous bodies previously mentioned (Chapter III, page 42). Albumoses and peptones may also occur as products of a post-mortem decomposition. Besides the other ordinary tissue-constituents, such as collagen, elastin, and nuclein, we find in the lymphatic glands also cholesterin, fat, glycogen, xanthin bodies and adenin (KRONECKER), and leucin. In the inguinal glands of an old woman OIDTMaNN found r13.84 p. m. water, $285 \mathrm{p} . \mathrm{m}$. organic and $1.16 \mathrm{p}$. m. inorganic substances.

The Spleen. The pulp of the spleen cannot be freed from blood. The mass which is separated from the spleen capsule and the structural tissue by pressure and which ordinarily serves as material for chemical investigations is therefore a mixture of blood and spleen constituents. For this reason the albuminous bodies of the spleen are little known. As characteristic constituents we have albuminates containing iron and especially a protein substance which does not coagulate on boiling and which is precipitated by acetic acid and yields an ash containing much phosphoric acid and iron oxide (ScHERER). 
The pulp of the spleen, when fresh, has an alkaline reaction but quickly turns acid, due partly to the formation of free paralactic'acid and partly perhaps to glycero-phosphoric acid. Besides these two acids there have been found in the spleen also volatile fatty acids, as formic, acetic, and butyric acids, as well as succinic acid, neutral fats, cholesterin, traces of leucin, inosit (in ox-spleen), scyllit, a body related to inosit (in the spleen of plagiostoma), glycogen (in dog-spleen), uric acid, guanin, hypoxanthin; xanthin, adenin (KRON ECKER), and jecorin (BALDI).

Among the constituents of the spleen the deposit rich in iron, which consists of ferruginous granules or conglomerate masses of them, and closely studied by NASSE, is of special interest. These iron grains developed by the changing of the red corpuscles, and which also occur in old thrombi, are chiefly produced when stagnant blood-corpuscles are not dissolved, and they may be formed either extra-cellular or intracellular when the blood-corpuscles of the colorless cells are taken up. This deposit does not occur to the same extent in the spleen of all animals. It is found especially abundant in the spleen of the horse. NASSE on analyzing the grains (from the spleen of a horse) obtained $839.2 \mathrm{p} . \mathrm{m}$. organic and 160.8 p. m. inorganic substances. These last consisted of $566-726 \mathrm{p} . \mathrm{m}$. $\mathrm{Fe}_{2} \mathrm{O}_{3}, 205-388$ p. m. $\mathrm{P}_{2} \mathrm{O}_{5}$, and 57 p. m. earths. The organic substances consisted chiefly of proteids (660-800 p. m.), nuclein, 52 p. m. (maximum), a yellow coloring matter, extractive bodies, fat, cholesterin, and lecithin.

In regard to the mineral constituents it is to be observed that the amount of iron is strikingly large, and further that the amount of sodium and phosphoric acid is smaller than that of potassium and chlorine. The amount of iron in new-born and young animals is small (LAPICQUE), in adults more appreciable, and in old animals sometimes considerable. NAsSE found nearly $50 \mathrm{p.} \mathrm{m}$. in the dried pulp of the spleen of an old horse.

The quantitative analyses of the human spleen by OIDTMaNN give the following results: In men he found $750-694 \mathrm{p}$. m. Water and 250-306 p. m. solids. In that of a woman he found 774.8 p. m. water and 225.2 p. m. solids. The quantity of inorganic bodies was in men 4.9-7.4 p. m., and in women $9.5 \mathrm{p} . \mathrm{m}$.

In regard to the pathological processes going on in the spleeu 
we must specially recall the abundant re-formation of leucocytes in leucæmia and the appearance of amyloid substance (see page 39).

The physiological functions of the spleen are little known. Some consider the spleen as a melting organ of the red blood-corpuscles (KöLLIKER, ECKER), and the occurrence of the above-mentioned iron deposit seems to confirm this view. Some (GerLach, Funke, and others) regard the spleen as a blood-forming organ. Other investigators consider that steps in the modelling of the red blood-corpuscles occur in the spleen or that young red blood-corpuscles occur in the blood of the splenic vein.

The spleen has also been claimed to play an important part in digestion. The organ is known to enlarge after a meal, and this enlargement is thought by ScHIFF and Herzen to be dependent upon the filling of the pancreas with enzymes. According to the above-mentioned investigators, after the extirpation of the spleen the pancreas does not produce any enzyme, which digests albumin, but Heidenheim and Ewald have not been able to confirm this fact. According to later investigations of Herzen, an enzyme which digests albumin is produced in the spleen during its enlargement.

An increase in the quantity of uric acid eliminated occurs in leucæmia (Ranke, Salkowski, Fleischer and Penzoldt, and StadtHAGEN), while the reverse of this takes place under the influence of quinine, which produces an enlargement of the spleen. We have here a rather positive proof that there is a close relationship between the spleen and the formation of uric acid. If we assume that the xanthin bodies are steps to the formation of uric acid, then the increase in the uric acid in leucæmia may perhaps depend on the increased amount of xanthin bodies (hypoxanthin) in the spleen in this disease.*

The spleen has the same property as the liver of retaining foreign bodies, metals and metalloids.

The Thymus has been little studied. Besides proteids and substances belonging to the connective group, we find small quantities

* Horbaczewski has lately found in the spleen the first steps in the formation of uric acid, and he has also shown that when the spleen and blood of a calf are allowed to act on each other at the temperature of the blood and in the presence of air, large quantities of uric acid are formed. 
of fat, leucin, succinic acid, lactic acid, and glucose. The large quantity of xanthin bodies, chiefly adenin, is remarkable-179 p. m. in the fresh gland, or $19.19 \mathrm{p} . \mathrm{m}$. in the dried substance (KosseL and Schindler). Potassium and phosphoric acid are the prominent mineral constituents. OIDTMANN found $80 \% .06 \mathrm{p}$. m. water, $192.74 \mathrm{p}$. m. organic and $0.2 \mathrm{p} . \mathrm{m}$. inorganic substances in the gland of a 14-days-old child.

The Thyroid Gland. The chemical constituents of this gland are little known. BuBNow has obtained a protein substance called by him "thyreoproteine," by extracting the gland with common-salt solution or by very dilute caustic potash. This body has about the same amount of nitrogen but smaller amounts of carbon and hydrogen than the proteids in general. The fluid found in the vesicle sometimes contains a mucin-like substance which is precipitated by an excess of acetic acid. Besides these, other substances have been found in the extract of the glands, such as leucin, xanthin, hyperxanthin, lactic and succinic acids. OIdTmans found in the thyroid gland of an old woman 822.4 p. m. water, 176.7 p. m. organic and 0.9. p. m. inorganic substances. He found $772.1 \mathrm{p} . \mathrm{m}$. water, $223.4 \mathrm{p}$. m. organic and $4.5 \mathrm{p} . \mathrm{m}$. inorganic substances in an infant 14 days old.

In "STRUMA CYSTICA" HOPPE-SEYLER found hardly any albumin in the smaller glandular vessels, but an excess of mucin, while in the larger he found a great deal of albumin, 70-80 p. m. Cholesterin is regularly found in such cysts, sometimes in such large quantities that the entire contents form a thick mass of cholesterin plates. Crystals of calcium oxalate also occur frequently. The contents of the struma cysts are sometimes of a brown color due to decomposed coloring matter, methæmoglobin (and hæmatin?). Bile-coloring matters have also been found in such cysts. (In regard to the paralbumins and colloids which have been found in struma cysts and colloid degeneration, see Chapter XI.)

Little is known in regard to the functions of the thyroid gland. From a chemical standpoint the view is worth suggesting that the so-called myxœdema, which is a slimy infiltration of the subcutaneous cell-tissue of the head and throat (besides other disturbances) stands in connection with the failing of the activity of the thyroid gland. Horsley and Halliburtor found in monkeys, but not in 
pigs, that the amount of mucin in the tissue was increased after extirpating the thyroid gland.

The Suprarenal Body. Besides proteids, substances of the connective tissue and salts, we have found in the suprarenal body palmitin, lecithin, neurin, and glycero-phosphoric acid, which last gives the poisonous properties of the watery extract of the gland (MARINo-Zuco and GUARNIERI), and some leucin, which is probably a decomposition product. The statement that benzoic acid, hippuric acid, biliary acids, and taurin occur in this gland requires further confirmation. In the medulla there have been found one or more chromogens which are converted into a red coloring matter by the action of the air, light, warmth, haloid or metallic salts (Vulpian, Krukenberg). Pyrocatechin also probably occurs therein. Because of the amount of chromogen contained in the suprarenal body, a connection is claimed between the abnormal deposition of pigment in the skin, which is characteristic of ADDIsoN's disease, and the diseased changes which often occur in the suprarenal body. 


\section{CHAPTER VI.}

\section{THE LIVER.}

THE liver, which is the largest organ of the body, stands in close relationship to the blood-forming organs. The importance of this organ in the physiological composition of the blood is evident from the fact that the blood coming from the digestive tract, laden with absorbed bodies, must circulate through the liver before it is driven by the heart through the different organs and tissues. It has been proved, at least for the carbohydrates, that an assimilation of the absorbed nutritive bodies which are brought to the liver by the blood of the portal vein takes place in this organ. The occurrence of synthetical processes in the liver has been positively proved by special observations. It is possible that in the liver certain ammonia combinations are converted into urea or uric acid (in birds), while certain products of putrefaction in the intestines, such as phenol, may be converted by synthesis into ethereal sulphuric acids by the liver (PFLÜGER and Kochs). The liver has also the property of removing and retaining heterogeneous bodies from the blood, and this is not only true of metallic salts, which are often retained by this organ, but also, as ScHIFF, LAUTENBERGER, JACQUES, HÉGER, and ROGER have shown, the alkaloids are retained and are probably partially decomposed in the liver.

Even though the liver is of assimilatory importance and purifies the blood coming from the digestive tract, it is at the same time a secretory organ which eliminates a specific secretion, the bile, in the production of which the red blood-corpuscles are destroyed, or at least one of their constituents, the hæmoglobin, is transformed. It is generally admitted that the liver acts contrariwise during fœetal life, at that time forming the red blood-corpuscles. 
There is no doubt that the chemical operations going on in this organ are manifold and must be of the greatest importance for the organism; but unfortunately we know very little about the kind and extent of these processes. Among them are two principal ones which will be fully treated in this chapter, after we have first described the constituents and the chemical composition of the liver. One of these processes seems to be of an assimilatory nature and refers to the formation of glycogen, while the other refers to the production and secretion of the bile.

The reaction of the liver-cell is alkaline during life, but becomes acid after death. This change is probably due to the formation of lactic acid, causing a coagulation of the albumins of the protoplasm of the cell. A positive difference between the albuminous bodies of the dead and the living, non-coagulated protoplasm has not been observed.

The albuminous bodies of the liver were first carefully investigated by Plos'z. He found in the watery extract of the liver an albuminous substance which coagulates at $+45^{\circ} \mathrm{C}$., also a globulin which coagulates at $+75^{\circ} \mathrm{C}$, a nucleo-albumin (?) which coagulates at $+70^{\circ} \mathrm{C}$., and lastly an albuminous body which is nearly related to coagulated albumin and which is insoluble in dilute acids at the ordinary temperature, but dissolves on the application of heat, being converted into an albuminate.' ST. ZALESKI found in the liver an albuminous body containing iron, in which the iron is more or less strongly combined, but it is unknown what relation this bears to the albuminous bodies isolated by PLos'z.

The fat of the liver occurs partly as very small globules and partly, especially in nursing children and sucking animals, as also after food rich in fat, as rather large fat-drops. This infiltration of fat, which may be made so abundant by proper food that it appears similar in the highest degree to a pathological fatty liver, begins in the periphery of the acini and extends towards the centre. If the amount of fat in the liver is increased by an infiltration, the water decreases correspondingly, while the quantity of the other solids remains little changed. In fatty degeneration this is different. In this process the fat is formed from the protoplasm of the cell, and the quantity of the other solids is therefore diminished while the amount of water is only slightly changed. To 
illustrate this, we give below the results from a normal liver, and also the results obtained by PERLs in fatty degeneration and fatty infiltration. The results are in 1000 parts.

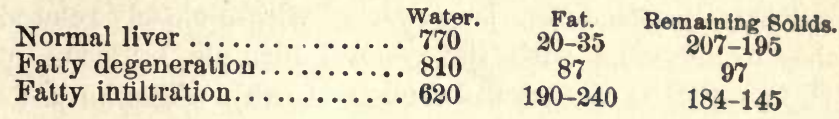

Among the extractive substances besides glycogen, which will be treated of later, we find rather large quantities of xanthin bodies. KoSSEL found in 1000 parts of the dried substance 1.97 p. m. guanin, 1.34 p. m. hypoxanthin, and 1.21 p. m. xanthin. Adenin is also contained in the liver. In addition there have been found urea and uric acid (especially in birds), and indeed in larger quantities than in the blood, paralactic acid, leucin, jecorin, and, in pathological cases, inosit, tyrosin, and cystin. The occurrence of bile-coloring matters in the liver-cell under normal conditions is doubtful; but in retention of the bile the cells may absorb the coloring matter and become colored thereby.

Jecorin was first found by DRECHSEL in the liver of a horse, and later by BALDI in the liver and spleen of other animals, in the muscles and blood of the horse, and in the human brain. It contains sulphur and phosphorus, but its constitution is not positively known. Jecorin dissolves in ether, but is precipitated from this solution by alcohol. It reduces copper oxide, and it solidifies after boiling with alkalies to a gelatinous mass. It may lead to errors in the investigations of organs or tissues, for it can easily be mistaken for lecithin on account of its solubilities and because it contains phosphorus.

The mineral bodies of the liver consist of phosphoric acid, potassium, sodium, alkaline earths, and chlorine. The potassium is in excess of the sodium. Iron is a regular constituent of the liver, but in very variable amounts, $0.3-11.8 \mathrm{p} . \mathrm{m}$. calculated for the dried substance of the liver (ST. Zaleski). A part of the iron exists as phosphate, and the greater part in combination with the protein bodies (ST. ZALESKI). Copper seems to be a physiological constituent. Foreign metals, such as lead, zinc, and others, are easily taken up and retained for a long time by the liver.

v. BIBRA found in 1000 parts of the liver of a young man who had suddenly died 762 p. m. water and $238 \mathrm{p}$. m. solids, consisting of $25 \mathrm{p} . \mathrm{m}$. fat, $152 \mathrm{p}$. m. albumin and gelatine-forming substance, and 61 p. m. extractive substances. 


\section{Glycogen and the Glycogen Formation.}

Glycogen was discovered in 1857 by BERNARD and HENSEN independently of each other. It is a carbohydrate closely related to the starches or dextrins with the general formula $\mathrm{C}_{6} \mathrm{H}_{10} \mathrm{O}_{5}$, perhaps $6\left(\mathrm{C}_{6} \mathrm{H}_{10} \mathrm{O}_{5}\right)+\mathrm{H}_{2} \mathrm{O}$ (KüLZ and BoRNTR ̈̈GER). The largest quantities are found in the liver of full-grown animals (BERNARD, HENSEN, and others), and smaller quantities in the muscles (NASSE, BRÜCKE, and others). It is found in very small quantities in many organs, such as the lungs, skin, the sheath of the roots of the hair (WIERSMA, BARFUTH), the middle coat of the arteries, and also in certain epithelial cells (Schiele, Wiersma). Its occurrence in lymphoid cells and in pus has been mentioned in the previous chapter. Glycogen has been shown by BERNARD and KüHNE to be very widely diffused in the embryonic tissue, and it seems habitually to be a constituent of tissues in which a rapid cell-formation and cell-development is taking place (HOPPE-SEYLER). It is also present in rapidly-formed pathological swellings (HOPPE-SEYLER). It has been found in several organs in diabetes mellitus. Glycogen is also found in the plant kingdom in the myxomycetæ.

The quantity of glycogen in the liver, as also in the muscles, depends essentially upon the food. In starvation it disappears after a short time, but more rapidly in small than in large animals. According to the old views (LUCHSINGER), it disappears earlier from - the muscles than from the liver; but according to more modern views (ALDEHOFF), the reverse of this takes place. After partaking of food especially rich in carbohydrates, the liver becomes rich again in glycogen, the greatest increment occurring 14 to 16 hours after eating (KüLz). The quantity of glycogen in the liver may be 100-120 p. m. or indeed even more after the consumption of food rich in carbohydrates. Ordinarily it is considerably less or $12-30$ to 40 p. m.

Glycogen forms an amorphous, white, tasteless, and inodorous powder. It gives an opalescent solution with water which, when allowed to evaporate in the water-bath, forms a pellicle over the surface that disappears again on cooling. The solution is dextro-gyrate, $(\alpha) \mathrm{D}=+211^{\circ}$ (KüLz). The specific rotary power is given somewhat differently by various investigators. A solution of glycogen 
is colored wine-red by iodine. It may hold copper oxyhydrate in solution in alkaline liquids, but does not reduce it. A solution of glycogen in water is not precipitated by potassium-mercuric iodide and hydrochloric acid, but is precipitated by alcohol or ammoniacal lead acetate. Glycogen seems to be changed somewhat by prolonged boiling with dilute caustic potash (VINTschaAu and Dietr). It is converted into glucose by diastatic enzymes and also by being boiled with dilute mineral acids.

The preparation of pure glycogen (simplest from the liver) is generally performed by the method suggested by BRÜCKE, of which the main points are the following: Immediately after the death of the animal the liver is thrown into boiling water, then finely divided and boiled several times with fresh water. The filtered extract is now sufficiently concentrated, allowed to cool, and the albumin removed by alternately adding potassium-mercuric iodide and hydrochloric acid. The glycogen is precipitated from the filtered liquid by the addition of alcohol until the liquid contains 60 vols. per cent. The glycogen is first washed on the filter with $60 \%$ and then with $95 \%$ alcohol, then treated with ether and dried over sulphuric acid. It is always contaminated with mineral substances. To be able to extract the glycogen from the liver or especially from muscles and other tissues completely, which is essential in a quantitative estimation, these parts must first be boiled for a few hours with a dilute solution of caustic potash, say $4 \mathrm{gms}$. KOH to $100 \mathrm{gms}$. liver (KüLz).

The quantitative determination is ordinarily performed according to the above method of BRÜCKE, hut care must be taken that the precipitate obtained by the potassium-mercuric iodide and hydrochloric acid is removed at least four times from the filter and stirred with water to which has been added a few drops of hydrochloric acid and potassium-mercuric chloride, and refiltered so as to be certain that all the glycogen is obtained in the filtrate (KüLz). The quantity of glycogen may also be determined by the polariscope or by titration after first converting the glycogen into glucose by boiling with an acid.

Numerous investigators have endeavored to determine the origin of glycogen in the body. The quantity of glycogen in the liver is increased after partaking of many substances, in the first place by the varieties of sugar and several other carbohydrates (PAVY and others), also by glycerin (VAN DEEN, WEISS, LUCHSINGER), gelatin (WoroschilofF), and the glucoside arbutin. Inosit (KuLz) and mannit (LUCHSINGER) have, on the contrary, no action. Fat is 
claimed by most investigators to have no influence. The views in regard to the importance of the proteids in the glycogen formation are somewhat divided. From many observations, particularly certain nutrition experiments with boiled meat (NAUNYN) or blood-fibrin (v. Merings), there is no doubt that the proteids are concerned in the formation of glycogen. WoLFFBerg has also found that he obtained a larger glycogen production from a diet of proteids and carbohydrates in proper proportions than from a diet consisting only of carbohydrates with very little proteids. It has been shown by many observers, and lately also by MoszeIK, that a diet of proteids with carbohydrates caused a greater increase in the glycogen than a diet of carbohydrates alone.

The great importance of the carbohydrates in the formation of glycogen has given rise to the opinion that the glycogen in the liver is produced from other carbohydrates (glucose) by a synthesis with the separation of water with a formation of anhydride (LUCHsinGER and others). This theory (anhydride theory) has found opponents because it neither explains the formation of glycogen from such different bodies as albumin, carbohydrates, glycerine, and others, nor the circumstance that the glycogen is always the same, independent of the properties of the carbohydrate introduced, whether it is dextro- or lævo-gyrate. It is therefore the opinion of many investigators that all glycogen is formed from proteid, and that this splits into two parts, one containing nitrogen and the other free from nitrogen: the latter is the glycogen. According to these views, the carbohydrates act only in that they spare the proteid and the glycogen produced therefrom (WEISS, WOLFFBERG, and others).

In many animal tissues we have proteids from which carbohydrates or closely-related bodies may be split off. The occurrence in the liver of such proteids, from which carbohydrates can be split off, is from certain observations not at all improbable, but still it has not been fully proved. Nor has the view of certain investigators, that in the ordinary sense we have a carbohydrate group preformed in the albuminous body, been conclusively established. Under such circumstances it is not easy to explain the formation of glycogen in the animal body from proteids. But as it scems to be certain that glycogen can be produced either from proteids or from carbohydrates, which has lately been further demonstrated by E. VoIT, 


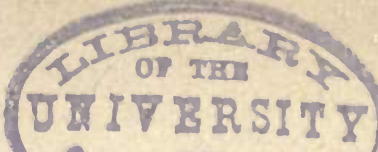 \\ THE LIVER. \\ Oy

the opinion expressed by Prü̈Ger has unquestionably been misleading. As the fat may be formed partly from proteids and partly from carbohydrates by a synthesis after previous splitting, PFLÜGER claims that the glycogen in the liver may also be produced from different substances by a complex splitting and synthesis. There is no doubt that the glycogen of the liver, which surrounds the nucleus of the liver-cells as amorphous masses, is formed in these cells. Where does the glycogen occurring in the other organs, such as the muscles, originate? Is the glycogen of the muscles formed on the spot or is it transmitted from the liver to the muscles by means of the blood? These questions cannot yet be answered with positiveness, and the investigations on this subject by different experimenters (on frogs by KüLZ and on birds by LAves and MinkowskY) have given contradictory results.

Glycogen is considered as a reserve nutritive substance deposited in the liver, and, according to the ordinary view, it is transported by the blood from the liver to the other organs, especially to the muscles, where it serves as a source of material for work. The importance of glycogen in the formation of heat follows from the fact that on cooling the animal body the glycogen is quickly exhausted. The possibility that fats may be formed from glycogen, as well as from other carbohydrates, cannot be denied.

The relationship of glycogen to the formation of sugar is of special interest. In a dead liver the glycogen is rapidly converted into sugar, and this fact naturally leads to the supposition that we have a sugar formation from glycogen in the liver during life under normal conditions, a vitale Glykogenie (AUTHOR). As proof of this, CL. BERNARD has found that the liver, under physiological conditions, always contains some sugar, and also that the blood from the hepatic vein is always somewhat richer in sugar than the blood from the portal vein. The correctness of either or both of these statements has been disputed by many investigators, such as PAvY, RrTter, Schiff, Eulenberg, Lussana, Abeles and others. It is not denied that the blood from the hepatic vein may not contain somewhat more sugar under certain circumstances, but it is probably due to the result of the experiment.

It is impossible to discuss more completely the numerous works which treat of this question, and it is perhaps sufficient to say here 
that the two above-mentioned opinions stand to-day in opposition to each other. The existence of a vital formation of sugar from the glycogen of the liver is denied by certain investigators, but admitted by others. Those who admit this formation claim that it is produced by the action of an enzyme which is formed in the blood, especially on the destruction of the red blood-corpuscles (TIEGEL). Other investigators, such as Forster, EvES, DASTRE and others, deny the action of an enzyme, and are of the opinion that the formation of sugar is produced by a vital action of the protoplasm of the living cell.

SEEGEN claims that the sugar formation in the liver occurs on a very large scale under physiological conditions, and that the blood. of the hepatic vein is considerably richer in sugar than the blood of the portal vein. He also claims that the sugar is not formed from the glycogen, but from the peptones and fat. The observations which form the basis of this theory have not been confirmed by other investigators (CHITTENDEN and LAMBERT).

The question as to a physiological formation of sugar in the liver is disputable and not settled. There is no doubt that in certain lesions of the nervous system, by poisoning, etc., an abundant formation of sugar may appear, which, at least in certain cases, is derived from the glycogen of the liver, and several investigators consider with CL. BERNARD this sugar formation as well as the elimination sugar in diabetes mellitus, as an increase in the normal formation of sugar from the glycogen.

A discussion of the different views in regard to glycosuria and diabetes mellitus is beyond the plan and scope of this book. The appearance of glucose in the urine is a symptom which under different conditions may have essentially different causes. Under all circumstances it is necessary to carefully differentiate between those diseased conditions on the one side which are grouped under the name diabetes mellitus and the experimental production of glycosuria on the other side. In diabetes, at least in most cases, we are more probably dealing with a decreased burning-up of sugar in the organism than an increased production of sugar from the glycogen of the liver, or a disturbed storing-up of glycogen in this organ. On the contrary, in experimental glycosuria in certain cases we have undoubtedly a formation of sugar 
from the glycogen of the liver. As proof thereof, in the so-called "Zuckerstich" (lesion of a certain part of the fourth ventricle of the brain) no glycosuria is produced in animals with glycogen-free livers (fasting animals), while in livers containing glycogen it is found rapidly disappearing with the formation of sugar after this operation (Hermans and Dock, Luchsinger). Von Merings has shown by experiments that we may have a glycosuria formed independently of the glycogen of the liver. This investigator has shown by experiments on dogs that in animals which have been starving for some time, so that the liver, as well as the muscles, is free from glycogen, a very considerable glycosuria is produced on administering the glucoside phloridzin. The elimination of sugar is considerably greater by this means than that produced by the decomposition of the glucoside itself. Von Merings has been able to produce diabetes by this glucoside in geese which have had their livers removed; and LANGENDORFF has also produced the same in frogs whose livers had been extirpated. In the so-called phloridzin diabetes the sugar is not formed from the glycogen of the liver, but to all appearances from the albumin (or proteids).

\section{The Bile and its Formation.}

By the employment of a biliary fistula, an operation which was first performed in 1844 by SchWANN, it is possible to study the secretion of the bile. This secretion takes place at a very low pressure; therefore an apparently unimportant hindrance in the outflow of the bile, namely, a stoppage of mucus in the exit or the secretion of large quantities of viscous bile, may cause stagnation and absorption of the bile by means of the lymphatic vessels (ab. sorption icterus).

The quantity of bile secreted during a specified time, say 24 hours, is rather difficult to determine with accuracy. The approximate amount for the dog, as determined by BIDDER and SCHMIDT, is about $20 \mathrm{grms}$., with, in round numbers, $1 \mathrm{grm}$. of solids per kilo of the weight of the body. For human beings RANKE has calculated an average of 14 grms., with 0.44 grms. solids. The amount is dependent upon the nutrition. In fasting the quantity decreases, but increases after taking food. The statements are contradictory in 
regard to the time necessary after partaking of food before the secretion reaches its maximum. Formerly it was held that the secretion of bile is increased by food rich in albumin; in later investigations, by ROSENBERG, it was found, on the contrary, that the fats give a greater stimulus to the secretion of bile than do the other nutritive substances. The drinking of water increases the secretion of bile. The statements of different investigators vary so much in regard to the action of different medicinal bodies in the secretion of bile that it is impossible to reach any conclusion on the subject. All investigators who have worked on this subject seem to agree that sodium salicylate is a true cathartic (RUtherford, VIgNal, Lewaschew, Prevost and Binet, Rosenberg). Also turpentine, which is a component of the so-called Durand's remedy, seems to increase the secretion (Lewaschew, Prevost and Binet, RosenBERG). Olive-oil is a very active cathartic (Rosenberg). By the increased secretion of bile the amount of solids does not, as a rule, increase at the same rate as the water, and the concentration of the bile decreases. An exception to this is found only in the influence exerted by the bile itself, acting as it does as a powerful cathartic by which also the concentration of the secreted bile is increased.

The bile is a mixture of the secretion of the liver-cells and the so-called mucus which is secreted by the glands of the biliary passages and by the mucous membrane of the gall-bladder. The secretion of the liver, which is generally poorer in solids than the bile from the gall-bladder, is thin and clear, while the bile collected in the gall-bladder is more ropy and viscous on account of the absorption of water and the admixture of "mucus," and cloudy because of the admixture of cells, pigments, and the like. The specific gravity of the bile from the gall-bladder varies considerably, in man between 1.010 and 1.040. Its reaction is alkaline. The color changes in different animals: golden yellow, yellowish brown, olive-brown, brownish green, grass-green, or bluish green. Bile obtained from an executed person immediately after death is golden yellow or yellow with a shade of brown. Still cases occur in which fresh human bile has a green color. The ordinary post-mortem bile has a variable color. The bile of certain animals has a peculiar odor; as example, ox-bile has an odor of musk, especially on warming. The taste of bile is also different in different 
animals. Human as well as ox bile has a bitter taste with a sweetish after-taste. The bile of the pig and rabbit has an intense persistent bitter taste. On heating bile to boiling it does not coagulate. It contains (in the ox) only traces of true mucin, and its ropy properties depend, it seerns, chiefly on the presence of a nucleoalbumin similar to mucin (PAIJKULL). The specific constituents of the bile are bile-acids combined with alkalies, bile-pigments, and besides small quantities of lecithin, cholesterin, soaps, neutral fats, urea, and mineral substances (sodium chloride, calcium and magnesium phosphate, and iron).

Bile-salts. All bile-acids can be divided into two groups, the glycocholic- and the taurocholic-acid groups. All glycocholic acids contain nitrogen, but are free from sulphur and can be split with the addition of water into glycocoll (amido-acetic acid) and an acid free from nitrogen, cholalic acid. All taurocholic acids contain nitrogen and sulphur and are split, with the addition of water, into taurin (amido-isethionic acid) containing sulphur and cholalic acid. The reason of the existence of different glycocholic and taurocholic acids depends on the fact that there are several cholalic acids.

The different bile-acids occur in the bile as alkali salts, generally in combination with sodium, but in sea-fishes as potassium salts. In the bile of certain animals we find almost solely glycocholic acid, in others only taurocholic acid, and in other animals a mixture of both (see below).

All alkali salts of the biliary acids are soluble in water and alcohol, but insoluble in ether. Their solution in alcohol is therefore precipitated by ether, and this precipitate, with the proper care in manipulation, gives, for nearly all kinds of bile thus far investigated, rosettes or balls of fine needles or 4-6-sided prisms (Plattrer's crystallized bile). Fresh human bile also crystallizes readily. The bile-acids and their salts are optically active and dextro-rotary. The former are dissolved by concentrated sulphuric acid at the ordinary temperature, forming a reddish-yellow liquid which has a beautiful green fluorescence. On carefully warming with concentrated sulphuric acid and a little cane-sugar, the bile-acids give a beautiful cherry-red or reddish-violet liquid. Petrenkofer's reaction for bile-acids is based on these facts. 
Pettenkofer's test for bile-acids is performed as follows. $\Lambda$ small quantity of bile in substance is dissolved in a small porcelain dish in concentrated sulphuric acid and warmed, or some of the liquid containing the bile-acids is mixed with concentrated sulphuric acid, taking special care in both cases that the temperature does not rise higher than $60-70^{\circ} \mathrm{C}$. Then a $10 \%$ solution of cane-sugar is added, drop by drop, continually stirring with a glass rod. The presence of bile is indicated by the production of a beautiful red liquid, whose color does not disappear at the ordinary temperature, but becomes more bluish violet in the course of a day. This red liquid shows a spectrum with two absorption-bands, the one at $F$ and the other between $D$ and $E$, near $E$.

This extremely delicate test fails, however, when the solution is heated too high or if an improper quantity-generally too muchof the sugar is added. In the last-mentioned case the sugar easily carbonizes and the test becomes brown or dark brown. 'The reaction readily fails if the sulphuric acid contains sulphurous acid or the lower oxides of nitrogen. Many other substances, such as albumin, oleic acid, amylacohol, morphin, and others, give a similar reaction, and therefore in doubtful cases the spectroscopic examination of the red solution must not be forgotten.

Pettenkofer's test for the bile-acids depends essentially on the fact that furfurol is formed from the sugar by the sulphuric acid, and this body can therefore be substituted for the sugar in this test (MYlius). According to MrLius and v. Udranszky a $1 \mathrm{p}$. m. solution of furfurol should be used. Dissolve the bile, which must first be purified by animal charcoal, in alcohol. To each c. c. of the alcoholic solution of bile in a glass add 1 drop of the furfurol solution and 1 c. c. conc. sulphuric acid, and cool when necessary so that the test does not become too warm. This reaction, when performed as described, will detect $\frac{1}{20}-\frac{3}{30}$ milligram cholalic acid (v. Udranszky). Other modifications of Pettenkofer's test have been proposed.

Glycocholic Acid. The constitution of that glycocholic acid occurring in human and ox bile, which has been most studied and which is identical with the cholic acid of STRECKER and GMELIN, is represented by the formula $\mathrm{C}_{26} \mathrm{H}_{43} \mathrm{NO}_{6}$. Glycocholic acid is absent or nearly so in the bile of carnivora. On boiling with acids or 
alkalies this acid, which is analogous to hippuric acid, is converted into cholalic acid and glycocoll.

Glycocholic acid crystallizes in fine, colorless needles or prisms. It is soluble with difficulty in water (in about 300 parts cold and 120 parts boiling water), and is easily precipitated from its alkalisalt solution by the addition of dilute mineral acids. It is readily soluble in strong alcohol, but with great difficulty in ether. The solutions have a bitter but at the same time sweetish taste. The salts of the alkalies and alkaline earths are soluble in alcohol and water. The salts of the heavy metals are mostly insoluble or soluble with difficulty in water. The solution of the alkali salts in water is precipitated by sugar of lead, copper-oxide and ferric salts, and silver nitrate.

The preparation of pure glycocholic acid may be performed in several ways. We may precipitate the bile, which has been freed from mucus by means of alcohol and the alcohol removed by evaporation, by a solution of lead acetate. 'The precipitate is then decomposed by a soda solution and heat, evaporated to dryness, and the residue extracted with alcohol, which dissolves the alkali glycocholate. The alcohol is distilled from the filtered solution and the residue dissolved in water; this solution is now decolorized by animal charcoal, and the glycocholic acid precipitated from the solution by the addition of a dilute mineral acid. The acid may be obtained in crystals either from boiling water, on cooling, or from strong alcohol by the addition of ether. 'The reader is referred to more exhaustive works for other methods of preparation.

Hyo-glycocholic Acid, $\mathrm{C}_{27} \mathrm{H}_{48} \mathrm{NO}_{6}$, is the crystalline glycocholic acid obtained from the bile of the pig. It is very insoluble in water. The alkali salts, whose solutions have an intense bitter taste without any sweetish after-taste, are precipitated by $\mathrm{CaCl}_{2}, \mathrm{BaCl}_{2}$, and $\mathrm{MgCl}_{2}$, and may be salted out like a soap by $\mathrm{Na}_{2} \mathrm{SO}_{4}$ when added in sufficient quantity. Besides this acid there occurs in the bile of the pig still another glycocholic acid (JoLIN).

The glycocholate in the bile of the rodent is also precipitated by the abovementioned salts, but cannot, like the corresponding salt in the human or ox bile, be precipitated on saturating with a neutral salt $\left(\mathrm{Na}_{2} \mathrm{SO}_{4}\right)$. Guano bilo8cid possibly belongs to the glycocholic-acid group, and is found in Peruvian guano but has not been thoroughly studied.

Taurocholic Acid. This acid, which is found in the bile of man, carnivora, oxen and a few other herbivora, such as sheep and goats, and which is identical with the choleic of STRECKER and DEMARGAY, has the constitution $\mathrm{C}_{26} \mathrm{H}_{45} \mathrm{NSO}_{7}$. On boiling with acids and alkalies it splits into cholalic acid and taurin. 
Taurocholic acid may be obtained, though only with difficulty, in fine needles which deliquesce in the air (PARKE). It is very soluble in water, and can hold the difficultly-soluble glycocholic acid in solution. This is the reason why a mixture of glycocholate with a sufficient quantity of taurocholate, which often occurs in oxbile, is not precipitated by a dilute acid. Taurocholic acid is readily soluble in alcohol but insoluble in ether. Its solutions have a bittersweet taste. Its salts are, as a rule, readily soluble in water, and the solutions of the alkali salts are not precipitated by copper sulphate, silver nitrate, or sugar of lead. Basic lead acetate gives, on the contrary, a precipitate which is soluble in boiling alcohol.

Taurocholic acid is best prepared from decolorized, crystallized dog-bile, which contains only taurocholate. The solution of this bile is precipitated by basic lead acetate and ammonia, and the washed precipitate dissolved in boiling alcohol. The filtrate is now treated with $\mathrm{H}_{2} \mathrm{~S}$, and this filtrate is evaporated at a gentle heat to a small volume, and treated with an excess of water-free ether. The acid sometimes partially crystallizes.

Cheno-taurocholic Acid. This is the most essential acid of goose-bile and has the formula $\mathrm{C}_{29} \mathrm{H}_{49} \mathrm{NSO}_{6}$. This acid, though little studied, is known to be amorphous and soluble in water and alcohol.

As repeatedly mentioned above, the two bile-acids split on boiling with acids or alkalies into non-nitrogenized cholalic acid and glycocoll or taurin. Therefore we will now describe the products of this splitting.

Cholalic Acid. The ordinary cholalic acid obtained as a decomposition product of human and ox bile, which occurs regularly in the contents of the intestines and in the urine in icterus, and which is identical with DEMARÇAY's cholic acid, has, according to STRECKER and nearly all recent investigators, the constitution $\mathrm{C}_{24} \mathrm{H}_{40} \mathrm{O}_{5}$; but others give as the formula $\mathrm{C}_{25} \mathrm{H}_{42} \mathrm{O}_{5}$ (LATsChinoFF). According to Mruius, cholalic acid is a monobasic alcohol-acid with a secondary and two primary alcohol groups. Its formula may there-

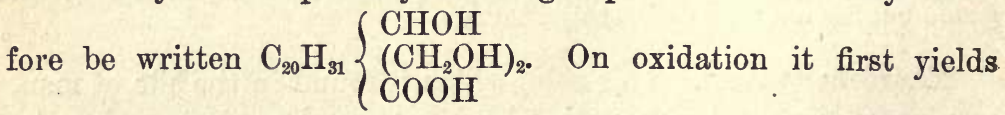
dehydrocholalic acid (AUTHOR), and then bilianic acid (CLEVE). The formulæ of these acids (when we take $\mathrm{C}_{24}$ for the cholalic acid) are $\mathrm{C}_{24} \mathrm{H}_{34} \mathrm{O}_{5}$ and $\mathrm{C}_{24} \mathrm{H}_{34} \mathrm{O}_{8}$. On reduction (in putrefaction) cholalic acid may yield desoxycholalic acid, $\mathrm{C}_{24} \mathrm{H}_{40} \mathrm{O}_{4}$ (MrLius). 
Cholalic acid crystallizes partly with one molecule of water, in rhombic plates or prisins, and partly in larger rhombic tetrahedra or octahedra with $1 \mathrm{~mol}$. of alcohol of crystallization (MYLIUs). These crystals become quickly opaque and porcelain-white in the air. They are quite insoluble in water (in 4000 parts cold and 750 parts boiling), rather soluble in alcohol, but soluble with difficulty in ether. The amorphous cholalic acid is less insoluble. The solutions have a sweetish-bitter taste. The crystals lose their alcohol of crystallization only after a lengthy heating to $100-120^{\circ} \mathrm{C}$. The acid free from water and alcohol melts at $+195^{\circ} \mathrm{C}$.

The alkali salts are readily soluble in water, but when treated with a concentrated caustic or carbonated alkali solution may be separated as an oily mass which becomes crystalline on cooling. The alkali salts are not readily soluble in alcohol, and on the evaporation of the alcohol they may crystallize. The specific rotary power of the sodium salt is $(\alpha) \mathrm{D}=+31.4^{\circ}$. The watery solution of the alkali salts, when not too dilute, is precipitated immediately or after some time by sugar of lead or by barium chloride. The barium salt crystallizes in fine, silky needles, and it is rather insoluble in cold, but somewhat easily soluble in warm water. The barium salt, as well as the lead salt which is insoluble in water, is soluble in warm alcohol.

To prepare cholalic acid we boil ox-bile for 18-36 hours with strong caustic alkali, or, better, with as much barium hydrate as the boiling liquid will dissolve. During the boiling add when necessary more barium hydrate. The boiling-hot liquid is strained and concentrated until a crystalline mass separates in large quantity. 'This mass is separated from the mother-liquor and strongly pressed and recrystallized a few times from boiling water. The recrystallized barium salt is now dissolved, and the solution decomposed by hydrochloric acid. The acid which separates is dissolved in boiling alcohol, and the acid generally separates as crystals immediately on cooling. By recrystallization from ethyl alcohol and finally from methyl alcohol the acid may be readily obtained in pure white crystals the size of a pea.

Choleic Acid, $\mathrm{C}_{25} \mathrm{H}_{42} \mathrm{O}_{4}$, is another cholalic acid named by LATSCHINOFF, which is obtained from ox-bile with ordinary cholalic acid, though in very. small amounts (hardly $\frac{1}{3}$ the quantity of the latter). The barium salt of this cholalic acid is 
more readily soluble than the barium salt of the ordinary cholalic acid. Choleic acid yields on oxidation, first, dehydrocholeic acid, $\mathrm{C}_{25} \mathrm{H}_{38} \mathrm{O}_{4}$, and then cholanic acid, $\mathrm{C}_{25} \mathrm{H}_{38} \mathrm{O}_{8}+\frac{1}{4} \mathrm{H}_{2} \mathrm{O}$ (LAtschinofF).

Fellic Acid, $\mathrm{C}_{23} \mathrm{H}_{40} \mathrm{O}_{4}$, is a cholalic acid, so called by ScHoтTEN, and which he obtained from human bile, along with the ordinary acid. This acid is crystalline, is insoluble in water, and yields barium and magnesium salts which are very insoluble. It does not give Pettenkofer's reaction easily and gives a more reddishblue color.

The hyo-glycocholic and the cheno-taurocholic as well as the glycocholic acid of the bile of rodents yield corresponding cholalic acids.

On boiling cholalic acids with acids, by putrefaction in the intestines, or by heating, they lose water and are converted into an anhydride, the so-called dyslysin. The dyslysin, $\mathrm{C}_{24} \mathrm{H}_{36} \mathrm{O}_{3}$, corresponding to ordinary cholalic acid, and which occurs in fæces, is amorphous, insoluble in water and alkalies. Choloidic acid is called the first anhydride, or an intermediate product in the formation of dyslysin. According to HoPPE-SEYler, choloidic acid is perhaps only a mixture of cholalic acid and dyslysin. On boiling dyslysin with caustic alkali it is reconverted into the corresponding cholalic acid.

Glycocoll, $\mathrm{C}_{2} \mathrm{H}_{5} \mathrm{NO}_{2}$, or amido-acetic acid, $\mathrm{NH}_{2} \cdot \mathrm{CH}_{2} \cdot \mathrm{COOH}$, also called glycine, or sugar of gelatine, has been found in the muscles of pecten irradians, but it is of special interest as a decomposition product of certain protein substances-gelatine and spongin -as also of hippuric acid or glycocholic acid on splitting them by boiling with acids.

Glycocoll forms colorless, often large, hard rhombic crystals or four-sided prisms. The crystals taste sweet and dissolve easily in cold (4.3 parts) water. They are insoluble in alcohol and ether; in warm spirits of wine they dissolve, but with difficulty. Glycocoll combines with acids and bases. Under the last-mentioned combinations we must mention those with copper and silver. Glycocoll dissolves copper hydroxide in alkaline liquids, but does not reduce it at the boiling temperature. A boiling-hot solution of glycocoll dissolves freshly-precipitated copper hydroxide, forming a blue liquid from which dark-blue needles crystallize on cooling, if 
the liquid is sufficiently concentrated. The combination of glycocoll with $\mathrm{HCl}$ is soluble in water and alcohol.

Glycocoll is best prepared from hippuric acid by ooiling it 10 12 hours with 4 parts of dilute sulphuric acid, 1:6. After cooling separate the benzoic acid, concentrate the filtrate, remove the remainder of the benzoic acid by shaking with ether, remove the sulphuric acid by $\mathrm{BaCO}_{3}$, and evaporate the filtrate to crystallization.

Taurin, $\mathrm{C}_{2} \mathrm{H}_{7} \mathrm{NSO}_{3}$, or amido-ethylsulphonic acid, $\mathrm{NH}_{2} \cdot \mathrm{C}_{2} \mathrm{H}_{4}$. $\mathrm{SO}_{2} \mathrm{OH}$. This body is well known as a splitting product of taurocholic acid, and may occur in small quantities in the contents of the intestines. It has also been found in the lungs and kidneys of oxen and in the blood and muscles of cold-blooded animals.

Taurin crystallizes in colorless, often in large, shining, 4-6 sided prisms. It dissolves in 15-16 parts of water at ordinary temperatures, but rather more easily in warm water. It is insoluble in absolute alcohol and ether; in cold spirits of wine it dissolves slightly, but more when warm. Taurin yields acetic and sulphurons acids, but no alkali sulphides, on boiling with strong caustic alkali. The amount of sulphur can be determined as sulphuric acid after fusing with saltpetre and soda. Taurin combines with metalic oxides. The combination with mercuric oxide is white, insoluble, and is formed when a solution of taurin is boiled with freshly-precipitated mercuric oxide (J. IAANG). This combination may be used in detecting the presence of tanrin. Taurin is not precipitated by metallic salts.

The preparation of taurin from bile is very simple. The bile is boiled a few hours with hydrochloric acid. The filtrate from the dyslysin and choloidic acid is concentrated well in the water-bath, and filtered so as to remove the common salt and other substances which have separated. Then evaporate to dryness, and treat the residue with strong alcohol, which dissolves the hydrochlorate of glycocoll, while the taurin remains. ('The alcoholic solution of hydrochlorate of glycocoll may be used in the preparation of $\mathrm{gly}$ cocoll by evaporating the alcohol and dissolving the residne in water, decomposing the solution with lead hydroxide, filtering, and freeing the solution from lead by $\mathrm{H}_{2} \mathrm{~S}$, and strongly concentrating this filtrate. The crystals which separate are dissolved and decolorized by animal charcoal, and the solution evaporated to crystallization.) The above-obtained residue containing the taurin is dissolved in as little water as possible, filtered warm, and treated 
with an excess of alcohol. The crystalline precipitate which immediately forms is filtered as soon as possible, and the taurin now separates, on cooling, into very long needles or prisms. These crystals may be purified by recrystallization from a little warm water.

Though the taurin shows no positive reactions, it is chiefly identified by its crystalline form, by its solubility in water and insolubility in alcohol, by its combination with mercuric oxide, by its non-precipitability by metallic salts, and above all by its containing sulphur.

The Detection of Bile-acids in Animal Fluids. To obtain the bile-acids pure so that PeTtenkofer's test can be applied to them, the albumins and fat must first be removed. 'The albumin is removed by making the liquid first neutral and then adding a great excess of alcohol, so that the mixture contains at least 85 vols. per cent of water-free alcohol. Now filter, extract the precipitated albumin with fresh alcohol, unite all filtrates, distil the alcohol, and evaporate to dryness. The residue is completely exhausted with strong alcohol, filtered, and the alcohol entirely evaporated from the filtrate. The new residue is dissolved in water, and filtered if necessary, and the solution precipitated by basic lead acetate and ammonia. The washed precipitate is dissolved in boiling alcohol, filtered while warm, and a few drops of soda solution added. Then evaporate to dryness, extract the residue with absolute alcohol, filter, and add an excess of ether. The precipitate now formed may be used for PetTenkofer's test. It is not necessary to wait for a crystallization; but one must not consider the crystals which form in the liquid as being positively-crystallized bile. It is also possible for needles of alkali acetate to be formed. For the detection of bileacids in urine see Chapter XIV.

Bile-pigments. The bile-coloring matters known thus far are relatively numerous, and in all probability there are still more. Most of the known bile-pigments are not found in the normal bile, but occur either in post-mortem bile or, principally, in the bile concrements. The pigments which occur under physiological conditions are the reddish-yellow bilirubin, the green biliverdin, and sometimes there is also observed in fresh human bile a pigment closely related to hydrobilirubin. The pigments found in gall-stones are (besides the bilirubin and biliverdin) bilifuscin, biliprasin, bilihumin, bilicyanin (and choletelin ?). Besides these, others have been observed in human and animal bile. The two above-mentioned physiological pigments, bilirubin and biliverdin, are those which serve to give the golden-yellow or orange-yellow or sometimes 
greenish color to the bile, or when, as is most frequently the case in ox-bile, the two pigments are present in the bile at the same time, producing the different shades between reddish brown and green.

Bilirubin. This pigment, according to the common acceptation, has the formula $\mathrm{C}_{16} \mathrm{H}_{18} \mathrm{~N}_{2} \mathrm{O}_{3}$ (MALY) and is designated by the

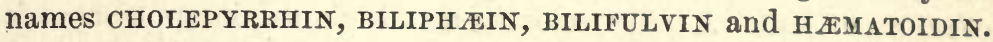
It occurs chiefly in the gall-stones as bilirubin-calcium. It is further found in the bile, especially in man and carnivora; sometimes, however, the latter when fasting or in a starving condition may have a green bile in the gall-bladder. It occurs also in the contents of the small intestines, in blood-serum of the horse, in old blood extravasations (as hæmatoidin), and in the urine and the yellowcolored tissue in icterus. The bilirubin is in all probability a formation from the hæmatin which it closely resembles. It is converted into hydrobilirubin, $\mathrm{C}_{32} \mathrm{H}_{40} \mathrm{~N}_{4} \mathrm{O}_{7}$ (MALY), by hydrogen in a nascent state. It is claimed by several investigators to be identical with the urinary pigment urobilin, as well as with stercobilin (Masius and VANLAIR), which is found in the contents of the intestines. There is no doubt that a great similarity exists between these pigments, but their identity is emphatically denied by MacMuns. On oxidation bilirubin yields biliverdin and other coloring matters (see below).

Bilirubin is partly amorphous and partly crystalline. The amorphous bilirubin is a reddish-yellow powder of nearly the same color as amorphous antimony sulphide; the crystalline bilirubin has nearly the same color as crystallized chromic acid. The crystals, which can easily be obtained by allowing a solution of bilirubin in chloroform to spontaneously evaporate, are reddish-yellow, rhombic plates, whose obtuse angles are often rounded.

Bilirubin is insoluble in water, slightly soluble in ether, somewhat more soluble in alcohol, easily soluble in chloroform, especially in the warmth, and less soluble in benzol, carbon disulphide, amyl alcohol, fatty oils, and glycerin. Its solutions show no absorptionbands, but only a continuous absorption from the red to the violet end of the spectrum, and they have, even on diluting greatly, (1:500000) a decided green color. The combinations of bilirubin with alkalies are insoluble in chloroform, and bilirubin may be separated from its solution in chloroform by shaking with dilute caustic alkali (differing from lutein). Solutions of bilirubin-alkali 
in water are precipitated by the soluble salts of the alkaline earths and also by metallic salts.

If an alkaline solution of bilirubin be allowed to stand in contact with the air, it gradually absorbs oxygen and green biliverdin is formed. Biliverdin is also formed from bilirubin by 'oxidation under other conditions. A green coloring matter similar in appearance is formed by the action of other reagents such as $\mathrm{Cl}, \mathrm{Br}$, and $\mathrm{I}$. In these cases it does not seem to be biliverdin, but a substitution product of bilirubin (THUDICHUM, MALY) which is obtained.

GMeLiN's Reaction for Bile-pigments. If we carefully pour under a solution of bilirubin-alkali in water nitric acid containing some nitrous acid, we obtain a series of colored layers at the juncture of the two liquids, in the following order from above downwards: green, blue, violet, red, and reddish yellow. This color reaction, GMELIN's test, is very delicate and serves to detect the presence of one part bilirubin in 80,000 parts liquid. The green ring must never be absent; and also the reddish violet must be present at the same time, otherwise the reaction may be confused with that for lutein, which gives a blue or greenish ring. The nitric acid must not contain too much nitrous acid, for then the reaction takes place too quickly and it does not become typical. Alcohol must not be present in the liquid, because, as is well known, it gives a play of colors, in green or blue, with the acid.

HUPPERT's Reaction. If a solution of bilirubin-alkali is treated with milk of lime or with calcium chloride and ammonia, a precipitate is produced consisting of bilirubin-calcium. If this moist precipitate, which has been washed with water, is placed in a testtube and the tube half filled with alcohol which has been acidified with sulphuric acid, and heated to boiling for some time, the liquid becomes emerald-green or bluish green in color. This reaction is a good and easily-performed test for bile-pigments.

In regard to the modifications of GMELIN's test and certain other reactions for bile-pigments, see Chapter XIV (Urine).

That the characteristic play of colors in GMeLIN's test is the result of an oxidation is generally admitted. The first oxidation step is the green biliverdin. Then follows a blue coloring matter which Heinsius and CampBell call bilicyanin and Stokvis calls cholecyanin, and which shows a characteristic absorption-spectrum. 
The neutral solutions of these coloring matters are, according to STokvis, bluish green or steel-blue with a beautiful blue fluorescence. The alkaline solutions are green and have no marked fluorescence. The neutral and alkaline solutions show three absorption-bands, one sharp and dark in the red between $C$ and $D$, nearer to $C$; a second, less defined, covering $D$; and a third, forming only a faint shadow, in the green, exactly in the middle, between $D$ and $E$. The strongly-acid solutions are violet-blue and show two bands, described by JAFFE, between the lines $C$ and $E$, separated from each other by a narrow space near $D$. The next oxidation step after these blue coloring matters gives a red pigment, and lastly a yellowish-brown pigment, called choletelin by MaLY, which shows no absorption-spectrum.

Bilirubin is best prepared from gall-stones of oxen, these concretions being very rich in bilirubin-calcium. The finely.powdered concrement is first exhausted with ether and then with boiling water, so as to remove the cholesterin and bile-acids. 'The powder is then treated with hydrochloric acid, which sets free the pigment. Wash thoroughly with water and alcohol, dry, and extract continuously with boiling chloroform. After distilling the chloroform from the solution, treat the powdered residue with absolute alcohol to remove the bilifuscin; dissolve the remaining bilirubin in a little chloroform; precipitate it from this solution by alcohol, and do this several times if necessary. The bilirubin is finally dissolved in boiling chloroform and allowed to crystallize on cooling. The quantitative estimation of bilirubin may be made by the spectro-photometrical method, according to the steps suggested for the blood-coloring matters.

Biliverdin, $\mathrm{C}_{8} \mathrm{H}_{9} \mathrm{NO}_{2}$. This body, which is formed by the oxidation of bilirubin, occurs in the bile of many animals, in vomited matter, in the placenta of the bitch (?), in the shells of birds' eggs, in the urine in icterus, and sometimes in gall-stones, although in very small quantities.

Biliverdin is amorphous, or at least it has not been obtained in well-defined crystals. It is insoluble in water, ether, and chloroform (this is true at least for the artificially-prepared biliverdin, while the green pigment of ox-bile is soluble in chloroform, according to MACMUNN), but is soluble in alcohol or glacial acetic acid, showing a beautiful green color. It is dissolved by alkalies giving a brownish-green color, and this solution is precipitated by 
acids, as well as by calcium, barium, and lead-salts. Biliverdin gives HUPPER's's and GMELIN's reactions; commencing with the blue color. It is converted into hydrobilirubin by nascent hydrogen. On allowing the green bile to stand, also by the action of ammonium sulphide, the biliverdin may be reduced to bilirubin (HAYCRAFT and SCOFIELD).

Biliverdin is most simply prepared by allowing a thin layer of an alkaline solution of bilirubin to stand exposed to the air in a dish until the color is brownish green. The solution is then precipitated by hydrochloric acid, the precipitate washed with water until no $\mathrm{HCl}$ reaction is obtained, then dissolved in alcohol and the pigment again separated by the addition of water. Any bilirubin present may be removed by means of chloroform.

Bilifuscin, so named by STÄDELER, is an amorphous brown pigment, soluble in alcohol and alkalies, nearly insoluble in water and ether, and soluble with great difficulty in chloroform (when bilirubin is not present at the same time). It is found in post-mortem bile and gall-stones. Biliprasin is a green pigment prepared by STÄDELER from gall-stones, which perbaps is only a nixture of biliverdin and bilirubin. Bilihumin is the name given by STÄDELER to that brownish amorphous residue which is left after extracting gall-stones with chloroform, alcohol, and ether. It does not give GMELIN's test. Bilicyanin is also found in human gall-stones (HEINsIUs and CAMPBELL). Cholo homatin, so called by MACMUNN, is a pigment often occurring in sheep-and ox-bile and characterized by four absorption-bands, and which is formed from hæmatin by the action of sodium amalgam. In the dried condition, obtained by the evaporation of the chloroform solution, it is green, and in alcoholic solution olive-brown.

GMELIN's and HuPPERT's reactions are generally used to detect the presence of bile-pigments in animal fluids or tissues. The first, as a rule, can be performed directly, and the presence of albumin does not interfere with it, but, on the contrary, it brings out the play of colors more strikingly. If blood-coloring matters are present at the same time, the bile-coloring matters are first precipitated by the addition of sodium phosphate and milk of lime. This precipitate containing the bile-pigments may be used directly in HUPPERT's reaction, or may be treated with water and some hydrochloric acid, and then shaken with chloroform free from alcohol, and this chloroform solution used in testing for the bile-pigments.

Besides the bile-acids and bile-pigments we also have in the bile cholesterin, lecithin, palmitin, stearin, olein, and soaps of the corresponding fatty acids. In some animals the bile contains a diastatic enzyme. Cholin and glycero-phosphoric acid, when they are present, may be considered as decomposition products of lecithin. Urea occurs, though only as traces, as a physiological constituent of human, ox, and dog bile. The mineral constituents 
of the bile are, besides the alkalies, to which the bile acids are united, sodium and potassium chloride, calcium and magnesium phosphate, and iron-0.04-0.11 p. m. in human bile, chiefly combined with phosphoric acid (YouNG). Traces of copper are habitu. ally present, and traces of zinc are often found. Sulphates are entirely absent or only occur in very small amounts.

Quantitative Composition of the Bile. Complete analyses of human bile have been made by HOPPE-SEYLER and his pupils. The bile was removed as fresh as possible from the gall-bladder of cadavers whose livers showed no remarkable change. The following figures of SocolofF are the average of six analyses, and those of HopPe-SEYLER of five analyses. The relationship between the glycocholate and taurocholate was found by fusing the precipitate consisting of biliary alkalies obtained by ether from the alcoholic extract with saltpetre and soda. On determining the amount of sulphur in the fused mass the taurocholic acid can be calculated from this. 100 parts $\mathrm{BaSO}$, correspond to 220.86 parts taurocholic acid. The figures are parts per 1000 .

\begin{tabular}{|c|c|c|c|c|}
\hline & & & SOCOLOFF & IOPPE-SE \\
\hline Mucin.... & 248 & 13.0 & & 12.9 \\
\hline Remaining bodies insol. in alcohol.. & 4.5 & $14.6\}$ & 37.20 & 1.4 \\
\hline Taurocholate................... & 7.5 & 19.2 & 15.67 & 8.7 \\
\hline Glycocholate.................. & 21.0 & 4.4 & 4904 & 30.3 \\
\hline Soaps......................... & 8.1 & 16.3 & 14.60 & 13.9 \\
\hline Cholesterin................ & 25 & 3.3 & $\ldots$ & 3.5 \\
\hline Lecithin... & $5 ?$ & 0.2 & $\ldots$ & 3.3 \\
\hline Fat. . . . . . . . . . . . & 5.2 & 3.6 & $\ldots$ & 7.3 \\
\hline Ferric phosphate $\ldots \ldots \ldots \ldots \ldots$ & . . & .. & $\ldots$ & 0.166 \\
\hline
\end{tabular}

Older and less complete analyses of human bile have been made by Frerichs and v. Gorup-Besanez. The bile analyzed by them was from perfectly healthy persons who had been executed or accidentally killed. The two analyses of Frerichs are, respectively, of (I) an 18-year-old and (II) a 32-year-old male. The analyses of v. GoRUP-BESANEZ are of (I) a man of 49 and (II) a woman of 29. The results are, as usual, in parts per 1000 .

\begin{tabular}{|c|c|c|c|c|}
\hline & & & v. GORUP- & SANEZ. \\
\hline Water. & 8600 & 859.2 & 822.7 & 898.1 \\
\hline $\begin{array}{l}\text { Water........ } \\
\text { Solids....... }\end{array}$ & 140.0 & 140.8 & 177.3 & 101.9 \\
\hline 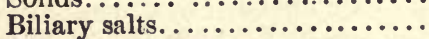 & 72.2 & 91.4 & 107.9 & 56.5 \\
\hline Mucus and pigments........... & 26.6 & 29.8 & 22.1 & 14.5 \\
\hline 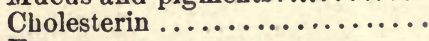 & 1.6 & $2.6\}$ & 47.3 & 30.9 \\
\hline 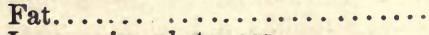 & 3.2 & $9.2\}$ & 10.8 & 6.3 \\
\hline Inorganic substances.......... & 6.5 & $7.7^{\circ}$ & 10.8 & \\
\hline
\end{tabular}


The bile of the gall-bladder is, as above stated, richer in solids than the bile of the liver. Human bile obtained by means of a fistula contained 22.4-22.8 p. m. solids, according to JACOBSEN, who determined the mineral substances in the same bile and found the following, calculated from the dry residue: $\mathrm{KCl} 12.85$, $\mathrm{NaCl}$ 245.1, $\mathrm{Na}_{3} \mathrm{PO}_{4} 59.8, \mathrm{Ca}_{3}\left(\mathrm{PO}_{4}\right)_{2} 16.7$, and $\mathrm{Na}_{2} \mathrm{CO}_{3} 41.8$ p. $\mathrm{m}$.

The relationship between the amounts of glycocholates and taurocholates in the human bile seems to be quite variable. According to the analyses and observations of the majority of investigators, the human bile is, in most cases, relatively richer in glycocholic acid and correspondingly poor in taurocholic acid.

In animals the relative proportion of the two acids varies very much. It has been found, on determining the amount of sulphur, that, so far as the experiments have gone, taurocholic acid is the prevailing acid in carnivorous mammalia, birds, snakes, and fishes. Among the herbivora sheep and goats have a predominance of taurocholic acid in the bile. Ox-bile sometimes contains taurocholic acid in excess, in other cases glycocholic acid predominates, and in a few cases the latter occurs almost alone. The bile of the rabbit, hare, and kangaroo contains, like the bile of the pig, almost exclusively glycocholic acid. A distinct influence on the relative amounts of the two bile-acids by different foods has not been detected. RitTer claims to have found a decrease in the quantity of taurocholic acid in calves when they pass from the milk to the plant diet.

The gases of the bile consist of a large quantity of carbon dioxide, which increases with the amount of alkalies, only traces of oxygen, and a very small quantity of nitrogen.

. Little is known in regard to the properties of the bile in disease. The quantity of urea is found to be considerably increased in uræmia. Leucin and tyrosin are observed in acute yellow atrophy of the liver and in typhus. Traces of albumin (without regard to nucleoalbumin) have several times been found in the human bile. The so-called pigmentary acholia, or the secretion of a bile containing bile-acids but no bile-pigments, has also been repeatedly noticed. In all such cases observed by RITTER he found a fatty degeneration of the liver-cells, in return for which, even in excessive fat infiltration, a normal bile containing pigments was secreted. The secretion of a bile nearly free from bile-acids has been observed by HOPPE-SEYLER in amylaceous degeneration of the iiver, and also by K. Mornere. A number of substances, such as turpentine, salicylic acid, potassium bromide and iodide, arsenic, iron, lead, and mercury (Prevost and Binet), are eliminited by the bile. In animals, dogs, and especially rabbits it has been observed 
that the blood-coloring matters pass into the bile in poisoning and in other cases, causing a destruction of the blood-corpuscles (WERTHimen and Mexer, Filehne).

Chemical Formation of the Bile. The first question to be answered is the following: Do the specific constituents of the bile, the bile-acids and bile-pigments, originate in the liver; and if this is the case, do they come from this organ only, or are they also formed elsewhere?

The investigations of the blood, and especially the comparative investigations of the blood of the portal and hepatic veins under normal conditions, have not given any answer to this question. To decide this, therefore, it is necessary to extirpate the liver of animals or isolate it from the circulation. If the bile constituents are not formed in the liver or at least not alone in this organ, but only eliminated from the blood, then, after the extirpation or removal of the liver from the circulation, an accumulation of the bile constituents is to be expected in the blood and tissues. If the bile constituents, on the contrary, are formed exclusively in the liver. then the above operation naturally would give no such result. It the choledochus duct is tied, then the bile constituents will be collected in the blood or tissues whether they are formed in the liver or elsewhere.

From these principles KöBNER has tried to demonstrate by experiments on frogs that the bile-acids are produced exclusively in the liver. While he was unable to detect any bile-acids in the blood and tissues of these animals after extirpation of the liver, still he was able to discover them on tying the choledochus duct. The investigations of LUDWIG and FLEISCHL show that in the dog the bile-acids originate in the liver alone. After tying the choledochus duct they observed that the bile constituents were absorbed by the lymphatic vessels and passed into the blood through the thoracic duct. - Bile-acids could be detected in the blood after such an operation, while they could not be detected in the normal blood. But when the choledochus and thoracic ducts were both tied at the same time, then not the least trace of bile-acids could be detected in the blood, while if they are also formed in other organs and tissues they should have been present. The general opinion is that the bile-acids are formed only in the liver; still there are investigators 
who have other views. BALDI claims that the formation of bileacids does not take place only in the liver, but in the entire organism, and he claims to have detected bile-acids in the normal, circulating blood of different organs.

It has been indubitably proved that the bile-pigments may ite formed in other organs besides the liver, for, as is generally admitted, the coloring matter hæmatoidin, which occurs in old blood extravasations, is identical with the bile-pigment bilirubin (see page 80). LATSChenberger has also observed in horses, under physiological conditions, a formation of bile-pigments from the bloodcoloring matters in the tissues. Also the occurrence of bilepigments in the placenta seems to depend on their formation in that organ, while the occurrence of small quantities of bile-pigments in the blood-serum of certain animals probably depends on an absorption of the same.

Though the bile-pigments may be formed in other organs, it still seems that their formation under physiological conditions occurs mainly in the liver. By experimenting on pigeons STERN was able to detect bile-pigments in the blood-serum five hours after tying the biliary passages alone, while after tying all the vessels of the liver and also the biliary passages no bile-pigments could be detected either in the blood or the tissues of the animal, which was killed 10-12 hours after the operation. Minkowski and NAUNyN have also found that poisoning with arseniuretted hydrogen produces a liberal formation of bile-pigments and the secretion, after a short time, of a urine rich in biliverdin in previously healthy geese. In geese with extirpated livers this does not occur. The great importance of the liver in the formation of bile-pigments seems to be settled, even though these bodies may be formed also in other organs.

In regard to the materials from which the bile-acids are produced, it may be said with certainty that the two components, glycocoll and taurin, which are both nitrogenized, are formed from the protein bodies. In regard to the origin of the non-nitrogenized cholalic acid, which was formerly considered as originating from the fats, we know nothing positively.

The blood-coloring matters are considered as the mothersubstance of the bile-pigments. If the identity of hæmatoidin and bilirubin were beyond doubt, then this view might be consid- 
ered as proved. Independently, however, of this identity, which is not admitted by all investigators, the view that the bile-pigments are derived from the blood-coloring matters has strong arguments in its favor. It has been shown by several experimenters (lately by LATSCHENBERGER) that a yellow or yellowish-red coloring matter can be formed from the blood-coloring matters which gives GMELIN's test, and which, though it may not form a complete bile-pigment, is at least a step in its formation (IAATSCHENBERGER). A further proof of the formation of the bile-pigments from the blood-coloring matters consists in the fact that hæmatin yields urobilin, which is identical with hydrobilirubin, on reduction (HOPPE-SEYLER). Other investigators (NENCKI and SIEBER and Le NoBeL) claim that the substance thus obtained is not true urobilin, but, all things considered, it seems to be very nearly related.

But even thongh the identity of urobilin with the hydrobilirubin obtained by the reduction of bilirubin is disputed by certain investigators (MACMUNN), still the substances may be so closely related that the relationship will serve as a proof of the formation of bilirubin from the blood-coloring matters. Further, hæmatoporphyrin (see page 78) and bilirubin are isomers, according to NeNCKI and SIEBER, and nearly allied. The formation of bilirubin from the blood-coloring matters is shown, according to the observations of several investigators, by the appearance of free hæmoglobin in the plasma-by the destruction of the red corpuscles by widely-differing influences (see below) or by the injection of hæmoglobin solution-causing an increased formation of bile-pigments. The amount of pigments in the bile is not only considerably increased (TARCHANOFF), but the bile-pigments may even pass into the urine under certain circumstances (icterus). After the injection of hæmoglobin solution into a dog either subcutaneously or in the peritoneal cavity, GonoDECKI observed in the secretion of pigments by the bile an increase of $60 \%$ which lasted for twenty hours.

If, then, iron-free bilirubin is derived from the hæmatin containing iron, then iron must be split off. This process may be represented by the following formula, according to NENCKI and SIEBER,

$$
\mathrm{C}_{32} \mathrm{H}_{32} \mathrm{~N}_{4} \mathrm{O}_{4} \mathrm{Fe}+2 \mathrm{H}_{2} \mathrm{O}-\mathrm{Fe}=2 \mathrm{C}_{16} \mathrm{H}_{18} \mathrm{~N}_{2} \mathrm{O}_{3} \text {, }
$$


though in reality it is probably more complicated. The question in what form or combination the iron is split off is of special interest, and also whether it is eliminated by the bile. This latter does not seem to be the case. In 100 parts of bilirubin which are eliminated by the bile there are only 1.4-1.5 parts iron, according to KUNKEL; while 100 parts hæmatin contain about 9 parts iron. MINKowsKI and BASERIN have also found that the abundant formation of bile-pigments occurring in poisoning by arseniuretted hydrogen does not increase the quantity of iron in the bile. The quantity apparently does not correspond with that in the decomposed blood-coloring matters.

On the contrary, it seems as if the iron, at least for a time, is retained by the liver as a pigment rich in iron. Such a pigment containing iron, which was formed by the decomposition of hæmoglobin, was observed by NAUNYN and MrNkowSKI in the livers of birds, in arseniuretted hydrogen icterus. LATsCheNBERGER claims that a yellow or yellowish red pigment, "choleglobin," is derived from the blood-coloring matters, and acts as a step in the formation of the bile-pigments; and besides this he mentions another body consisting of dark grains and containing iron, which he designates as melanin. NEUMANN has observed in blood extravasations, besides hæmatoidin, a pigment containing iron, for which he has proposed the name homatosiderin.

An absorption of bile from the liver by the lymphatic vessels and the passage of the bile-constituents into the blood and urine occurs in retarded discharge of the bile, and usually in the different forms of hepatogenic icterus. But bile-pigments may also pass into the urine under other circumstances, especially in animals where a solution or destruction of the red blood-corpuscles takes place through injection of water or a solution of biliary salts, through poisoning by ether, chloroform, arseniuretted hydrogen, or toluylendiamin; and in other cases. This occurs also in man in grave infectious diseases. We have therefore a second form of icterus, in which the blood-coloring matters are transformed into bilepigments elsewhere than in the liver, blood, and tissues-a homatogenic, chemical, or anhepatogenic icterus. Only bile-pigments occur in the urine in these cases, while in hepatogenic icterus the urine contains the bile-pigments and bile-acids at the 
same tine (LEYDEN). This distinction, however, cannot be main. tained. It is certainly true that the presence of more than traces of bile-acids in the urine indicates the existence of hepatogenic icterus; but cases of absorption icterus undoubtedly occur in which no bile-acids can be detected in the urine.

It has been clearly demonstrated by the above-mentioned observations of MINkowsKI and NAUNYN on geese with extirpated livers that the bile-pigments are formed in the liver in arseniuretted hydrogen icterus. It has also been demonstrated by STADELMANN and AFANASSIEW that the icterus, after poisoning by arseniuretted hydrogen or by toluylendiamin, must be considered as an absorption icterus. Perhaps the icterus depends in these cases upon the fact that the viscosity of the abundantly-secreted bile may form a check to its outflow, which counteracts the low secretion pressure, so that a stowing and absorption occurs (polycholic icterus). It is also possible that other cases of so-called hæmatogenic icterus may be of an analogous kind, and that every icterus is consequently hepatogenic. The occurrence of hæmatogenic icterus cannot be considered as proved beyond doubt, and it is questioned by several recent investigators.

\section{Appendix to the Bile. Bile Concretions.}

The concrements which occur in the gall-bladder vary considerably in size, form, and number, and are of three kinds, depending upon the kind and nature of the bodies forming their chief mass. One group of gall-stones contains lime-pigment as chief constituent, the other cholesterin, and the third calcium carbonate and phosphate. The concrements of the last-mentioned group occur very seldom in man. The so-called cholesterin stones are those which occur most frequently in man, while the lime-pigment stones are not found very often in man, but often in oxen.

The pigment-stones are generally not large in man, but in oxen and pigs they are sometimes found the size of a walnut or even larger. In most cases they consist chiefly of bilirubin-calcium with little or no biliverdin. Sometimes also small black or greenish black, metallic-looking stones are found, which consist chiefly of bilifuscin along with biliverdin. Iron and copper seem to be reg- 
ular constituents of pigment-stones. Manganese and zinc have also been found a few times. The pigment-stones are generally heavier than water.

The cholesterin-stones, whose size, form, color, and structure may vary greatly, are often lighter than water. The fractured surface is radiated, crystalline, and frequently shows crystalline, concentric layers. The cleavage fracture is waxy in appearance, and the fractured surface when rubbed by the nail also becomes like wax. By rubbing against each other in the gall-bladder they often become faceted or take other remarkable shapes. Their surface is sometimes nearly white and waxlike, but generally their color is variable. The quantity of cholestrin in the stones varies from 642 to $981 \mathrm{p} . \mathrm{m}$. (RITTER). The cholesterin-stones also sometimes contain variable amounts of lime-pigments which give them a very changeable appearance.

Cholesterin, $\mathrm{C}_{26} \mathrm{H}_{44} \mathrm{O}$, or, according to ReinitzeR, $\mathrm{C}_{27} \mathrm{H}_{47} \mathrm{O}$. Cholesterin is generally considered as a monatomic alcohol of the formula $\mathrm{C}_{26} \mathrm{H}_{43} . \mathrm{OH}$. It yields a colored hydrocarbon (cholesteriline or cholesterone), of the formula $\mathrm{C}_{26} \mathrm{H}_{42}$, with sulphuric acid, and this hydrocarbon is claimed by WEYL to be nearly related to the terpene group It has also been claimed that it is closely allied to cholalic acid.

Cholesterin occurs in small amounts in nearly all animal fluids and juices. It occurs only rarely in the urine, and then in very small quantities. It is also found in the different tissues and organsespecially abundant in the brain and the nervous system,-further in the yolk of the egg, in semen, and in wool-fat. It appears (together with isocholesterin) in the contents of the intestines, in excrements, and in the meconium. It occurs pathologically especially in gall-stones, as well as in atheromatous cysts, in pus, in tuberculous masses, old transudations, cystin fluids, excrements, and tumors. Several kinds of cholesterin seem to occur in the plant world

Cholesterin which crystallizes from warm alcohol on cooling, and that which is present in old transudations, contains 1 mol. of water of crystallization, melts at $137^{\circ} \mathrm{C}$, and forms colorless, transparent plates whose sides and angles frequently appear broken and whose acute angle is often $76^{\circ} 30^{\prime}$ or $87^{\circ} 30^{\prime}$. In large quantities 
it appears as a mass of white plates which shine like mother-ofpearl and have a greasy feel.

Cholesterin is insoluble in water, dilute acids and alkalies. It is neither dissolved nor changed by boiling caustic alkali. It is easily soluble in boiling alcohol and crystallizes on cooling. It dissolves readily in ether, chloroform, and benzol, and also in the volatile or fatty oils. It is dissolved to a slight extent by alkali salts of the bile, acids. For the detection of cholesterin we make use of its action with concentrated sulphuric acid, which, as above stated, gives a colored hydrocarbon with this acid.

If a mixture of five parts sulphuric acid and one part water acts on a cholesterin crystal, this crystal will show colored rings, first a bright carmine-red and then violet. This fact is made use of in the microscopic detection of cholesterin. Another test, and one very good for the microscopical detection of cholesterin, consists in treating the crystal first with the above dilute acid and then with some iodine solution. The crystals will be gradually colored violet, bluish green, and a beautiful blue.

Salkowski's Reaction. The cholesterin is dissolved in chloroform and then treated with an equal volume of concentrated sulphuric acid. The cholesterin solution becomes first bluish red, then gradually more violet-red, while the sulphuric acid appears dark red with a greenish fluorescence. If the chloroform solution is poured into a porcelain dish it becomes violet, then green, and finally yellow.

ScHIFr's Reaction. If a little cholesterin is placed in a porcelain dish with the addition of a few drops of a mixture of two to three vols. conc. hydrochloric acid or sulphuric acid and one vol. of a medium solution of ferric chloride, and carefully evaporated to dryness over a small flame, a reddish-violet residue is first obtained and then a bluish violet.

If a small quantity of cholesterin is evaporated to dryness with a drop of concentrated nitric acid, we obtain a yellow spot which becomes deep ornngered with ammonia or caustic soda (not a characteristic reaction).

Isocholesterin. This body, so called by ScHOLTZE, is isomeric with the ordinary cholesterin and occurs in wool-fat, and is therefore found in abundant quantities in so-called lanolin. It does not give SALKowski's or ScurFr's reactions.

We make use of the so-called cholesterin-stones in the preparation of cholesterin. The powder is first boiled with water and then repeatedly boiled with alcohol. The cholesterin which on cooling separates from the warm filtered solution is boiled with 
a solution of caustic potash in alcohol so as to saponify any fat. After the evaporation of the alcohol we extract the cholesterin from the residue with ether, by which the soaps are not dissolved, filter, evaporate the ether, and purify the cholesterin by recrystallization from alcohol-ether. The cholesterin may be extracted from tissues and fluids by first extracting with ether and then purifying as above.

It is detected and determined quantitatively in tissue, etc., by this same method. It is ordinarily easily detected in transudations and pathological formations by means of the microscope. 


\section{CHAPTER VII.}

\section{DIGESTION.}

THE purpose of the digestion is to separate those constituents of the food which serve as the nutriment of the body from those which constitute the waste, and to separate each in such a form that it may be easily taken up by the blood from the alimentary canal and conveyed to its proper organism. This demands not only mechanical but also chemical action. The first action which essentially depends on the physical properties of the food consists in a tearing, cutting, crushing, or grinding of the food, and serves chiefly to convert the nutritive bodies into a soluble and easilyabsorbed form, or in the splitting of the same into simpler combinations for use in the animal synthesis. The solution of the nutritive bodies may take place in certain cases by the aid of water alone, but in most cases a chemical metamorphosis or splitting is necessary, and is effected by means of the acids or alkalies, or by the fluids secreted by the glands. The study of the processes of digestion from a chemical standpoint must therefore begin with the digestive fluids, their qualitative and quantitative composition, as well as their action on the nutriments and foods.

\section{The Salivary Glands and the Saliva.}

The salivary glands are partly albuminous-glands (as the parotid in man and mammalia and the submaxillary in rabbits), partly mucous-glands (as some of the small glands in the buccal cavity and the sublingual and submaxillary glands of many animals), and partly mixed glands (as the submaxillary gland in man). The alveoli of the albumin glands contain cells which are rich in albumin, but contain no mucin. The alveoli of the mucin-glands

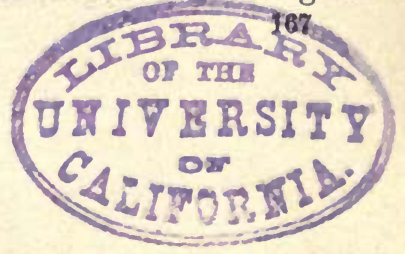


contain cells rich in mucin but poor in albumin. A cell rich in albumin may also occur in the submaxillary and sublingual glands between the mucus-cells and the membrana propria, which in a few cases akes the form of a half-moon (lunula, according to Gianuzzi), and in other cases the cells rich in mucin are surrounded as by a ring, and sometimes certain alveoli may be com pletely filled. By continuous secretion the mucin-cells seem to give up all their mucin (EwALd, STöHR), so that only albumin-cells are to be seen (HeIDENHAIN). During rest the mucin-cells are re-formed. According to the analyses of OIDTMAN, the salivary glands of a dog contain 790 p. m. water, 200 p. m. organic and 10 p. m. inorganic solids. Among the solids, in addition to albumin, we find, nucleoalbumin and mucin, diastatic enzyme, in certain animals nuclein, extractive bodies, leucin, traces of xanthin bodies, and mineral substances.

The saliva is a mixture of the secretion of the abore-mentioned groups of glands; therefore it is proper that we first study each of the different secretions by itself, and then the mixed saliva.

The submaxillary saliva in man may be easily collected by introducing a canula into the Wharton's duct.

The submaxillary saliva has not always the same composition or properties; these depend essentially upon the conditions under which the secretion takes place. That is to say, the secretion is partly dependent on the cerebral, partly on the sympathetic, nervous system. In consequence of this dependence the two distinct varieties of submaxillary secretion are distinguished as chordaand sympathetic saliva. A third kind of saliva, the so-called paralytic saliva, is secreted after poisoning with curara or after the severing of the glandular nerves.

The difference between chordal and sympathetic saliva (in dogs) consists chiefly in their quantitative constitution, namely, the less abundant sympathetic saliva is more viscous and richer in solids, especially in mucin, than the more abundant chordal saliva. The specific gravity of the chordal saliva of the dog is 1.0039-1.0046 and contains from 12 to $14 \mathrm{p}$. m. solids (ЕскнARD). The sympathetic has a specific gravity of 1.0075-1.01.8, with 16-28 p. m. solids. The gases of the chordal saliva have been investigated by PFLüGER. He found $0.5-0.8 \%$ oxygen, $0.9-1 \%$ nitrogen, and $64.73-85.13 \%$ carbon 
dioxide-all results calculated at $0^{\circ} \mathrm{C}$. and $760 \mathrm{~mm}$. pressure. The greater part of the carbon dioxide was chemically combined.

The two kinds of submaxillary secretion just named have not thus far been separately studied in man. The secretion may be excited by a psychological conception, by mastication, and by irritating the mucous membrane of the mouth, especially with acidtasting substances. The submaxillary saliva in man is ordinurily clear, rather thin, a little ropy, and froths easily. Its reaction is alkaline. The specific gravity is $1.002-1.003$, and it contains $3.6-4.5$ p. m. solids. We find as organic constituents mucin, traces of albumin and diastatic enzyme is absent in several species of animals. The inorganic bodies are alkali chlorides, sodium and magnesium phosphates, besides bicarbonates of the alkalies and calcium. OEHL finds $0.036 \mathrm{p}$. m. potassium sulphocyanide in this saliva.

The Sublingual Saliva. The secretion of this saliva is also influenced by the cerebral and the sympathetic nervous system. The chordal saliva, which is secreted only to a small extent, contains numerous salivary corpuscles, but is otherwise transparent and very ropy. Its reaction is alkaline and contains, according to Heidenhain, 27.5 p. m. solids (in dogs).

The sublingual secretion in man has been investigated by $0 \mathrm{EHL}$. It was clear, mucilaginous, more alkaline than the submaxillary saliva, and contained mucin, diastatic enzymes, and potassium sulphocyanide.

Buccal mucus can only be obtained pure from animals by the method of BIDDER and SCHMIDT, which consists in tying the exit to all the large salivary glands and cutting off their secretion from the mouth. The quantity of liquid secreted under these circumstances (in dogs) was so very small that the investigators named were able to collect only 2 grms. buccal mucus in the course of twenty-four hours. It is a thick, ropy, sticky liquid containing mucin; it is rich in form-elements, above all in flat epitheliumcells, mucous cells, and salivary corpuscles. The quantity of solids in the buccal mucus of the $\operatorname{dog}$ is, according to BIDDER and SсHмidT, 9.98 p. m.

Parotid Saliva. The secretion of this saliva is also partly dependent on the cerebral nervous system (n. glossopharyngeus) and partly on the sympathetic. The secretion may be excited by 
mental emotions and by irritation of the glandular nerves, either directly (in animals) or reflexly, by mechanical or chemical irritation of the mucous membrane of the mouth. Among the chemical irritants the acids take first place, while alkalies and pungent substances have little action. Sweet-tasting bodies, such as honey, are said to have no effect. Mastication has great influence in the secretion of parotid saliva, which is especially marked in certain herbivora.

Human parotid saliva may be collected by the introduction of a canula into the parotid duct. This saliva is thin, less alkaline than the submaxillary saliva (the first drops are sometimes neutral or acid), without special odor or taste. It contains a little albumin but no mucin, which is to be expected from the construction of the gland. It also contains a diastatic enzyme, which, however, is absent in many animals. The quantity of solids varies between 5 and $16 \mathrm{p}$. $\mathrm{m}$. The specific gravity is 1.003-1.012. Potassium sulphocyanide seems to be present, though it is not a constant constituent. KüLz found $1.46 \%$ oxygen, 3.2\% nitrogen, and in all $66.7 \%$ carbon dioxide in human parotid saliva. The quantity of closely-combined carbon dioxide was $62 \%$.

The mixed buccal saliva in man is a colorless, faintly opalescent, slightly ropy, easily frothing liquid without special odor or taste. It is made turbid by epithelium cells, mucous and salivary corpuscles, and often by food residues. Like the submaxillary and parotid saliva, on exposure to the air it becomes covered with an incrustation consisting of calcium carbonate and a small quantity of an organic substance, or it gradually becomes cloudy. Its reaction is alkaline, but occasionally also acid. According to STICKER, fresh saliva may be acid a few hours after a meal. Two or three hours after breakfast and four to five hours after dinner the maximum of acidity occurs, and it may also be faintly acid from midnight to morning. The specific gravity varies between 1.002 and 1.009 , and the quantity of solids between 5 and $10 \mathrm{p} . \mathrm{m}$. The solids, irrespective of the form-constituents mentioned, consist of albumin, mucin, ptyalin, and mineral bodies. It is also claimed that urea is a normal constituent of the saliva. The mineral bodies are alkali chlorides, bicarbonates of the alkalies and calcium, phosphates, and traces of sulphates and sulphocyanides. 
Sulphocyanides, which, although not constant, occur in the saliva of man and certain animals, may be easily detected by first acidifying the saliva with hydrochloric acid and treating with a very dilute solution of ferric chloride. 'To make the test more conclusive it is best, as control, to take an equal quantity of acidified water and then add ferric chloride. Another, simpler method, proposed by GSCHEIDLEN, consists in putting in a drop or two of the saliva on filter-paper which has previously been dipped in an ambercolored solution of ferric chloride containing hydrochloric acid, and then dried. Each drop of saliva containing sulphocyanide will give a reddish spot. If the quantity of sulphocyanide is so small that it cannot be detected directly, concentrate the saliva after the addition of a little alkali, acidify strongly with hydrochloric acid, and shake repeatedly with ether, evaporating the latter after the addition of water containing alkali over a gentle heat; then test the remaining liquid.

Ptyalin, or salivary diastase, is the amylolytic ferment of the saliva. This ferment is found in human saliva, but not in that of animals. It occurs not only in adults, but also in new-born infants. ZwEIFEL claims that the ptyalin in new-born infants occurs only in the parotid gland, but not in the submaxillary. In the latter it appears only two months after birth.

Ptyalin has not been isolated in a pure form up to the present time. It was obtained purest by precipitation with calcium phosphate (CoHNHEIM). For the study or the demonstration of the action of ptyalin we may use a watery or glycerin extract of the salivary glands, or, more simply, the saliva itself.

Ptyalin, like other enzymes, is characterized by its action. This consists in converting starch into dextrin and sugar. According to the common acceptation, soluble starch is first formed by this action and then erythro-dextrin, which is further changed so that we obtain at the end achroo-dextrin and maltose with a small admixture of glucose. Like starch, glycogen is also split by ptyalin into dextrin and sugar (apparently maltose) upon the addition of water. Ptyalin is not identical with malt diastase; while the first acts with greatest energy at about $+40^{\circ} \mathrm{C}$., diastase acts best at $+50^{\circ}$ to $55^{\circ} \mathrm{C}$. (ChitTENDEN and Martin).

Ptyalin acts in faintly alkaline, in nentral, and in extremely weak acid solutions. It seems to act most energetically in neutral or, in a few cases, in very faint acid reaction (ChITTENden and SchMidT). 
The amount of action in the latter case is dependent upon several circumstances, such as the degree of dilution and the presence of proteids or peptone (CHITTENDEN). Of the greatest physiological importance, however, is the fact that even very small quantities of free acids-not only a degree of acidity of about $1 \mathrm{p} . \mathrm{m} . \mathrm{HCl}$, which often occurs in the gastric juice, but even a smaller quantity of hydrochloric acid (organic acids are less active)-at once prevents the action of the ptyalin and destroys the enzyme. It is also of interest that boiled starch (starch-paste) is more quickly converted into sugar than unboiled. The time required to change unboiled starch varies with the kind.

The rapidity with which ptyalin acts increases, at least under conditions otherwise favorable, with the amount of enzyme and a temperature a little above $+40^{\circ} \mathrm{C}$. Foreign substances such as metallic salts, have a different effect. Certain salts even in small quantities completely arrest the action; for example, $\mathrm{HgCl}_{2}$ accomplishes this result by the presence of only $0.05 \mathrm{p}$. m. Other salts, such as magnesium sulphate, in small quantities $(0.25 \mathrm{p} . \mathrm{m}$.) accelerate, and in larger quantities $(5$ p. m.) check the action. The accumulation of the products of the amylolytic decomposition also checks the action of the saliva.

To show the action of saliva or ptyalin on starch the three ordinary tests for glucose may be used, namely, Moone's or Trommen's test or the Bismuth test (see Chap. XIV). It is also necessary, as a control, to first test the starch-paste and the saliva for the presence of glucose.

The quantitative composition of the mixed saliva must vary considerably, not only because of individual differences, but also because under varying conditions there may be an unequal division of the secretion from the different glands. Analyses of the composition of human saliva are given in the table on the opposite page. The figures are parts per 1000 .

The quantity of saliva secreted during 24 hours cannot be exactly determined, but has been calculated by BIDDER and Schmidt to be 1500 grms. The most abundant secretion occurs during meal-times. According to the calculations and determinations of TuczeK in man, $1 \mathrm{grm}$. of gland should yield $13 \mathrm{grms}$. secretion in the course of one hour during mastication. These figures 


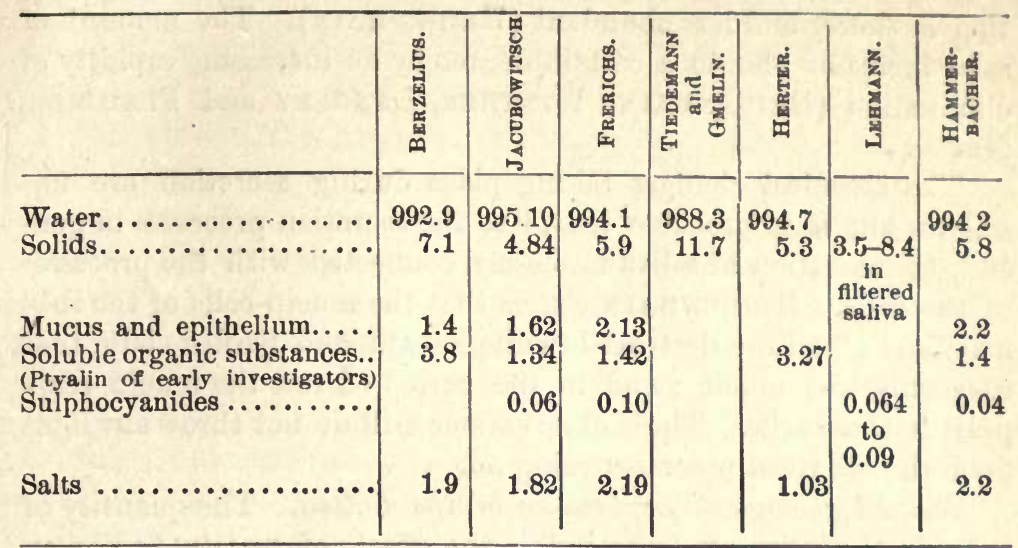

HAMmerbacher found in 1000 parts of the ash from human saliva (potash 457.2, soda 95.9 , iron oxide 50.11, magnesia 1.55 , sulphuric anhydride $\left.\mathrm{SO}_{3}\right) 63.8$, phosphoric anhydride $\left(\mathrm{P}_{2} \mathrm{O}_{3}\right) 188.48$, and chlorine 183.5\%.

correspond fairly well with those representing the average secretion from 1 grm. of gland in animals, namely, 14.2 grms. in the horse and 8 grms. in oxen. The quantity of secretion per hour may be 8 to 14 times greater than the entire mass of glands, and there is probably no gland in the entire body, as far as we know at present-the kidneys not excepted-whose ability of secretion under physiological conditions equals that of the salivary glands. A remarkably abundant secretion of saliva is induced by pilocarpin, while atropin, on the contrary, prevents it.

Though an abundant secretion of saliva is produced, as a rule, by an increased supply of blood, still it is not a simple filtration process, as seen from the following circumstances. The secretionpressure is greater than the blood-pressure in the carotid, and in poisoning by atropin, which paralyzes the secretory nerves, an increased supply of blood is produced by irritation of the chorda, but no secretion (HEIDENHAIN). The salivary glands have moreover a specific property of eliminating certain substances, such as potassium salts (SALKowski), iodine and bromine combinations, but not others, such as iron combinations. It is also noticeable that the saliva is richer in solids when it is eliminated quickly by graduallyincreased irritation, and in larger quantities than when the secre. 
tion is slower and less abundant (HeIDenhais). The amount of salts increases also to a certain degree by an increasing rapidity of elimination (Heidenhain, Werther, Langley and Fletcher, NovY).

The chemical changes taking place during secretion are unknown, but it is probable that, like the secretion processes in general, the secretion of saliva is closely connected with the processes in the cells. HeIdenhain claims that the mucin-cells of the submaxillary gland are destroyed (while EWALD and SToHR claim that they only lose mucin), and in the period of rest the mucin reappears in these cells. These observations still do not throw any light upon the chemical processes going on.

The Physiological Importance of the Saliva. The quantity of water in the saliva renders possible the effects of certain bodies on the organs of taste, and it also serves as a solvent for a part of the nutritive substances. The importance of the saliva in mastication is especially marked in herbivora, and there is no question of its importance in facilitating the act of swallowing. The power of converting starch into sugar does not belong to the saliva of all animals, and even when it possesses this property the intensity varies in different animals. In man, whose saliva forms sugar rapidly, a formation of sugar from (boiled) starch undoubtedly takes place in the mouth, but how far this action goes on after the morsel has entered the stomach depends upon the rapidity with which the acid gastric juice mixes with the swallowed food, and also upon the relative amounts of the gastric juice and food in the stomach. The large quantity of water which is swallowed with the saliva must be absorbed and pass into the blood, and it must go through an intermediate circulation in the organism. Thus the organism possesses in the saliva an active medium by which a constant stream, conveying the dissolved and finely-divided bodies, passes into the blood from the intestinal canal during digestion.

Salivari Calculi. The so-called tartar is yellow, gray, yellowish gray, brown or black, and has a stratified structure. It may contain more than 200 p. m. organic substances, which consist of mucin, epithelium, and LEPTOTHRIX. The chief part of the inorganic constituents consists of calcium carbonate and phosphate. The salivary stone may vary in size from the size of a small grain to that of a pea or still larger (a salivary stone has been found weighing 18.6 grms.), and it contains a variable quantity of organic substances, 50-380 p. m., which remain on extracting the stone with hydrochloric acid. The chief inorganic constituent is calcium phosphate. 


\section{The Glands of the Mucous Membrane of the Stomach, and the Gastric Juice.}

Since of old, the glands of the mucous coat of the stomach have been divided into two distinct kinds. The kind occurring in the greatest abundance, and the most important in size, especially in the fundus, has been named fundus glands, also RENNET or PEPTONe glands. The other, which is found in the neighborhood of the pylorus, has received the name of pyloric glands, sometimes also, though incorrectly, mucous glands. The mucous coating of the stomach is covered to its entire extent with a layer of cylindrical epithelium, which is considered as consisting exclusively of follicles, small cup-shaped cavities which by a mucus-like metamorphosis produce the protoplasm.

The fundus glands contain two kinds of cells: ADELOMORPHIC or principal cells, and DELOMORPHIC or PARIETAL cells, the latter formerly called RENNET or pepsin cells. Both kinds consist of protoplasm rich in proteids; but their relationship to coloring matters seems to show that the two albuminous bodies are not identical. The nucleus must consist chiefly of nuclein. Besides the above-mentioned constituents the fundus glands contain as more specific constituents two zymogens, which are the mother-substances of the pepsin and the rennet, besides a small quantity of fat and cholesterin.

The pyloric glands contain cells which are generally considered as related to the above-mentioned chief cells of the fundus glands. As these glands were formerly thought to contain a larger quantity of mucin, they were also called mucous glands. According to Heidentain, independent of the cylindrical epithelium of the excretory passages, they take no part worthy of mention in the formation of mucus, which, according to his views, is effected by the epithelium covering the mucous membrane. The pyloric glands also seem to contain the zymogens referred to above. Alkali chlorides, alkali phosphates, and calcium phosphates are found in the mucous coating of the stomach.

The Gastric Juice. The experiments of HeLM and BeAumont on persons with gastric fistula led to the suggestion that gastric fistulas be made on animals, and this operation was first performed 
by BAssow in 1842 on a dog. VERNEUIL performed the same on a man in $187 \%$ with successful results. These fistulas in animals afford an excellent means of studying the secretion of gastric juice and also the stomachic digestion.

In a fasting condition the mucous coat is often nearly dry; sometimes, especially on certain herbivora, it is covered with a layer of viscid so-called mucus. If food is introduced into the stomach, or if the mucous membrane is irritated in some way, then a secretion of a thin, acid fluid, the real gastric juice, takes place. 'The secretion may be produced by mechanical or thermal irritation (introduction of cold water or pieces of ice into the stomach), or by chemical irritants. Among the latter, alcohol and ether when in too great concentration do not produce a physiological secretion, but a transudation of a neutral or faintly alkaline fluid containing albumin (CL. BERNARD). To this class of irritants belong carbon dioxide and hydrochloric acid; the last especially increases the secretion of pepsin (JAworskY), spices, meat extracts, neutral salts, such as $\mathrm{NaCl}$ (which acts like alcohol in too great concentration), and alkali carbonates. The alkali carbonates are supposed by certain investigators to first neutralize, and then produce a continuous secretion of, acid gastric juice. The statements in regard to the action of different bodies in the secretion of gastric juice are still rather uncertain and often contradictory.

Several investigators state that the secretion of gastric juice may be stimulated by reflex means. After the introduction of water into the stomach a proportionally poor and scanty secretion takes place, while on the contrary the introduction of digestible foods causes a more abundant and continuous secretion (SCHIFF, Heidenhain). But in these cases the secretion does not take place immediately, but only after the absorption of the soluble bodies has commenced. This fact justifies the usual custom of commencing a meal with fluid nutritives such, as soup.

The Qualitative and Quantitative Composition of the Gastric Juice. The gastric juice, which can hardly be obtained pure and free from residues of the food or from mucus and saliva, is a clear, or only very faintly cloudy, and in man nearly colorless fluid of an insipid, acid taste and strong acid reaction. It contains, as form- 
elements, glandular cells or their nuclei, mucus-corpuscles, and more or less changed cylindrical epithelium.

The acid reaction of the gastric juice depends on the presence of free acid, which, as we have learned from the investigations of C. SCHMIDT, RICHET, and others, seems, under physiological conditions, to consist only of hydrochloric acid. Under special conditions, as after a meal rich in carbohydrates, lactic acid occurs in the contents of the stomach, and even acetic and butyric acid. The quantity of free hydrochloric acid in the gastric juice of sheep is about $1.2 \mathrm{p} . \mathrm{m}$., and in dogs $3 \mathrm{p} . \mathrm{m}$. Richet found as average for 80 determinations of human gastric juice $1.7 \mathrm{p}$. $\mathrm{m}$. free hydrochloric acid, with a variation between 0.5 and 3 p. $\mathrm{m}$. According to SzABo and EWALD and BOAS, the human gastric juice contains usually about $2-3 \mathrm{p} . \mathrm{m}$. $\mathrm{HCl}$.

The gastric juice seems to contain no coagulable albumin, but contains traces of peptone or albumose (?). Among the organic bodies a little mucin is found, and besides, especially in man, two enzymes, pepsin and rennet.

The specific gravity of gastric juice is low, 1.001-1.010. It is therefore correspondingly poor in solids. As examples of the composition of different kinds of gastric juice the analyses of C. Schmid are here given. It must be remarked that the human gastric juice analyzed was diluted by saliva and water and should therefore not be considered as normal. The figures are parts per 1000.

\begin{tabular}{|c|c|c|c|c|}
\hline & $\begin{array}{c}\text { Human Gas- } \\
\text { tric Juice } \\
\text { mixed with } \\
\text { Saliva. }\end{array}$ & $\begin{array}{c}\text { Gastric } \\
\text { Juice from } \\
\text { Dog free } \\
\text { from Saliva. }\end{array}$ & $\begin{array}{c}\text { Gastric } \\
\text { Juice from } \\
\text { Dog contain- } \\
\text { ing Saliva. }\end{array}$ & $\begin{array}{l}\text { Gastric } \\
\text { Juice of } \\
\text { Sheep. }\end{array}$ \\
\hline 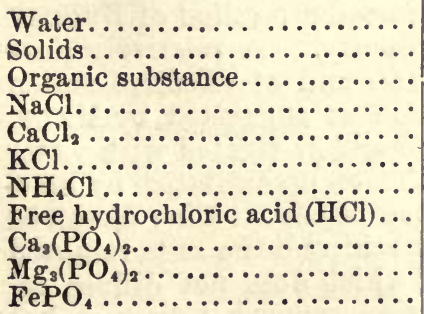 & $\begin{array}{r}994.40 \\
5.60 \\
3.19 \\
1.46 \\
0.06 \\
0.55 \\
\cdots 0.20 \\
0.12\end{array}$ & $\begin{array}{r}973.0 \\
27.0 \\
17.1 \\
2.5 \\
0.6 \\
1.1 \\
0.5 \\
3.1 \\
1.7 \\
0.2 \\
0.1\end{array}$ & $\begin{array}{r}971.2 \\
28.8 \\
17.3 \\
3.1 \\
1.7 \\
1.1 \\
0.5 \\
2.3 \\
2.3 \\
0.3 \\
0.1\end{array}$ & $\begin{array}{r}986.15 \\
13.85 \\
4.05 \\
4.36 \\
0.11 \\
1.52 \\
0.47 \\
1.23 \\
1.18 \\
0.57 \\
0.33\end{array}$ \\
\hline
\end{tabular}


The physiologically important constituents, besides free nyarochloric acid, are pepsin and rennet.

Pepsin. This enzyme is found, with the exception of certain fishes, in all vertebrata thus far investigated.

Pepsin occurs in adults and in new-born infants. This condition is different in new-born animals. While in a few herbivora, such as the rabbit, pepsin occurs in the mucous coat before birth, this enzyme is entirely absent at the birth of those carnivora which have thus far been examined, such as the dog and cat.

In various invertebrates a ferment has also been found which has a proteolytic action in acid solutions. KRUKENBERG has shown that this enzyme, nevertheless is not in all animals, identical with ordinary pepsin. DARwIN has further found that certain plants which feed upon insects secrete an acid juice which dissolves albumin, but it is still doubtful whether these plants contain any pepsin. v. GoRUP. BESANEz has isolated from vetch-seed an enzyme which acts like pepsin, but whose identity with pepsin is doubtful.

Pepsin is as difficult to isolate in a pure condition as other enzymes. The purest pepsin was that prepared by BRücKE and SUNDBERG; this gave negative results with most reagents for albumin. Pepsin, therefore, does not seem to be a true albuminous substance. It is, at least in the impure condition, soluble in water and glycerin. It is precipitated by alcohol, but only slowly destroyed. It is quickly destroyed by heating its watery solution to boiling; in the dry state it can, on the contrary, be heated to over $100^{\circ} \mathrm{C}$. without losing its physiological action. The only property which is characteristic of pepsin is that it dissolves albuminous bodies in acid, but not in neutral or alkaline, solutions with the formation of albumoses and peptones.

The methods for the preparation of relatively pure pepsin depend, as a rule, upon its property of being thrown down with finely-divided precipitates of other bodies, such as calcium phosphate or cholesterin. The rather complicated method of BR̈̈CKE and SundBerg is based upon this property. A relatively pure pepsin solution intended for digestion tests and of effective action may be prepared by the following method as suggested by MALY. The mucous membrane (of the pig's stomach) is treated with water containing phosphoric acid, and the filtrate precipitated by limewater; the precipitate, which contains the pepsin, is then dissolved in water by the addition of hydrochloric acid, and the salts removed by dialysis, by which means the pepsin which does not diffuse remains in the dialyzer. A pepsin solution somewhat impure but 
active and not liable to spoil may be obtained if, as suggested by v. WitTICHS, we extract the finely-divided mucous membrane with glycerin, or better with glycerin which contains $1 \mathrm{p} . \mathrm{m}$. HCl. To each part by weight of the mucous coat add 10-20 parts glycerin. This is filtered after 8-14 days. The pepsin (together with much albumin) may be precipitated by alcohol from this extract. If this extract is to be used directly for digestion tests, then to $100 \mathrm{c}$. c. of water which has been acidified with 1-4 p. m. $\mathrm{HCl}$ add $2-3$ c. c. of the extract.

For digestion tests an infusion of the mucous membrane of the stomach may be used directly in many cases. 'The mucous coat is carefully washed with water (if a pig's stomach is used) and finely cut; if a calf's stomach is employed, only the upper layer of the mucous coat is scraped off with a watch-glass or the back of a knife. The pieces of mucous membrane or the slimy masses obtained by scraping are rubbed with pure quartz-sand, treated with acidified water, and allowed to stand for 24 hours in a cool place and then filtered.

In the preparation of artificial gastric juice that part only of the mucous coat richest in pepsin is used, the pyloric is of little value. A strong, impure infusion may generally be obtained from the pig's stomach, while a relatively pure and powerful infusion is obtained from the stomach of birds (hens). The stomachs of fish (pike) also yield a tolerably pure and active infusion. An active and rather pure artificial gastric juice may be prepared by scraping the inner layers of a calf's stomach from which the pyloric end has been removed. For a medium-sized calf's stomach 1000 c. c. of acidified water must be used.

The degree of acidity required in the infusion depends upon the use to which the gastric juice is to be put. If it is to be employed in the digestion of fibrin, an acidity of $1 \mathrm{p} . \mathrm{m}$. HCl must be selected, while, on the contrary, if it is to be used for the digestion of hard-boiled-egg albumin, an acidity of $2-3 \mathrm{p} . \mathrm{m}$. HCl is preferable. This last-mentioned degree of acidity is generally the better, because the infusion is preserved thereby, and at all events it is so rich in pepsin that it may be diluted with water until it has an acidity of $1 \mathrm{p} . \mathrm{m}$. $\mathrm{HCl}$ without losing any of its solvent action on unboiled fibrin.

The Action of Pepsin on Proteids. Pepsin is inactive in neutral or alkaline reactions, but in acid liquids it dissolves coagulated albuminous bodies. The proteid always swells and becomes transparent before it dissolves. Unboiled fibrin swells up in a solution containing $1 \mathrm{p} . \mathrm{m} . \mathrm{HCl}$, forming a gelatinous mass, and does not dissolve at ordinary temperature within a couple of days. Upon 
the addition of a little pepsin, however, this swollen mass dissolves quickly at an ordinary temperature. Hard-boiled-egg albumin, cut in thin pieces with sharp edges, is not perceptibly changed by dilute acid $(2-4 \mathrm{p} . \mathrm{m} . \mathrm{HCl})$ at the temperature of the body in the course of several hours. But the simultaneous presencc of pepsin causes the edges to become clear and transparent, blunt and swollen, and the albumin gradually dissolves.

From what has been said above in regard to pepsin, it follows that albumin may be employed as a means of detecting pepsin in liquids. - Fibrin may be employed as well as hard-boiled-egg albumin, which latter is used in the form of slices with sharp edges. As the fibrin is easily digested at the normal temperature, while the pepsin test with egg-albumin requires the temperature of the body, and as the test with fibrin is somewhat more delicate, it is often preferred to that with egg-albumin. When we speak of the "pepsin test" without further explanation, we ordinarily understand it as the test with fibrin.

This test nevertheless requires care. The unboiled fibrin may be dissolved by acid alone without pepsin, but this generally requires more time. In testing with unboiled fibrin at normal temperature, it is advisable to make a control test with another portion of the same fibrin with acid alone. Since at the temperature of the body unboiled fibrin is easier dissolved by acid alone, it is best always to work with boiled fibrin.

As pepsin has not, thus far, been prepared in a positively pure condition, it is impossible to determine the absolute quantity of pepsin in a liquid. It is only possible to compare the relative amounts of pepsin in two or more liquids, which may be done in several ways. As the best of these we give the following method as suggested by BRÜcKE.

If two pepsin solutions $A$ and $B$ are to be compared with each other relatively to the amounts of pepsin they contain, they must first be brought to the proper degree of acidity, about $1 \mathrm{p} . \mathrm{m}$. HCl, care being taken that one is not more diluted than the other. Then prepare a large number of specimens of each solution by diluting with hydrochioric acid of $1 \mathrm{p} . \mathrm{m}$. $\mathrm{HCl}$, so that they contain respectively $\frac{1}{2}, \frac{1}{4}, \frac{1}{8}, \frac{1}{16}, \frac{1}{32}$, and so on. the amount of pepsin in the original liquid being 1 . If the original quantity of pepsin in the two liquids is designated by $p$ and $p^{\prime}$, we then have the two series of liquids:

$\begin{array}{cc}A & B \\ 1 p & 1 p^{\prime} \\ \frac{1}{2} p & \frac{1}{2} p^{\prime} \\ \frac{1}{4} p & \frac{1}{4} p^{\prime} \\ \frac{1}{8} p & \frac{1}{8} p^{\prime} \\ \frac{1}{16} p & \frac{1}{16} p^{\prime} \\ \frac{1}{32} p & \frac{1}{32} p^{\prime}\end{array}$


Then a small piece of boiled-egg albumin, obtained by cutting thin slices with a cork-cutter, is placed in each test, or a small flake of fibrin is added. Of course care must be taken to add the same-sized slice of egg-albumin or flake of fibrin. Now observe and note exactly the time when each test of the two series begins to digest and when it ends, and it will be found that certain tests of one series make about the same progress as certain tests of the other series. It may be inferred from this that they contain about the same quantity of pepsin. As example, it is found in one series of tests that the digestive rapidity of the tests $p \frac{1}{8}, p \frac{1}{16}, p \frac{1}{8}$ is about the same as the tests $p^{\prime} \frac{1}{2}, p^{\prime} \frac{1}{4}, p^{\prime} \frac{1}{8}$; therefore we conclude that the liquid $A$ is about four times as rich in pepsin as the liquid $B$.

The rapidity of the pepsin digestion depends on several circumstances. Thus different acids are unequal in their action; hydrochloric acid shows a more powerful action than any other, whether an organic or an inorganic acid. The degree of acidity is also of the greatest importance. The most effective degree of acidity is not the same with hydrochloric acid for different albuminous bodies. For fibrin it is $0.8-1 \mathrm{p}$. m., for myosin, casein, and regetable albumin about 1 p. m., for hard-boiled-egg albumin, on the contrary, about $2.5 \mathrm{p} . \mathrm{m}$. The rapidity of the digestion increases, at least to a certain point, with the quantity of pepsin present, unless the pepsin added is contaminated by a large quantity of products of digestion, which may prevent its action. The accumulation of products of digestion disturb the digestion. At low temperatures the pepsin acts slowly in warm-blooded animals, and it is nearly without action at a temperature below $+3^{\circ} \mathrm{C}$. With increasing temperature the rapidity of digestion increases, and at about $40^{\circ} \mathrm{C}$. it is greatest. The pepsin of cold-blooded animals also acts in the neighborhood of $0^{\circ} \mathrm{C}$. If the swelling up of the proteid is prevented, as by the addition of neutral salts, such as $\mathrm{NaCl}$ in sufficient amounts, or by the addition of bile to the acid liquid, the digestion can be prevented. Foreign bodies of different kinds can produce different actions, in which naturally the variable quantities in which they are added are of the greatest importance. Salicylic acid and carbolic acid hinder the digestion, while arsenious acid promotes it (Chittender), and hydrocyanic acid is relatively indifferent. Alcohol in large quantities (10\% and above) disturbs the digestion, while small quantities act indifferently. Metallic salts in very small quantities may indeed sometimes accelerate digestion, but otherwise they tend to retard it. The action of metallic salts in different cases can be explained in different ways, but they often seem to form 
with proteids insoluble or difficultly-soluble combinations. The alkaloid compounds may also decrease the pepsin digestion (CHITTENDEN and ALLEN). A very large number of observations have been made in regard to the action of foreign substances on artificial pepsin digestion, but as these observations have not given any direct result in regard to the action of these same substances on natural digestion, we will not here further discuss them.

The Products of the Digestion of Proteids by Means of Pepsin and Acid. In the digestion of nucleo-albumin an insoluble residue of nuclein always remains. Fibrin also yields an insoluble residue, which consists, at least in great part, of nuclein, derived from the form-elements enclosed in the blood-clot. This residue which remains in the digestion of certain albuminous bodies is called dyspeptone by MEISSNER. If the solution is filtered after a complete digestion and neutralized, it gives in different cases a more or less abundant precipitate of acid albuminate, or a mixture of albuminates called parapeptone by MEIssNer. After filtering this precipitate and concentrating the filtrate again, some proteid often separates. If this precipitate be filtered, the filtrate will now contain albumoses and peptones in the ordinary sense, while the socalled true peptone of KüHNE may sometimes be entirely absent, and in general is obtained in quantity worth mentioning only after a more continuous and intensive digestion. The relationship between the albumoses and peptones in the ordinary sense changes very much in different cases and in the digestion of various albuminous bodies. For instance, a larger quantity of primary albumoses is obtained from fibrin than from hard-boiled-egg albumin or from the proteids of meat. In the digestion of unboiled fibrin an intermediate product may be obtained in the earlier stages of the digestion-a globulin which coagulates at $+55^{\circ} \mathrm{C}$. (HASEBROEK). For information in regard to the different albumoses and peptones which are formed in pepsin digestion, the reader is referred to previous pages (25-28.).

KüHNE claims that the albumoses and peptones are the final products in the pepsin digestion. HoPPe-SEYLER claims, on the contrary, that amido-acids, leucin and tyrosin, are also found. HIRSCHLER has tried to confirm this view by his investigations. The methods used by him do not seem to be quite trustworthy 
(NeUMeister), and according to KüHNE and NeUMeister the amido-acids found in the specified cases as products of digestion were derived from the contamination of the gastric juice employed.

Action of Pepsin Hydrochloric Acid on other Bodies. 'The gelatine-forming substance of the connective tissue, of the cartilage and of the bones, from which last the acid only dissolves the inorganic substances, is converted into gelatine by digesting with gastric juice. The gelatine is further changed so that it loses its property of gelatinizing and is converted into a so-called gelatine peptone (see page 38). True mucin (from the submaxillary) is dissolved by the gastric juice and yields a substance similar to peptone and a reducible substance similar to that obtained by boiling with a mineral acid.' Elastin is dissolved more slowly and yields the above-mentioned substance (page 36). Keratin and the epidermis formation are insoluble. Nuclein is not dissolved and the cellnucleus is therefore insoluble in gastric juice. The animal cellmembrane is, as a rule, more easily dissolved the nearer it stands to elastin, and it dissolves with greater difficulty the more closely it is related to keratin. The membrane of the plant-cell is not dissolved. The oxyhcomoglobin is changed into hæmatin and acid albuminate, the latter undergoing further digestion. It is for this reason that blood is changed into a dark-brown mass in the stomach. The gastric juice does not act on fat, but, on the contrary, on fatty tissue in which it dissolves the cell-membrane, setting the fat free. Human gastric juice, according to LEUBE, can convert cane-sugar into grape-sugar. Pepsin hydrochloric acid does not seem to take any part in the decompositions and clearages, which the carbohydrates may undergo in the stomach.

Pepsin alone, as above stated, has no action on proteids, and an acid of the intensity of the gastric juice can only very slowly, if at all, dissolve coagulated albumin at the temperature of the body. Pepsin and hydrochloric acid together not only act more quickly, but qualitatively they act otherwise than the acid alone. If liquid proteid is digested with hydrochloric acid of $2 \mathrm{p} . \mathrm{m}$., it is converted into acid albuminates; but if the acid is replaced by pepsin, the formation of syntonin takes place essentially slower under the same conditions (MEISsNer). From this it is inferred that a part of the hydrochloric acid is combined with the pepsin, and we have 
here a proof of the existence of a paired acid, called by C. ScHMIDT pepsin hydrochloric acid.

It has been further suggested that this hypothetical acid is possibly decomposed into pepsin and a free acid, which in statu nascendi dissolves proteids to a certain degree. The pepsin set free reunites with a new portion of acid, forming pepsin hydrochloric acid, and in contact with proteids is further decomposed as above described. It is hardly necessary to mention that this statement is only an unproved hypothesis.

Rennet or CHYMOSIN (DESCHAMPS) is the second enzyme of the gastric juice. According to BoAs, it is found in human gastric under physiological conditions, but may be absent under special pathological conditions, such as carcinoma, atrophy of the mucous membrane, and certain chronic catarrhs (BoAs, JoHnson, KLEMPERER). It is habitually found in the neutral, watery infusion of the fourth stomach of the calf and sheep, especially in an infusion of the fundus part. In other mammalia and in birds it is seldom found, and in fishes hardly ever in the neutral infusion. Instead of this rennet-forming substance a rennet zymogen, from which the rennet is formed by the action of an acid is always found.

Rennet is just as difficult to prepare in a pure state as the other enzymes. The purest rennet enzyme thus far obtained did not give the ordinary albumin reactions. On heating its solutions it is destroyed, and indeed more easily in acid than in neutral solutions. If an active and strong infusion of a mucous coat in water containing $3 \mathrm{p} . \mathrm{m} . \mathrm{HCl}$ is heated to $37-40^{\circ} \mathrm{C}$. for 48 hours, the rennet is destroyed, while the pepsin remains. A pepsin solution free from rennet can be obtained in this way. - Rennet is characterized by its physiological action, which consists in coagulating milk or a casein solution containing lime, if neutral or faintly alkaline.

Rennet may be carried down by other precipitates like other enzymes, and thus may be obtained relatively pure. It may also be obtained, contaminated with a great deal of proteids, by extracting the mucous coat of the stomach with glycerin.

A comparatively pure solution of rennet may be obtained in the following way. An infusion of the mucous coat of the stomach in hydrocholoric acid is prepared and then neutralized, after which it is repeatedly shaken with new quantities of magnesium carbonate until the pepsin is precipitated. The filtrate, which should act strongly on milk, is precipitated by basic lead acetate, the precipitate decomposed with very dilute sulphuric acid, the acid liquid filtered and treated with a solution of stearin soap. The rennet is 
thrown down by the fatty acids set free, and when these last are placed in water and removed by shaking with ether, the rennet re. mains in the watery solution.

A fasting animal cannot secrete a strongly-acid gastric juice The acid of the gastric juice then cannot be derived from the foods, but must originate in the mucous coat. As the pyloric glands, which contain no delomorphic cells, secrete an alkaline secretion, while the fundus glands, in which such cells occur, yield an acid secretion, it is generally assumed with HeIdenhain that the delomorphic cells are of special importance in the secretion of free hydrochloric acid-a statement which other observations tend to confirm. That the hydrochloric acid must originate from the chlorides of the blood is evident; and these latter must therefore undergo a decomposition with the setting free of hydrochloric acid. This decomposition has been considered as an electrolysis, but the opinion has also been held that it may be caused by some organic acid formed in the mucous membrane (BR̈̈CKE).

MALY has called attention to the fact that, on account of the presence of a large quantity of free carbon dioxide in the blood and the avidity of the same, there must be present among the numerous combinations of acids and bases which exist in the serum traces of free hydrochloric acid in addition to acid salts. As these traces of hydrochloric acid are separated from the blood by means of rapid diffusion by the glands, the action of the carbon dioxide must set free new traces of hydrochloric acid in the blood, and in this way may be explained the secretion in the blood of large quantities of hydrochloric acid. But though the occurrence of traces of free hydrochloric acid in the alkaline blood is not denied, it does not fol. low that the hydrochloric acid passes by diffusion from the blood to the gastric juice. Similar processes in other animal glands render it probable that here, as in other cases of secretion, we have to deal with a yet unexplained specific secretory action of the glandular cells. The process going on in the secretion of hydrochloric acid is not yet understood.

After an abundant meal, when the store of pepsin in the stomach is completely exhausted, ScHIFF claims that certain bodies, especially dextrin, have the property of causing a supply of pepsin in the mucous membrane. This "charge theory," though experi- 
mentally proved by several investigators, has nevertheless not yet been confirmed. On the contrary, the statement of ScHiff that a substance forming pepsin, a "pepsinogen" or " propepsin," occurs in the ventricle has been proved. LANGLEY has shown positively the existence of such a substance in the mucous coat. This substance, propepsin, shows a comparatively strong resistance to dilute alkalies (a soda solution of $5 \mathrm{p} . \mathrm{m}$.) which easily destroys pepsin (LANGLEY). Pepsin, on the other hand, withstands better than propepsin the action of carbon dioxide, which quickly destroys the latter (LANGLEY). The occurrence of a rennet zymogen in the mucous coat has been mentioned above.

The question in which cells the two zymogens, especially the propepsin, are produced has been extensively discussed for several years. Formerly it was the general opinion that the delomorphic cells were pepsin-cells, but at the present time the theory universally prevails, based chiefly on the experiments of HeIDENHAIN and his pupils, supported by LANGLEY and others, that the formation of pepsin goes on in the adelomorphic or principal cells.

The Pyloric Secretion. 'That part of the pyloric end of the dog's stomach which contains no fundus glands was dissected by Klemensiewicz, one end being sewed together in the shape of a blind sack and the other sewed into the stomach. From the fistula thus created he was able to obtain the pyloric secretion of a living animal. This secretion is alkaline, viscous, jelly-like, rich in mucin, of a specific gravity of 1.009-1.010, and containing 16.5-20.5 p. m. solids. It has no effect on fat, but acts, though very slowly, on starch, converting it into sugar, and habitually contains pepsin in rather large amounts. HEIDENHaIN has observed the same in permanent pyloric fistula.

The secretion of the juices of the stomach is dependent to a great extent upon the excitement acting on the mucous coat of the stomach, and it follows from this that the quantity of secretion under different conditions must vary considerably. The statements of the quintity of gastric juice secreted in a certain time are therefore so unreliable that they need not be taken into account.

The Chyme and the Digestion in the Stomach. By the movements of the walls of the stomach the contents are kneaded and the food-particles pressed against each other and divided. By means of 
this mechanical irritation of the mucous coat of the stomach, as well as by the chemical irritation caused by the food and saliva, an increased secretion of gastric juice occurs. The food is thereby freely mixed with liquid and is gradually converted into a pulpy mass, the chyme. This mass is acid in reaction, and with the exception of the interior of large pieces of meat or other solid foods, the chyme is acid throughout. The metabolism products in the digestion of proteids and carbohydrates may always be detected in the chyme; likewise more or less changed undigested residues of swallowed food, which indeed form the chief mass of the constituents of the chyme.

In the chyme morsels of FLESH more or less changed are found which, when unboiled flesh is partaken of, may be much swollen and slippery. Muscle and Cartilage are also often swollen and slippery, while pieces of BONE sometimes show a rough and uneven surface after the digestion has continued for some time, which depends upon the fact that the gelatinous substances of the bone are attacked more quickly by the gastric juice than the earthy parts. MrLK coagulates in the stomach by the combined action of the rennet enzyme and the acid, but in certain cases by the action of the acid alone. From the relative quantities of the swallowed milk to the other food either large and solid lumps of cheese are formed or smaller lumps or grains which are divided in the pulpy mass. Cow's milk regularly yields large, solid masses or lumps; human milk gives, on the contrary, a fine, loose coagulum or a fine precipitate which is immediately dissolved in part by the acid liquid. The milk-sugar may pass into lactic-acid fermentation, and this according to RICHET, is the reason why the acid reaction of the contents of the stomach is greater at the end of the digestion of a meal consisting mainly of milk.

BREAD, especially when not too fresh, is converted rather easily into a pulpy mass in the stomach. Other vegetable foods, such as POTATOES, may, if not sufficiently masticated, often be found in the contents of the stomach, very little changed, several hours after a meal.

StarCH is not converted into sugar by the gastric juice, but in the first phases of the digestion, before a large quantity of hydrochloric acid has accumulated, it seems that the action of the saliva 
continues, and therefore the presence of dextrin and sugar can be detected in the contents of the stomach. Besides this the car. - bohydrates in the stomach may in part undergo a lactic-acid fer. mentation, probably caused by the micro-organisms present.

According to the investigations of ELLENBERGER and HoFFMEISTER on horses and pigs, after a meal rich in amylaceous bodies in the first phase of the digestion, an AMYLOLYSE takes place with the formation of lactic acid; then gastric juice containing hydrochloric acid is secreted, then follows a second phase in which the proteolyse takes place. As a rule, the formation of lactic acid decreases as the secretion of hydrochloric acid increases. EWALd and BoAs claim that a similar condition also exists in human beings. They claim that there is in the first stage of digestion a predominance of lactic acid in the stomach, in the second a simultaneous occurrence of lactic and hydrochloric acids, and in the third stage almost exclusively hydrochloric acids. KJAERGAARD has lately formed the same conclusions from his investigations on children and robust persons. In persons with altered blood-vessels due to senility the contents of the stomach show chiefly the presence of lactic acid. Such persons digest large amounts of carbohydrates, while the digestion of albuminous bodies is decreased.

The FATS which are not fluid at the ordinary temperature melt in the stomach at the temperature of the body and become fluid. In the same way the fat of the fatty structure is set free in the stomach by the gastric juice which digests the cell-membrane. The gastric juice itself seems to have no action on fats, but, according to recent statements, a splitting of the neutral fats into fatty acids and glycerin takes place, though not to a great extent. This splitting is not dependent on the bacteria of the contents of the stomach, or at least only to a small extent (KLeMperer and Scheurlen). The soluble salts of the food naturally are found dissolved in the liquids of the contents of the stomach; but the insoluble salts may also be dissolved by the acid of the gastric juice.

Since the hydrochloric acid of the gastric juice prevents the contents of the stomach from fermenting with the generation of gas, those gases which occur in the stomach probably depend, at least in great measure, upon the swallowed air and saliva, and upon those gases generated in the intestines and returned through 
the pyloric valve. Planer found in the stomach-gases of a dog $66-68 \% \mathrm{~N}, 25-33 \% \mathrm{CO}_{2}$, and only a small quantity, $0.8-6.1 \%$, of oxygen.

According as the food is finely or coarsely divided it passes sooner or later through the pylorus into the intestines. From Buscr's observations on a human intestinal fistula, it required generally 15-30 minutes after eating for undigested food, such as pieces of meat, to pass into the upper part of the intestines. Among the cases of duodenal fistula in human beings observed by $K \ddot{U} H N E$, one is mentioned in which he saw, ten minutes after eating, uncurdled but still coagulable milk and small pieces of meat pass out of the fistula. The time in which the stomach unburdens itself of its contents depends, however, upon the rapidity with which the quantity of hydrochloric acid increases, for it seems to act as a sort of irritant and causes the opening of the pylorus (EwaLd and others). Many other conditions also come into play, namely, the activity of the gastric juice, the quantity and character of the food, etc., etc., and therefore the time required to empty the stomach must be variable. RICHET observed in a case of stomach fistula that in man the quantity of food which is in the stomach the first three hours is not essentially changed, but that in the course of a quarter of an hour nearly all is driven out, so that only a small residue remains. KüHNE has made about the same observations on dogs and human beings. He found, indeed, in dogs that in the first hour small quantities of meat passed into the intestines every ten minutes; but he also observed that in dogs, on an average, about five hours after eating, in nıan somewhat earlier, a free emptying into the intestines takes place. According to other investigators (EWALD and BOAS), the emptying of the human stomach does not take place suddenly, but gradually. BEAUMONT found in his extensive observations on the Canadian hunter, ST. MarTin, that the stomach, as a rule, is emptied $1 \frac{1}{2}-5 \frac{1}{2}$ hours after a meal, depending upon the character of the food.

The time in which different foods leave the stomach depends upon their digestibility. In regard to the unequal digestibility in the stomach of foods rich in proteids, which really form the object of the action of the gastric juice, a distinction must be made between the rapidity with which the proteids are converted into 
albumoses and peptones and the rapidity with which the food is converted into chyme, or at least so prepared that it may easily pass into the intestines. This distinction is especially important from a practical standpoint. When a proper food is to be decided upon in cases of diminished stomachic digestion, it is important to select such foods as, independent of the difficulty or ease with which their proteid is peptonized, leave the stomach easily and quickly, and which require as little action as possible on the part of this organ. From this point of view those foods, as a rule, are most digestible which are fluid from the start or may be easily liquefied in the stomach; but these foods are not always the most digestible in the sense that their proteid is most easily peptonized. As an example, hard-boiled white of egg is more easily peptonized than fluid white of egg at a degree of acidity of 1-2 p. m. $\mathrm{HCl}$; nevertheless we consider, and justly, that an unboiled or soft-boiled egg is easier to digest than a hard-boiled one. Likewise uncooked meat, when it is not chopped very fine, is not more quickly but more slowly peptonized by the gastric juice than the cooked, but if it is divided sufficiently fine it is often more quickly peptonized than the cooked.

The greater or less facility with which the different albuminous foods are peptonized by the gastric juice has been comparatively little studied, and as the conditions in the stomach are more complicated, results obtained with artificial gastric juice are often of no value for the practising physician and should in any case be used only with the greatest caution. Under these circumstances we cannot enter more deeply into this subject, but the reader is referred to text-books on dietetics and the theory of foods.

As our knowledge of the digestibility of the different foods in the stomach is slight and dubious so also our knowledge of the action of other bodies, such as alcoholic drinks, bitter principles, spices, etc., on the natural digestion is very uncertain and imperfect. The difficulties which stand in the way of this kind of investigation are very great, and therefore the results obtained thus far are often ambiguous or conflict with each other. For example, one investigator has seen that small quantities of alcohol or alcoholic drinks do not prevent but rather facilitate digestion; others observe only a preventive action ; while other investigators believe 
to have found that the alcohol first acts somewhat as a preventive, but afterwards, as a rule, it is absorbed, producing an abundant secretion of gastric juice, and thereby facilitating digestion (CL. Bernard, Gluzinski, Chittenden).

The digestion of sundry foods is not dependent on one organ alone, but divided among several. For this reason it is to be expected that the various digestive organs can act for one another to a certain point, and that therefore the work of the stomach could be taken up more or less by the intestines. This in fact is the case. Thus the stomach of a dog has been almost completely extirpated (CzERNY), and even that part necessary in the digestive process has been eliminated by plugging the pyloric opening (LUDWIG and OGATA), and in both cases it was possible to keep the animal alive, well fed, and strong. In these cases it is evident that the digestive work of the stomach was taken up by the intestines. That the stomach nevertheless, during normal conditions, bears an essential part of the process of digestion may be inferred from the fact that the products of the proteolyse can generally be detected in the contents of the human stomach even shortly after a meal. By tests on dogs that had been given meat-powder, CAHN found large quantities of peptone in the stomach, and this absorption, as shown by SCHMIdT-MÜLHEIM, requires about the same steps as digestion.

It is meanwhile quite generally assumed that no peptonization of the proteids worth mentioning occurs in the stomach, and that the albuminous foods are only prepared in the stomach for the real digestive processes in the intestines. That the stomach serves in the first place as a storeroom follows from its shape, and this function is of special value in certain new-born animals, for instance in dogs and cats. In these animals the secretion of the stomach contains only hydrochloric acid but no pepsin, and the casein of the milk is converted by the acid alone into solid lumps or a solid coagulum which fills the stomach. Small portions of this coagulum pass into the intestines only little by little and an overburdening of the intestines is thus prevented. In other animals, such as the snake and certain fishes, which swallow their food entire, it is certain that the major part of the process of digestion takes place in the stomach. The importance of the stomach in digestion cannot at once be decided. It varies for different animals, and it may vary in the same animal, depending upon the division of the food, the rapidity 
with which the peptonization takes place, the more or less rapid increase in the amount of hydrochloric acid, and so on.

It is a well-known fact that the contents of the stomach may be kept without decomposing for some time by means of hydrochloric acid, while, on the contrary, when the acid is neutralized a fermentation commences by which lactic acid and other organic acids are formed. The hydrochloric acld of the gastric juice has unquestionably an anti-fermentive action, and also, like dilute mineral acids, an antiseptic action. This action is of importance, as many disease micro-organisms may be destroyed by the gastric juice. The bacillus of cholera is killed by the normal acid gastric juice, while if it is introduced into the stomach after an injection of a soda solution it may remain active (КосH, NicATi and RIETSCH). Also varieties of streptococcus infecting wounds and the staphylococcus pyog. aureus are killed by the acid gastric juice (ALAPY). Still the gastric juice does not act on all micro-organisms, and especially in the state of spores they can withstand its action. As an example, the tubercle-virus is not destroyed by the gastric juice (FALK), and the spores of the anthrax bacteria do not seem to be always destroyed by the hydrochloric acid of the gastric juice.

After death, if the stomach still contains food, digestion goes on of itself not only in the stomach but also in the neighboring organs during the slow cooling of the body. This leads to the question, why does the stomach not digest itself during life? Ever since PAvy has shown that after tying the smaller bloodvessels of the stomach of dogs the corresponding part of the mucous membrane was digested, efforts have been made to find the cause in the neutralization of the acid of the gastric juice by the alkali of the blood. That the reason for the non-digestion during life is to be sought for in the normal circulation of the blood cannot be contradicted; it is more probably found in the fact that the living mucous coat nourished by the alkaline blood shows quite different absorption, diffusion, and filtration properties than the dead mucous coat. This last was shown long ago by RANKE and HALENKE.

Under pathological conditions irregularities in the secretion as well as in the absorption and in the mechanical work of the stomach may occur. Pepsin is almost always present, but the 
absence of the rennet enzyme, as above stated, may occur in many cases (Bons, Johnson, Klemperen). In regard to the acid, it should be mentioned that sometimes this secretion may be increased so that an abnormally acid gastric juice is secreted, and sometimes may be decreased so that little or hardly any hydrochloric acid is secreted. A hypersecretion of acid gastric juice sometimes occurs. In the secretion of too little hydrochloric acid the same conditions appear as after the neutralization of the acid contents of the stomach outside of the organism. Fermentation processes now appear in which, besides lactic acid, there appear also volatile fatty acids, such as butyric and acetic acids, etc., and gases like hydrogen. These fermentation products are therefore often found in the stomach in cases of chronic catarrh of the stomach, which may give rise to belching, heart-burn, and other symptoms.

Among the foreign substances found in the contents of the stomach we have UREA, or ammonium carbonate derived therefrom in uræmia; BLOOD, which generally forms a dark-brown mass through the presence of hæmatin, due to the action of the gastric juice ; BILE, which, especially during vomiting, easily finds its way through the pylorus into the stomach, but whose presence seems to be without importance.

If it is desired to test the gastric juice or the contents of the stomach for pepsin, fibrin may be employed. If this is thoroughly washed immediately after beating the blood, well pressed and placed in glycerin, it may be kept in serviceable condition an indefinitely long time. The gastric juice or the matter contained in the stomach-the latter, if necessary, having been previously diluted with 1 p. m. hydrochloric acid, is filtered and tested with fibrin at ordinary temperature. (It must not be forgotten that a control test must be made with acid alone and another porton of the same fibrin.) If the fibrin is not noticeably digested within one or two hours, no pepsin is present, or at most there are only slight traces.

In testing for rennet enzyme the liquid must be first carefully neutralized. To $10 \mathrm{cc}$. unboiled amphoteric (not acid) reacting cow's milk add $1-2$ cc. of the filtered neutralized liquid; but care must be taken not to add too much of the liquid from the stomach, for the coagulation may be retarded or prevented by diluting the milk. In the presence of rennet the milk should coagulate to a solid mass at the temperature of the body in the course of 10-20 minutes without changing its reaction. If the milk is diluted too much by the addition of the liquid of the stomach, only coarse

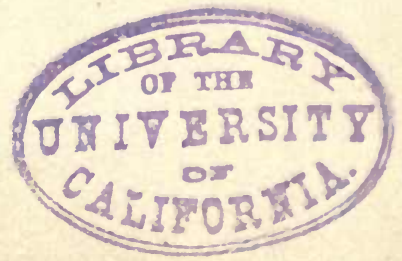


flakes are obtained and no solid coagulum. Addition of lime salts is to be avoided, as they in great excess may produce a partial coagulation even in the absence of rennet.

In many cases it is especially important to determine the degree of acidity of the gastric juice. This may be done by the ordinary titration methods. Phenol phthalein must not be used as an indicator, for we get too high results in the presence of large quantities of albumin. Good results may be obtained, on the contrary, by using very delicate litmus-paper. As the acid reaction of the contents of the stomach may be caused simultaneously by several acids, still the degree of acidity is here, as in other cases expressed in only one acid, e. g., HCl. Generally the acidity is expressed by the number of cc. of $\frac{N}{10}$ caustic soda which is required to neutralize the several acids in $100 \mathrm{cc}$. of the liquid of the stomach. An acidity of $43 \%$ means that $100 \mathrm{cc}$. of the liquid of the stomach required $43 \mathrm{cc}$. of $\frac{\mathrm{N}}{10}$ caustic soda to neutralize it.

It is also important to be able to ascertain the nature of the acid or acids occurring in the contents of the stomach. For this purpose, and especially for the detection of free hydrochloric acid, a great number of color reactions have been proposed, which are all based upon the fact that the coloring substance gives a characteristic color with very small quantities of hydrochloric acid, while lactic acid and the other organic acids do not give these colorations, or only in a certain concentration, which can hardly' exist in the contents of the stomach. These reagents are a mixture of FERRIC ACETATE and POTASSIUM SULPHOCYANIDE solution (MOHR's reagent is modified by several investigators), METHYL, ANILINVIOLET, TROP EOLIN 00, CONGO RED, MALACHITE-GREEN, PHLOROGLUCIN-VANILLIN, BENZOPURPURIN $6 \mathrm{~B}$, and others. As reagents for free lactic acid UFFELMANN suggests a strongly diluted, amethyst-blue solution of FERRIC CHLORIDE and CARBOLIC ACID or a strongly diluted, nearly colorless solution of FERRIC CHLORIDE. These give a yellow with lactic acid, but not with hydrochloric acid or with volatile fatty acids.

The value of these reagents in testing for free hydrochloric acid or lactic acid is still disputed. Among the reagents for free hydrochloric acid, MoHR's test (even though not very delicate), GUNzBURG's test with phloroglucin-vanillin, and the test with tropæolin 00, performed in moderate heat as suggested by BoAs, seem to be the most valuable. If these tests give positive results, then the presence of hydrochloric acid may be considered as proved. A negative result does not eliminate the presence of hydrochloric acid, as the delicacy of these reactions has a limit, and also the simul- 
taneous presence of albumin, peptones and other bodies influence the reactions more or less. 'The reactions for lactic acid may also give negative results in the presence of comparatively large quantities of hydrochloric acid in the liquid to be tested. Sugar and other bodies (FR. MÜLLER) may act with these reagents similarly to lactic acid. As the above-mentioned reactions for hydrochloric acid and organic acids are not claimed to be sufficient in exact investigations, while they may serve in many cases for clinical purposes, it will suffice to refer the reader to other text-books and especially to "Klinische Diagnostik innerer Krankheiten," by R. v. JAKSCH, $2 \mathrm{~d}$ edition, 1889 , for the performance and the relative value of these tests.

The method suggested by CAHN and v. MeHRING for the detection and simultaneous quantitative estimation of hydrochloric acid in the presence of lactic acid and volatile fatty acids in the contents of the stomach will be here given. The chief points are the following: First the volatile acids are distilled and their quantity determined in the distillate by titration. The lactic acid is removed from the liquid remaining in the retort by repeated shaking with large quantities of ether, and after the evaporation of the ether the quantity of lactic acid in the residue is determined by titration. The liquid which has been shaken out with the ether is either directly titrated for hydrochloric acid, or, as suggested by RABUTEAU, combines the hydrochloric acid with cinchonin by digestion therewith at a gentle heat until the reaction is neutral. The cinchonin combination is then agitated with chloroform and the chloroform evaporated, and from the amount of chlorine in this residue we may calculate the quantity of previously-free hydrochloric acid. This somewhat expensive and lengthy method is still not free from errors, and it is made useless by that following.

The method of K. MörNer and SJöQvisT depends on the following principle: When the gastric juice is evaporated to dryness with barium carbonate and then calcined the organic acids burn up and give insoluble barium carbonate, while the hydrochloric acid forms soluble barium chloride. From the quantity of this the original amount of hydrochloric acid can be calculated. $10 \mathrm{cc}$. of the filtered contents of the stomach is mixed in a small platinum or silver dish, by means of a clean, sharp knife-point, with the harium carbonate free from chlorides, and evaporated to dryness. The residue is burnt and allowed to glow for a few minutes. 'The cooled carbon is gently rubbed with water and completely extracted with boiling water, and the filtrate (about $50 \mathrm{cc}$.) treated with an equal volume of alcohol and $3-4 \mathrm{cc}$. sodium acetate solution (10\% acetic acid and $10 \%$ acetate). The amount of barium in the filtrate is determined by titration with a solution of potassium bichromate, 
in which the alcohol facilitates the precipitation of the barium chromate, while the acetate prevents in part the precipitation of the calcium carbonate and in part the setting free of hydrochloric acid. The potassium-bichromate solution should contain about $8.5 \mathrm{grms}$. potassium dichromate to the litre. Its titre must exactly correspond with an $\frac{\mathrm{N}}{10}$ barium-chloride solution, and the procedure is the same as in the titration of the $\mathrm{BaCl}_{2}$ solution obtained from the contents of the stomach. A paper moistened with tetramethylparaphenylendiamin is used as indicator; this is colored blue by a bichromate in acetic-acid solution. In titrating we add chromate solution as long as the barium chromate precipitated does not apparently increase, then test with the indicator paper after each addition until it gives a decided blue coloration within one minute, and stop adding chromate solution. As the titre of the chromate solution has been determined by an $\frac{\mathrm{N}}{10} \mathrm{BaCl}_{2}$ solution, it is easy to calculate the quantity of $\mathrm{HCl}$ in $10 \mathrm{cc}$. of the gastric juice corresponding to the number of cc. of the chromate solution used. If the total acidity is determined in a second portion of the gastric juice, then the quantity of lactic acid or other organic acids represented as $\mathrm{HCl}$ may be calculated. This method, which is the most exact known at present, gives not only the amount of free hydrochloric acid, but also the hydrochloric acid combined with the albumin and peptone.

In testing for volatile fatty acids the contents of the stomach should not be directly distilled, as volatile fatty acids may be formed by the decomposition of other bodies, such as albumin and hæmoglobin. The neutralized contents of the stomach are therefore precipitated with alcohol at ordinary temperature, filtered quickly, pressed, and repeatedly extracted by alcohol. The alcoholic extracts are made faintly alkaline by soda, and the alcohol distilled. The residue is now acidified by sulphuric or phosphoric acid and distilled. The distillate is neutralized by soda and evaporated to dryness on the water-bath. The residue is extracted with absolute alcohol, filtered, the alcohol distilled off, and this residue dissolved in a little water. This solution may be directly tested for acetic acid with sulphuric acid and alcohol or with ferric chloride. Formic acid may be tested for by silver nitrate, which quickly gives a black precipitate; and butyric acid is detected by the odor after the addition of an acid. In regard to the methods for more fully investigating the different volatile fatty acids, the reader is referred to other text-books. 


\section{The Glands of the Mucous Membrane of the Intes- tines and their Secretions.}

The Secretion of Brunner's Glands. These glands are partly considered as small pancreas glands and partly as mucous or salivary glands, but according to GRÜTZNER they are related to the pyloric tglands. They are assumed to contain pepsin (GröTzner and diastatic enzymes (COSTA, BUDGE and KroLOW). The diffculty of collecting the secretions of these glands free from contamination, however, makes these assumptions somewhat unreliable.

The Secretion of Lieberkuhn's Glands. The secretion of these glands has been studied by the aid of a fistula in the intestines according to the method of Thiry and VelLA. Very little if any secretion takes peace in fasting animals (dog) when the mucous membrane is not irritated. The secretion begins in the first hour after partaking of food, but the maximum varies with the quantity and character of the food (Heidenhain). Mechanical, chemical, or electrical irritation excites a secretion or increases that already begun (Thiry). Laxatives do not increase the secretion, while pilocarpin produces a very abundant one (MASLOFF and VeLLA). The quantity of this secretion in the course of 24 hours has not been exactly determined.

In the upper part of the small intestines of the dog this secretion is scanty, slimy, and gelatinous; in the lower part it is more fluid, with gelatinous lumps or flakes (RömanN). Intestinal juice has a strong alkaline reaction, generates carbon dioxide on the addition of an acid, and contains (in dogs) nearly a constant quantity of $\mathrm{NaCl}$ and $\mathrm{Na}_{2} \mathrm{CO}_{3}, 4.8-5$ and $4.5 \mathrm{p}$. m. respectively (Gumilewski, Röhmans). It contains albumina (ThIry found $8.01 \mathrm{p} . \mathrm{m}$.), the quantity decreasing with the duration of the elimination. The quantity of solids varies. In dogs the quantity of solids is $12.2-24.1$ p. m., and in sheep $46-47$ p. m. The specific gravity of the intestinal juice of the dog, according to the observations of ThIRY, is 1.010-1.010\%.

The action of intestinal juice has not been exhaustively studied and the opinions concerning it are somewhat at variance. According to most investigators, starch is converted into sugar, a statement 
which has lately been confirmed by BASTIANELLI. Intestinal juice or an infusion of the mucous coat inverts cane-sugar (PASCHUTIN, BASTIANELLI), and this inversion is, according to BRowN and Heron, produced even more quickly by the mucous membrane itself. Maltose seems to be quickly changed into glucose, and this action depends especially on PEYER's group of glands. The action on carbohydrates takes place more quickly and in greater quantity in the upper part of the intestine, and correspondingly the absorption of starch and sugar occurs more quickly in the upper part than in the lower section of the intestine (LANNoIs and LÉPINE, RöHMaNe).

Intestinal juice does not split neutral fats, but it has the property, like other alkaline fluids, of emulsifying the fats. In regard to its action on albuminous bodies most investigators agree that the intestinal juice has no action on boiled albumin or flesh, while it dissolves fibrin according to THIRY. Albumoses are not converted into peptones (WENz, BASTIANELLI). Contrary to other investigators, ScHIFF claims that the juice from a successful fistula. operation digests not only coagulated albumin and lumps of casein, but also unboiled and boiled flesh. The lack of action on albumins, which was observed by other investigators, SCHIFF attributes to the abnormal juice with which they experimented. ScHIFF also obtained from an operation not entirely successful a juice whose action on albumin and meat was no greater than that studied by THIRY and other investigators.

Human intestinal juice in a case of anis prceternaturalis has been investigated by Demant. This juice showed itself entirely inactive on albuminous bodies, even on fibrin and on fats. It only showed a very faint action on boiled starch. Those tests on the action of the intestinal juice which are made, in isolated stoppage of the intestine in animals or human intestine in cases of anus proternaturalis, on the food which has been introduced into the intestine, generally give no positive results, because of the putrefaction processes going on in the intestine.

The secretion of the glands in the large intestine seems to consist chiefly of mucus. Fistulas have also been introduced into these parts of the intestine, which are chiefly if not entirely to be considered as absorption organs. The investigations on the action of 
this secretion on nutritive bodies have not as yet yielded any positive results.

\section{Pancreas Gland and Pancreatic Juice.}

In invertebrata, which have no pepsin digestion and which also have no formation of bile, the pancreas, or at least an analogous organ, seems to be the essential digestion gland. On the contrary, an anatomically characteristic pancreas is absent in certain vertebrata and in certain fishes. Those functions which should be performed by this organ seem to be performed in these animals by the liver, which may be rightly called HEPATOPANCREAS. In man and in most vertebrata the formation of bile and of certain secretions containing enzymes important for digestion is divided between the two organs, the liver and the pancreas.

The pancreas gland is similar in certain respects to the parotid gland. The elements secreted by the former consist of nucleated cells whose basis forms a mass rich in albumin, expand in water and in which two distinct zones exist. The outer zone is more homogeneous, the inner cloudy due to a quantity of granular. substance. The nucleus lies about midway between the two zones, but this position may change with the varying relative size of the two zones. According to HEIDENHAIN, the inner part of the cells diminishes in size during the first stages of digestion, in which the secretion is active, while at the same time the outer zone enlarges owing to the absorption of new material. In a later stage, when the secretion has decreased and the absorption of the nutritive bodies has taken place, the inner zone enlarges at the expense of the outer, the substance of the latter having been converted into that of the former. Under physiological conditions the glands are undergoing a constant change, at one time consuming from the inner part and at another time growing from the outer part. The inner granular zone is converted into the secretion, and the outer, more homogeneous zone, which contains the repairing material, is then converted into the granular substance.

Besides considerable quantities of proteids, globulin, nucleoalbumin, and albumin, we find in this gland three enzymes, or, 
more correctly, three zymogens, which will be described later. We also find in this gland nuclein, leucin (butalanin), tyrosin (not in the perfectly fresh gland), xanthin, 1-8 p. m., hypoxanthin, 3-4 p. m., guanin, 2-7.5 p. m. (all figures are calculated for the dried substance, KOSSEL), adenin, inosit, lactic acid, volatile fatty acids, fats, and mineral substances. According to the investigations of OIDTMAN, the human pancreas contains $750-760 \mathrm{p}$. m. water, 240250 p. m. organic and $3.7-9.5$ p. m. inorganic substances.

Pancreatic Juice. This secretion may be obtained by adjusting a fistula in the excretory duct, according to the methods suggested by Bernard, Ludwig, and Heidentain. If the operation is performed with sufficient rapidity and dexterity on an animal which has been well fed a few hours before, there is obtained from the fistula, as a rule, immediately after the operation (temporary fistula) a secretion rich in solids, viscid, very active, and which may be considered as normal pancreatic juice. Ordinarily, however, the gland becomes diseased in a few hours or days after the operation, and the secretion which then flows out of the fistula (permanent fistula) is more liquid, deficient in solids, and in certain other respects different from the secretion obtained immediately after the operation. Still a permanent fistula may also sometimes yield a normal secretion for a long time (HeIDENHaIN), while the temporary fistula in careless operations may give no secretion or only an abnormal juice.

In herbivora, such as rabbits, whose digestion is uninterrupted, the secretion of the pancreatic juice is continuous. In carnivora it seems, on the contrary, to be intermittent and dependent on the digestion. During starvation the secretion almost stops, but commences again after partaking of food. Food seems to act in a twofold manner. First, it may, with the more abundant flow of blood during the digestion, which is seen by the red color of the gland, convey a larger quantity of nutritive material to the gland, and thereby cause the secretion of a juice rich in solid nutritive bodies. In another way the food may also, by the irritation which it produces on the mucous coat of the stomach and the duodenum, cause an increased secretion. That the food indeed acts in these two ways follows from the fact that other substances, such as ether, may also cause a secretion of pancreatic juice, but by this means 
in starvation a thin fluid is secreted, and after partaking of food a thick fluid is produced. According to the observations of Bernstein, Heidenhain, and others, the secretion increases rapidly after eating, and it reaches its maximum in the course of the first three hours. From this time the secretion diminishes, but may again increase from the 5th-7th hour, when generally large quantities of food pass from the stomach to the intestine. 'Then it again decreases continuously from the 9 th-11th hour, and stops entirely at the 15 th-16th hour.

The statements as to the amount of pancreatic juice secreted in the course of 24 hours are variable and not trustworthy. It seems positively proved that the permanent fistula yields a considerably larger quantity of secretion than the temporary. While Keferstein and Hallwachs, and SchmidT and Kröger, find that the quantity of juice secreted from the first is $45-100$ grms. per kilo during 24 hours, Bidder and Schmidt and Bidder and SKREBITZKY claim that the quantity from the temporary fistula is 2.5-5 grms. per kilo in the same time.

In regard to the constituents and composition of the pancreatic juice, a distinction must be made between the secretion of a temporary and of a permanent fistula. The secretion flowing from the former is in dogs a clear, colorless, nearly-sirupy, odorless fluid of an alkaline reaction which is very rich in albumin and sometimes containing so large a quantity that it coagulates like white of egg when heated. Besides albumin the juice contains also three.enzymes-one diastatic, one fat-splitting, and one which dissolves proteids. The last-mentioned has been called trypsin by KüHNE. Besides the above-mentioned bodies the pancreatic juice habitually contains small quantities of leucin, fat, and soaps. As mineral constituents it contains chiefly alkali chlorides, also alkali carbonates, and some phosphoric acid, lime, magnesia, and iron.

The secretion from the permanent fistula always contains less solids, especially albumin and enzymes, than that from a temporary fistula. A long time after the operation it is more fluid, more strongly alkaline, and the property which the juice from the temporary fistula has of dissolving albumin is often absent, or the secretion shows it in only a slight degree. As an example of the different composition of the juice from a temporary and from a 
permanent fistula, we give below the analyses of C. Schmidt. The figures represent parts per 1000 .

\begin{tabular}{|c|c|c|c|c|c|}
\hline \multirow{3}{*}{ Vater } & \multirow{2}{*}{\multicolumn{2}{|c|}{$\begin{array}{l}\text { Juice from a Temporary } \\
\text { Fistula. }\end{array}$}} & \multicolumn{3}{|c|}{ Juice from a Permanent } \\
\hline & & & a. & b. & \\
\hline & 900.8 & 884.4 & 976.8 & 979.9 & 984.6 \\
\hline & 99.2 & 115.6 & 23.2 & 20.1 & 15.4 \\
\hline nic substance & 90.4 & $\ldots \ldots$ & 16.4 & 12.4 & 9.2 \\
\hline (1) & 8.8 & $\ldots \ldots$ & 6.8 & 7.5 & 6.1 \\
\hline
\end{tabular}

The mineral constituents of the secretion from the temporary fistula consisted chiefly of $\mathrm{NaCl}, 7.4 \mathrm{p}$. m.

In the pancreatic juice of rabbits 11-26 p. m. solids have been found, and in that from sheep 14.3-36.9 p. m. In the pancreatic juice of the horse 9-17.5 p. m. solids have been found; in that of the pigeon, 12-14 p. m.

The human pancreatic juice has been analyzed by HERTER in a case of stoppage of the exit of the juice by the pressure of a cancer. This juice, which could hardly be considered as normal, was clear, alkaline, without odor, and contained the three enzymes. It contained peptone, but no other proteid. The quantity of solids was $24.1 \mathrm{p} . \mathrm{m}$. Of these $6.4 \mathrm{p} . \mathrm{m}$. were soluble in alcohol. It contained $11.5 \mathrm{p}$. m. peptones (and enzymes) and $6.2 \mathrm{p} . \mathrm{m}$. mineral substances.

Among the constituents of the pancreatic juice, the three enzymes are the most important.

The pancreatic diastase, which according to Konowin and ZWEIFEL is not found in new-born infants and does not appear until more than one month after birth, seems, although not identical with ptyalin, to be nearly related to it. The pancreatic diastase acts very energetically upon boiled starch, especially at $+37^{\circ}$ to $40^{\circ} \mathrm{C}$., and besides dextrin yields maltose with only a small quantity of glucose (Musculus and v. Mering).

If the natural pancreatic juice is not to be obtained, then the gland, best after it has been exposed a certain time ( 24 hours) in the air, may be treated with water or glycerin. 'This infusion or the glycerin extract diluted with water (when a glycerin has been used which has no reducing action) may be tested directly with starch-paste. It is safer, however, to first precipitate the enzyme from the glycerin extract by alcohol, and wash with this liquid, dry the precipitate over sulphuric acid, and extract with water. The enzyme is dissolved by the water. The detection of sugar may be made in the same manner as in the saliva.

The fat-splitting enzyme. The action of the pancreatic juice on fats is twofold. First, the neutral fats are split intö fatty acids and glycerin, which is an enzymotic process; and secondly, it has also the property of emulsifying the fats. 
The action of the pancreatic juice in splitting the fats may be shown in the following way. Shake olive-oil with caustic soda and ether, siphon off the ether and filter if necessary, then shake the ether repeatedly with water and evaporate at a gentle heat. In this way we obtain a residue of fat free from fatty acids which is completely neutral, and which dissolves in acid-free alcohol and is not colored red by alkanet tincture. If such fat is mixed with quite fresh alkaline pancreatic juice or with a freshly-prepared infusion of the fresh gland and treated with a little alkali or with a fuintly alkaline glycerin extract of the fresh gland (9 parts glycerin and 1 part soda solution of $1 \%$ for each gramme of the gland) and some litmus tincture added and the mixture warmed to $+37^{\circ} \mathrm{C}$., the alkaline reaction will gradually disappear and an acid one takes its place. This acid reaction depends upon the conversion of the neutral fats by the enzyme into glycerin and free fatty acids.

The splitting of the neutral fats may also be shown more exactly by the following method. The mixture of neutral fats (absolutely free from fatty acids). aud pancreatic juice or pancreas infusion is digested at the temperature of the body and treated with some soda and repeatedly shaken with fresh quantities of ether until all the unsplit neutral fats are removed. Then it is made acid with sulphuric acid, after which shake the acid liquid with ether, evaporate the ether, and test the residue for fatty acids.

Another simple process for the demonstration of the fat-splitting action of the pancreas glands is the following (CL. BERNARD). A small portion of the perfectly-fresh, finely-divided gland substance is first soaked in alcohol (of 90\%). Then the alcohol is removed as far as possible by pressing between blotting-paper, after which the pieces of gland are covered with an ethereal solution of neutral butter-fat (which may be obtained by shaking milk with caustic soda and ether). After the evaporation of the ether the pieces of gland covered with butter-fat are pressed between two watch-glasses and then gently heated to $37^{\circ}$ to $40^{\circ} \mathrm{C}$. in this position. After a certain time a marked odor of butyric acid appears.

The action of the pancreatic juice in splitting fats is a process analogous to that of saponification, the neutral fats being decomposed, by the addition of the elements of water, into fatty acids and glycerin according to the following formula: $\mathrm{C}_{3} \mathrm{H}_{5} \cdot \mathrm{O}_{3} \cdot \mathrm{R}_{3}$ (neutral fat) $+3 \mathrm{H}_{2} \mathrm{O}=\mathrm{C}_{3} \mathrm{H}_{5} \cdot \mathrm{O}_{3} \cdot \mathrm{H}_{3}$ (glycerin) $+3(\mathrm{H} . \mathrm{O} . \mathrm{R})$ (fatty acid). This depends upon a hydrolytic splitting, which was first positively proved by BERNARD and BERTHELOT. The pancreas enzyme also decomposes other esters just as it does the neutral fats (NENCKI). The pancreas-enzyme which decomposes fats has been less studied 
than the other pancreas-enzymes, and it has indeed been questioned whether or not the decomposition of the neutral fats in the intestine may not be effected through lower organisms. According to the investigations of NENCKI, it seems that the pancreas actually contains an enzyme which decomposes fats. 'This enzyme, which is still little known, appears to be very sensitive to acids, and it is often absent in acid glands not perfectly fresh. If a watery infusion of the gland prepared cold be treated with calcined magnesia, then the enzyme in question will, according to DaNILEWSKI, be retained by the magnesia precipitate.

The fatty acids which are split off by the action of the pancreatic juice combine in the intestine with the alkalies, forming soaps which have a strong emulsifying action on the fats, and thus the pancreatic juice becomes of great importance in the emulsification and the absorption of the fats.

Trypsin. The action of the pancreatic juice in digesting proteids was first observed by BERNARD, but first proved by CoRviSART. It depends upon a special enzyme called by KüHNE trypsin. Strictly speaking, this enzyme does not occur in the gland itself. In the gland, more probably, a zymogen occurs from which the enzyme is split off or formed by the secretion, also by the action of water, acids, alcohol, and other substances. According to AlberTONI, this zymogen is found in the gland in the last third of the intra-uterine life.

The purest trypsin thus far prepared, isolated by KüHNE, is soluble in water but insoluble in alcohol or glycerin. The less pure enzyme, on the contrary, is soluble in glycerin. If the solution of the enzyme in water is heated to the boiling point with the addition of a little acid, it decomposes into coagulated albumin and peptone (KüHNE). It is destroyed by gastric juice. Like other enzymes, trypsin is characterized by its physiological action. This action consists in its power of dissolving albumin, and especially easily fibrin, in alkaline, neutral, and even in faintly acid-reacting solutions.

The production of pure trypsin has been tried by various experimenters, DANILEWSKI, HÜFNER, KÜHNE, LOEW, and others. The purest seems to have been prepared according to the rather complicated method of KüHNE. In studying the action of trypsin a 


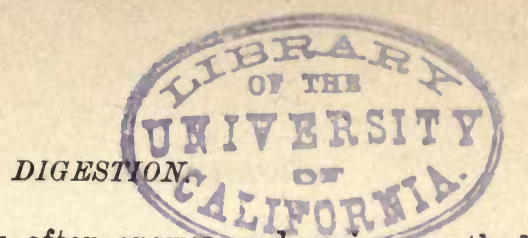

less pure preparation may often answer, and variotis methods of preparing such have been proposed, but we cannot describe all of them. For the production of a glycerin extract the gland should be rubbed with glass powder or pure quartz-sand, this mass carefully mixed with acetic acid of $1 \%$ ( 1 cc. to each grm. of gland), then for each part of the gland-mass add 10 parts of glycerin, and filter after about three days. By precipitating the glycerin extract with alcohol and redissolving the precipitate in water, we obtain a solution which has a powerful digestive action (HEIDENHAIN). A watery infusion of the gland may be made only after it has been exposed to the air for 24 hours, and 5-10 parts of water for each part by weight of the gland should be used. The simplest method is to cut the gland fine and place it in a flask and allow it to digest with water to which 5-10 cc. of chloroform (SALKowskI) or ether has been added for each litre. After a few days we obtain in this way a powerful infusion which keeps.

The action of trypsin on proteids is best demonstrated by the use of fibrin. Very considerable quantities of this albuminous body are dissolved by a small amount of trypsin at $37^{\circ}-40^{\circ} \mathrm{C}$. It is always necessary to make a control test with fibrin alone, with or without the addition of alkali. Fibrin is dissolved by trypsin without any putrefaction; the liquid has a pleasant odor somewhat like bouillon. To completely exclude putrefaction a little thymol, chloroform, or ether should be added to the liquid. Trypsin digestion differs essentially from pepsin digestion in that the first takes place in neutral or alkaline reaction and not, as is necessary for pepsin digestion, in an acidity of 1-2 p. m. $\mathrm{HCl}$, and further by the fact that the proteids dissolve in the trypsin digestion without previous expansion.

Many circumstances exert a marked influence on the rapidity of the trypsin digestion. With an increase in the quantity of enzyme present the digestion is hastened at least to a certain point, and the same is also true of an increase in temperature at least to about $+40^{\circ} \mathrm{C}$, at which temperature the albumin is very rapidly dissolved by the trypsin. The reaction is also of the greatest importance. Trypsin acts energetically in neutral, or still better in alkaline, solutions, and best in an alkalinity of 3-4 p. m. $\mathrm{Na}_{2} \mathrm{CO}_{3}$. Free mineral acids, even in very small quantities, completely prevent the digestion. If the acid is not actually free, but combined with albuminous bodies, then the digestion may take place quickly when 
the acid combination is not in too great excess (ChITTENDEN and Cummins). Organic acids act less disturbingly, and in the presence of $0.2 \mathrm{p} . \mathrm{m}$. lactic acid and the simultaneous presence of proteid, bile, and common salt the digestion may indeed proceed more quickly than in a faintly-alkaline liquid (LINDBERGER). Foreign bodiex, such as borax and potassium cyanide, may promote digestion, while bodies such as mercuric, fenic and other salts (CHITTENdes and Cummins), or salicylic acid in large quantities (KüHNE), may act preventively. The nature of the proteids is also of importance. Unboiled fibrin is, relatively to most other albuminous bodies, dissolved so very quickly that the digestion test with raw fibrin gives an incorrect idea of the power of trypsin to dissolve coagulated albuminous bodies in general. An accumulation of digestion products tends to hinder the trypsin digestion.

The Products of the Trypsin Digestion. In the digestion of unboiled fibrin a globulin which coagulates at $+55^{\circ}-56^{\circ} \mathrm{C}$. may be obtained as an intermediate product (Herrmans). Moreover from fibrin, as well as from other albuminous bodies, albumoses and peptones, leucin, tyrosin and aspartic acid, a little ammonia (HIRSCHLER), and a substance whose nature is not known and which gives a beautiful purple-red liquid with chlorine or bromine water in acid solution, have been obtained. When putrefaction has not been entirely prevented numerous other bodies appear which will be spoken of later in connection with the putrefaction process going on in the intestine. In the trypsin digestion, in contrast to the pepsin digestion, pure peptones, not precipitated by ammonium sulphate, are relatively easily and quickly formed. The peptone, according to KüHNE consists entirely of antipeptone, and the abovementioned decomposition products, leucin and the others, are formed by the decomposition of the hemipeptone. We will now consider leucin and tyrosin decomposition products formed by the trypsin digestion of proteids.

Leucin, $\mathrm{C}_{6} \mathrm{H}_{13} \mathrm{NO}_{2}$, or AMIDO-CAPROIC ACID, $\mathrm{C}_{5} \mathrm{H}_{10}\left(\mathrm{NH}_{2}\right) \mathrm{COOH}$, besides occurring in the trypsin digestion of proteids, is derived from the protein substances by their decomposition on boiling with diluted acids or alkalies, by melting with alkali hydrates, and by putrefaction. Because of the ease with which leucin and tyrosin are formed in the decomposition of protein substances, it is difficult 
to positively decide whether these bodies when found in the tissues are constituents of the living body or are only to be considered as decomposition products formed after death. Leucin has been found in the pancreas and its secretion, in the spleen, thymus and lymphglands, in the thyroid gland, in the salivary glands, in the kidneys, brain, and liver (but mostly in disease). It also occurs in the wool of sheep, in dirt from the skin (inactive epidermis) and between the toes, and its decomposition products have the disagreeable odor of the perspiration of the feet. It is found pathologically in atheromatous ulcers, ichthyosis scales, pus, blood, and urine (in diseases of the liver). Leucin also occurs in the vegetable kingdom.

Leucin may be prepared synthetically most simply by the action of ammonia on monobrom-caproic acid (HüFNER). On heating with fuming hydriodic acid to $140^{\circ} \mathrm{C}$. it splits into ammonia and caproic acid. On heating leucin alone it decomposes with the formation of carbon dioxide, ammonia, and amylamin. On fusing with caustic alkali, as also on putrefaction, it yields valerianic acid and ammonia.

Leucin crystallizes when pure in shining, white, very thin plates, usually forming round knobs or balls, either appearing like hyalin or alternating light or dark concentric layers which consist of radial groups of crystals. Leucin as obtained from the animal fluids and tissues is very easily soluble in water and rather easily in alcohol. Pure leucin is soluble with difficulty; it dissolves in 27 parts cold water, in 1040 parts cold and in 800 parts boiling alcohol. It is easily dissolved by alkalies and acids. On slowly heating to $170^{\circ} \mathrm{C}$. it melts and sublimes in white, woolly flakes which are similar to sublimed zinc oxide. A marked odor of amylamin is generated at the same time.

The solution of leucin in water is not, as a rule, precipitated by metallic salts. The boiling-hot solution may, however, be precipitated by a boiling-hot solution of copper acetate. If the solution of leucin is boiled with sugar of lead and then ammonia be added to the cooled solution, shining crystalline leaves of leucin-lead oxide separate. When boiled with leucin, copper oxyhydrate is dissolved without reduction.

Leucin is recognized by the appearance of the balls or knobs under the microscope, by its action when heated (sublimation test), 
and by Scherer's test. This last consists in the lencin yielding a colorless residue when carefully evaporated with nitric acid on platinum-foil, and this residue when warmed with a few drops of caustic soda gives a color varying from a pale yellow to brown (depending on the purity of the leucin), and on further concentrating over the flame it agglomerates into an oily drop which rolls about on the foil.

Tyrosin, $\mathrm{C}_{9} \mathrm{H}_{11} \mathrm{NO}_{3}$, or $p$. OXYPHENYLAMIDOPROPRIONIC ACID, HO. $\mathrm{C}_{6} \mathrm{H}_{4} \cdot \mathrm{C}_{2} \mathrm{H}_{3}\left(\mathrm{NH}_{2}\right) \cdot \mathrm{COOH}$, is derived from most protein substances (not gelatin) under the same conditions as leucin, which it habitually accompanies. It is especially found with leucin in large

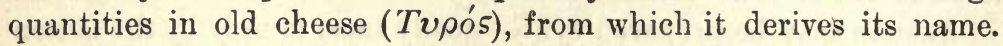
Tyrosin has not with certainty been found in perfectly fresh organs, with the exception, perhaps, of the spleen and pancreas of cattle. It occurs in the intestine in the digestion of albuminous substances, and it has about the same physiological and pathological importance as leucin.

Tyrosin was prepared by ERLENMEYER and LiPP from p. amidophenylalanin by the action of nitrous acid. . On fusing with caustic alkali it yields p. oxybenzoic acid, acetic acid, and ammonia. By putrefaction it may yield $\mathrm{p}$. hydrocoumaric acid, oxyphenyl-acetic acid, and p. cresol.

Tyrosin in a very impure state may be in the form of balls similar to leucin. The purified tyrosin, on the contrary, appears as colorless, silky, fine needles which are often grouped into tufts or balls. It is soluble with difficulty in water, being dissolved by 2454 parts water at $+20^{\circ} \mathrm{C}$. and 154 parts boiling water, separating, however, as tufts of needles on cooling. It dissolves more easily in the presence of alkalies, ammonia, or a mineral acid. It is difficultly soluble in acetic acid. Crystals of tyrosin separate from an ammoniacal solution on the spontaneous evaporation of the ammonia. It is not soluble in alcohol or ether. Tyrosin is known by its crystalline form and by the following reactions :

PIrIA's Test. Tyrosin is dissolved in concentrated sulphuric acid by the aid of heat, by which tyrosin sulphuric acid is formed; it is allowed to cool, diluted with water, neutralized by $\mathrm{BaCO}_{3}$, and filtered. On the addition of a solution of ferric chloride, the filtrate gives a beautiful violet color. This reaction is impeded by 
the presence of free mineral acids and by the addition of too much ferric chloride.

HoFMaNN's Test. If some water is poured on a small quantity of tyrosin in a test-tube and a few drops of Millow's reagent added and then the mixture boiled for a certain time, the liquid becomes a beautiful red and then yields a red precipitate. Or mercuric nitrate may first be added, then, after this has boiled, nitric acid which contains some nitrous acid.

ScHerer's Test. If tyrosin is carefully evaporated to dryness with nitric acid on platinum-foil, a beautiful yellow residue (nitrotyrosin nitrate) is obtained, which gives a deep reddish-yellow color with caustic soda. This test is not characteristic, as other bodies give a similar reaction.

Leucin and tyrosin may be prepared in large quantities by boiling albuminous bodies or albuminoids with dilute mineral acids. Ordinarily we boil hoof-shavings ( 2 parts) with dilute sulphuric acid ( 5 parts concentrated acid and 13 parts water) for 24 hours. After boiling the solution it is diluted with water and neutralized while still warm with milk of lime and then filtered. The calcium sulphate is repeatedly boiled with water, and the several filtrates are united and concentrated. The lime is precipitated from the concentrated liquid by oxalic acid and the precipitate filtered off, repeatedly boiled with water, all filtrates united and evaporated to crystallization. What first crystallizes consists chiefly of tyrosin with only a little lencin. By concentration a new crystallization may be produced in the mother-liquor, which consists of leucin with some tyrosin. To separate leucin and tyrosin from each other their different solubilities in water may be taken advantage of in preparing them on a large scale, but surer and better results are obtained by the following method of HLASIWETZ and HABERMANN. The crystalline mass is boiled with a large quantity of water and enough ammonia to dissolve it. To this boiling-hot solution enough basic lead acetate is added until the precipitate formed is nearly white; now filter, heat the light yellow filtrate to boiling, neutralize with sulphuric acid, and filter while boiling hot. After cooling, nearly all the tyrosin is precipitated, while the leucin remains in the solution. The tyrosin may be purified by recrystallizing from boiling water or from ammoniacal water. The above-mentioned mother-liquor rich in leucin is treated with $\mathrm{H}_{2} \mathrm{~S}$, the filtrate concentrated and boiled with an excess of freshly-precipitated copper oxyhydrate. A part of the leucin is precipitated, and the residue remains in the solution and partly crystallizes as a cuprous compound on cooling. The copper is removed from the 
precipitate and solution by means of $\mathrm{H}_{2} \mathrm{~S}$, the filtrate decolorized when necessary with animal charcoal, strongly concentrated and allowed to crystallize. The lencin obtained from the precipitate is quite pure, while that from the solution is somewhat contaminated.

If one is working with small quantities, the crystals, which consist of a mixture of the two bodies, may be dissolved in water and this solution precipitated with basic lead acetate. The filtrate is treated with $\mathrm{H}_{2} \mathrm{~S}$, the new filtrate evaporated to dryness, and the residue treated with warm alcohol which dissolves the leucin but not the tyrosin. The remaining tyrosin is purified by recrystallization from ammoniacal alcohol. Leucin may be purified by recrystallization from boiling alcohol or by precipitating it as leucin lead oxide, treating the precipitate suspended in water with $\mathrm{H}_{2} \mathrm{~S}$ and evaporating the filtered solution to crystallization.

To detect the presence of leucin and tyrosin in animal fluids or tissues the albumin must first be removed by coagulation with the addition of acetic acid and then precipitated by basic lead acetate. The filtrate is treated with $\mathrm{H}_{2} \mathrm{~S}$, this filtrate evaporated to a syrup or to dryness, and the two bodies in the residue are separated from each other by boiling alcohol and then purified as above stated.

Aspartic Acid, $\mathrm{C}_{4} \mathrm{H}_{7} \mathrm{NO}_{4}$, or AMIDO-SUCCINIC ACID, $\mathrm{C}_{2} \mathrm{H}_{3}\left(\mathrm{NH}_{2}\right)$. $(\mathrm{COOH})_{2}$. This acid has been obtained in the trypsin digestion of fibrin (RADZIEJEWSKI and SALKOWSKI) and of glutin (v. KNIERIEM). It may also be obtained by the decomposition of albuminous bodies or albuminoids with acids (see Chap. II). It has also , been found in beet-root molasses, and lastly it is very widely diffused in the vegetable kingdom as the amid ASPARAGINE (amidosuccinic-acid amid), which seems to be of the greatest importance in the development and formation of the albuminous bodies.

Aspartic acid dissolves in boiling water and crystallizes on cooling into rhombic prisms. It is optically active, and is dextrogyrate in a solution strongly acid with nitric acid. It forms with copper oxide a crystalline combination which is soluble in boiling-hot water and nearly insoluble in cold water, and which may be used in the preparation of the pure acid from a mixture with other bodies.

The action of trypsin on other bodies has not been thoroughly studied. An enzyme has been found in the pancreas-gland of the pig and certain herbivora which causes a coagulation of neutral or alkaline milk (KÜHNE and RoBERTS). Gelatin is dissolved by the pancreatic juice and is converted into gelatin peptone. In tests with a very impure infusion (self-digestion of the gland in the pres- 
ence of gelatine), besides gelatin peptone Nזxcki obtained leucin, glycocoll, ammonia, a base, $\mathrm{C}_{8} \mathrm{H}_{11} \mathrm{~N}$, and other products. The pure enzyme, according to KüHNE, gives neither glycocoll nor leucin with gelatin. The gelatin-giving substance of the connective tissues is not directly dissolved by trypsin, but only after it has been treated with acid or after soaking in water at $+r 0^{\circ} \mathrm{C}$. By the action of trypsin on hyalin cartilage the cells dissolve, leaving the nucleus. The basis is softened and shows an indistinctly-constructed network of collagenous substance (KüHNE and EWALD). The elastic substance, the structureless membrane, and the membrane of the fat-cells are also dissolved. Parenchymatous organs, such as the liver and the muscles, are dissolved to the nucleus, and also connective tissue, fat-corpuscles, and the remainder of the nervous tissue. If the muscles are boiled, then the connective tissue is also dissolved. Trypsin seems to be without action on chitin and horny substance. Oxyhomoglobin is decomposed by trypsin with the splitting off of hæmatin. Homoglobin, on the contrary, when the access of oxygen is completely prevented, is not decomposed by trypsin (HoPPE-SeYler). Trypsin does not act on fats or carbohydrates.

It was already brought out above that trypsin does not exist ready formed in the gland, but more likely, as HeIDENHAIN has shown, the gland contains a corresponding zymogen. The maximum quantity of such zymogen in the gland occurs 14-16-18 hours after an abundant meal, and the minimum 6-10 hours after. This zymogen is not changed by glycerin so that it forms trypsin, but is easily changed by water and acids. A soda solution of 1-1.5\%, on the contrary, prevents almost entirely the changing of the zymogen. If we allow the gland to lie in the air it gradually becomes acid, and this leads to the formation of an enzyme in which the oxygen seems to be active, as is usual in the conversion of the zymogen into trypsin. It is very probable also that the two other enzymes are formed from corresponding zymogens, and this has been shown to be plausible in regard to the diastatic enzymes by LIVERSIDGE.

After a plentiful meal Heidenhain found in dogs in the first stages of digestion, when the secretion of pancreatic juice was most active, that the glandular cells became smaller owing to the 
consumption of the inner granular zone, while the outer zone at the same time took up new material and became larger. In these stages the quantity of zymogen is smallest. At a later period, 12-20 hours after a meal, the inner zone is re-formed from the outer, and the larger this zone is the larger the quantity of zymogen in the gland seems to be. The zymogen consequently belongs to the inner zone, and the secretion consists therefore, at least in part, in a destruction or dissolution of this zone whereby the substance of the gland itself is changed into the secretion (HeIDenhain). 'This view, however, is in opposition to that of LEwASCHEw, who observed that in animals which have starved and whose pancreas-glands are nearly free from zymogen, the inner granular zone is just as much developed as under normal conditions and contains abundant quantities of zymogen. We are still completely in the dark regarding the nature of the chemical processes which take place in the conversion of the zymogen into the euzyme.

\section{The Chemical Processes in the Intestine.}

The action which belongs to each digestive secretion may be essentially changed by mixing with other digestive fluids; and since the digestive fluids which flow into the intestine are mixed with still another fluid, the bile, it will be readily understood that the combined action of all these fluids in the intestine makes the chemical processes going on therein very complicated.

As the acid of the gastric juice acts destructively on ptyalin, this enzyme has no further diastatic action, even after the acid of the gastric juice has been neutralized in the intestine. The bile has, at least in certain animals, a faint diastatic action which in itself can hardly be of any great importance, but which shows that the bile has not a preventive but rather a beneficial influence on the energetic diastatic action of the pancreatic juice and the faint diastatic action of the intestinal juices. Martin and Williams observed, in experiments made recently, a beneficial action of the bile on the diastatic action of the pancreas infusion. To this may be added that the organized ferments which occur habitually in the intestine and sometimes in the food have partly a diastatic 
action and partly produce a lactic-acid and butyric-acid fermentation.

The maltose which is formed from the starch seems to be converted into glucose in the intestine. The cellulose, especially the finer and more tender, is undoubtedly partly dissolved in the intestine; the products formed hereby are not very well known. It has been shown by TAPPENIER that cellulose may produce a marsh-gas fermentation in the intestines caused by the action of a microorganism; but we do not know to what extent the cellulose is destroyed and what part is valueless for the organism.

Bile possesses the power of dissolving fats in so slight a degree that it is scarcely worthy of mention. It is, however, without doubt of greater importance that the bile, as NENCKI has shown, facilitates the fat-splitting action of the paucreatic juice. This splitting of the fats into fatty acids and glycerin is an important factor in the absorption of the fats. The fatty acids combine with the alkalies of the bile and most readily with the alkalies of the intestinal and pancreatic juices, producing soaps which may be partly absorbed as such and partly exercise a powerful action on the absorption of the fats. There is no doubt that the chief part of the fats in the foods is absorbed as a fine emulsion, and for this reason the soaps are of such importance in the formation of this emulsion.

If to a soda solution of about 2 p. m. $\mathrm{Na}_{2} \mathrm{CO}_{3}$ we add pure, actually neutral olive-oil in not too large quantity, we obtain, after vigorous shaking, a transient emulsion. If, on the contrary, we add to the same quantity of soda solution an equal amount of commercial olive-oil (which always contains free fatty acids), we need only turn the vessel over for the two liquids to mix and immediately we have a very finely-divided and permanent emulsion making the liquid appear like milk. The free fatty acids of the always somewhat rancid commercial oil combine with the alkali forming soaps, which act to emulsify the fats (BRÜCKE, GAD). This emulsifying action of the fatty acids split off by the pancreatic juice is undoubt edly assisted by the habitual occurrence of free fatty acids in the food, and also by the splitting off of fatty acids from the neutral fats by the putrefaction in the intestine. These fatty acids must also combine with the alkalies in the intestine and form soaps.

This emulsification of fats by means of the action of the pan- 
creatic juice or by soaps formed in other ways can only take place in an alkaline solution. In the contents of the intestine, as long as they are acid, such an emulsion can hardly occur. On the contrary, it undoubtedly occurs at the point where the fat comes in contact with an alkaline secretion under a mucous membrane, or in general where it meets with sufficient alkali to form an emulsion. In the acid contents of the intestines of dogs, which had been kept on food rich in fat, LUDWIG and CASH observed no emulsion. After tying the two pancreas excretory ducts they found a remarkably fine emulsion in the chylous vessels, though the fat in the contents of the intestine was not emulsified. In this case it is possible that the free fatty acid which is hardly ever absent in the fat of the food, and which may be produced also by putrefaction in the intestine, forms soaps with the alkali of the mucous coat of the intestine and produces the emulsion in the chylous vessels.

Claude Bernard found long ago in his experiments on rabbits, in which animals the choledochus duct to the small intestine was inosculated above the pancreas passages, that when their food contained a large proportion of fat the chylous vessels of the intestine above the pancreas passages were transparent, but below the same they were milky-white, and from this concluded that the bile alone, without the pancreatic juice, does not emulsify fats. DASTrE tried the reverse experiment in dogs, namely, tying the choledochus duct and producing a biliary fistula, through which the bile would flow into the intestine below the mouth of the pancreatic passages. When the animals were killed after a meal rich in fat, the chylous vessels were first milky-white below the opening of the biliary fistula. DASTRE draws the following conclusion from this: that combined action of the bile and the pancreatic juice is necessary for the absorption of the fats-a deduction which coincides with the above-mentioned observations of NENCKI.

Bile has no solvent action on proteids, but still it may have an influence on their digestion. The acid contents of the stomach, containing an abundance of proteids, give with the bile a precipitate of proteids and bile-acids. This precipitate carries a part of the pepsin with it, and for this reason and on account of the partial or complete neutralization of the acid of the gastric juice by the alkali of the bile and the pancreatic juice the pepsin digestion can- 
not proceed further in the intestine. On the contrary, the bile does not disturb the digestion of albumin by means of the pancreatic juice in the intestine. The action of these digestive secretions, as above stated, is not disturbed by the bile, especially not by the faintly-acid reaction due to organic acids which are habitually found in the upper parts of the intestine. In a dog killed while digestion is going on, the faintly-acid, bile-containing matter of the intestine shows a strong digestive action on albumin.

The precipitate formed on the mixing of the acid contents of the stomach and the bile dissolves easily-partly by the acid reaction-in an excess of the bile, also in the $\mathrm{NaCl}$ produced by the neutralization of the hydrochloric acid of the gastric juice. Since in man the exit passages of the bile and the pancreatic juice open near one another, and therefore the acid contents of the stomach are probably immediately neutralized by the bile as soon as it enters, it is doubtful whether a precipitation of albumin by the bile occurs in the intestine.

Besides the previously-mentioned processes caused by enzymes, there are others of a different nature going on in the intestine, namely, the putrefaction processes caused by micro-organisms. These are less intense in the upper parts of the intestine, but increase in intensity towards the lower part of the same, and decrease in the large intestine because of the absorption of water. A positive proof that the micro-organisms are active in these processes lies in the fact that they occur very abundantly in the contents of the intestine; and it is to be remarked that these organisms occur in largest quantities in the lower parts of the intestine, where the contents have the most disagreeable odor. No putrefaction occurs, on the contrary, in the intestinal canal of the fœtus, which follows from the fact, proved by ZweIfeL, Hoppe-Seyler, and Senator, that in the contents of the same only undecomposed bile-acids and bile-pigments occur, while the otherwise regularly-occurring products of putrefaction in the intestinal canal are absent.

The putrefaction processes in the intestine are somewhat different from those of the pancreas digestion; and these two processes are essentially different from each other in the products which they yield. In the pancreatic digestion there are formed, so far as is known, besides albumoses and peptones, amido-acids and ammonia. 
In the putrefaction of the proteids we have, indeed, the same products formed at the beginning, but the decomposition proceeds considerably further and a number of products are developed, which have become known through the labors of numerous investigators, Nencki, Baumann, Brieger, H. and E. Salkowski. The products which are formed in the putrefaction of proteids are (in addition to albumoses, peptones, amido-acids, and ammonia) indol, skatol, paracresol, phenol, phenyl-propionic acid, and-phenylacetic acid, also paraoxyphenyl-acetic acid and hydroparacoumaric acid (besides paracresol, produced in the putrefaction of tyrosin), volatile fatty acids, carbon dioxide, hydrogen, marsh-gas, and sulphuretted hydrogen. In the putrefaction of gelatin neither tyrosin nor indol is formed, while glycocoll is produced.

Among these products of decomposition a few are of special interest because of their behavior within the organism, and because after their absorption they pass into the urine. A few, such as the oxyacids, pass unchanged into the urine, while others, such as phenol, are transformed into ethereal sulphuric acids by synthesis, and are eliminated by the urine ; others, on the contrary, such as indol and skatol, are only converted into ethereal sulphuric acids after oxidation (for details see Chapter XIV). The quantity of these bodies in the urine varies also with the extent of the putrefaction processes in the intestine ; at least this is true for the ethereal sulphuric acids. Their quantity increases with a stronger putrefaction, and the reverse takes place, as BAUMaNN has shown by experiments on dogs, when the intestine has been disinfected by calomel, as then they disappear from the urine.

Among the products of putrefaction developed in the intestine, indol and skatol must be carefully discussed.
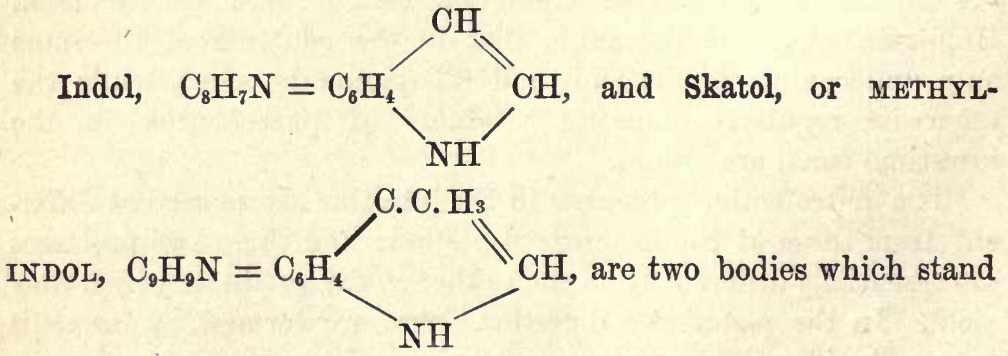
in close relationship to the indigo substances, and which are formed from the albuminous bodies by their putrefaction, or by fusion with caustic alkali. Hence they occur habitually in the human intestinal canal and after oxidation into indoxyl and skatoxyl respectively, pass, at least partly, into the urine as the corresponding ethereal sulphuric acids, but also as glycuronic acids.

These two bodies have been prepared synthetically in many ways. Both may be obtained from indigo by reducing it with tin and hydrochloric acid and heating this reduction product with zinc-dust (BAEYER). Indol may be formed from skatol by passing it through a red-hot tube. Indol suspended in water is in part oxidized into indigo-blue by ozone (NeNCKI).

Indol and skatol crystallize in shining leaves, and their melting points are $+52^{\circ}$ and $95^{\circ}$ respectively. Indol has a peculiar excrementitious odor, while skatol has an intense fetid odor (skatol obtained from indigo should be odorless). Both bodies are easily volatilized by steam, skatol more easily than indol. They may both be removed from the watery distillate by ether. Skatol is the more insoluble of the two in boiling water. Both are easily soluble in alcohol, and give with picric acid a combination consisting of red crystalline needles. If a mixture of the two picrates be distilled with ammonia, they both pass over without decomposition; but if they are distilled with caustic soda, the indol is decomposed but not the skatol. The watery solution of indol gives with fuming nitric acid a red liquid, and then a red precipitate of nitroso-indol nitrate (BAEYER). It is better to first add two or three drops of nitric acid, and then a $2 \%$ solution of potassium nitrite, drop by drop (SALKowskI). Skatol does not give this reaction. An alcoholic solution of indol treated with hydrochloric acid colors a pine chip cherry-red. Skatol does not give this reaction. Skatol dissolves in concentrated hydrochloric acid with a violet coloration.

For the detection of indol and skatol in, and their preparation from, excrement and putrefying masses, the nlain points of the usual method are as follows: The mass is distilled after acidifying with acetic acid; the distillate is then treated with alkali (to combine with any phenol which may be present at the same time) and again distilled. From this second distillate the two bodies, after 
the addition of hydrolichloric acid, are precipitated by picric acid. The picrate precipitate is then distilled with ammonia. The two bodies are obtained from the distillate by repeated shaking with ether and evaporation of the several ethereal extracts. The residue, containing indol and skatol, is dissolved in a very small quantity of absolute alcohol and treated with 8-10 vols. of water. Skatol is precipitated, but not the indol. The further treatment necessary for their separation and purification will be found in other works.

The gases which are produced by the putrefactive processes are mixed in the intestinal tract with the atmospheric air swallowed with the saliva, and as the gas generated by different foods vary, so the mixture of gases after various foods should have a dissimilar composition. This is found to be true. Oxygen is only found in very faint traces in the intestine; this may be accounted for in part by the formation of reducing substances in the fermentation processes which combine with the oxygen, and partly, perhaps chiefly, to a diffusion of the oxygen through the tissues of the walls of the intestine. To show that these processes take place mainly in the stomach the reader is referred to page 189, on the composition of the gases of the stomach. Nitrogen is habitually found in the intestine, and it is probably due chiefly to the swallowed air, or perhaps in part, as BUNGE claims, to a diffusion of nitrogen from the tissues of the intestinal walls to the intestine. The carbon dioxide originates partly from the putrefaction of the proteids, partly from the lactic-acid and butyric-acid fermentation of carbohydrates, and partly from the setting free of carbon dioxide from the alkali carbonates of the pancreatic and intestinal juices by their neutralization by the hydrochloric acid of the gastric juice and by organic acids formed in the fermentation. Hydrogen occurs in largest quantities after a milk diet and in smallest quantities after a purely meat diet. This gas seems to be formed chiefly from the butyric-acid fermentation of carbohydrates, although it may occur in large quantities in the putrefaction of proteids under certain circumstances. There is no doubt that the sulphuretted hydrogen which occurs normaliy in the intestine originates from the proteids. The marsh-gas undoubtedly originates in the putrefaction of proteids. As proof of this RUGE found 26.45\% marshgas in the human intestine after a meat diet. He found a still 
greater quantity of this gas after a diet consisting of leguminous plants; this coinsides with the observation that marsh-gas may bo produced by a fermentation of carbohydrates, but especially of cellulose (Hoppe-Seyler, TAPPENIER). Such an origin of marshgas, especially in herbivora, is to be expected. A small part of the marsh-gas and carbon dioxide may also depend on the decomposition of lecithin (HASEBROEK).

Putrefaction in the intestine not only depends upon the composition of the food, but also upon the albuminous secretions and the bile. Among the constituents of bile which are changed or decomposed, we have not only the pigments-produced from the bilirubin, as is generally assumed, hydrobilirubin and brown pigments-but also the bile-acids, especially taurocholic acid. Glycocholic acid is more stable and a part is found unchanged in the excrement of certain animals, while taurocholic acid is so completely decomposed that it is entirely absent in the fæces. In the foetus, in whose intestinal tract no putrefaction processes occur, we find, on the contrary, undecomposed bile-acids and bile-pigments in the contents of the intestine. That the secretion rich in albumin is an important element in putrefaction in the intestine follows from the fact that putrefaction may also continue during fasting. From the observations of MüLLER on CETTI it was found that the elimination of indican during hunger rapidly decreased and after the third day of starvation it had entirely disappeared, while the phenol elimination, which at first decreased so that it was nearly minimum, increased again from the fifth day of starvation and on the eighth or ninth day it was three to seven times as much as in man under ordinary circumstances. In dogs, on the contrary, the elimination of indican during starvation is considerable, but the phenol elimination is minimum. Among the secretions which undergo putrefaction in the intestine, the pancreatic juice, which putrifies most readily, takes first place. Pisenti found, in his experiments on dogs, that the elimination of indican by the urine greatly diminished after tying the pancreatic passages, but that it increased again when the animal was given pancreas peptones or pancreatic juice.

From the foregoing facts we conclude that the products formed by the putrefaction in the intestine are in part the same as those 
formed by digestion. The putrefaction may be of benefit to the organism so far as the formation of such products as albumoses, peptones, and perhaps also certain amido-acids is concerned. On the contrary, the formation of further splitting products is to be considered as a loss of valuable material, and it is therefore important that putrefaction in the intestine is kept within certain limits. If an animal is killed while digestion in the intestine is going on, the contents of the small intestine give out a peculiar but not putrescent odor. Also the odor from the contents of the large intestine is far less offensive than a putrefying pancreas infusion or a putrefying mixture rich in albumin. From this we may conclude that putrefaction in the intestine is ordinarily not nearly so intense as ontside of the organism.

It seems thus to be provided, under physiological conditions, that putrefaction shall not proceed too far, and the factors which here come under consideration are probably of different kinds. Absorption is one of the more important of them, and it has been proved by actual observation that the putrefaction increases, as a rule, as the absorption is checked and fluid masses accumulate in the intestine. The character of the food also has an unmistakable influence, and it seems as if a large quantity of carbohydrates in the food acts against putrefaction (HIRSCHLER). A specially strong action tending to prevent putrefaction is observed in the bile. This anti-putrid action does not occur in neutral or faintly-alkaline bile, which itself easily putrefies, but in the free bile-acids, especially in taurocholic acid (MALY and EMICH, LINDBERGER). There is no question that the free bile-acids have a strong preventive action on putrefaction outside of the organism, and it is therefore difficult to deny such an action in the intestine. Notwithstanding this the anti-putrid action of the bile in the intestine is contradicted by certain investigators (VoIT, RöHMANN).

Biliary fistulæ have been introduced so as to study the importance of the bile in digestion (SchwanN, Blondlot, Bidder and Schmidt, and others). As a result it has been observed that from fatty foods an imperfect absorption of fat regularly takes place, and the excrements contain, therefore, an excess of fat and have a light-gray or pale color. How long after the operation the deviation from the normal appears depends essentially upon the char- 
acter of the food. If an animal is fed on meat and fat, then the quantity of food must be considerably increased after the operation, otherwise the animal will become very thin, and indeed die with symptoms of starvation. In these cases the excrements have the odor of carrion, and this was considered a proof of the action of the bile in checking putrefaction. The emaciation and the increased want of food depend, naturally, upon the imperfect absorption of the fats, whose high calorific value is reduced and must be replaced by the taking up of larger quantities of other nutritive bodies. If the quantity of proteids and fats be increased, then this last, which can be only very incompletely absorbed, accumulates in the intestine. This accumulation of the fats in the intestine only renders the action of the digestive juices on proteids more difficult, and these last increase the amount of putrefaction. This explains the appearance of stinking fæces, whose pale color is not due to a lack of bile-pigments, but to a surplus of fat (RöHMaNs, VorT). If the animal is, on the contrary, fed on meat aud carbohydrates, it may remain quite normal, and the leading off of the bile does not cause any increased putrefaction. The carbohydrates may be absorbed unprevented in such large quantities that they replace the fat of the food, and this is the reason why the animal on such a diet does not become emaciated. If with this diet the putrefaction in the intestine is no greater than under normal conditions even though the bile is absent, it would seem that the bile in the intestine exercise no preventive action on putrefaction.

We must remember, however, that the presence of free acids counteracts putrefaction, and further that the carbohydrates yield free acids by acid fermentation within the intestine. It is therefore conceivable that to the carbohydrates, which, according to HIRSCHLER, are capable of checking putrefaction without entering into an acid fermentation, the antiseptic action of the bile is due. It cannot be denied that the bile under ordinary conditions, with a mixed diet deficient in carbohydrates, has a preventive action on the putrefaction in the intestine. LIмвобR日 has shown that it acts in an antiseptic sense, so that the decay of the proteids, giving rise to simpler products less valuable, or perhaps even injurious, in the organism, are checked.

Although the question how the putrefactive processes in the 
intestine under physiological conditions are kept within certain limits cannot be answered positively, still it may be asserted that the acid reaction of the upper parts of the intestine and the absorption of water in the lower parts are important factors.

Excrements. It is evident that the residue which remains after completed digestion and absorption in the intestine must be different, both qualitatively and quantitatively, according to the variety and quantity of the food. In man the quantity of excrement from a mixed diet is $120-150$ grms., with $30-37$ grms. solids, per 24 hours, while the quantity from a vegetable diet, according to Vorr, was 333 grms., with 75 grms. solids. With a strictly meat diet the excrements are scanty, pitch-like, and colored nearly black by hæmatin and iron sulphide. The scanty excrements in starvation have a similar appearance. A large quantity of coarse bread yields a great amount of light-colored excrement. If there is a large proportion of fat, it takes a lighter, clayey appearance. The decomposition products of the bile-pigments seem to play only a small part in the normal color of the fæces.

The constituents of the fæces are of different kinds. We find in the excrements digestible or absorbable constituents of the food, such as muscular fibres, connective tissues, lumps of casein, grains of starch, and fat which have not had sufficient time to be completely digested or absorbed in the intestinal tract. In addition the excrements contain indigestible bodies, such as remains of plants, keratin substances, nuclein, and others; also form-elements originating from the mucous coat and the glands; constituents of the different secretions, such as mucin, cholalic acid, dyslysin, and cholesterin; mineral bodies of the food and the secretions; and lastly, products of putrefaction or of the digestion, such as skatol, indol, volatile fatty acids, lime, and magnesia soaps. Occasionally, also, parasites of different kinds occur; and lastly, the excrements contain micro-organisms, fungi of different kinds, sometimes in such large quantities that the chief mass of the excrements seems to consist of micro-organisms ( $\mathrm{v}$. J $\mathrm{AKSCH}$ ).

The reaction of the excrements is very changeable. It is often acid in the inner part, while the outer layers in contact with the mucous coat have an alkaline reaction. In nursing infants it is 
habitually acid (WEGSCHEIDER). The odor is perhaps chiefly due to skatol, which was first found in the excrements by BRIEGER, and so named by him. Indol and other substances also take part in the production of odor. The color is ordinarily lighter or darker brown, and depends above all upon the nature of the food. Medicinal bodies may give the fæces an abnormal color. The excrements are colored black by iron and bismuth, yellow by rhubarb, and green by calomel. This last-mentioned color was formerly accounted for by the formation of a little mercury sulphide, but now it is said that calomel checks the putrefaction and the decomposition of the bile-pigments, so that a part of the bile-pigments pass into the fæces as biliverdin. According to LeSAGE, a green color of the excrements in children is caused partly by biliverdin and partly by a pigment produced from a bacillus. In the yolk-yellow or greenish-yellow excrements of nursing infants we can detect bilirubin. Neither bilirubin nor biliverdin seems to exist in the excrements of mature persons under normal conditions. On the contrary, we find stercobilin (Masius and Vanlair), which, according to certain investigators, is identical with hydrobilirubin (MALY), which is obtained from bilirubin by a reduction process, and urobilin (JAFFÉ) - a view contested by MACMUNN. Bilirubin may occur in pathological cases in the fæces of mature persons. It has been observed in a crystallized state (as hæmatoidin) in the fæces of children as well as of grown persons (UFFELMANN, v. $\mathrm{J}_{\mathrm{AKSCH}}$.

The absence of bile (acholic fæces) causes the excrements to have, as above stated, a gray color, due to large quantities of fat; this may, however, be partly attributed to the absence of bile-pigments. In these cases a large quantity of crystals has been observed (GERHARDT, v. JAKSCH) which consist chiefly of magnesia soaps (Oesterlen) or sodium soaps (Stadelyans). Hemorrhage in the upper parts of the digestive tract yields, when it is not very abundant, a dark-brown excrement, due to hæmatin.

EXCRETIN, so named by MARCET, is a crystalline body occurring in human excrement, but which, according to HoPPE-SEYLER, is perhaps only impure cholesterin. Excretolic ACID is the name given by MaRCET to a body similar to oil and with an excrementiteous odor.

In consideration of the very variable composition of excrements their quantitative analyses are of little value and therefore will be omitted. 
Meconium is a dark brownish-green, pitchy, mostly acid mass without any strong odor. It contains greenish-colored epithelium cells, cell-detritus, numerous fat-globules, and cholesterin plates. The amount of water and solids is respectively 720-800 and 280200 p. m. Among the solids we find mucin, bile-pigments and bile-acids, cholesterin, fats, soaps, calcium and magnesium phosphates. Sugar and lactic acid, albuminous bodies (?) and peptones, also leucin and tyrosin and the other products of putrefaction occurring in the intestine are absent. Meconium may contain undecomposed taurocholic acid, bilirubin and biliverdin, but it does not contain any hydrobilirubin, which is considered as proof of the non-existence of putrefactive processes in the digestive tract of the fœtus.

In medico-legal cases it is sometimes necessary to decide whether spots on linen or other substances are caused by meconium. In such cases we have the following conditions. The spot caused by meconium has a brownish-green color and can be easily separated from the material because, on account of the ropy property of the meconium, it is difficult to wet through. When moistened with water it does not develop any special odor, but on warming with dilute sulphuric acid it has a somewhat fetid odor. It forms with water a slimy, greenish-yellow liquid containing brown flakes. The solution gives with an excess of acetic acid an insoluble precipitate of mucin; on boiling it does not coagulate. The filtered, watery extract gives GMELIN's, but still better HUPPERT's, reaction for bile-pigments. The liquid precipitated by an excess of milk of lime gives a nearly colorless fitrate, which after concentration gives Pettenkofer's reaction.

The contents of the intestine under abnormal conditions are perhaps less the subject of chemical analysis than of an inspection or microscopical investigation. On this account the question as to the properties of the contents of the intestine in different diseases cannot be thoroughly treated here. The question as to the different processes which, so far as they are dependent on secretion and absorption, cause an abnormal consistency, a thinning of the excrements, possesses a certain interest. Such excrements may in part be produced by arrested absorption of liquid from the intestine for some reason or other, and in part caused by an increased secretion or a transudation of liquids into the intestine. 
A diminished absorption (of water) may be caused by a more active movement of the intestine, which causes their contents to pass quickly, and in this way the action of laxatives is often explained. A diminished absorption may also be due to a decreased activity of the absorbing cells. In absorption, which is generally accepted to-day, the cells of the mucous coat take an active part, and anything which acts disturbingly on the protoplasm of these cells must also exercise an influence on the absorption. This condition with regard to the action of laxatives has been especially noted by Hoppe-SeYler. According to him, it is also probable that such laxatives, of which only traces are required for absorption, by a direct action on the intestinal epithelium-whether the absorption is made more difficult, or a transudation made possible, or whether the action of these two is simultaneous-cause watery evacuations. According to RöHmavs, concentrated salt solutions act by a decreased absorption activity.

A thin evacuation may be produced by an increased elimination of fluid into the intestine, and there are many investigators who consider it positively proved that a transudation of liquid into the intestine is caused by the action of saline laxatives.

The character of the intestinal epithelium is undoubtedly an important factor in the production of such a transudation, and when this is caused by the saline laxatives it probably is produced by action on the epithelium. We must admit with HoPPE-SEYLER and other investigators that the most important regulator of the flow of liquid through the intestinal mucous membrane is the intestinal epithelium. It is the epithelium which renders possible the stream of fluid contrary to the laws of osmose, and which under normal conditions prevents a transudation into the intestines. Bodies which affect the epithelium may therefore cause a transudation, and this is found to be especially abundant after ejection of the intestinal epithelium. The most striking example of this is observed in Asiatic cholera, in which the epithelium is largely expulsed and an extraordinarily abundant transudation takes place. 


\section{Appendix. Intestinal Concrements.}

Calculi occur very seldom in human intestine or in the intestine of carnivora, but they are quite common in herbivora. Foreign bodies or indigested residues of food may, when for some reason or other they are retained in the intestine for some time, become incrusted with salts, especially ammonium-magnesium phosphate or magnesium phosphate, and these salts form usually the chief constituent of the concrements. In man they are sometimes oral or round, yellow, yellowish-gray, or brownish-gray, of variable size, consisting of concentric layers and containing chiefly ammoniummagnesium phosphate, calcium phosphate, besides a small quantity of fat or pigment. The nucleus ordinarily consists of some foreign body, such as the stone of a fruit, a fragment of bone, or something similar. In those countries where bread made from oat-bran is an important food, we often find in the large intestine balls similar to the so-called hair-balls (see below). Such calculi contain calcium and magnesium phosphate (about $70 \%$ ), oat-bran (15-18\%), soaps and fat (about 10\%). Concretions which contain very much (about 74\%) fat occur seldom, and those consisting of fibrin clots, sinews, or pieces of meat incrusted with phosphates are also rare.

Intestinal calculi occur often in animals, especially in horses fed on bran. These calculi, which attain a very large size, are hard and heavy (as much as 8 kilos) and consist in great part of concentric layers of ammonium-magnesium phosphate. Another variety of concrements which occurs in horses and cattle consists of gray colored, often very large, but relatively light stones which contain plant-remains and earthy phosphates. Stones of a third variety are sometimes cylindrical, sometimes spherical, smooth, shining, brownish on the surface, consisting of matted hairs and plantfibres, and termed hair-balls. The so-called “ AGAGROPILA," which probably originate from the ANTILOPUS RUPICAPRA, belong to this group, and they are generally considered as nothing else than the hair-balls of cattle.

The so-called oriental bezoar-stone belongs also to the intestinal concrements, and probably originates from the CAPRA EGAGRUS and ANTILOPE DORCAS. We may have two varieties of bezoar- 
stones. One is olive-green, faintly shining, formed of concentric layers. On heating it melts with the development of an aromatic odor. It contains as chief constituent LITHOFELLIC acid, $\mathrm{C}_{90} \mathrm{H}_{30} \mathrm{O}_{4}$, which is related to cholalic acids, and besides this a bile-acid, LITHOBILIC ACID. The others are nearly blackish brown or dark green, very glossy, consisting of concentric layers, and do not melt on heating. They contain as chief constituent ELLAGIC ACID, a derivative of tannic acid, of the formula $\mathrm{C}_{14} \mathrm{H}_{6} \mathrm{O}_{8}$, which gives a deep blue color with an alcoholic solution of ferric chloride. This lastmentioned bezoar-stone originates, to all appearances, from the food of the animal.

Ambergris is generally considered an intestinal concrement of the sperm. whale. Its chief constituent is AMBRAIN, which is a non-nitrogenious substance perhaps related to cholesterin. Ambrain is insoluble in water and is not changed by boiling alkalies. It dissolves in alcohol, ether, and oils.

\section{Absorption.}

In discussing the absorption processes we must treat of the form into which the different foods are transformed before absorption, of the manner in which this is accomplished, and, lastly, of the force which acts in these processes.

Starch and the other carbohydrates are chiefly absorbed as sugar, in part also as organic acids (lactic acid), and perhaps in small quantities as dextrin. Fats may indeed be partly absorbed as soaps; still the quantity absorbed in this way is very small compared with that absorbed as an emulsion. Emulsion is undoubtedly the most important form under which fat is absorbed, and the neutral fats, also the free fatty acids, are emulsified when they occur in large quantities in the intestines.

Peptone, as above stated, is the final product of the digestion of albuminous bodies. Now as peptone is a very soluble and a relatively easily diffusible modification of proteids, it is not difficult to admit the deduction that proteids must be changed into peptone in order that it may be readily absorbed. Certain observations of Funkes on animals confirm this view. He found in an untied intestinal knot of a living animal that the peptone (in the old sense of the word) was absorbed considerably faster than other proteids. There is also no doubt that a part of the proteids is 
invariably absorbed from the intestinal canal as peptones, or more correctly perhaps as albumoses and peptones. But it has been positively settled by the investigations of BRÜCKE, BAUER and Voit, EichHorst, Czerny and Latschenberger, that peptonized albumin, casein, myosin, and alkali albuminates cannot be absored from the intestines-a matter which is of practical importance, especially with regard to the nutritive clysters. If the albumin can be absorbed partly as such and partly as peptone, then the question arises, how much more can it be absorbed in one form than in the other? This question cannot be decisively answered. The investigations by ScHMidT-MÜLHEIM of the contents of the stomach and intestine of dogs show that the stomach contains a considerably larger amount of peptone than of simple dissolved proteids, which seems to indicate that the greatest part of the proteids is absorbed as peptones (or albumoses).

The soluble salts are also absorbed with the water. The albumin and peptone which can dissolve a considerable quantity of salts insoluble in alkaline water are of great importance in the absorption of such salts.

The soluble constituents of the digestive secretions may, like other dissolved bodies, be absorbed, as is demonstrated by the passage of peptone into urine; the enzymes may also be absorbed. The occurrence of urobilin in urine attests to the absorption of the bile-constituents under physiological conditions notwithstanding that, according to certain investigators (MACMUNN), it is not identical with hydrobilirubin, and contrariwise, according to the observations of COPEMAN and WINSTON on a woman with a biliary fistula, it also occurs in the urine when no bile comes into the intestine. The question as to the occurrence of very faint traces of bile-acids in normal urine is also contradicted, and from the behavior of the urine it is therefore difficult to draw any positive conclusion as to a possible absorption of the bile-constituents from the intestine. On the other hand, the absorption of bile-acids from the intestine seems to be established by other observations. TAPPEINER introduced a solution of bile-acid salts of a known concentration into an untied intestinal knot, and after a time investigated the contents. He found that in the jejunum and the ileum, but not in the duodenum, an absorption of bile-acids took 
place, and further that of the two bile-acids only the glycocholic acid was absorbed in the jejunum. Further, ScHIFr long ago expressed the opinion that bile undergoes an intermediate circula. tion, in such wise that it is absorbed from the intestine, then carried to the liver by the blood, and lastly eliminated from the blood by this organ. Although this view has met with some opposition, still its correctness seems to be established by the researches of various investigators, and more recently by PREvosT and BINET, at least to the extent that after the introduction of foreign bile into the intestine of an animal the foreign bile-acids appear again in the secreted bile.

There are two possible ways in which the absorbed bodies may enter the blood-stream. They may pass into the blood through the chylous vessels and the thoracic duct indirectly, or they may, after they have passed the intestinal epithelium, pass into the blood-capillaries of the villous membranes and so directly into the blood. By the investigations of LUDWIG and his pupils, RöHRIG, ZaWilsky, v. Mering and. Schmidt-MüLheim, as well as by those of HeIDENHAIN and his pupils, it has now been proved that the fat is driven through the chylous vessels and the thoracic duct to the blood, while on the contrary the bodies soluble in water, such as sugar and salts, are taken up by the blood of the capillaries of the villous membrane and in this way pass into the blood. The reason why the dissolved bodies do not pass into the chylous vessels in larger quantities is explained by HeIdenHaIN to lie in the anatomical situation, - the arrangement of the capillaries close under the layer of epithelium. Ordinarily these capillaries find the necessary time for the taking up of the water and the solids dissolved in it. But when a large quantity of liquid, such as a sugar solution, is introduced into the intestine at once, this is not possible, and in these cases a part of the dissolved bodies pass into the chylous vessels and the thoracic duct (GINSBERG and Röhmans).

The question of the absorption of the peptones, albeit in most cases these have not been differentiated from albumoses, has been the subject of numerous investigations. Ludwig and ScHMDTMüLHEIM tied the jugular and humeral arteries and lymphatic ressels of both sides of a dog so that the section showed later a complete cutting off of the chyle from the blood-circulation. They 
found that the absorption of proteids from the intestine was not influenced thereby, and from this concluded that the proteids as well as the other nutritive bodies soluble in water pass directly into the blood through the walls of the intestinal capillaries. If this were the case, then we might expect to find peptone in solution in the blood during or after digestion. This, however, is not the case. Schmidt-Mülheim and Hofmeister found only traces of peptone in serum or blood, and according to NEÜMEISTER it does not occur even as traces. Chyle does not contain any peptone.

Then where does the peptone which is absorbed from the intestine remain? If peptone in solution is introduced into the circulating blood, it is quickly eliminated therefrom with the urine (Plos'z and Gyergyai, Hofmeister, Schmint-Mülheim). The same happens also after injecting peptone subcutaneously. Normal urine does not contain any peptone, and the absence of this body in the blood after digestion cannot be explained by assuming that it is eliminated by the kidneys. Since the peptone introduced into the blood is quickly eliminated by the kidneys, while the peptone formed in the intestine does not pass into the urine, it may perhaps be thought that this peptone is retained normally by the liver and is absorbed, and only that peptone which finds its way into the circulating blood by evasion from this organ passes into the urine. This supposition, however, is untenable. NEUMEISTER has investigated the portal blood of rabbits in whose stomachs large quantities of albumoses and peptones had been introduced, and found therein only traces of the body in question. $\mathrm{He}$ has also shown that when we supply the liver of a dog with the portal-blood peptone (ampho-peptone), this is not retained by the liver but is eliminated with the urine. Peptone seems to pass neither into the blood nor the chylous vessels, but to be changed in some way by the walls of the intestine. HoFMEISTER, according to whom the walls of the stomach and the intestine are the only parts of the body in which peptones occur constantly during digestion, has also made the observation that peptone (at the temperature of the body) after a time disappeared from the excised but apparently still living mucous coat of the stomach. Peptone seems to undergo a change in the mucous membrane of the digestive canal, and the following observation of LUDWIG and SALvioli 
bears out that assumption. These investigators introduced a peptone solution into a doubly-tied, removed knot of the small intestine which was kept active by passing through defibrinated blood, and observed that the peptone disappeared from the intestine, but that the blood passing through did not contain any peptone.

If, then, peptone already disappears in the mucous coat, or at least in the walls of the digestive tract, the question naturally arises, what becomes of the peptone in the mucous membrane? The experiments of Maly, Plos'z and Gyergyai, Adamkiewicz, ZuNTz and Pollitzer have established that the.albumoses and peptones may be substituted for albumin in the food, and may also be converted into ordinary albumin. We must then assume that peptone is already converted into albumin in the mucous membrane of the digestive canal.

According to HoFMEISTER, a considerable increase of leucocytes occurs in the adenoid tissues during digestion, an observation which is in close accord with that of PoHL, who found that in dogs kept on an albuminous diet the venous blood of the intestine contains more leucocytes than the arterial blood. According to HoFMEISTER, leucocytes play an important part in the absorption and assimilation of the peptones. They may take up the peptones and be the means of transporting them to the blood, and secondly by their growth, regeneration, and increase may stand in close relation to the transformation and assimilation of the peptones. HeIdENHAIN, who considers that the transformation of peptone into albumin in the mucous membrane is positively settled, does not, attribute so great an importance to these last in the absorption of the peptones as HoFMEIsTer, chiefly on the ground of equalization of the quantity of absorbed peptones and leucocytes. He considers it most probable that the reconversion of the peptones into albumin takes place in the epithelium layers.

Little is known concerning the forces taking part in absorption. Osmose and filtration were formerly considered as the most important factors. But as in regard to the peptones, whose formation in the digestion was considered as taking place especially in the interest of a facilitated osmosis and filtration, but whose conditions have been found quite different and much more complicated, so in 
the absorption theory there is a still greater contrast between former and present views, the latter inclining to the theory that absorption is a process connected with the vital properties of the cells. Investigations in this direction have been made by HeIDENHaIN and his pupils, RöhmanN and Gumilewsky; and these investigations have shown that the cells take an active part in the absorption, and that this action is independent of the processes caused by an unequal diffusibility of the different bodies. For example, in a solution which contains equal quantities of grapesugar and sodium sulphate the sugar will be almost completely absorbed in a certain time, while the salt, which has the greater diffusibility, still remains in considerable amounts in the intestine. Certain coloring matters are not absorbed, and the cells seem to have the property of discriminating between the different substances. The absorption of dissolved bodies seems to be connected with a specific activity of the living cell, the living protoplasm.

In the absorption of bodies not dissolved, of the emulsified fats forces take part which are not known. That the bile performs the most important part in the absorption of fats is very generally admitted, but how the bile acts in this process is not yet determined. v. WistinghaUSEN has found that fat rises higher in a capillary tube when it has been moistened with bile than when with water, and further that fluid fat filters more easily through a dead membrane dipped in bile than when dipped in water. From these observations, whose correctness has lately been disputed by GAD and GRöper, the inference has been drawn that bile facilitates the capillary attraction and thereby accelerates the absorption of the fats. The epithelium layer of the intestinal mucous membrane cannot be compared with a dead membrane soaked in water, and it is therefore doubtful if the above-mentioned action of bile can have any influence on the absorption of fats in the intestine. That the absorption of fats is caused by the lymphoidal migratory cells (ZaWARYKin, SchæFfer) is disputed by Gruenhagen and Heidenhain. According to them, the fat takes its way chiefly through the epithelium cells. How these last act is, like the nature of the action in absorption, enveloped in darkness. 


\section{CHAPTER VIII.}

\section{TISSUES OF THE CONNECTIVE SUBSTANCE.}

\section{The Connective Tissues.}

THE form-elements of the typical connective tissues are cells of various kinds, of a not very well known chemical composition, and gelatine-yielding fibrils. Besides these, elastic formations are often found in variable amounts, though sometimes in such predominant quantities that the connective tissue passes nearly into an elastic tissue. Mucin is also found in the connective tissue, serum globulin and serum albumin occurring in the parenchymatous fluid (LOEBISCH).

If finely-divided tendons are extracted in cold water, the albuminous bodies soluble in the nutritive fluid in addition to a little mucin are dissolved. If the residue is extracted with half-saturated lime-water, then the mucin is dissolved (RoLLETT, LoEBISCH) and may be precipitated from the filtered extract by saturating with acetic acid. The digested residue contains the fibrils of the connective tissue together with the cells and the elastic substance.

The fibrils of the connective-tissue consist of collagen. They are elastic, swell slightly in water, somewhat more in diluted alkalies or acetic acid, but on the other hand are shrivelled by the action of certain metallic salts, such as ferrous sulphate or mercuric chloride, and by the action of tannic acid, which forms with collagen an insoluble combination. Among these combinations, which prevent the putrefaction of collagen, that with tannic acid has been found of great value in the preparation of leather. In regard to tendon mucin see page 32 , and in regard to collagen, glutin, and elastin see pages $36-38$. 
The tissues described under the names mucous or gelatinous tissues are characterized more by their physical than their chemical properties and have been but little studied. So much, however, is known, that the mucous or gelatinous tissues contain, at least in certain cases, as in the acalephæ, no mucin.

The nmbilical cord is the most accessible material for the investigation of the chemical constituents of the gelatinous tissues. The mucin occurring therein has been described on page 32. C. TH. Mörner has found a mucoid in the vitreous humor which contains $12.20 \%$ nitrogen and $1.19 \%$ sulphur.

\section{Cartilage.}

Cartilaginous tissue consists of cells and of a basic substance originally hyaline, which, however, may become changed in such wise that there appears in it a network of elastic fibres or connective-tissue fibrils.

Those cells that offer great resistance to the action of alkalies and acids have not been carefully studied. According to former views, the basic substance was considered as consisting of a body analogous to collagen, the so-called chondrigen, which under similar conditions passes, like collagen, into a corresponding gelatine called chondrin or cartilage-gelatine. The more recent investigations of Morochowetz and others, but especially those of C. TH. Mörnen, have shown that the basic substance of the cartilage consists of a mixture of collagen with other bodies.

The tracheal, thyroideal, cricoidal, and arytenoidal cartilages of full-grown cattle contain, according to MörNer, four constituents in the basic substance, namely, chondromucoid, chondroitic acid, collagen, and albumoid.

Chondromucoid. This body, according to Mörner, has the composition $\mathrm{C} 47.30, \mathrm{H} 6.42, \mathrm{~N} 12.58, \mathrm{~S} 2.42, \mathrm{O} 31.28$ per cent. Sulphur is in part loosely combined and may be split off by the action of alkalies, and a part separates as sulphuric acid when boiled with hydrochloric acid. Chondromucoid is decomposed by dilute alkalies and yields alkali albuminate, peptone substance, chondroitic acid, alkali sulphides, and some alkali sulphates. On 
boiling with acids it yields acid albuminate, peptone substance, chondroitic acid, and on account of the further decomposition of this last body sulphuric acid and a reducing substance are formed.

Chondromucoid is a white, amorphous, acid-reacting powder which is insoluble in water, but dissolves easily on the addition of a little alkali. This solution is precipitated by acetic acid in great excess and by small quantities of mineral acids. The precipitation may be retarded by neutral salts or by chondroitic acid. The solution containing $\mathrm{NaCl}$ and acidified with $\mathrm{HCl}$ is not precipitated by potassium ferrocyanide. Precipitants for chondromucoid are alum, ferric chloride, sugar of lead or basic lead acetate. Chondromucoid is not precipitated by tannic acid, and it may by its presence prevent the precipitation of gelatine by this acid. It gives the usual color reactions for albuminous bodies; namely, with nitric acid, with copper sulphate and alkali, with MrLlos's and ADAMkIEwICZ's reagents.

Chondroitic Acid. This acid, which thus far has not been obtained free, but as acid salts, has, according to MörNer. the following composition : $\mathrm{C} 35.28, \mathrm{H} 4.68, \mathrm{~N} 3.15, \mathrm{~S} 6.33,050.56$ per cent. On boiling with dilute hydrochloric acid all the sulphur splits off as $\mathrm{H}_{2} \mathrm{SO}_{4}$, and a reducing substance is formed at the same time whose nature is not known.

This acid (more correctly the acid alkali salts) appears as a white amorphous powder which dissolves very easily in water, forming an acid solution and when sufficiently concentrated, a sticky liquid similar to a solution of gum arabic. The neutralized solution is precipitated by tin chloride, basic lead acetate, neutral ferric chloride, and by alcohol in the presence of a little neutral salt. The solution, on the other hand is not precipitated by acetic acid, tannic acid, potassium ferrocyanide and acid, sugar of lead, mercuric chloride, or silver nitrate. Chondroitic acid does not give the color reactions for albuminous bodies.

Preparation of chondromucoid and chondroitic acid. If very finely-cut cartilage is extracted with water, the preformed chondroitic acid, as well as some chondromucoid, is dissolved. In this watery extract the chondroitic acid prevents the precipitation of this chrodromucoid by means of an acid. If $2-4 \mathrm{p} . \mathrm{m} . \mathrm{HCl}$ is added to this watery extract and warmed on the water-bath, the 
chondromucoid gradually separates, while the chondroitic acid and the rest of the chondromucoid remain in the filtrate. If the cartilage, which has been lixiviated, at the temperature of the body, with water, is extracted with hydrochloric acid of $2-3$ p. m. until the collagen is converted into soluble gelatine, the remaining chondromucoid may be removed from the insoluble residue by dilute alkali and precipitated from the alkaline extract by an acid. It may be purified by repeatedly redissolving in water with the aid of a little alkali, precipitating by an acid and then treating with alcohol and ether.

The chondroitic acid originally formed, or that formed by the decomposition of chondromucoid, is obtained by lixiviating the cartilage with a 5\% caustic-alkali solution. The alkali albuminate formed by the decomposition of the chondromucoid can be removed from the solution by neutralization, then the peptone precipitated by tannic acid, the excess of this acid removed with sugar of lead, and the lead separated from the filtrate by $\mathrm{H}_{2} \mathrm{~S}$. If further purification is necessary, the acid is precipitated with alcohol, the precipitate dissolved in water, this solution dialyzed and precipitated again with alcohol, this dissolving in water and precipitating with alcohol being repeated a few times, and lastly the acid is treated with alcohol and ether.

The collagen of the cartilage gives, according to MörNER, a gelatine which contains only $16.4 \% \mathrm{~N}$ and which can hardly be considered identical with ordinary glutin.

On the above-mentioned cartilages of full-grown animals the chondroitic acid and chondromucoid, perhaps also the collagen, are found surrounding the cells as round balls or lumps. These balls (MörNeR's chondrin-balls), which give a blue color with methyl-violet, lie in the meshes of an albumoid structure, which is colored when brought in contact with tropæolin.

This albumoid is a nitrogenized body which contains looselycombined sulphur. It is soluble with difficulty in acids and alkalies, and resembles keratin in many respects, but differs from it by being soluble in gastric juice. In other respects it is more similar to elastin, but differs from this substance by containing sulphur. This albumoid gives the color reactions of the albuminous bodies.

The preparation of cartilage-gelatine and albumoid may be performed according to the following method of MörNER. First remove the chondromucoid and chondroitic acid by extraction with dilute caustic potash $(0.2-0.5 \%)$, remove the alkali from the re- 
maining cartilage by water, and then boil with water in a PAPIN's digestor. The collagen passes into solution as gelatine, while the albumoid remains undissolved (contaminated by the cartilage-cells). The gelatine may be purified by precipitating with sodium sulphate, which must be added to saturation in the faintly-acidified solution, redissolving the precipitate in water, dialyzing well, and precipitating with alcohol.

According to Mörner, no albumoid is found in young cartilage, but only the three first-mentioned constituents. Nevertheless the young cartilage contains about the same amounts of nitrogen and mineral substances as the old.

HOPPE-SEYLER found in fresh rib cartilage 676.7 p. m. water, $301.3 \mathrm{p}$. m. organic and $22 \mathrm{p}$. m. inorganic substance. In the cartilage of the knee-joint 735.9 p. m. water, 248.7 p. m. organic and $15.4 \mathrm{p}$. m. inorganic substance have been found. The ash of cartilage contains considerable amounts (even 800 p. m.) of alkali sulphate, which probably did not exist originally as such, but is produced in great part by the calcining of the chondroitic acid and the chondromucoid. The analyses of the ash of cartilage therefore cannot give a correct idea of the quantity of mineral bodies existing in this substance. Petersen and Soxhlet have found 940 p. m. $\mathrm{NaCl}$ in the ash from the cartilage of a shark, and such cartilage can scarcely contain quantities of chondromucoid or chondroitic acid worth mentioning. The cartilage of the ray (Raja batis LIN.), which has been investigated by LöNNBERG, contains no albumoid and only a little chondromucoid, but a large proportion of chondroitic acid and collagen.

The Cornea. The corneal tissue, which is considered by many investigators to be related to cartilage in a chemical sense, contains traces of albumin and a collagen as chief constituent, which $\mathrm{C}$. Tr. MörNER claims contains $16.94 \% \mathrm{~N}$. According to him it contains, a mucoid which has the composition C 50.16, H 6.98, N 12.8, and $\mathrm{S} 2.05$ per cent. On boiling with dilute acid this mucoid yields a reducing substance.

In the cornea of oxen His found $758.3 \mathrm{p} . \mathrm{m}$. water, $203.8 \mathrm{p} . \mathrm{m}$. gelatin forming substance, 28.4 p. m. other organic substance, besides $8.1 \mathrm{p}$. m. soluble and $1.1 \mathrm{p} . \mathrm{m}$. insoluble salts. 


\section{Bone.}

The bony structure proper, when free from other formations occurring in bones, such as marrow, nerves, and blood-ressels, consists of cells and a basic substance.

The cells have not been closely studied in regard to their chemical constitution. On boiling with water they yield no gelatine. They contain no keratin, which is not usually present in the bony structure (HeRbert SMITH), but they may contain a substance which is similar to elastin.

The basic substance of the bony structure contains two chief constituents, namely, an organic substance, ossein, and the so-called bone-earths enclosed in or combined with it. If bones are treated with dilute hydrochloric acid at the ordinary temperature, the limesalts are dissolved and the ossein remains as an elastic mass, preserving the shape of the bone. This ossein is generally considered identical with the collagen of the connective tissue. The ossein in the bones of certain aquatic fowls and fishes can hardly be considered identical with this collagen (FrEMY).

The inorganic constituents of the bony structure, the so-called bone-earths, which remain after the complete calcination of the organic substance as a white, brittle mass, consist chiefly of calcium and phosphoric acid, but also include carbon dioxide and, in smaller amounts, magnesium, chlorine, and fluorine. Alkali sulphate and iron, which are found in the bone-ash, do not seem to belong exactly to the bony substance, but to the nutritive fluids or to the other constituents of bones.

The opinions of investigators differ somewhat as to the manner in which the mineral bodies of the bony structure are combined with each other. Chlorine and fluorine are present in the same form as in apatite $\left(\mathrm{CaFl}_{2}, 3 \mathrm{Ca}_{3} \mathrm{P}_{2} \mathrm{O}_{8}\right)$. Proceeding from the chlorine and fluorine, it is possible that the other mineral bodies form the combination $3\left(\mathrm{Ca}_{3} \mathrm{P}_{2} \mathrm{O}_{8}\right) \mathrm{CaCO}_{3}$.

Analyses of bone-earths have shown that the mineral constituents are related to each other in nearly constant proportions, and this applies not only to man but also to the different animals. As 
example of the constitution of bone-earth we give here the analyses of ZALEWSKY. 'The figures represent parts per thousand.

\begin{tabular}{|c|c|c|c|c|}
\hline & Man. & Ox. & Tortolse. & \\
\hline phosphate, $\mathrm{Ca}_{3} \mathrm{~F}$ & 838.9 & 860.9 & 859.8 & 873.8 \\
\hline hosphate, $\mathrm{Mg}_{3} \mathrm{P}_{2} \mathrm{O}_{8} \ldots \ldots$ & 10.4 & 10.2 & 13.6 & 10.5 \\
\hline conbined with $\mathrm{CO}_{2}, \mathrm{Fl}$, and $\mathrm{Cl}$. & 76.5 & 73.6 & 63.2 & 70.3 \\
\hline$\ldots \ldots \ldots \ldots, \ldots, \ldots, \cdots$ & 57.3 & 62.0 & 52.7 & \\
\hline ne.............. & 1.8 & 2.0 & & 1.3 \\
\hline luorine...$\ldots \ldots \ldots$. & 2.3 & 3.0 & 2.0 & \\
\hline
\end{tabular}

Some of the $\mathrm{CO}_{2}$ is always lost on calcining, so that the bone-ash does not contain the entire $\mathrm{CO}_{2}$ of the bony substance.

The quantity of organic substance in the bones, calculated from the loss of weight in burning, varies somewhat between 300 and $520 \mathrm{p} . \mathrm{m}$. This variation may in part be explained by the difficulty in obtaining the bony substance entirely free from water, and partly by the very variable amount of blood-vessels, nerves, marrow, and the like in different bones. The unequal amounts of organic substance found in the compact and in the spongy parts of the same bone, as well as in bones at different periods of development in the same animal, depend probably upon the varying quantities of these above-mentioned formations. Dentin, which is comparatively pure bony structure, contains only 260 to $280 \mathrm{p}$. m. organic substance, and HOPPE-SEYLER therefore thinks it probable that entirely pure bony substance has a constant composition and contains only about $250 \mathrm{p}$. m. organic substance. The question whether these substances are chemically combined with the bone-earths or only intimately mixed has not been decided.

The nutritive fluids which circulate through the bones have not been isolated, and we only know that they contain some albumin and some $\mathrm{NaCl}$ and alkali sulphate. The yellow marrow contains chiefly fat, which consists of olein, palmitin, and stearin. Albumin has been found especially in the socalled red marrow of the spongy bones. Besides these substances, the marrow contains so-called extractive bodies, such as lactic acid, hypoxanthin, and cholesterin, but mostly bodies of an unknown character.

The diverse quantitative composition of the various bones of the skeleton depends probably on the varying quantities of other formations, such as marrow, blood-vessels, etc., they contain. The same reason explains, to all appearances, the larger quantity of organic substance in the spongy parts of the bones as compared with the more compact parts. ScHRODT has made comparative analyses of different parts of the skeleton of the same animal (dog) 
and has found an essential difference. The quantity of water in the fresh bones varies between 138 and $443 \mathrm{p} . \mathrm{m}$. The bones of the extremities and the skull contain 138-222, the vertebræ 168443 , and the ribs $324-356 \mathrm{p}$. m. water. The quantity of fat varies between 13 and 269 p. m. The largest amount of fat, 256-269 p. m., is found in the long tubular bones, while only $13-175 \mathrm{p} . \mathrm{m}$. fat is found in the small short bones. The quantity of organic substance, calculated from fresh bones, was 150-300 p. m., and the quantity of mineral substances $290-563 \mathrm{p}$. m. Contrary to the general supposition the greatest amount of bone-earths was not found in the femur, but in the three first cervical vertebræ. In geese the largest amount of bone-earth was found in the humerns (HILLER).

We do not possess trustworthy statements in regard to the composition of bones at different ages. There is no question, however, that the mineral constituents increase until a certain age is reached, at which time the bones attain their necessary solidity. On the other hand, it is not certainly known whether this increase stops at a certain point or whether, as was formerly thought, it continues, even if slowly, uninterrupted from childhood to old age.

The composition of bones of animals of different species is but little known. The bones of birds contain, as a rule, somewhat more water than those of mammalia, and the bones of fishes contain the largest quantity of water. The bones of fishes and amphibians contain a greater amount of organic substance. The bones of pachydermata and cetaceæ contain a large proportion of calcium carbonate ; those of granivorous birds always contain silicic acid. The bone-ash of amphibians and fishes contains sodium sulphate. The bones of fishes seem to contain more soluble salts than the bones of other animals.

A great many tests have been made to determine the exchange of material in the bones-for instance, with food rich in lime and with food deficient in lime-but the results have always been doubtful or contradictory. The attempts, also, to substitute other alkaline earths or clay for the lime of the bones have given contradictory results. KRAPP found that the bones of the animals under experimentation were tinged with red after a few days or weeks; but these tests have not led to any positive conclusion in regard to the growth or exchange of material in the bones.

Under pathological conditions, as in rachitis and softening of the bones, an ossein has been found which does not give any 
typical gelatine on boiling with water. Otherwise the pathological conditions seem to affect chiefly their quantitative composition, and especially the relationship between the organic and the inorganic substance. In exostosis and osteomalacosis the quantity of organic substance is generally increased. Attempts have been made to produce rachitis in animals by the use of food deficient in lime. From experiments on fully-developed animals contradictory results have been obtained. In young, undeveloped animals ERwiN VoIT produced, by lack of lime-salts, a change similar to rachitis. In full-grown animals the bones were changed after a long time because of the lack of lime-salts in the food, but did not become soft, only thinner, and atrophied. The experiment of removing the lime-salts from the bones by the addition of lactic acid to the food has led to no positive results (Heitzmans, Heiss, Baginsky). A few investigators are of the opinion that in rachitis, as in osteomalacosis, a solution of the lime-salts by means of lactic acid takes place. 'This was suggested by the fact that 0 . WEBER and C. Schmidt found lactic acid in the cyst-like, altered bony substance in osteomalacia.

Well-known investigators have disputed the possibility of the lime-salts being washed from the bones in osteomalacosis by means of lactic acid. They have given special prominence to the fact that the lime-salts held in solution by the lactic acid must be deposited on neutralization of the acid by the alkaline blood. This objection is not very important, as the alkaline stream of blood has the property to a high degree of holding earthy phosphates in solution, which can be easily proved.

In rachitis the amount of organic substance has been found to vary between 664 and $811 \mathrm{p}$. m. The quantity of inorganic substance was $189-336 \mathrm{p} . \mathrm{m}$. In opposition to rachitis, osteomalacosis is often characterized by a considerable amount of fat in the bones, $230-290 \mathrm{p}$. m.; but as a rule the composition varies so much that the analyses are of little value.

The tooth-structure is nearly related, from a chemical stand- point, to the bony structure.

Of the three chief constituents of the teeth, dentin, enamel, and cement, the last-mentioned, the cement, is to be considered as true bony structure, and as such has been spoken of to a certain extent. Dentin has the same composition as the bony structure, 
but contains somewhat less water. The organic substance yields gelatine on boiling; but the dental tubes are not dissolved, therefore they cannot consist of collagen. In dentin 260-280 p. m. organic substance has been found. Enamel is an epithelium for mation containing a large proportion of lime-salts. The fact that the organic substance of enamel does not yield any gelatine corresponds to its nature and origin. Completely-developed enamel contains the least water, the greatest quantity of mineral substances, and is the hardest of all the tissues of the body. In fullgrown animals it contains hardly any water, and the amount of organic substance amounts to only $20-40 \mathrm{p}$. m. The relative amounts of calcium and phosphoric acid are, according to the analyses of HOPPE-SEYLER, about the same as in bone-earths. According to the determination of Berzelius, the enamel may contain 40 p. m. calcium fluoride.

\section{The Fatty Tissue.}

The membranes of the fat-cells withstand the action of alcohol and ether. They are not dissolved by acetic acid nor by dilute mineral acids, but are dissolved by artificial gastric juice. They may possibly consist of a substance closely related to elastin. The contents of the fat-cells are fluid during life, but solidify after death and become more or less solid, depending upon the character of the fats. Besides fat, the fat-cells contain a yellow pigment which in emaciation does not disappear so rapidly as the fat; and this is the reason that the subcutaneous cellular tissue of an ema. ciated corpse has a dark orange-red color. The cells deficient in or nearly free from fat, which remain after the complete disappearance of the latter, seem to have an albuminous protoplasm rich in water.

The less water the fatty tissue contains, the richer it is in fat. SCHULTZE and REINECKE found in 1000 parts:

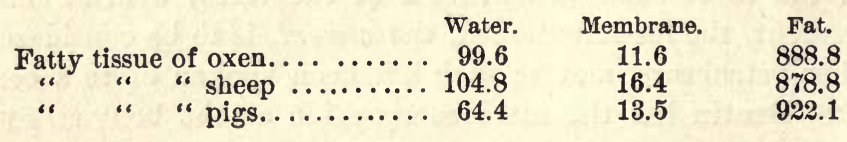


The fat contained in the fat-cells consists chiefly of triglycerides of stearic, palmitic, and oleic acids. Besides these, especially in the less solid kinds of fat, there are glycerides of caproic, valerianic, and other fatty acids which have not been so closely investigated. In all animal fats there are besides these, as Hormans has shown, free, non-volatile fatty acids, although in very small amounts. Fats from different species of animals, and even from different parts of the same animal, have an essentially different consistency, depending upon the relative amounts of the different fats. In solid fats-as tallow-tristearin and tripalmitin are in excess, while the less solid fats are characterized by a greater abundance of palmitin and triolein. This last-mentioned fat is found in greater quantities proportionally in cold-blooded animals, and this accounts for the fat of these animals remaining fluid at temperatures at which the fat of warm-blooded animals solidifies. Human fat from different organs and tissues contains, in round numbers, 670 800 p. m. olein. The melting-point of different fats depends upon the composition of the mixtures, and it not only varies for fat from different tissues of the same animal, but also for the fat from the same tissues in various kinds of animals.

Fat occurs in all organs and tissues of the animal organism, though the quantity may be so variable that a tabular exhibit of the amount of fat in different organs is of little interest. The marrow contains the largest quantity proportionally, having over $960 \mathrm{p} . \mathrm{m}$. The three most important deposits of fat in the animal organism are the intermuscular connective tissue, the fatty tissue in the abdominal carity, and the subcutaneous connective tissues.

The average composition of animal fat is as follows: C 76.5, $H$ 12.0, and 011.5 per cent. Neutral fats are colorless or yellowish and, when perfectly pure, odorless and tasteless. They are lighter than water, on which they float when in a molten condition. They are insoluble in water, dissolve in boiling alcohol, but separate on cooling,- - often in crystals. They are easily soluble in ether, benzol, and chloroform. The fluid neutral fats give an emulsion when shaken with a solution of gum or albumin. With water alone they give an emulsion only after vigorous and prolonged shaking, but the emulsion is not persistent. The presence of some soap causes a very fine and permanent emulsion to form 
easily. Fat produces spots on paper which do not disappear; it is not volatile; it boils at about $300^{\circ} \mathrm{C}$. with partial decomposition, and burns with a luminous and smoky flame. The fatty acids have most of the above-mentioned properties in common with the neutral fats, but differ from them in being soluble in alcohol-ether, in having an acid reaction, and by not giving the acrolein test. The neutral fats generate a strong irritating vapor of acrolein, due to the decomposition of glycerine, $\mathrm{C}_{3} \mathrm{H}_{5}(\mathrm{OH})_{3}-2 \mathrm{H}_{2} \mathrm{O}=\mathrm{C}_{3} \mathrm{H}_{4} \mathrm{O}$, when heated alone, or more easily when heated with potassium bisulphate or with other substances removing water.

The neutral fats may be split by the addition of the constituents of water according to the following equation: $\mathrm{C}_{3} \mathrm{H}_{5}(\mathrm{OR})_{3}+$ $3 \mathrm{H}_{2} \mathrm{O}=\mathrm{C}_{3} \mathrm{H}_{5}(\mathrm{OH})_{3}+3 \mathrm{HOR}$. This splitting may be produced by the pancreatic enzyme or by superheated steam. We most frequently decompose the neutral fats by boiling them with caustic alkali not too concentrated, or, still better (in zoochemical researches), with an alcoholic potash solution. By this procedure, which is called saponification, the alkali salts of the fatty acids (soaps) are formed. If the saponification is made with lead oxide, then lead-plaster, lead-salts of the fatty acids, is produced.

Stearin, or TRISTEARIN, $\mathrm{C}_{3} \mathrm{H}_{5}\left(\mathrm{C}_{18} \mathrm{H}_{35} \mathrm{O}_{2}\right)_{3}$, occur's especially in the solid varieties of tallow, but also in the vegetable fats.

Stearic acid, $\mathrm{C}_{18} \mathrm{H}_{36} \mathrm{O}_{2}$, is found in the free state in decomposed pus, in the expectorations in gangrene of the lungs, and in cheesy tuberculous masses. It occurs as lime-soap in excrements and adipocere, and in this last product also as an ammonia soap. It perhaps exists as sodium soap in the blood, transudations, and pus.

Stearin is the hardest and most insoluble of the three ordinary neutral fats. It is nearly insoluble in cold alcohol and soluble with great difficulty in cold ether (225 parts). It separates from warm alcohol on cooling as rectangular, less frequently as rhombical plates. In regard to the melting-point, which may be changed by alternately warming and cooling, opinions are somewhat divergent; for stearin from the fatty tissues it is often stated as $63^{\circ} \mathrm{C}$.

Stearic acid crystallizes (on cooling from boiling alcohol) in large, shining, long-rhombical scales or plates. It is less soluble than the other fatty acids and melts at $69.2^{\circ} \mathrm{C}$. Its barium salt contains $19.49 \%$ barium. 
Palmitin, tripalmitin, $\mathrm{C}_{3} \mathrm{H}_{5}\left(\mathrm{C}^{16} \mathrm{H}_{31} \mathrm{O}_{2}\right)_{3}$. Of the two solid varieties of fats, palmitin is the one which occurs in predominant quantities in human fat (LANGER). Palmitin is present in all animal fats and in several kinds of vegetable fats. A mixture of sterin and palmitin was formerly called MARGARIN. ${ }^{1}$

Palmitic acid, $\mathrm{C}_{16} \mathrm{H}_{32} \mathrm{O}_{2}$. As to occurrence about the same remarks apply as to stearic acid. The mixture of these two acids has been called margaric acid, and this mixture occurs-often as very long, thin, crystalline plates-in old pus, in expectorations from gang. rene of the lungs, etc.

Palmitin crystallizes, on cooling from a warm saturated solution in ether or alcohol, into starry rosettes of fine needles. The mixture of palmitin and stearin, called margarin, crystallizes, on cooling from a solution, as balls or round masses which consist of short or long, thin plates or needles which often appear like blades of grass. Palmitin, like stearin, has a variable melting and solidifying point, depending upon the way it has been previously treated. The melting-point is often given as $+62^{\circ}$ and the solidifying-point as $+45^{\circ} \mathrm{C}$.

Palmitic acid crystallizes from an alcoholic solution in tufts of fine needles. It melts at $+62^{\circ} \mathrm{C}$; ; still the admixture with stearic acid, as Heintz has shown, essentially changes the melting and solidifying points according to the relative amounts of the two acids. Palmitic is somewhat more soluble in cold alcohol than stearic acid; but they have about the same solubility in boiling alcohol, ether, chloroform, and benzol.

Olein, TRIOLEIN, $\mathrm{C}_{3} \mathrm{H}_{5}\left(\mathrm{C}_{18} \mathrm{H}_{33} \mathrm{O}_{2}\right)_{3}$, is present in all animal fats and in greater quantities in plant fats. It is a solvent for stearin and palmitin. Oleic acid, ELAIC ACID, $\mathrm{C}_{18} \mathrm{H}_{34} \mathrm{O}_{2}$, occurs probably as soaps in the intestinal canal during digestion and in the chyle.

Olein is, at ordinary temperatures, a nearly colorless oil of a specific gravity of 0.914 , without odor or marked taste. It solidifies in crystalline needles at $-5^{\circ} \mathrm{C}$. It becomes rancid quickly if exposed to the air. It dissolves with difficulty in cold alcohol, but more easily in warm alcohol or in ether. It is converted into its isomer, ELAIDIN, by nitrous acid.

1 This mixture must not be confounded with the synthetically-prepared neutral fat called trimargarin. 
Oleic acid forms at ordinary temperature a colorless, tasteless, and odorless oily liquid which crystallizes at about $+4^{\circ}$ C. On being heated it yields, besides volatile fatty acids, SEBACIC ACID; $\mathrm{C}_{10} \mathrm{H}_{18} \mathrm{O}_{4}$, which crystallizes in shining plates and melts at $f-127^{\circ} \mathrm{C}$. Oleic acid is converted by nitrous acid into its isomer, ELAIDIC ACID, which is a solid, melting at $+45^{\circ} \mathrm{C}$. Oleic acid is insoluble in water but dissolves in alcohol, ether, and chloroform. With concentrated sulphuric acid and some cane-sugar it gives a beautiful red or reddish-violet liquid whose color is similar to that produced in Pettenkofer's test for bile-acids.

If the watery solution of the alkali combinations of oleic acid is precipitated with lead acetate, a white, tough, sticky mass of oleate of lead is obtained which is not soluble in water and only slightly in alcohol, but is soluble in ether (differing from the lead-salts of the other two fatty acids).

An acid related to oleic acid, DoglingIC ACID, which is solid at $0^{\circ} \mathrm{C}$., liquid at $+16^{\circ}$, and soluble in alcohol, is found in the blubber of the BALANA Rostrata.

To detect the presence of fat in an animal fluid or tissue the fat must first be extracted with ether. After the evaporation of the ether the residue is tested for fat and the acrolein test must not be neglected. If this test gives positive results, then neutral fats are present; if the results are negative, then only fatty acids are present. If the above residue after evaporation gives the acrolein test, then a small portion is dissolved in alcohol-ether free from acid and which has been colored bluish violet by tincture of alkanet. If the color becomes red, a mixture of neutral fat and fatty acids is present. In this case the fat is treated in the warmth with a soda solution and evaporated on the water-bath, constantly stirring until all the water is removed The fatty acids are hereby combined with the alkali as soaps, while the neutral fats are not saponified under these conditions. If this mixture of soaps and neutral fats is treated with water and then shaken with pure ether, the neutral fats are dissolved, while the soaps remain in the watery solution. The fatty acids may be separated from this solution by the addition of a mineral acid which sets the acid free.

The neutral fats separated from the soaps by means of ether are contaminated with some cholesterin, which must be separated in quantitative determinations by saponification with alcoholic caustic potash.

The cholesterin is not attacked by the caustic alkali while the neutral fats are saponified. After the evaporation of the alcohol 
the residue is dissolved in water and shaken with ether, which dissolves the cholesterin. The fatty acids are separated from the watery solution of the soaps by the addition of a mineral acid. If a mixture of soaps, neutral fats, and fatty acids is originally present, it is treated first with water, then agitated with ether free from alcohol, which dissolves the fat and fatty acids, while the soaps remain in the solution, with the exception of a very small amount which is dissolved by the ether.

To detect and to separate the different varieties of neutral fats from each other it is best first to saponify them with alcoholic potash. After the evaporation of the alcohol they are dissolved in water and precipitated with sugar of lead. The oleate of lead is then separated firom the other two lead-salts by repeated extraction with ether. 'The residue insoluble in ether is decomposed on the water-bath with an excess of soda solution, evaporated to dryness, finely pulverized, and extracted with boiling alcohol. 'The alcoholic solution is then fractionally precipitated by barium acetate or barium chloride. In one fraction the amount of barium is determined, and in the other the melting-point of the fatty acid set free by a mineral acid. The fatty acids occurring originally in the animal tissues or fluids as free acids or as soaps are converted into barium salts and investigated as above.

The derivation of the fats in the organism may occur in various ways. The fat of the animal body may consist partly of absorbed fat of the food deposited in the tissues and partly of fat formed in the organism from other bodies, such as albuminous bodies or carbohydrates.

That the fat of the food which is absorbed in the intestinal canal may be retained by the tissues has been shown in several ways. LEBEDEFF and MUNK have fed dogs with various fats, such as linseed-oil, mutton-tallow, and rape-seed-oil, and have afterwards found the administered fat in the tissues. Hormans starved dogs until they appeared to have lost their fat, and then fed them upon large quantities of fat and only little proteids. When the animals were killed he found so large a quantity of fat that it could not have been formed from the administered proteids alone, but the greatest part must have been derived from the fat of the food. Pettenkofer and Voit arrived at similar results in regard to the behavior of the absorbed fats in the organism, though their experi. ments were of another kind. Munk has found that on feeding with free fatty acids, these are deposited in the tissues, not, how- 
ever, as such; but they are transformed by synthesis with glycerin into neutral fats on their passage from the intestines to the thoracic duct. According to EwALD, such a synthesis may be produced by the surviving mucous membrane of the intestine.

Albuminous bodies and carbohydrates may be considered as the mother-substance of the fats formed in the organism.

The formation of the so-called CORPSE-WAX, ADIPOCERE, into which parts of the corpse rich in proteids are sometimes converted, and which consists of abundant quantities of fatty acids, ammonia, and lime-soaps, is often given as a proof of the formation of fats from proteids. The provableness of this observation has, however, been disputed, and many other explanations of the formation of this substance have been offered. According to the recent experiments of Kratter and K. B. Lehmann, it seems as if it were possible by experimental means to convert animal tissue rich in proteids (muscles) into adipocere by the continuous action of water. That the formation of fatty acids in this case actually takes place at the expense of the proteids may be inferred from the investigations of LehmanN.

Another proof of the formation of fat from proteids, taken from pathological chemistry, is fatty degeneration. On this point also all investigators are not united; but the investigations of BAUER seem to show that at least in acute poisoning by phosphorus the fatty degeneration actually consists of a formation of fat from proteids.

As a more direct proof of fat-formation from proteids the investigations of Petrenkofer and Vort are often quoted. These investigators fed dogs with large quantities of meat containing the least possible proportion of fat, and found all of the nitrogen in the excreta but only a part of the carbon. As an explanation of these conditions it has been assumed that the proteid of the organism splits into a nitrogenized and a non-nitrogenized part, the former changing into the nitrogenized final product, urea, the other, on the contrary, being retained in the organism as fat (PETTENKOFER and VOIT).

Another more direct proof of a fat formation from proteid has been given by Hofmans. He experimented with fly-maggots. A number of these were killed and the quantity of fat determined. 
The remainder were allowed to develop in blood whose proportion of fat had been previously determined, and after a certain time they were killed and analyzed. He found in them from 7 to 19 times as much fat as the maggots first analyzed and the blood together contained.

While, then, in view of this experiment, the question of the formation of fat from proteids can hardly be disputed, we do not yet know the maximum amount of fat formed from the proteids nor the chemical processes concerned in its formation. DrEchsel, mindful of the products which are formed by the decomposition of albumin with barium hydrate, has called attention to the fact that the albumin molecule probably originally contains no radical with more than six or nine carbon atoms. If fat is formed from albumin in the animal body, then, according to DRECHSEL, such formation is not a splitting off from the albumin, but rather a synthesis from primarily-formed splitting products of albumin which are deficient in carbon.

The theory of the formation o fat from carbohydrates in the animal body was first adopted by LIEBIG. This was combated for some time, however, and until lately it was the general opinion that a direct formation of fat from carbohydrates had not been proved, and also that it was improbable. The undoubtedly great influence which Liebig has shown the carbohydrates to exert on the formation of fat has been explained by C. v. VorT upon the assumption that these carbohydrates are consumed instead of the fat absorbed or formed from the albumins, and therefore have an action tending to economize the fat. By means of a series of nutrition tests with foods especially rich in carbohydrates, LAwes and GILBERT, Soxhlet, Tscherwinsky, Meissl and Stromer (on pigs), B. Schultze, Chaniewski, E. Voit and C. Lehmann (on geese), J. MUNK and M. RUBNER (on dogs) apparently prove that a direct formation of fat from carbohydrates does actually occur. The processes by which this formation takes place are still unknown. As the carbohydrates do not contain as complicated a molecule as the fats, the formation of fat from carbohydrates must consist of a synthesis, in which, the group $\mathrm{CHOH}$ is converted into $\mathrm{CH}_{2}$, a reduction must also take place.

When food contains an excess of fat, the superfluous amount is 
stored up in the fatty tissue, and on partaking of food deficient in fat this accumulation is quickly exhausted. There is perhaps no one of the various tissues that decreases so much in starvation as the fatty tissue. The organism, then, possesses in this tissue a depot where there is stored during proper alimentation a substance of great importance in the development of heat and vital force, which substance, on insufficient nutrition, is given off as may be needed. The fatty tissues, on account of their low conducting power, become of great importance in regulating the loss of heat from the body. They also serve to fill cavities, and as a protection and support to certain internal organs.

\section{Appendix to the Fatty Tissue.}

Spermaceti. In the living spermaceti or white whale there is found in a large cavity in the skull an oily liquid called spermaceti, which on cooling after death separates into a solid crystalline part, ordinarily called SPERMACET, and into a liquid, spermacetr-orl. This last is separated by pressure. Spermaceti is also found in other whales and in certain species of dolphin.

'The purified, solid spermaceti, which is called CETIN, is a mixture of esters of fatty acids. The chief constituent is the cetyl palmitic ester mixed with small quantities of compound ethers of lauric, myrisitic, and stearic acids with radicals of the alcohols, LETHAL, $\mathrm{C}_{12} \mathrm{H}_{26} . \mathrm{OH}$, METHAL, $\mathrm{C}_{14} \mathrm{H}_{29} . \mathrm{OH}$, and STETHAL, $\mathrm{C}_{18} \mathrm{H}_{37}$. OH.

Cetin is a snow-white mass shining like mother-of-pearl, crystallizing in plates, brittle, fatty to the touch, and which has a varying melting-point of $+30^{\circ}$ to $50^{\circ} \mathrm{C}$., depending upon its purity. Cetin is insoluble in water, but dissolves easily in cold ether or volatile and fatty oils. It dissolves in boiling 'alcohol, but crystallizes on cooling. It is saponified with difficulty by a solution of caustic potash in water, but with an alcoholic solution it saponifies readily and the above-mentioned alcohols are set free.

Ethal, or cetyl alcohol, $\mathrm{C}_{18} \mathrm{H}_{38} . \mathrm{OH}$, which also occurs in the coccygeal gland of ducks and geese (DE JONGE) and in smaller quantities in beeswax, forms white, transparent, odorless, and tasteless crystals which are insoluble in water but dissolve easily in alcohol and ether. Ethal melts at $49.5^{\circ} \mathrm{C}$.

SPERMACETi-oIL yields on saponification valerianic acid, small amounts of solid fatty acids, and PHYSETOLEIC ACID. This acid forms colorless and odorless, needle-shaped crystals which easily dissolve in alcohol and ether and melt at $+34^{\circ} \mathrm{C}$.

BEESWAX may be treated here as concluding the subject of fats. It contains three chief constituents. 1. CEROTIC ACID, $\mathrm{C}_{27} \mathrm{H}_{54} \mathrm{O}_{2}$, which occurs as cetyl ether in chinese wax and as free acid in ordinary wax. It dissolves in boiling alcohol and separates as crystals on cooling. The cooled alcoholic extract of wax contains (2) CEROLEIN, which is probably a mixture of several bodies, and (3) MYRISIN, which forms the chief constituent of that part of wax which is insoluble in warm or cold alcohol. Myrisin consists chiefly of palmitic-acid ether of melissyl (myricyl) alcohol, $\mathrm{C}_{80} \mathrm{H}_{61} . \mathrm{OH}$. This alcohol is a silky, shining, crystalline body melting at $+85^{\circ} \mathrm{C}$. 


\title{
CHAPTER IX.
}

\author{
MUSCLE.
}

\section{Striated Muscles.}

In the study of the muscles the chief problem for physiological chemistry is to isolate their different morphological elements and to investigate each element separately. By reason of the complicated structure of the muscles this has been thus far almost impossible, and we must be satisfied at the present time with the investigation of the chemical composition of the muscular fibres and with a few micro-chemical reactions.

Each muscle-tube or muscle-fibre consists of a sheath, the SARCOLEMMA, which apparently is composed of a substance similar to elastin, and the contents containing a large proportion of ALbUMIns. This last-mentioned substance, which in life is possessed of contractile force, has in the inactive muscle an alkaline reaction, or, more correctly speaking, an amphoteric reaction with a predominating action on red litmus-paper. This reaction depends on a mixture of mono- and di-potassium phosphate with a preponderance of diphosphate. The dead muscle has, on the contrary, an acid reaction depending on the presence of potassium monophosphate and free lactic acid.

If we disregard the disputed statements relative to the finer structure of the muscles, we can differentiate in the striated muscles between the two chief components, the doubly refracting-anisotropous - and the singly refracting-isotropous-substance. If the muscular fibres are treated with reagents which dissolve albumins, such as dilute hydrochloric acid, soda solution, or gastric juice, they swell greatly and break up into "Bowman's disks." By the action of alcohol, chromic acid, boiling water, or in general such 
reagents as cause a shrinking, the fibres split longitudinally into fibrils; and this behavior shows that several chemically different substances of various solubilities enter into the construction of the muscular fibres.

The albuminous body, myosin, is generally considered as the principal constituent of the diagonal disks, consisting of a doublyrefracting substance, while the chief mass of the remaining albuminous bodies found in the muscles, also most of the extractive matter, is contained in the isotropous substance. According to the observations of DANILEWSKY, myosin may be completely extracted from the muscle without changing its structure, by the use of a 5\% solution of ammonium chloride. This is contrary to the above statements. DANILEWSKY claims that in the structure of the muscles another substance enters, similar to albumin, which has not been closely studied and which does not dissolve in ammonium chloride solution, but only expands. Albuminous bodies constitute an important part of the muscular structure, of which it forms the chief mass of its solids.

\section{Albuminous Bodies of the Muscles.}

Like the blood which contains a fluid, the blood-plasma, which spontaneously coagulates, separating fibrin and yielding blood-serum, so also the living muscle contains, as first shown by KüHNE, a spontaneously coagulating liquid, the muscle-plasma, which coagulates quickly, separating an albuminous body, myosin, and yielding also a serum. That liquid which is obtained by pressing the living muscle is called muscle-plasma, while that obtained from the dead muscle is called muscle-serum. These two fluids also contain different albuminous bodies.

The muscle-plasma was first prepared by KüHNE from frogmuscles, and lately HaLlibuRTon has prepared it according to the same method from the muscles of warm-blooded animals, especially rabbits. The principle of this method is as follows: The blood is removed from the muscles immediately after the death of the animal by passing through them a strongly-cooled common-salt solution of 5 to $6 \mathrm{p}$. $\mathrm{m}$. Then the quickly-cut muscles are immediately thoroughly frozen so that they can be ground in this state to a fine mass - " muscle-snow." This pulp is strongly pressed in the cold, and 
the liquid which drips off, the muscle-plasma, is faintly yellowish in color, alkaline, and spontaneously but slowly coagulates at a little above $0^{\circ} \mathrm{U}$., but very quickly at the temperature of the body. In the muscle-plasma of the frog the reaction does not change immediately with the coagulation, but the alkaline reaction is gradually changed into an acid one. The liquid which is pressed from the clot, the muscle-serum, is always acid. The albuminous body which forms the clot has been called myosin. Besides this, another albuminous body, musculin or paramyosin (HALLIBURTON), is found in the clot.

Myosin was first discovered by KÜHNE, and constitutes the principal mass of the albuminous bodies of the dead muscle, and according to a few investigators it forms the greatest part of the contractile protoplasma. The statements as to the occurrence of myosin in other organs besides the muscles require further proof. The amount of myosin in the muscles of different animals varies, according to DANILEWSKY, between $30-110 \mathrm{p}$. m.

Myosin is a globulin whose elementary composition, according to Chittenden and Cummins, is, on an average, the following : C 52.82, $\mathrm{H} \% .11, \mathrm{~N} 16.1 \%, \mathrm{~S} 1.2 \%, 022.03$ per cent. If the myosin separates as fibres, or if a myosin solution with a minimum quantity of alkali is allowed to evaporate on a microscope-slide to a gelatinous mass, doubly-refracting myosin may be obtained. Myosin has the general properties of the globulins. It is completely precipitated by saturating with $\mathrm{NaCl}$, also by $\mathrm{MgSO}_{4}$, in a solution containing $94 \%$ of the salt with its water of crystallization (HALLI. BURTON). Like fibrinogen it coagulates at $+56^{\circ} \mathrm{C}$. in a solution containing common salt, but differs from it since under no circumstances can it be converted into fibrin. "The coagulation temperature, according to CHITTENDEN and CuMmins, not only varies for myosin of different origin, but also for the same myosin in different salt solutions.

Myosin may be prepared in the following way, as suggested by HALliburton. The muscle is first extracted by a 5\% magnesium sulphate solution. The filtered extract is then treated with magnesium sulphate in substance until $100 \mathrm{cc}$. of the liquid contains about 50 grms. of the salt. The so-called paramyosin or musculin separates. The filtered liquid is then treated with magnesium 
sulphate until each $100 \mathrm{cc}$. of the liquid holds 94 grms. of the salt in solution. The myosin which now separates is filtered, dissolved in water by aid of the retained salt, precipitated by diluting with water, and, when necessary, purified by redissolving in dilute-salt solution and precipitating with water.

The older and perhaps the usual method of preparation consists, according to DANILEWSKY, in extracting the muscle with a 5-10\% ammonium-chloride solution, precipitating the myosin from the filtrate by strongly diluting with water, redissolving the precipitate in ammonium-chloride solution, and the myosin obtained from this solution is either reprecipitated by diluting with water or by removing the salt by dialysis.

As the coagulation of the blood-plasma is considered by most investigators as an enzymotic process, so certain observations seem to show that the coagulation of the muscle-plasma is an analogous process. From muscles which had been kept for a long time in alcohol HALLIBURToN obtained, by extracting the mass with water, a soluble substance contaminated with albumose which, although not identical with fibrin ferment, had the property of accelerating the coagulation of the muscle-plasma. This substance was called by him "myosin-ferment."

As in the blood-plasma we have a mother-substance of fibrin, fibrinogen, so also it is considered that in the muscle-plasma we have a mother-substance of myosin, myosinogen. This body has not thus far been isolated with certainty. HALLIBURToN found that a solution of purified myosin in dilute-salt solution (5\% $\left.\mathrm{MgSO}_{4}\right)$, and sufficiently diluted with water, coagulates after a certain time, and at the same time becomes acid and a typical myosin-clot separates. This coagulation, which is accelerated by warming or by the addition of myosin-ferment, is, according to HALLIBURToN, a process analogous to the coagulation of the muscle-plasma. According to this same investigator, myosin when dissolved in water by the aid of a neutral salt is reconverted into myosinogen, while after diluting with water myosin is again produced from the myosinogen. These observations may, however, be explained in other ways. In these cases the separation of the myosin is evidently closely connected with the liquid becoming acid, while the separation of myosin from the muscle-plasma, at least from the muscle-plasma of the frog, is independent of this acidity, for it may 
take place before the liquid becomes acid. The mother-substance of myosin and the chemical processes of the myosin coagulation are questions which must not be considered as settled.

Musculin, called paramyosinogen by Halliburton, is a globulin which is characterized by its low coagulation temperature, about $+47^{\circ} \mathrm{C}$., which may vary in different species of animals $\left(+45^{\circ}\right.$ in frogs, $+51^{\circ} \mathrm{C}$. in birds). It is more easily dissolved than myosin by $\mathrm{NaCl}$ or $\mathrm{MgSO}_{4}$ (salt containing $50 \%$ water of crystallization). Musculin is separated simultaneously with myosin in the coagulation of the muscle-plasma, and it is therefore found in the clot. A solution which contains musculin and no myosin does not coagulate on the addition of the myosin ferment (HALLIBURTON). If the dead muscle is extracted with water, the musculin passes in part into solution. The musculin may be isolated by fractional precipitation with magnesium sulphate (50 grms. to each 100 cc. liquid), and may be identified by its low coagulation temperature.

Myoglobulin. After the separation of the musculin and the myosin from the salt extract of the muscle by means of $\mathrm{MgSO}_{4}$ the myoglobulin may be precipitated by saturating the filtrate with the salt. It is similar to serum globulin, but coagulates at $+63^{\circ} \mathrm{C}$. (Halliburton). Myoalbumin, or muscle-albumin, seems to be identical with serum-albumin (serum-albumin $\alpha$, according to HALLIBURTON), and is prepared according to the same method. Myoalbumose (a denteroalbumose) is found in small quantities in the muscles, and may be obtained by extracting with water the finelydivided mass of muscle which has previously been coagulated by keeping in alcohol for a long time (HALLIBURTON).

After the complete removal from the muscle of all albuminous bodies which are soluble in water and ammonium chloride, DANILEWSKY claims that an insoluble albuminons body remains which only swells in ammonium-chloride solution and which forms with the other insoluble constituents of the muscular fibre the " musclestroma." According to DANILEWSKY, the amount of such stroma substance is connected with the muscle activity. He maintains that the muscles contain a greater amount of this substance, compared with the myosin present, when the muscles are quickly contracted and relaxed. 
Muscle-syntonin, which may be obtained by extracting the muscles with hydrochloric acid of $1 \mathrm{p}$. m., and which, according to K. MöRNER, is less soluble and has a greater aptitude to precipitate than other acid albumins, seems not to occur pre-existent in the muscles.

Muscle-coloring Matters. There is no question that the red color of the muscles even when completely freed from blood depends in part on hæmoglobin, though it is contested by many. MACMUNN claims that the muscles contain also another coloring substance which is nearly related to the blood-coloring matters and whose spectrum is very similar to that of hæmochromogen. This coloring matter has been called myohamatin. According to LEVY and HOPPE-SEYLER, this myohæmatin is nothing but hæmochromogen, which is produced from oxyhæmoglobin by decomposition and reduction. Nevertheless MAcMunN still adheres to his view that myohæmatin is an independent coloring substance, and in support of his opinion he adduces the fact that myohæmatin is found also in the muscles of insects in which no hæmoglobin occurs.

The reddish-yellow coloring matter of the muscles of the salmon has been little studied. Traces of enzymes, such as pepsin and diastatic enzymes, have been found in them. The so-called "myosin-ferment," and probably an enzyme producing lactic-acid fermentation, are also found in these muscles.

\section{Extractive Bodies of the Muscles.}

The nitrogenized extractives consist chiefly of creatin, in the proportion of $2-4$ p. m., in the fresh muscles containing water, also the xanthin bodies, hypoxanthin and xanthin, besides guanin and carnin. The average quantity of hypoxanthin, xanthin, and guanin in 1000 parts of the dried substance of the muscles of oxen is, according to KossEL, respectively $2.30,0.53$, and 0.20 grms., and in the embryonic ox-muscles respectively $3.59,1.11$, and 4.12 grms.

Besides these we must also consider as an extractive body the syrupy inosic acid $\left(\mathrm{C}_{10} \mathrm{H}_{14} \mathrm{~N}_{4} \mathrm{O}_{11}\right)$, of which only traces are found in certain animals. This acid was first prepared by LIEBIG, but not closely studied. LIMPRICHT has found another in the flesh of certain cyprindea, namely, the nitrogenized protic acid. Uric acid, urea, taurin, and leucin are found as traces in the muscles in certain cases only, in a few species of animals. In regard to the amount of these different extractives in the muscles, KRUKENBERG and WAGNER have shown that it varies greatly in different animals. A large amount of urea is found in the muscles of the shark and ray; uric acid is found in alligators ; 
taurin in cephalopoda ; glycocoll in mollusks, pecten irradians; and creatinin in luvarus imperialis, etc., etc.

The xanthin bodies, with the exception of carnin, have been treated on pages $47-52$, and therefore among the extractive bodies we will first consider the discussion of creatin.

Creatin, $\mathrm{C}_{4} \mathrm{H}_{2} \mathrm{~N}_{3} \mathrm{O}_{2}+\mathrm{H}_{2} \mathrm{O}$ or METHYLGUANIDIN-ACETIC ACID, $\mathrm{NH}: \mathrm{C}\left(\mathrm{NH}_{2}\right) \cdot \mathrm{N}\left(\mathrm{CH}_{3}\right) \cdot \mathrm{CH}_{2} \cdot \mathrm{COOH}+\mathrm{H}_{2} \mathrm{O}$, occurs in the muscles of vertebrate animals in variable amounts in different species; the largest amount is found in birds. It is also found in the brain, blood, transudations, and the amniotic fluid. Creatin may be prepared synthetically from cyanamid and sarcosine (methylglycocoll). On boiling with baryta-water it decomposes, with the addition of water, and yields urea, sarcosine, and certain other products. Because of this behavior several investigators consider creatin as a step in the formation of urea in the organism. On boiling with acids creatin is easily converted, with the elimination of water, into creatinin, whick occurs in urine, and which has also been found in the muscles of the dog by MoNari (see Chapter XIV).

Creatin crystallizes in hard, colorless, monoclinic prisms which lose their water of crystallization at $100^{\circ} \mathrm{C}$. It dissolves in 74 parts of water at the ordinary temperature and 9410 parts absolute alcohol. It dissolves more easily with the aid of heat. Its watery solution has a neutral reaction. Creatin is not dissolved by ether. If a creatin solution is boiled with precipitated mercuric oxide, this is reduced to mercury and oxalic acid, and the disgusting-smelling methyluramin (methylguanidin) is developed. A solution of creatin in water is not precipitated by basic-lead acetate but gives a white, flaky precipitate with mercurous nitrate. When boiled for an hour with dilute hydrochloric acid creatin is converted into creatinin, and may be identified by its reactions.

The preparation and detection of creatin is best performed by the following method of NeUBAUER, which was first used in the preparation of creatin from muscles. Finely-cut flesh is extracted with an equal weight of water at $+55^{\circ}$ to $60^{\circ} \mathrm{C}$. for $10-15$ minutes, pressed and extracted again with water. The albumin is removed from the united extracts as far as possible by coagulation at boiling heat, the filtrate precipitated by the careful addition of basic-lead acetate, the lead removed from this filtrate by $\mathrm{H}_{2} \mathrm{~S}$ and carefully ooncentrated to a small volume. The creatin, which crystallizes in 
a few days, is collected on a filter, washed with alcohol of $88 \%$, and purified, when necessary, by recrystallization. The quantitative estimation of creatin is performed according to the same method.

Carnin, $\mathrm{C}_{7} \mathrm{H}_{8} \mathrm{~N}_{4} \mathrm{O}_{3}+\mathrm{H}_{2} \mathrm{O}$, is one of the substances found by WEIDEL in American meat extract. It has also been found by KRUKENBERG and WAGNER in frog-muscles and in the flesh of fish, and by Podcher in urine. As previously stated (page 48), carnin may be converted into hypoxanthin by means of oxidation.

Carnin has been obtained as a white crystalline mass. It dissolves with difficulty in cold water, but dissolves easily in warm. It is insoluble in alcohol and ether. It dissolves in warm hydrochloric acid and yields a salt crystallizing in shining needles, which gives a double combination with platinum chloride. Its watery solution is precipitated by silver nitrate, but this precipitate is neither dissolved by ammonia nor by warm nitric acid. Carniu does not give the so-called WEIDEL's xanthin reaction. Its watery solution is precipitated by basic-lead acetate; still the lead combinations may be dissolved on boiling.

We must also include among the nitrogenized extractives those bodies which were discovered by GAUTIER, and which occur in very small quantities, namely, the leucomaines : xanthocreatinin, $\mathrm{C}_{5} \mathrm{H}_{10} \mathrm{~N}_{4} \mathrm{O}$, crusocreatinin, $\mathrm{C}_{8} \mathrm{H}_{8} \mathrm{~N}_{4} \mathrm{O}$, amphicreatin, $\mathrm{C}_{9} \mathrm{H}_{19} \mathrm{~N}_{7} \mathrm{O}_{4}$, and pseudoxanthin, $\mathrm{C}_{4} \mathrm{H}_{5} \mathrm{~N}_{5} \mathrm{O}$.

The non-nitrogenized extractive bodies of the muscles are inosit, glycogen, sugar, and lactic acid.

Inosit, $\mathrm{C}_{6} \mathrm{H}_{12} \mathrm{O}_{6}+\mathrm{H}_{2} \mathrm{O}$. This body, discovered by ScHeRER, is not a carbohydrate, but belongs to the aromatic series and seems to be hexahydroxybenzol (MAquenNe). With hydroiodic acid it yields benzol and tri-iodophenol, and on oxidation with nitric acid, tetra-oxychinon. Inosit is found in the muscles, liver, spleen, kidneys, super-renal cavity, lungs, brain, testicles, and in the urine in pathological cases. It is found very widely distributed in the vegetable kingdom, especially in unripe fruits, in green beans, (phaseolus vulgaris), and therefore it is also called PHASEOMANNIT.

Inosit crystallizes in large, colorless, rhombical crystals of the monoclinic system, or, if not pure and if only a small quantity crystallizes, it forms groups of fine crystals similar to cauliflower. It looses its water of crystallization at $110^{\circ} \mathrm{C}$, also if exposed to the air for a long time. Such exposed crystals are non-transparent and milk-white. The crystals melt at $217^{\circ} \mathrm{C}$. Inosit dissolves in six parts of water at ordinary temperature, and the solution has a sweetish taste. It is insoluble in strong alcohol and in ether. Inosit does not ferment with beer-yeast; it dissolves copper oxyhydrate in alkaline solutions, but does not reduce on boiling. It gives negative results with MOORE's or BöTTGER-ALMEN's bismuth tests. 
If inosit is evaporated to dryness on a platinum foil with nitric acid and the residue treated with ammonia and a drop of calcium. chloride solution and carefully re-evaporated to dryness, a beautiful rose-red residue is obtained (ScheneR's inosit test). If we evaporate an inosit solution to incipient dryness and moisten the residue with a little mercuric-nitrate solution, we obtain a yellowish residue on drying, which becomes a beautiful red on strongly heating. The coloration disappears on cooling, but it reappears on gently warming (GALLOIS's inosit test).

To prepare inosit from a liquid or from a watery extract of a tissue, the albumin is first removed by coagulating at boiling heat. The filtrate is precipitated by sugar of lead, this filtrate boiled with basic-lead acetate and allowed to stand 24-48 hours. The precipitate thus obtained, which contains all the inosit, is decomposed in water by $\mathrm{H}_{2} \mathrm{~S}$. The filtrate is strongly concentrated, treated with 2-4 vols. hot alcohol, and the liquid removed as soon as possible from the tough or flaky masses which ordinarily separate. If no crystals separate from the liquid within 24 hours, then treat with ether until the liquid has a milky appearance and allow it to stand. In the presence of a sufficient quantity of ether, crystals of inosit separate within 24 hours. The crystals thus obtained, as also those which are obtained from the alcoholic solution directly, are recrystallized by redissolving in very little boiling water and the addition of 3-4 vols. alcohol.

Glycogen is a constant constituent of the living muscle, while it may be absent in the dead muscle. The amount of glycogen varies in the different muscles of the same animal. BöHM found $10 \mathrm{p.m}$. glycogen in the muscles of cats, and moreover he found a greater amount in the muscles of the extremities than in those of the rump. GRÜTZNER obtained more glycogen from the white muscles of mammalia than from the red ones. The food also has a great influence. ВöHM found 1-4 p. m. glycogen in the muscles of fasting animals and $7-10 \mathrm{p}$. m. after partaking of food. LUCHSINGER maintains an opinion, formerly generally accepted, that in starvation, or if there is a lack of carbohydrates in the food, glycogen disappears more quickly from the muscles than from the liver; but according to ALDEHOFF, exactly the reverse takes place. The glycogen disappears more quickly in starvation from the liver than from the muscles, not only in hens, as observed by WEIss, but also in other animals, such as the pigeon, rabbit, cat, and horse. 
Muscle-sugar, of which traces only occur in the living muscle and which is probably formed after the death of the muscle from the nuscle-glycogen, is perhaps grape-sugar (glucose). As an intermediate step in this sugar formation we must mention dextrin, which is sometimes found in the muscles. Perhaps this dextrin has been confounded with glycogen.

Lactic Acids. Of the four acids of the formula $\mathrm{C}_{3} \mathrm{H}_{6} \mathrm{O}_{3}$ there is one, hydracrylic acid, which is not found in the animal organism. The statements as to the occurrence of another, ethylen lactic acid (in meat extract), are disputed. Ethidene lactic acid, $\mathrm{CH}_{3} \cdot \mathrm{CH}(\mathrm{OH}) \cdot \mathrm{COOH}$, is of physiologico-chemical importance. Two modifications occur-the optically inactive FERMENTATION LACTIC ACID, and the optically active, dextro-gyrate PARALACTIC ACID, Or SARCOLACTIC ACID.

The fermentation lactic acid, which is formed from milk-sugar by allowing milk to sour and by the acid fermentation of other carbohydrates, is considered to exist in small quantities in the muscles (Heintz), in the gray matter of the brain (GscheidLen), and in diabetic urine. During digestion this acid is also found in the contents of the stomach and intestines, and as alkali lactate in the chyle. The paralactic acid is, at all events, the true acid of meat extracts, and this alone has been found with certainty in dead muscle. That lactic acid which is found in the spleen, lymphatic glands, thymus, thyroid gland, blood (traces), bile, pathological transudations, osteomalicous bones, in perspiration in puerperal fever, and in the urine after excessive marches, in acute yellow atrophy of the liver, in poisoning by phosporus, especially after extirpation of the liver (in geese, according to Minkowski, in frogs by MARCUSE and WerTher), seems to be paralactic acid.

The lactic acids are amorphous. They have the appearance of colorless or faintly yellowish, acid-reacting syrups which mix in all proportions with water, alcohol, or ether. The salts are soluble in. water, and most of them in alcohol. The two acids are differentiated from each other by their different optical properties-paralactic acid being dextro-gryate, while fermentation lactic acid is optically inactive-also by their different solubilities and the different amounts of water of crystallization of the calcium and zinc salts. The zinc salt of fermentation lactic acid dissolves in 58-63 parts of 
water at $14^{\circ}-15^{\circ} \mathrm{C}$. and contains $18.18 \%$ water of crystallization, corresponding to the formula $\mathrm{Zn}\left(\mathrm{C}_{3} \mathrm{H}_{5} \mathrm{O}_{3}\right)_{2}+3 \mathrm{H}_{2} \mathrm{O}$. The zinc salt of paralactic acid dissolves in 17.5 parts of water at the above temperature and contains ordinarily $12.9 \%$ water, corresponding to the formula $\mathrm{Zn}\left(\mathrm{C}_{3} \mathrm{H}_{5} \mathrm{O}_{3}\right)_{2}+2 \mathrm{H}_{2} \mathrm{O}$. The calcium salt of fermentation lactic acid dissolves in 9.5 parts water and contains $29.22 \%$ (=5 mol.) water of crystallization, while the calcium paralactate dissolves in 12.4 parts water and contains 24.83 or $26.21 \%$ ( $=4$ or $4 \frac{1}{2}$ mol.) water of crystallization. Both calcinm salts crystallize, not unlike tyrosin in spheres or tufts of very fine microscopic needles.

Lactic acids may be detected in organs and tissues in the following manner: After complete extraction with water the albumin is removed by coagulation at boiling temperature and the addition of a small quantity of sulphuric acid. The liquid is then exactly neutralized while boiling with caustic baryta, and then evaporated to a syrup after filtration. The residue is precipitated with absolute alcohol, and the precipitate completely extracted with alcohol. The alcohol is entirely distilled from the united alcoholic extracts, and the neutral residue is shaken with ether to remove the fat. The residue is taken up by water and phosphoric acid added, and repeatedly shaken with fresh quantities of ether, which dissolve the lactic acid. The ether is now distilled from the several ethereal extracts, the residue dissolved in water, and this solution carefully warmed on the water-bath to remove the last traces of ether and volatile acids. A solution of zinc lactate is prepared from this filtered solution by boiling with zinc carbonate, and this is evaporated until crystallization commences and then allowed to stand over sulphuric acid.

Fat is never absent in the muscles. Some fat is always found in the inter-muscular connective tissue; but the muscle-fibres themselves also contain fat. The amount of fat in the real muscle substance is always small, usually amounting to about $10 \mathrm{p}$. m. or somewhat more. A considerable amount of fat in the muscle-fibres is only found in fatty degeneration. Lecithin is also habitually found in the muscles.

The Mineral Bodies of the Muscles. We have no complete analyses of the mineral substances of the pure, blood-free muscle substance. The ash remaining after burning the muscle, which amounts to about 10-15 p. m., calculated on the moist muscle, is acid in reaction. The largest constituents are potassium and phos- 
phoric acid. Next in amount we have sodium and magnesium, and lastly calcium, chlorine, and iron oxide. Sulphates only exist as traces in the muscles, but are formed by the burning of the proteids of the muscles, and therefore occur in abundant quantities in the ash. The muscles contain such a large quantity of potassium and phosphoric acid that potassium phosphate seems to be unquestionably the predominating salt. Chlorine is found in such insignificant quantities that it is perhaps derived from a contamination with blood or lymph. The quantity of magnesium is about double that of calcium. These two bodies, as well as iron, occur only in very small amounts.

The gases of the muscles consist of large quantities of carbon dioxide, besides traces of nitrogen.

Rigor Mortis of the Muscles. If the influence of the circulating oxygenated blood is removed from the muscles, as after death of the animal or by tying the aorta or the muscle-arteries (STENsoN's test), rigor mortis, sooner or later takes place. The ordinary rigor appearing under these circumstances is called the spontaneous or the fermentive rigor, because it seems to depend in part on the action of an enzyme. A muscle may also become stiff for other reasons. The muscles may become momentarily stiff by warming, in the case of frogs to $40^{\circ}$, in mammalia to $48^{\circ}-50^{\circ}$, and in birds to $53^{\circ} \mathrm{C}$. (heat-rigor). Distilled water may also produce a rigor in the muscles (water-rigor). Acids, even when very weak, such as carbon dioxide, may quickly produce a rigor (acid-rigor), or hasten its appearance. A number of chemically different substances, such as chloroform, ether, alcohol, ethereal oils, caffein, and many alkaloids, produce a similar effect. That rigor which is produced by means of acids or other agents which, like alcohol, coagulate albumin must be considered as produced by entirely different processes than the spontaneous rigor.

The time within which the spontaneous rigor occurs depends upon the temperature; a low temperature retarding and a high temperature hastening its appearance. Muscular activity also exercises an appreciable influence on the rigor of the muscles, for a previous active contraction accelerates the rigor of the muscles; the mechanical abuse, of the muscles acts in the same way. The appearance of spontaneous rigor is under the influence of the central nerv- 
ous system, and a muscle whose nerve has been severed stiffens more slowly than one whose continuity with the central nervous system has not been destroyed (HermanN and his pupils v. EiselBerg, v. Gender and Bierfreund). The nervous system seems also to have a similar influence on the post-mortem acidification of the muscles (Gross). HermanN and his pupils consider the rigor mortis as a slowly-proceeding muscular contraction, identical with the ordinary muscular contraction, but it is difficult at this time to determine as to the correctness of this view from a chemical standpoint.

When the muscle passes into rigor mortis it becomes shorter and thicker, harder and non-transparent, less ductile and acid. The chemical processes which take place in this step are the following : By the coagulation of the plasma a myosin-clot is produced which is the cause of the hardening and of the diminished transparency of the muscle. The appearance of this clot may be hastened by the simultaneous occurrence of lactic acid. Carbon dioxide is also formed, which does not seem to be a direct oxidation product. Hermann claims that carbon dioxide is produced in the removed muscle, even in the absence of oxygen, when it passes into rigor mortis. The quantity of carbon dioxide and lactic acid produced on the acidification of the muscle is not dependent upon the quickness or slowness of the rigor mortis (RANKE, HermanN). It is not known from what mother-substance these acids are formed. The most probable explanation is that carbon dioxide and lactic acid are produced from glycogen, and the fact that the glycogen in the muscle decreases on stiffening has been asserted positively (NASSE, Werther). On the other side, BöHM has shown that cases are found in which the glycogen is not diminished on stiffening, and he has also found that the quantity of lactic acid produced is not proportional to the quantity of glycogen. Under these circumstances, as the muscles of starving pigeons, which are free from glycogen, yield, according to DAMANT, lactic acid after death, it is hardly possible that the two above acids are formed from glycogen. The only remaining explanation is that they originate from the proteids or certain other not well-known constituents of the muscles.

After the muscles have been stiff for some time they relax again and become softer. This may partly depend on their becom- 
ing strongly acid and the myosin-clot dissolving in the acid, and partly, and in all probability mainly, upon a putrefaction.

Exchange of Material in the Inactive and Active Muscles. It is admitted by a number of prominent investigators, PFLÜGER and Colasanti, Zuntz and RöHrig, and others, that the exchange of material in the muscles is regulated by the nervous system. When at rest, when there is no mechanical exertion, we have a condition which ZUNTZ and RöHRIG have designated "chemical tonus." This tonus seems to be a reflex tonus, for it may be reduced by discontinuing the connection between the muscles and the central organ of the nervous system by cutting through the spinal marrow or the muscle-nerves, or by paralyzing the same by means of curara poison. It may also be reduced or checked by adjusting the temperature between the skin and the surrounding. medium; or it may be increased by the reverse, by irritating the nerves of the skin by cooling. The possibility of reducing the chemical tonus of the muscles by any of the above-mentioned means, but especially by the action of curara, offers an important means of deciding the extent and kind of chemical processes going on in the muscles when at rest. In comparative chemical investigation of the processes in the active and in the inactive muscles several ways of procedure have been adopted. The removed homologous, active and inactive muscles have been compared, also the arterial and venous muscle-blood in rest and in activity, and lastly the total exchange of material, the receipts and expenditures of the organism, have been investigated under these two conditions.

By investigations according to these several methods, it has been found that the active muscle takes up oxygen from the blood and returns to it carbon dioxide, and also that the quantity of oxygen taken up is greater than the oxygen contained in the carbon dioxide eliminated at the same time. The muscle, therefore, holds in some form of combination a part of the oxygen taken up while at rest. During activity the exchange of material in the muscle, and therewith the exchange of gas, is increased. The animal organism takes up considerably more oxygen in activity than when at rest, and eliminates also considerably more carbon dioxide (REgNaUlt and REISET and others). The quantity of oxygen which leaves the body as carbon dioxide is, during activity, 
considerably larger than the quantity of oxygen taken up at the same time; and the venous muscle-blood is poorer in oxygen and richer in carbon dioxide during activity than during rest (LUDWIG and Sczelkow and others). The exchange of gases in the muscles during activity is the reverse to when at rest, for the active muscle gives up a quantity of carbon dioxide which does not correspond to the quantity of oxygen taken up, but is considerably greater. It follows from this that in muscular activity not only oxidation takes place, but also splitting processes. This follows also from the fact that removed blood-free muscles, when placed in an atmosphere devoid of oxygen, can labor for some time and also yield carbon dioxide (HermanN).

During muscular inactivity a consumption of glycogen takes place. This is inferred from the observations of several investigators that the quantity of glycogen is increased and its corresponding consumption reduced in those muscles whose chemical tonus is lowered either by cutting through the nerve or for other reasons (Bernard, McDonnel, Chandelon, and others. After cutting the nerve MaNCHÉ obtained no doubtful results). In activity this consumption of glycogen is increased, and it has been positively proved by the researches of several investigators (NASSE, BRÜCKE and Weiss, Külz, Marcuse, Manché) that the quantity of glycogen in the muscles in activity quickly and abundantly decreased. By in restigating with the muscles in situ, especially on the levator labii superioris of a horse, CHaUveaU and KaUfmann have not only confirmed the above facts in regard to the exchange of gas during rest and activity, but they also found that the muscles remove sugar from the blood, and indeed considerably more during activity than when at rest. They found (calculating the amount found in 1 gramme of muscle per minute to 1 kilo per hour) that 1 kilo of muscle removes 2.186 grms. sugar from the blood per hour during rest, while it removes 8.416 grms. per hour in activity. QUINQUAUD has also observed a consumption of sugar from the blood during activity. On comparing the amount of carbon which the muscles remove from the blood as sugar with the amount they give off as carbon dioxide, CHAUveaU and KaUfMaNe found that in rest more carbon was taken up than given off, while during activity this condition was reversed. From these facts it is claimed

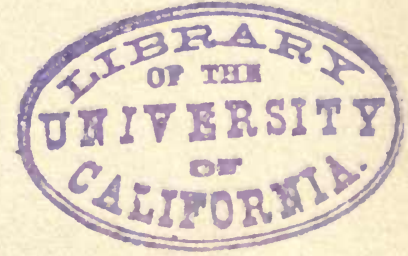


by them that during rest glycogen is formed from the sugar, and also that in activity other bodies besides sugar are transformed in the muscles.

The faintly alkaline or amphoteric reaction of the inactive muscles is changed during activity to an acid reaction (DuBorsREYMOND and others), and the acid reaction to a certain point increases with the work (HeIdenHAIN). The quickly-contracting pale muscles produce, according to GLEISS, more acid during activity than the more slowly-contracting red muscles. The acid reaction appearing during activity was formerly considered due to the formation of lactic acid, a view which has been contradicted by Astaschewsky, Pfü̈ger and Warren, who found less lactic acid in the tetanized muscle than when at rest. According to the recent and more carefully conducted investigations of MARCUSE, which have since been confirmed by WERTHER, there is no doubt that free lactic acid is actually produced in the muscle during activity. According to WEYL and ZEITLER, the active muscle contains a larger amount of phosphoric acid (formed at least in part from the decomposition of lecithin) than the resting muscle; and the acid reaction of the active muscle may, therefore, in part be due to the acid phosphate.

The amount of albumin in the removed muscles is, according to RANKE and NAWROcKI, decreased by work. The correctness of this statement is, however, disputed by other investigators. Also the older statements in regard to the nitrogenized extractive bodies of the muscle in rest and in activity are uncertain. According to the recent researches of MoNARI, the total quantity of creatin and creatinin is increased by work; and indeed by an excess of muscular activity, the amount of creatinin is especially augmented. The creatinin is formed essentially from the creatin. In excessive activity MoNarI also found xantho-creatinin in the muscle, and the quantity was one tenth of that of the creatinin. The quantity of xanthin bodies is, according to MoNaRI, decreased under the influence of work. It seems to have been positively shown that the active muscle contains a smaller quantity of bodies soluble in water and a larger quantity of bodies soluble in alcohol than the resting muscle (HeLmHoltz).

An attempt has been made to solve the question relative to the 
behavior of the nitrogenized constituents of the muscle at rest and during activity, by determining the total quantity of nitrogen eliminated under these different conditions of the body. While we in the past agreed with the views of LIEBIG that the elimination of nitrogen by the urine was increased by muscular work, the researches of several experimenters, especially those of VoIT on dogs and Vort and Pettenkofer on man, have led to quite different results. They have shown that during work no increase or only a very insignificant increase in the elimination of nitrogen takes place. We must not conceal the fact that we have a series of experiments which show a significant increase in the exchange of proteids during or after work. We have as example the observations of Flint ${ }^{1}$ and PAví on a pedestrian, v. WolfF, v. Funke, Kreuzhage and KeLLNer on a horse, and lately those of ARgutinsky on himself, which show an undoubted increase in the elimination of nitrogen during or after work.

The elimination of nitrogen is mainly dependent upon causes which will be spoken of later (Chapter XV), such as the quantity and composition of the food, the condition of the adipose tissue, the action of work on the respiratory mechanism, etc., etc., all of which can hardly have received sufficient consideration in the lastmentioned experiments. The strong proof which the very careful experiments of VoIT and of PetTenkofer and VoIT furnish in support of this theory is hardly destroyed by these investigations; though we must admit that this question is still somewhat unsettled. Even if we consider that muscular work does not cause any increase in the elimination of nitrogen, which has been quite positively proved, still we do not exclude the possibility of an increased exchange of proteids in the muscle. It is possible on account of the functional exchange action of the organs, of which RANKE has made a special study, that an increased exchange of proteid in the muscles may be compensated by a simultaneous decreased exchange

The results obtained by Prof. A. FLINT were derived from experiments made upon the pedestrian Weston, who in 1870, in the American Institute Building (New York), travelled a distance of 317 $\frac{1}{2}$ miles in five days. The chemical part of the work was performed under the immediate supervision of Prof. R. O. Donemus. For fifteen days, five before, five during, and five after the walk, all ingesta and excreta were weighed or measured and analyzed. The experiments of Dr. PAVY were made in Iondon in 1876. - Translator. 
of proteid in other organs. But however this may be, the modern view is, notwithstanding, that the exchange of proteid in the muscle is not increased by activity.

The investigations on the amount of fat in removed muscles during activity and at rest have not led to any definite results. The experiments of VoIT on a starving dog, and those of PetTenKOFER and VoIT on a man, offer strong proofs to show that an increased decomposition of the fat takes place during activity.

If the results from the investigations thus far made of the chemical processes going on in the active and inactive muscle were collected together, we should find the following characteristics for the active muscle. The active muscle takes up more oxygen and gives off more carbon dioxide than the inactive muscle; still the elimination of carbon dioxide is increased considerably more than the absorption of oxygen. In work a consumption of carbohydrates, glycogen, and sugar takes place. A consumption of sugar seems only to have been shown in muscle with blood circulation, while a consumption of glycogen also has been observed in removed muscle. Lactic acid is formed in activity, which is carried off by the blood and taken up and consumed by the liver. Acid-alkali phosphates also seem to be produced by work. In regard to the behavior of fats in removed muscles nothing is known with certainty, though an increase in the consumption of fat in the organism has been observed during activity. An increase in the nitrogenized extractive bodies of the creatin group seems also to occur. In regard to the albuminous bodies the views are contradictory; but an increased elimination of nitrogen as a direct consequence of muscular exertion has thus far not been positively proved.

In close connection with the above-mentioned facts we have the question as to the origin of muscular activity so far as it has its origin in chemical processes. In the past the generally-accepted opinion was that of LIEBIG, that the source of muscular action consisted of a metabolism of the albuminous bodies; to-day another view prevails. FICK and WisLicenus climbed the Faulhorn and calculated the amount of mechanical force expended in the attempt. With this they compared the mechanical equivalent transformed in the same time, from the proteids, calculated from the nitrogen eliminated with the urine, and found that the work really performed 
was not by any means compensated by the consumption of proteid. It was therefore proved by this that albumin alone cannot be the source of muscular activity, and that this depends in great measure on the metabolism of non-nitrogenized substances. Many other observations have led to the same result, especially the experiments of Vort, of Petrenkofer and Vort, and of other investigators, whose experiments show that while the elimination of nitrogen remains unchanged, the elimination of carbon dioxide during work is very considerably increased. It is also generally considered as positively proved, that muscular work is produced, at least the greatest part, by the metabolism of non-nitrogenized substances. Nevertheless we are not warranted in the statement that muscular activity is produced entirely at the cost of the non-nitrogenized substance, and that the albuminous bodies are without importance as a source of force.

Among the non-nitrogenized bodies we must accord to carbohydrates, glycogen, and sugar the first place as sources of force. The fact that the carbohydrates are consumed during exertion has been previously discussed, and their great importance as a source of activity is generally admitted. The most important is glycogen, -not only that which pre-exists in the muscles, but also that which is contained by the liver and conveyed by the blood to the muscles as sugar to be used in muscular work. If the consumption of carbohydrates in activity is positively proved, then the question arises whether the quantity of glycogen or carbohydrates which is at the disposition of the muscles is sufficient for the development of the living force produced within them. Though it is difficult to give a positive answer to this question, it still seems certain that, at least in some particular cases, the carbohydrates are not sufficient for the development of this force. According to statements whose correctness cannot be doubted, muscles free from glycogen can perform work (BERNARD, LUCHSINGER).

Even though no direct investigations on removed muscles have shown any increase in the consumption of fat during activity, still, from the above-mentioned experiments on the exchange of material, we conclude that the fats are also undoubtedly sources of muscular force. In regard to the albuminous bodies we have no positive direct observations which tend to establish the importance attrib- 
uted to them by LIEBIG in muscular activity; but when we consider that from the albuminous bodies not only the fats proceed but also the carbohydrates, glycogen, it is difficult to see why the source of muscular force is not in part due to a metabolism of the proteids. Though it is probable that in work the carbohydrates in the first place, and then the fats, are consumed by an increased exchange, it is therefore not improbable that the albuminous bodies also take part, and that all three chief groups of organic foods or muscle-constituents undergo an increased exchange during activity (RANKE).

Quantitative Composition of the Muscle. A large number of analyses have been made of the flesh of various animals for purely practical purposes, in order to determine the nutritive value of different varieties of meat; but we have no exact scientific analyses with sufficient regard to the quantity of different albuminous bodies and the remaining muscle-constituents; or these analyses being incomplete are of little value.

To give the reader some idea of the variable composition of muscle-substance we give the following summary, chiefly obtained from K. B. HofmaNs's book. The figures are parts per 1000.

\begin{tabular}{|c|c|c|c|}
\hline 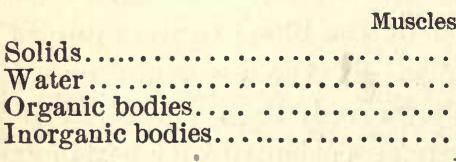 & $\begin{array}{l}\text { from Mammalia. } \\
217-255 \\
745-783 \\
208-245 \\
9-10\end{array}$ & $\begin{array}{l}\text { Birds. } \\
227-282 \\
717-773 \\
217-263 \\
10-19\end{array}$ & $\begin{array}{c}\text { Cold-blooded } \\
200 \\
800 \\
180-190 \\
10-20\end{array}$ \\
\hline 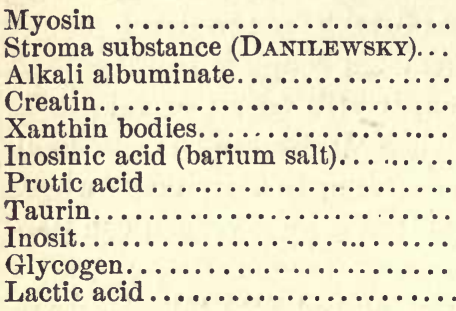 & $\begin{array}{l}35-106 \\
78-161 \\
29-30 \\
2 \\
0.4-0.7 \\
0.1 \\
\overline{0.7} \text { (horse) } \\
0.03 \\
4-5 \\
0.4-0.7\end{array}$ & $\begin{array}{l}29.8-111 \\
88.0-184 \\
- \\
3.4 \\
0.7-1.3 \\
0.1-0.3 \\
= \\
= \\
=\end{array}$ & $\begin{array}{l}29.7-87 \\
70.0-121 \\
\overline{2.3} \\
\overline{-} \\
7.0 \\
\overline{1.1} \\
\overline{3-5}\end{array}$ \\
\hline 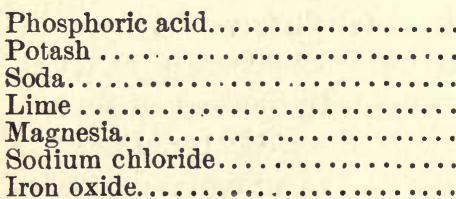 & $\begin{array}{l}3.4-4.8 \\
3.0-4.0 \\
0.4 \\
0.2 \\
0.4 \\
0.04-0.1 \\
0.03-0.1\end{array}$ & & \\
\hline
\end{tabular}


In these tables, which have little value because of the variation in the composition of the muscles, we have no results as to the estimates of fat. Because of the variable quantity of fat in meat it is hardly possible to quote a positive average for this body. After most careful efforts to remove the fat from the muscles without chemical means, it has been found that a variable amount of inter-muscular fat, which does not really belong to the muscular tissue, always remains. The smallest quantity of fat in the muscles from lean oxen is, according to Grouven, $6.1 \mathrm{p}$. m., and according to Petersen, $7.6 \mathrm{p}$. m. This last observer also found habitually a smaller amount of fat, 7.6-8.6 p. m., in the fore quarter of oxen, and a greater amount, 30.1-34.6 p. m., in the hind quarter of the animal. A lower amount of fat has also been found in the muscles of wild animals. B. KöNIG and FARWICK found in the muscles of the extremities of the hare $10.7 \mathrm{p}$. m. fat, and $14.3 \mathrm{p} . \mathrm{m}$. in the muscles of the partridge. The muscles of pigs and fattened animals are, when all the appending fat is removed, very rich in fat, amounting to $40-90 \mathrm{p}$. m. The muscles of certain fishes also contain a large amount of fat. According to Almén, the flesh of the salmon, mackerel, and eel contain respectively 100, 164, and 329 p. m. fat.

The quantity of WATER in the muscle is liable to considerable variation. The amount of fat has a special influence on the quantity of water, and we find, as a rule, that the flesh which is deficient in water is correspondingly rich in fat. The quantity of water does not depend alone upon the amount of fat, but upon many other circumstances, among which we must mention the age of the animal. In young animals the organs in general, and therefore also the muscles, are poorer in solids and richer in water. In man the amount of water decreases until mature age, but increases again towards old age. Work and rest also influence the amount of water, for the active muscle contains more water than the inactive. The uninterruptedly active heart should therefore be the muscle richest in water. That the amount of water may vary independently of the amount of fat is strikingly shown by comparing the muscles of different species of animals. In cold-blooded animals the muscles generally have a greater amount of water, in birds a lower. The comparison of the flesh of cattle and fish shows very 
strikingly the different amounts of water (independent of the amount of fat) in the flesh of different animals. According to the analyses of Almén, the muscles of lean oxen contain $15 \mathrm{p}$. m. fat and $767 \mathrm{p}$. m. water; the flesh of the pike contains only 1.5 fat and 839 p. m. water.

For certain purposes, as, for example, for the tests of the exchange of material, it is important to know the amount of nitrogen in the flesh. In general, it may be considered on an average as about $3.4 \%$ of the fresh lean flesh (VoIT). According to NowaK and Huppert this quantity may vary about $0.6 \%$, and in more exact investigations it is therefore necessary to determine the nitrogen.

\section{Non-striated Muscles.}

The smooth muscles have a neutral or alkaline reaction (DUBoIs-REYMOND) when at rest. During activity they are acid, which is inferred from the observations of BERNSTEIN, who found that the nearly continually contracting sphincter muscle of the Anodonta is acid during life. The smooth muscles may also, according to HEIDENHAIN and KüHNE, pass into rigor mortis and thereby become acid. Because of this behavior it is believed that among the albuminous bodies of the smooth muscles there is also a substance forming myosin. A spontaneously coagulating plasma has not thus far been obtained, but it may be considered as the juice obtained by pressing the muscles of the Anodonta and which coagulates immediately at $+45^{\circ} \mathrm{C}$. or within 24 hours at the ordinary temperature. Myosin has not been found in the smooth muscles. Heidenhain and Hellwig have obtained from the smooth muscles of a dog an albuminous body which coagulates at $+45^{\circ}-$ $49^{\circ} \mathrm{C}$. and which is analogous to musculin. The smooth muscles contain large amounts of alkali albuminates besides an albumin coagulating at $+75^{\circ} \mathrm{C}$.

Hcemoglobin occurs in the smooth muscles of certain animals, but is absent in others. Creatin has been found by Lehmans. According to Frémy and Valenciennes, the muscles of the Cephalopods contain taurin besides creatinin (creatin?). Of the nonnitrogenized substances, glycogen and lactic acid have been found without doubt. The mineral constituents show the remarkable fact that the sodium combinations exceed the potassium combinations. 


\section{CHAPTER $X$.}

\section{BRAIN AND NERVES.}

ON account of the difficulty of making a mechanical separation and isolation of the different tissue elements of the nerrous central organ and the nerves, we must resort to a few micro-chemical reactions, chiefly to qualitative and quantitative investigations of the different parts of the brain, in order to study the different chemical composition of the cells and the nerve-tubes. The chemical investigation of this part is accompanied with the greatest difficulty; and although our knowledge of the chemical composition of the brain and nerves has been somewhat extended by the investigations of modern times, still we must admit that this chapter is yet to-day one of the most obscure and complicated in physiological chemistry.

Albuminous bodies of different kinds have been shown to be chemical constituents of the brain and nerves. A part of these are insoluble in water and dilute neutral-salt solutions, and part are soluble therein. Among the latter we find albumin and globulin. Nucleoalbumin, which is often considered as an alkali albnminate, also occurs. It seems unquestionable that the albuminous bodies belong chiefly to the gray substance of the brain and to the axiscylinders. The same remarks apply to nuclein which $\mathrm{v}$. JAKSCH found in large quantities in the nerve subtances. Neurokeratin (see page 35), which was first detected by KüHNE, and which partly forms the neuroglia, and which as a double sheath envelops the outside of the nerve medulla under ScHwanN's sheath and the inner axis-cylinders, chiefly occurs in the white substance (KÜHNE and ChitTENDEN, Baumstark).

The phosphorized substance protagon must be considered as one of the chief constituents, perhaps the only constituent (BAUMsTARK), of the white substance. This last-mentioned substance 
yields easily, as decomposition products, lecithin, fatty acids, and a nitrogenized substance, cerebrin; this last probably does not occur preformed in the brain, but is more likely a product of transformation (BAUMSTARK). That lecithin also is pre-existent in the brain and nerves can hardly be doubted. The investigations thus far made have not shown decidedly whether it is more abundant in the gray or white substance. Fatty acids and neutral fats may be prepared from the brain and nerves; but as these may be readily derived from a decomposition of lecithin and protagon, which exist in the fatty tissue between the nerve-tubes, it is difficult to decide what part the fatty acids and neutral fats play as constituents of the real nerve-substance. Cholesterin is also found in the brain and nerves, a part free and a part in chemical combination of which we know nothing about (BAUMSTARK). Cholesterin seems to occur in greater abundance in the white substance. Besides these substances the nerve tissue, especially the white substance, contains doubtless a number of other constituents not well known, and among which are several containing phosphorus. THUDICHUM asserted that he had isolated a number of phosphorized substances from the brain which he divided into three principal groups: kepalines, myelines, and lecithines. But thus far this assertion has not been confirmed by other investigators.

By allowing water to act on the contents of the medulla, round, or long double-contoured drops or fibres, not unlike double-contoured nerves, are formed. This remarkable formation, which can also be seen in the medulla of the dead nerve, has been called "myeline forms," and they were formerly considered as produced from a special body, "myeline." Myeline forms may, however, be obtained from other bodies, such as protagon, lecithin, fat, and impure cholesterin, and they depend on a decomposition of the constituents of the medulla, chiefly the protagon.

The extractive bodies seem to be almost the same as in the muscles. We find: creatin, which may, however, be absent (BAUMSTARK), xanthin bodies, inosit, lactic acid (also fermentation lactic acid), uric acid, jecorin (according to BALDI, in the human brain), and neuridin, discovered by BRIEGER and which is most interesting because of its appearance in the putrefaction of animal tissues. Under pathological conditions leucin and urea have been found in 
the brain. The latter is also a physiological constituent of the brain of cartilaginous fishes.

Of the above-mentioned constituents of the nerve-substance, protagon and its decomposition product, cerebrin, must be specially described.

Protagon. This body, which was discovered by LIEBreich, is a nitrogenized and phosphorized substance whose elementary composition, according to GAMGEE and BLANKENHorN, is $\mathrm{C} 66.39, \mathrm{H}$ 10.69, $\mathrm{N} 2.39$, and $\mathrm{P} 1.068$ per cent, and whose empirical formula is $\mathrm{C}_{160} \mathrm{H}_{308} \mathrm{~N}_{5} \mathrm{PO}_{35}$. On boiling with baryta-water, protagon yields the decomposition products of lecithin, namely, fatty acids, glycerophosphoric acid, and cholin (neurin?), and also cerebrin. On boiling with dilute mineral acids, it yields among other products a substance which is lævo-gyrate, reducible, and fermentable.

Protagon appears, when dry, as a white loose powder. It dissolves in alcohol of 85 vols. per cent at $+45^{\circ} \mathrm{C}$., but separates on cooling as a snow-white, flaky precipitate, consisting of balls or groups of fine crystalline needles. It decomposes on heating even below $100^{\circ} \mathrm{C}$. ' It is hardly soluble in cold alcohol or ether, but dissolves on warming. It swells in little water, decomposes partly, and gives myaline forms. With more water it swells to a gelatinous or pasty mass, which with much water yields an opalescent liquid. On fusing with saltpetre and soda, alkali phosphates are obtained.

Protagon is prepared in the following way: An ox-brain as fresh as possible, with the blood and membranes carefully removed, is ground fine and then extracted for several hours with alcohol of 85 vols. per cent at $+45^{\circ} \mathrm{C}$., filtered at the same temperature, and the residue extracted with warm alcohol until the filtrate does not yield a precipitate at $0^{\circ} \mathrm{C}$. 'The several alcoholic extracts are cooled to $0^{\circ} \mathrm{C}$. and the precipitates united and completely extracted with cold ether, which dissolves the cholesterin and lecithin-like bodies. The residue is now strongly pressed between filter-paper and allowed to dry over sulphuric acid or phosphoric anhydride. It is now pulverized, digested with alcohol at $+45^{\circ} \mathrm{C}$., filtered and slowly cooled to $0^{\circ} \mathrm{C}$. The crystals which separate may be purified when necessary by recrystallization.

The same steps are taken when we wish to detect the presence of protagon.

Cerebrin. Under this name W. MüLLER first described a nitrogenized substance, free from phosphorus, which he obtained by 
extracting a brain-mass which had been previously boiled with baryta-water, with boiling alcohol. Following a method essentially the same, but differing somewhat, GEOGHEGAN prepared from the brain a cerebrin with the same properties as MüLLER's, but containing less nitrogen. According to GAMGEE, this cerebrin of GEOGHEGAN is a mixture of the cerebrin produced by the decomposition of the protagon and a substance called by GAMGEE " pseudocerebrin." Furthermore, THUDICHUM has collected together under the name "cerebrine" several nitrogenized bodies free from phosphorus, such as MüLLER's cerebrin and the newer bodies phrenosine and kerasene. Lastly, Parcus has endeavored to prove that the cerebrin described by both MULLER and GEOGHEGAN is a mixture of three bodies, "cerebrin," "homocerebrin," and "encephalin."

From the above statements it is seen that the composition of the cerebrins is still unsettled, so that we cannot admit the numerical results of any investigator as authoritative, nor can we even take an average as correct, and we therefore give below a summary of the results thus far obtained :

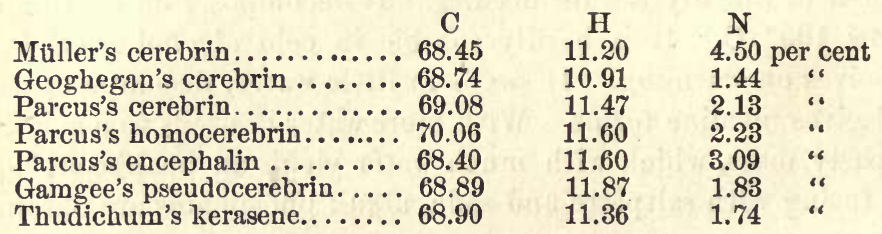

As GEOGHEGAN's cerebrin has the lowest amount of nitrogen, and as the analyses of this investigator agree well with each other, it is difficult to understand how this cerebrin can be, as Parcts' claims, a mixture of bodies richer in nitrogen which were prepared by this last-named investigator. On the other hand, Parcus has found a constant composition for the cerebrin irrespective whether it was recrystallized two, five, or eight times, and it is therefore quite as unjustifiable to question his results. Future researches are required to elucidate this question. GAMGEE's pseudocerebrin and THUDICHUM's kerasene have, with the exception of the amount of hydrogen, so similar a composition that they may be considered as identical.

The products of decomposition of the cerebrins possess a certain interest. By the action of concentrated sulphuric acid GEOGHEGAN obtained a lævogyrate, reducible substance, which is not sugar but an acid. As chief product a substance is obtained which he called "cetylid," $\mathrm{C}_{22} \mathrm{H}_{42} \mathrm{O}_{5}$, and which on fusing with caustic potash yields marsh-gas, hydrogen, and palmitic acid. According to him, this cetylid is probably a derivative of cetyl-alcohol.

Of special interest is the proof, as first shown by THUDICHUM and lately substantiated by THIERFELDER, that on heating the socalled cerebrin with dilute sulphuric acid a glucose is split off. 
This glucose 1s, as ThIERfelder has proved, identical with GaLACTOSE.

If we put aside the above differences in the composition and certain somewhat differing statements in regard to the qualitative reactions of cerebrin, there are still a few general reactions for all cerebrin preparations which may be employed in their detection. From these lately-described properties it has been claimed that, besides in the brain and nerves, cerebrin occurs in the pus-cells, and in the electric organ of the ray. GEOGHEGAN has also found it in a cancer of the liver.

Cerebrin, as generally described, is in the dry state a loose, purely while, odorless and tasteless powder. On heating it becomes brown at about $80^{\circ} \mathrm{C}$., puffs up, on continuing the heat melts and gradually decomposes. It is insoluble in water, cold alcohol, or ether, also in dilute caustic alkali or baryta-water. It swells in boiling water, forming a pasty mass. It dissolves in boiling alcohol (also ether), but on cooling a flocculent precipitate separates, which on microscopic examination is found to consist of a series of balls or grains. Cerebrin is chiefly characterized by these properties, and by its yielding a reducible substance on boiling with dilute mineral acids.

The Parcus's cerebrin differs from the ordinary in the following particulars : it is not soluble in boiling ether; on warming it melts without decomposing (which takes place at $145^{\circ}-160^{\circ} \mathrm{C}$.); it gives a light yellow solution with concentrated sulphuric acid, and it swells very little in cold water.

The Homocerebrin and ENCEPhalin of PARCus remain in the mother liquor after the precipitation of the impure cerebrin from the alcohol. These bodies have a tendency to separate as gelatinous mass. Homocerebrin, which, according to PARCUs, is homologous to cerebrin, is similar to it, but dissolve's more easily in warm alcohol and also in warm ether. It may also be obtained as extremely fine needles. Encephalin is claimed by Parcus to be a product of the metamorphosis of cerebrin. In the perfectly pure state it crystallizes in small plates. It swells in warm water, forming a pasty mass. Like the cerebrin and the homocerebin, it yields a reducible substance on boiling with dilute acids.

GAMGEE's PSEUDOCEREBIIN, which has thus far not been closely studied, is obtained as a by-product in the recrystallization of protagon.

The cerebrins are generally prepared according to MÜLLER'S method. The brain is first stirred with baryta-water until it appears like thin milk, and then it is boiled. The insoluble parts 
are removed, pressed, and repeatedly boiled with alcohol, which is filtered while boiling hot. The impure cerebrin which separates on cooling is freed from cholesterin and fat by means of ether, and then purified by repeated solution in warm alcohol. According to Parcus, this repeated solution in alcohol is continued until no gelatinous separation of homocerebrin or encephalin takes place.

According to GEoGHegaN's method, the brain is first extracted with cold alcohol and ether and then boiled with alcohol. 'The precipitate which separates on the cooling of the alcoholic filtrate is treated with ether and then boiled with baryta-water. 'The insoluble residue is purified by repeated solution in boiling alcohol.

The cerebrin may also be obtained from other organs by employing the above methods. The quantitative estimation, when such is desired, may be performed in the same way.

Neuridin, $\mathrm{C}_{6} \mathrm{H}_{14} \mathrm{~N}_{2}$, is a non-poisonous diamin discovered by BRIEGER, and which was obtained by him in the putrefaction of meat and gelatine. It also occur's under physiological conditions in the brain, and as traces in the yolk of the egg.

Neuridin dissolves in water, and yields on boiling with alkalies a mixture of dimethylamin and trimethylamin. It dissolves with difficulty in amyl-alcohol. It is insoluble in ether or absolute alcohol. In the free state neuridin has a peculiar odor, suggesting semen. With hydrochloric acid it gives a combination crystallizing in long needles. With platinic chloride or gold chloride it gives crystallizable double combinations which are valuable in its preparation and detection.

The so-called CoRpuscula AMYLACEA, which occur on the upper surface of the brain and in the pituitary gland, are colored more or less pure violet by iodine and more blue by sulphuric acid and iodine. They consist, perhaps, of the same substance as certain prostatic calculi, but they have not been closely investigated.

Quantitative Composition of the Brain. The quantity of water is greater in the gray than in the white substance, and greater in newborn or young individuals than in grown ones. The brain of the fotus contains 879-926 p. m. water. According to the observations of WEISBACH, the amount of water in the several parts of the brain (and in the medulla) varies at different ages. The following figures are in 1000 parts ; $A$ for men and $B$ for women.

\begin{tabular}{|c|c|c|c|c|c|c|c|c|}
\hline \multirow{3}{*}{$\begin{array}{l}\text { White substance of } \\
\text { the brain........ }\end{array}$} & \multicolumn{2}{|c|}{ 20-30 Years. } & \multicolumn{2}{|c|}{ 30-50 Years. } & \multicolumn{2}{|c|}{ 50-r0 Years. } & \multicolumn{2}{|c|}{ 70-94 Years, } \\
\hline & $\boldsymbol{A}$. & $B$. & $\boldsymbol{A}$. & B. & A. & $B$. & $A$. & $B$. \\
\hline & 695.6 & 682.9 & 6831 & 703.1 & 701.9 & 689.6 & 726.1 & 722.0 \\
\hline 7ray ditto & 833.6 & 826.2 & 836.1 & 830.6 & 838.0 & 838.4 & 847.8 & 889.5 \\
\hline & 784.7 & 792 & 795.9 & 772.9 & 79 & & 2.3 & 801.7 \\
\hline & 788.3 & 794 & 778.7 & 789 & & & 3.4 & 797.9 \\
\hline & 734.6 & 740.3 & 725.5 & 722.0 & 720.1 & 714 & 727.4 & 724.4 \\
\hline Fivaga & 744 & 740.7 & 732.5 & 729.8 & 722.4 & 730.6 & 736.2 & 736.7 \\
\hline
\end{tabular}


Quantitative analyses of the brain have also been made by Petrowsky on an ox-brain and by Baumstark on the brain of a horse. In the analyses of Petrowsky the protagon has not been considered, and all organic, phosphorized substances were calculated as lecithin. On these grounds these analyses are not of much value from a certain standpoint. In BAUMSTARK's analyses the gray and the white substance could not be sufficiently separated, and these analyses, on this account, show partly an excess of white and partly an excess of gray substance; nearly one half of the organic bodies, chiefly consisting of bodies soluble in ether, could not be exactly analyzed. Neither do these analyses give sufficient explanation of the quantitative composition of the brain.

The analyses made up to the present time give, as above stated, an equal division of the organic constituents in the gray and white substance. In the analyses of PETROWSKY the quantity of albumin and gelatine-forming substances in the gray matter was somewhat more than one half, and in the white about one quarter of the solid organic substances. The quantity of cholesterin in the white was about one half, and in the gray substance about one fifth, of the solid bodies. A greater quantity of soluble saits and extractive bodies was found in the gray substance than in the white (BAUMSTARK). The following analyses of BAUMSTARK give the most important known constituents of the brain calculated in 1000 parts of the fresh, moist brain. $A$ represents chiefly the white, and $B$ chiefly the gray, substance.

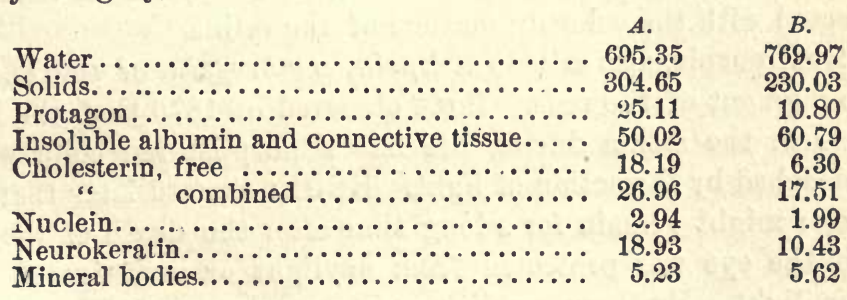

The remainder of the solids probably consists chiefly of lecithin and other phosphorized bodies. Of the total amount of phosphorus, $15-20 \mathrm{p}$. m. belongs to the nuclein, 50-60 p. m. to the protagon, $150-160 \mathrm{p}$. m. to the ash, and $790 \mathrm{p}$. m. to the lecithin and the other phosphorized organic substances. 
The quantity of neurokeratin in the nerves and in the different parts of the brain has been carefully determined by KüHNE and ChitTenden. They found $3.16 \mathrm{p} . \mathrm{m}$. in the plexus brachialis, $3.12 \mathrm{p} . \mathrm{m}$. in the edge of the cerebellum, $22.434 \mathrm{p} . \mathrm{m}$. in the white substance of the large brain, 25.72-29.02 p. m. in the white substance of the corpus callosum, and $3.27 \mathrm{p} . \mathrm{m}$. in the gray substance of the edge of the large brain (when free as possible from white substance).

The quantity of mineral constituents in the brain amounts to 2.95-\%.08 p. m., according to GEOGHEGAN. He found in 1000 parts of the fresh, moist brain $0.43-1.32 \mathrm{Cl}, 0.956-2.016 \mathrm{PO}_{4}, 0.244-0.796$ $\mathrm{CO}_{3}, 0.102-0.220 \mathrm{SO}_{4}, 0.01-0.098 \mathrm{Fe}_{2}\left(\mathrm{PO}_{4}\right)_{2}, 0.005-0.022 \mathrm{Ca}, 0.016-$ $0.072 \mathrm{Mg}, 0.58$ to $1.778 \mathrm{~K}, 0.450-1.114 \mathrm{Na}$. The gray substance yields an alkaline ash, the white an acid ash.

\section{Appendix.}

\section{The Tissue and Fluids of the Eye.}

The retina contains in all $816-880$ p. m. water, $72-102$ p. m. proteid bodies-myosin, albumin, and mucin (?), 9-32 p. m. lecithin, and 2.7-10.6 p. m. salts (Hoppe-Seyler and KAHN). The mineral bodies consist of 420 p. m. $\mathrm{Na}_{2} \mathrm{HPO}_{4}$ and 350 p. m. $\mathrm{NaCl}$.

Those bodies which form the different segments of the rods have not been closely studied, and the greatest interest is therefore connected with the coloring matters of the retina.

Visual purple, also called rhodopsin, erythropsin, or VISUAL RED, is the pigment of the rods. BoLL observed in $18 \% 6$ that the layer of rods in the retina during life had a purplish-red color which was bleached by the action of light. KüHNE showed later that this red color might remain for a long time after the death of the animal if the eye was protected from daylight or investigated by a sodium light. By these conditions it was also possible to isolate and closely study this substance.

Visual red (BOLL) or visual purple (KüHNE) has become known mainly by the investigations of KüHNE. The pigment occurs chiefly in the rods and only in their outer parts. In animals whose retina has no rods the visual purple is absent, and is also necessarily 
absent in the macula lutea. In a variety of bat (rhinolophus hipposideros), in hens, pigeons, and new-born rabbits, no visual purple has been found in the rods.

A solution of visual purple in water which contains $2-5 \%$ crystallized bile, the best solvent for it, is purple-red in color, quite clear, and not fluorescent. On evaporating this solution in vacuo, we obtain a residue similar to ammonium carminate which contains violet or black grains. If the above solution is dialyzed with water, the bile is diffused and the visual purple separates as a violet mass. Under all circumstances, even when still in the retina, the visual purple is quickly bleached by direct sunlight, and with diffused light with a rapidity corresponding to the intensity of the light. It passes from red and orange to yellow. Red light bleaches the visual purple slowly; the ultra-red light does not bleach it at all. A solution of visual purple shows no special absorption-bands, but only a general absorption which extends from the red side, beginning at $D$, to the line $G$. The strongest absorption is found at $E$.

Visual purple when heated to $52^{\circ}-53^{\circ} \mathrm{C}$. is destroyed after several hours, and almost instantly when heated to $+76^{\circ} \mathrm{C}$. It is also destroyed by alkalies, acids, alcohol, ether, and chloroform. On the contrary, it resists the action of ammonia or alum solution.

As the visual purple is easily destroyed by light, it must there. fore also be regenerated during life. KüHNE has also found that the retina of the eye of the frog becomes bleached when exposed for a long time to strong sunlight, and that its color gradually returns when the animal is placed in the dark. This regeneration of the visual purple is a function of the living cells in the layer of the pigment epithelium of the retina. This may be inferred from the fact that a detached piece of the retina which has been bleached by light may have its visual purple restored if the detached piece of the retina be carefully laid on the chorioildea having layers of the pigment epithelium attached. The regeneration has, it seems, nothing to do with the dark pigment, the melanin or fuscin, in the epithelium cells. A partial regeneration seems, according to KÜHNE, to be possible in the completely-removed retina. On account of this property of the visual purple of being bleached by light during life, we may, as KüHNe has shown, under special conditions and by observing special precautions, obtain after death by 
the action of intense light or more continuous light the picture of bright objects, such as windows and the like, so-called optograms.

The physiological importance of visual purple is unknown. It follows that the visual purple is not essential to sight, since it is absent in certain animals and also in the cones. HoLMGreN has further shown that in the eye of a frog or rabbit, whose retina has been previously bleached by continuous light, a flow of nega. tive electricity is continually observed in the optic nerve when the eye is exposed to the action of light.

Visual purple must always be prepared exclusively in a sodium light. It is extracted from the net membrane by means of a watery solution of crystallized bile. The filtered solution is evaporated in vacuo or dialyzed until the visual purple is separated.

The pigments of the cones. In the inner segments of the cones of birds, reptiles, and fishes a small fat-globule of varying color is found. KüHNE has isolated from this fat a green, a yellow, and a red pigment called respectively chlorophan, xanthophan, and rhodophan.

The dark pigment of the epithelium cells of the net membrane, which was formerly called melanin, but since named fuscin by KüHNE, dissolves in concentrated caustic alkalies or concentrated sulphuric acid on warming, but, like melanine in general (see Chapter XIII), has been little studied. The pigment occurring in the pigment-cells of the chorioïdea seems to be identical with the fuscin of the retina.

The vitreous humor is often considered as a variety of gelatinous tissue. It contains albumin and, as C. TH. MörNer has shown, a mucoid (see page 234). Among the extractives we find a little urea -according to PICARD, 5. p. m., and according to RöHMANN, 0.64 p. $m$. The reaction of the vitreous humor is alkaline, and the quantity of solids amounts to about $11 \mathrm{p}$. m. The quantity of mineral bodies is about $9 \mathrm{p}$. m., and the albuminous bodies $0.7 \mathrm{p.} \mathrm{m}$. In regard to the aqueous humor see page 126 .

The crystalline lens. That substauce which forms the capsule of the lens has not had its chemical nature closely investigated, but gives a reducible body when boiled with dilute acids (C. TH. Mörner). The real substance of the lens, the lens-fibres, contains as chief constituent a globulin which is nearly related to vitellin and to which Berzelius gave the name crystallin. This globulin may be partially precipitated from a watery extract of the lens by means of $\mathrm{CO}_{2}$, and it may be completely separated by saturating with $\mathrm{MgSO}_{4}$. The statements as to the occurrence of an albumin besides the globulin in the lens are disputed; that at least 
in the lens of cattle an albumin occurs is nevertheless easy to demonstrate.

A. BÉchamp distinguishes the two following albuminous bodies in the watery extract of the crystalline lens. Phacozymase, which coagulates at $+55^{\circ}$ C., contains a diastatic enzyme and has a specific rotary power of $(\alpha) j=-41^{\circ}$, and the crystalbumin, with a specitic rotary power of $\left(\alpha \hat{b}=-80.3^{\circ}\right.$. BÉCHAMP extracted from the residue of the lens, which was insoluble in water, by means of hydrocoloric acid, an albuminous body which had a specific rotary power of $(\alpha) j=-80.2^{\circ}$ which be called crystal. fibrin.

The lens does not seem to contain any albuminous body which coagulates spontaneously like fibrinogen. That cloudiness which appears after death depends, according to KüHNE, upon the unequal changing of the concentration of the contents of the lenstubes. This change is produced by the altered ratio of diffusion. A cloudiness of the lens may also be produced in life by a rapid removal of water, as, for example, when a frog is plunged into a salt or sugar solution (KUNDE). The appearance of cloudiness in diabetes has been attributed by some to the removal of water. The views on this subject, however, do not accord.

The average results of four analyses made by LAPTSCHINSKY of the lens of oxen are here given, calculated in parts per 1000:

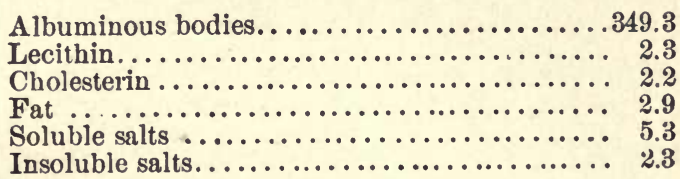

In cataract the amount of albumin is diminished and the amount of cholesterin increased.

The corneal tissue has been previously treated of (page 237). The sclerotica has not been closely investigated, and the chlorioidea is chiefly of interest because of the coloring matter, melanine, it contains (see Chap. XIII).

TEARS consist of a water-clear, alkaline fluid of a saltish taste. According to the analyses of LERCH, they contain $982 \mathrm{p}$. m. water, 18 p. m. solids, with 5 p. m. albumin and 13 p. m. NaCl. 
The Fluids of the Inner Ear.

The perilymph and endolymph are alkaline fluids which, besides salts, contain-in the same amounts as in transudations-traces of albumin, and in certain animals (torsk) also mucin. The quantity of mucin is greater in the perilymph than in the endolymph.

Otoliths contain $745-795 \mathrm{p}$. m. inorganic substance which consists chiefly of crystallized calcium carbonate. The organic substance is very like mucin. 


\section{CHAPTER XI.}

\section{ORGANS OF GENERATION.}

\section{(a) Male Generative Secretions.}

THE testes have been little investigated chemically. We find in the testes of animals albuminous bodies of different kinds, serum albumin, alkali albuminate (?), and an albuminous body related to Rovidas' Tyaline substance, also leucin, tyrosin, creatin, xanthin bodies, cholesterin, lecithin, inosit, and fat. In regard to the occurrence of glycogen the statements are somewhat contradictory. DARESTE found in the testes of birds granules similar to starch, which were colored blue with difficulty by iodine.

The semen as ejected is a white or whitish-yellow, viscous, sticky fluid of a milky appearance, with whitish, non-transparent lumps. The milky appearance is due to semen-fibres. Semen is heavier than water, contains albumin, has a neutral or faintlyalkaline reaction, and a peculiar specific odor. The semen of bulls has an acid reaction. Soon after ejection semen becomes gelatinous, as if it were coagulated, but afterwards becomes more fluid. When diluted with water white flakes or shreds separate (HENLE's fibrin). According to the analyses of VAUquelin, the human semen contains $900 \mathrm{p}$. m. water and $100 \mathrm{p}$. m. solids, with $60 \mathrm{p} . \mathrm{m}$. organic and $40 \mathrm{p}$. m. inorganic substance, of which $30 \mathrm{p.}$. is calcium phosphate. Among the albuminous bodies PosNer claims that propeptone occurs even in the absence of the semen-fibres.

The semen in the vas deferens differs chiefly from the ejected in that it is without the peculiar odor. This last depends on the admixture with the secretion of the prostate. This secretion, according to IVERSEN, has a milky appearance and ordinarily an alkaline reaction, very rarely a neutral one, contains small amounts 
of proteids and mineral bodies, especially $\mathrm{NaCl}$. Besides these it contains a crystalline combination of phosphoric acid with a base, $\mathrm{C}_{2} \mathrm{H}_{5} \mathrm{~N}$. This combination has been called BöTTCHER's spermine crystals, and it is claimed that the specific odor of the semen is due to a partial decomposition of these crystals.

The crystals which appear on slowly-evaporating the semen, and which are also observed in anatomical preparations kept in alcohol, and in desiccated egg-albumin, are identical, according to Schreiner, with Charcot's crystals found in the blood, and in the lymphatic glands in leucaemia. They are, according to ScHreINER, a combination of phosphoric acid with a base, $\mathrm{C}_{2} \mathrm{H}_{5} \mathrm{~N}$, which he discovered, and this base is, according to LADENBURG and ABEL, probably ethylenimin.

The semen-fibres (spermatozoa) show a great resistance to chemical reagents in general. They do not dissolve completely in concentrated sulphuric acid, nitric acid, acetic acid, nor in boilinghot soda solutions. They are dissolved by a boiling-hot causticpotash solution. They resist putrefaction, and after drying they may be obtained again in their original form by moistening them with a 1\% common-salt solution. By careful heating and burning to an ash the shape of the spermatozoa may be seen in the ash. The quantity of ash is about $50 \mathrm{p}$. m. and consists mainly ( $\left(\frac{3}{4}\right)$ of potassium phosphate.

The spermatozoa show well-known movements, but the cause of this is not known. This movement may continue for a very long time, as under some conditions it may be observed for several days in the body after death, and in the secretion of the uterus longer than a week. Acid liquids stop these movements immediately; they are also destroyed by strong alkalies, especially ammoniacal liquids, also by distilled water, alcohol, ether, etc: The movements continue for a longer time in faintly-alkaline liquids, especially in alkaline animal secretions, and also in properly-diluted neutral salt-solutions.

According to the investigations of MIESCHER, there are lecithin and nuclein but no cerebrin in the SPERMATOZOA of BULLS. The head of the spermatozoa contains nuclein, which forms probably the outer part of the head; albumin, which forms the contents of the head; and lastly a substance rich in sulphur which has not been studied. The tail dissolves in gastric juice after continuous 
digestion, and seems to consist of albuminous bodies, or bodies closely related, which show a variable resistance towards pepsinhydrochloric acid. The semen of bulls does not contain any adenin, but the other three xanthin bodies treated of on pages 49-51 (Kossel and SchindLer) are present.

The spermatozon of the RHINe salmon show, according to Mrescher, a great resistance. With caustic-potash and soda solutions they give a cloudy, gelatinous mass which is precipitated as shreds by acids; but these shreds do not dissolve in an excess of the acid. They are strongly attacked by a $10-15 \%$ solution of $\mathrm{NaCl}$ or $\mathrm{NaNO}_{3}$, and the semen is converted by such a solution into a stiff gelatin. The head is attacked, but not the tail or the middle part. This last-mentioned part, like the tail, contains albumin, which is dissolved by hydrochloric acid of $1 \mathrm{p} . \mathrm{m}$., but not in $\mathrm{NaCl}$. Mrescher also found lecithin, fat, cholesterin, guanin, and sarkin in relatively large amounts in the salmon-semen. The organic constituent occurring in the largest amount in the salmonsemen is, according to MrescHer, a combination of nuclein with the base protamin, $\mathrm{C}_{9} \mathrm{H}_{20} \mathrm{~N}_{5} \mathrm{O}_{2} \cdot \mathrm{OH}$, which is soluble in water but insoluble in alcohol or ether.

According to Mrescher and PrCCARD, the spermatozoa of salmon contain 487 p. m. nuclein-protamin (268 p. m. protamin), $103 \mathrm{p} . \mathrm{m}$. albuminous bodies, 75 p. m. lecithin, $60-80$ p. m. guanin and sarkin, $22 \mathrm{p} . \mathrm{m}$. cholesterin, and $45 \mathrm{p}$. m. fat. KosseL and SCHINDLER found no guanin, but xanthin and large amounts of adenin and hypoxanthin, in the SEMEN of the CARP.

Spermatin is a name which has been given to a constituent similar to alkali albuminate, but it has not been closely studied.

Prostatic concrements are of too kinds. One is very small, generally oval in shape with concentric layers. In young but not in older persons they are colored blue by iodine (IVERSEN). According to PAULICKY, they yield sugar when warmed with dilute sulphuric acid; but this statement has not been substantiated by IvERsEN. The other kind is larger, sometimes the size of the head of a pin, and consisting chiefly of calcium phosphate (about 700 p. m.) with only a very small amount, about 160 p. m., organic substance.

\section{(b) Female Generative Organs.}

The stroma of the ovaries are of little interest from a physiologico-chemical standpoint, and the most important constituent of the ovaries, the Graaffian vesicles with the ovum, have thus far not 
been the subject of a careful chemical investigation. The fluid in the vesicles (of the cow) do not contain, as has been stated, the peculiar bodies, paralbumin or metalbumin, which are found in certain pathological ovarial fluids, but seems to be a serous liquid. The corpora lutea are colored yellow by an amorphous coloring-matter called lutein. Besides this another coloring-matter sometimes occurs which is not soluble in alkali ; it is crystalline, but not identical with bilirubin or hæmatoidin ; but it may be identified as a lutein by its spectroscopic behavior (PICCOLO and LIEBEN, KüHNE and EWALD).

The cysts often occurring in the ovaries are of special pathological interest, and these may have essentially different contents, depending upon their variety and origin.

The serous cysts (HYDROPS FOLLICULORUM GRAAFII), which are formed by a dilation of the Graafian vesicles, contain a serous liquid which has a specific gravity of 1.005-1.022. A specific gravity of 1.020 is less frequent. Generally the specific gravity is lower, 1.005-1.014, with 10-40 p. m. solids. As far as is known, the contents of these cysts do not essentially differ from other serous liquids.

The proliferous cysts (MYXOID CYSTS, COLLOID CYSTS). The contents of these cysts have variable properties.

We sometimes find in small cysts a semi-solid, transparent, or somewhat cloudy or opalescent mass which appears like solidified glue or quivering jelly and which has been called colloid because of its physical properties. In other cases the cysts contain a thick, tough mass which can be drawn out into long threads, and as this mass in the different cysts is more or less diluted with serous liquids their contents may have a variable consistency. The color of the contents is also variable. In certain cases they are bluish white, opalescent, and in others yellow, yellowish brown, or yellowish with a shade of green. They are oiten colored more or less chocolate-brown or red-brown, due to the decomposed blood-coloring matters. The reaction is alkaline or nearly neutral. The specific gravity, which may vary considerably, is generally $1.015-1.030$, but may in few cases be 1.005-1.010 or 1.050-1.055. The amount of solids is variable. In rare cases they amount to only 10-20 p. m.; 
ordinarily they vary between $50-70-100$ p. m. In a few cases 150-200 p. m. solids have been found.

As form-elements we find red and colorless blood-corpuscles, granular cells, partly fat-degenerated epithelium and partly large so-called GLUGE's corpuscles, fine granular masses, epithelium-cells, cholesterin-crystals, and colloid corpuscles-large, circular, refractive formations.

Though the contents of the proliferous cyst may have a variable composition, still it may be characterized in typical cases by its slimy or ropy consistency; by its grayish-yellow, chocolate-brown, sometimes whitish-gray color, and by its relatively high specific gravity, 1.015-1.025. Such a liquid does not ordinarily show a spontaneous fibrin-coagulation.

We cousider colloid, metalbumin and paralbumin as characteristic constituents of these liquids.

Colloid. This name does not designate any particular chemical substance, but is given to the contents of tumors with certain physical properties similar to gelatinous glue. Colloid is found as a diseased product in several organs.

Colloid is a gelatinous mass, insoluble in water and acetic acid; it is dissolved by alkalies and gives a liquid which is not precipitated by acetic acid or by acetic acid and potassium ferrocyanide. Sometimes a colloid is found which, when treated with a very dilute alkali, gives a solution similar to a mucin solution. On boiling with acids colloid gives a reducible substance. It is related to mucin, and it is considered by certain investigators as a transformed mucin. A colloid found by WURTz in the lungs contains $\mathrm{C} 48.09, \mathrm{H} \% .4 \%, \mathrm{~N} \% .00$, and $03 \% .44 \%$. Colloids of difforent origin seem to have an unequal composition.

Metalbumin. This name ScHERER gave to a proteid substance found by him in an ovarial fluid. The metalbumin was considered by ScHerer to be an albuminous body, but it belongs to the mucin group and it is therefore called by the author pseudomucin.

Pseudomucin. 'This body, which, like mucin, gives a reducible substance when boiled with acids, is a mucoid of the following composition: C 49.75, H 6.98, N 10.28, S 1.25, O 31.74\% (AUTHOR). With water pseudomucin gives a slimy, ropy solution, and it is this substance which gives the fluid contents of the ovarial cysts their 
typical ropy property. Its solutions do not coagulate on boiling but only become milky-opalescent. Unlike mucin solutions, pseudomucin solutions are not precipitated by acetic acid. With alcohol they give a coarse flocculent or thready precipitate which only dissolves in water or alcohol after having been kept in these liquids for a long time.

Paralbumin is another substance discovered by SCHERER and which occurs in ovarial liquids and also in ascites fluids with the simultaneous presence of ovarial cysts and rupture of the same. It is therefore only a mixture of pseudomucin with variable amounts of albumin, and the reactions of paralbumin are correspondingly variable.

The detection of metalbumin and paralbumin is naturally connected with the detection of pseudomucin. A typical ovarial fluid containing pseudomucin is, as a rule, sufficiently characterized by its physical properties, and a special chemical investigation is only necessary in cases where a serous fluid contains very small amounts of pseudomucin. We proceed in the following way: The albumin is removed by heating to boiling with the addition of acetic acid; the filtrate is strongly concentrated and precipitated by alcohol. The precipitate is carefully washed with alcohol and then dissolved in water. A part of this solution is digested. with saliva at the temperature of the body and then tested for glucose (derived from glycogen or dextrin). If glycogen is present, it will be converted into glucose by the saliva; precipitate again with alcohol and then proceed as in the absence of glycogen. In this last-mentioned case, first add acetic acid to the solution of the alcohol precipitate in water so as to precipitate any existing mucin. The precipitate produced is filtered, the filtrate treated with $2 \%$ $\mathrm{HCl}$ and warmed on the water-bath until the liquid is deep brown in color. In the presence of pseudomucin this solution gives 'T'ROMMER's test.

The other proteid bodies which have been found in cystic fluids are serum-globulin and serum-albumin, peptone (?), mucin, and mucin-peptone (?). Fibrin only occurs in exceptional cases. The quantity of mineral bodies on an average amount to about 10 p. m. The amount of extractive bodies (cholesterin and urea) and fat is ordinarily $2-4 \mathrm{p} . \mathrm{m}$. The remaining solids, which constitute the chief mass, are albuminous bodies and pseudomucin.

The intraligamentary, papillary cysts contain a yellow, Jellowish- 
green, or brownish-green liquid which either contains no pseudomucin or-with the simultaneous degeneration of colloid-very little. The specific gravity is generally rather high, 1.032-1.036 with 90-100 p. m. solids. The principal constituents are the albuminous bodies of blood-serum.

The rare tubo-ovarial cysts contain as a rule a,watery, serous fluid containing no pseudomucin.

The parovarial cysts or the CYSTS of the LIGAMENTA LATA may attain a considerable size. In general the contents are watery, mostly very pale yellow-colered, water-clear or only slightly opalescent liquids. The specific gravity is low, 1.002-1.009; and the solids only amount to 10-20 p. m. Pseudomucin does not occur as a typical constituent; albumin is sometimes absent, and when it does occur the quantity is very small. The principal part of the solids consist of salts and extractive bodies. In exceptional cases the fluid may be rich in albumin, and may show a higher specific gravity.

\section{- The Egg.}

The small ova of man and mammalia cannot, for evident reasons, be the subject of a searching chemical investigation. Up to the present time the eggs of birds, amphibians, and fishes have been investigated, but above all the hen's egg. We will here occupy ourselves with the constituents of this last.

The yolk of the hen's egg. In the so-called white yolk, which forms the germ with a continuation reaching to the centre of the yolk (latebra) and also a layer found between the yolk and yolkmembrane, we find albumin, nuclein, lecithin, and potassium (LirebermanN). The occurrence of glycogen is doubtful. The yolk-membrane consists of an albumoid similar in certain respects to keratin (LIEBERMANN).

The principal part of the yolk-the nutritive yolk or yellow-is a viscous, non-transparent, pale- or orange-yellow alkaline emulsion of a mild taste. The yolk contains vitellin, lecithin, cholesterin, fat, coloring matters, traces of neuridin (BRIEGER), glucose in very small quantities, and mineral bodies. The occurrence of cerebrin 
and of granules similar to starch (DARESTE) has not been positively proved.

Ovovitellin. This body is generally considered as a globulin, but it resembles a nucleoalbumin more. The question as to what relationship other proteid substances, such as the aleurone crystals of certain semen, and the so-called "dotterplättchen" in the eggs of certain fishes and amphibians which are related to ovovitellin, bear to this substance, is a question which requires further investigation.

The ovovitellin which has been prepared from the yolk of eggs is not a pure albuminous body, but always contains lecithin. HOPPE-SEYLER found 25\% lecithin in vitellin and also some nuclein. The lecithin may be removed by boiling alcohol, but the vitellin is changed thereby and it is therefore probable that the lecithin is chemically united with the vitellin (HOPPE-Seyler). Bunge prepared a nuclein by digesting the yolk with gastric juice, and this nuclein, according to him, is of great importance in the formation of the blood, and on these grounds he called it homatogen. This hæmatogen-whose composition is as follows: $\mathrm{C} 42.11, \mathrm{H} 6.08, \mathrm{~N}$ 14.73, S $0.55, \mathrm{P} 5.19, \mathrm{Fe} 0.29$ and $\mathrm{O} 31.05 \%$ seems to be a product of the decomposition of vitellin.

Vitellin is similar to the globulins in that it is insoluble in water ; but on the contrary soluble in dilute neutral-salt solutions (although the solution is not quite trausparent). It is also soluble in hydrochloric acid of $1 \mathrm{p}$. m. and in very dilute solutions of alkalies or alkali carbonates. It is precipitated from its salt solution by diluting with water, and when allowed to stand some time iu contact with water the vitellin is gradually changed, forming a substance more like the albuminates. The coagulation temperature for the solution containing salt $(\mathrm{NaCl})$ lies between $+70^{\circ}$ and $75^{\circ} \mathrm{C}$. or, when heated very rapidly, at about $+80^{\circ} \mathrm{C}$. Vitellin differs from the globulins in yielding nuclein by pepsin digestion. On this account vitellin, as previously stated (page 14), is considered as a nucleoalbumin even though it differs from them in general by the coagulation of its neutral solutions containing salt, at a tomperature below $100^{\circ} \mathrm{C}$; ; also by certain solubilities and precipitations. Because of the difficulty of removing lecithin without changing the properties of vitellin it is necessary, since lecithin may 
essentially change the solubility and precipitating capacity of the albuminous body, to wait for further investigations before we place vitellin definitely in the globulin or nucleoalbumin group.

The chief points in the preparation of ovovitellin are as follows: The yolk is thoroughly agitated with ether; the residue is dissolved in a $10 \%$ common-salt solution, filtered, and the vitellin precipitated by adding an abundance of water. 'The vitellin is now purified by repeatedly redissolving in dilute common-salt solutions and precipitating by water.

The yolk also contains, besides vitellin, alkali-albuminate and albumin.

The $f a t$ of the yolk of the egg is, according to Liebermann, a mixture of a solid and a liquid fat. The solia fat consists chiefly of tripalmitin with some stearin. On the saponification of the egg-oil LiEbermanN obtained 40\% oleic acid, $38.04 \%$ palmitic acid and 15.21\% stearic acid. The fat of the yolk of the egg contains less carbon than other fats, which may depend on the presence of mono and diglycerides or on a quantity of fatty acid deficient in carbon (LIEBERMANN).

Lutein. Yellow or orange-red amorphous coloring matters occur in the yellow of the egg and in several other places in the animal organism, for instance in the blood-serum and serous fluids, fatty tissues, milk-fat, corpora lutea, and in the fat-globules of the retina. These coloring matters, which also occur in the vegetable kingdom (THUDICHUM), have been called luteines or lipochromes.

The luteines, which among themselves show somewhat different properties, are all soluble in alcohol, ether, and chloroform. They differ from the bile-coloring matter, bilirubin, in that they are not separated from their solution in chloroform by water containing alkali, and also in that they do not give the characteristic play of colors with nitric acid containing a little nitrous acid, but give a transient blue color, and lastly they give an absorption-spectrum of ordinarily two bands, of which one covers the line $F$ and the other lies between the lines $F$ and $G$. The luteines withstand the action of alkalies so that they are not changed when we remove the fats present by means of saponification.

The lutein from the yolk of the egg has not been prepared in a pure state. According to CHevreul and GoBLEY, the yolk contains a partly red and partly yellow pigment. MaLY has found two pigments free from iron in the eggs 
of the water-spider (maja squinado), one a red, vitellorubin, and the other a yellow pigment, vitellolutein. Both of these pigments are colored blue by nitric acid containing nitrous acid, and beautifully green by concentrated sulphuric acid. The absorption-bands, especially of the vitellolutein, correspond very nearly with those of ovolutein.

The mineral bodies of the yolk of the egg consist, according to PoLECK, of 51.2-65.7 parts soda, 89.3-80.5 pótash, 122.1-132.8 lime, 20.7-21.1 magnesia, 14.5-11.90 iron oxide, 638.1-66\%.0 phosphoric acid, and 5.5-14.0 parts silicic acid in 1000 parts of the ash. We find phosphoric acid and lime the most abundant, and then potash, which is somewhat greater in quantity than the soda. These results are not, however, quite correct, first, because no dissolved phosphate occurs in the yolk (LIEBERMANN), and secondly, in burning, phosphoric and sulphuric acids are produced and these drive away the chlorine, which is not accounted for in the preceding analyses.

The yolk of the hen's egg weighs about 12-18 grms. The quantity of water and solids amounts, according to PARKES, to 4 \%1.9 p. m. and 528.1 p. m. respectively. Among the solids he found 156.3 p. m. albumin, 3.53 p. m. soluble and 6.12 p. m. insoluble salts. The quantity of fat, according to PARKES, is 228.4 p. m., the lecithin, calculated from the amount of phosphorus in the organic substance in the alcohol-ether extract, was $10 \% .2 \mathrm{p} . \mathrm{m}$., and the cholesterin $17.5 \mathrm{p} . \mathrm{m}$.

The white of the egg is a faint-yellowish albuminous fluid enclosed in a framework of thin membranes; and this fluid is in itself very liquid, but seems viscous because of the presence of these fine membranes. That substance which forms the membranes, and of which the chalaza consists, seems to be a body nearly related to horn substances (LiEBERMANN).

The white of the egg has a specific gravity of 1.045 and always nas an alkaline reaction. It contains $850-880$ p. m. water, $100-130$ p. m. albuminous bodies, and 7 p. m. salts. Among the extractive bodies LeHMANN found a fermentable kind of sugar which amounted to $5 \mathrm{p}$. m., or according to MeIssner, 80 p. m. Besides these, we find in the white of the egg traces of fats, soaps, lecithin, and cholesterin.

The white of the egg during incubation becomes transparent on boiling and acts in many respects like alkali-albuminate. This albumin TARCHANOFF called "tatalbumin." 
The albuminous bodies of the white of the egg belong partly to the globulin and partly to the albumin group.

'The egg-globulin is, according to DILLNER, closely related to serum-globulin. On diluting the white of the egg with water it partly separates. It is also precipitated by magnesium sulphate. The quantity of globulins in the white of the egg is on an average $6.67 \mathrm{p}$. m., or about $67 \mathrm{p}$. m. of the total proteids. According to CorIN and BERARD, we have two globulins in the white of the egg, one coagulating at $+5 \% .5^{\circ} \mathrm{C}$., and the other at $+67^{\circ} \mathrm{C}$.

Ovalbumin, or the albumin of the white of the egg. The composition of this albumin is $\mathrm{C} 52.25, \mathrm{H} 6.9, \mathrm{~N} 15.25$, and $\mathrm{S} 1.93 \%$. The ovalbumin has the properties of the albumins in general, but differs from serum-albumin in the following: The specific rotary power is lower $\alpha(D)=-38^{\circ}$. Solutions containing medium amounts of the albumin coagulate at $+56^{\circ} \mathrm{C}$., irrespective whether the amount of salt present is large or small. On the contrary, the temperature of coagulation changes with a constant amount of salt, but with variable amounts of albumin in the solution. Ovalbumin is quickly rendered insoluble by alcohol. It is precipitated by a sufficient quantity of nitric or hydrochloric acid, but it dissolves with more difficulty in an excess of these acids than the serum-albumin. Ovalbumin in solution, when introduced into the bloodcirculation, passes into the urine, which is not the case with serumalbumin.

According to GaUtier and BÉCHAMr, ovalbumin is a mixture of two albumins with a coagulation temperature $60^{\circ}-63^{\circ}$ and $71^{\circ}-74^{\circ} \mathrm{C}$. respectively. According to CoRIN and BERARD, it is a mixture of three albumins with a coagulation-temperature respectively $67^{\circ}, 72^{\circ}$, and $82^{\circ} \mathrm{C}$. Perhaps the fact has been overlooked that the coagulation-temperature of ovalbumin is changed by variable amounts of albumin in solution.

Ovalbumin is obtained by precipitating the globulins with $\mathrm{MgSO}_{4}$ at $+20^{\circ} \mathrm{C}$. and saturating the filtrate with $\mathrm{Na}_{2} \mathrm{SO}_{4}$ at the same temperature. The ovalbumin which separates is filtered, pressed, dissolved in water, and freed from salts by dialysis. The dialyzed solution is then evaporated in a vacuum or at $40^{\circ}-50^{\circ} \mathrm{C}$. If precipitated with alcohol, albumin becomes quickly insoluble.

The mineral bodies of the white of the egg have been analyzed by Poleck and Weber. They found in 1000 parts of the ash: 276.6-284.5 grms. potash, 235.6-329.3 soda, 17.4-29 lime, 16-31.' 
magnesia, 4.4-5.5 iron oxide, 238.4-285.6 chlorine, 31.6-48.3 phosphoric acid $\left(\mathrm{P}_{2} \mathrm{O}_{5}\right), 13.2-26.3$ sulphuric acid, 2.8-20.4 silicic acid and 96.7-116 grms. carbon dioxide. Traces of fluorine have also been found (NICKLÉs). The ash of the white of the egg contains, as compared with the yolk, a greater amount of chlorine and alkalies, and a smaller amount of lime, phosphoric acid, and iron.

The Shell-membrane and the Egg-shell. The shell-membrane consists, as above stated (page 35), of a keratin substance. The shell contains very little organic substance, $36-65 \mathrm{p} . \mathrm{m}$. The chief mass, more than 900 p. m., consists of calcium carbonate; besides this there are very small amounts of magnesium carbonate and earthy phosphates.

The different coloring of birds' eggs depends, as WiCkE, Sorbr, LIEBERMANN and KrUKeNBERg have shown, upon several coloring matters. Among these we find a red or reddish-brown pigment called "oorodein" by SoRBY, which is perhaps identical with hæmatoporphyrin (SoRBY, KRUKENBERG). The green or blue coloring matter, SonBY's oocyan, seems, according to Liebermann, and Krukenbera, to be partly biliverdin and partly a blue derivative of the bile-pigments.

The eggs of birds have a space at their blunt end filled with gas; this gas contains on an average 23.3 rols. per cent oxygen (BIschoFf).

The weight of a hen's egg varies between 40-60 grammes and may weigh sometimes 70 grms. The shell and shell-membrane together when carefully cleaned, but still in the moist state, weigh 5-8 grms. The yolk weighs 12-18 and the white 23-34, or about double.

The white of the egg of cartilaginous and bone fishes contains only traces of true albumin, and the cover of the frog's egg consists, according to Giacosa, of mucin. The crystalline formations (yolk-spherules or dotterplättchen) which have been observed in the egg of the tortoise, frog, ray, shark, and other fishes, and which are described by Valenciennes and Fremy under the names, emydin, ichthin, ichthidin, and ichthulin, contain lecithin, nuclein, and albumin. They probably correspond to the yellow yolk-globule in the nutritive yolk of the hen's egg. The egg of the river crab and the lobster contain the same pigment as the shell of the animal. This pigment, called cyanoerystallin, becomes red on boiling in water.

In fossil eggs (of APTENODYTES, PELECANUS, and HALIEUS) in old guano deposits a yellowish-white, silky, lamiated combination, has been found which is called guanovulit, $\left(\mathrm{NH}_{4}\right)_{2} \mathrm{SO}_{4}+2 \mathrm{~K}_{2} \mathrm{SO}_{4}+3 \mathrm{KHSO}_{4}+4 \mathrm{H}_{2} \mathrm{O}$, and which is easily soluble in water but is insoluble in alcohol and ether.

Those eggs which develop outside of the mother-organism must contain all the elements necessary for the young animals. One 
finds, therefore, in the yolk and white of the egg an abundant quantity of albuminous bodies of different kinds, and especially a phosphorized albumin in the yolk. Further, we also find lecithin in the yolk, which seems to habitually occur in the developing cell. The occurrence of glycogen is doubtful, and the carbohydrates, which have no direct value as tissue-builders, are perhaps represented by a very small amount of glucose. On the contrary, the egg contains a large proportion of fat, which doubtless is an important source of nutrition and respiration for the embryo. The cholesterin and the lutein can hardly have a direct influence on the development of the embryo. The egg also seems to contain the mineral bodies necessary for the development of the young animal. The lack of phosphoric acid is compensated by an abundant amount of phosphorized organic substance, and the nucleoalbumin containing iron, from which the hæmatogen (see page 292) is formed, is doubtless, as BUNGE claims, of great importance in the formation of the hæmoglobin containing iron. The silicic acid necessary for the development of the feathers is also found in the egg.

During the period of incubation the egg loses weight, chiefly due to loss of water. The quantity of solids, especially the fat and the albumins, diminishes and the egg gives off not only carbon dioxide, but also, as LIEBERMANN has shown, nitrogen or a nitrogenized substance. This loss is compensated by the absorption of oxygen, and it is found that during incubation a respiratory exchange of gas takes place. While the quantity of dry substance in the egg during this period always decreases, the quantity of mineral bodies, albumin, and fat always increases in the embryo. The increase in the amount of fat in the embryo depends, according to LIEBERMANN, in great part upon a taking up of the nutritive yolk in the abdominal cavity. The weight of the shell and the quantity of lime salts contained therein remains unchanged during incubation. The yolk and white together contain the necessary quantity of lime for development.

The most complete and careful chemical investigation on the development of the embryo of the hen has been made by LIEBERMANN. From his researches we may quote the following: In the earlier stages of the development, tissues very rich in water are formed, but on the continuation of the development the quantity of 
water decreases. The absolute quantity of bodies soluble in water increases with the development, while their relative quantities, as compared to the other solids, continually decreases. The quantity of bodies soluble in alcohol quickly increases. A specially important increase is noticed in the fat, whose quantity is not very great even on the fourteenth day, but after that it becomes considerable. The quantity of albuminous bodies and albuminoid insoluble in water grows continually and regularly in such a way that their absolute quantity increases while their relative quantity remains nearly unchanged. LiebermanN found noglutin in the embryo of the hen. The embryo does not contain any gelatin-forming substance until the tenth day, and from the fourteenth day on it contains a body which when boiled with water gives a substance similar to chondrin. A body similar to mucin occurs in the embryo when about six days old, but then disappears. The quantity of hæmoglobin shows a continual increase compared to the weight of the body. LIEBERMANN found that the relationship of the hæmoglobin to the bodily weight was 1: 728 on the eleventh day and 1:421 on the twentyfirst day.

The tissue of the placenta has not thus far been the subject of detailed chemical investigations. In the edges of the placenta of bitches and of cats a crystallizable orange-colored pigment (bilirubin ?) has been found, and also a green amorphous pigment, MECKEL's hamatochlorin, which is considered as biliverdin by ETri. Preyer questions the identity of these pigments with biliverdin.

From the cotyledons of the placenta in ruminants a white or faint rosecolored creamy fluid, the uterine milk, can be obtained by pressure. It is al kaline in reaction, but becomes acid quickly. Its specific gravity is 1.0331.040. It contains as form-elements fat-globules, small granules, and epithelium-cells. We have found $81.2-120.9 \mathrm{p}$. m. solids, $61.2-105.6 \mathrm{p} . \mathrm{m}$. albumin, about $10 \mathrm{p}$. m. fat, and $3.7-8.2 \mathrm{p}$. m. ash in the uterine milk.

The fluid occurring in the so-called GRAPE-MOLE (mola racemosa) has a low specific gravity, 1.009-1.012, and 19.4-26.3 p. m. solids with 9-10 p. m. protein bodies and 6-7 p. m. ash.

The amniotic fluid is in women thin, whitish, or pale yellow; sometimes it is somewhat yellowish brown and cloudy. White flakes separate. The form-elements are mucus-corpuscles, epithelium-cells, fat-drops, and lanugo hair. The odor is stale, the reaction neutral or faintly alkaline. The specific gravity is 1.002-1.028.

The amniotic fluid contains the constituents of ordinary transudations. The amount of solids at birth is hardly $20 \mathrm{p.m}$. In the earlier stages of pregnancy the fluid contains more solids, especially 
proteids. Among the albuminous bodies WEYL found one substance similar to vitellin and with great probability also serum albumin, besides small quantities of mucin. Glucose is regularly found in the amniotic fluid of cows, but not in human beings. On the contrary, the human amniotic fluid contains some urea and allantoin. The quantity of these may be increased in hydramnion (Prochownick, Harnack), which depends on an increased secretion by the kidneys and skin of the foetus. Creatin and lactates are questionable constituents of the amniotic fluid. The quantity of urea in the amniotic fluid is, according to Pвоснош NICK, 0.16 p. $m$. In the fluid in hydramnion PROCHOWNICK and HaRNack found respectively 0.34 and $0.48 \mathrm{p}$. $\mathrm{m}$. urea. The chief mass of the solids consists of salts. The quantity of chlorides $(\mathrm{NaCl})$ is 5. 7-6.6 p. m. 


\section{CHAPTER XII.}

MILK.

THE chemical constituents of the mammary glands have bees little studied. The protoplasm of the cells is rich in proteids and, as is generally admitted, consists in great part of casein or a-substance nearly related. If all the milk is removed from the mammary gland by thorough washing, the cells still contain a large quantity of proteids which swell up to a slimy, ropy, or fibrous mass when very dilute alkali $(1-2 \mathrm{p} . \mathrm{m} . \mathrm{KOH})$ is added. These proteids consists mainly of nucleoalbumin, which is gradually changed by the action of the alkali. If the mammary gland is boiled with water, the protoplasm of the cell is decomposed and a nucleoalbumin passes into solution, which may be precipitated by the addition of acetic acid, and which is characterized by its greater insolubility in acetic acid, compared with casein. This nucleoalbumin, which may well be considered as a protoplasm-nucleoalbumin changed by heat, also gives on boiling with dilute mineral acids a reducible substance whose nature is not known. The relation the above-mentioned nucleoalbumin, which is more correctly designated as a proteid when the mother-substance of the reducible body does not occur as an impurity, bears to the sugar of milk or the mother-substance of the same, has not been determined. According to BERT, the secreting glands contain a body which on boiling with dilute mineral acids yields a reducible substance. Such a substance, which acts as a step towards the formation of milk-sugar, has also been observed by Thierfelder. Fat seems to be a never-failing constituent of the cell, at least in the secreting gland, and this fat may be ohserved in the protoplasm as large or small globules similar to milk-globules. The extractive bodies of 
the mammary glands have been little investigated, but among them we find considerable amounts of xanthin bodies.

As human milk and milk of animals are essentially of the same constitution, it seems best to speak first of the one most thoroughly investigated, namely, cow's milk, and then of the essential properties of the remaining important varieties of milk.

\section{Cow's Milk.}

Cow's milk forms, as all milks do, an emulsion which consists of very finely-divided fat suspended in a solution consisting chiefly of albuminous bodies, milk-sugar, and salts. Milk is non-transparent, white, whitish yellow, or in thin layers somewhat bluish white, of a faint, insipid odor and mild, faintly-sweetish taste. The reaction is regularly amphoteric, sometimes with a stronger action on the red and sometimes on the blue litmus-paper. The specific gravity is 1.028 to 1.0345 at $+15^{\circ} \mathrm{C}$.

Milk gradually changes when exposed to the air, and its reaction becomes more acid. This depends on a transformation of the milk-sugar into lactic acid, which is produced partly by the presence of a special enzyme originating in the glands but not yet positively detected, but which is chiefly produced by microorganisms.

Entirely fresh, amphoteric milk does not coagulate on boiling, but forms a skin consisting of coagulated casein and lime-salts, which rapidly re-forms after being removed. Even after passing a current of carbon dioxide through the fresh milk it does not coagulate on boiling. In proportion as the formation of lactic acid advances this behavior changes, and soon a stage is reached when the milk, which has previously had carbon dioxide passed through it, coagulates on boiling. At a second stage it coagulates alone on heating; then it coagulates by passing carbon dioxide alone without boiling; and lastly, when the formation of lactic acid is sufficient, it coagulates spontaneously at the ordinary temperature, forming a solid mass. It may also happen, especially in the warmth, that the casein-clot contracts and a yellowish or yellowish-green acid liquid (acid whey) is separated.

If the milk is sterilized by heating and contact with micro- 
organisms prevented, the formation of lactic acid may be entirely stopped. The formation of acid may also be prevented, at least for some time, by many antiseptics, such as salicylic acid (1:5000), thymol, boracic acid, and other bodies.

If milk is allowed to stand for a long time at a temperature of $0^{\circ} \mathrm{C}$. it remains fluid for several weeks, but coagulates at last. In this case the coagulation is not caused by the formation of lactic acid, but is more likely due to the formation of fatty acids caused by an oxidation (HoPPE-SEYLER).

If frcshly-milked amphoteric milk is treated with rennet, it coagulates quickly, especially at the temperature of the body, to a solid mass (cheese), from which a yellowish fluid (sweet whey) is gradually pressed out. This coagulation of milk occurs without any change in its reaction; it may also take place with the very faintest alkaline reaction; therefore it is distinct from the acid coagulation.

Milk sometimes undergoes a peculiar kind of coagulation, being converted into a thick, ropy, slimy mass (thick milk). This conversion depends, according to SCHMIDT-MüLHEIM, upon a peculiar change of the milk-sugar in which this last is converted into a slimy product. This conversion is caused by a special organized ferment.

In cow's milk we find as form-elements a few colostrum corpuscles (see Colostrum) and a few pale nucleated cells. The number of these form-elements is very small compared with the immense amount of the most essential form-constituents, the milkglobules.

The Milk-globules. These consist of extremely small drops of fat whose number is, according to BoHR, 2.6-11.4 or an average of 5.6 million in $1 \mathrm{c}$. $\mathrm{mm}$. and whose diameter is $0.00014-0.0063$ $\mathrm{mm}$. (BoHr). It is unquestionable that milk-globules contain fat, and we consider it as positive that all the milk-fat is found in them. Another and disputed question is whether milk-globules consist entirely of fat or whether they also contain albumin.

According to an observation of AscHerson, the drops of fat are covered with a fine albuminous coat, a so-called haptogen-membrane, when placed in an alkaline albumin solution. As milk on shaking with ether does not give up its fat, or only very slowly by a great excess of ether, and as this takes place very easily after the addition of acids or alkalies which dissolve albumin, it was formerly thought that the fat-globules of the milk were enveloped in an albuminous 
coat. A true membrane has not been detected; and since, when no means of dissolving the albumin is resorted to-for example, when the milk is precipitated by carbon dioxide after the addition of very little acetic acid (SoxHLET), or when it is coagulated by rennet-the fat can be very easily extracted by ether, the theory of a special albuminous membrane for the fat-globules has been generally abandoned. The observations of QUINCKES on the behavior of the fat-globules in an emulsion prepared with gum have led, at the present time, to the conclusion that each fat-globule in the milk is surrounded by a stratum of casein solution by means of molecular attraction, and this prevents the globules from uniting with each other. Everything that changes the physical property of the casein in the milk or precipitates it must necessarily help the solution of the fat in ether, and it is in this way that the alkalies, acids, and rennet work.

If we accept this view, which requires further proof, we must not overlook the fact that the fat-globules remain unchanged when the milk under agitation is coagulated with rennet. In this case we find an immense amount of unchanged milk-globules in the whey, and if we wish to admit of a stratum of proteids around the fat-globules proceeding from the molecular attraction, we must not consider that it is entirely due to casein, but also to albumin.

If the fat-globules are filtered off and washed on a filter, we always obtain (RADENHAUSEN and DANILEwSKY) after their treatment with ether a residue consisting of albumin. From this behavior the deduction has been made that the fat-globules, even though they have no real membrane, consist, nevertheless, of fat and albumin. The extreme difficulty of completely removing the albuminous bodies of the milk by washing the fat on the filter renders it necessary to exercise great caution in drawing a conclusion. The question as to the composition of the milk-globules, and especially as to the possible amount of albumin, cannot be decided at present.

The milk-fat has a rather variable specific gravity, which according to BoHR, is $0.949-0.996$ at $-15^{\circ} \mathrm{C}$. The milk-fat, which is obtained under the name of butter, consists in great part of the neutral fats palmitin, olein, and stearin. Besides these it contains, as tri-glycerides, small quantities of butyric acid and caproic acid, traces of caprylic and capric acids (lauric acid probably also occurs), myristic and arachiclic acids. Butter which has been exposed to the action of sunlight contains also formic acid (DUCLAUx). Milk-fat also contains a small quantity of lecithin and cholesterin, also a yellow coloring matter. The quantity of volatile fatty acids in butter is, according to DUCLAUX, on an average about $70 \mathrm{p.} \mathrm{m}$., of which $37-51 \mathrm{p}$. $\mathrm{m}$. is butyric acid and $20-33 \mathrm{p}$. m. is caproic 
acid. The non-volatile fat consists of $\frac{3}{10}$ to $\frac{4}{10}$ olein, and the remainder of a mixture of palmitin and stearin.

The quantity of volatile fatty acids in butter-fat is of great practical importance in the methods for detecting the presence of foreigu fats in butter. This detection is performed generally according to REICHER'T's process based on HEHNER and ANGELL's method. The fat is saponitied with alcoholic potash and the alcohol evaporated. The soaps are dissolved in water, and then distilled with an excess of phosphcric acid. The quautity of volatile fatty acids in the distillate is determined by titration with deci-normal alkali. With butter of proper composition 2.5 grms. should yield a distillate requiring 14-13 cc. for neutralization, and at least not less than $12 \mathrm{cc}$. of the deci-normal alkali. In proportion as the butter contains a greater quantity of foreign fats the quantity of alkali required becomes smaller.

The milk-plasma, or that fluid in which the fat-globules are suspended, contains at least three different albuminous bodies, casein, lactoglobulin, and lactalbumin, and two carbohydrates, of which only one, the milk-sugar, is of great importance. The milk-plasma also contains extractive bodies, traces of urea, creatin, creatinin, hypoxanthin (?), lecithin, cholesterin, about 1 p. m. citric acid (SoxHLET and HENKEL); a still greater amount according to SöLdner, and lastly also mineral bodies and gases.

Casein. This protein substance, which thus far has been detected positively in milk, belongs to the nucleoalbumins, and differs from the albuminates by its containing phosphorus and by its behavior with the rennet enzyme. Casein from cow's milk has the following composition: C 53.0, H 7.0, N 15.7, S 0.8, P 0.85, and 022.65 per cent. Its specific rotation is, according to HoPPESEYLER somewhat variable; in neutral solution it is $\alpha(D)=-80^{\circ}$. The question whether the casein from different varieties of milk is identical or if there are several different caseins has not been positively determined.

Casein, when dry, appears like a fine white powder which, after heating to $100^{\circ} \mathrm{C}$. or somewhat above, shows the properties and solubilities of freshly-precipitated, still-moist casein. Casein is only slightly soluble in water or in neutral-salt solutions. It acts Tike a rather strong acid, dissolves readily in water on the addition of very little alkali, forming a neutral or acid liquid, and lastly it dissolves in water in the presence of calcium carbonate, from which it expels the carbon dioxide. If casein is dissolved in lime-water and this solution treated with very dilute phosphoric acid until it is neutral 
in reaction, the casein appears to remain in solution, but is probably only swollen as in milk, and the liquid contains at the same time a large quantity of calcium phosphate without any precipitate or any visible suspended particles. The casein solutions containing lime are opalescent and have on warming the appearance of milk deficient in fat. Therefore it is not impossible that the white color of the milk is due partly to the casein and calcium phosphate.

Casein solutions do not coagulate on boiling, but are covered, as milk, with a skin. It is precipitated by very little acid, but the presence of neutral salts retards the precipitation. A casein solution containing salt, or ordinary milk, requires, therefore, more acid for precipitation than a salt-free solution of casein of the same concentration. The precipitated casein dissolves very easily again in a small excess of the acid, but less easily in an excess of acetic acid. The acid solutions are precipitated by mineral acids in excess. Casein is precipitated from neutral solutions or from milk by common salt or magnesium sulphate in substance without changing its properties. Metallic salts, such as copper sulphate, completely precipitate the casein from neutral solutions.

The property which is the most characteristic of casein is that it coagulates with rennet in the presence of a sufficiently great amount of lime-salts. In solutions free from lime-salts the casein does not coagulate. According to SoxHLET and SöLDNER, the soluble limesalts are only of essential importance in coagulation, while the calcium phosphate is without importance. The chemical processes which take place in the rennet coagulation have not been thoroughly investigated; still several observations seem to show that casein splits partly into a difficultly soluble body paracasein or cheese, whose composition closely resembles that of casein and which forms the chief product, and partly into an easily-soluble substance, similar to albumose, whey-albumin, which is deficient in carbon and nitrogen (50.3\% C and 13.2\% N, KösTNER) and which is produced in very small quantities. Paracasein is not further changed by the rennet enzyme, and it has not the same property of holding calcium phosphate in solution as casein has. If rennet be added to a casein solution free from lime, the solution does not coagulate, but the casein is changed so that the solntion, after the enzyme has been destroyed by rapid heating, acts like a paracasein solution, on the 
addition of lime-salts after cooling. The action of the rennet enzyme on casein takes place also in the absence of lime-salts, and these last are only necessary for the coagulation or the precipitation of the paracasein.

Casein may be prepared in the following way: The milk is diluted with 4 vols. water and the mixture treated with acetic acid to 0.75 to $1 \mathrm{p}$. $\mathrm{m}$. Casein thus obtained is purified by repeated solutions in water with the aid of the smallest quantity of alkali possible, by filtrating and reprecipitating with acetic acid, and thoroughly washing with water. Most of the milk-fat is retained by the filter on the first filtration, and the casein contaminated with traces of fat is purified by treating with alcohol and ether.

Lactoglobulin was obtained by SEBELIEN from cow's milk by saturating it with $\mathrm{NaCl}$ in substance (which precipitatad the casein), and saturating the filtrate with magnesium sulphate. As far as it has been investigated it had the properties of serum-globulin, with which it is perhaps identical.

Lactalbumin was first prepared in a pure state from milk by Sebelien. Its composition is, according to Sebelien, C 52.19, $\mathrm{H} \% 18, \mathrm{~N} 15.7 \%$, S 1.73, 023.13 per cent. Lactalbumin has the properties of the albumins. It coagulates, according to the concentration and the amount of salt in solution, at $+72^{\circ}$ to $84^{\circ} \mathrm{C}$. It is similar to serum albumin, but differs from it in having a considerably lower specific rotary power; $\alpha(D)=-37^{\circ}$.

The principle of the preparation of lactalbumin is the same as for the preparation of serum-albumin from serum. The casein and the globulin are removed by $\mathrm{MgSO}_{4}$ in substance and the filtrate treated as previously stated (page 61).

The occurrence of other albuminous bodies, such as albumoses and peptones, in milk has not been positively proved. These bodies are easily produced as laboration products from the other albuminous bodies of the milk. Such a laboration product is MrLuon's and ComaluLe's lactoprotein, which is a mixture of a little casein with changed albumin, and which is formed by the chemical operations.

Milk-sugar, LACTose, $\mathrm{C}_{12} \mathrm{H}_{22} \mathrm{O}_{11}+\mathrm{H}_{2} \mathrm{O}$. This sugar with the absorption of water can be split into two glucoses, dextrose and galactose. It yields by the action of dilute nitric acid, besides carbon dioxide, oxalic acid, tartaric acid, saccharic acid, and racemic acid, a crystallizable mucic acid, which is nearly insoluble in cold water and in alcohol, and which may also be obtained from dulcite, 
gum, and vegetable mucus. By the action of sodium amalgam on milk-sugar we obtain dulcite, mannite, lactic acid, and other products. By the action of alkalies we obtain lactic acid among other products.

Milk-sugar occurs in all milks. It has also been found in the urine of pregnant women. According to Bouchardat, it also occurs in the ripe fruit of the achras sapota.

Milk-sugar occurs ordinarily as colorless rhombic crystals with $1 \mathrm{~mol}$. of water of crystallization, which is driven off by slowly heating to $100^{\circ} \mathrm{C}$., but more easily at $130^{\circ}-140^{\circ} \mathrm{C}$. At $170^{\circ}$ to $180^{\circ} \mathrm{C}$. it is converted into a brown amorphous mass, lactocaramel, $\mathrm{C}_{6} \mathrm{H}_{10} \mathrm{O}_{6}$. Milk-sugar dissolves in 6 parts cold and in 2.5 parts boiling water; it has a faint sweetish taste. It does not dissolve in ether or absolute alcohol. Its solutions are dextro-gyrate. The rotary power, which, on heating the solution to $100^{\circ} \mathrm{C}$. becomes constant, is $\alpha(D)$ $=+52.5^{\circ}$. Milk-sugar combines with bases; the alkali combinations are insoluble in alcohol.

Milk-sugar is not fermentable with pure yeast. It undergoes, on the contrary, alcoholic fermentation by the action of certain schizomycetes, and lactic acid is then produced thereby. The preparation of milk-wine, "kumyss," from mare's milk and "kephir" from cow's milk is based upon this fact. Micro-organisms produce a lactic fermentation in lactose, and this explains the ordinary souring of milk.

Lactose responds to the reactions of grape-sugar, such as MOoRE's or 'TROMMER's and the bismuth test, which will all be described in Chapter XIV on the urine. It also reduces mercuric oxide in alkaline solutions. After warming with phenylhydrazin acetate it gives on cooling a yellow crystalline precipitate of phenyllactosazon, $\mathrm{C}_{24} \mathrm{H}_{32} \mathrm{~N}_{4} \mathrm{O}_{2}$ (see Chap. XIV on sugar in the urine). It differs from cane-sugar by giving positive reactions with Moore's and the bismuth test, and also that it does not darken when heated with anhydrous oxalic acid to $100^{\circ} \mathrm{C}$. It differs from grape-sugar and maltose by its solubility and crystalline form ; but especially by its not fermenting with yeast and by yielding mucic acid with nitric acid.

For the preparation of milk-sugar we make use of the by-product in the preparation of cheese, the sweet whey. The albumin is re- 
moved by coagulation with heat and the filtrate evaporated to a syrup. The crystals which separate after a certain time are recrystallized from water after decolorizing with animal charcoal. A pure preparation may be obtained from the commercial milk-sugar by repeated recrystallization. The quantitative estimation of milksugar may in part be performed by the polaristrobometer and partly by means of titration with FeHLING's solution. 10 cc. of FeHLING's solution corresponds to $0.06 \%$ grm. milk-sugar (in regard to FEHLING'S solution and the titration of sugar, see Chapter XIV).

Ritthausen has found another carbohydrate in milk which is soluble in water, non-crystallizable, which has a faint reducing action, and which yields on boiling with an acid a body having a greater reducing power. LANDWEHR considers this as an animal gum.

The mineral bodies of milk will be treated in connection with its quantitative composition.

The methods for the quantitative analysis of milk are numerous, and as they all cannot be treated of here, we will give the chief points of a few of the most trustworthy and most frequentlyemployed methods.

In determining the solids a carefully-weighed quantity of milk is mixed with an equal weight of heated sand, fine glass powder, or asbestos. The evaporation is first done on the water-bath and finished in a current of carbon dioxide or hydrogen not above $100^{\circ} \mathrm{C}$.

The mineral bodies are determined by ashing the milk, using the precautions suggested in the text-books. The results obtained for the phosphoric acid are incorrect on account of the burning of phosphorized bodies, such as casein and lecithin. We must therefore, according to SöLDNER, subtract 25\% from the total phosphoric acid found in the milk. The quantity of sulphate in the ash also depends on the burning of the albumins.

In the determination of the total amount of albuminous bodies we make use of RITTHAUSEN's method, namely, precipitate the milk with copper sulphate. This method gives incorrect results because the copper hydroxide does not give up all its water of hydration on drying the precipitate, but only after ashing the same. The results for the proteids are therefore somewhat too high.

The method of Puls and Stenberg consists in first diluting the neutralized milk with some water and then treating with alcohol until the mixture contains 70-85 vols. per cent alcohol. The precipitate is collected on a filter, washed with warm $70 \%$ alcohol, extracted with ether, dried, weighed, burnt, and the residue reweighed. The traces of albumin which remain in the filtrate and 
wash-liquor are precipitated by tannic acid (see page 21). $63 \%$ of the tannic acid precipitate is considered as albumin, and this must be added to the albumin found directly. This method gives exact and good results.

According to the method of SEBELIEN, 3-5 grms. of milk are diluted with an equal volume of water, a little common-salt solution added, and precipitated with an excess of tannic acid. The precipitate is washed with cold water, and then the quantity of nitrogen determined by KJELDAHL's method. The total nitrogen found when multiplied by 6.37 (casein and lactalbumin contain both 15.\% nitrogen) gives the total quantity of albuminous bodies. 'This easily-executed method gives very good results, but until the quantity of nitrogen in the albuminous bodies of other varieties of milk has been exactly determined this method cannot be used except for the analysis of cow's milk.

To determine the casein and albumins separately we may make use of the method first suggested by HOPPE-SEYLER and TOLMATSCHEFF, in which the casein is precipitated by magnesium sulphate. According to SEBELIEN, the milk is diluted with its own volume of a saturated magnesium-sulphate solution, then saturated with the salt in substance, the precipitate filtered and washed with a saturated magnesium-sulphate solution. The nitrogen is determined in the precipitate by KJELDAHL's method, and the quantity of casein determined by multiplying the result by $6.3 \%$. The quantity of lactalbumin may be calculated as the difference between the casein and the total albumin found. The lactalbumin may also be precipitated by tannic acid from the filtrate containing $\mathrm{MgSO}_{4}$ from the casein precipitate, diluted with water, and the nitrogen determined by KJELDAHL's method and the result multiplied by $6.3 \%$. SEBELIEN's method is only suited for cow's milk.

The quantity of globulins in milk cannot be exactly determined. A minimum result can be obtained by first precipitating the casein completely by $\mathrm{NaCl}$ in substance, and then precipitating the globulins in the filtrate by magnesium sulphate (SEBELIEN). The casein may also be precipitated from the diluted milk, and the globulin precipitated after neutralization by means of $\mathrm{MgSO}_{4}$. In these cases we obtain somewhat high results, because of the presence of traces of casein which remain behind.

The fat is determined directly by thoroughly extracting the dried milk with ether, evaporating the ether from the extract, and weighing the residue. The fat may be determined by aerometric means by adding alkali to the milk, shaking with ether, and determining the specific gravity of the fat solution by means of SoxHLET's apparatus. In determining the amount of fat in a large number of samples the lactokrit of DE LAVAL may be used with success. The milk is first mixed with an equal volume of a mix- 
ture of acetic acid and concentrated sulphuric acid, warmed 7-8 minutes on the water-bath, the mixture placed in graduated tubes, and these in the centrifugal machine at $+50^{\circ} \mathrm{C}$. The height of the layer of fat gives its quantity.

In determining the milk-sugar first the albumin is removed. For this purpose we precipitate either with alcohol, which must be evaporated from the filtrate, or by diluting with water, and removing the casein by the addition of little acid, and the lactalbumin by coagulation at a boiling heat. The sugar is determined by titration with FeHLING's or KNapP's solution (see Chap. XIV). The principle of titration is the same as for the titration of sugar in urine: 10 c.c. of FeHLING's solution corresponds to 0.067 grm. milk-sugar; 10 c.c. of KNAPP's solution corresponds to 0.0311-0.0310 grm. milk-sugar, when the saccharine liquid contains about $\frac{1}{2}-1 \%$ sugar. In regard to the modus operandi of the titration we must refer the reader to more complete works and to Chapter XIV.

Instead of the volumetric determinations the following steps may be taken: A measured quantity of the milk-sugar solution is treated with an excess of FeHLING's solution, boiled, the copper suboxide filtered and reduced in a current of hydrogen, and the metallic copper weighed. SoxHLET has given a table (Journal für praktische Chemie, 1880) which simplifies the calculations in such cases.

The sugar may also be determined by the polariscope, and with ease, because the filtrates containing milk-sugar are generally colorless. The determination is quickly performed but does not give exact results.

The quantitative composition of cow's milk is variable. The average obtained by KöNIG is as follows in 1000 parts :

$\begin{array}{lcccccc}\text { Water. } & \text { Solids. } & \begin{array}{c}\text { Casein. } \\ \text { 874.2 }\end{array} & \text { Albumin. } & \text { Fats. } & \text { Sugar. } & \text { Salt. } \\ & \underbrace{28.8}_{34.1} \quad 5.3 & & 36.5 & 48.1 & 7.1\end{array}$

The quantity of mineral bodies in 1000 parts of cow's milk is, according to the analyses of SöLDNER, as follows: $\mathrm{K}_{2} \mathrm{O} 1.72$, $\mathrm{Na}_{2} \mathrm{O}$ 0.51, $\mathrm{CaO}$ 1.98, $\mathrm{MgO} 0.20, \mathrm{P}_{2} \mathrm{O}_{5} 1.82$ (after correction for the nucleins), $\mathrm{Cl} 0.98$ grms. Bunge found 0.0035 grm. $\mathrm{Fe}_{2} \mathrm{O}_{3}$. According to SöLDNER, the $\mathrm{K}, \mathrm{Na}$ and $\mathrm{Cl}$ are found in the same quantities in whole milk as in milk-serum. Of the total phosphoric acid $36-56 \%$ is not dissolved and also $53-72 \%$ of the lime. A part of this lime is combined with the casein; the remainder is found 
nnited with the phosphoric acid as a mixture of di-and tri-calcium phosphate, which is kept dissolved or suspeuded by the casein. 'The bases are in excess of the mineral acids in the milk-serum. The excess of the first is combined with organic acids which correspond to $2.5 \mathrm{p}$. m. citric acid (SöLdNER).

The gases of the milk consist chiefly of $\mathrm{CO}_{2}$, besides a little $\mathrm{N}$ and traces of $O$. PFLÜGER found 10 vols. per cent $\mathrm{CO}_{2}$ and $0.6 \mathrm{vol}$. per cent $\mathrm{N}$, calculated at $0^{\circ} \mathrm{C}$. and $760 \mathrm{~mm}$. pressure.

The variation in the composition of cow's milk depends on several circumstances.

The colostrum, or the milk which is secreted before calving and in the first few days after, is yellowish, sometimes alkaline, but often acid, of high specific gravity, 1.046-1.080, and richer in solids than ordinary milk. The colostrum contains, besides fat-globules, an abundance of colostrum-corpuscles-nucleated granular cells $0.005-$ $0.025 \mathrm{~mm}$. in diameter with abundant fat-grains and fat-globules. The fat of colostrum has a somewhat higher-melting point and is poorer in volatile fatty acids than the fat from ordinary milk (Nilson). 'The quantity of cholesterin and lecithin is generally greater. The most apparent difference between it and ordinary milk is that colostrum coagulates on heating to boiling because of the absolute and relatively greater quantities of globulin and albumin it contains. The quantity of the first of these two albuminous bodies may indeed amount to several per cent (SEBELIEN). The composition of colostrum is very variable. KöNIG gives as average the following figures in 1000 parts:

$\begin{array}{lcccccc}\text { Water. } & \text { Solids. } & \text { Casein. } & \text { Albumin and Globulin. } & \text { Fat. } & \text { Sugar. } & \text { Salts. } \\ 740.5 & 259.5 & 46.6 & 136.2 & 34.3 & 26.6 & 15.8\end{array}$

The properties of milk are changed during lactation and it becomes richer in casein. A change in the amount of fat is also sometimes observed (Emmerling). Otherwise we often say that the milk becomes poorer in fat during lactation and the fat deficient in volatile fatty acids. The evening milk 18 richer in fat than the morning milk (Alex. Müller and Eisenstuck; Nilson). The race of the animal also has a great influence on the milk.

The influence food exercises upon milk will be discussed in connection with the chemistry of the milk secretion. 
In the following we give the average composition of skimmed milk and certain other preparations of milk (KöNlG).

\begin{tabular}{|c|c|c|c|c|c|}
\hline Water. & Proteids. & Fat. & Sugar. & Lactic Acid. & Salts. \\
\hline ilk... 906.6 & 31.1 & 7.4 & 47.5 & $\ldots$ & 7.4 \\
\hline$\cdots \cdots{ }_{902.7}^{655.1}$ & $\begin{array}{l}36.1 \\
40.6\end{array}$ & 267.5 & 35.2 & $\because$ & 6.1 \\
\hline $\begin{array}{c}\cdots \\
\cdots\end{array}$ & $\begin{array}{r}40.6 \\
8.5\end{array}$ & $\begin{array}{l}9.3 \\
2.3\end{array}$ & $\begin{array}{l}37.3 \\
47.0\end{array}$ & $\begin{array}{l}3.4 \\
3.3\end{array}$ & 6.7 \\
\hline
\end{tabular}

KUMYss and KEPHIR are obtained as above stated, by the alcoholic and lactic acid fermentation of the milk-sugar, the first from mare's milk and the last from cow's milk. Large quantities of carbon dioxide are formed hereby, and also the albuminous bodies of the milk are partly converted into albumoses and peptones, which increases the digestibility. The quantity of lactic acid in these preparations may be about 10-20 p. m. The quantity of alcohol varies from 10 to $35 \mathrm{p}$. $\mathrm{m}$.

Milk from other Animals. GoAT's milk has a more yellowish color and another, more specific, odor than cow's milk. The coagulation obtained by acid or rennet is more solid and is harder than that from cow's milk. SHEEP's milk is similar to goat's milk, but has a higher specific gravity and contains a greater amount of solids.

MARE's milk is alkaline and contains a casein which is not precipitated by acids in lumps or solid masses, but, like the casein from woman's milk, in fine flakes. This casein is only incompletely precipitated by rennet, and it is very similar also in other respects to the casein of human milk. According to BIEL, the casein from mare's and cow's milk is the same, and the different behavior of the two varieties of milk is due to different amounts of salts and to a different relation between the casein and the albumin. The milk of the Ass is similar to human milk.

The milk of CARNIVORA, the bitch and cat, are acid in reaction and very rich in solids. The composition of the milk of these animals varies very much with the composition of the food.

To illustrate the composition of the milk of other animals the following figures, the compilation of KöNIG, will be given. As the milk of each variety of animal may have a variable composition, these figures may only be considered as examples of the composition of milk of different kinds.

$\begin{array}{rrrcccr}\text { Milk of the } & \text { Water. } & \text { Solids. } & \text { Proteids. } & \text { Fat. } & \text { Sugar. } & \text { Salts. } \\ \text { Dog..... } & 754.4 & 245.6 & 99.1 & 95.7 & 31.9 & 7.3 \\ \text { Cat..... } & 816.3 & 183.7 & 90.8 & 33.3 & 49.1 & 5.8 \\ \text { Goat.... } & 869.1 & 130.9 & 36.9 & 40.9 & 44.5 & 8.6 \\ \text { Sheep... } & 835.0 & 165.0 & 57.4 & 61.4 & 39.6 & 6.6 \\ \text { Cow .... } & 874.2 & 125.8 & 34.1 & 36.5 & 48.1 & 7.1 \\ \text { Hor... } & 900.6 & 99.4 & 18.9 & 10.9 & 66.5 & 3.1 \\ \text { Ass..... } & 900.0 & 100.0 & 21.0 & 13.0 & 63.0 & 3.0 \\ \text { Pig..... } & 823.7 & 167.3 & 60.9 & 64.4 & 40.4 & 10.6\end{array}$

\section{Human Milk.}

Woman's milk generally differs from cow's milk in having an alkaline reaction and larger fat-globules. Their number, according to BouchuT, is in most cases 1-2 millions in $1 \mathrm{c.mm}$. The specific 
gravity of woman's milk is between 1.026 and 1.035 , but varies generally between 1.028 and 1.034. 'This milk in general has a less inclination to turn acid, and it therefore does not coagulate distinctly.

The fat from human milk has not been thoroughly investigated. According to HoPPE-SEYLER, it is richer in fluid fats than the fat from cow's milk.

The essential qualitative difference between woman's and cow's milk seems to lie in the proteids, or in the more accurately determined casein. A number of investigators, such as BERZELIUS, Simon, Biedert, LangGard, MaKris, and others, claim that the casein from woman's milk has other properties than that from cow's milk. The essential differences are the following: The casein from woman's milk is precipitated with greater difficulty with acids or salts; it does not coagulate regularly in the milk after the addition of rennet; it may be precipitated by gastric juice, but dissolves completely and easily in an excess of the same; the casein precipitate produced by an acid is more easily soluble in an excess of the acid; and lastly, the clot formed from the casein does not appear in such large and coarse masses as the casein from cow's milk, but is more loose and flocculent. This last-mentioned fact is of great importance, since it explains the generally-admitted easy digestibility of the casein from woman's milk. The question as to whether the above-mentioned differences depend on a decided difference in the two caseins or only on an unequal relationship between the casein and the salts in the two varieties of milk, or upon other circumstances, has not been sufficiently investigated, and doubtless further experiments will be of great value. We have not, up to the present time, any quite trustworthy analyses of the casein from woman's milk, but it seems probable that the caseins from woman's and cow's milk are not identical albuminous bodies. Besides casein, woman's milk contains lactalbumin, and certain investigators maintain that they have found relatively large amounts of albumoses and peptones. According to other statements (Dogiel and Hofmeister), no peptones occur in woman's milk, and the methods employed for detecting albumoses seem to have given no positive results. The albuminous bodies of woman's milk require more thorough investigation. The total quantity of 
milk secreted by both mammary glands amounts to $500-1500$ grms. in two hours.

The quantitative composition of woman's milk is, even after those differences are eliminated which depend on the imperfect analytical methods employed, variable to such an extent that it is impossible to give any average results. Eliminating certain of the older, incorrect analyses, we here give only examples from the average results of a few modern investigators, taken from a very large number of analyses (PFEIFFER, LEEDS). The following figures are parts per 1000 .

\begin{tabular}{|c|c|c|c|c|c|c|c|}
\hline Water. & Solids. & Proteids. & Fat. & $\begin{array}{l}\text { Choles } \\
\text { terin. }\end{array}$ & Sugar. & Salts. & \\
\hline 876.0 & 124.0 & 22.10 & 38.10 & & 60.90 & 2.90 & BIEL \\
\hline & & 23.60 & 25.60 & 0.32 & 55.60 & $\ddot{m} \ddot{\rho}$ & $\begin{array}{l}\text { TOLMATSCHEFF } \\
\text { GERBER }\end{array}$ \\
\hline $\begin{array}{l}91.0 \\
72.4\end{array}$ & $\begin{array}{l}109.0 \\
127.6\end{array}$ & $\begin{array}{l}17.90 \\
19.00\end{array}$ & $\begin{array}{l}33.00 \\
43.20\end{array}$ & $\cdots \cdots$ & $\begin{array}{l}53.90 \\
59.80\end{array}$ & $\begin{array}{l}4.20 \\
2.60\end{array}$ & $\begin{array}{l}\text { GERBER } \\
\text { CHRISTENN }\end{array}$ \\
\hline 892.0 & 108.0 & 16.13 & 32.28 & $\ldots \ldots$ & 57.94 & 1.65 & $2(1)-30$ years old ) \\
\hline 890.6 & 109.4 & 17.24 & 2915 & & 59.92 & 2.09 & $30-40$ " " $\}$ PFEIFFER \\
\hline 877.90 & & 25.30 & 38.90 & & 55.40 & 2.50 & MENDES DE LEON \\
\hline 867. & 132.68 & 19.95 & 41.31 & $\ldots \ldots$ & 69.36 & 2.00 & LEEDS \\
\hline
\end{tabular}

Although the composition of woman's milk is very variable, and notwithstanding that in a few cases higher results (about 40 p. m.) have been obtained, by later analyses, for albuminous bodies, still it seems that woman's milk in general contains less proteids and more sugar than cow's milk. The quantity of casein is not only absolutely but also relatively smaller in proportion to the quantity of albumin in woman's than in cow's milk.

A further difference between woman's and cow's milk is that the first is richer in lecithin but poorer in mineral bodies, especially $\mathrm{CaO}$ and $\mathrm{P}_{2} \mathrm{O}_{5}$ (it contains only $\frac{1}{6}$ and $\frac{1}{4}$, respectively, of the corresponding quantity of these mineral bodies in cow's milk).

In regard to the quantity of mineral bodies in woman's milk the analyses of BUNGE are most reliable. He analyzed the milk of a woman, fourteen days after delivery, whose diet contained very little common salt for four days previous to the analysis $(A)$, and again three days later after a daily addition of $30 \mathrm{grms}$. $\mathrm{NaCl}$ to the 
food $(B)$. BUNGE found the following figures in 1000 parts of the milk :

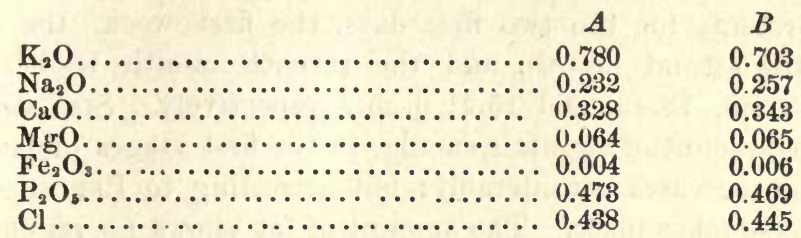

The relationship of the two bodies, potassium and sodium, to each other may, according to BUNGE, vary considerably (1.3-4.4 equivalents potash to 1 of soda). By the addition of salt to the food the quantity of sodium and chlorine in the milk increases, while the quantity of potassium decreases. The gases of woman's milk have not been investigated.

The proper treatment of cow's milk by diluting with water and by certain additions in order to render it a proper substitute for woman's milk in the nourishment of babes cannot be determined before the difference in the albuminous bodies of these two kinds of milk has been completely studied.

The period of lactation acts essentially the same on woman's as on cow's milk.

The colustrum has a higher specific gravity, 1.040-1.060, a greater quantity of coagulable proteids, and a deeper yellow color than ordinary woman's milk. Even a few days after delivery the color becomes less yellow, the quantity of albumin less, and the number of colostrum-corpuscles diminishes. ClemM has analyzed the colostrum at different periods before and after delivery, and the following are his results in parts per 1000:

\begin{tabular}{|c|c|c|c|c|c|c|}
\hline & \multicolumn{2}{|c|}{$\begin{array}{c}\text { Four Weeks before } \\
\text { Delivery. }\end{array}$} & \multirow{2}{*}{$\begin{array}{c}\text { Seventeen } \\
\text { Days } \\
\text { before } \\
\text { Delivery. }\end{array}$} & \multirow{2}{*}{$\begin{array}{c}\text { Nine Days } \\
\text { before } \\
\text { Delivery. }\end{array}$} & \multirow{2}{*}{$\begin{array}{c}\text { Twenty- } \\
\text { four Hours } \\
\text { after } \\
\text { Delivery. }\end{array}$} & \multirow{2}{*}{$\begin{array}{c}\text { Two Days } \\
\text { after } \\
\text { Delivery. }\end{array}$} \\
\hline & 1 & 2 & & & & \\
\hline Water. & 945.2 & 852.0 & 851.7 & 858.8 & 843.0 & 867.9 \\
\hline Solids. & 54.8 & 148.0 & 148.3 & 141.2 & 157.0 & 132.1 \\
\hline Casein ........... & & & & & ....... & 21.8 \\
\hline Albumin.......... & 28.8 & 69.0 & 74.8 & 80.7 & $\ldots \ldots$ & \\
\hline 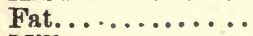 & 7.3 & 41.3 & 30.2 & 23.5 & $\ldots$ & 48.6 \\
\hline Milk-sugar........ & 17.3 & 39.5 & 43.7 & 36.4 & $\ldots \ldots$ & 61.0 \\
\hline 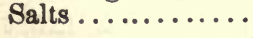 & 4.4 & 4.4 & 4.5 & 5.4 & 5.1 & $\cdots \cdots$ \\
\hline
\end{tabular}


The total quantity of albumins seems to decrease with the duration of lactation. Pfeiffer found the average figures for the total proteids for the two first days, the first week, the second week, the second month, and the seventh month to be 86.04 , $34.42,22.88,18.43$, and 15.21 p. m., respectively. SIMoN claims that the amount of casein is smaller in the first stages of lactation and then increases considerably; but according to PfeIffer, just the reverse takes place. The amount of fat shows no regular and constant variation during lactation. According to Vernors and Becquerel, the quantity of milk-sugar decreases in the first months, but increases in the eighth to the tenth month. According to PFEIfFer, the quantity of sugar increases from the delivery to the third to fourth month, and then it is somewhat variable.

The two mammary glands of the same woman may yield somewhat different milk, as shown by SoURdat and later by BRUnNer. Also the different portions of milk from the same milking may have different compositions. The first portions are always poorer in fat (PARMentien, Peligot, and others).

According to L'Héritier, Vernois, and Becquerel, the milk of blonds contains less casein than that of brunettes, a difference which TOLMATsCHEFF could not substantiate. Women of weak constitutions yield a milk richer in solids, especially in casein, than women with strong constitutions (V. and B.).

According to VERNOIS and BECQUEREL, the age of the woman bas an effect on the composition of the milk, so that we find a greater quantity of proteids and fat in women 15-20 years old and a smaller quantity of sugar. The smallest quantity of proteids and the greatest quantity of sugar are found at 20 or from 25-30 years of age. According to V. and B., the milk with the first-born is richer in water-with a proportionate diminution of the quantity of casein, sugar, and fat-than after several deliveries.

The influence of menstruation seems to slightly diminish the milk-sugar and to considerably increase the fat and casein (V. and B.).

Witch's Milk is the secretion of the mammary glands of new-born children of both sexes immediately after birth. This secretion has from a qualitative standpoint the same constitution as milk, but may show important differences and variations from a quantitative point of view. ScHLOssBERGER and HAUFF, GUBLer and QUEVENNE, and v. GESNER have made analyses of this milk and give the following results : $10.5-28 \mathrm{p}$. m. proteids, $8.2-14.6 \mathrm{p}$. m. fat, and 9-60 p. m. sugar.

As milk is the only form of nourishment during a certain period of the life of man and mammalia, it must contain all the nutritious bodies necessary for life. This fact is shown by the milk-containing representives of the three chief groups of organic-nutritive substances, proteids, carbohydrates, and fat; and all milk seems to contain also some lecithin. The mineral bodies in milk must 
also occur in proper proportion, and on this point the observations of BUNGE on dogs are of special interest. He found that the mineral bodies of the milk occur in about the same relative proportion as they do in the body of the sucking animal. BUNGE found in 1000 parts of the ash the following results ( $A$ represents results from the new-born $\operatorname{dog}$ and $B$ the milk from the bitch):

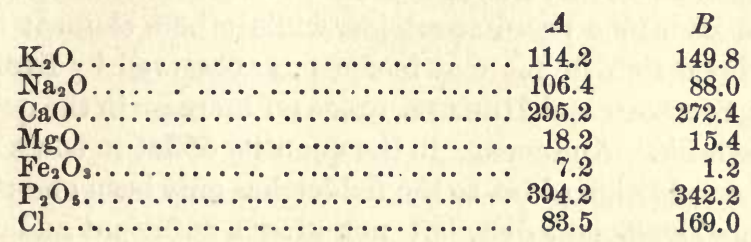

BUNGE explains the fact that the milk-ash is richer in potash and poorer in soda than the new-born animal by saying that in the growing animal the growing muscles rich in potash relatively increase and the cartilage rich in soda relatively decreases. BUNGE seeks to explain the high amount of chlorine in the milk-ash also teleologically by the statement that the chlorides not only serve to build up the tissues, but are indispensable in the secretions of the kidneys. In regard to the amount of iron we find an unexpected condition, the ash of the new-born animal containing six times as much as the milk-ash. This condition Bunge explains by the fact founded on his and ZALESKY's experiments, that the quantity of iron in the total organism is highest at birth. The new-born animal has therefore a storage of iron for the growth of its organs even at its birth.

The influence of the food on the composition of the milk is of interest from many points of view and has been the subject of many investigations. From these investigations we learn that in human beings as well as in animals an insufficient diet decreases the quantity of milk, and the quantity of solids in the same, while abundant food increases both. From the observations of DeCAISNE on nursing women during the siege of Paris in 1871, the quantity of casein, fat, sugar, and salts, but especially the fat, was found to decrease with insufficient food, while the quantity of lactalbumin was found to be somewhat increased. Food rich in proteids increases the quantity of milk, and also the solids contained, especially 
the fat (as shown in women by ZaLesky, in sheep by Stolzmann, Weiske, Schrodt and Dehmel and Munk, in dogs by Poggiale and Ssubbotin, in cows-at least in most cases-by KüHN and his pupils). An increase in the quantity of casein, and a decrease in the albumin and the sugar in cow's milk after food containing an excess of proteids has been observed by KüHN and his pupils. The quantity of sugar in woman's milk is found by certain investigators to be increased after food rich in proteids, while others claim it is diminished. Food rich in fat may in sheep, as observed by Stolzmann WeIske, Schrodt and DeHMEL, cause an increase in the quantity of fat in the milk. An increase in the quantity of fat in cow's milk because of an addition of fat to the fodder has only been observed after a previous insufficient diet, but not after a sufficient and rich diet (KüHN and FleischmanN). After feeding with palm-oil cake a one-sided increase in the fat of cow's milk was observed by KüHN. The presence of large quantities of carbohydrates in the food seems to cause no constant, direct action on the quantity of the milk-constituents. In carnivora the secretion of milk-sugar proceeds uninterrupted on a diet consisting exclusively of lean meat. Watery food gives a milk containing an excess of water of little value. In the milk from cows which were fed on distillers' grains CommaIlle found 906.5 p.m. water, 26.4 p.m. casein, 4.3 p.m. albumin, 18.2 p. m. fat, and 33.8 p.m. sugar. Such milk has a peculiar sour, sharp after-taste.

Chemistry of the Milk-Secretion. That the actually-dissolved constituents occurring in milk pass into the secretion, not alone by filtration or diffusion, but more likely are secreted by a specific secretory activity of the glandular elements, is shown by the fact that milk sugar, which is not found in the blood, is to all appearances formed in the glands themselves. A further proof lies in the fact that the lactalbumin is not identical with serum-albumin (Sebelien); and lastly, as Bunge has shown, the mineral bodies secreted by the milk are in quite different proportions than to those in the blood-serum.

Little is known in regard to the formation and secretion of the specific constituents of milk. The older theory, that the casein was produced from the lactalbumin by the action of an enzyme, is incorrect and originated probably from mistaking an alkali-albuminate 
for casein. Better founded is the statement that the casein originates from the protoplasm of the gland cells, which seem to consist of casein or a substance related to it. 'I'he previously (page 300) mentioned nucleoalbumin of the gland-cells appears to be related to casein, and it may possibly form its mother substance. There does not seem to be any doubt that the protoplasm of the cells takes part in the secretion in such a manner that it becomes itself a constituent of the secretion. According to Heidenhain, the alveoles contain a simple layer of cells, which, in the inactive gland, are flat, polyhedrous, and with single nucleus, while in the active gland they often have several neuclei, are rich in albumin, and are high and cylindrical in form. In the inner part of the cell turned towards the cavity of the acinus, single fat-granules are formed during the secretion which are broken off with the edge of the cells. The broken-off or destroyed cell-substance in the secretion dissolves in the milk, filling the lumen of the acinus, while the cells take up nutrition by their outer parts, and grow, and replace the inner parts used in the secretion. This reminds us of the action of the pancreas-cells in the secretion of the pancreatic juice. The colostrumcorpuscles are not, according to HeIDENHAIN, degenerated fatcells, but are contractile elements originating from the epithelium, which take up finely-divided fat and thereby obtain their quantity of fat-globules.

That the milk-fat is produced by a formation of fat in the protoplasm, and that the fat-globules are set free by their destruction, is a generally-admitted opinion which, however, does not exclude the possibility that the fat is in part taken up by the glands from the blood and eliminated with its secretion. A formation of fat from carbohydrates in the animal organism is at the present day considered as positively proved, and it is also possible that the milk-glands also produce fats from the carbohydrates brought to them by the blood. It is a well-known fact that an animal gives off for a long time, daily, considerably more fat in the milk than it receives as food, and this proves that at least a part of the fat secreted by the milk is produced from proteids or carbohydrates, or perhaps from both. The question as to how far this fat is produced directly in the milk-glands, or from other organs and tissues, and brought to the gland by means of the blood, cannot be decided. 
The origin of the milk-sugar is not known. Müstz calls attention to the fact that a quantity of very widely-diffused bodies in the vegetable kingdom-vegetable mucus, gums, pectin bodiesyield galactose as products of decomposition, and he believes, therefore, that the milk-sugar may be formed in herbivora by a synthesis from dextrose and galactose. This origin of milk-sugar does not answer for carnivora, as they produce milk-sugar when fed on food consisting entirely of lean meat. The observations of BERT and Thierfelder that a mother-substance of the milk-sugar, a saccharogen, occurs in the glands cannot, as the nature of this mother-substance is still unknown, give further explanation as to the formation of milk-sugar. The question whether the above (page 300) mentioned proteid, which yields a reducible substance when boiled with dilute acids, has anything to do with the formation of milk-sugar cannot be answered until further thorough investigations have been made on this subject.

'The passage of foreign substances into the milk stands in close connection with the chemical processes of the milk-secretion.

It is a well-known fact that milk acquires a foreign taste from the food of the animal, which is in itself a proof that foreign bodies pass into the milk. This fact becomes of special importance in reference to such injurious substances as may be introduced into the organism of the nursing child by means of the milk.

Among these substances may be mentioned opium and morphine, which after large doses pass into the milk and act on the child. Alcohol may pass into the milk in such large quantities that it produces a stupefying effect on the child. Milk from cattle fed on distiller's grains may also contain alcohol.

Among the inorganic bodies we find iodine, arsenic, bismuth, antimony, zinc, lead, mercury, and iron in the milk. After inunction-cures PASCHKIS and VAJDA detected mercury in the milk. In icterus neither the bile-acids nor bile-pigments pass into the milk.

Under diseased conditions no constant change has been found in woman's milk. In isolated cases SchLossberger, JoLY and FILHoL have observed indeed an essential declining composition, but no positive conclusion can be derived therefrom.

The changes in cow's milk have also been little studied. In tuberculosis of the udder STORCH found tubercule bacilli in the milk, and he also found that the milk became more and more diluted during the disease with a serous liquid 
similar to blood-serum, so that the glands, instead of yielding milk, only gave b ood-serum or a serous fluid. Husson found the milk from cows sick with murrain contained more proteids but considerably less fat and (in difficult cases) less sugar than normal milk.

The milk may be blue or red in color, due to the development of microorganisms.

The formation of concrements in the exit-passages of the cow's udder are often observed. They consist chiefly of calcium carbonate, or of carbonate and phosphate with only a small amount of organic substances. 


\section{CHAPTER XIII.}

\section{THE SKIN AND ITS SECRETIONS.}

Is the structure of the skin of man and vertebrates many different kinds of substances occur which have already been treated of, such as the constituents of the epidermis formation, the connective and fatty tissues, the nerves, muscles, etc. Among these the different horn-formations, the hair, nails, etc., whose chief constituent, keratin, has been spoken of in another chapter (Chap. II), are of special interest.

The cells of the horny formation show, in proportion to their age, a different resistance to chemical reagents, especially fixed alkalies. The younger the horn-cell the less resistance it has to the action of alkalies; with advancing age the resistance becomes greater, and the cell-membranes of many horn-formations are nearly insoluble in caustic alkalies. Keratin occurs in the horn formation mixed with other bodies, from which it is isolated with difficulty. Among these bodies the mineral constituents in many cases occupy a prominent place because of their quantity. Hair leaves on burning 5-70 p. m. ash which contains in 1000 parts 230 parts alkali sulphates, 140 parts calcium sulphate, 100 parts iron oxide, and 400 parts silicic acid. Dark hair seems generally, but not always, to yield more iron oxide on burning than blond. The nails are rich in calcium phosphate, and the feathers rich in silicic acid.

The skin of invertebrates has been the subject, in a few cases, of chemical investigation, and in these animals several substances have been found, of which a couple, though little studied, are worth discussing. Among these bodies tunicin, which is found especially in the tunic of the tunicata, and the widely-diffused 


\section{chitin, found in the cuticle-formation of invertebrates, are of interest.}

Tunicin, or animal cellulose, occurs, as above mentioned, in the tunicata. According to BERTHELOT, tunicin differs from ordinary cellulose, belng colored yellow by iodine, and by its slower conversion into sugar on boiling with acids. Otherwise it is similar to ordinary cellulose. The sugar obtained by boiling tunicin with acids is grape sugar, according to FraNcHIMONT.

Chitin is not found in vertebrates. The horny layer which covers the inner side of the gizzard of birds, may perhaps consist of a substance related to chitin. In invertebrates the chitin occurs in many classes of animals; it cannot be positively asserted that true, typical chitin is found elsewhere than in articulated animals ; in these it forms the chief organic constituent of the shell, etc.

According to SUNDWIK, the composition of chitin is probably $\mathrm{C}_{60} \mathrm{H}_{100} \mathrm{~N}_{8} \mathrm{O}_{30}$. $+n\left(\mathrm{H}_{2} \mathrm{O}\right)$, where $n$ may vary from 1 to 4 . On boiling with mineral acids it decomposes and yields, as LEDDERHOSE has shown, glucosamine. According to SuNDwIK, a glucose is also probably hereby produced. He also claims that chitin is an amido derivative of a carbohydrate of the formula $\mathrm{C}_{60} \mathrm{H}_{100} \mathrm{O}_{30}$.

In the dry state chitin forms a white, brittle mass retaining the form of the original tissue. It is insoluble in boiling water, alcohol, ether, acetic acid, dilute mineral acids, and dilute alkalies. It is soluble in concentrated acids. It is dissolved without decomposing in cold concentrated hydrochloric acid, but is decomposed by boiling hydrochloric acid. When chitin is dissolved in concentrated sulphuric acid and the solution dropped into boiling water and then botled, we obtain a.substance (glucosamine or glucose) which reduces copper suboxide in alkaline solutions.

Chitin may be easily prepared from the wings of insects or from the shells of the lobster or the crab, the last mentioned having first been extracted by an acid so as to remove the lime-salts. The wings or shells are boiled with caustic alkali until they are white, afterward washed with water, then with dilute acid and water, and lastly extracted with alcohol and ether.

Hyalin is the chief organic constituent of the walls of hydatid cysts. From a chemical point of view it stands close to chitin, or between it and the albumins. In old and more transparent sacks it is tolerably free from mineral bodies, but in younger sacks it contains a great quantity $(16 \%)$ of lime-salts (carbonate, phosphate, and sulphate).

According to LückE, its composition is :

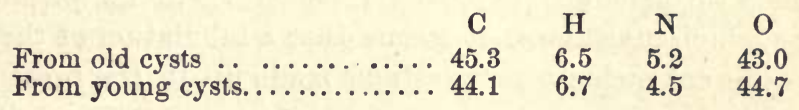

It differs from keratin on the one hand and from albumin on the other by the absence of sulphur, also by its yielding a variety of sugar in large quantities $(50 \%)$, which is reducible, fermentable, and dextro-gyrate when boiled with dilute sulphuric acid. It differs from chitin by the property of being gradually dissolved by caustic potash or soda, or by dilute acids; also by its solubility on heating with water to $150^{\circ} \mathrm{C}$.

The coloring matters of the skin and horn-formations are of different kinds, but have been but little studied. Those occurring in the Malpighian layer of the skin, especially of the negro, and 
the black or brown pigment occurring in the hair oelong to the group of coloring matters which have received the name melanin.

Melanins. This group includes several different varieties of amorphous black or brown pigments which are insoluble in water, alcohol, ether, chloroform, and dilute acids, and which occur in the skin, hair, epithelium-cells of the retina, in sepia, in certain pathological formations, and in the blood and urine in disease. Of these pigments there are a few, such as the melanin of the eye and that from the melanotic tumor of horses, the hippomelanin. (NENCKI and BERDEZ), which are soluble with difficulty in alkalies, while others, such as the pigment of the hair and the coloring matter of certain pathological swellings in man, the phymatorusin (NENCKI and BERDEZ), are easily soluble in alkalies.

Among the melanins there are a few, for example the choroid pigment, which are free from sulphur; others, on the contrary, as the pigment of the hair and of horse-hair, are rather rich in sulphur $(2-4 \%)$, while the phymatorusin found in certain swellings and in the hair (NENCKI and BERDEZ, K. MÖRNER) is very rich in sulphur (8-10\%). If any of these pigments, especially the phymatorusin, contains any iron or not is an important though disputed point, for it leads to the question whether these pigments are formed from the blood-coloring matters (NENCKI and Sieber, K. MörNER). The difficulties which attend the isolation and purification of the melanins have not been overcome in certain cases, while in others it is questionable whether the final product obtained has not another composition than the original coloring matter, owing to the energetic chemical processes resorted to in its purification. Under such circumstances it seems that a tabulation of the analyses of different melanin preparations made up to the present time are of secondary importance.

Among the above-mentioned bodies belonging to the melanin group, phymatorosin prepared by NENCKI and SIEBER from melanotic tumors, and by $K$. MörNer from the tumors and the urine of a patient, seems to be of special interest. Phymatorusin is an amorphous dark-brown coloring matter soluble in alkalies or alkali carbonates, but insoluble in warm 50- $75 \%$ acetic acid. In alkaline solution it shows no absorption-bands. According to NENCKI and Steber, it is free from iron, but MöRNER, on the contrary, claims 
that it does contain iron. MökNer found for this coloring matter from tumors (A) and from urine (B) the following composition calculated on the substance considered as ash-free :

A

C
H
N
S

C

$55.32-56.13$

$5.65-6.33$

12.30

7.97

$0.063-0.081$
B

55.76

5.95

12.27

9.01

0.20

NeNCKI and Sieber have also shown that other melanins, not identical with phymatorusin, occur in melanotic sarcoma of man.

The coloring matter or matters of human hair contain a low amount of nitrogen, $8.5 \%$ (SIEBER), and a variable but high amount of sulphur, 2.71-4.10\%. The considerable quantity of iron oxide found in the ash does not seem to belong to the coloring matters.

In addition to the coloring matters of the human skin we may also here treat of the pigments found in the skin or epidermis-formation of animals.

The beautiful color of the feathers of many birds depends in certain cases on purely physical causes (interference-phenomena), but in other cases on coloring matters of various kinds. Such a coloring matter is the amorphous reddish-violet turacin, which contains copper, and whose spectrum is very similar to that of oxyhæmoglobin. KRUKENBERG found a large number of coloring matters in birds' feathers, namely, zooerythrin, zoofulvin, turacoverdin, zooru. bin, psittacofulvin, and others which cannot be enumerated here.

Tetronerythrin, so named by WURM, is a red amorphous coloring matter which is soluble in alcohol and ether, and which occurs in the red warty spots over the eyes of the heath-cock and the grouse, and which is very widely spread among the invertebrates (Halliburton, De Merejkowski, MacMunn). Besides tetronerythrin MacMunN found in the shells of crabs and lobsters a blue coloring matter, cyanocrystallin, which turns red with acids and by boiling water. Homatoporphyrin, according to MACMUNN, also occurs in the integuments of certain lower animals.

In addition to the coloring matters thus far mentioned a few others found in certain auimals (though not in the skin) will be spoken of.

Carminic acid, or the red coloring matter of cochineal, has the composition $\mathrm{C}_{1}, \mathrm{H}_{18} \mathrm{O}_{10}$. It gives sugar on boiling with acids, but this does not correspond with the recent statements of LiEBERMANN. The beautiful purple solution of ammonium carmivate has two absorption-bands between $D$ and $E$ which are similar to those of oxyhæmoglobin. These bands lie nearer to $E$ and closer together and are less sharply defined. Purple is the evaporated residue from the purple-violet secretion, caused by the action of the sunlight, from the socalled "purple gland" of the tunic of certain species of murex and purpura. Its chemical nature has not been investigated.

Among the remaining coloring matters found in invertebrata we may mention blue stentorin, actiniochrom, bonellin, polyperythrin, pentucrinin, antedonin, crustaceorubin, janthinin, and cllorophyll. 
Sebum, when freshly secreted, is an oily semi-fluid mass which solidifies on the upper surface of the skin, forming a greasy coating. The quantity is very different in different persons. HOPPE-SEYLER has found a body similar to casein, besides albumin and fat, in the sebum. Cholesterin is also found in this fat, and in especially large quantities in the vernix caseosa. The solids of the sebum consist chiefly of fat epithelium-cells and protein bodies; the vernix caseosa consists chiefly of fat.

Cerumen is a mixture of the secretion of the sebaceous and sweat glands of the cartilaginous part of the outer organs of hearing. It contains chiefly soaps and fat, and besides these a red substance easily soluble in alcohol and with a sweetish-bitter taste.

The preputial secretion, smegma prceputii, contains chiefly fat, also cholesterin and ammonium soaps, which probably are produced from decomposed urine. The hippuric acid, benzoic acid, and calcium oxalate found in the smegma of the horse have probably the same origin.

We may also consider as a preputial secretion the castoreum, which is secreted by two peculiar glandular sacks in the prepuce of the beaver. This castoreum is a mixture of proteids, fat, resins, traces of phenol (volatile oil), and a non-nitrogenized body, castorin, crystallizing in four-sided needles from alcohol, insoluble in cold water, but somewhat soluble in boiling water, and whose composition is little known.

Wool-fat, or the so-called fat-sweat of sheep, is a mixture of the secretion of the sudoriparous and sebaceous glands. We find in the watery extract a large quantity of potassium which is combined with organic acid, volatile and nonvolatile fatty acids, benzoic acid, phenol-sulphuric acid, lactic acid, malic acid, succinic acid, and others (BuIsINE). The fat contains among other bodies abundant quantities of ethers of fatty acids with cholesterin and isocholesterin.

WEBER found that from the glands of the skin of a kangaroo and a dwarfantelope a colorless secretion was eliminated which in the first case, when exposed to the air, formed a red and in the second case a blue coloring matter. The secretion of the coccygeal glands of ducks and geese contains a body similar to casein, besides albumin, nuclein, lecithin, and fat, but no sugar (DE JoNGE). Poisonous bodies have been found in the secretion of the skin of the salamander and the toad, respectively, samandarin (ZALESKY) and bufidin (JorNARA and CASALI).

The Sweat. A disproportionally large part of the secretions of the skin whose quantity amounts to about $\frac{1}{64}$ of the weight of the body consists of water. Next to the kidneys the skin is the most important means for the elimination of water. As the glands of the skin and the kidneys stand near to each other in regard to 
their functions, they may to a certain extent act vicariously for one another.

Various conditions affect both the character and quantity of the sweat secreted. The secretion differs for different parts of the skin, and it has been stated that the perspirations of the cheek the palm of the hand, and under the arm stand to each other as 100: $90: 45$. From the unequal secretion on different parts of the body it follows that no results as to the amount of secretion for the entire surface of the body can be calculated from the amount secreted by a small part of the skin in a given time. In determining the total amount a stronger secretion is as a rule produced, and as the glands can with difficulty work for a long time with the same energy, it is hardly correct to estimate the quantity of secretion per 24 hours from a strong secretion enduring only a short time. FAVRE obtained 2560 grms. sweat in $1 \frac{1}{2}$ hours from the entire surface of the body in a steam-bath and with abundant drinking of water.

The perspiration obtained for investigation is never quite pure, but contains cast-off epidermis-cells, also cells and fat-globules from the sebaceous glands. Filtered sweat is a clear, colorless fluid with a salty taste and of different odors from different parts of the body. The physiological reaction is acid, according to most statements; still after continuous secretion the sweat may be alkaline (FAvre and Gillibert, Trümpy and Luchsinger). An alkaline reaction may also depend on a decomposition with the formation of ammonia. According to a few investigators, the physiological reaction is alkaline, and an acid reaction depends, according to these investigators, upon an admixture of fatty acids from the sebum. Morigaia found that the sweat from herbivora was ordinarily alkaline, while that from carnivora was generally acid. The specific gravity of sweat is 1.003-1.005.

Perspiration contains $977.4-995.6$ p. m., average 988.2 p. m., water, and 4.4-22.6 p. m., average 11.80 p. m., solids. The organic bodies are neutral fats, cholesterin, volatile fatty acids, traces of albumin (according to LECLERC habitually in horses; sometimes in man after hot baths, in BRIGHT's disease, and after the use of pilocarpin), also creatinin (CAPRANICA), aromatic oxyacids, ethereal sulphuric acids of phenol and skatoxyl (KAST), but not of indoxyl, and lastly urea. The amount of urea, which according to Funke 
is $1.99 \mathrm{p}$. m., has been more exactly determined in later times by ARGUTINSKY. In two steam-bath experiments, in which in the course of $\frac{1}{2}$ and $\frac{3}{4}$ hour he obtained respectively 225 and $330 \mathrm{c.c}$. sweat, he found 1.61 and $1.24 \mathrm{p}$. m. urea. In uræmia, and in ischuria in cholera, urea may be secreted in such quantities by the sweat-glands that crystals deposit upon the skin. The mineral bodies consist chiefly of sodium chloride with some potassium chloride, alkali sulphate, and phosphate. The relative amounts of these in perspiration differ materially from the amounts in the urine (FAVRE, KAST). The relationship, according to KAST, is as follows:

$\begin{array}{lcc:c} & \text { Chlorine : Phosphate : Sulphate } \\ \text { In perspiration } & 1 & : 0.0015 & : 0.009 \\ \text { In urine } & 1 & : & 0.1320\end{array}$

KAST found that the proportion of ethereal sulphuric acid to the sulphate sulphuric in sweat was 1:12. After the administration of aromatic substances the ethereal sulphuric acid does not increase to the same extent in the sweat as in the urine (see Chap. XIV).

Sugar may pass into the sweat in diabetes, but the passage of the bile-coloring matters has not been positively shown in this secretion. Benzoic acid, succinic acid, tartaric acid, iodine, arsenic, mercuric chloride, and quinine pass into the sweat. Uric acid has also been found in the sweat in gout, and cystin in cystinura.

Chromhidrosis has been called the secretion of colored sweat. Sometimes sweat has been observed to be colored blue by indigo (Bizlo), by pyocyanin (Fondos), or by ferro-phosphate (KollumaNN). 'True blood-sweat, in which blood-corpuscles exude from the openings of the glands, bas also been observed.

The exchange of gas through the skin in man is of very little importance compared to the exchange of gas by the lungs. 'The absorption of oxygen by the skin, which was first shown by REGNAULT and REISET, is very small. The quantity of carbon dioxide eliminated by the skin increases with the rise of temperature (AUbert, Röhrig, Fubini, and Ronchi). It is also greater in light than in darkness. It is greater during digestion than when fasting, and greater after a vegetable than after an animal diet (Fubini and Ronchi). According to Scharling it is 10 grms., and according to AUBERT 3.9 grms., in 24 hours. In certain ani- 
mals, as in frogs, the exchange of gas through the skin is of great importance.

As the exchange of gas through the skin in man and mammalia is very small, it follows that the injurious and dangerous effects caused by covering the skin with varnish, oil, or the like, can hardly depend on a prevented exchange of gas. After varnishing the skin there is a considerable loss of heat, and the animal quickly dies. If the animal, on the contrary, be guarded from this loss of heat, it may be saved, or kept at least alive, for a longer time. This effect was supposed to be due to a poisoning caused by a retention of one or more substances of the perspiration (perspirabile retentum), accompanied by fever and increased loss of heat through the skin; but this statement has not been substantiated. This phenomenon seems to be due to other causes, and at least in certain animals (rabbits) death seems to ensue from the paralyzation of the vasomotor nerves. In anastomosis the loss of heat through the skin seems to be increased to such an extent that the animal dies from the lowered temperature.

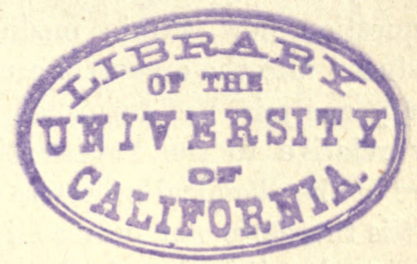




\section{CHAPTER XIV.}

\section{THE URINE.}

THE urine is the most important excretion of the animal organism; it is the means of eliminating the nitrogenized products of exchange, also the water and the soluble mineral substances; and in many cases it furnishes important data relative to the exchange of material; quantitatively, by its variation, and qualitatively by the appearance of foreign bodies in the excretion. Also in many cases we are able from the chemical or morphological constituents which the urine abstracts from the kidneys, ureters, bladder, and urethra to judge of the condition of these organs; and lastly, urinary analysis affords an excellent means of deciding the question how certain medicines or other foreign snbstances introduced into the organism are absorbed and chemically changed. Urinary analysis has furnished very important particulars especially relative to the last-mentioned question in regard to the nature of the chemical processes taking place within the organism, and it is therefore not only an important aid in diagnosis to the physician, but it is also of the greatest importance to the toxicologist and the physiological chemist.

In studying the secretions and excretions the relationship must be sought between the chemical structure of the secreting organ and the chemical composition of its secreted products. Investigations with respect to the kidneys and the urine have led to very few results from this standpoint. Although the anatomical relation of the kidneys has been carefully studied, their chemical composition has not been the subject of thorough analytical research. In cases in which a chemical investigation of the kidneys has been undertaken, it has only been in general on the organ as such, and not on the different anatomical parts. An enumeration of the 
chemical constituents of the kidneys known at the present time . can, therefore, only have a secondary value.

In the kidneys we find albuminous bodies of different kinds, namely, globulin, albumin, and nucleoalbumin, also a gelatineforming and elastic substance, and lastly a body similar to mucin. The question as to whether pure mucin really exists in the kidneys has not been decided. The body similar to mucin, which is a nucleoalbumin and which gives no reducible substance when boiled with acids (LöNNBERG), belongs chiefly to the papillæ, while the nucleoalbumin dissimilar to mucin belongs to the cortical substance. Fat only occurs in very small amounts in the cells of the tortuous urinary passages. Among the extractive bodies of the kidneys we find xanthin bodies, also adenin (KRONECKER), urea, and traces of uric acid, glycogen, leucin, inosit, taurin, and cystin (in ox-kidneys). The quantitative analyses of the kidneys thus far made possess little interest. The quantity of water in human kidneys is $828.4 \mathrm{p}$. m. according to FrERICHS, and $834.5 \mathrm{p}$. m. according to VoLKMANN.

The fluid collected under pathological conditions, as in hydronephrosis, is thin with a variable but generally low specific gravity. Usual!y it is strawyellow or paler in color, and sometimes colorless. Most frequently it is clear, or only faintly cloudy from white blood-corpuscles and epithelium-cells; in a few cases it is so rich in form-elements that it appears like pus. Albumin occurs generally only in small amounts; sometimes it is entirely absent, and in a few rare cases the amount is nearly as large as in the blood-serum. Urea occurs sometimes in considerable amounts when the parenchyma of the kidneys is only in part atrophied; in complete atrophy the urea may be entirely absent.

\section{Physical Properties of Urine.}

Consistency, transparency, odor, and taste of urine. Urine is under physiological conditions a thin liquid and gives, when shaken with air, a froth which quickly subsides. Human urine or urine from carnivora, which is habitually acid, appears clear and transparent, often faintly fluorescent immediately after voiding. When allowed to stand for a little while human urine shows a light cloud (nubecula) which consists of the so-called "mucus" and generally also contains a few epithelium-cells, mucus-corpuscles, and urate-granules. The presence of a larger quantity of urates (uric-acid salts) renders the urine cloudy, and a clay-yellow, 
yellowish-brown, rose-colored, or often brick-red precipitate (sedimentum lateritium) settles on cooling because of the greater insolubility of the urates at the ordinary temperature than at the temperature of the body. This cloudiness disappears on gently warming. In new-born infants the cloudiness of the urine during the first 4-5 days is due to epithelium, mucus-corpuscles, uric acid, and urates. The urine of herbivora, which is habitually neutral or alkaline in reaction, is very cloudy on account of the carbonates of the alkaline earths present. Human urine may sometimes be alkaline under physiological conditions. In this case it is made cloudy by the earthy phosphates, and this cloudiness does not disappear on warming, differing in this respect from the sedimentum lateritium. Urine has a salty and faintly-bitter taste produced by sodium chloride and urea. 'The odor of urine is peculiarly aromatic; the bodies which produce this odor are unknown.

The color of urine is normally pale yellow when the specific gravity is 1.020. The color otherwise depends on the concentration of the urine and varies from pale straw-yellow, when the urine contains small amounts of solids, to a dark reddish yellow or reddish brown in stronger concentration. As a rule, the intensity of the color corresponds to the concentration, but under pathological conditions exceptions occur, and such an exception is found in diabetic urine, which contains a large amount of solids and has a high specific gravity and a pale yellow color.

The reaction of urine depends essentially upon the composition of the food. The carnivora void an acid, the herbivora a neutral or alkaline, urine. If a carnivora is put on a vegetable diet, its urine may become less acid or neutral, while the reverse occurs when an herbivora is starved, that is, when it lives upon its own flesh, as then the urine voided is acid.

The urine of a healthy man on a mixed diet has an acid reaction, and the sum of the acid equivalents is greater than the sum of the base equivalents. This depends on the fact that in the physiological burning of neutral substances (proteids and others) within the organism acids are produced, chiefly sulphuric acid, but also phosphoric and organic acids, such as hippuric, uric, and oxalic acid, also aromatic oxyacids and others. From this it follows that the acid reaction is not due to one acid alone. We do not know to 
what extent any one acid takes part in the acid reaction; but as the .sum of the base equivalents is greater than, or at least the same as, the sum of the inorganic acid equivalents, the acid reaction must be due in greatest part to organic acids or acid salts. It is generally considered that the acid reaction of human urine is caused by double-acid alkali-phosphate (monophosphate). The amount of acid-reacting bodies or combinations eliminated by the urine in 24 hours, when calculated as oxalic acid or hydrochloric acid, is respectively 2-4 and 1.15-2.3 grms. (VOGEL).

The composition of the food is not the only influence which affects the degree of acidity of human urine. For example, after taking food, at the beginning of digestion, when a larger amount of gastric juice containing hydrochloric acid is secreted, the urine may be neutral or even alkaline. The greatest amount of acid or acidsalts per hour is secreted, according to VoGEL, during the night, while according to Hoffmans, it is in the afternoon. The smallest amount is voided, according to HoFFMAN during the night, but QUINCKE claims that it is in the morning. It has not infrequently been observed that perfectly healthy persons in the morning void a neutral or alkaline urine which is cloudy from earthy phosphates. The effect of muscular activity on the acidity of urine is yet undetermined. According to HoffmanN and Ringstedt, muscular work raises the degree of acidity, but ADUCCO claims that it decreases it. Abundant perspiration reduces the acidity (HoFFMANN).

In man and carnivora it seems that the degree of acidity of the urine cannot be increased above a certain point, even though mineral acids or organic acids which are burnt up with difficulty are taken in large quantities. When the supply of carbonates of the fixed alkalies stored up in the organism for this purpose is not sufficient to combine with the excess of acid, then ammonia is split from the proteids or their decomposition products, and the excess of acid combines therewith, forming ammonium-salts which pass into the urine. In herbivora this splitting of ammonia and formation of ammonia-salts does not seem to take place, and the herbivora therefore soon die when acids are given. Nevertheless, the degree of acidity of human urine may be easily diminished so that the reaction is neutral or alkaline. This occurs after the taking of 
carbonates of the fixed alkalies or of such salts of vegetable acidstartaric acid, citric-acid, and malic-acid salts-as are easily burnt into carbonates in the organism. Under pathological conditions, as in the absorption of alkaline transudations, the urine may become alkaline (QUINCKE).

The degree of acidity cannot be determined by the ordinary acidimetric process, since the urine contains di-hydrogen phosphate, $\mathrm{MH}_{2} \mathrm{PO}_{4}$, besides hydrogen di-phosphate $\mathrm{M}_{2} \mathrm{HPO}_{4}$. In the titration the di-hydrogen phosphate is changed gradually into $\mathrm{M}_{2} \mathrm{HPO}_{4}$, and we obtain at a certain point a mixture of the two phosphates in variable proportions, which mixture is not neutral but amphoteric. Since it is generally admitted that the acid reaction of urine is due to the di-hydrogen phosphate, it is therefore best to express the degree of acidity by the amount of di-hydrogen phosphate present.

If we wish to calculate the degree of acidity of the urine as di-hydrogen phosphate or, still more simply, as phosphoric anhydride, $\mathrm{P}_{2} \mathrm{O}_{5}$, contained in this salt, the titration is performed according to the method of MALY and HoFFMaNN, which is as follows: The urine (100-200 c. c.) is treated with an exactly-measured quantity of $\frac{1}{4}$ normal caustic-soda solution, which is more than sufficient to convert all the phosphate into basic phosphate, or, in other words, enough to make the urine strongly alkaline. 'Then an approximate $\frac{3}{4}$ normal $\mathrm{BaCl}_{2}$ solution (142.8 grms. $\mathrm{BaCl}_{2}, 2 \mathrm{HO}_{2}$ in a litre) is added until no further precipitate is formed. By this means all the phosphoric acid is precipitated from the urine. Filter through a dry filter, measure a quantity corresponding to 50 or 100 c.c. of the original urine from the filtrate, and titrate with $\frac{1}{4}$ normal sulphuric acid until a neutral reaction is obtained, using litmus-paper as an indicator. If the amount found by this titration be subtracted from the original amount of caustic soda added to this volume of urine, the difference is the amount of caustic soda necessary to convert the existing di-hydrogen and hydrogen di-phosphates into normal phosphate. If we designate this by $a$, and the quantity of total $\mathrm{P}_{2} \mathrm{O}_{5}$ in milligrammes in the same quantity of urine, as determined by a method which will be described later, by $g$, then we obtain the quantity of $\mathrm{P}_{2} \mathrm{O}_{5}$ in milligrammes in the di-hydrogen phosphate $s$ by the following formula: $s=17.75 a-g$ (HUPPERT).

If, for example, in a case in which the conversion of both phosphates into normal phosphate in 100 c.c. of the urine required 20 c.c. caustic soda, while the total quantity of $\mathrm{P}_{2} \mathrm{O}_{5}$ in 100 c.c. urine was 275 milligrammes, then: $s=17.75 \times 20-275=80$ milligrammes. The quantity of $\mathrm{P}_{2} \mathrm{O}_{5}$ as simple acid phosphate was therefore 195 milligrammes. 
A urine with an alkaline reaction caused by fixed alkalies has a very different diagnostic value than one whose alkaline reaction is caused by the presence of ammonium carbonate. In the latter case we have to deal with a decomposition of the urea of the urine by the action of micro-organisms.

If we wish to determine whether the alkaline reaction of the urine is due to ammonia or fixed alkalies, we dip a piece of red litmus-paper into the urine and allow it to dry exposed to the air or to a gentle heat. If the alkaline reaction is due to ammonia, the paper becomes red again; but if it is caused by fixed alkalies, it remains blue.

The specific gravity of urine, which is dependent upon the relationship existing between the quantity of water secreted and the solid urinary constituents, especially the urea and sodium chloride, may vary considerably, but is generally 1.017-1.020. After drinking large quantities of water it may fall to 1.002, while after profuse perspiration or after drinking very little water it may rise to 1.035-1.040. In new-born infants the specific gravity is low, 1.00\%-1.005. The determination of the specific gravity is an important means of learning the average amount of solids eliminated from the organism with the urine, and on this account the determination becomes of true value only when at the same time the quantity of urine voided in a given time is determined. The different portions of urine voided in the course of the 24 hours are collected, mixed together, the total quantity measured, and then the specific gravity taken.

The determination of the specific gravity is most accurately obtained with the pyknometer. For ordinary cases the specific gravity may be determined with sufficient accuracy by means of areometers. The areometers found in the trade, or urinometers, are graduated from 1.000 to 1.040 ; for exact observations it is better to use two urinometers, one graduated from 1.000 to 1.020 , and the other from 1.020 to 1.040. A special urinometer is that of HELLER, which is graduated according to Baumé's scale, from 0 to 8 . Each degree corresponds to $\%$ degrees of the ordinary urinometer, and as the zero-point of HELLER's urinometer corresponds to the figure 1000 , then the $1,1.5,2,2.5,3$, etc., degrees of HeLleR's urinometer correspond to $1.00 \%, 1.0105,1.014,1.01 \% 5,1.024$, etc., of the ordinary specific gravity. 
To determine the specific gravity of urine, if necessary filter the urine, or if it contains a urate sediment, first dissolve it by gentle heat, then pour the clear urine into a dry cylinder, avoiding the formation of froth. Air-bubbles or froth, when present, must be removed with a glass rod or filter-paper. 'The cylinder, which must be about $\frac{4}{5}$ full, must be wide enough to allow the urinometer to swim freely in the liquid without touching the sides. The cylinder and urinometer should both be dry or previously washed with the urine. On reading, the eye is brought on a level with the lower meniscus-which occurs when the surface of the liquid and the lower limb of the meniscus coincide; the reading is then made from the point where this curred line cuts the scale of the urinometer. If the eye is not in the same horizontal plane with the convex line of the meniscus, but is too high or too low, the surface of the liquid assumes the shape of an ellipse, and the reading in this position is incorrect. Before reading press the urinometer gently down into the liquid and then allow it to rise, and wait until it is at rest.

If the quantity of urine at disposal is not sufficient to fill the cylinder to the proper height it may be diluted, according to circumstances, with an equal volume or several volumes of water. This does not give quite accurate results, and with small quantities of urine it is best to determine the specific gravity by means of the pyknometer.

Each urinometer is graduated for a certain temperature, which is marked on the instrument, or at least on the best. If the urine is not at the proper temperature, the following corrections must be made: For every three degrees above the normal temperature one unit of the last order is added to the reading, and for every three degrees below the normal temperature one unit (as above) is subtracted from the specific gravity observed. For example, when a urinometer graduated for $+15^{\circ} \mathrm{C}$. shows a specific gravity of $1.01 \%$ at $+24^{\circ} \mathrm{C}$, , then the specific gravity at $+15^{\circ} \mathrm{C} .=1.017+0.003$ $=1.020$.

\section{Organic Physiological Constituents of the Urine.}

Urea, $\stackrel{+}{\mathrm{H}} \mathrm{r}$, which is ordinarily considered as carbamid, $\mathrm{CO}\left(\mathrm{NH}_{2}\right)_{2}$, may be synthetically prepared in several different ways, namely, from carbonyl-chloride, or carbonic-acid ethyl-ether and ammonia, $\mathrm{COCl}_{2}+2 \mathrm{NH}_{3}=\mathrm{CO}\left(\mathrm{NH}_{2}\right)_{2}+2 \mathrm{HCl}$, or $\left(\mathrm{C}_{2} \mathrm{H}_{5}\right)_{2} \cdot \mathrm{O}_{2} \cdot \mathrm{CO}+2 \mathrm{NH}_{3}=$ $2\left(\mathrm{C}_{2} \mathrm{H}_{5} . \mathrm{OH}\right)+\mathrm{CO}\left(\mathrm{NH}_{2}\right)_{2}$; by the metameric decomposition of ammonium cyanate, $\left(\mathrm{NH}_{4}\right) \cdot 0 . \mathrm{CN}=\mathrm{CO}\left(\mathrm{NH}_{2}\right)_{2} \quad$ (WöHLER, 1828); and in many other ways. It is also formed by the decomposition 
or oxidation of certain bodies found in the animal organism, such as creatin and uric acid.

Urea is found most abundant in the urine of carnivora and man, but in smaller quantities in that of herbivora. The quantity in human urine is ordinarily $20-30 \mathrm{p}$. $\mathrm{m}$. It has also been found in the urine of certain birds and amphibians. Urea occurs in the perspiration in small quantities, about $2 \mathrm{p}$. m., and as traces in the blood and in most of the animal fluids. It is also found in certain tissues and organs, especially in the liver (in mammalia) and the spleen, but not in the muscles. Under pathological conditions, as in obstructed secretion of urine, the urea in the animal fluids and tissues may increase to a considerable amount. Under these circumstances it may also occur in the muscles.

The quantity of urea which is voided in 24 hours on a mixed diet is in a grown man about 30 grammes, for women somewhat less. Children void absolutely less, but relative to their bodyweight the excretion is larger than in grown persons. The physiological significance of urea lies in the fact that this body forms in man and carnivora, from a quantitative standpoint the most important nitrogenized final product of the metabolism of proteid bodies. On this account the elimination of urea varies to a great extent with the amount of proteid transformed, and above all with the quantity of absorbable proteids in the food taken. 'The elimination of urea is greatest after an exclusive meat diet, and lowest, indeed less than during starvation, after the consumption of bodies free from nitrogen. for these diminish the metabolization of the proteids of the body.

If the consumiption of the proteids of the body is increased, then the elimination of urea is correspondingly increased. This is found to a rather high degree in certain diseases with fever; also, though to a less extent, after taking common salt or after abundant drinking of water (VoIT). The elimination of urea is also somewhat increased by several medicines; and lastly it is also increased after poisoning with arsenic, antimony, and phosphorus, by the diminished supply of oxygen-as in severe and continuous dyspnoa-poisoning with carbon monoxide, hemorrhage, etc. In these cases an exact difference has not always been made between the urea and the total quantity of nitrogen in the urine, and it is just this last which is 
often the cause of the increase found in these cases. The amount of nitrogen contained in the urea does not correspond with the total amount of nitrogen in the urine, since, as PFLÜGER has shown, the amount of nitrogen which under physiological conditions occurs combined with other combinations than urea is, on an average, $13.4 \%$, sometimes indeed $16 \%$, of the total nitrogen. In disease this relationship may be essentially changed and, as an example, in a case of phosphorus poisoning FraEnkel observed that the amount of nitrogen contained in the urea was less than one half of the total amount of nitrogen in the urine.

A diminished elimination of urea occurs in a reduced consumption of proteids, and also-a fact which is of interest in regard to the importance of the liver in the formation of urea-in certain diseases of the liver, as in acute yellow atrophy and sometimes in interstitial cirrhosis. Under these conditions the amount of ammonia in the urine as compared to the urea may be increased (HALLervorden, StadelmaNe). In diseases of the kidneys which disturb or destroy the integrity of the epithelium of the tortuous urinary passage the elimination of urea is considerably diminished.

Formation of urea in the organism. The experiments to produce urea directly from the proteids by oxidation have not led to any positive results. We often obtain amido-acids as decomposition products of albumin (see page 17), and we therefore conclude that the amido-acids are intermediate steps in the formation of urea from albumin. It has also been shown that leucin and glycocoll (Schultzen and Nencki, SAlkowski), and asparaginic acid (v. KNIERIEM) may be transformed into urea within the organism. The nature of the chemical processes by which these transformations are effected is unknown. Many other ways for the formation of urea have been suggested, but the only one which has been demonstrated is the formation from ammonium carbonate in the liver. After the researches of v. KNieriem, Salkowski, Feder, Munck, Schmiedeberg and Walter, and Hallervorden had shown that ammonium carbonate, or such ammonium-salts as are burnt up in the system into carbonate, is converted into urea in the body of carnivora and herbivora, v. SCHRöDER furnished a decisive proof of the formation of urea from ammonium carbonate in the liver of mammalia. By passing blood which had been treated with 
ammonium carbonate or ammonium formate through a dog's liver, he found that a considerable formation of urea took place. The very careful observations of SALOMON confirm this, and also the above-mentioned decrease of urea and increase of ammonia in the urine in certain diseases of the liver.

It cannot be denied that the formation of urea in the liver is of great physiological importance; but still it does not follow that all the urea has its origin in this organ. The possibility of a formation of urea from creatin or xanthin bodies is not to be rejected without further inquiry, and other possibilities as to its formation are conceivable.

The question in which organ urea is formed has been the subject of much discussion. From the researches of numerous investigators, Prevost and Dumas, Meissner, Voit, Gréhant, Gscheidlen, SALKowski, and v. SchröDer, it has been found that the extirpation of the kidneys causes a considerable increase in the quantity of urea in the blood. The kidneys, therefore, although they may produce urea generally, are not the only organs which produce it. By experiments performed on the removed kidneys, which were analogous to the above-mentioned experiments on the removed liver, v. SCHRöDER has shown that neither the kidneys nor the muscles nor the remaining tissues of the lower extremities of the dog have the property of forming urea from ammonium carbonate. The only organ in which a formation of urea has been proved with certainty, thus far, is the liver, but this does not exclude the possibility that urea may also be formed in other organs, perhaps from other material than ammonium carbonate and in other ways.

Properties and Reactions of Urea. Urea crystallizes in needles or in long, colorless, four-sided, often hollow, anhydrous rhombic prisms. It has a neutral reaction and produces a cooling sensation on the tongue like saltpetre. It melts at $130^{\circ}-132^{\circ} \mathrm{C}$, but decomposes already at about $100^{\circ} \mathrm{C}$. At ordinary temperatures it dissolves in equal weight of water and in five parts alcohol; it requires one part boiling alcohol for solution; it is sparingly soluble in ether. If urea in substance is heated in a test-tube it melts, decomposes, gives off ammonia, and leaves finally a non-transparent white residue which, among other substances, contains biuret and 
which dissolves in water, giving a beautiful reddish-violet liquid with copper sulphate and alkali (biuret reaction). On heating with baryta-water or caustic alkali, urea splits into carbon dioxide and ammonia with the addition of water; this also takes place by the action of micro-organisms, which produces the so-called alkaline fermentation of urine. The same decomposition is produced when urea is heated with concentrated sulphuric acid. An alkaline solution of sodium hypobromite decomposes urea into nitrogen, carbon dioxide, and water according to the equation

$$
\mathrm{CON}_{2} \mathrm{H}_{4}+3 \mathrm{NaOBr}=3 \mathrm{NaBr}+\mathrm{CO}_{2}+2 \mathrm{H}_{2} \mathrm{O}+\mathrm{N}_{2} \text {. }
$$

Urea forms with many acids crystalline combinations. Among these the one with nitric acid and the one with oxalic acid are the most important.

Urea Nitrate, $\mathrm{CO}\left(\mathrm{NH}_{2}\right)_{2} \cdot \mathrm{HNO}_{3}$. This combination on crystallizing quickly forms thin rhombic or six-sided, overlapping tiles, colorless plates, whose point has an angle of $82^{\circ}$. On slowly crystallizing, larger and thicker rhombic pillars or plates are obtained. This combination is rather easily soluble in pure water, but is considerably less soluble in water containing nitric acid; it may be obtained by treating a concentrated solution of urea with an excess of strong nitric acid free from nitrous acid. On heating this combination it volatilizes without leaving a residue.

This compound may be employed with advantage in detecting small amounts of urea. A drop of the concentrated solution is placed on a microscope-slide and the cover-glass placed upon it ; a drop of nitric acid is then placed on the side of the cover-glass and allowed to flow under. The formation of crystals begins where the solution and the nitric acid meet. Alkali nitrates may crystallize very similarly to urea nitrate when they are contaminated with other bodies ; therefore, in testing for urea, the crystals must be identified as urea nitrate by heating and by other means.

Urea Oxalate, 2.CO $\left(\mathrm{NH}_{2}\right)_{2} \cdot \mathrm{H}_{2} \mathrm{C}_{2} \mathrm{O}_{4}$. This compound is more sparingly soluble in water than the nitric-acid compound. It is obtained in rhombic or six-sided prisms or plates on adding a saturated oxalic-acid solution to a concentrated solution of urea.

Urea also forms combinations with mercuric nitrate in variable proportions. If a very faintly-acid mercuric-nitrate solution is added to a two-per-cent solution of urea, and the mixture carefully neutralized, a combination is obtained of a constant composition 
which contains for every 10 parts of urea 7.2 parts mercuric oxide.

- This compound serves as the basis of Liebig's titration method. Urea combines also with salts, forming mostly crystallizable combinations, as, for instance, with sodium chloride, with the chlorides of the heavy metals, etc. An alkaline but not a neutral solution of urea is precipitated with mercuric chloride.

The method of preparing urea from urine is chiefly as follows: Concentrate the urine, which has been faintly acidified with sulphuric acid, at a low temperature, add an excess of nitric acid, at the same time keeping the mixture cool, press the precipitate well, decompose it in water with freshly-precipitated barium carbonate, dry on the water-bath, extract the residue with strong alcohol, decolorize when necessary with animal charcoal, and filter while warm. The urea which crystallizes on cooling is purified by recrystallization from warm alcohol. A further quantity of urea may be obtained from the mother-liquor by concentration. The urea is purified from contaminating mineral bodies by redissolving in alcohol-ether. If it is only necessary to detect the presence of urea in urine, it is sufficient to concentrate a little of the urine on a watch-glass and after cooling treat with an excess of nitric acid. In this way we obtain crystals of urea nitrate.

Quantitative Estimation of Urea in urine. The methods suggested for this purpose are those of LIEBIG, by titration, of HEINTZ and RAGSKY, also that of KJELDAHL, by which the total nitrogen is determined, and those of BUNSEN and KNOP-HÜFNER, where urea is intended to be determined as such. Among these methods, that of LIEBIG, which is perhaps the one most frequently employed by physicians, will here be carefully explained. In regard to the others, whose chief points only will be spoken of here, the student is referred to other text-books.

LIEBIG'S METHOD is based upon the fact that a dilute solution of mercuric nitrate under proper conditions precipitates all the urea forming a compound of constant composition. As indicator, a soda solution or a thin paste of sodium bicarbonate is used. An excess of mercuric nitrate produces herewith a yellow or yellowishbrown combination, while the combination of urea and mercury is white. PFL ÜGER has given full particular's of this method; therefore we will describe PFLÜGER's modification of LIEBIG's method.

As phosphoric acid is also precipitated by the mercuricnitrate solution, this must be removed from the urine by the addi- 
tion of a baryta solution before titration. PFLÜGER also suggested that the acidity produced by the mercury solution be neutralized with a soda solution. The liquids necessary for the titration are the following:

1. Mercuric Nitrate Solution. This solution is calculated for a $2 \%$ urea solution, and 20 c. c. of the first should correspond to 10 c. c. of the latter. Each c. c. of the mercury solution corresponds to $0.01 \mathrm{grm}$. urea. As a small excess of $\mathrm{HgO}$ is necessary in the urine to make the final reaction (with alkali carbonate or bicarbonate) appear, each c. c. of the mercury solution must contain $0.07 \% 2$ instead of $0.0720 \mathrm{grm}$. HgO. The mercury solution contains therefore 77.2 grms. $\mathrm{HgO}$ in one litre.

The solution may be prepared from pure mercury or mercuric oxide by dissolving in nitric acid. The solution, freed as completely as possible from an excess of acid, is diluted by the careful addition of water, stirring mean while, until it has a specific gravity of 1.10 or a little higher at $+20^{\circ} \mathrm{C}$. The solution is standardized with a $2 \%$ solution of pure urea which bas been dried over sulphuric acid, and the operation conducted as will be described later. If the solution is too concentrated, it is corrected by the careful addition of the necessary amount of water, avoiding basic sult, and titrated again. The solution is correct if $19.8 \mathrm{c}$. c. of it is added at once to $10 \mathrm{c}$. c. of the urea solution and the necessary quantity of normal soda solution(11-12 c. c. or more) to completely neutralize the liquid, gives the final reaction when $20 \mathrm{c}$. c. of the mercury solution have been employed.

2. Baryta Solution. This consists of 1 vol. barium-nitrate and 2 vols. barium-hydrate solution, both saturated at the ordinary temperature.

3. Normal Soda Solution. This solution contains 53 grms. pure anhydrous carbonate in 1 litre of water. According to PFIÜGER, a solution having a specific gravity of 1.053 is sufficient. The amount of this soda solution necessary to completely neutralize the acid set free during the titration is determined by titrating with a pure $2 \%$ urea solution. To facilitate operations a table can be made showing the quantity of soda solution necessary when from 10 to 35 c. c. of the mercury solution is used.

Before the titration the following must be considered. The chlorides of the urine interfere with the titration in that a part of the mercuric nitrate is transformed into mercuric chloride, which does not precipitate the urea. The chlorides of the urine are therefore removed by a silver-nitràte solution, which also removes any bromine or iodine combinations which may exist in the urine. If the urine contains albumin in noticeable amounts, it must be removed by coagulation and the addition of acetic acid, but care must be taken that the concentration and the volume of the urine is not changed during these operations. If the urine contains ammonium carbonate in notable quantities, caused by alkaline 
fermentation, this titration method cannot be applied. The same is true of urine containing leucin, tyrosin or medicinal preparations, precipitated by mercuric nitrate.

In cases where the urine is free from albumin or sugar and not specially poor in chlorides, the quantity of urea, and also the approximate quantity of mercuric nitrate necessary for the titration, may be learned from the specific gravity. A specific gravity of 1.010 corresponds to about $10 \mathrm{p}$. m., the specific gravity 1.015 generally somewhat less than $15 \mathrm{p}$. m., and the specific gravity 1.015-1.020 about $15-20$ p. m. urea. With a specific gravity higher than 1.020 the urine generally contains more than $20 \mathrm{p}$. m. of urea, and above this point the amount of urea increases much more rapidly than the specific gravity, so that at a specific gravity of 1.030 it contains over $40 \mathrm{p}$. m. urea. In fevers, urine with a specific gravity above 1.020 sometimes contains $30-40$ p. m. urea, or even more.

Preparation for the Titration. If a large amount of urea is suspected from a high specific gravity, the urine must first be diluted with a carefully-measured quantity of water, so that the amount of urea is reduced below $30 \mathrm{p}$. $\mathrm{m}$. In a special portion of the same urine the amount of chlorides is determined by one of the methods which will be given later, and the number of c. c. of silvernitrate solution necessary is noted. Then a larger quantity of urine, say 100 c. c., is mixed with one half or, if this is not sufficient to precipitate all the sulphuric and phosphoric acids, with an equal volume of the baryta solution, it is then allowed to stand a little while, and the precipitate is filtered through a dried filter. From the filtrate containing the urine diluted with water a proper quantity, corresponding to about $60 \mathrm{c.}$. c. of the original urine, is measured, and exactly neutralized with nitric acid added from a burette, so that the exact quantity employed is known. The neutralized mixture of urine and baryta is treated with the proper quantity of silver-nitrate solution necessary to completely precipitate the chlorides, which was ascertained by a previous determination. The mixture containing a known volume of urine is now filtered through a dried filter into a flask, and from the filtrate an amount is measured corresponding to $10 \mathrm{c}$. c. of the original urine.

Execution of the Titration. Nearly the total quantity of mercuric-nitrate solution to be used, and which is known from the specific gravity of the urine, is added at once, and immediately afterwards the quantity of soda solution necessary, as indicated by the table. If the mixture becomes yellowish in color, then too much mercury solution has been added and another determination must be made. If the test remains white, and if a drop taken out and placed on a glass plate with a dark background and stirred with a drop of a thin paste of sodium bicarbonate does not give a yellow 
color, the addition of mercury solution is continued by adding $\frac{1}{2}$ and then $\frac{1}{10}$ c. c., and testing after each addition in the following way: A drop of the mixture is placed on a glass plate with a dark background beside a small drop of the bicarbonate paste. If the color after stirring the two drops together is still white after a few seconds, then more mercury solution must be added; if, on the contrary, it is yellowish, then-if not too much mercury solution has been added by inattention-the result to $\frac{1}{10}$ c. c. has been found. By this approximate determination, which is sufficient in many cases, we have learned the minimum amount of mercury solution necessary to add to the quantity of urine in question, and we now proceed to the final determination.

A second quantity of the filtrate, corresponding to $10 \mathrm{c}$. c. of the original urine, is filtered, and the same quantity of mercury solution added at one time as was found necessary to produce the final reaction, and immediately after the corresponding amount of soda solution, which must not indicate the end of the reaction. Then add the mercury solution in $\frac{1}{10}$ c. c. without neutralizing with soda, until a drop taken out and mixed with the soda solution gives a yellow coloration. If this final reaction is obtained after the addition of 0.1-0.2 c. c., then the titration may be considered as finished. If, on the contrary, a larger quantity is necessary, the addition of the mercury solution must be continued until a final reaction is obtained with simple carbonate, and the titration repeated again, adding the quantity of mercury solution used in the previous test at one time, and also adding the corresponding amount of soda solution. If we obtain the end reaction by the addition of $\frac{1}{10}$ c. c., we may consider the titration as finished.

If in each titration a quantity of filtrate containing urine and baryta corresponding to $10 \mathrm{c}$. c. of the primitive urine is used, then the calculations are very simple, since 1 c. c. of mercuric-nitrate solution corresponds to $0.01 \mathrm{grm}$. of urea. As the mercury solution is made for a $2 \%$ urea solution, the filtrate of urine and baryta being generally deficient in urea (if the quantity of urea is above $2 \%$, it is easy to avoid any mistake by diluting the urine at the beginning of the operation), a mistake occurs here which can be corrected in the following way, according to PFLÜGER: To the measured volume of the filtrate from the urine (the filtrate with baryta after neutralization with nitric acid, precipitation with silver nitrate and filtration) the quantity of normal soda solution employed is added, and from this sum the volume of mercury solution used is subtracted. The remainder is then multiplied by 0.08 , and the product subtracted from the number of c. c. of mercury solution used. For example, if the filtrate (urine and baryta + nitric acid + silver nitrate) measured 25.8 c. c., and the number of c. c. of soda solution used in the titration, 13.8 c. c., and the mercury solution, 20.5 
c. c., we have then $20.5-\{(39.6-20.5) \times 0.08\}=20.5-1.53=$ 18.97 , and the corrected quantity of mercury solution is therefore ' 18.97 c. c. If the measured c. c. of the filtrate (in this case 25.8 c. c.) corresponds to $10 \mathrm{c}$. c. of the original urine, then the amount of urea is $18.97 \times 0.01=0.1897=18.97 \mathrm{p} . \mathrm{m}$. urea.

Besides the urea other nitrogenized constituents of the urine are precipitated by the mercury solution. By the titration we really do not obtain the quantity of urea, but, as PFLÜGER has shown, the total quantity of nitrogen in the urine expressed as urea. As the urea contains 46.67 p. c. N, the total quantity of nitrogen in the urine may be calculated from the quantity of urea found.

The results obtained by LIEBIG-PFLÜGER's titration method for the total nitrogen, PFLÜGER has shown, correspond well with the results obtained by K'JELDAHL's method, which was first (1861) used by ALMÉn for urea determinations, and modified by PFLÜGER and BoHLAND. This method consists in heating the urine a certain time with an excess of concentrated or fuming sulphuric acid (5 c. c. urine and 40 c. c. sulphuric acid) until all the nitrogen has been converted into ammonia, and after the addition of an excess of caustic soda the ammonia is distilled into $\frac{n}{10}$ sulphuric acid and the amount of ammonia distilled over is determined by titration.

Bunsen's Urea Determination. The principle of this method consists in heating the urine or urea solution in a sealed glass tube to a high temperature with an alkaline barium-chloride solution. The urea splits into carbon dioxide and ammonia, which may be determined separately. This method has been very carefully tested by PfLüGER and his pupils BoHLANd and BleibTREu, and essentially improved. They found that very accurate results can be obtained by this method if the other nitrogenized constituents of the urine are first precipitated by a mixture of hydrochloric acid and phospho-tungstic acid, and then the filtrate made faintly alkaline with milk of lime, and lastly heated with alkaline barium-chloride solution in a sealed tube. The carbon dioxide and the ammonia can be determined (by distilling with magnesia and receiving the distillate in $\frac{n}{10}$ acid and titrating). In the last 
case a correction must be made (according to ScHLÖsING's method) for the ammonia pre-existing in the urine. PfLüger and BleibTREU have essentially changed this method in the following way: They precipitate the other nitrogenized urinary constituents with hydrochloric acid and phospho-tungstic acid, make the filtrate faintly alkaline with milk of lime, determine the pre-existing ammonia in a part of this filtrate according to ScHLösING's method (observing certain precautions), aud then placing the other part of the filtrate (about 15 c. c.) in a large flask which contains 10 grms. crystallized phosphoric acid, heat to $230^{\circ}-260^{\circ} \mathrm{C}$. for about three hours. All the urea is decomposed, and the ammonia split off combines with the phosphoric acid. After cooling, an excess of caustic soda is added and the ammonia distilled into a titrated acid, which must then be retitrated. After subtracting the quantity of pre-existing ammonia very accurate results are obtained for the ammonia originating from the urea (and perhaps from an unknown ureid present in the urine).

KNOP-HÜFNER'S METHOD is based on the fact that urea by the action of sodium hypobromite splits into water, carbon dioxide (which dissolves in the alkali), and nitrogen, whose volume is measured (see page 340). This method is less accurate than the preceding ones, and therefore in scientific work it is discarded. It is of value to the physician and for practical purposes because of the ease and rapidity with which it may be performed, even though it may not give very accurate results. For practical purposes a series of different apparatus have been constructed to facilitate the use of this method. Among these the ureometer of EsBACH deserves to be especially mentioned. ${ }^{1}$ In regard to the reagents

${ }^{1}$ Dr. Chas. A. Donemus has constructed a ureometer of the very simplest kind. It consists of two parts. First, a vertical glass tube closed at the top aud bent sharply below, where it expands into a bulb having an orifice at its upper part. Secondly, a pipette with an elastic rubber nipple. Both parts are graduated; the vertical tube so as to show the quantity of urea (as indicated by the volume of nitrogen) in each cubic centimetre of urine tested, while the pipette is graduated so as to show one cubic centimetre. The instrument is used thus: First the vertical tube is filled with the alkaline sodium-hypobromite solution in the following manner: holding it vertically, the operator pours the solution into the bulb, and when it is rather more than half full he inclines the apparatus horizontally until the entire tube is filled and a little left in the bulb-say one third or thereabouts. Then he restores it to the vertical position. He now draws into the pipette, by means of the 
necessary for the determination of urea, and also for instructions in -the use of this instrument, we must refer the reader to the directions accompanying the apparatus. For pure urea solutions EsBACH's apparatus gives quite exact results. The determination of urea in urine by this method always gives results somewhat too low, and as a rule a result is obtained which on an average is about 0.1\% lower than that obtained with LIEBIG's titration method.

Creatinin, $\mathrm{C}_{4} \mathrm{H}_{7} \mathrm{~N}_{3} \mathrm{O}$, or $\mathrm{NH}: \mathrm{C}<\frac{\left.\mathrm{NH}-\mathrm{CH}_{3}\right) \cdot \dot{\mathrm{C}} \mathrm{H}_{2}}{\mathrm{~N}}$, is generally considered as the anhydride of creatin (see page 257) found in the muscles. It occurs in human urine and in that of certain mammalia. It has also been found in ox-blood (VorT), milk, though in very small amounts (WEYL), and in the flesh of certain fishes.

The quantity of creatinin in human urine is for a grown man, voiding a normal quantity of urine in the 24 hours, $0.6-1.3$ grms. (NeUbauER), or on an average $1 \mathrm{grm}$. The quantity is dependent on the food, and decreases in starvation. Sucklings do not generally eliminate any creatinin, and it only appears in the urine when the milk is replaced by other food. The quantity of creatinin in urine varies as a rule with the quantity of urea, although it. is increased, more by flesh (because the flesh contains creatin) than by albumin. Grocco claims that the elimination of creatinin is increased by muscular activity, which is contrary to the statements of HoFMANN and others. 'The behavior of creatinin in disease is little known. By increased exchange of material the amount is increased, while by decreased exchange of material, as in anæmia and cachexia, it is diminished.

Creatinin crystallizes in colorless, shining monoclinic prisms which differ from creatin crystals in not becoming white with loss of water when heated to $100^{\circ} \mathrm{C}$. It dissolves in 11.5 parts cold water, but more easily in warm water. It requires nearly 100 parts

rubber nipple, a cubic centimetre of the urine to be tested, which should be taken from the total excretion of the twenty-four hours, the exact quantity of which should be noted. The end of the pipette is passed into the bulb until the point is exactly under the vertical tube, and slowly compress the nipple. The urine being lighter than the hypobromite solution rises through it, and on its way the contained urea is decomposed and its nitrogen set free. The quantity of urea contained in the one cubic centimetre of urine used is read off on the instrument.-TRANSLATOR. 
cold absolute alcohol for solution, but it is more soluble in warm alcohol. It is nearly insoluble in ether. In alkaline solution creatinin is converted into creatin very easily on warming.

Creatinin gives an easily-soluble crystalline combination with hydrochloric acid. A solution of creatinin acidified with mineral acids gives crystalline precipitates with phospho-tungstic or phospho-molybdic acids, even in very dilute solutions $(1: 10,000)$ (KERNER). It is precipitated, like urea, with mercuric-nitrate solution. Among the compounds of creatinin, that with zinc chloride, creatinin zinc chloride $\left(\mathrm{C}_{4} \mathrm{H}_{7} \mathrm{~N}_{3} \mathrm{O}\right)_{2} \mathrm{ZnCl}_{2}$, is of special interest. This combination is obtained when a sufficiently concentrated solution of creatinin in alcohol is treated with a concentrated, faintly-acid solution of zinc chloride. Free mineral acids dissolve the combination, but this, however, is prevented, when they are present, by an addition of sodium acetate. In the impure state, as ordinarily obtained from urine, creatinin zinc chloride forms a sandy, yellowish powder which under the microscope appears as fine needles forming concentric groups, mostly complete rosettes or yellow balls or tufts, or grouped as brushes. On slowly crystallizing, or when very pure, more sharply-defined prismatic crystals are obtained. This combination is sparingly soluble in water.

Creatinin acts as a reducing agent. Mercuric oxide is reduced to metallic mercury, and oxalic acid and methylguanidin (methyluramin) are formed. Creatinin also reduces copper hydroxide in alkaline solution, forming a colorless soluble combination, and only after continuous boiling with an excess of copper salts is free suboxide of copper formed. . Creatinin interferes with 'Trommen's test for sugar, partly because it has a reducing action and partly by retaining the copper suboxide in solution. The combination with copper suboxide is not soluble in a saturated-soda solution and if a little creatinin is dissolved in a cold, saturated-soda solution and then a few drops of FeHLING's reagent added, a white flocculent combination separates after heating to $50^{\circ}-60^{\circ} \mathrm{C}$. and then cooling

- (v. MASCHKE's reaction). An alkaline bismuth solution (see Sugar 'l'ests) is not reduced by creatinin.

If we add a few drops of a freshly-prepared very dilute sodium nitroprusside (sp. gr. 1.003) to a dilute creatinin solution (or to the urine) and then a few drops of caustic soda, a ruby-red liquid 
is obtained which quickly turns yellow again (WEYL's reaction). If we use ammonia instead of caustic soda in this reaction, the red color is not obtained (differing from aceton and ethyl-diacetic acid, LE NOBEL). If the above solution, which has become yellow, is treated with an excess of acetic acid and heated, the solution becomes first green and then blue (SALKowSKI); finally a precipitate of Prussian blue is obtained. If a solution of creatinin in water (or urine) is treated with a watery solution of piciric acid and a few drops of a dilute soda solution, a red coloration lasting several hours occurs immediately at the ordinary temperature, and which turns yellow on the addition of acid (JAFFE's reaction). Acetone gives a more reddish-yellow color. Grape-sugar gives with this reagent a red coloration only after heating.

In preparing creatinin from urine the creatinin zinc chloride is first prepared according to NEUBAUER's method, and this method is also employed for the quantitative estimation of creatinin. In making a quantitative estimation 200-300 c. c. of urine freed from albumin (by boiling with acid) and from sugar (by fermentation with yeast) are measured, alkalized with milk of lime, and treated with $\mathrm{CaCl}_{2}$ solution until all the phosphoric acid is precipitated; it is filtered and washed with water, the filtrate and the wash-water united, and evaporated to a syrup after acidifying with acetic acid. This syrup is mixed while hot with 50 c. c. of $95 \%-97 \%$ alcohol. This mixture is transferred to a beaker, and the residue in the evaporating-dish is completely and carefully removed and added. The liquid is allowed to stand covered for at least eight hours in the cold. Then it is filtered through a small filter, the precipitate washed with alcohol, the filtrate evaporated if necessary until the volume is $50-60 \mathrm{c}$. c., then allowed to cool and $\frac{1}{2}$ c. c.of an acidfree zinc-chloride solution of an sp. gr. of 1.20 is added; it is stirred, and the covered beaker is left standing in a cool place for two or three days. The precipitate is collected on a small dried and weighed filter, using the filtrate to wash the crystals from the beaker. After allowing the crystals to completely drain off, they are washed with a little alcohol until the filtrate gives no reaction for chlorine, and dried at $100^{\circ} \mathrm{C} .100$ parts creatinin zinc chloride contain 62.42 parts creatinin. As the precipitate is never quite pure, the quantity of zinc must be carefully determined, in exact. experiments, by evaporating with nitric acid, heating, washing the oxide of zinc with water (to remove any $\mathrm{NaCl}$ ), drying, heating, and weighing. 22.4 parts zinc oxide correspond to 100 parts creatinin zinc chloride. 
The preparation of creatinin zinc chloride on a large scale from urine is done in the same way. The creatinin is obtained from the creatinin zinc chloride by boiling with lead hydroxide, filtering, decolorizing the filtrate with animal charcoal, evaporating, treating the residue with strong alcohol (which leaves the creatin undissolved), evaporating to crystallization, redissolving in water, and recrystallizing.

Xanthocreatinin. This body, which has been spoken of in a previous chapter on the muscles, has been found in dog's urine after the injection of creatinin into the abdominal cavity (MONARI), and in human urine after several hours of continuous marching. The correctness of these observations is disputed by STADTHAGEN.

Uric Acid, $\overline{\mathrm{Ur}}, \mathrm{C}_{5} \mathrm{H}_{4} \mathrm{~N}_{4} \mathrm{O}_{3}$. The structural formula of this acid, according to Medreus, is $\mathrm{CO}\left\langle\begin{array}{c}\mathrm{NH} . \mathrm{C} . \mathrm{NH} \\ \ddot{\mathrm{C} .} \mathrm{NH}\end{array}>\mathrm{CO}\right.$, and this acid may therefore be considered, from its constitution as a derivate of acrylic acid, as acrylic acid diureid.

Uric acid has been synthetically prepared by HoRBACZEWSKI in several ways. On fusing urea and glycocoll, uric acid is formed according to the formula $3 \mathrm{CON}_{2} \mathrm{H}_{4}+\mathrm{C}_{2} \mathrm{H}_{5} \mathrm{NO}_{2}=\mathrm{C}_{5} \mathrm{H}_{4} \mathrm{~N}_{4} \mathrm{O}_{3}+$ $2 \mathrm{H}_{2} \mathrm{O}+3 \mathrm{NH}_{3}$, and in this reaction hydantoin and biuret are formed as intermediate products. On melting methylhydantoin with urea or methylhydantoin with biuret or with allophanic-acid amyl-ester HoRBACZEWSKI obtained methyl-uric acid. He also obtained uric acid on heating trichlor-lactic acid, or still better trichlor-lactic acidamid, with an excess of urea. If we eliminate from the reaction the numerous by-products (cyanuric acid, carbon dioxide, etc.), then this process may be expressed by the formula $\mathrm{C}_{3} \mathrm{Cl}_{3} \mathrm{H}_{4} \mathrm{O}_{2} \mathrm{~N}+$ $2 \mathrm{CON}_{2} \mathrm{H}_{4}=\mathrm{C}_{5} \mathrm{H}_{4} \mathrm{~N}_{4} \mathrm{O}_{3}+\mathrm{H}_{2} \mathrm{O}+\mathrm{NH}_{4} \mathrm{Cl}+2 \mathrm{HCl}$.

On strongly heating uric acid it decomposes with the formation of UREA, HYDROCYANIC ACID, CYANURIC ACID, and AMMONIA. On heating with concentrated hydrochloric acid in sealed tubes to $1 \% 0^{\circ} \mathrm{C}$. it splits into GLYCOCOLL, CARBON DIOXIDE, and AMMONIA. By the action of oxidizing agents a splitting and oxidation takes place, and either monoureid or diureid is produced. By oxidation with lead peroxide, CARBON DIOXIDE, OXALIC ACID, UREA, and ALLANToIN, which last is glyoxyldiureid, are produced (see below). By oxidation with nitric acid in the cold UREA and a monoureid, the 
mesoxalyl urea or ALLOXAN, are obtained, $\mathrm{C}_{5} \mathrm{H}_{4} \mathrm{~N}_{4} \mathrm{O}_{3}+\mathrm{O}+\mathrm{H}_{2} \mathrm{O}=$ . $\mathrm{C}_{4} \mathrm{H}_{2} \mathrm{~N}_{2} \mathrm{O}_{4}+\left(\mathrm{NH}_{2}\right)_{2} \mathrm{CO}$. On warming with nitric acid, uric acid yields alloxan, carbon dioxide, and oxalyl urea or PARABANIC ACID, $\mathrm{C}_{3} \mathrm{H}_{2} \mathrm{~N}_{2} \mathrm{O}_{3}$. By the addition of water the parabanic acid passes into OXALURIC ACID, $\mathrm{C}_{3} \mathrm{H}_{4} \mathrm{~N}_{2} \mathrm{O}_{4}$, traces of which are found in the urine and which easily split into oxalic acid and urea.

Uric acid occurs most abundantly in the urine of birds and of scaly amphibians, in which animals the greater part of the nitrogen of the urine appears in this form. Uric acid occurs frequently in the urine of carnivorous mammalia, but is sometimes absent; in urine of herbivora it is habitually present, though only as traces; in human urine it occurs in greater but still small and variable amounts. Traces of uric acid are also found in several organs and tissues, as in the spleen, lungs, heart, pancreas, liver (especially in birds), and in the brain. It habitually occurs in the blood of birds (Meissner). Traces have been found in human blood under normal conditions (ABELES), but especially in gout (GARROD). Uric acid also occurs in large quantities in "chalk-stones," certain urinary calculi, and in guano. It has also been detected in the urine of insects and certain snails.

The amount of uric acid eliminated with the human urine is subject to considerable variation, but amounts on an average to $0.7 \mathrm{grm}$. during 24 hours on a mixed diet. On a vegetable diet the amount is smaller, and on an abundant meat diet it may rise to 2 grms. and over. The relationship of the uric acid to the urea on a mixed diet is on an average $1: 50-1: 70$. In new-born infants and in the first days of life the elimination of uric acid is increased (MARÈs), and the relation between the uric acid and urea is about 1:13-14. After taking glycerin the amount of uric acid is increased (HORBACZEWSKI and KANĚRA), while it is not increased by sodium acrylate (HoRBACZEWSKI).

Uric acid when introduced into the organism of a dog is in great part converted into urea, and as urea is also formed by the action of oxidizing agents on uric acid outside of the body, uric acid has been often considered as a step towards the formation of urea in the organism. Such a view is not, however, well founded, and the statement that an incomplete supply of oxygen and diminished oxidation cause an increased formation of uric acid has not been 
proved. With regard to the pathological relations we really only know two conditions in which the elimination of uric acid is increased, namely, in fever and leucæmia. In fevers the elimination of uric acid is increased because of an increased waste of the organism. In leucæmia the elimination is increased absolutely as well as relatively to the urea (RANke, SALKowsKi, Fleischer and Penzoldt, Stadthagen, and others), and the relationship of the elimination of uric acid to the urea may be 1:16-1:12. The elimination of uric acid may be diminished in gout shortly before and during the attack, because the uric acid is retained in the body. A decrease in the elimination of uric acid is observed also in a diminished exchange of material, also after the use of quinine, caffeine, and certain other medicines.

Formation of Uric Acid in the organism. The formation of uric acid in birds is increased by the administration of ammonia-salts (v. ScHRöDER). Urea acts in the same way (MEYER and JAFFÉ), while in the organism of mammalia uric acid is more or less completely converted into urea. MiNkowsKI observed in geese with their livers extirpated a very significant decrease in the elimination of uric acid, while the elimination of ammonia is increased to a corresponding degree. This indicates a participation of ammonia in the formation of uric acid in the organism of birds ; and as MINKowski has also found after the extirpation of the liver that considerable amounts of lactic acid occur in the urine, it is probable that the uric acid in birds is produced in the liver, perhaps from lactic acid and ammonia by synthesis. Amido-acids-leucin, glycocoll, and aspartic acid-increase the elimination of uric acid in birds (v. KNIERIEM), but whether the amido-acids are first decomposed with the splitting off of ammonia is still unknown. V. MACH has shown that a small part of the uric acid in birds originates from hypoxanthin, and a similar origin for the uric acid of mammalia is also very probable (MіNкоwsкi).

After the extirpation of the kidneys of snakes and birds v. SCHRöDER has observed an accumulation of uric acid in the blood and tissues. This shows that the kidneys of these animals are not the only source of uric acid, and any direct proof of the formation of this acid in the kidneys has not to the present time been demonstrated. A direct relationship between the spleen and the forma- 
tion of uric acid, also in man, has been sought by many investigators - (RANKE, KÜHNE), and in support of this view they claim that the elimination of uric acid is increased in diseases in which the spleen is enlarged, also that the elimination of uric acid by the urine is decreased when the volume of the spleen is diminished by the administration of large quantities of quinine. That uric acid is formed in the spleen receives additional proof from the investigations of HoRBACZEWSKI. He found a considerable new formation of uric acid when he allowed the pulp of the spleen and blood of calves to act upon each other at the ordinary temperature, at the same time passing a stream of air through the mass. He also obtained extracts from the pulp of the spleen with boiling water, which yielded uric acid after the action of the blood. This production must depend chiefly upon decomposition products of nuclein (xanthin bodies). HorbaczewsKI claims that probably the lymphatic elements are here concerned, a statement which coincides with the increased elimination of uric acid in lineal leucæmia, also with the parallelism existing between the digestion-leucocytes and the increased elimination of uric acid occurring after taking food. We have no positive basis for the statement that uric acid is formed in the liver of man and mammalia, but the formation of uric acid in the liver of birds is shown to be highly probable by the researches of Minkowski.

Properties and Reactions of Uric Acid. Pure uric acid is a white, odorless, and tasteless powder consisting of very small rhombical prisms or plates. Impure uric acid is easily obtained as somewhat larger, colored crystals.

In quick crystallization, small, apparently colorless, thin, foursided rhombic prisms, which can only be seen by the aid of the microscope, are formed, and these sometimes appear as spools because of the rounding of their obtuse angles. The plates are sometimes six-sided, irregularly developed; in other cases they are rectangular with partly straight and partly jagged sides; and in other cases they show still more irregular forms, the so-called dumbbells, etc. In slow crystallization, as when the urine deposits a sediment or when treated with acid, large, always colored crystals separate. Examined with the microscope these crystals appear always yellow or yellowish brown in color. The most ordinary 
form is the whetstone shape formed by the rounding off of the obtuse angles of the rhombic plate. The whetstones are numerous, connected together, two or more crossing each other. Besides these forms, rosettes of prismatic crystals, irregular crosses, brown-colored rough masses of crystals which assume the shape of needles and prisms occur, also other different forms.

Uric acid is insoluble in alcohol and ether; it is rather easily dissolved in boiling glycerin, very difficultly soluble in cold water (14,000-15,000 parts); and difficultly soluble in boiling water (in 1800-1900 parts), it is soluble in a warm solution of sodium diphosphate, and in the presence of an excess of uric acid monophosphate and acid urate are produced. Sodium phosphate is considered as a solvent for the uric acid in the urine. Uric acid is dissolved without decomposing in concentrated sulphuric acid. It is completely precipitated from the urine by picric acid (JAFFÉ).

Uric acid forms with bases two series of salts, neutral and acid. Of the alkali urates the neutral potassium and lithium salts dissolve the most easily, and the ammonium salt dissolves with the most difficulty. The acid-alkali urates are very insoluble and separate as a sediment (sedimentum lateritium) from concentrated urine on cooling. The salts with alkaline earths are very insoluble.

If a little uric acid in substance is treated with a few drops of nitric acid, the uric acid dissolves on warming with a strong development of gas, and after thoroughly drying on the water-bath a beautiful red residue is obtained, which turns a purple-red (purpurate of ammonium) on the addition of a little ammonia. If, instead of the ammonia, we add a little caustic soda (after cooling), the color becomes more blue or bluish violet. This color disappears quickly on warming, differing from certain xanthin bodies. This reaction is called the murexide test.

Uric acid does not reduce an alkaline solution of bismuth, but does, on the contrary, an alkaline copper-hydroxide solution. In the presence of only a little copper salt we obtain a white precipitate consisting of copper urate. In the presence of more copper salt red suboxide separates.

If a drop of uric acid dissolved in sodium carbonate is placed on a piece of filter-paper which has been previously treated with silver-nitrate solution, a reduction of silver oxide occurs producing 
a brownish-black or, in the presence of only 0.002 milligram uric - acid, a yellow spot (SchIFF's test).

Preparation of Uric Acid from Urine. Filtered normal urine is treated with $20-30$ c. c. of $25 \%$ hydrochloric acid for each litre of urine. After forty-eight hours collect the crystals and purify them by redissolving in dilute alkali, decolorizing with animal charcoal and reprecipitating with hydrochloric acid. Large quantities of uric acid are easily obtained from the excrements of serpents by boiling them with dilute caustic potash until no more ammonia is developed. A current of carbon dioxide is passed through the filtrate until it barely has an alkaline reaction; dissolve the separated and washed acid potassium urate in caustic potash, and precipitate the uric acid by addition of an excess of hydrochloric acid to the filtrate.

Quantitative Estimation of Uric Acid in the urine. The older method of HeINTZ, somewhat modified by ScHwANERT, is in its main points as foliows: The filtered urine free from albumin and any sediment of urates (dissolved by warming) is concentrated when too dilute to a sp. gr. of $1.020,200 \mathrm{c}$. c. measured off and this treated with 10-20 c. c. hydrochloric acid of a sp. gr. of 1.12. After allowing this mixture to stand forty-eight hours in a cool place the precipitated uric acid is collected on a small weighed filter (5-6 cm. diameter), and the crystals which have adhered to the sides of the glass are removed by means of a glass rod tipped with a piece of rubber tubing, using the filtrate to wash with. After all the liquid has passed through, fill the filter with water and allow it to run through completely before adding more water; continue until the wash-water does not give a chlorine reaction, then dry and weigh. A part of the uric acid always remains dissolved in the filtrate. The filtrate, including the wash-water, must therefore be measured, and for each $10 \mathrm{c}$. c. of filtrate (and washwater) $0.00048 \mathrm{grm}$. uric acid must be added. With this correction this determination gives the same results as the following more complicated method.

In SALKOWSKI and LUDWIG's method the uric acid is precipitated from the urine by silver-nitrate solution, treated with magnesium mixture, and the uric acid removed from the silver precipitate and weighed. Uric-acid determinations according to this method are often performed according to the treatment suggested by E. LUDWIG, which requires the following solutions:

1. An AMMONIACAL sILVER-NITRATE solution which contains in one litre 26 grms. silver nitrate and a quantity of ammonia sufficient to completely redissolve the precipitate produced by the first addition of ammonia. 2. Magnesium mixture. Dissolve 100 grms. crystallized magnesium chloride in water and add enough ammonia so that the liquid smells strougly of it, 
and then add sufficient ammonium chloride to dissolve the precipitate and lastly dilute to 1 litre. 3. SoDIUM-sULPHIDE solution. Dissolve $10 \mathrm{grms}$. caustic soda which is free from nitric and nitrous acids in 1 litre of water. One half of this solution is completely saturated with hydrogen sulphide and then mixed with the other half.

The solutions are such that 10 c. c. of each is sufficient for 100 c. c. of the urine.

100-200 c. c., according to concentration, of the filtered urine freed from albumin (by boiling after the addition of a few drops of acetic acid) are poured into a beaker. In another vessel mix 10-20 c. c. of the silver solution with 10-20 c. c. of the magnesium mixture, and add ammonia, and when necessary also some ammonium chloride, until the mixture is clear. This solution is added to the urine while stirring, and the mixture allowed to stand quietly for $\frac{1}{2}$ hour. The precipitate is collected on a filter, washed with ammoniacal water, and then returned to the same beaker by the aid of a glass rod and a spirit-bottle without destroying the filter. Now heat to boiling-point 10-20 c. c. of the alkaline sulphide solution, which has previously been diluted with an equal amount of water, and allow this solution to flow through the above filter into the beaker containing the silver precipitate, wash with boiling water, and warm the contents of the beaker on the water-bath for a time, stirring constantly. After cooling filter into a porcelain dish, wash with boiling water, acidify the filtrate with hydrochloric acid, evaporate to about 15 c. c., add a few drops more of hydrochloric acid, and allow it to stand for 24 hours. 'The uric acid which has crystallized is collected on a small weighed filter, washed with water, alcohol, ether, and carbon disulphide, dried at $100^{\circ}-110^{\circ}$ C., and weighed. For each $10 \mathrm{c}$. c. of the watery filtrate we must add $0.00048 \mathrm{grm}$. uric acid to the amount found directly. Instead of the weighed filter-paper a glass tube filled with glass wool may be substituted. This tube was constructed by LUDwIG, and is described in other hand books.

HAYCRAFT'S METHOD: 25 c. c. of the urine are first treated with $1 \mathrm{grm}$. bicarbonate, then made strongly alkaline by ammonia, and lastly precipitated by an ammoniacal silver solution. The carefully washed precipitate is dissolved in $20-30 \%$ nitric acid and this solution titrated with a $\frac{n}{100}$ sulphocyanide solution according to VolHARD's method. Each c. c. of this solution corresponds to $0.00168 \mathrm{grm}$. uric acid. This method has been modified by CzAPEK. After the addition of a known amount of silver solution of known strength, the amount of silver salts remaining in the urine after all the uric acid has been precipitated is titrated with alkali sulphide. The advantage of HAYCrAFT's method is the ease and rapidity with which it can be performed, and it is therefore recom- 
mended for clinical purposes. For exact determinations it is not quite reliable, because the amount of silver in the precipitate of 'silver urate is not constant (SALKowSKI). Since the value of this method has been the subject of much adverse criticism, we will not give further particulars.

Oxaluric ACID, $\mathrm{C}_{3} \mathrm{H}_{4} \mathrm{~N}_{2} \mathrm{O}_{4}=\left(\mathrm{CON}_{2} \mathrm{H}_{3}\right) \cdot \mathrm{CO} . \mathrm{COOH}$. This acid, whose relation to uric acid and urea has been spoken of above, occurs only as traces in the urine as ammonium salts. This salt is not directly precipitated by $\mathrm{CaCl}_{2}$ and $\mathrm{NH}_{2}$, but after boiling, when it is decomposed into urea and oxalate. In preparing oxaluric acid from urine the latter is filtered through animal charcoal. The oxalurate retained by the charcoal may be obtained by boiling with alcohol.

\section{Oxalic Acid, $\mathrm{C}_{2} \mathrm{H}_{2} \mathrm{O}_{4}$, or $\stackrel{\mathrm{COOH}}{\mathrm{COOH}}$, occurs under physiological}

conditions in very small amounts in the urine, about $0.02 \mathrm{grm}$. in 24 hours (FüRBRINGER). According to the generally-received opinion it is found in the urine as calcium oxalate, which is kept in solution by the acid phosphates present. Calcium oxalate is a frequent constituent of the urinary sediments, and occurs also in certain urinary calculi.

The origin of the oxalic acid in the urine is not well known. Oxalic acid, when administered, is eliminated by the urine unchanged, and as many regetables and fruits, such as cabbage, spinach, asparagus, sorrel, apples, grapes, etc., contain oxalic acid, it is possible that a part of the oxalic acid of the urine originates directly from the food. Another part is certainly formed in the body from the proteids and fat or by the incomplete combustion of the carbohydrates. The formation of oxalic acid from proteids (or fat) is inferred from the fact that oxalic acid is eliminated by the urine after food consisting entirely of flesh and fat, as also in starvation. It has also been considered, but without sufficient reason, that the oxalic acid of the urine is an oxidation product of uric acid.

An increased elimination of oxalic acid may occur in diabetes. The question whether it may occur as an independent disease (oxaluria, oxalic-acid diathesis) has not been positively decided.

The properties and reactions of oxalic acid and calcium oxalate are well known. The calcium oxalate as a constituent of urinary sediments will be described later.

Detection and quantitative estimation of oxalic acid in urine. The presence of oxalic acid in solution in urine is determined 
according to the method suggested by NEUBAUER, who treats 500-600 c. c. of the urine with $\mathrm{CaCl}_{2}$ solution, makes alkaline with ammonia and then faintly acid with acetic acid. After 24 hours the precipitate is collected on a small filter, washed with water, treated with hydrochloric acid (which leaves the uric acid undissolved on the filter), and washed again with water. The filtrate, including the wash-water, is treated with an excess of ammonia and allowed to stand 24 hours. Calcium oxalate separates as quadratic octahedra. 'The quantitative estimation is performed after the same principle. The oxalate is converted into quick-lime by heat, and weighed as such.

Allantoin or GLYOXYLDIUREID, $\mathrm{C}_{4} \mathrm{H}_{6} \mathrm{~N}_{4} \mathrm{O}_{3}$ or
$\mathrm{CO}\left\langle\begin{array}{l}\mathrm{NH} . \mathrm{CH} . \mathrm{NH} \text {.CO. } \mathrm{NH}_{2} \text {, occurs in the urine of children within } \\ \mathrm{NH} . \dot{\mathrm{CO}}\end{array}\right.$ the first eight days after birth, and in very small amounts also in the urine of grown persons (GUSSERow, ZIEGLER and HermanN). It is found in rather abundant quantities in the urine of pregnant women (GUSSERow). Allantoin has also been found in the urine of sucking calves (WöHLER), and sometimes in the urine of other animals (MEISSNER). It is also found in the amniotic fluid and allantoic fluid of the cow (hence the name). Allantoin is formed, as above stated, by the oxidation of uric acid. The increased elimination of allantoin which SALKowsKI observed in dogs after the administration of uric acid shows that the formation of allantoin from uric acid in the organism is not improbable.

Allantoin is a colorless substance often crystallizing in prisms, difficultly soluble in cold water, easily soluble in boiling water and also in warm alcohol, but not soluble in cold alcohol or ether. It combines with acids, forming salts. A watery allantoin solution gives no precipitate with silver nitrate alone, but by the careful addition of ammonia a white flocculent precipitate is formed, $\mathrm{C}_{4} \mathrm{H}_{5} \mathrm{AgN}_{4} \mathrm{O}_{3}$, which is soluble in an excess of ammonia and which consists after a certain time of very small, transparent microscopic globules. The dried precipitate contains $40.75 \%$ silver. A watery allantoin solution is precipitated by mercuric nitrate.

Allantoin is most easily prepared by the oxidation of uric acid with lead peroxide. In preparing allantoin from calves' urine, concentrate the urine on the water-bath to a syrup and allow it to stand in the cold for several days. 'The crystals which are sepa- 
rated from the precipitate by washing are dissolved in boiling water with the addition of some animal charcoal, and filtered while - hot; then acidify the filtrate faintly with hydrochloric acid (so as to keep the phosphates in solution) and allow it to crystallize. Allantoin is detected in human urine by the method first suggested by Meissner. It consists chiefly of the following points: Precipitate the urine with baryta-water, filter, remove the baryta with sulphuric acid, filter, precipitate the allantoin with $\mathrm{HgCl}_{2}$ in alkaline solution, decompose the precipitate with sulphuretted hydrogen, concentrate strongly, purify the erystals which separate by recrystallization, and lastly prepare the silver combination.

Xanthin Bodies. The xanthin bodies which habitually occur in human urine are xanthin, hypoxanthin (SALOMON), guanin (Pouchet), carnin (PoucheT), and the newly-discovered bodies paraxanthin ('THUdichum, SALOMON) and heteroxanthin (SALOMON). The quantity of these bodies in the urine is very small. The quantity of xanthin bodies in the urine is increased especially in leucæmia, in which disease adenin is also found in the urine (STADTHAGEN). Xanthin also occurs as a constituent of a variety of rare calculi (MARCET). It is also sometimes found as a constituent of urinary sediments (BENCE JoNES).

Paraxanthin, $\mathrm{C}_{7} \mathrm{H}_{8} \mathrm{~N}_{4} \mathrm{O}_{2}$ (dimethylxanthin), and heteroxanthin, $\mathrm{C}_{6} \mathrm{H}_{6} \mathrm{~N}_{4} \mathrm{O}_{2}$ (methylxanthin), do not give the xanthin reaction with nitric acid and alkali, but give WeIDEL's reaction (see page 50). They differ from other xanthin bodies by forming crystalline combinations with alkalies, which are diffcultly soluble. Amorphous heteroxanthin separates on neutralizing the sodium combination, but paraxanthin, on the contrary, separates in a crystalline condition. Paraxanthin gives an easily-soluble combination with hydrochloric acid, while heteroxanthin forms an insoluble, beautiful crystalline combination.

In preparing xanthin bodies from the urine, it is supersaturated with ammonia and precipitated by a silver-nitrate solution. The precipitate is then decomposed with sulphuretted hydrogen. The boiling-hot filtrate is evaporated to dryness and the dried residue treated with $3 \%$ sulphuric acid. The xanthin bodies are dissolved, while the uric acid remains undissolved. This filtrate is saturated with ammonia and precipitated by silver-nitrate solution. The different xanthin bodies may be separated from each other by treating the silver precipitate with boiling-hot nitric acid of a sp. gr. of 1.1 (see page 51).

Hippuric acid, or BENZOYL-AMIDO ACETIC ACID, $\mathrm{C}_{9} \mathrm{H}_{9} \mathrm{NO}_{3}$ or $\mathrm{C}_{6} \mathrm{H}_{5} \cdot \mathrm{CO} \cdot \mathrm{NH} \cdot \mathrm{CH}_{2} \cdot \mathrm{COOH}$. This acid decomposes into benzoic acid and glycocoll on boiling the urine with mineral acids or alkalies, this also takes place in putrefaction. The reverse of this occurs if these two components are heated in a sealed tube, according 
to the following equation: $\mathrm{C}_{6} \mathrm{H}_{5} \mathrm{COOH}+\mathrm{NH}_{2} \cdot \mathrm{CH}_{2} \cdot \mathrm{COOH}=$ $\mathrm{C}_{6} \mathrm{H}_{5} \cdot \mathrm{CO} \cdot \mathrm{NH} \cdot \mathrm{CH}_{2} \cdot \mathrm{COOH}+\mathrm{H}_{2} \mathrm{O}$. This acid may be synthetically prepared from benzamid and monochlor-acetic acid, $\mathrm{C}_{6} \mathrm{H}_{5} . \mathrm{CO} . \mathrm{NH}_{2}$ $+\mathrm{CH}_{2} \mathrm{Cl} \cdot \mathrm{COOH}=\mathrm{C}_{6} \mathrm{H}_{5} \cdot \mathrm{CO} \cdot \mathrm{NH} \cdot \mathrm{CH}_{2} \cdot \mathrm{COOH}+\mathrm{HCl}$, also in other different ways.

Hippuric acid occurs in large amounts in the urine of herbivora, but only in small quantities in that of carnivora. The quantity of hippuric acid eliminated in human urine on a mixed diet is usually less than $1 \mathrm{grm}$. per 24 hours; as an average it is $0.7 \mathrm{grm}$. After eating freely of vegetables and fruit, especially such fruit as plums, the quantity may be more than 2 grms. Hippuric acid is also found in the perspiration, blood, suprarenal capsule of oxen, and in the ichthyosis scales. Nothing is positively known in regard to the quantity of hippuric acid in the urine in disease.

The Formation of Hippuric Acid in the organism. Benzoic acid and also the substituted benzoic acids are converted into hippuric acid and substituted hippuric acids within the body. Also, those bodies are transformed into hippuric acid which by oxidation (toluol, cinnamic acid, hydrocinnamic acid) or by reduction (quinic acid) are converted into benzoic acid. The question of the origin of hippuric acid is therefore connected with the question of the origin of benozic acid; for the formation of the second component, glycocoll, from the protein substances in the body is without question.

Hippuric acid is found in the urine of starving human beings (SchultzeN) and dogs (SALKowSKI), also in dog's urine after a diet consisting entirely of meat (Meissner and Shepard, SalkowSKI, and others). It is evident that the benzoic acid originates in these cases from the proteids. Benzoic acid may indeed be produced outside of the body by the oxidation of the albumins; the benzoic acid produced on a diet consisting entirely of meat seems to be derived from the putrefaction of the proteids in the intestines. Among the products of the putrefaction of albumin outside of the body SALKowSKI has found phenylpropionic acid, $\mathrm{C}_{6} \mathrm{H}_{5} \cdot \mathrm{CH}_{2} \cdot \mathrm{CH}_{2}$. $\mathrm{COOH}$, which is oxidized in the organism to benzoic acid and eliminated as hippuric acid after combining with glycocoll. Phenylpropionic acid seems to be formed from the amidophenylpropionic acid, which is prepared only from the plant proteids, and the sup- 
position that the phenylpropionic acid is produced from tyrosin by putrefaction in the intestines has not been substantiated by the researches of BAUMANN, SchotTen and BAas. The importance of putrefaction in the intestines in producing hippuric acid is evident from the fact that after thoroughly disinfecting the intestines of dogs with calomel the hippuric acid disappears from the urine (BAUMANN).

The large quantity of hippuric acid present in the urine of herbivora is partly explained by the fact that vegetable proteids yield perhaps larger amounts of amidophenylpropionic acid, and partly by the specially active processes of putrefaction going on in the intestines of herbivora. These circumstances do not entirely explain this excess of hippuric acid (SALKowSKI). The abundant elimination of hippuric acid by herbivora may in part depend on the great amount of aromatic substances in the food of these animals which is converted into benzoic acid. There is hardly any doubt that the hippuric acid in human urine after a mixed diet, and especially after a diet of vegetables and fruits, has in part a similar origin.

The kidneys may be considered in dogs as special organs for the synthesis of hippuric acid (Schmiedeberg and BuNGE). In other animals, as in rabbits, the formation of hippuric acid seems to take place in other organs, such as the liver and muscles. The synthesis of hippuric acid is therefore not exclusively limited to any special organ, though perhaps in some species of animals it may be more abundant in one organ than in another.

Properties and reactions of hippuric acid. This acid crystallizes in semi-transparent, milk-white, long, four-sided rhombic prisms or columns, or in needles by rapid crystallization. They dissolve in 600 parts cold water, but more easily in hot water. They are easily soluble in alcohol, but with difficulty in ether. They are more easily soluble (about 12 times) in acetic ether than in ethyl ether. Petroleum ether does not dissolve them.

On heating hippuric acid it first melts to an oily liquid which crystallizes on cooling. By continuing the heat it decomposes, producing a red mass and a sublimate of benzoic acid, with the generation, first, of a peculiar pleasant odor of hay, and then an odor of hydrocyanic acid. Hippuric acid is easily differentiated from ben- 
zoic acid by this behavior, also by its crystalline form and its insolubility in petroleum ether. Hippuric acid and benzoic acid both give LücKE's reaction, namely, they generate an intense odor of nitrobenzol when evaporated with nitric acid to dryness and when the residue is heated. Hippuric acid forms crystallizable salts, in most cases, with bases. 'The combinations with alkalies and alkaline earths are soluble in water and alcohol. The silver, copper, and lead salts are soluble with difficulty in water; the ironoxide salt is insoluble.

Hippuric acid is best prepared from the fresh urine of a horse or cow. The urine is boiled a few minutes with an excess of milk of lime. The liquid is filtered while hot, concentrated and then. cooled, and the hippuric acid precipitated by the addition of an excess of hydrochloric acid. The crystals are pressed, dissolved in milk of lime by boiling, and treated as above; the hippuric acid is precipitated again from the concentrated filtrate by hydrochloric acid. The crystals are purified by recrystallization and decolorized, when necessary, by animal charcoal.

The quantitative estimation of hippuric acid in the urine may be performed by the following method (BUNGE and ScHMiEdeBERG): 'T'he urine is first made faintly alkaline with soda, evaporated nearly to dryness, and the residue thoroughly extracted with strong alcohol. After the evaporation of the alcohol, dissolve in water, acidify with sulphuric acid, and completely extract by agitating (at least five times) with fresh portions of acetic ether. The acetic ether is then repeatedly washed with water, which is removed by means of a-separatory funnel, then evaporated at a medium temperature, and the dry residue treated repeatedly with petroleum ether, which dissolves the benzoic acid, oxyacids, fat and phenol, while the hippuric acid remains undissolved. This residue is now dissolved in a little warm water and evaporated at $50^{\circ}-60^{\circ} \mathrm{C}$. to crystallization. The crystals are collected on a small weighed filter. The mother-liquor is repeatedly shaken with acetic ether. This last is removed and evaporated; the residue is added to the above crystals on the filter, dried and weighed.

Phenaceturic Acid, $\mathrm{C}_{10} \mathrm{H}_{11} \mathrm{NO}_{3}=\mathrm{C}_{6} \mathrm{H}_{5} \cdot \mathrm{CH}_{2} \cdot \mathrm{CO} \cdot \mathrm{NH} \cdot \mathrm{CH}_{2} \cdot \mathrm{COOH}$. This acid, which is produced in the animal body by a grouping of the phenylacetic acid, $\mathrm{C}_{6} \mathrm{H}_{5} . \mathrm{CH}_{2} . \mathrm{COOH}$, formed by the putrefaction of the proteids with glycocoll, has been prepared from horse's urine by SALKowsKI, but it probably also occurs in human urine.

Benzoic Acid, $\mathrm{C}_{7} \mathrm{H}_{6} \mathrm{O}_{2}$ or $\mathrm{C}_{6} \mathrm{H}_{5} . \mathrm{COOH}$, is found in rabbit's urine and sometimes, though in small amounts, in dog's urine (WEYL and v. ANREP). According to JAARSVELD and STOKVIS and to KRONECKER, it is also found in human urine in diseases of the kidneys. The occurrence of benzoic acid in 
the urine seems to be due to a fermentative decomposition of hippuric acid. Such a decomposition may very easily occur in an alkaline urine or one con- taining albumin (VAN DE VELDE and STOKVIs). In certain animals-pigs and dogs-the kidneys, according to Schmiedeberg and MrNkowski, contain a special enzyme, Schmiedererg's histozym, which splits the hippuric acid with the separation of benzoic acid.

Ethereal Sulphuric Acids. Phenol, whose mother-substance is considered to be tyrosin, is produced by the putrefaction of albumin in the intestines; indol and skatol are also produced. The phenol and the two last-mentioned bodies, after they have been oxidized into indoxyl and skatoxyl, pass into the urine as ethereal sulphuric acids after uniting with sulphuric acid. The most important of these ethereal acids are phenol- and cresol-sulphuric acid-which were formerly called phenol-forming substance-indoxyl-and skatoxyl-sulphuric acid. To this group belong also the pyrocatechin-sulphuric acid, which only occurs in very small amounts in human urine, and hydrochinon-sulpluric acid, which appears in the urine after poisoning with phenol, and perhaps under physiological conditions other ethereal acids occur which have not been isolated. The ethereal sulphuric acids of the urine were discovered and specially studied by BAUMANN. The quantity of these acids in the urine is small. The quantity of grouped sulphuric acid per twenty-four hours is on an average $0.25 \mathrm{grm}$., but varies between 0.094 and $0.620 \mathrm{grm}$. The relationship of the sulphate-sulphuric acid $A$ to the grouped sulphuric acid $B$ in health is on an average as $10: 1$, but varies from $6: 1$ and $15: 1$. After taking phenol, also after increased putrefaction within the organism, this relationship may be essentially changed by an increased elimination of ethereal sulphuric acids. The urine of the horse is considerably richer in ethereal sulphuric acids than human urine.

Phenol- and p-Cresol-sulphuric Acid, $\mathrm{C}_{6} \mathrm{H}_{6} \cdot 0 . \mathrm{SO}_{2} \cdot \mathrm{OH}$ and $\mathrm{C}_{7} \mathrm{H}_{7} \cdot \mathrm{O} \cdot \mathrm{SO}_{2} \cdot \mathrm{OH}$. These acids are found as alkali salts in human urine, in which also orthocresol has been detected. The quantity of cresol-sulphuric acid is considerably greater than phenol-sulphuric acid. In the quantitative estimation the phenols set free from the two ethereal acids are determined together as tribromphenol. The quantity of phenol' which is separated from the ethereal sulphuric acids of the urine amounts to $17-51$ milligrammes in the twenty-four hours (MUNK). After a vegetable diet the 
amount is greater than after a meat diet. After taking carbolic acid, which is in great part converted by synthesis within the organism into phenol-ethereal sulphuric acid, pyrocatechin- and hydrochinon-sulphuric acid, and also when the amount of sulphuric acid is not sufficient to combine with the phenol forming phenylglycuronic acid, the amount of phenol and ethereal sulphuric acids in the urine is considerably increased at the expense of the sulphatesulphuric acid.

An increased elimination of phenol-sulphuric acid occurs in active putrefaction in the intestines with stoppage of the contents of the intestine, as in ileus, diffused peritonitis with atonia of the intestine, or tuberculous enteritis, but not in simple obstruction. The elimination is also increased by the absorption of the products of putrefaction from purulent wounds or abscesses. An increased elimination of phenol has been observed in a few other cases of diseased conditions of the body.

The alkali salts of phenol- and cresol-sulphuric acids crystallize in white plates, similar to mother-of-pearl, which are rather freely soluble in water. They are soluble in boiling alcohol, but only slightly soluble in cold. On boiling with dilute mineral acids they are decomposed into sulphuric acid and the corresponding phenol.

Phenol-sulphuric acids have been synthetically prepared by BAUMANN from potassium pyrosulphate and phenol- or p-cresol-potassium. For the method of their preparation from urine, which is rather complicated, the reader' is referred to other text-books. 'The quantitative estimation of these ethereal sulphuric acids is done by BAUMANN and BRIEGER by determining the amount of phenol which may be separated from the urine as tribromphenol. In this determination, when the urine is not specially rich in phenol, about one fourth of the total quantity in the twenty-four hours is used; it is acidified with concentrated hydrochloric acid-5 c. c. for every 100 c. c. of urine-and distilled until a portion of the distillate does not give the slighest reaction for phenol with MiLlon's reagent or with bromine-water. The distillate is now carefully neutralized with soda solution (which combines with the benzoic acid, etc.) and again distilled until a portion of the distillate is free from phenol, as shown by the above-mentioned reagents. This distillate is treated with bromine-water until a permanent yellow color is produced, and then allowed to stand for about twenty-four hours in the cold; the crystalline precipitate then is collected on a small 
weighed filter, washed with dilute bromine-water, dried over sulphuric acid without the use of a vacuum and weighed (331 parts tribromphenol correspond to 94 parts phenol). Paracresol is gradually converted into tribromphenol by this treatment with brominewater. 'The methods for the separate determination of the coupled sulphuric acid and the sulphate-sulphuric acid will be spoken of later in connection with the determination of the sulphuric acid of the urine.

Pyrocatechin-sulphuric Acid (and Prrocatechis). This acid was first found in horses' urine in rather large quantities by BAUMANN. It only occurs in human urine in the very smallest quantities and perhaps not constantly, but it occurs abundantly in the urine after taking phenol, pyrocatechin, or protocatechinic acid.

On an exclusive meat diet this acid does not occur in the urine, and it therefore originates from the vegetable food. It probably originates from the protocatechuic acid, which, according to PREUSSE, passes in part into the urine as pyrocatechin-sulphuric acid. 'This acid may also perhaps depend on oxidation of phenol within the organism (Baumann and Preusse).

Pyrocatechin, or o-DioxyBenzoL, $\mathrm{C}_{6} \mathrm{H}_{4}(\mathrm{OH})_{2}$, was first observed in the urine of a child (EPstein and J. MüLlen). The reducible body alcapton, first found by BöDEKER in human urine and which was considered for a long time as identical with pyrocatechin, is UROLEUCIC ACID, according to KIRK.

Pyrocatechiu crystallizes in prisms which are soluble in alcohol, ether, and water. It melts at $102^{\circ}-104^{\circ} \mathrm{C}$. and sublimes in shining plates. The watery solution becomes green, brown, and ultimately black in the presence of alkali and the oxygen of the air. If very dilute ferric chloride is treated with tartaric acid and then made alkaline with ammonia and this added to a watery solution of pyrocatechin, we obtain a violet or cherry-red liquid which becomes green by saturating with acetic acid. Pyrocatechin is precipitated by lead acetate. It reduces an ammoniacal silver solution at the ordinary temperature and reduces alkaline copper-oxide solutions with heat, but does not reduce bismuth oxide.

A urine containing pyrocatechin, if exposed to the air, especially when alkaline, quickly becomes dark and reduces alkaline copper solutious with heat. In detecting pyrocatechin in the urine it is concentrated when necessary, filtered, boiled with the addition of sulphuric acid to remove the phenols, and repeatedly shaken after cooling with ether. The ether is distilled from the several ethereal extracts, the residue neutralized with barium carbonate and shaken again with ether. The pyrocatechin which remains after evaporating the ether may be purified by recrystallization from benzol.

Hydrochinon, or P-DroxYBENZOL, $\mathrm{C}_{6}^{\prime} \mathrm{H}_{4}(\mathrm{OH})_{2}$, often occurs in the urine after the use of phenol (BAdMans and Preusse). The dark color which certain urines, so-called "carbolic urines," take in the air is due to decomposition products. Hydrochinon does not occur as a normal constituent of urine, but after the administration of hydrochinon; according to v. MERING and LEwIN, it passes into the urine of rabbits as ethereal sulphuric acid, as a decomposition product of arbutin.

Hydrochinon forms rhombical crystals which are readily soluble in water, alcohol, and ether. It melts at $169^{\circ} \mathrm{C}$. Like pyrocatechin. it easily reduces metallic oxides. It acts like pyrocatechin with alkalies, but is not precipitated with lead acetate. It is oxidized into chinon by ferric chloride and other oxidizing agents, and chinon is detected by its peculiar odor. Hydrochinonsulphuric acid is detected in the urine by the same methods as pyrocatechinsulphuric acid. 
Indoxyl-sulphuric acid, $\mathrm{C}_{8} \mathrm{H}_{7} \mathrm{NSO}_{4}$ or $\mathrm{C}_{8} \mathrm{H}_{6} \mathrm{~N}$.0. $\mathrm{SO}_{2} \cdot \mathrm{OH}$, also called URINE INDICAN, formerly called UROXANTHIN (HELLER), occurs as alkali-salt in the urine. This compound is the mothersubstance of a great part of the indigo of the urine. The quantity of indigo which can be separated from the urine is considered as a measure of the quantity of indoxyl-sulphuric acid (and indoxylglycuronic acid) contained in the urine. This amount, according to JAFFÉ, for man is 5-20 milligrammes per 24 hours. Horse's urine contains about 25 times as much indigo-forming substance as human urine.

Indoxyl-sulphuric acid is derived, as above mentioned (page 216), from indol, which is first oxidized in the body into indoxyl and then is coupled with sulphuric acid. After subcutaneous injection of indel the elimination of indican is considerably increased (JAFFÉ, BAUMANN and BRIEGER). It is also increased by the introduction of orthonitrophenylpropiolic acid in the organism of animals (G. Hoppe-SerLeR). Indol is formed by the putrefaction of proteids, and it is therefore easy to understand why the quantity of indoxyl-sulphuric acid is greater with a meat than with a vegetable diet. The putrefaction of secretions rich in albumin in the intestine explains also the occurrence of indican in the urine during starvation. Gelatine, on the contrary, does not increase the elimination of indican. An abnormally-increased elimination of indican occurs in such diseases as obstruct the small intestine, causing an increased putrefaction, thus producing an abundant formation of indol. Such an increased elimination of indican occurs on tying the small intestine of a dog, but not the large intestine (JAFFÉ).

The simple obstruction of the human colon does not increase the indican in the urine. The obstruction of the large intestine may, when it causes a considerable disturbance in the motion of the contents of the upper ileum, produce an increased elimination of indican. Like the putrefaction in the intestine, the putrefaction of proteids in other organs and tissues of the body may cause an increase in the indican of the urine.

An increased elimination of indican has been observed in many diseases, such as in ileus, cholera, acute general peritonitis, abscess, - and carcinoma of the stomach, intestinal catarrh, multiple lym- 
phoma, fetid bronchitis, ichorous pleural exudations, diabetes mellitus, and others. The increase of indican in the urine observed in consumption and inanition depends probably upon the disturbed digestion. In increased elimination of indican the elimination of phenol is also increased ; a urine rich in phenol is, on the contrary, not always rich in indican.

The potassium-salt of indoxyl-sulphuric acid which was prepared by BAUMANN and BRIEGER from the urine of a dog fed on indol, crystallizes in colorless, shining plates or leaves which are easily soluble in water but less readily in alcohol. It is split by mineral acids into sulphuric acid and indoxyl. The latter without access of air passes into a red compound, indoxyl-red, but in the presence of oxidizing reagents is converted into indigo-blue: $2 \mathrm{C}_{8} \mathrm{H}_{7} \mathrm{NO}+2 \mathrm{O}=\mathrm{C}_{16} \mathrm{H}_{10} \mathrm{~N}_{2} \mathrm{O}_{2}+2 \mathrm{H}_{2} \mathrm{O}$. The detection of indican is based on this last fact.

For the rather complicated preparation of indoxyl-sulphuric acid as potassium-salt from urine the reader is referred to other text-books. For the detection of indican in urine in ordinary cases the following method of JAFFE, which also serve as an approximate test for the quantity of indican, is sufficient.

JAFFE's Indican Test. 20 c. c. of urine are treated in a glass with 2-3 c. c. chloroform and mixed with an equal volume of concentrated hydrochloric acid. Immediately after a concentrated chloride-of-lime solution or a $\frac{1}{2} \%$ potassium-permanganate solution is added drop by drop, and after each drop the mixture is thoroughly shaken. The chloroform is gradually colored faintly or strongly blue. An excess of oxidizing reagent, especially chloride of lime, interferes with the reaction and must therefore be avoided. 'The test is repeated with somewhat varying amounts of oxidizing material until a point is found at which the maximum coloration of the chloroform takes place. From the intensity of the color the quantity of indigo is determined.

An exact determination of the amount of indigo in urine is very rarely made. The methods suggested for this purpose are very complicated, and even then they are not quite accurate; therefore the reader is referred to other text-books for their description.

Indol seems also to pass into the urine as a glycuronic acid, indoxyl-glycuronic acid (SchMIEdEBERG). Such an acid has been found in the urine of animals after the administration of the sodium-salt of o-nitrophenylpropiolic acid (G. HOPPE-SEYLER). 
Skatoxyl-sulphuric Acid, $\mathrm{C}_{9} \mathrm{H}_{9} \mathrm{NSO}_{4}$ or $\mathrm{C}_{9} \mathrm{H}_{8} \cdot \mathrm{N} \cdot \mathrm{O} \cdot \mathrm{SO}_{2} \cdot \mathrm{OH}$. The potassium-salt of this acid seems to occur generally in human urine as a chromogen, which yields a red or violet coloring matter on decomposing with strong acids, and an oxidizing reagent. This salt has been prepared by OrTo from diabetic human urine. Little is known of the quantity of this skatolchromogen, to which probably also the skatoxyl-glycuronic acid must be counted, under physiological and pathological conditions.

Skatoxyl-sulphuric acid originates from skatol formed by putrefaction in the intestine, which is coupled with sulphuric acid after oxidation into skatoxyl. That skatol introduced into the body passes partly as an ethereal sulphuric acid in to the urine has been shown by BRIEGER. Indol and skatol act differently, at least in dogs; indol producing a considerable amount of ethereal sulphuric acid, while skatol only gives a small quantity (Mester). Skatol seems partly to pass into the urine as a skatoxyl-glycuronic acid.

The potassium-salt of skatoxyl-sulphuric acid is crystalline; it dissolves in water, but with difficulty in alcohol. A watery solution becomes deep violet with ferric chloride, and red with concentrated nitric acid. The salt is decomposed by concentrated hydrochloric acid with the separation of a red precipitate. The nature of this red coloring matter produced by the decomposition of skatoxylsulphuric acid is not well known; neither is the relationship existing between this and other red coloring matters in the urine known. On distillation with zinc-dust the skatol coloring matter yields skatol.

Urines containing skatoxyl are colored dark red to violet by JAFFÉ's indican test even after the addition of hydrochloric acid; with nitric acid they are colored cherry-red, and on warming with ferric chloride and hydrochloric acid red. The coloring matter which yields skatol with zinc-dust may be removed from the urine by ether. Urines rich in skatoxyl darken when allowed to stand, and may become reddish, violet, or nearly black.

SALKowski has shown that the occurrence of skatol-carbonic acid, $\mathrm{C}_{9} \mathrm{H}_{8}$. $\mathrm{N} . \mathrm{COOH}$, in normal urine is probable. This is also a putrefaction product.

Aromatic 0xyacids. In the putrefaction of proteids in the intestine, paraoxyphenyl-acetic acid, $\mathrm{C}_{6} \mathrm{H}_{4}(\mathrm{OH}) \cdot \mathrm{CH}_{2} \mathrm{COOH}$, and 
paraoxyphenyl-propionic acid, $\mathrm{C}_{6} \mathrm{H}_{4}(\mathrm{OH}) \cdot \mathrm{C}_{2} \mathrm{H}_{4} \cdot \mathrm{COOH}$, are formed from tyrosin as intermediate steps, and these pass unchanged into the urine. They were first detected by Baumans. The quantity of these acids is usually very small. They are increased by the same circumstances as phenol, especially in acute phosphorus-poisoning, in which the increase is considerable. In acute atrophy of the liver another oxyacid, oxymandel-acid, has been found in the urine (Schultzen and Riess).

The above two acids are soluble in ether. On warming with MrLlon's reagent they give a beautiful red color. To detect the presence of these oxyacids proceed in the following way (BAUMANN): Warm the urine for a while on the water-bath with hydrochloric acid, in order to drive off the volatile phenols. After cooling shake three times with ether, and then shake the ethereal extracts with dilute soda solution, which dissolves the oxyacids, while the residue of the phenols soluble in ether remains. The alkaline solution of the oxyacids is now faintly acidified with sulphuric acid, shaken again with ether, the ether removed and allow to evaporate, the residue dissolved in a little water, and the solution tested with Miluon's reagent. The two oxyacids are best differentiated by their different melting-points. The reader is referred to other works for the method of isolating and separating these two oxyacids.

Uroleucic Acid, $\mathrm{C}_{9} \mathrm{H}_{30} \mathrm{O}_{6}$. This acid in a pure state was first prepared by Marshall from urine, but named and specially studied by KIrk. In an impure state it forms the reducible substance ALCAPTON discovered by BOEDEKER. This acid is especially found in children's urine. Such urine reduces FenLing's reagent, but not an alkaline bismuth or picric-acid solution. It is fermentable, optically inactive, and is colored deep brown in the air, especially in an alkaline solution. In these respects it differs from a urine containing sugar.

Urinary Coloring Matters and Chromogens. The yellow color of normal urine depends apparently upon several coloring matters (VIERORDT) which have not been isolated and studied. Besides these bodies, uRoBILIN sometimes occurs in fresh normal urine, but by no means always. Instead of urobilin, normal urine often contains a mother-substance of the same, a chromogen or UROBILINOGEN, from which the urobilin is gradually formed by oxidation on allowing the urine to stand exposed to the air (JAFFÉ, STokvis, DisquÉ, and others). Besides this chromogen, urine contains various other bodies from which coloring matters may be produced by the action of chemical agents. Humous substances (perhaps in part from the carbohydrates of the urine) may be formed by the action of acids (v. UDRANSZKY and HoPPE-SEYLER) without regard to the fact that such substances may sometimes originate from the reagents used, as from impure amyl-alcohol (v. Udranszky and Hoppe- 
SEYLER). To these humin bodies developed by the action of acid in normal urine when exposed to the air must be added the UROPHAIN of HELLER, the various UROMELANINS, and other bodies described by different investigators (Plos'z, Thudichum, SCHUNCK). Indigo-blue (UROGLAUCIN of HELLER, UROCYANIN, CYANURIN, and other coloring matters of older investigators) is split off from the indoxyl-sulphuric acid or indoxyl-glycuronic acid. Red coloring matters may be formed from the coupled indoxyl and skatoxyl acids, and uroHodin (Heller), urorubin (Plos'z), UROH zMATIN (HARLEY), and perhaps also UROROSEIN (NENCKI and SIEBER) probably have such an origin.

We cannot enter into too many details of the different coloring matters obtained as decomposition products from normal urine; and as the preformed physiological coloring matters of urine have not been closely studied, we can only discuss the most carefullyinvestigated urinary pigment, urobilin.

Urobilin was first prepared from urine by JAFFÉ. This coloring matter occurs in urine especially in fevers, and it is therefore designated FEBRILE UROBILIN by MACMUNN. The urobilin occurring in normal urine is somewhat different from an optical standpoint from the above, and is called NORMAL UROBILIN by MacMunn. As above stated, a mother-substance of urobilin, a UROBILINOGEN, occurs in the urine, from which urobilin is produced by the action of the air.

Many investigators claim that urobilin is identical with hydrobilirubin (MALY) and corresponds to the composition $\mathrm{C}_{32} \mathrm{H}_{40} \mathrm{~N}_{4} \mathrm{O}_{7}$. Also, that urobilin is formed by a reduction of bilirubin in the intestine. The correctness of this view is disputed by others (MacMunn, Le Nobel). According to MacMunn, hydrobilirubin and the urinary urobilin are not identical bodies, because he obtained normal urobilin by the action of peroxide of hydrogen upon a solution of hæmatin in alcohol containing sulphuric acid.

Coloring matters similar to urobilin, though not identical, have been obtained from the biliary and from the blood coloring matters. Besides the hydrobilirubin prepared by MaLY from bilirubin, STokvis obtained a choletelin from a biliary pigment, cholecyanin, by the action of zinc chloride and tincture of iodine, or by boiling 
with a little lead peroxide. This choletelin acts like urobilin, but that obtained from bilirubin by the action of nitric acid does not. "Bodies similar to urobilin have also been obtained by HoppeSEYLER, by the reduction of hæmatin and hæmoglobin with zinc and hydrochloric acid; by LE NOBEL, by treating an acid-alcoholic or alkaline solution of hæmatoporphyrin with tin or zinc; and lastly by NeNCKI and SIEBER, by treating hæmatoporphyrin with zinc and hydrochloric acid. From the observations of LE NoBeL and Nencki and Sieber it follows that these coloring matters artificially prepared from the blood-coloring matters are not identical, even though they are closely related from an optical standpoint. It must be left undecided whether these bodies are identical with each other or with the urinary urobilin, or if the observed difference is only due to a contamination with other bodies.

Because of our imperfect knowledge of the urobilin of the urine and the URobILINoIdin (this name has been given by Le Nobel to the substance artificially prepared by him) it is difficult to say anything positive in regard to the occurrence of urobilin in the urine in disease. During the absorption of large blood extravasations, as also in diseases connected with destruction of the bloodcorpuscles or of the appearance of methæmoglobin in the bloodplasma, the urine becomes dark in color, which generally depends upon an increased elimination of urobilin. The question whether it depends on an increased elimination of urinary urobilin or, as is more probable, upon the urobilinoidin produced from the bloodcoloring matters is still doubtful. In icterus the elimination of urobilin is often increased, and indeed cases occur in which the urobilin is almost the only coloring matter which can be detected in icteric urines (UROBILINICTERUS). In these cases we are probably dealing with a urobilinoid substance produced from the bile-coloring matters.

The urobilin obtained from a fever urine is, according to JAFFÉ, amorphous, red, dingy-red, or reddish yellow, according to the method of preparation. It dissolves easily in alcohol, amylalcohol, and chloroform, but less readily in ether. It is less soluble in water, but the solubility is angmented in the presence of a neutral salt. It may be precipitated from a solution saturated 
with ammonium sulphate by the addition of sulphuric acid (Мي́нY). It is soluble in alkalies and is incompletely precipitated from the alkaline solution by the addition of acid. It is partly dissolved by chloroform from an acid (watery-alcoholic) solution; alkali solutions remove the urobilin from the chloroform. The alkaline solutions of urobilin give insoluble combinations with salts of the heavy metals, such as zinc and lead. Urobilin does not give GMELIN's test for bile-pigments.

Neutral alcoholic urobilin solutions are in strong concentration brownish yellow, in great dilution yellow or rose-colored. They have a strong green fluorescence. The acid-alcoholic solutions are, according to concentration, brown, reddish yellow, or rose-red. They are not fluorescent, but show a faint absorption-band, $\gamma$, between $b$ and $F$, which borders on $F$, or in greater concentration extends over $F$. The alkaline solutions are, according to concentration, brownish yellow, yellow, or (the ammoniacal) yellowish green. If some zinc-chloride solution is added to an ammoniacal solution, it becomes red and shows a beautiful green fluorescence. This solution, as also that made alkaline with fixed alkalies, shows a darker and more sharply-defined band, $\delta$, almost midway between $b$ and $F$.

The urobilin obtained by MACMUNN according to other methods, and that obtained by JAFFE, differ from each other mainly in the following: A solution of normal urobilin becomes deeper red with soda, while the febrile urobilin becomes yellow. The band $\gamma$ of the normal urobilin disappears on the addition of alkali, while the corresponding band of the febrile moves towards the left. The ethereal solution of febrile urobilin shows two faint absorption-bands on each side of $D$ which are not to be seen in the watery solution nor in the urine. Febrile urobilin is a brownishred and the normal a yellowish-brown powder. Febrile urobilin is, according to MACMUNN, converted into normal urobilin by potassium permanganate.

In preparing urobilin from normal urine, precipitate the urine with basic lead acetate (JAFFÉ), wash the precipitate with water, dry at the ordinary temperature, then boil it with alcohol, and decompose it when cold with alcohol containing sulphuric acid. The filtered alcoholic solution is diluted with water, saturated with 
ammonia, and then treated with zinc-chloride solution. This new -precipitate is washed free from chlorine with water, boiled with alcohol, dried, dissolved in ammonia, and this solution precipitated with sugar of lead. 'This precipitate, which is washed with water and boiled with alcohol, is decomposed by alcohol containing sulphuric acid, the filtered alcoholic solution is mixed with $\frac{1}{2}$ vol. chloroform, diluted with water, and shaken repeatedly, but not too energetically. 'The urobilin is taken up by the chloroform. This last is washed once or twice with a little water and then filtered, leaving the urobilin, which is purified from a contaminating red coloring matter by means of ether.

According to JAFFE, the coloring matter can be directly precipitated from a fever urine rich in urobilin by ammonia and zinc chloride, and this precipitate treated as above. MÉHY faintly acidifies the urine with sulphuric acid (1-2 grms. per litre), then saturates with ammonium sulphate, washes the precipitate on a filter with an acidified ammonium-sulphate solution, presses the filter, and extracts the coloring matter with absolute alcohol at a gentle heat after the addition of a few drops of ammonia. MACMUNN precipitates the urine with sugar of lead and basic lead acetate, decomposes the precipitate with acidified alcohol, dilutes the solution with water, shakes with chloroform, evaporates this last, and dissolves the residue repeatedly with chloroform. The method of preparation, according to MACMUNN, is the same for both urobilins, the normal and the febrile.

The color of the acid or alkaline solution, the beautiful fluorescence of the ammoniacal solution treated with zinc chloride, and the absorption-bands of the spectrum, all serve as means of detecting urobilin. In fever urines the urobilin may be detected directly or after the addition of ammonia and zinc chloride by its spectrum. It may also be detected sometimes in normal urine directly or after the urine has stood exposed to the air until the chromogen has been converted into urobilin. If it cannot be detected by means of the spectroscope, then the urine may be treated with a mineral acid and shaken with ether. The ethereal solution may be, directly or after concentration, tested with the spectroscope. It is often better to dissolve the residue, after the evaporation of the ether, in absolute alcohol, and use this for the spectroscopic investigation. According to SALKOWSKI, the urobilin may be directly extracted by gently shaking with ether free from alcohol. If the urobilin cannot be detected by the above-described methods, then precipitate the urine with basic lead acetate, decompose the precipitate with acidified alcohol, test this solution or extract the coloring matter by diluting with water and shaking with chloroform.

The urochrom (THUDICHUM) seems to be a mixture of several bodies. Uroerythrin is that coloring matter which often colors the urinary sediment (sedimentum lateritium) beautifully red. It occurs especially in fevers and other diseases, but it is not found in the urine of perfectly bealthy persons. 
Volatile fatty acids, such as formic acid, acetic acid, and perbaps also butyric acid, occur under normal conditions in human urine (v. J in that of dogs and herbivora (ScHo'TTEN). The acids poorest in carbou, formic acid, and acetic acid are more constant in the body than those richer in carbon, and therefore the relatively greater part pass unchanged into the urine (SchotTen). Normal human urine contains besides these bodies others which yield acetic acid when oxidized by potassium dichromate and sulphuric acid (v. JAKSCH). The quantity of volatile fatty acids in normal urine is, according to v. JAKSCH, $0.008-0.009$ grm. per 24 hours, and according to v. RoBITANSKY, $0.054 \mathrm{grm}$. The quantity is increased by exclusive farinaceous food, also in fever and in certain diseases of the liver (v. $\mathrm{JAKSCH}_{\mathrm{A}}$. It is also increased in leucæmia and in many cases of diabetes ( $\mathrm{v}$. J $\mathrm{AKSCH}$ ). Large amounts of volatile fatty acids are produced in alkaline fcrmentation of the urine, and the quantity is 15-16 times as large as in normal urine (SALKowskI).

Paralactic Acid. It is claimed that this acid occurs in the urine of healthy persons after very fatiguing marches (Colasanti and Moscatelli). It is found in larger amounts in the urine in acute phosphorus poisoning or acute yellow atrophy of the liver (SchuLtzen and RIESs), also in osteomalacia (Mörs and Muck). After the extirpation of the liver of birds it is found in large quantities in their urine (MINkowsKI). Glycero-phosphoric acid occurs as traces in the urine, and it is probably a decomposition product of lecithin. The occurrence of succinic acid in normal urine is the subject of discussion.

Carbohydrates and Reducing Substances in the Urine. The occurrence of grape-sugar as traces in normal urine is highly probable, as the investigations of BRÜCKE, ABELES and v. UDRANSZKI show. The last has also shown the habitual occurrence of carbohydrates in the urine, and their presence has been positively proved by the investigations of BAUMANN and WEDENSKI. Besides this, the urine contains traces of a carbohydrate similar to dextrin (animal gum) (LANDWEHR, WEDENSKI). Besides traces of sugar and the previously-mentioned reducing substances, uric acid and creatinin, the urine contains still other reducing substances. These last are probably (FLÜCKIGER) coupled combinations of glycuronic acid, $\mathrm{C}_{6} \mathrm{H}_{10} \mathrm{O}_{7}$, which closely resembles sugar. The reducing power of normal urine corresponds according to FLÜCKIGER to 1.5-2.5 p. m. grape-sugar, according to SALKowSKI 4.08, according to MUNK an average of 3.0 , and according to WORM MÜLLER about 4.0 p. m.

Glycuronic Acid, $\mathrm{C}_{6} \mathrm{H}_{10} \mathrm{O}_{7}$ or $\mathrm{CHO} .(\mathrm{CH} . \mathrm{OH})_{4} . \mathrm{COOH}$. This acid may be converted into saccharic acid, $\mathrm{C}_{6} \mathrm{H}_{10} \mathrm{O}_{8}$, by the action of bromine (THIERFELDER), and it seems to occupy an intermediate position between this acid and gluconic acid, $\mathrm{C}_{6} \mathrm{H}_{12} \mathrm{O}_{7}$, obtained by the oxidation of glucose or cane-sugar with chlorine or bromine. Glycuronic acid probably only occurs normally in very small quanti- 
ties in human urine as coupled combinations with indoxyl, skatoxyl, and phenols. It is also found in the artists' color "jaune indien," which contains the magnesium-salt of euxanthonic acid. On heating this acid with water to $120^{\circ}-125^{\circ} \mathrm{C}$. it splits into euxanthin and glycuronic acid.

This acid may pass into the urine in larger quantities as coupled glycuronic acids after the administration of various medicines or other substances (see below). Thus after the administration of chloral hydrate, naphthalin, camphor, and turpentine, respectively, urochloralic acid, naphthol-glycuronic acid, campho-glycuronic acid, and terpen-glycuronic acid appear in the urine. The conpled glycuronic acids turn the plane of polarization to the left, while glycuronic acid itself is dextro-gyrate. With the absorption of water they may split into glycuronic acid and other coupled bodies. A few of the coupled glycuronic acids, such as the urochloralic acid, reduce copper oxide and certain other metallic oxides in alkaline solution, and therefore they may interfere with the detection of sugar in the urine.

Glycuronic acid is not crystalline, but is obtained only as a syrup. It dissolves in alcohol and is easily soluble in water. If the watery solution is boiled for an hour, the acid is in part (20\%) converted into the anhydride GLYCURON, $\mathrm{C}_{6} \mathrm{H}_{8} \mathrm{O}_{6}$, which is crystalline, soluble in water but insoluble in alcohol. The potassium-salt of this acid crystallizes in fine needles. The neutral barium-salt is amorphous, soluble in water, but is precipitated by alcohol. If a concentrated solution of the acid is saturated with barium hydrate, the basic barium-salt separates. The neutral lead-salt is soluble in water, but the basic salt is, on the contrary, insoluble. The acid is dextrogyrate, reduces copper, silver, and bismuth salts. It gives a crystalline combination with phenylhydrazin.

Glycuronic acid may be prepared from urochloralic acid or campho-glycuronic acid by boiling with a mineral acid. It may be prepared more easily by heating euxanthonic acid with water in PAPIN's digestor to $120^{\circ}-125^{\circ} \mathrm{C}$. for an hour and evaporating the watery solution at $+40^{\circ} \mathrm{C}$. The anhydride which crystallizes gradually is removed, the mother-liquor diluted with water and boiled for a time to convert a second portion of acid into anhydride, and then evaporated at about $+40^{\circ} \mathrm{C}$. This is continued until nearly 
all the acid is converted into anhydride. The anhydride may then be further purified.

Organic combinations containing sulphur of unknown kind, which may in small part consist of sulphocyanides, 0.04 (GschridLEN) - $0.11 \mathrm{p}$. m. (J. MUNK), cystin, or bodies related to it, and protein bodies, are found in human as well as in animal urines. The sulphur of these mostly unknown combinations has been called " neutral," to differentiate it from the "acid " sulphur of the sulphate and ethereal-sulphuric acids (SALKowski). The neutral sulphur in normal urine as determined by SALKowskI is 15\%, by STADTHAGEN 13.3-14.5\%, and by LÉPINE 20\% of the total sulphur. An increase in the quantity of neutral sulphur has been observed in icterus (LÉPINE), and in cystinuria (STADTHAGEN).

The total quantity of sulphur in the urine is determined by fusing the solid urinary residue with saltpetre and caustic alkali. The quantity of neutral sulphur is determined as the difference between the total sulphur and the sulphur of the sulphate and ethereal sulphuric acids.

Sulphuretted hydrogen occurs in urine only under abnormal conditions or as a decomposition product. Sulphuretted hydrogen may be produced from the neutral sulphur of the organic substances of the urine by the action of certain bacteria (Fr. MÜLLER, SALKOWSKI). Other investigators (ROSENHEIM and Gutzmans) have given hyposulphites as the source of the sulphuretted hydrogen. The occurrence of hyposulphites in normal human urine, which is asserted by HEFFTER, is disputed by SALKowski. In cat's and dog's urine the hyposulphites are, on the contrary, constant.

Organic combinations containing phosphorus (glycero-phosphoric acid, etc.), which yield phosphoric acid on fusing with saltpetre and caustic alkali, are also found in urine (ZüLZER, LÉPINE, EYroNNET, and AUBERT).

Enaymes of various kinds have been isolated from the urine. Among these we may mention pepsin (BRÜCKE and others), diastatic enzyme (CoHNHEIM and others), and rennet (Grützner, Holovtschiner, Helwes). The occurrence of trypsin in the urine is doubtful.

Substances similar to mucin (nucleoalbumin ?) from the urinary passages and the bladder are generally present in the urine, though in very small amounts. According to several investigators (Leube, Hofmeister, PosNer), normal human urine also contains traces of albumin.

Plomaines and leucomaines or poisonous substances of an unknown kind, which are often described as alkaloidal substances, occur in normal urine (Podchet, Bouchard, AdUcCo, and others). Under pathological conditions the quantity of these substances may be increased (BOUCHARD, LEPINE and GUERIN, Villiers, and others). Within the last few years the poisonous properties of urine have been the subject of more thorough investigation, especially by Bouchand. He found that the night urine is less poisonous than the day urine, and that the poisonous constituents of the day and night urines have not the same action.

BAUMANN and $v$. UDRANSzKY have shown that ptomaines may occur in the urine under pathological conditions. They demonstrated the presence of the two ptomaines discovered and first isolated by BRIEGER-putrescine, $\mathrm{C}_{4} \mathrm{H}_{12} \mathrm{~N}_{2}$ (tetramethylendiamin), and cadaverin, $\mathrm{C}_{6} \mathrm{H}_{14} \mathrm{~N}_{3}$ (pentamethylendiamin)-in the urine of a patient suffering from cystinuria and catarrh of the bladder. Brieger, v. Udranszky and BaUmanN and StadThageN have shown that not only these but other diamins occur under physiological conditions. The occurrence in normal urine of any "urine poison" is denied by certain investigators, such as Feltz and RitTer and Stadthagen. The poisonous action of the urine, according to them, is due in great part to the potassium salts. 
Many substances have been observed in animal urine which are not found in human urine. To these belong: cynurenic acid, $\mathrm{C}_{2} \mathrm{H}_{7} \mathrm{NO}_{3}$, occurring in dog's urine and which is an oxychinolin carbonic acid ; urocanic acid (JAFFÉ), first found in dog's urine; damaluric acid and damolic acid (according to SchotTen, probably a mixture of benzoic acid with volatile fatty acids), obtained by the distillation of cow's urine; and lastly the lithuric acid, found in the urinary concrements of certain animals.

\section{Inorganic Constituents of Urine.}

Chlorides. The chlorine occurring in urine is undoubtedly combined with the bases contained in this excretion; the chief part is combined with sodium. In accordance with this, the amount of chlorine in the urine is generally expressed as $\mathrm{NaCl}$.

The amount of chlorine combinations in the urine is subject to considerable variation. In general the quantity for a healthy grown person on a mixed diet is $10-15$ grms. $\mathrm{NaCl}$ per 24 hours. The quantity of common salt in the urine depends chiefly upon the quantity of salt in the food, with which the elimination of chlorine increases and decreases. Abundant drinking of water also increases the elimination of chlorine, which is greater during activity than during rest (during night). Certain organic chlorine combinations, such as chloroform, may increase the elimination of inorganic chlorides by the urine (ZeLler, MYLiUS, KaST).

In diarrhoa, in quick formation of large transudations and exudations, also in specially-marked cases of acute febrile diseases, at the time of the crisis, the elimination of common salt is significantly decreased. The elimination is abnormally increased in the first days after the crisis and during the absorption of extensive exudations. A diminished elimination of chlorine is found in acute and chronic diseases of the kidneys accompanied with albuminuria. In chronic diseases the elimination of chlorine in general keeps pace with the nutritive condition of the body and the activity of the secretion of the urine. As a rule the chlorine is diminished in chronic diseases.

The quantitative estimation of chlorine in urine is most simply performed by titration with silver-nitrate solution. The urine must not contain either albumin (which if present must be removed by coagulation) or iodine or bromine compounds.

In the presence of bromides or iodides evaporate a measured quantity of the urine to dryness, fuse the residue with saltpetre and soda, dissolve the 
fused mass in water, and remove the iodine or bromide by the addition of dilute sulphuric acid and some nitrite, and thoroughly shake with carbon disulphide. The liquid thus obtained may now be titrated with silver nitrate according to VoLHaRD's method. The quantity of bromide or iodide is calculated as the difference between the quantity of silver-nitrate solution used for the titration of the solution of the fused mass and the quantity used for the corresponding volume of the original urine.

The otherwise beautiful titration method of MoHR, according to which we titrate with silver nitrate in neutral liquids, using neutral potassium chromate as an indicator, cannot be used directly on the urine in careful work. Organic urinary constituents are also precipitated by the silver-salt, and the results are therefore somewhat high for the chlorine. If we wish to use this method, the organic urinary constituents must first be destroyed. For this purpose evaporate to dryness 5-10 c.c. of the urine, after the addition of $1 \mathrm{grm}$. of chlorine-free soda and 1-2 grms. chlorine-free saltpetre, and carefully fuse. The mass is dissolved in water, acidified faintly with nitric acid, and then nentralized exactly with pure lime carbonate. This neutral solution is used for the titration. The silver-nitrate solution may be a $\frac{\mathrm{N}}{10}$ solution. It is often made of such a strength that each c. c. corresponds to $0.006 \mathrm{grm}$. $\mathrm{Cl}$ or $0.01 \mathrm{grm}$. NaCl. This last-mentioned solution contains 29.075 grms. $\mathrm{AgNO}_{3}$ in 1 litre.

VoLHARD's METHOD. Instead of the preceding determination, VolHARD's method, which can be performed directly on the urine, may be employed. The principle is as follows: All the chlorine from the urine acidified with nitric acid is precipitated by an excess of silver nitrate, filtered, and in a measured part the quantity of silver added in excess is determined by means of a sulphocyanide solution. This excess of silver is completely precipitated by the sulphocyanide, and a solution of some ferric salt, which, as is well known, gives a blood-red reaction with the smallest quantity of sulphocyanide, is used as an indicator.

We require the following solutions for this titration : 1. A silvernitrate solution which contains $29.0 \% 5$ grms. $\mathrm{AgNO}_{3}$ per litre and of which each c. c. corresponds to $0.01 \mathrm{grm}$. NaCl or $0.00607 \mathrm{grm}$. $\mathrm{Cl}$; 2. A saturated solution at the ordinary temperature of chlorine-free iron alum or ferric sulphate; 3. Chlorine-free nitric acid of a specific gravity of $1.2 ; 4$. A potassium sulphocyanide solution which contains 8.3 grms. KCNS per litre, and of which 2 c. c. corresponds to $1 \mathrm{c}$. c. of the silver-nitrate solution.

About 9 grms. of potassium sulphocyanide are dissolved in water and diluted to one litre. The amount of KCNS contained in this solution is determined by the silver-nitrate solution in the following way: Measure exactly $10 \mathrm{c}$. c. of the silver solution and treat with $5 \mathrm{c}$. c. of nitric acid and 
1-2 c. c. of the ferric-salt solution, and dilute with water to about $100 \mathrm{c.c}$. Now the sulphocyanide solution is added from a burette, constantly stirring, until a permanent faint red coloration of the liquid takes place. The amount of sulphocyanide found in the solution by this means indicates how much it must be diluted to be of the proper strength. Titrate once more with $10 \mathrm{c}$. $\mathrm{c}$. $\mathrm{AgNO}_{3}$ solution and correct the sulphocyanide solution by the careful addition of water until $20 \mathrm{c}$. c. exactly correspond to $10 \mathrm{c}$. c. of the silver solution.

The determination of the chlorine in the urine is performed by this method in the following way: Exactly $10 \mathrm{c}$. c. of the urine are placed in a flask which has a mark corresponding to 100 c. c. ; 5 c. c. nitric acid are added; dilute with about 50 c. c. water, and then allow exactly $20 \mathrm{c}$. c. of the silver-nitrate solution to flow in. Close the flask with the thumb and shake well, slide off the thumb and wash it with distilled water into the flask, and fill the flask to the 100-c. c. mark with distilled water. Close again with the thumb, carefully mix by shaking, and filter through a dry filter. Measure off 50 c. c. of the filtrate by means of a pipette, add 3 c. c. ferric-salt solution, and allow the sulphocyanide solution to flow in nntil the liquid above the precipitate has a permanent red color. The calculation is very simple. For example, if $4.6 \mathrm{c}$. c. of the sulphocyanide solution were necessary to produce the final reaction, then for 100 c. c. of the filtrate ( $=10$ c. c. urine) 9.2 c. c. of this solution are necessary. 9.2 c. c. of the sulphocyanide solution corresponds to $4.6 \mathrm{c}$. c. of the silver solution, and since $20-4.6$ $=15.4$ c. c. of the silver solution were necessary to completely precipitate the chlorides in 10 c. c. of the urine, then 10 c. c. contain $0.154 \mathrm{grm}$. NaCl. The amount of sodium chloride in the urine is therefore $1.54 \%$ or $15.4 \%$. If we always use 10 c. c. for the determination, and always 20 c.c. $\mathrm{AgNO}_{3}$, and dilute with water to 100 c. c., we find the amount of $\mathrm{NaCl}$ in 1000 parts of the urine by subtracting the number of c. c. of sulphocyanide (R) required with 50 c. c. of the filtrate from 20 . The quantity of $\mathrm{NaCl} \mathrm{p.} \mathrm{m.} \mathrm{is}$ therefore under these circumstances $=20-R$, and the percentage of $\mathrm{NaCl}=\frac{20-\mathrm{R}}{10}$.

The approximate estimation of chlorine in the urine (which must be free from albumin) is made by strongly acidifying with nitric acid and then adding to it, drop by drop, a concentrated silver-nitrate solution $(1: 8)$. In a normal amount of chlorides the drop sinks to the bottom as a rather compact cheesy lump. In diminished amounts of chlorides the precipitate is less compact and coherent, and in the presence of very little chlorine a fine whits precipitate or only a cloudiness or opalescence is obtained.

Phosphates. Phosphoric acid occurs in acid urines partly as double-, $\mathrm{MH}_{2} \mathrm{PO}_{4}$, and partly as simple-acid, $\mathrm{M}_{2} \mathrm{HPO}_{4}$, phosphates, 
both of which are found in acid urines at the same time. OTr found that on an average $60 \%$ of the total phosphoric acid was double- and $40 \%$ was simple-acid phosphate. The total quantity of phosphoric acid is very variable and depends on the kind and the quantity of food. The average amount of $\mathrm{P}_{2} \mathrm{O}_{5}$ is in round numbers 2.5 grms., with a variation of 1-5 grms., per 24 hours. The phosphoric acid of the urine originates to a small extent from the burning of organic compounds, nuclein, protagon and lecithin, within the organism. The greater part originates from the phosphates of the food, and the quantity of eliminated phosphoric acid is greater when the food is rich in alkali phosphates in proportion to the quantity of lime and magnesia phosphates. If the food contains much lime and magnesia, large amounts of earthy phosphates are eliminated by the excrements; and even though the food contains considerable amounts of phosphoric acid in these cases, the quantity of phosphoric acid in the urine is small. Such a condition is found in the herbivora, whose urine is habitually poor in phosphates. The extent of the elimination of phosphoric acid by the urine depends not only upon the total quantity of phosphoric acid in the food, but also upon the relative amounts of alkaline earths and the alkali salts in the food.

From the transformation of tissues rich in proteid or of phosphorized nerve-substance in the body we might perhaps expect an equal relation between the nitrogen and the phosphoric acid in the urine. Many investigations have been made upon this subject by ZUelzer, Strübing, and Edlefssen; but as all the conditions which affect the elimination of phosphoric acid are not yet sufficiently known, it is difficult to draw any definite conclusions from the observations thus far made.

Little is known in regard to the elimination of phosphoric acid in disease. In febrile diseases the amount of phosphoric acid is considerably decreased as compared with the urea (ZUELZER). In diseases of the kidneys the activity of these organs in eliminating the phosphates is considerably diminished (FleIscher). In meningitis, on the contrary, a marked increase in the phosphates is observed in the urine. Teissièn has described a special form of polyuria, in which abundant quantities of earthy phosphates, 10-20-30 grms. per 24 hours, were eliminated. This polyuria was 
called phosphate diabetes by Teissièr. The statements in regard to the amount of phosphate in the urine in rachitis and in osteomalacia are somewhat contradictory. A diminished elimination of phosphoric acid has been observed by STokvis in arthritis.

Quantitative estimation of phosphoric acid in the urine. This estimation is most simply performed by titrating with a solution of uranium acetate. The principle of the titration is as follows: A warm solution of phosphates containing free acetic acid gives a whitish-yellow precipitate of uranium phosphate with a solution of a uranium salt. This precipitate is insoluble in acetic acid, but dissolves in mineral acids, and on this account we always add in titrating a certain quantity of sodium acetate solution. Potassium ferrocyanide is used as the indicator, which does not act on the uranium-phosphate precipitate, but gives a reddish-brown precipitate or coloration in the presence of the smallest amount of soluble uranium salt. The solutions necessary for the titration are: 1 . A solution of a uranium salt of which each c. c. corresponds to 0.005 grm. $\mathrm{P}_{2} \mathrm{O}_{5}$ and which contains 20.3 grms. uranium oxide per litre. 20 c. c. of this solution corresponds to $0.100 \mathrm{grm}$. $\mathrm{P}_{2} \mathrm{O}_{5}$. 2. A solution of sodium acetate; 3 . A freshly-prepared solution of potassium ferrocyanide.

The uranium solution is prepared from uranium nitrate or acetate. Dissolve about 35 grms. uranium acetate in water, add some acetic acid to facilitate solution, and dilute to one litre. The strength of this solution is determined by titrating with a solution of sodium phosphate of known strength $(10.085$ grms. crystallized salt in 1 litre, which corresponds to 0.100 grm. $\mathrm{P}_{2} \mathrm{O}_{6}$ in 50 c. c.). Proceed in the same way as in the titration of the urine (see below) and correct the solution by diluting with water, and titrate again until 20 c.c. of the uranium solution correspond exactly to $50 \mathrm{c}$. c. of the above phosphate solution.

The sodium-acetate solution should contain 10 grms. sodium acetate and 10 grms. conc. acetic acid in 100 c. c. For each titration 5 c. c. of this solution is used with 50 c. c. of the urine.

In performing the titration, mix $50 \mathrm{c}$. c. of filtered urine in a beaker with $5 \mathrm{c}$. c. of the sodium acetate, cover the beaker with a watch-glass, and warm over the water-bath. Then allow the uranium solution to flow in from a burette, and, when the precipitate does not seem to increase, place a drop of the mixture on a porcelain plate with a drop of the potassium-ferrocyanide solution. If the amount of uranium solution employed is not sufficient; the color remains pale yellow and more uranium solution must be added; but as soon as the slightest excess of uranium has been used, the color becomes faint reddish brown. When this point has been obtained, warm the solution again and add another drop. If the color remains of the same intensity, the titration is ended; but if 
the color varies, add more uranium solution, drop by drop, until a permanent coloration is obtained after warming, and now repeat the test with another $50 \mathrm{c.c}$. of the urine. The calculation is so simple that it is unnecessary to give an example.

In the above manner we determine the total quantity of phosphoric acid in the urine. If we wish to know the phosphoric acid combined with alkaline earths or with alkalies, we first determine the total phosphoric acid in a portion of the urine and then remove the earthy phosphates in another portion by ammonia. The precipitate is collected on a filter, washed, transferred in a beaker with water, treated with acetic acid, and dissolved by warming. This solution is now diluted to 50 c. c. with water, and 5 c. c. sodiumacetate solution added, and titrated with uranium solution. The difference between the two determinations gives the quantity of phosphoric acid combined with the alkalies.

Sulphates. The sulphuric acid of the urine originates only to a very small extent from the. sulphates of the food. A disproportionally greater part is formed by the burning of the proteids containing sulphur within the body, and it is chiefly this formation of sulphuric acid from the proteids which gives rise to the previouslymentioned excess of acids over the bases in the urine. The quantity of sulphuric acid eliminated by the urine amounts to about 2.5 grms. $\mathrm{H}_{2} \mathrm{SO}_{4}$ per twenty-four hours. As the sulphuric acid chiefly originates from the proteids, it follows that the elimination of sulphuric acid and the elimination of nitrogen are nearly parallel, and the relationship $\mathrm{N}: \mathrm{H}_{2} \mathrm{SO}_{4}$ is about $5: 1$. Sulphuric acid occurs in the urine partly preformed (sulphate-sulphuric acid) and partly as ethereal sulphuric acid.

The quantity of total sulphuric acrd is determined in the following way, but at the same time the precautions described in other works must be observed: 100 c. c. of filtered urine are treated with $5 \mathrm{c}$. c. concentrated hydrochloric acid and boiled for fifteen minutes. While boiling precipitate with 2 c. c. of a saturated $\mathrm{BaCl}_{2}$ solution and warm for a little while until the barium sulphate has completely settled. The precipitate must then be washed with water or with alcohol and ether (to remove resinous substances) and then treated according to the usual method.

The separate determination of the sulphate-sulphuric acid and the ethereal sulphuric acid may be done, according to BAUMANN's method, by first precipitating the sulphate-sulphuric acid from the urine acidified with acetic acid, by $\mathrm{BaCl}_{3}$, and then decomposing the ethereal sulphuric acid by boiling after the addition of hydro- 
chloric acid, and then determining the sulphuric acid set free as barium sulphate. A still better method is the following suggested , by SALKOWSKI :

200 c. c. of urine are precipitated by an equal volume of a barium solution which consists of 2 vols. barium hydrate and 1 vol. barium-chloride solution, both saturated at the ordinary temperature. Filter through a dry filter, measure off 100 c. c. of the filtrate which contains only the ethereal sulphuric acid, treat with 10 c. c. hydrochloric acid of a specific gravity 1.12 , boil for fifteen minutes, and then warm on the water-bath until the precipitate has completely settled and the supernatant liquid is entirely clear. Wash with warm water and with alcohol and ether and proceed according to the generally-prescribed method. The difference between the ethereal sulphuric acid found and the total quantity of sulphuric acid as determined in a special portion of urine is considered as the quantity of sulphate-sulphuric acid.

Nitrates occur in small quantities in human urine (SсHöNBEIN), and they probably originate from the drinking-water and the food. According to WEYL and CITRON, the quantity of nitrates is smallest with a meat diet and greatest with vegetable food. The average amount is about 42.5 milligrammes per litre.

Potassium and Sodium. The quantity of these bodies eliminated by the urine by a healthy full-grown person on a mixed diet is, according to SALKowsKI, 3-4 grms. $\mathrm{K}_{2} \mathrm{O}$ and 5-7.5 grms. $\mathrm{Na}_{2} \mathrm{O}$. The proportion of $\mathrm{K}$ to $\mathrm{Na}$ is ordinarily as $3: 5$. The amount depends above all upon the food. In starvation the urine may become richer in potassium than in sodium, which results from the lack of common salt and the destruction of tissue rich in potassium. The quantity of potassium may be relatively increased during fever, while after the crisis the reverse is the case.

The quantitative estimation of these bodies is performed by the gravimetric methods as described in analytical works.

Ammonia. Some ammonia is habitually found in human urine and in that of carnivora. This ammonia may represent, as above stated (page 338), on the formation of urea from ammonia, the small amount of ammonia which, because of the excess of acids formed by the combustion, as compared to the fixed alkalies, is united with such acids and in this way excluded from the synthesis to urea. These views are confirmed by the observations of CoRANDA, who found that the elimination of ammonia was smaller on a vegetable diet and larger on a rich meat diet than when on a 
mixed diet. On a mixed diet the arerage amount of ammonia eliminated by the urine is about $0.7 \mathrm{grm} . \mathrm{NH}_{\mathrm{s}}$ per twenty-four hours (NEUBAUER).

The quantity of ammonia in human urine and that of carnivora is increased by the introduction of mineral salts and also in diseases in which an increased formation of acid takes place due to an increased metabolism of proteids. This is the case in fevers and diabetes. In the last-mentioned disease an organic acid, $\beta$-oxybutyric acid, is produced (Minkowski, KüLz, StadelmaNN) which passes into the urine combined with ammonia. In diseases of the liver, as in acute yellow atrophy and interstitial hepatitis (HALLERVORDEN, STADELMANN), the formation of urea may decrease and the elimination of ammonia increase. In these cases the proportion of $\mathrm{NH}_{3}: \stackrel{+}{U}^{+}$, which, according to STADELMANN, is normally $2.8: 100$, may be changed. The same may also be observed in acute phosphorus-poisoning. In such a case K. MörNer found the relation $5.2: 100$.

The detection and quantitative estimation of ammonia is performed according to the method suggested by Schlösing. The principle of this method is that the ammonia from a measured amount of urine is set free by lime-water in a closed vessel and absorbed by a measured amount of $\frac{\mathrm{N}}{10}$ sulphuric acid. After the absorption of the ammonia the quantity is determined by titrating the remaining free sulphuric acid with a $\frac{\mathrm{N}}{10}$ caustic alkali. This method gives low results, and in exact work we must proceed as suggested by BoHLand (PFLÜGER's Archiv, vol 43, page 32). Other methods have been suggested by SchMIEDEBERG and by LATSCHENBERGER.

Calcium and magnesium occur in the urine for the most part as phosphates. The quantity of earthy phosphates eliminated daily is somewhat more than $\mathbf{1}$ gr., and of this amount $\frac{2}{3}$ is magnesium and $\frac{1}{3}$ calcium phosphate. In acid urines the simple- as well as the double-acid earthy phosphates are found, and the solubility of the first, among which the calcium-salt, $\mathrm{CaHPO}_{4}$, is especially insoluble, is particularly augmented by the psesence of double-acid alkali phosphate and sodium chloride in the urine (Отт). The quan- 
tity of alkaline earths in the urine depends on the composition of the food. Nothing is known with positiveness in regard to the constant and regular change in the elimination of these substances in disease.

The quantity of calcium and magnesium is determined according to the ordinary well-known methods.

Iron occurs in the urine only in small amounts, and, as it seems, not as a salt, but as an organic combination-part perhaps as pigment or chromogen (Konkel, Gracosa) and part in other forms. According to Magnier, the quantity of iron in 1 litre of urine is $3-11$ milligrms. According to GotTLIEB, the elimiuation of iron by the healthy human urine amounts to 2.59 milligrms. per day. Iron-salts introduced into the intestine do not pass into the urine at all, or only in very small amounts. The quantity of silicic acid, according to the ordinary statements, amounts to about $0.03 \mathrm{p} . \mathrm{m}$. Traces of hydrogen peroxide also occur in the urine.

The gases of the urine are carbon dioxide, nitrogen, and traces of oxygen. The quantity of nitrogen is not quite 1 vol. per cent. The carbon dioxide varies considerably. In acid urines it is hardly one half as great as in neutral or alkaline urines.

\section{The Amount and Quantitative Composition of Urine.}

A direct participation of the kidney substance in the formation of the urinary constituents is proved at least for hippuric acid. It is hardly to be doubted that the kidneys as well as the tissues generally have a certain part to play in the formation of other urinary constituents, but their chief task consists in separating and removing urinary constituents dissolved in the blood which have been taken up by it from other organs and tissues.

It has been shown by the experiments of numerous investigators, Heidenhain, v. Wittich, Nussbaum, Neisser, UstimoWITSCH, J. MUNK, and others, that the elimination of water and the remaining urinary constituents is not alone produced by simple diffusion and filtration. It is generally conceded that the processes of urinary secretion depend essentially upon a specific activity of the cells of the epithelium of the urinary passages, besides which also processes of filtration and diffusion take part. The process of the secretion of urine in man and the higher animals is generally considered to proceed chiefly as follows: The water together with 
a small amount of the salts passes through the glomeruli Malpighii, while the chief part of the solids is secreted by the epithelium of the urinary passages. A secretion of solids without a simultaneous secretion of water is not possible, and therefore a part of the water must be secreted by the epithelium-cells of the urinary passages. The passage of the chief part of the water through the glomeruli is rather generally considered as a filtration due to bloodpressure. According to Heidenhain, the thin cell-layers of the glomeruli have a secretory action.

The amount and the composition of urine is liable to great variation. Those circumstances which under physiological conditions exercise a great influence are the following: the blood-pressure, and the rapidity of the blood-current in the glomeruli; the quantity of urinary constituents, especially water in the blood; and lastly, the condition of the secretory glandular elements. Above all, the amount and concentration of the urine depend on the elimination of water. That this last may vary with the amount of water in the blood, with changed blood-pressure, and with circulatory conditions is evident; but under ordinary circumstances the amount of water eliminated by the kidneys depends essentially upon the quantity of water which is brought to them by the blood, or which leaves the body by other exits. The elimination of urine is increased by abundant drinking or if the amount of water removed in other ways is lessened; but it is decreased by a diminished introduction of water, or by a greater loss of water in other ways. Ordinarily in man just as much water is eliminated by the kidneys as by the skin, lungs, and intestines together. At lower temperatures and in moist air, since under these conditions the elimination of water by the skin is diminished, the elimination of urine may be considerably increased. Diminished introduction of water or diminished secretion of water-as in violent diarrhœa, violent vomiting, or abundant perspiration-greatly diminishes the elimination of urine. For example, the urine may sink as low as $500-400$ c. c. per day in intense summer-heat, while after copious draughts of water the elimination of 3000 c. c. of urine has been observed during the same time. The average quantity of urine secreted in the course of 24 hours for healthy grown men is 1500 c. c., and for women 1200 c. c. The minimum secretion occurs 
during the night between $2-4$ o'clock; the maximum, in the first hours after awakening and from 1-2 hours after a meal.

The quantity of solids secreted in the course of 24 hours is rather constant even though the quantity of urine may vary, and it is more constant when the manner of living is regular. Therefore the percentage of solids in the urine is naturally in an inverse proportion to the quantity of urine. The average quantity of solids per 24 hours is calculated as 60 grms. The quantity may be calculated with approximate accuracy by means of the specific gravity, if the second and third decimals of the specific gravity be multiplied by HäsER's coefficient 2.33. The product gives the amount of solids in 1000 c.c. of urine, and if the quantity of urine eliminated in the 24 hours be measured, the quantity of solids in the 24 hours may be easily calculated. For example, 1050 c.c. of urine of a sp. gr. 1.021 was eliminated in the 24 hours; therefore the quantity of solids eliminated is $21 \times 2.33=48.9$, and $\frac{48.9 \times 1050}{1000}=51.35$ grms. The urine in this case contained 48.9 p. m. solids and 51.35 grms. in the daily secretion.

Those bodies which, under physiological conditions, affect the density of the urine are common salt and urea. The specific gravity of the first is 2.15 and the last only 1.32 , so it is easy to understand, when the relative proportion of these two bodies essentially deviates from the normal, why the above calculation from the specific gravity is not exact. The same is the case when a urine poor in a normal constituent contains large amounts of foreign bodies, such as albumin or sugar.

As above stated, the percentage of solids in the urine generally decreases with a greater elimination, and an abundant secretion (polyuria) has therefore, as a rule, a lower specific gravity. An important exception to this rule is observed in urine containing sugar (diabetes mellitus), in which there is a very abundant secretion of a very high specific gravity due to the sugar. In cases where very little urine is secreted (oliguria), as when the perspiration is profuse, in diarrhoea, and in fevers, the specific gravity is as a rule high, the percentage of solids high, and the color dark. Sometimes, as, for example, in certain cases of albuminuria, the 
reverse of the above may be observed even though the urine has a low specific gravity, a pale color, and is poor in solids.

It is difficult to give a tabular view of the composition of urine, on account of its variation. For certain purposes the following table may be of some value, but it must not be overlooked that the results are not given for 1000 parts of urine, but only approximate figures for the amounts of the most important constituents which are eliminated in the course of 24 hours in a quantity of 1500 c.c.

Daily amount of solids $=60$ grms.

Organic constituents..... $=35$ grms.

Urea................... 30

Uric acid................ 0.7 “

Creatinin .............. 1.0 “

Hippuric acid........... 0.7 “"

Remaining organic bodies 2.6

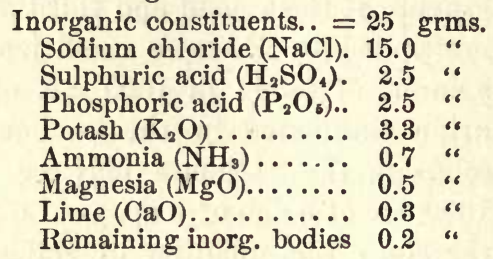

Urine contains on an average 40 p. m. solids. The amount of urea is about $20 \mathrm{p} . \mathrm{m}$. and common salt about $10 \mathrm{p}$. m.

\section{Casual Urinary Constituents.}

The casual appearance in the urine of medicines or of urinary constituents resulting from the introduction of foreign substances into the organism is of practical importance, "because such constituents may interfere in certain urinary investigations and also because they afford a good means of determining whether certain substances have been introduced into the organism or not. From this point of view a few of these bodies will be spoken of in a following section (on the pathological urinary constituents). The presence of these foreign bodies in the urine is of special interest in those cases in which they serve to elucidate the chemical transformations certain substances undergo within the body. As inorganic substances generally leave the body unchanged, they are of very little interest from this standpoint, but the changes which certain organic substances undergo may be studied by this means so far as these transformations are shown by the urine.

The bodies belonging to the fatty series, though not without exceptions, fall mostly into a combustion leading towards the end. 
products of the exchange of material; still, often a smaller or greater part of the body in question withdraws itself from oxidation and appears unchanged in the urine. A part of the organic acids, which are otherwise burnt into water and carbonates and render the urine neutral or alkaline act in this way. 'The volatile fatty acids poor in carbon are less easily burnt than those rich in carbon, and they therefore pass in large amounts unchanged into the urine. This is especially true of formic and acetic acids (ScHotTen, GréHANT and QuinquaND). Oxalic acid passes completely or almost completely unchanged into the urine (GAGLIO).

The acid amides appear not to be changed in the body (Schultzen and Nencki). A small part of the amido-acids seem indeed to be eliminated unchanged, but otherwise they are, as stated above (page 338) for leucin, glycocoll and aspartic acid, decomposed within the body, and they may therefore cause an increased elimination of urea. Sarcosin (methylglycocoll), $\mathrm{NH}\left(\mathrm{CH}_{3}\right) \cdot \mathrm{CH}_{2} \cdot \mathrm{COOH}$, also perhaps passes in small part into the corresponding uramidoacid, methylhydantoinic acid, $\mathrm{NH}_{2} \cdot \mathrm{CO} \cdot \mathrm{N}\left(\mathrm{CH}_{3}\right) \cdot \mathrm{CH}_{2} \cdot \mathrm{COOH}$. Also taurin, amido-ethylsulphonic acid, which acts somewhat differently in different animals (SALKowSKI), passes in human beings, at least in part, into the corresponding uramido-acid, taurocarbaminic acid, $\mathrm{NH}_{2}$.CO.NH. $\mathrm{C}_{2} \mathrm{H}_{4} \cdot \mathrm{SO}_{2} \cdot \mathrm{OH}$. A part of the taurin appears as such in the urine. In rabbits, when taurin is introduced into the stomach, nearly all its sulphur appears in the urine as sulphuric and sulphurous acids. After subcutaneous injection the taurin appears again in great part unchanged in the urine.

Creatin passes, at least in part, into creatinin. A part may perhaps appear as urea (see page 257). Hypoxanthin passes into uric acid.

A coupling with glycocoll may also occur. Furfurol or the anhydride of pyromucic acid, $\mathrm{C}_{5} \mathrm{H}_{4} \mathrm{O}_{2}$, passes to a great extent coupled with glycocoll as pyromucuric acid, $\mathrm{C}_{7} \mathrm{H}_{7} \mathrm{~N}_{4} \mathrm{O}$, in to the urine of rabbits and dogs and to a less extent as furfuracrylic acid coupled with glycocoll as furfuracryluric acid, $\mathrm{C}_{9} \mathrm{H}_{9} \mathrm{~N}_{4} \mathrm{O}$ (J AFFÉ and СонN). In birds (hens) this condition is different. In these animals furfurol gives pyromucic acid and a coupled acid, pyromucinornithuric acid, $\mathrm{C}_{15} \mathrm{H}_{16} \mathrm{~N}_{2} \mathrm{O}_{6}$, which decomposes on warming with concentrated hydrochloric acid into pyromucic acid and ornithin, $\mathrm{C}_{5} \mathrm{H}_{12} \mathrm{~N}_{2} \mathrm{O}_{2}$ (JAFFÉ and CoHN). 
Coupling with glycuronic acid occurs in certain substituted alcohols, aidehydes and ketones (?), which probably first pass over into alcohol (SUNDVIK). Chloral hydrate, $\mathrm{C}_{2} \mathrm{Cl}_{3} \mathrm{OH}+\mathrm{H}_{2} \mathrm{O}$, passes, after it has been converted into trichlorethyl-alcohol by a reduction, into a lævo-gyrate reducible acid, urochloralic acid or trichlorethyl-glycuronic acid, $\mathrm{C}_{2} \mathrm{Cl}_{3} \mathrm{H}_{2} \cdot \mathrm{C}_{6} \mathrm{H}_{9} \mathrm{O}_{7}$ (Musculus and v. Mering). Trichlorbutyl-alcohol and butyl-chloral hydrate also pass into trichlorbutyl-glycuronic acid. Tertiary amyl-and butylalcohol also undergo (in rabbits but not in man) a coupling with glycuronic acid. In animals which have starved until the glycogen has disappeared from the muscles and liver and which are given chloral hydrate or dimethyl carbinol, coupled glycuronic acids appear in the urine (THIERFELDER). On account of these facts the albuminous bodies are considered the origin of the glycuronic acid. It may perhaps originate from such bodies as the proteids which are found widely diffused in the body and from which carbohydrates or near-related acids may be split.

The aromatic combinations pass as a rule, so far as we know, into the urine after a previous partial oxidation or after a synthesis with other bodies. The question whether the benzol ring is destroyed in the body is still undecided, but at least in certain cases such a destruction is very probable.

The fact that benzol may be oxidized outside of the body into carbon dioxide, oxalic acid, and volatile fatty acids has been known for a long time, and we may refer the reader to the investigations of Drechsel, mentioned in the first chapter, in which this experimenter obtained, by the electrolysis of phenol, normal caproic acid and afterward substances in which the amount of carbon decreased constantly until he obtained the end-products of the exchange of material. As in these experiments a splitting of the benzol ring must take place before the formation of the body of the fatty series, also when aromatic bodies are burnt in the animal body, we must admit that first a rupture of the benzol ring takes place with the formation of fatty bodies. If this does not take place, then the benzol nucleus is eliminated with the urine as an aromatic combination of one kind or another. As the difficultlyburnt benzol nucleus can protect from destruction a substance belonging to the fatty series and coupled with it, which is the case 
with the glycocoll of hippuric acid, it seems also that the aromatic nucleus itself may be protected from destruction in the organism by syntheses with other bodies. The aromatic ethereal sulphuric acids are examples of this kind.

The difficulty in deciding whether the benzol ring itself is destroyed in the body lies in the fact that we do not know all the different aromatic transformation products which may be produced by the introduction of any aromatic substance in the organism and which we must seek for in the urine. On this account it is also impossible to learn by exact quantitative estimations whether or not an aromatic substance introduced or absorbed appears again in its entirety in the urine. Certain observations render it probable that the benzol ring, as above mentioned, is at least in certain cases destroyed in the body. Schotten and BAUMANN have found that certain amido-acids, such as tyrosin, phenylamido-propionic acid and amido-cinnamic acid, when introduced into the body cause no increase in the quantity of known aromatic substances in the urine; this makes a destruction of these amido-acids in the animal body seem probable. JUvALTA also made an experiment on dogs with phthalic acid and found that $5 \% .5-68.76 \%$ of the acid introduced into the body disappeared, or more correctly was not found again. According to Juvalita, this acid does not undergo any synthesis, nor does it yield any aromatic transformation products; and if this supposition be correct, we have here a proof of the destruction of the benzol nucleus of a part of the phthalic acid introduced into the organism of the dos.

An oxidation in the side chain of aromatic compounds is often found and may also occur in the nucleus itself. As an example, benzol is first oxidized to oxybenzol (Schultzen and NAUNYN), and this is then in part converted into dyoxybenzols (BAUMANN and Preusse). Naphthalin appears to be converted into oxynaphthalin and probably a part also into dioxynaphthalin (LESNIK and M. NENCKI). Anilin, $\mathrm{C}_{6} \mathrm{H}_{5} \cdot \mathrm{NH}_{2}$, passes into paramidophenol, which passes into the urine as ethereal sulphuric acid, $\mathrm{H}_{2} \mathrm{~N} \cdot \mathrm{C}_{6} \mathrm{H}_{4} \cdot 0 \cdot \mathrm{SO}_{2} \cdot \mathrm{OH}$ (F. MÚLLER).

If the aromatic substance has a side chain belonging to the fatty series, this last is generally oxidized. For example, toluol, $\mathrm{C}_{6} \mathrm{H}_{5} \cdot \mathrm{CH}_{3}$ (Schultzen and NAUNYN), ethyl-benzol, $\mathrm{C}_{6} \mathrm{H}_{5} \cdot \mathrm{C}_{2} \mathrm{H}_{5}$, and propyl-

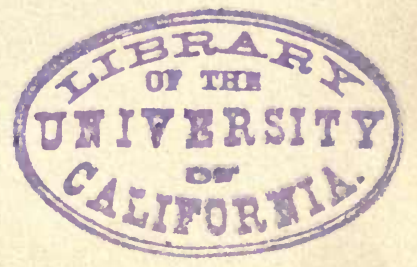


benzol, $\mathrm{C}_{6} \mathrm{H}_{5} \cdot \mathrm{C}_{3} \mathrm{H}_{7}$ (NenckI and Gracosa), also many other bodies are oxidized into benzoic acid. If the side chain has several mentbers, the behavior is somewhat different. Phenyl-acetic acid, $\mathrm{C}_{6} \mathrm{H}_{5}$. $\mathrm{CH}_{2} . \mathrm{COOH}$, in which only one curbon atom exists between the benzol nucleus and the carboxyl, is not oxidized, but is eliminated after coupling with glycocoll as phenaceturic acid (SALKowsKr). Phenyl-propionic acid, $\mathrm{C}_{6} \mathrm{H}_{5} \cdot \mathrm{CH}_{2} \cdot \mathrm{CH}_{2} \cdot \mathrm{COOH}$, with two carbon atoms between the benzol nucleus and the carboxyl is, on the contrary, oxidized into benzoic acid. Aromatic amido-acids with three carbon atoms in the side chain, and where the $\mathrm{NH}_{2}$ group is bound to the middle one, as in tyrosin, $\alpha$-oxyphenylamido-propionic acid, $\mathrm{C}_{6} \mathrm{H}_{4}(\mathrm{OH}) \cdot \mathrm{CH}_{2} \cdot \mathrm{CH}\left(\mathrm{NH}_{2}\right) \cdot \mathrm{COOH}$, and $\alpha$-phenylamido-propionic acid, $\mathrm{C}_{6} \mathrm{H}_{5} \cdot \mathrm{CH}_{2} \cdot \mathrm{CH}\left(\mathrm{NH}_{2}\right) \cdot \mathrm{COOH}$, seem to be in great part burnt within the body (Schotren and Baumane). Phenylamido-acetic acid, which has only two carbon atoms in the side chain, $\mathrm{C}_{6} \mathrm{H}_{5} \cdot \mathrm{CH}\left(\mathrm{NH}_{2}\right)$. $\mathrm{COOH}$, acts otherwise, passing into mandelic acid, phenyl-glycolic acid, $\mathrm{C}_{6} \mathrm{H}_{5} \cdot \mathrm{CH}(\mathrm{OH}) . \mathrm{COOH}$ (SchotTeN).

If several side chains are present in the benzol nucleus, then only one is always oxidized into carboxyl. Thus xylol, $\mathrm{C}_{6} \mathrm{H}_{4}\left(\mathrm{CH}_{3}\right)_{2}$, is oxidized into toluic acid, $\mathrm{C}_{6} \mathrm{H}_{4}\left(\mathrm{CH}_{3}\right) \mathrm{COOH}$ (SchultzeN and NAUNYN), mesitylen, $\mathrm{C}_{6} \mathrm{H}_{3}\left(\mathrm{CH}_{3}\right)_{3}$, into mesitylenic acid, $\mathrm{C}_{6} \mathrm{H}_{3}\left(\mathrm{CH}_{3}\right)_{2}$. $\mathrm{COOH}$ (L. NeNCKI), and cymol into cumic acid (M. NenckI and ZIEGLER).

Syntheses of aromatic substances with other atomic groups occur frequently. To these syntheses belongs, in the first rank, the coupling of benzoic acid with glycocoll to form hippuric acid, first discovered by WöHLER. All the numerous aromatic substances which are converted into benzoic acid in the body are voided as hippuric acid. This statement is not true for all classes of animals. According to the observations of JAFFÉ, benzoic acid does not pass into hippuric acid in birds, but into another more nitrogenized acid, ornithuric acid, $\mathrm{C}_{19} \mathrm{H}_{20} \mathrm{~N}_{2} \mathrm{O}_{4}$. This acid yields as splitting products, besides benzoic acid, a basic body, ornithin (see page 389). Oxybenzoic acids (salicylic acid passes partly into salicyluric acid) and the substituted benzoic acids form pairs with glycocoll corresponding to hippuric acid, but also with the abovementioned acids, toluic, mesitylenic, cumic, and phenyl-acetic acid. 
These acids are voided as toluric, mesitylenuric, cuminuric, and phenaceturic acid.

Another synthesis of aromatic substances is that of the ethereal 'sulphuric acids. Phenol and chiefly the hydroxylated aromatic hydrocarbons are voided as ethereal sulphuric acids, according to BaUmann, Herter, and others.

A pairing of aromatic substances with glycuronic acid, which last is protected from burning, occurs rather often. Camphor, $\mathrm{C}_{10} \mathrm{H}_{16} \mathrm{O}$, when given to a dog, is first converted by oxidation into camphorol, $\mathrm{C}_{10} \mathrm{H}_{15}(\mathrm{OH}) \mathrm{O}$, and by coupling with glycuronic acid, campho-glycuronic acid is produced (SCHMIEDEBERG). Borneol and menthol give directly, with the elimination of water, the corresponding glycuronic acids (Pellacani). Phenol may also be partly voided directly as a coupled glycuronic acid (SCHMIEDEBERG). Naphthol appears in great part to pass into the urine as coupled glycuronic acids (LESNIK and M. NeNCKI). Orthonitrotoluol in dogs passes first into orthonitrobenzyl-alcohol and then into a coupled glycuronic acid (JAFFÉ). Indol (SchmiedeberG) and skatol (MEster) seem, as above mentioned, to be partly voided by the urine as coupled glycuronic acids. The same is true also for many other aromatic substances.

A synthesis in which a compound containing sulphur, mercapturic acid, occurs is produced by the introduction of chlorine or bromine derivatives of benzol into the organism of dogs (BAUMANN and Preusse, JAFFÉ). Chlorbenzol combines with cystein, a body which seems to be a decomposition product of the albumins, and which is nearly related to cystin (see below), forming chlorphenylmercapturic acid, $\mathrm{C}_{11} \mathrm{H}_{12} \mathrm{ClSNO}_{3}$. On boiling with a mineral acid this compound is decomposed into acetic acid and chlorphenylcystein, $\mathrm{C}_{6} \mathrm{H}_{4} \mathrm{Cl}_{2} \mathrm{C}_{3} \mathrm{H}_{6} \mathrm{NSO}_{2}$.

Pyridin, $\mathrm{C}_{5} \mathrm{H}_{5} \mathrm{~N}$, which does not combine either with glycuronic acid or with sulphuric acid after previous oxidation, shows a special behavior. It takes up a methyl group and forms an ammonium combination, methylpyridyl-ammonium hydroxyl (HIs). Several alkaloids, such as quinin, morphin, and strychnin, may pass into the urine. After taking turpentine, balsam of copaiva, and resins these may pass into the urine as resin acids (MaLY). Different kinds of coloring matters, such as alizarin, crysophanic acid, after 
the use of rhubarb or senna, and the coloring matter of the blueberry, may pass into the urine. After taking rhubarb, senna or santonin the urine takes a yellow or greenish-yellow color, which is transformed into a beautiful red color by the addition of alkali. Phenol produces, as above mentioned, a dark-brown or dark-green color which depends mainly on the decomposition products of hydrochinon or humin substances (v. UDRANSZKY). After the use of naphthalin the urine has a dark color, and several other medicines produce a special coloration. Thus cairin gives often a yellowish-green hue, or the urine darkens when exposed to the air; thallin gives a greenish-brown color which is marked green in thin layers, and antipyrin gires a yellow to blood-red. After the administration of balsam of copaiva the urine becomes, when strongly acidified with hydrochloric acid, gradually rose and purple-red (QUINCKE). After the use of naphthalin or naphthol the urine gives with concentrated sulphuric acid (1 c. c. concentrated acid and a few drops of urine) a beautiful emerald-green color (PeNZOLDT), which is probably due to naphthol-glycuronic acid. Odoriferous bodies also pass into the urine. After eating asparagus the urine acquires a sickly disagreeable odor. After taking turpentine the urine may have a peculiar odor similar to that of violets.

\section{Pathological Constituents of Urine.}

Albumin. The appearance of slight traces of albumin in the urine of apparently healthy persons has been observed in many cases by several investigators (Leube, Hofmeister, Posner, and others), but still we must not conceal the fact that other investigators consider these traces of albumin as the first symptoms, though very mild, of a diseased condition of the urinary apparatus, or as a symptom of a transitory disturbance in the circulation. Frequently traces are found in the urine of a substance similar to nucleoalbumin which can easily be mistaken for mucin and which is probably identical with nucleoalbumin. This substance has been isolated from the pupillary part of the kidneys and from the mucous membrane of the bladder by LöNNBERG. In diseased conditions albumin occurs in the urine in a variety of cases. The albuminous bodies which most often occur are serum-globulin and serum- 
albumin. Albumoses and peptones also sometimes occur. The amount of albumin in the urine is in most cases less than 5 p. m., , rarely $10 \mathrm{p}$. m., and only very rarely does it amount to $50 \mathrm{p}$. $\mathrm{m}$. or over.

Among the many reactions proposed for the detection of albumin in urine, the following are to be recommended :

The Heat Test. Filter the urine and test its reaction. An acid urine may, as a rule, be boiled without further treatment, and only in especially acid urines is it necessary to first treat with a little alkali. An alkaline urine is made neutral or faintly acid before heating. If the urine is poor in salts, add $\frac{1}{10}$ vol. of a saturated common-salt solution before boiling; then heat to boiling point, and if no precipitation, cloudiness, or opalescence appears, the urine in question contains no coagulable albumin, but it may contain albumoses or peptones. If a precipitate is produced on boiling, this may consist of albumin, or of earthy phosphates, or of both. The simple-acid calcium phosphate decomposes on boiling, and normal phosphate may separate (Stokvis, SALKowski, OTT). The proper amount of acid is now added to the urine, so as to prevent any mistake caused by the presence of earthy phosphates, and to give a better and more flocculent precipitate of the albumin. If acetic acid is used for this, then add 1-2-3 drops of a 25\% acid to each 10 c. c. of the urine, and boil after the addition of each drop. On using nitric acid, add 1-2 drops of the 25\% acid to each c. c. of the boiling-hot urine.

On using acetic acid, when the amount of albumin is very small, and especially when the urine was originally alkaline, the albumin may sometimes remain in solution on the addition of the above quantity of acetic acid. If, on the contrary, less acid is added, the precipitate of calcium phosphate, which forms in amphoteric or faintly-acid urines, is liable not to dissolve completely, and this may cause it to be mistaken for an albumin precipitate. If nitric acid is used for the heat test, the fact must not be overlooked that after the addition of only a little acid a combination between it and the albumin is formed which is soluble while boiling and which is only precipitated by an excess of the acid. On this account the large amount of nitric acid as suggested above must be added, but in this case a small part of the albumin is liable to be dissolved by the excess of the nitric acid. When the acid is added after boiling, which is absolutely necessary, the liability of a mistake is not so great. It is on these grounds that the heat test, although it gives very good results in the hands of experts, is not recommended to physicians as a positive test for albumin.

A confounding with mucin, when this body occurs in the urine, 
is easily prevented in the heat test with acetic acid, by acıdifying another portion with acetic acid at the ordinary temperature. Mucin and nucleoalbumin substances similar to mucin are hereby precipitated. If in the performance of the heat and nitric acid test a precipitate first appears on cooling or is strikingly increased, then this shows the presence of albumoses in the urine, either alone or mixed with coagulable albumin. In this case a further investigation is necessary (see below). In a urine rich in urates a precipitate consisting of uric acid separates on cooling. This precipitate is colored, sandy, and hardly to be mistaken for an albumose or albumin precipitate.

HELLER's test is performed as follows (see page 19): The urine is very carefully floated on the surface of nitric acid in a test-glass. The presence of albumin is shown by a white ring between the two liquids. With this test a red or reddish-violet ring is always obtained with normal urine; it depends on the indigo coloring matters and can hardly be mistaken for the white or whitish albumin ring, and this last must not be mistaken for the ring produced by bile-pigments. In a urine rich in urates another complication may occur, due to the formation of a ring produced by the precipitated uric acid. 'The uric-acid ring does not lie, like the albumin ring, between the two liquids, but somewhat higher. For this reason we may often have two simultaneous rings with urines rich in urates and yet not containing very much albumin. 'The disturbance caused by uric acid is easily prevented by diluting the urine with 1-2 vol. water before performing the test. The uric acid now remains in solution, and the delicacy of HELLER's test is so great that after dilution only in the presence of insignificant traces of albumin does this test give negative results. In a urine very rich in urea a ring-like separation of urea nitrate may also appear. This ring consists of shining crystals, and it does not appear in the previously-diluted urine. A confusfon with resinous acids, which also give a whitish ring with this test, is easily prevented, since these acids are soluble on the addition of alcohol. A liquid which contains pure mucin does not give a precipitate with this test, but it gives a more or less strongly opalescent ring, which disappears on stirring. The liquid does not contain any precipitate after stirring, but is clear or somewhat opalescent. If we bear in mind the above-mentioned possible mistakes and the means by which they may be prevented, there is hardly another test for albumin in the urine which is at the same time so easily performed, so delicate, and so positive as HELLER's. With this test even $0.02 \mathrm{p} . \mathrm{m}$. albumin may be detected without difficulty. In performing this test the (primary) albumoses are also precipitated.

The reaction with metaphnsphoric acid (see page 19) is very convenient and easily performed. It is not quite so delicate and 
positive as HELLER's test. The albumoses are also precipitated by this reagent.

Reaction with Acetic Acid and Potassium Ferrocyanide. Treat the urine first with acetic acid of about $2 \%$ and then add drop by drop a potassium ferrocyanide solution (1:20), carefully avoiding an excess. This test is very good, and in the hands of experts it is even more delicate than HeLLER's. In the presence of very small amounts of albumin it requires more practice and dexterity than HELLER's, as the relative quantities of reagent to the albumin act on the result of the test. The amount of salts in the urine also seems to have an influence. This reagent also precipitates albumoses.

The different color reactions cannot be directly used, especially in deep-colored urines which only contain little albumin. The common salt of the urine has a disturbing action on MiLLoN's reagent. To prove more positively the presence of albumin, the precipitate obtained in the boiling test may be filtered, washed, and then tested with Millos's reagent. The precipitate may also be dissolved in dilute alkali and the biuret test applied to the solution. The presence of albumoses or peptones in the urine is directly tested for by this last-mentioned test. In testing the urine for albumin one must never be satisfied with one test alone, but one must at least apply the heat test and HeLLER's test or the potassium-ferrocyanide test. In using the heat test alone the albumoses may be easily overlooked, but these are detected, on the contrary, by HeLLER's test. If we are satisfied with this last test or the potassium-ferrocyanide test alone, we have no sufficient intimation of the kind of albumin present, whether it consists of albumoses or coagulable albumin.

For practical purposes several dry reagents for albumin have been recommended. Besides the metaphosphoric acid may be mentioned : PAvY's reagent, which consists of small disks or plates of citric acid and sodium ferrocyanide; STUTZ's or FÜRBRINGER's gelatine capsules, which contain mercuric chloride, sodium chloride, und citric acid; and GeIssLen's albumin-test papers, which consist of strips of filter-paper which have been dipped in a solution of citric acid and also mercuric chloride and potassium-iodide solution and then dried.

If the presence of albumin has been positively proved in the urine by the above tests, it then remains necessary to determine the variety.

The detection of globulin and albumin. In detecting serumglobulin the urine is exactly neutralized, filtered; and treated with magnesium sulphate in substance until it is completely saturated at the ordinary temperature, or with an equal volume of a saturated neutral solution of ammonium sulphate. In both cases a white, flocculent precipitate is formed in the presence of globulin. In 
using ammonium sulphate with a urine rich in urates a precipitate consisting of ammonium urate may separate. This precipitate does not appear immediately, but only after a certain time, and it must not be mistaken for the globulin precipitate. In detecing serum albumin heat the filtrate from the globulin precipitate to boilingpoint or add about 1\% acetic acid to it at the ordinary temperature.

In detecting albumoses, whose occurrence in the urine was first shown by BENCE JONES and by KüHNE and later observed by many investigators in different diseased conditions, first remove all coagulable albumins, if any are present, by boiling with the addition of acetic acid. The filtrate is then tested by the biuret test, and when this gives positive results apply the three above-mentioned albumose reagents (page 26), nitric acid, acetic acid, and potassium ferrocyanide, and saturate with common salt with the addition of acid. The albumoses may also be precipitated by saturating with ammonium sulphate in substance.

Peptones, as shown by the experiments of several investigators, among whom should be especially mentioned Hofmeister, v. JAKSCH, MAIXNer, and FISCHEL, may appear in the urine as soon as it occurs dissolved in the blood. This is especially the case in an abundant destruction of the pus-cells with absorption of the peptones originating therefrom, as also in pneumonia, in purulent pleuritis, etc. (PYogene Peptonuria, Hofmeister, MaIXner, v. $\mathrm{J}_{\mathrm{AKSCH}}$ ). Peptone also passes into the urine when the normal absorption and assimilation of the peptones is disturbed so that the peptones pass directly through the destroyed part of the intestine into the blood, as in ulcerous processes in the intestine (ENTERogentc peptonuria, Mixner), also in puerperal peptonuria (FISCHEL), in acute phosphorus-poisoning and certain diseases of the liver (HEPATOGENIC PEPTONURIA), in effusion of blood in difficult cases of scurvy (HæMATOGENIC PEPTONURIA, v. JAKSCH), etc. etc.

These statements refer only to the peptone in the old sense, and the question as to" whether we are dealing with "secondary albumoses" or with "pure peptones" or a mixture of both is still undecided. Until we are agreed as to the importance of the peptones and albumoses it is hardly possible to give positive statements in regard to the occurrence of peptones in the urine, or to give accurate methods for their detection and quantitative estimation.

The urine to be tested for peptone in the old sense must be free from mucin and from albumin so that it does not give either the potassium-ferro- 
cyanide reaction or Hellen's test. Such a urine may be tested directly; if, on the contrary, it contains albumin, this must first be removed. This is ordinarily done according to HOFMEISTER's method. At least half a litre of , the urine is treated with an excess of a lead-acetate solution, or with only a quantity sufticient to produce a dense flocculent precipitate in order to separate some so-called mucin present as well as a part of the albumin and coloring matters. If the operation hus been well conducted, the filtrate, which gives a precipitate with more lead-acetate solution, is tested for albumin. In the absence of this it is directly tested for peptone; but if albumin be present, it must first be removed by boiling with ferric-acetate solution. For this purpose treat the filtrate with a concentrated sodium-acetate solution (about 10 c. c. for $\frac{1}{2}$ litre urine) and then with ferric-chloride solution until it has a blood-red color. The acid-reacting liquid is then rendered neutral or falntly acid by means of the addition of alkali, boiled strongly, and filtered after cooling. The filtrate should be free from albumin; but if that is not the case, it must be repeatedly treated with sodium acetate and ferric chloride. If the urine to be tested for peptones is rather rich in albumin at first, the albumin must be removed as far as possible by boiling with the addition of acetic acid before it is treated with lead-acetate solution as above described.

A small portion of the filtrate entirely free from albumin is taken, made strongly acid with acetic acid and then treated with an acetic-acid solution of phospho-tungstic acid. If the test remains clear for some time, the urine contains no peptones; but if a milky cloudiness occurs, peptones may be present and the filtrate must be further treated.

For this purpose add $\frac{1}{20}-\frac{1}{10}$ vol. concentrated hydrochloric acid and then add a phospho-tungstic acid solution treated with acid as long as a precipitate is produced. This is quickly filtered and treated with water which contains 3-5\% concentrated sulphuric acid, washed, until the filtrate is colorless. The still moist precipitate is thoroughly rubbed with an excess of solid barium hydrate, some water added, gently warmed for a time, and filtered. The peptones (and secondary albumoses) are detected in the filtrate by applying the previously-mentioned reactions. Special stress must be laid on the biuret reaction, which is used also as a colorimetric quantitative test for peptones.

In detecting pure peptones the solution must be saturated with ammonium sulphate while boiling, and filtered at the same temperature. After cooling remove the liquid from the deposited crystals, dilute strongly, precipitate the peptone by the careful addition of tannic acid, treat the precipitate with an excess of barium hydrate, and use the filtrate in which the excess of dissolved barium hydrate has been removed by $\mathrm{CO}_{2}$ for the biuret test. Still by this procedure the peptones may be contaminated by some albumoses. We have no very exact method for the quantitative estimation of albumose or peptones in the urine.

Quantitative Estimation of Albumin in Urine. Of all the methods proposed thus far, the COAGULATION METHOD (boiling with the addition of acetic acid) when performed with sufficient care gives the best results. The average errors need never amount to more than $0.01 \%$, and it is generally smaller. In using this method it is best to first find how much acetic acid must be added to a small portion of urine, which has been previously heated on the water-bath, to completely separate the albumin, so that the filtrate does not respond to HeLLER's test. Then coagulate 20-50-100 c. c. of the urine. Pour the urine into a beaker and heat on the water-bath, add the required quantity of acetic acid slowly, stirring 
constantly and heating at the same time. Filter while warm, wash first with water, then with alcohol and ether, dry and weigh, ash. and weigh again. In exact determinations the filtrate must not give HELLER's test.

'The separate estimation of GLOBULINS and ALBUMINS is done by carefully neutralizing the urine and precipitating :with $\mathrm{MgSO}_{4}$ added to saturation (AUTHOR), or simply by adding an equal volume of a saturated neutral solution of ammonium sulphate (Hofmeister and POHs). The precipitate consisting of globulin is thoroughly washed with a saturated magnesium sulphate or halfsaturated ammonium-sulphate solution, dried continuously at $110^{\circ}$ C., boiled with water, extracted with alcohol and ether, then dried, weighed, ashed, and weighed again. The quantity of albumin is calculated as the difference between the quantity of globulins and the total proteids.

Approximate Estimation of Albumin in Urine. Of the methods suggested for this purpose none has been more extensively employed than EsBACH's.

EsBAcH's method. The acidified urine (acidified with acetic acid) is poured into a specially-graduated tube to a certain mark and then the reagent (a 2\% citric-acid and 1\% picric-acid solution in water) is added to a second mark, the tube is closed with a rubber stopper and carefully shaken, avoiding the production of froth. The tube is allowed to stand twenty-four hours, and then the height of the precipitate in the graduated tube is read off. The reading gives directly the quantity of albumin in 1000 parts of the urine. Urines rich in albumin must first be diluted with water. The results obtained by this method are, however, dependent upon , the temperature; and a difference in temperature of $5^{\circ}$ to $6.5^{\circ} \mathrm{C}$. may in urines containing a medium quantity of albumin cause an error of $0.2-0.3 \%$ deficiency or excess (CHRISTENSEN and MYGGE). This method is only to be used in a room in which the temperature may be kept nearly constant. The directions for the use of the apparatus accompany it.

Christensen's and Mrgae's method. 5 c. c. of urine, after being acidified with 2 drops of acetie acid, are poured into a somewhat modified burette and precipitated with a certain quantity of a $1 \%$ tannic-acid solution and then treated with 1 c. c. of mucilage. After the addition of water to a certain mark and after inverting the tube several times a uniform emulsion is produced. A cylindrical glass filled one half or one third with water is now placed on a white surface having a number of close black lines traced upon it, and the contents of the burette are gradually added to the water with constant stirring, until by close observation the black lines cannot even be distinguished from the white spaces. The reading of the amount of urine emulsion employed gives directly the 
amount of albumin in the urine: This method is claimed to give very good results. A special description accompanies each apparatus.

The method proposed by RoBerts and SToLnikow and further developed by BRANDBERG, though somewhat more difficult to perform, also gives satisfactory results. The density methods of LANG, HUPPERT, and ZAHOR are also very good. The last consists in determining the specific gravity before and after the coagulation of the albumins.

Mucin occurs in the urine under normal conditions partly dissolved and partly in a strongly-distended, finely-divided state. It appears in greatest amounts in catarrhal affections of the urinary passages. The occurrence of pure mucin in the urine has thus far not been positively shown.

To detect mucin in urine first dilute with water, partly to prevent the precipitation of uric acid on the subsequent addition of acid, and partly to diminish the solubility of the mucin in the common salt of the urine. Now add an excess of acetic acid. The precipitate formed is purified by dissolving in water with the addition of a little alkali and reprecipitated with acetic acid. The precipitate is tested with the ordinary mucin reagents. To avoid mistaking mucin for nucleoalbumin, which is similar to mucin, the precipitate must be tested in regard to its behavior on boiling with dilute mineral acids. If no reducible substance is formed by this treatment, it contains no mucin.

Blood and Blood-coloring Matters. The urine may contain blood from hemorrhage in the kidneys or other parts of the urinary passages (H жMATURIA). In these cases, when the quantity of blood is not very small, the urine is more or less cloudy and colored reddish, yellowish red, dirty red, brownish red, or dark brown. In recent hemorrhages, in which the blood has not decomposed, the color is nearer blood-red. Blood-corpuscles may be found in the sediment, sometimes also blood-cylinders and smaller or larger blood-clots.

In certain cases the urine contains no blood-corpuscles, but only dissolved blood-coloring matters, hæmoglobin, or, and indeed quite often, methæmoglobin (H\&MOGLOBINURIA). The blood-coloring matters appear in the urine under different conditions, as in dissolution of blood in poisoning with arseniuretted hydrogen, chlorates, etc., after serious burns, after transfusion of blood, and also in the periodic appearance of hæmoglobinuria with fever. The urine may in hæmoglobinuria also have an abundant grayish-brown sediment rich in albumin which contains the remains of the stromata of the red blood-corpuscles. In animals hæmoglobinuria may be produced by many causes which force free hæmoglobin into the plasma.

To detect blood in the urine we make use of the microscope, 
spectroscope, the guaiacum test, and Heller's or HellerTEICHManN's test.

Microscopic Investigation. The blood-corpuscles may remain undissolved for a long time in acid urine; in alkaline urine, on the contrary, they are easily changed and dissolved. They often appear entirely unchanged in the sediment ; in some cases they are distended, and in others unequally pointed or jagged like a thornapple. In hemorrhage of the kidneys a cylindrical clot is sometimes found in the sediment which is covered with numerous red blood-corpuscles, forming casts of the urinary passages. These formations are called BLOOD-CYLINDERS.

The spectroscopic investigation is naturally of very great value; and if it be necessary to determine not only the presence but also the kind of coloring matter, this method is indispensable. In regard to the optical behavior of the various blood-coloring matters we must refer to Chapter IV.

Almés's Guaiacum Test. Mix in a test-tube equal volumes of tincture of guaiacum and old turpentine which has become strongly ozonized by the action of air under the influence of light. To this mixture, which must not have the slightest blue color, add the urine to be tested. In the presence of blood or blood-coloring matters, first a bluish-green and then a beautiful blue ring appears where the two liquids meet. On shaking the mixture it becomes more or less blue. Normal urine or one containing albumin does not give this reaction. For the reason of this we must refer the reader to Chapter IV, page 71 . Urine containing pus, although no blood is present, gives a blue color with these reagents; but in this case the tincture of guaiacum alone, without turpentine, is colored blue by the urine (VITALLI). This is at least true for a tincture that has been exposed for some time to the action of air and sunlight. The blue color produced by pus differs from that produced by blood-coloring matters by disappearing on heating the urine to boiling. A urine alkaline by decomposition must first be made faintly acid before performing the reaction. The turpentine should be kept exposed to sunlight, while the tincture of guaiacum must be kept in a dark glass bottle. These reagents to be of use must be controlled by a liquid containing blood. This test, it is true, in positive results is not absolutely decisive, because other bodies may 
give a blue reaction ; but when properly performed it is so extremely delicate that when it gives negative results any other test for blood is superfluous.

Heller-Teichmans's Test. If a neutral or faintly-acid urine containing blood is heated to boiling, we always obtain a mottled precipitate consisting of albumin and hæmatin. If caustic soda is added to the boiling-hot test, the liquid becomes clear and turns green when examined in thin layers (due to hæmatin alkali), and a red precipitate, appearing green by reflected light, re-forms which consists of earthy phosphates and hæmatin. This reaction is called HeLLen's blood-test. If this precipitate is collected after a time on a small filter, it may be used for the hæmin test (see page 79). If the precipitate contains only a little blood-coloring matter with a larger quantity of earthy phosphates, then wash it with dilute acetic acid, which dissolves the earthy phosphates, and use the residue for the preparation of 'TeIchmanN's hæmin crystals. If, on the contrary, the amount of phosphates is very small, then first add a little $\mathrm{CaCl}_{2}$ solution to the urine, heat to boiling, and add simultaneously with the caustic potash some sodiumphosphate solution. In the presence of only very small amounts of blood, first make the urine very faintly alkaline with ammonia, add tannic acid, acidify with acetic acid, and use the precipitate in the preparation of the hæmin crystals (STRUVE).

Melanin. In the presence of melanotic cancers sometimes dark coloring matters are eliminated with the urine. K. MörNer has isolated two coloring matters from such a urine, of which one was soluble in warm 50-75\% acetic acid and the other, on the contrary, was insoluble. The one seemed to be phymatorusin (see page 324). Usually the urine does not contain any melanin, but a chromogen of melanin, melanogen. In such cases the urine gives EISELT's reaction, becoming dark-colored with oxidizing agents such as conc. nitricacid, potassium bichromate, and sulphuric acid, as well as with free sulphuric acid. Urine containing melanin or melanogen is colored black by ferricchloride solution (JAKSCH).

BAUMSTARK found in a case of leprosy two characteristic coloring matters in the urine, "urorubrohæmatin" and "urof uscohæmatin," which, as their names indicate, seem to stand in close relationship to the blood-coloring matters. Urorubrohamatin, $\mathrm{C}_{68} \mathrm{H}_{94} \mathrm{~N}_{8} \mathrm{Fe}_{2} \mathrm{O}_{28}$, contains iron and shows an absorptionband in front of $D$ and one broader back of $D$. In alkaline solution it shows four bands, behind $D$, at $E$, beyond $F$, and behind $G$. It is not soluble either in water, alcohol, ether, or chloroform. It gives a beautiful brownish-red non-dichroitic liquid with alkalies. Urofuscohcematin, $\mathrm{C}_{88} \mathrm{H}_{106} \mathrm{~N}_{8} \mathrm{O}_{28}$, which is free from iron shows no characteristic spectrum ; it dissolves in alkalies, producing a brown color.

Urorosein, so named by NENCKI, is a urinary coloring matter, occurring in various diseases, which appears on the acidification of the urine with a mineral acid, and which is taken up by shaking with amyl-alcohol. The solution shows an absorption-band between $D$ and $E$. Uroerythrin, which gives a rose-red color to the urinary sediments especially in fevers, seems to occur also in urine under physiological conditions. It has not been thoroughly studied. A 
coloring matter nearly related to homatoporphyrin has been found by NEUSSER in two pathological urines. MACMUNN has found a coloring matter, urohcematin, in rheumatism and ADDIson's disease ; he also prepared it artificially from hæmatin. This coloring matter seems also to stand in close relationship to hæmatoporphyrin.

Pus occurs in the urine in different inflammatory affections, especially in catarrh of the bladder and in inflammation of the membrane of the kidneys or the urethra.

Pus is best detected by means of the microscope. The pus-cells are rather easily destroyed in alkaline urines. In detecting pus we make use of DoNNÉ's pus-test, which is performed in the following way: Pour off the urine from the sediment as carefully as possible, place a small piece of caustic alkali on the sediment, and stir. If the pus-cells have not been previously changed, the sediment is converted by this means into a slimy tough mass.

The pus-corpuscles swell up in alkaline urines, dissolve, or at. least are so changed that they cannot be recognized under the microscope. The urine in these cases is more or less slimy or fibrous, and it is precipitated in large flakes by acetic acid, so that it may possibly be mistaken for mucin. The closer investigation of the precipitate produced by acetic acid, and especially the appearance or non-appearance of a reducible substance after boiling it with a mineral acid, demonstrates the nature of the precipitated substance. Urine containing pus always contains albumin.

Bile-acids. The statements in regard to the occurrence of bileacids in the urine under physiological conditions do not agree. According to VogeL and DRAGENDORFF and HöNe, traces of bileacids occur in the urine; according to HOPPE-SEYLER and $\nabla$. UdRANSZKx, they do not. Pathologically they are present in the urine in hepatogenic icterus, although not always.

Detection of Bile-acids in the urine. Pettenkofer's test gives always the most decisive reaction; but as it gives similar color reactions with other bodies, it must be supplemented by the spectroscopic investigation. The direct test for bile-acids is easy after the addition of traces of bile to a normal urine. But the direct detection in a colored icteric urine is more difficult and gives very misleading results; the bile-acid must therefore always be isolated from the urine. This may be done by the following method of HoPPESEYLER, which is slightly modified in non-essential points.

HopPe-Seyler's METHOD. Strongly concentrate the urine, and extract the residue with strong alcohol. The filtrate is freed from alcohol by evaporation and then precipitated by basic lead acetate and ammonia. The washed precipitate is treated with boiling alcohol, filtered hot, the filtrate treated with a few drops of soda solu- 
tion, and evaporated to dryness. The dry residue is extracted with absolute alcohol, filtered, and an excess of ether added. The amorphous or, after a longer time, crystalline precipitate consisting of - alkali-salts of the biliary acids is used in performing PetTenKOFER's test.

Bile-coloring matters occur in the urine in difioront forms of icterus. A urine containing bile-coloring matters is always abnormally colored-yellow, yellowish brown, deep brown, greenish yellow, greenish brown, or nearly pure green. On shaking it froths, and the bubbles are yellow or yellowish green in color. As a rule icteric urine is somewhat cloudy, and the sediment is frequently, especially when it contains epithelium-cells, rather strongly colored by the bile-coloring matters. In regard to the occurrence of urobilin in icteric urine see page 371 .

Detection of Bile-coloring Matters in urine. Many tests have been proposed for the detection of bile-coloring matters. Ordinarily we obtain the best results either with GMELIN's or with HUPPERT's test.

GMeLIN's test may be applied directly to the urine; but it is better to use Rosenbach's modification. Filter the urine, which is deep-colored from the retained epithelium-cells and bodies of that kind, through a very small filter. After the liquid has entirely passed through apply to the inside of the filter a drop of nitric acid which contains only very little nitrous acid. A pale-yellow spot will be formed which is surrounded by colored rings which appear yellowish red, violet, blue, and green. This modification is very delicate, and it is hardly possible to mistake indican and other coloring mat. ters for the bile-pigments. Several other modifications of GMELIN's test on the urine directly, as with concentrated sulphuric acid and nitrate, etc., have been proposed, but they are neither simpler nor more accurate than RosENBACH's modification.

HUPPERT's Reaction. In a dark-colored urine or one rich in indican we do not always obtain good results with GMELIN's test. In such cases, as also in urines containing blood-coloring matters at the same time, the urine is treated with lime-water, or first with some $\mathrm{CaCl}_{2}$ solution, and then with a solution of soda or ammonium carbonate. The precipitate which contains the bile-coloring matters is filtered and used for HUPPERT's test (see page 154).

The precipitate consisting of lime-pigments may also be shaken out with chloroform after washing in water and after being acidified with acetic acid. The bilirubin is taken up by the chloroform, which is colored yellow thereby, while the acetic acid solution is colored green by the bilirubin. Both solutions may then be used 
for GMELIN's test (HoPPE-SEYLER), and small amounts of bile-coloring matters may be detected in this way. The lime-pigments may, according to HILGER, also be used directly for GMELIN's test in the following way: Spread them on a porcelain dish in a thin layer, and add carefully a drop of nitric acid. The reaction generally appears very beautiful.

SToKvis's reaction is especially valuable in those cases in which the urine contains only very little bile-coloring matter together with larger amounts of other coloring matters. 'The test is performed as follows : $20-30$ c. c. urine are treated with 5-10 c. c. of a solution of zinc acetate $(1: 5)$. The precipitate is washed on a small filter with water and then dissolved in a little ammonia. The new filtrate gives, directly or after it has stood a short time in the air until it has a peculiar brownish-green color, the absorption-bands of bilicyanin (see page 155).

Many other reactions for bile-coloring matters in the urine have been proposed; but as the above-mentioned are sufficient, it is perhaps only necessary to give here a few of the other reactions without entering into details.

ULTZMANN's reaction consists in treating about $10 \mathrm{c}$. c. of the urine with 3-4 c. c. concentrated caustic-potash solution and then acidifying with hydrochloric acid. The urine will become a beautiful green.

Smitr's Reaction. Pour carefully over the urine tincture of iodine, whereby a green ring appears between the two liquids. You may also shake the urine with tincture of iodine until it has a green color.

EHRLICH's Test. First mix the urine with an equal volume of dilute acetic acid and then add drop by drop a solution of sulpho-diazobenzol. The acid mixture becomes dark red in the presence of bilirubin, and this color becomes bluish violet on the addition of glacial acetic acid. The sulpho-diazobenzol is prepared with $1 \mathrm{grm}$. sulphanilic acid, $15 \mathrm{c}$. c. hydrochloric acid, and 0.1 grm. sodium nitrite; this solution is diluted to 1 litre with water.

Medicinal Coloring MatTers produced from sautonin, rhubarb, senna, etc., may give an abnormal color to the urine, which may be mistaken for the bile-coloring matters, or in alkaline urines perhaps for blood-coloring matters. If hydrochloric acid is added to such a urine, it becomes yellow or pale yellow, while on the addition of an excess of alkali it becomes more or less beautifully red.

\section{Sugar in Urine.}

Grape-sugar, $\mathrm{C}_{6} \mathrm{H}_{12} \mathrm{O}_{6}$, also called GLUCose, Dextrose, and DIABETIC sugar, occurs chiefly in the vegetable kingdom; but it is found in very small amounts in the blood, on an average of $1.5 \mathrm{p} . \mathrm{m}$., and also as traces in other animal fluids and organs. The occurrence of traces of grape-sugar in the urine of perfectly healthy persons can hardly be disputed. If sugar appears in the urine in constant 
and in specially large amounts, it must be considered as an abnormal constituent.

Small amounts of glucose may pass into the urine from an excessive supply of sugar when the body, by absorption from the intestines, takes up more sugar than it can assimilate (Worm Müller; F. Hofmeister, De Jong). Also after starchy food HOFMEISTER observed in dogs, whose power of assimilating sugar had been greatly reduced by the almost complete withdrawal of food for several days, that glycosuria appeared (STARVATION-DIABETES according to HoFMEISTER). In man the appearance of glucose in the urine has been observed in numerous and various pathological conditions, such as lesions of the brain and especially of the medulla oblongata, abnormal circulation in the abdomen, heart and lung diseases, cirrhosis of the liver, cholera, etc., etc. Glycosuria has also been produced in animals in various ways, as by puncture of the fourth ventricle (piqûre), cutting through the spinal marrow, irritation of the pneumogastric nerve, poisoning with carbon monoxide, curare, amyl-nitrite, o-nitro-phenyl-propiolic acid, phloridzin, and many other substances, also by the injection of dilute common-salt solution into the blood-vessels, and in many other ways. The observation of v. Mering and Minkowski that in dogs after total extirpation of the pancreas a very copious and continuous secretion of sugar, a true diabetes, appears is of special interest.

The continuous appearance of sugar in human urine, sometimes in very considerable amounts, occurs in DIABETES MELLITUS. In this disease there may be an elimination of 1 kilogramme of grapesugar during the 24 hours, or even more. In the beginning of the disease, when the quantity of sugar is still very small, the urine often does not appear abnormal. In more developed, typical cases the quantity of urine voided increases considerably to 3-6-10 litres per 24 hours. The percentage of the physiological constituents is as a rule very low, while their absolute daily quantity is increased. The urine is pale, but of a high specific gravity, 1.030-1.040 or even higher. The high specific gravity depends upon the sugar present, which varies in different cases, but may even amount to 10\%. The urine is therefore characterized in typical cases of dia- 
betes by the very large quantity voided, by the pale color and high specific gravity, and by its containing sugar.

That the urine after the introduction of certain medicines or poisonous bodies into the system contains reducing bodies, paired glycuronic acids, which may be mistaken for sugar, has been mentioned in the previous pages.

Properties of Grape-sugar. Grape-sugar crystallizes sometimes with 1 mol. water of crystallization in warty masses or small leaves or plates, and sometimes when free from water in needles. The sugar containing water of crystallization melts even below $100^{\circ} \mathrm{C}$. and loses its water of crystallization at $110^{\circ} \mathrm{C}$. The anhydrous sugar melts at $146^{\circ} \mathrm{C}$. and is converted into glucosan, $\mathrm{C}_{6} \mathrm{H}_{10} \mathrm{O}_{5}$, at $170^{\circ} \mathrm{C}$. with the elimination of water. On strongly heating it is converted into caramel and then decomposed.

Grape-sugar is readily soluble in water. This solution, which is not as sweet as a cane-sugar solution of the same strength, is dextro-gyrate. The specific rotary power of a watery solution of $1-15 \%$ anhydrous glucose at $+20^{\circ} \mathrm{C}$. is indeed somewhat variable, $52.52^{\circ}-52.9^{\circ}$ (LANDOLT), but the average may be considered as $+52.6^{\circ}$. Anhydrous glucose dissolves sparingly in cold, but more freely in boiling, alcohol. 100 parts $85 \%$ alcohol dissolves 1.94 parts glucose at $+17.5^{\circ} \mathrm{C}$. and 21.7 parts at the boiling temperature (ANTHON). Glucose is insoluble in ether. If an alcoholic caustic-alkali solution is added to an alcoholic solution of glucose, an amorphous precipitate of insoluble saccharate is formed. On warming the saccharate decomposes easily with the formation of a yellow or brownish color which is the basis of the following reaction.

Moore's Test. If a glucose solution is treated with about $\frac{1}{4}$ of its volume of caustic potash or soda and warmed, the solution becomes first yellow, then orange, yellowish brown, and lastly dark brown. It has at the same time a faint odor of caramel, and this odor is more pronounced on acidification.

Glucose forms many crystallizable combinations with $\mathrm{NaCl}$, of which the easiest to obtain is $\left(\mathrm{C}_{6} \mathrm{H}_{12} \mathrm{O}_{6}\right)_{2} \cdot \mathrm{NaCl}+\mathrm{H}_{2} \mathrm{O}$, which forms large colorless six-sided double pyramids or rhomboids with $13.40 \%$ $\mathrm{NaCl}$.

Glucose in neutral or very faintly-acid (by an organic acid) 
solution passes into alcoholic fermentation with beer-yeast, $\mathrm{C}_{6}^{\prime} \mathrm{H}_{12} \mathrm{O}_{6}=2 \mathrm{C}_{2} \mathrm{H}_{5} \cdot \mathrm{OH}+2 \mathrm{CO}_{2}$. The most favorable temperature for this fermentation is about $25^{\circ} \mathrm{C}$. Besides the alcohol and 'carbon dioxide there are formed, especially at higher temperatures, small quantities of homologous alcohols (amyl-alcohol), glycerin, and succinic acid. In the presence of acid milk or cheese the grapesugar passes, especially in the presence of a base such as $\mathrm{ZnO}$ or $\mathrm{CaCO}_{3}$, into lactic-acid fermentation: $\mathrm{C}_{6} \mathrm{H}_{12} \mathrm{O}_{6}=2 \mathrm{C}_{3} \mathrm{H}_{6} \mathrm{O}_{3}$. (This process is even more complicated and $\mathrm{CO}_{2}$ is formed thereby, BouTRON, HUEPPE.) The lactic acid may then pass into butyric acidfermentation: $2 \mathrm{C}_{3} \mathrm{H}_{6} \mathrm{O}_{3}=\mathrm{C}_{4} \mathrm{H}_{8} \mathrm{O}_{2}+2 \mathrm{CO}_{2}+4 \mathrm{H}$.

Grape-sugar reduces several metallic oxides, such as copper oxide, bismuth oxide, mercuric oxide, in alkaline solutions, and the most important reactions for sugar are based on this fact.

Trommer's test is based on the property that glucose possesses of reducing copper-hydrated oxide in alkaline solution into suboxide. Treat the glucose solution with about $\frac{1}{5}-\frac{1}{8}$ vol. caustic soda and then carefully add a dilute copper-sulphate solution. The copper-hydrated oxide is thereby dissolved, forming a beautiful blue solution, and the addition of copper sulphate is continued until a very small amount of hydrate remains undissolved in the liquid. This is now warmed and a yellow hydrated suboxide or red suboxide separates even below the boiling-point. If too little copper salt has been added, the test will be yellowish brown in color as in MOoRE's test; but if an excess of copper-salt has been added, the excess of hydrate is converted on boiling into a dark-brown hydrate poor in water which interferes with the test. To prevent these difficulties the so-called FeHLing's solution may be employed. This reagent is obtained by mixing before use equal volumes of an alkaline solution of Rochelle salts and a copper-sulphate solution (see Quantitative Estimation of Sugar in the Urine in regard to concentration). This solution is not reduced or noticeably changed by boiling. The tartrate holds the excess of copperhydrate oxide in solution, and an excess of the reagent does not interfere in the performance of the test. In the presence of sugar this solution is reduced.

BöTTGER-ALMÉN's test is based on the property glucose possesses of reducing bismuth oxide in alkaline solution. The reagent best 
adapted for this purpose is obtained, according to NYLANDER's modification of AlMÉN's original test, by dissolving 4 grms. Rochelle salt in 100 parts caustic soda of $10 \% \mathrm{NaHO}$ and adding $2 \mathrm{grms}$. bismuth subnitrate and digesting on the water-bath until as much of the bismuth salt is dissolved as possible. If a glucose solution is treated with about $\frac{1}{10}$ vol., or with a larger quantity of the solution when large quantities of sugar are present, and boiled for a few minutes, the solution becomes first yellow, then yellowish brown, and lastly nearly black, and after a time a black deposit of bismuth (?) settles.

On heating with PHENYLHYDRAZIN a grape-sugar solution gives a precipitate consisting of fine yellow crystalline needles which are nearly insoluble in water but soluble in boiling alcohol, and which separate again on treating the alcoholic solution with water. The crystalline precipitate consists of phenylglucosazone (E. Fischer): $\mathrm{C}_{6} \mathrm{H}_{12} \mathrm{O}_{6}+2 \mathrm{C}_{6} \mathrm{H}_{6} \cdot \mathrm{N}_{2} \mathrm{H}_{3}=\mathrm{C}_{18} \mathrm{H}_{22} \mathrm{~N}_{4} \mathrm{O}_{4}+2 \mathrm{H}_{2} \mathrm{O}+2 \mathrm{H}$. This compound melts when pure at $204^{\circ}-205^{\circ} \mathrm{C}$.

Glucose is not precipitated by a lead-acetate solution, but is almost completely precipitated by an ammoniacal basic lead-acetate solution. On warming the precipitate becomes flesh-color or rosered.

If a watery solution of grape-sugar is treated with BENzoyLCHLORIDE and an excess of caustic soda and shaken until the odor of benzoylchloride has disappeared, a precipitate of benzoic-acid ester of glycose will be produced which is insoluble in water or alkali (BAUMANN).

If $\frac{1}{2}-1$ c. c. of a dilute watery solution of glucose is treated with a few drops of a 15\% alcoholic solution of $\alpha$-naphthol, the liquid is colored a beautiful violet on the addition of 1-2 c. c. concentrated sulphuric acid (MoLISCH). This reaction depends on the formation of furfurol from the sugar by the action of the sulphuric acid.

DiAzoBenzol-sULPHonic ACID gives with a sugar solution made alkaline with a fixed alkali a red color gradually changing after 10-15 minutes to violet (Penzoldt and Frscher). Orthonitrophenyl-propiolic aCID yields indigo when boiled with a small quantity of sugar and sodium carbonate, and this is converted into indigo-white by an excess of sugar (BAEYER). An alkaline solution of grape-sugar is colored deep red on being warmed with a dilute solution of PICRIC ACID (BRANN).

The detection of sugar in urine is ordinarily, in the presence of not too little sugar, a very simple opperation. The presence of 
only very small amounts may make its detection sometimes very difficult and laborious. A urine containing albumin must first have the albumin removed by coagulation with acetic acid and heat - before it can be tested for sugar.

The tests which are most frequently employed and are especially recommended are as follows:

Trommen's test. In a typical diabetic urine or one rich in sugar this test succeeds well, and it may be performed in the manner suggested on page 409. 'This test may lead to very great mistakes in urines poor in sugar, especially when they have at the same time normal or increased amounts of physiological constituents, and therefore it cannot be recommended to physicians or to persons little practised in such work. Normal urine contains reducible substances, such as uric acid, creatinin, and others, and therefore a reduction takes place with all urine on using this test. We generally do not have a separation of copper suboxide, but still if we vary the proportion of the alkali to the copper sulphate and boil, we often have an actual separation of suboxide in normal urines, or we obtain a peculiar yellowish-red liquid due to finelydivided hydrated suboxide. This occurs especially on the addition of much alkali or too much copper sulphate, and by careless manipulation the inexperienced worker may therefore sometimes obtain apparently positive results in a normal urine. Also as urine contains substances such as creatinin and ammonia (from the urea), which in the presence of only little sugar may keep the copper suboxide in solution, therefore in inexperienced hands small amounts of sugar may be overlooked.

Trommer's test may indeed be made positive and useful, even in the presence of very small amounts of sugar, by using the modification suggested by WORM MÜLLER. As this modification is rather complicated, and besides this requires much practice and exactness, it is probably rarely employed by the busy physician. The following test is to be preferred:

ALMÉN's bismuth test, which recently has been incorrectly called NYLANDER's test, is performed with the alkaline bismuth solution prepared as above described (page 410). For each test 10 c. c. of urine are taken and treated with 1 c. c. of the bismuth solution and boiled for a few minutes. In the presence of sugar the urine becomes darker yellow or yellowish brown. Then it grows darker, cloudy, dark brown, or nearly black, and non-transparent. After a shorter or longer time a black deposit appears, the supernatant liquid gradually clears, but, still remains colored. In the presence of only very little sugar the test is not black or dark brown, but is only deeper-colored, and after a longer time we only see on the upper layer of the phosphate precipitate a dark or black edge (of bismuth?). In the presence of much sugar a larger amount of 
reagent may be used without disadvantage. In a urine poor in sugar we must only use 1 c. c. of the reagent for every 10 c. c. of the urine.

This test shows the presence of $1-0.5 \mathrm{p} . \mathrm{m}$. sugar in the urine. The sources of error which interfere in 'Irommen's test, such as the presence of uric acid and creatinin, entirely disappear in this test. The bismuth test is, besides this, more easily performed, and it is therefore to be recommended to the physician. Small amounts of albumen do not interfere with this test; large amounts may give rise to an error by forming bismuth sulphide, and therefore must be removed by coagulation.

In using this method it must not be overlooked that it is, like Trommer's test, a reduction test, and it consequently may show, besides sugar, certain other reducing substances. Such bodies are certain coupled glycuronic acids which may appear in the urine. SALKOWsKI obtained a bluish-black precipitate with this reagent in a urine after the use of rhubarb, and black precipitates have been obtained with the bismuth test after the use of turpentine and certain other medicines. From this it follows that we should, especially when the reduction is not very great, never be satisfied with this test alone. As a control at least one of the following tests must be performed. Among these the fermentation test is of special value.

Fermentation Test. On using this test we must proceed in various ways, according as the bismuth test gives small or large results. If a rather strong reduction is obtained, the urine may be treated with yeast and the presence of sugar determined by the generation of carbon dioxide. In this case the acid urine, or otherwise faintly acidified with tartaric acid, is treated with yeast which has previously been washed by decantation with water. Pour this urine to which the yeast has been added into a ScHRöTteR's gas-burette, or glass tube with the open end ground, close with the thumb, and open under the surface of mercury contained in a dish. As the fermentation proceeds, the carbon dioxide collects in the upper part of the tube, while a corresponding quantity of liquid is expelled below. As a control in this case, two other similar tests must be made, one with normal urine and yeast to learn the amount of gas usually developed, and the other with a sugar solution and yeast to determine the activity of the yeast.

If, on the contrary, we find only a faint reduction with the bismuth test, no positive conclusion can be drawn from the absence of any carbon dioxide or the appearance of a very insignificant amount. In this case proceed in the following way: Treat the acid urine, or the urine which has been faintly acidified with tartaric acid, with yeašt whose activity has been tested by a special test on a sugar solution, and allow it to stand 24-48 hours at the 
temperature of the room, or, better, at a little higher temperature. After this time test again with the bismuth test, and if the reaction now gives negative results, then sugar was previously present. - But if the reaction continues to give positive results, then it shows -if the yeast is active - the presence of other reducing, fermentable bodies. There may indeed be a possibility that the urine also contains some sugar besides these bodies. This possibility is decided by the following test :

Phenylhydrazin Test. According to $\mathrm{v}$. JAKscH, this test is performed in the following way: Add in a test-tube containing 8-10 c. c. of the urine two knife-points of phenylhydrazin hydrochloride and three knife-points sodium acetate, and when the added salts do not dissolve on warming add more water. The mixture is heated in boiling water and kept there for one hour to avoid a confusion with phenylhydrazin-glycuronic acid ( $\mathrm{v}$. JAKSCH and HIRSCHL). It is then poured into a beaker of cold water. If the quantity of sugar present is not too small, a yellow crystalline precipitate is now obtained. If the precipitate appears amorphous, then on looking at it under the microscope it appears partly as single and partly as groups of yellow needles. If very little sugar is present, pour the test into a conical glass and examine the sediment. In this case at least a few phenylglucosazone crystals are found, while the occurrence of smaller and larger yellow plates or highly-refrac. tive brown globules do not show the presence of sugar. According to $\nabla$. $J_{\mathrm{AKSCH}}$, this reaction is very reliable, and by it the presence of 0.3 p. m. sugar can be detected (Rosenberg, GEYer). A confounding with glycuronic acid is, according to HIRSCHL, not to be apprehended when it is not heated in the water-bath for too short a time (one hour). In doubtful cases where we wish to be quite sure, prepare the crystals from a large quantity of urine, dissolve them on the filter by pouring over them hot alcohol, treat the filtrate with water, and boil off the alcohol. If the characteristic yellow crystalline needles, whose melting-point $\left(204^{\circ}-205^{\circ} \mathrm{C}\right.$.) is also determined, are now obtained, then this test is quite decisive.

Polarization. The polariscopic investigation is of great value, especially as in many cases it quickly differentiates between sugar and other reducible, often lævo-gyrate substances. In the presence of only very little sugar the value of this test depends on the delicacy of the instrument and the dexterity of the observer; therefore this method is perhaps inferior in most cases to the bismuth test or to the phenylhydrazin test.

If small quantities of sugar are to be isolated from the urine, precipitate the urine first with sugar of lead, filter, precipitate the filtrate with ammoniacal lead acetate, wash this precipitate with water, decompose it with $\mathrm{H}_{2} \mathrm{~S}$ when suspended in water, concentrate the filtrate, treat it with strong alcohol until it is $80 \mathrm{vol}$. per cent, filter 
when necessary, and add an alcoholic caustic-alkali solution. Dissolve the precipitate consisting of saccharates in a little water, precipitate the potash by an excess of tartaric acid, neutralize the filtrate with calcium carbonate in the cold, and filter. 'The filtrate may be used for testing with the polariscope as well as in the fermentation, bismuth, and phenylhydrazin tests. The presence of grape-sugar may be detected by this same process in animal fluids or tissues, from which the albumins have first been removed by coagulation or by the addition of alcohol.

For the physician, who naturally wants specially simple and quick methods, the bismuth test must be especially recommended, as this may be controlled when necessary by the fermentation or phenylhydrazin test.

Other tests for sugar, as, for example, the reaction with orthonitrophenylpropiolic acid, picric acid, diazobenzol-sulphonic acid, are superfluous. The reaction with $\alpha$-naphthol, which is a reaction for carbohydrates in general, for glycuronic acid and mucin, may, because of its extreme delicacy, give rise to nistakes, and is therefore not to be recommended to physicians.

Quantitative Estimation of Sugar in the urine. The urine for such an estimation must first be tested for albumin, and if any be present it must be removed by coagulation and the addition of acetic acid, care being taken not to increase or diminish the original volume of urine. 'The quantity of sugar may be determined by TITRATION with FEHLING's or KNAPP's solution, by FERMENTATION or by POLARIZATION.

The titration liquids not only react with sugar, but also with certain other reducing substances, and on this account the titration methods give rather high results. When large quantities of sugar are present, as in typical diabetic urine, which generally contains a lower percentage of normal reducing constituents, this is indeed of little account; but when small amounts of sugar are present in an otherwise normal urine, the mistake may, on the contrary, be . important, as the reducing power of normal urine may correspond to $4 \mathrm{p}$. m. grape-sugar (see page 374). In such cases the titration method must be employed in connection with the fermentation method, which will be described later. It is to be remarked that in typical diabetic urines with considerable amounts of sugar the titration with FEHLING's solution is just as reliable as with KNAPP's solution. When the urine, on the contrary, contains only little sugar with normal amounts of physiological constituents, then the titration with FeHLING's solution is more difficult, indeed in certain cases almost impossible, the results being very uncertain. In such cases KNAPP's method gives good results, according to WoRM MüLLER and his pupils.

The titration with Fehling's solution depends on the power of sugar to reduce copper oxide in alkaline solutions. For this we 
formerly employed a solution which contained a mixture of copper sulphate, Rochelle salt, and sodium or potassium hydrate ( $\mathrm{F}$ EHLING's solution); but as such a solution readily changes, we now prepare a copper-sulphate solution and an alkaline Rochelle-salt solution separately, and mix equal volumes of the two solutions before using.

The concentration of the copper-sulphate solution is such that 10 c. c. of this solution is reduced by $0.05 \mathrm{grm}$. grape-sugar. 'The copper-sulphate solution contains 34.65 grms. pure, crystallized, nonefflorescent copper sulphate in 1 litre. The sulphate is crystallized from a hot siturated solution by cooling and stirring; and the crystals are separated from the mother-liquor and pressed between blotting-paper until dry. 'The Rochelle-salt solution is prepared by dissolving 173 grms. of the salt in 350 c. c. water, adding 600 c. c. of a caustic-soda solution of a specific gravity of 1.12 , and diluting with water to 1 litre. According to WORM MÜLLER, these three liquids-Rochelle-salt solution, caustic soda, and watershould be separately boiled before mixing together. For each titration mix in a small flask or porcelain dish exactly 10 c. c. of the copper-sulphate solution and 10 c. c. of the alkaline Rochellesalt solution and add 30 c. c. water.

The urine free from albumin is diluted before the titration with water so that 10 c. c. of the copper solution requires between 5 and $10 \mathrm{c}$. c. of the diluted urine, which corresponds to between 1. and $\frac{1}{2} \%$ sugar. A urine of a specific gravity of 1.030 may be diluted five times; one more concentrated, ten times. The urine so diluted is poured into a burette and allowed to flow into the boiling copper-sulphate and Rochelle-salt solution until the copper oxide is completely reduced. This has taken place when, after boiling, the blue color of the solution disappears. It is very difficult and requires some practice to exactly determine this point, especially when the copper suboxide settles with difficulty. To determine whether the color has disappeared, allow the copper suboxide to settle a little below the meniscus formed by the surface of the liquid. If this layer is not blue, the operation is repeated, adding 0.1 c. c. less of urine; and if, after the copper suboxide has settled, the liquid has a blue color, the titration may be considered as completed. Because of the difficulty in obtaining this point exactly another end-reaction has been suggested. This consists in filtering immediately after boiling a small portion of the treated urine through a small filter into a test-tube which contains a little acetic acid and a few drops of potassium-ferrocyanide solution and water. The smallest quantity of copper is shown by a red coloration. If the operation is quickly conducted so that no oxidation of the suboxide into oxide takes place, this end-reaction is of value for urines which are rich in sugar and poor in urea and which 
have been strongly diluted with water. In urines poor in sugar which contain the normal amount of urea and which have not been strongly diluted, a rather abundant formation of ammonia from the urea may take place on boiling the alkaline liquid. This ammonia dissolves the suboxide in part, which easily passes into oxide thereby, and besides this the dissolved suboxide gives a red color with potassium ferrocyanide. In just those cases in which the titration is most difficult this end-reaction is the least reliable. Practice also renders it unnecessary, and it is therefore best to depend simply upon the appearance of the liquid.

To facilitate the settling of the copper suboxide and thereby clearing the liquid, MUNK has lately suggested the addition of a little calcium-chloride solution and boiling again. A precipitate of calcium tartrate is produced which carries down the suspended copper suboxide with it, and the color of the liquid can then be better seen. This artifice succeeds in many cases, but unfortunately there are urines in which the titration with FeHLING's solution in no way gives exact results.

The necessary conditions for the success of the titration under all circumstances are, according to SoxHLET, the following: The copper-sulphate and Rochelle-salt solution must, as above, be diluted to 50 c. c. with water; the urine must only contain between $0.5 \%$ and 1\% sugar, and the total quantity of urine required for the reduction must be added to the titration liquid at once and boiled with it. From this last condition it follows that the titration is dependent upon minute details, and several titrations are required for each determination.

It is best to give here directions for the titration. The proper amount of copper-sulphate and Rochelle-salt solution and water (total volume $=50$ c. c.) is heated to boiling in a flask: the color must remain blue. The urine diluted five times is now added to the boiling-hot liquid, 1 c. c. at a time; after each addition of urine boil for a few seconds, and look for the appearance of the end-reaction. If you find, for example, that $3 \mathrm{c}$. c. is too little but that $4 \mathrm{c}$. c. is too much (the liquid becoming yellowish), then the urine has not been sufficiently diluted, for it should require between 5 and $10 \mathrm{c.c}$. of the urine to produce the complete reduction. The urine is now diluted ten times, and it should now require between 6 and $8 \mathrm{c}$. c. for a total reduction. Now prepare four new tests, which are boiled simultaneously to save time, and add at one time respectively $6,6 \frac{1}{2}, 7$, and $7 \frac{1}{2}$ c. c. of urine. If it is found that between $6 \frac{1}{2}$ and $7 \mathrm{c}$. c. are necessary to produce the end-reaction, then make four other tests, to which add respectively $6.6,6.7,6.8$, and 6.9 c. c. of urine. If in this case the liquid is still somewhat bluish with 6.7 c. c. and completely decolorized with 6.8 c. c., we then consider the average figure 6.75 c. c. as correct. 
The calculation is simple. The 6.75 c. c. used contain $0.05 \mathrm{grm}$. sugar, and the percentage of sugar in the dilute urine is therefore $(6.75: 0.05=100: x)=\frac{5}{6.75}=0.74$. But as the urine was diluted with ten times its volume of water, the undiluted urine contained $\frac{5 \times 10}{6.75}=7.4 \%$. The general formula on using $10 \mathrm{c}$. c. copper-sulphate solution is therefore $\frac{5 \times n}{k}$, in which $n$ represents the number of times the urine has been diluted and $k$ the number of c. c. used for the titration of the diluted urine.

The TITRATION ACCORDING To KNaPP depends on the fact that mercuric cyanide is reduced into metallic mercury by grape-sugar. The titration liquid should contain 10 grms. chemically pure dry mercuric cyanide and 100 c. c. caustic-soda solution of a specific gravity of 1.145 per litre. When the titration is performed as described below (according to WORM MüLLER and OTTO), 20 c. c. of this solution should correspond to exactly 0.05 grm. grape-sugar.

Also in this titration the quantity of sugar in the urine should be between $\frac{1}{2} \%$ and $1 \%$, and here also the extent of dilution necessary must be determined by a preliminary test. To determine the endreaction as described below, the test for excess of mercury is made with sulphuretted hydrogen.

In performing the titration allow $20 \mathrm{c}$. c. of KNAPP's solution to flow into a flask and dilute with $80 \mathrm{c}$. c. water, or when you have reason to think that the urine contains less than $0.5 \%$ of sugar, then only with 40-60 c. c. After this heat to boiling and allow the dilute urine to flow gradually into the hot solution, at first 2 c. c., then $1 \mathrm{c}$. c., then 0.5 c. c., then 0.2 c. c., and lastly 0.1 c. c. After each addition let it boil $\frac{1}{2}$ minute. When the end-reaction is approaching, the liquid begins to clarify and the mercury separates with the phosphates. The end-reaction is determined by taking a drop of the upper layer of the liquid into a capillary tube and then blowing it out on pure white filter-paper. The moist spot is first held over a bottle containing fuming hydrochloric acid and then over strong sulphuretted hydrogen. The presence of a minimum quantity of mercury-salt in the liquid is shown by the spot becoming yellowish, which is seen best when it is compared with a second spot which has not been exposed to sulphuretted hydrogen. The end-reaction is still clearer when a small part of the liquid is filtered, acidified with acetic acid, and tested with sulphuretted hydrogen (ОTTO). The calculations are just as simple as for the previous method.

This titration, unlike the previous one, may be performed not only in daylight, but also in artificial light. KNAPP's method has 
the following advantages over FeHLING's method. It is applicable even when the quantity of sugar in the urine is very small and the amount of the other urinary constituents is normal. It is more easily performed, and the titration liquids may be kept without decomposing for a long time (WORM MÜLLER and his pupils). 'The views of different investigators on the value of this titration method are still somewhat contradictory.

Estimation of the Quantity of Sugar by Fermentation. This may be done in various ways ; the simplest, and at the same time sufficiently exact for ordinary cases, is RoBERTs' method. This method consists in determining the specific gravity of the urine before and after fermentation. In the fermentation of sugar, carbon dioxide and alcohol are formed as chief products and the specific gravity is lowered, partly on account of the disappearance of the sugar and partly on account of the production of alcohol. RoBERTs found, and this has been substantiated later by several other investigators (WORM MÜLLER and others), that a decrease of 0.001 in the specific gravity corresponded to $0.23 \%$ sugar. If the urine, for example, had a specific gravity of 1.030 before fermentation and 1.008 after, then the quantity of sugar contained therein was $22 \times 0.23=5.06 \%$.

In performing this test the specific gravity must be taken at the same temperature before and after the fermentation. The urine must be faintly acid, and when necessary it should be acidified with a little tartaric-acid solution. The activity of the yeast must, when necessary, be controlled by a special test. Place $200 \mathrm{c}$. c. of the urine in a 400-c. c. flask and add a piece of compressed yeast the size of a pea, and subdivide the yeast through the liquid by shaking, close the flask with a stopper provided with a finely drawn-out open glass tube, and allow the test to stand at the temperature of the room, or still better at $+20-25^{\circ} \mathrm{C}$. After $24-48$ hours the fermentation is ordinarily ended, but this must be verified by the bismuth test. After complete fermentation filter through a dry filter, bring the filtrate to the proper temperature, and determine the specific gravity.

If the specific gravity be determined with a good pyknometer supplied with a thermometer and an expansion-tube, this method, when the quantity of sugar is not less than 4-5 p. m., gives, according to WORM MüLLER, very exact results, but this has been disputed by BUDDE. For the physician this method in this form is not serviceable. Even if the specific gravity is determined by a delicate urinometer which can give the density to the fourth decimal, we do not obtain quite exact results because of the principal errors of the method (BUDDE); but the error is usually smaller than those which occur in titrations made by unpractised hands. Among the methods proposed and closely tested for the quantitative esti- 
mation of sugar, we have none which are at the same time easily performed and which give positive results in other than practised hands.

When the quantity of sugar is less than $5 \mathrm{p} . \mathrm{m}$. these methods cannot be used. Such a small amount of sugar cannot, as above mentioned, be determined by titration directly, because the reduction power of normal urine corresponds to 4-5 p. m. In such cases, according to WORM MÜLLER, first determine the reduction power of the urine by titration with KNAPP's solution, then ferment the urine with the addition of yeast, and titrate again with KNAPP's solution. The difference found between the two titrations calculated as sugar gives the true amount of sugar.

Estimation of Sugar by Polarization. In this method the urine must be clear, not too deeply colored, and, above all, must not contain any other optically-acting substances besides glucose. By using a delicate instrument and with sufficient practice very exact results can be obtained by this method (K. MöRNER, H. HUPPERT). For the physician, RoBers' fermentation test, which requires no expensive apparatus and no special practice, is to be preferred. Under such circumstances, and as the estimation by means of polarization can be performed with exactitude only by speciallyinstructed chemists, it is hardly necessary to give this method in detail, and the reader is referred to special works for instructions in the use of the apparatus.

Levulose. Lævo-gyrate urines containing sugar have been observed by Ventzke, Zimmer and Czapek, SeEgen, and others. The nature of the substance causing this action is difficult to exactly describe, but there is hardly any doubt that the urine, at least in certain cases, as in those observed by SEEGEN, contains levulose. LEO once found in a diabetic urine a lævo-gyrate, reducible, non-fermentable, and non-crystallizable substance which was considered by him as a peculiar variety of sugar.

The presence of levulose in a urine containing sugar is only probable when the urine is lævo-gyrate or optically inactive, or when it shows a reduction power not corresponding (less) to the dextro-rotary power, or when it contains no other lævo-gyrate substances ( $\beta$-oxybutyric acid, coupled glycuronic acids, protein bodies, or cystin).

MILK-SUGAR. The appearance of milk-sugar in the urine of nursing mothers has been made known especially by the investigations of De Sinety and F. Hofmeister. Aftcr taking large quantities of milk-sugar some lactose was found in the urine by WorM MüLleR, and DE Jong found also glucose under the same circumstances.

The positive detection of milk-sugar in the urine is difficult, because this sugar is, like glucose, dextro-gyrate and also gives the usual reduction tests. If urine contains a dextro-gyrate, non-fer- 
mentable sugar which reduces bismuth solutions, then it is very probable that it contains milk-sugar. The most certain means for its detection is to isolate the sugar from the urine. This may be done by the following method, suggested by F. HoFmeister.

Precipitate the urine with sugar of lead, filter, wash with water, unite the fitrate and wash-water, and precipitate with ammonia. The liquid filtered from the precipitate is again precipitated by sugar of lead and ammonia, until the last filtrate is optically inactive. 'The several precipitates with the exception of the first, which contains no sugar, are united and washed with water. The washed precipitate is decomposed in the cold with sulphuretted hydrogen and filtered. The excess of sulphuretted hydrogen is driven off by a current of air ; the acids set free are removed by shaking with silver oxide. Now filter, remove the dissolved silver by sulphuretted hydrogen, treat with barium carbonate to unite with any free acetic acid present, and concentrate. Before the evaporated residue is syrupy it is treated with $90 \%$ alcohol until a flocculent precipitate is formed which settles quickly. The filtrate from this when placed in a desiccator deposits crystals of milk-sugar, which are purified by recrystallization, decolorizing with animal charcoal and boiling with $60-$ $70 \%$ alcohol.

INOSIT occurs only rarely, and only in small quantities, in the urine in albuminuria and in diabetes mellitus. After excessive drinking of water inosit is found in the urine. According to HOPPE-SEYLER, traces of inosit occur in all normal urines.

In detecting inosit the albumin is first removed from the urine. Then concentrate the urine on the water-bath to $\frac{1}{4}$ and precipitate with sugar of lead. The filtrate is warmed and treated with lead acetate as long as a precipitate is formed. The precipitate formed after 24 hours is washed with water, suspended in water, and decomposed with sulphuretted hydrogen. A little aric acid may separate from the filtrate after a short time. The liquid is filtered, concentrated to a syrupy consistency, and treated while boiling with 3-4 vols. alcohol. The precipitate is quickly separated. After the addition of ether to the cooled filtrate, crystals separate after a time, and these are purified by decolorization and recrystallization. with these crystals perform the tests mentioned on page 258 .

Aceton and Aceto-acetic Acid. These bodies were first observed in urine in diabetes mellitus (Peters, KA ULICH, v. JAKSCH, GERHARDT). Both of these bodies occur sometimes simultaneously in diabetic urine, sometimes only one of them. Acetone may give the diabetic urine as well as the expired air the odor of apples or fruit. According to $\mathrm{v}$. $\mathrm{JAKSCH}_{\mathrm{A}}$, acetone is a normal urinary con. stituent, though it may only occur in very small amounts $(0.1 \mathrm{grm}$. 
in the 24 hours). LE NOBEL claims that it only appears in healthy urine after taking alcohol or after food rich in proteids. Acetone as well as aceto-acetic acid seems to be a decomposition product of the albumins, and acetonuria may be caused by food very rich in proteids.

In regard to the occurrence of these bodies under diseased conditions, a great many observations have been made, especially by v. Jaksch, Kaulich, Cantani, Deichmüller, Frerichs, Penzoldt, Le Nobel, Seifert, Gerhardt, and others. Acetonuria occurs in certain cases of diabetes, and especially in such pathological processes as are accompanied by an increased destruction of the tissues. It also occurs in fevers, in cachectic diseases, sometimes in cancer of the organs of digestion, also in inanition and in psychosis. Acetonuria occurs especially often in children.

Aceto-acetic acid never occurs in the urine as a physiological constituent, but appears under the same circumstances as acetone. It occurs frequently in children, in high fevers, acute exanthema. etc.

Acetone, dimethyl ketone, $\mathrm{C}_{3} \mathrm{H}_{6} \mathrm{O}$ or $\mathrm{CO} .\left(\mathrm{CH}_{3}\right)_{2}$, is a thin waterclear liquid boiling at $56.5^{\circ} \mathrm{C}$. and with a pleasant odor of fruit. It is lighter than water, with which it mixes in all proportions, also with alcohol and ether. The most important reactions for acetone are the following:

LIEBEN's Iodoform Test. When a watery solution of acetone is treated with alkali and then with some iodine-potassium-iodide solution and gently warmed a yellow precipitate of iodoform is formed, which is known by its odor and by the appearance of the crystals (six-sided plates or stars) under the microscope. This reaction is very delicate, but it is not characteristic of acetone. GunNing's modification of the iodoform test consists in using an alcoholic solution of iodine and ammonia instead of the iodine dissolved in potassium iodide and alkali hydrate. In this case, besides iodoform, a black precipitate of iodide of nitrogen is formed, but this gradually disappears on standing, leaving the iodoform visible. This modification has the advantage that it does not give any iodoform with alcohol. On the other hand, it is not quite so delicate, but still it detects 0.01 milligramme acetone in $1 \mathrm{cc}$.

REYNOLD'S mercuric-oxide test is based on the power of acetone 
to dissolve freshly-precipitated $\mathrm{HgO}$. A mercuric-chloride solution is precipitated by alcoholic caustic potash. To this add the liquid to be tested for acetone, shake well and filter. In the presence of acetone the filtrate contains mercury, which may be detected by ammonium sulphide. This test has about the same delicacy as GUNNING's test.

LEGAL's Sodium-nitroprusside Test. If an acetone solution is treated with a few drops of a freshly-prepared sodium-nitroprusside solution and then with caustic-potash or soda solution, the liquid is colored ruby-red. Creatinin gives the same color; but if we saturate with acetic acid, the color becomes carmine or purplish red in the presence of acetone, but yellow and then gradually green and blue in the presence of creatinin. If we use ammonia instead of the caustic alkali (LE NOBEL), the reaction takes place with acetone but not with creatinin. LEGAL's test indicates even 0.1 milligl'm. acetone.

Penzoldt's indigo test depends on the fact that orthonitrobenzaldehyde in alkaline solution with acetone yields indigo. A warm saturated and then cooled solution of the aldehyde is treated with the liquid to be tested for acetone and then with caustic soda. In the presence of acetone the liquid first becomes yellow, then green, and lastly indigo separates; and this may be dissolved with a blue color by shaking with chloroform. 1.6 milligrms. acetone can be detected by this test.

Aceto-acetic Acid, or Diacetic AcID, $\mathrm{C}_{4} \mathrm{H}_{6} \mathrm{O}_{3}$ or $\mathrm{C}_{2} \mathrm{H}_{3} \mathrm{O} . \mathrm{CH}_{2}$. $\mathrm{COOH}$. This acid is a colorless, strongly-acid liquid which mixes with water, alcohol, and ether in all proportions. On heating to boiling with water, and especially with acids, this acid decomposes into carbon dioxide and acetone, and therefore gives the abovementioned reactions for acetone. It differs from acetone in that it gives a violet-red or brownish-red color with a dilute ferric-chloride solution. This color decreases even at the ordinary temperature within 24 hours, and more quickly on boiling. It differs in this from phenol, salicylic acid, acetic acid, or sulphocyanides.

Detection of Acetone and Aceto-acetic Acid in the urine. Before testing for aceto-acetic acid test for acetone, and as this acid gradually decomposes on allowing the urine to stand, the urine must be as fresh as possible. In the presence of aceto-acetic acid the urine 
gives the so-called GerhardT's reaction, showing a wine-red color on the addition of a dilute, not too acid, ferric-chloride solution. Treat $10-50 \mathrm{c}$. c. of the urine with ferric chloride as long as it gives a precipitate, filter the precipitate of ferric phosphate, and add some more ferric chloride to the filtrate. In the presence of the acid a claret-red color is produced. After this heat a second, similar portion of the urine to boiling until faint acid reaction, and repeat the test on cooling, which should now give negative results. A third portion of urine is acidified with sulphuric acid and shaken with ether (which takes up the acid). Now shake the removed ether with a very dilute watery solution of ferric chloride, and the watery layer becomes violet-red or claret-red. The color disappears on warming.

In the absence of aceto-acetic acid the acetone may be tested for directly. This may be done directly on the urine by LEGAL's and Penzoldt's tests. 'These tests, which are only approximate tests, are only of value when the urine contains a considerable amount of acetone. For a more accurate test we distil at least $250 \mathrm{c}$. c. of the urine faintly acidified with sulphuric acid, care being taken to have a good condensation. Most of the acetone is contained in the first $10-20$ c. c. of the distillate. This distillate is tested for acetone by the above tests. In testing for acetone in the simultaneous presence of aceto-acetic acid, first make the urine faintly alkaline, and shake it carefully with ether free from alcohol and acetone in a separatory funnel. The removed ether is then shaken with water, which takes up the actone, and then the watery liquid is tested.

$\beta$-0xybutyric Acid, $\mathrm{C}_{4} \mathrm{H}_{8} \mathrm{O}_{3}$ or $\mathrm{CH}_{3} \cdot \mathrm{CH}(\mathrm{OH}) \cdot \mathrm{CH}_{2} \cdot \mathrm{COOH}$. The appearance of this acid in the urine was first positively shown by Mrnkowski, Külz and Stadelamans. It occurs especially in all difficult cases of diabetes, but it has also been observed in scarlet fever and in measles, in scurvy, and in diseases of the brain. $\beta$-oxybutyric acid is accompanied by aceto-acetic acid in the urine.

$\beta$-oxybutyric acid forms an odorless syrup which mixes readily with water, alcohol, and ether. This acid is optically active and indeed lævo-gyrate, and it therefore interferes with the estimation of sugar in the urine by means of polarization. It is not precipitated either by lead acetate or by ammoniacal basic lead acetate. On boiling with water, especially in the presence of a mineral acid, this acid decomposes into $\alpha$-CROTONIC ACID, which melts at $71^{\circ}-72^{\circ} \mathrm{C}$., and water: $\mathrm{CH}_{3} \cdot \mathrm{CH}(\mathrm{OH}) \cdot \mathrm{CH}_{2} \cdot \mathrm{COOH}=\mathrm{H}_{2} \mathrm{O}+\mathrm{CH}_{3} \cdot \mathrm{CH}$ : 
CH.COOH. It yields acetone on oxidation with a chromate mixture.

Detection of $\beta$-Oxybutyric Acid in the urine. If a urine is still lævo-gyrate after fermentation with yeast, the presence of oxybutyric acid is probable. A further test may be made, according to KULZ, by evaporating the fermented urine to a syrup, and, after the addition of an equal volume of concentrated sulphuric acid, distilling directly without cooling. $\alpha$-crotonic acid is produced which is distilled, and after strongly cooling the distiilate is collected in a glass; crystals, which melt at $+72^{\circ} \mathrm{C}$., separate. If no crystals are obtained, then shake the distillate with ether, and test the melting-point with the residue, which has been washed with the water obtained after evaporating the ether. According to MrNKowSKI, the acid may be isolated as a silver-salt (see SCHMIEDEBERG's Archiv, 18, 35, or FresenIUs' Zeitschrift, 24, 153).

EHRLICH's Urine Test. Mix 250 c. c. of a solution which contains 50 c. c. $\mathrm{HCl}$ and $1 \mathrm{grm}$. sulphanilic acid in one litre with 5 c. c. of a $\frac{1}{2} \%$ solution of sodium nitrite (which produces very little of the active body, sulphodiazobenzol). In performing this test treat the urine with an equal volume of this mixture, and then supersaturate with ammonia. Normal urine will become yellow thereby, or orange after the addition of ammonia (aromatic oxyacids may sometimes give after a certain time red azo bodies which color the upper layer of phosphate sediment). In pathological urines we sometimes have (and this is the characteristic diazo reaction) a primary yellow coloration, with a very marked secondary red coloration on the addition of ammonia, and the froth is also tinged with red. The upper layer of the sediment becomes greenish. The body which gives this reaction is unknown, but it occurs especially in the urine of typhus patients (EHRLICH). Views are divided in regard to the significance of this reaction (Ehrlich, Penzoldt, Petri, Escherik).

FAT in the urine. The elimination of a urine which in appearance and richness in fat resembles chyle is called chyluria. It contains habitually albumin, and often fibrin. Chyluria occurs mostly in the inhabitants of the tropics. Lipuria, or the elimination of fat with the urine, may appear in apparently healthy persons, sometimes with and sometimes without albuminuria, in pregnancy, and also in certain diseases, as in diabetes, poisoning with phosphorus, and fatty degeneration of the kidneys.

Fat is usually detected by the microscope. It may also be dissolved with ether, and it may always be detected by evaporating the urine to dryness and extracting the residue with ether.

ChOLESTERIN is also sometimes found in the urine in chyluria and in a few other cases.

Leucin and Tyrosin. These bodies are found in the urine, especially in acute yellow atrophy of the liver, in acute phosphoruspoisoning, and in difficult cases of typhus and smallpox.

Detection of leucin and tyrosin. Tyrosin occurring as sediment may be identified by means of the microscope ; but if a positive proof is desired, a recrystallization of the same from ammonia or ammoniacal alcohol is necessary. 'To detect both these bodies when they occur in solution in the urine, pro- 
ceed in the following manner: The urine free from albumin is precipitated by basic lead acetate, the lead removed from the filtrate by $\mathrm{H}_{2} \mathrm{~S}$, and concen. trated as much as possible. The residue is extracted with a small quantity of absolute alcohol to remove the urea. The residue is then boiled with faintly. ammoniacal alcohol, filtered, the filtrate evaporated to a small volume and allowed to crystallize. If no tyrosin crystals are obtained then dilute with water, precipitate again with lead acetate, and proceed as before. If tyrosin crystals now separate, they are filtered and the filtrate still further concentrated to obtain the leucin crystals.

Cystin, $\left(\mathrm{C}_{3} \mathrm{H}_{6} \mathrm{NSO}_{2}\right)_{2}$. This body is, according to BAUMANN, to

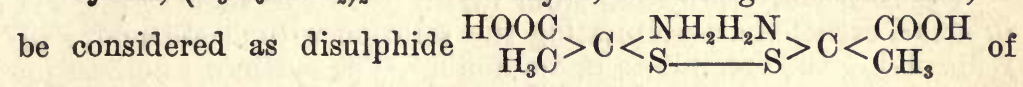
the previously-mentioned cystein, $\mathrm{C}_{3} \mathrm{H}_{7} \mathrm{NSO}_{2}$ (page 393). This last substance, containing sulphur, is considered by BAUMANN as pyrotartaric acid, whose ketone-oxygen atom is replaced by both the monatomic groups $\mathrm{NH}_{2}$ and $\mathrm{SH}$ or $\underset{\mathrm{HS}}{\mathrm{H}_{2} \mathrm{~N}}>\mathrm{S}<\mathrm{CH}_{3}$.

BAUMANN and GoLDManN claim that a substance similar to cystin occurs in very small amounts in normal urine. Cystin itself is found with positiveness only, and even then very rarely, in urinary calculi and in pathological urines, from which it may separate as a sediment. Cystinuria occurs oftener in men than in women. Cystin seems to be an abnormal splitting product of the albumins. BAUMANN and v. UDRANSzKY found in urine in cystinuria the two diamins, cadaverin (pentamethylen-diamin) and putrescin (tetramethylen-diamin), which are produced in the putrefaction of albumin (BRIEGER). These two diamins were also found in the contents of the intestine in cystinuria; under other conditions they are not usually present. The author, therefore, considers that some connection exists between the formation of diamins in the intestine by the peculiar putrefaction in cystinuria, and cystinuria itself. Diamins have also been found in the contents of the intestines in cystinuria by STADTHAGEN and BRIEgEr.

Cystin crystallizes in thin, colorless, six-sided plates. It is not soluble either in water, alcohol, ether, or acetic acid, but dissolves in mineral acids and oxalic acid. It also dissolves in alkalies and in ammonia, but not in ammonium carbonate. Cystin is optically active and strongly læro-rotary. If cystin is boiled with caustic alkali it decomposes, yielding among other products alkali sulphides, which may be detected by lead acetate or sodium nitroprusside. On 
treating cystin with tin and hydrochloric acid, only a little sulphuretted hydrogen is evolved and cystein is produced. On shaking a solution of cystin in an excess of caustic soda with benzoyl-chloride a voluminous precipitate of benzoyl-cystin is produced (BAUMANN and Goldmans). On heating on a platinum foil, cystin does not melt but ignites and burns with a bluish-green flame accompanied by a peculiar sharp odor.

Cystin is easily prepared from cystin calculi by dissolving them in alkali carbonate, precipitating the solution with acetic acid, and redissolving the precipitate in ammonia. The cystin crystallizes on the spontaneous evaporation of the ammonia. The cystin dissolved in the urine is detected, in the absence of albumin and sulphuretted hydrogen, by boiling with alkali and testing with lead salt or sodium nitroprusside. To isolate cystin from the urine, acidify the urine strongly with acetic acid. The precipitate containing cystin is collected after 24 hours and digested with hydrochloric acid, which dissolves the cystin and calcium oxalate, leaving the uric acid undissolved. Filter, supersaturate the filtrate with ammonium carbonate, and treat the precipitate with ammonia, which dissolves the cystin and leaves the calcium oxalate. Filter again and precipitate with acetic acid. The precipitated cystin is identified by the microscope and the above-mentioned reactions. Cystin as a sediment is identified by the microscope. It must be purified by dissolving in ammonia and precipitating with acetic acid and then tested. Traces of dissolved cystin may be detected by the production of benzoyl-cystin, according to BAUMANN and GoldMANN.

\section{Urinary Sediments and Calculi.}

Urinary sediment is the more or less abundant deposit which is found in the urine after standing. 'This deposit may consist partly of organized and partly of non-organized constituents. The first, consisting of cells of various kinds, yeast-fungus, bacteria, spermatozoa, casts, etc., must be investigated by means of the microscope, and the following only applies to the non-organized deposits.

As above mentioned (page 331), the urine of healthy individuals may sometimes, even on voiding, be cloudy on account of the phosphates present, or become so after a little while because of the separation of urates. As a rule, urine just voided is clear and after cooling shows only a faint cloud (nubecula), which consists of 
so-called mucus, a few epithelium-cells, mucous corpuscles, and urate particles. If an acid urine is allowed to stand it will gradually change ; it becomes darker and deposits a sediment consisting of uric acid or urates and sometimes also calcium-oxalate crystals, in which yeast-fungus and bacteria are often to be seen. The cause of this change, which the earlier investigators called "ACID FERMENTATION OF THE URINE," is, according to SCHERER, the mucus, which acts like an enzyme or ferment, producing an aceticacid or lactic-acid fermentation, precipitating free uric acid or acid urates. According to NeUBAUER, an actual acid fermentation may occur in diabetic urine, but this change in the urine is now generally explained in other ways. According to VoIT and HoFMANN, a separation of free uric acid and acid urates may be produced without any increase in the acid reaction, by an exchange of the di-hydrogen alkali phosphates with the alkali urate on cooling and on standing. Simple acid phosphate and, according to the conditions, acid urate or free uric acid are formed. A gradual precipitation of uric acid may occur not only without an increase in the acid reaction, but, because of the alkaline reaction of the di-alkali phosphate, it may occur with a simultaneous decrease of the same.

Earlier or later, sometimes only after several weeks, the reaction of the original acid urine changes and becomes neutral or alkaline. The urine has now passed into the "AlKaline Fermentation," which consists in the decomposition of the urea into carbon dioxide and ammonia by means of lower organisms, micrococcus ureæ, bacteria ureæ, and other bacteria. Musculus has isolated an enzyme from the micrococcus ureæ which decomposes urea and is soluble in water. During the alkaline fermentation volatile fatty acids, especially acetic acid, may be produced, chiefly by the fermentation of the carbohydrates of the urine (SALKowSKI).

If the alkaline fermentation has only advanced so far as to render the reaction neutral, then we often find in the sediment fragments of uric-acid crystals, sometimes covered with prismatic crystals of alkali urate; dark-colored globules of ammonium urate, often crystals of calcium oxalate, and sometimes crystallized calcium phosphate are also found. Crystals of ammonium-magnesium phosphate (triple phosphate) and globules of ammonium urate are 
specially characteristic of the alkaline formentation. The urine in alkaline fermentation becomes paler and is often covered with a fine membrane which contains amorphous calcium phosphate and glistening crystals of triple phosphate and numerous micro-organisms.

\section{Non-organized Sediments.}

Uric Acid. This acid occurs in acid urines as colored crystals which are identified partly by their form and partly by their property of giving the murexid test. On warming the urine they are not dissolved. On the addition of caustic alkali to the sediment the crystals dissolve, and when a drop of this solution is placed on a microscope-slide and treated with a drop of hydrochloric acid, small crystals of uric acid are obtained which are easily seen under the microscope.

Acid Urates. These only occur in the sediment of acid or neutral urines. They are amorphous, clay-yellow, brick-red, rosecolored, or brownish red. They differ from other sediments in that they dissolve on warming the urine. They give the murexid test, and small microscopic crystals of uric acid separate on the addition of hydrochloric acid. Crystalline alkali urates occur very rarely in the urine, and as a rule only in such as have become neutral but not alkaline by the alkaline fermentation. The crystals are somewhat similar to those of neutral calcium phosphate, but are not dissolved by acetic acid but give a cloudiness therewith due to small crystals of uric acid.

Ammonium urate may indeed occur as a sediment in a neutral urine which at first was strongly acid and has become neutralized by the alkaline fermentation, but it is only characteristic of ammoniacal urines. This sediment consists of yellow or brownish, rounded globules which are often covered with thorny-shaped prisms and, because of this, are rather large and resemble the thornapple. It gives the murexid test. It is dissolved by alkalies with the development of ammonia, and crystals of uric acid separate on the addition of hydrochloric acid to this solution.

Calcium oxalate occurs in the sediment generally as small, shining, strongly-refractive-quadratic octahedra, which on microscopical examination remind one of a letter-envelope. The crys- 
tals can only be mistaken for small, not fully-developed crystals of ammonium-magnesium phosphate. They differ from these by their insolubility in acetic acid. The oxalate may also occur as flat, oval, or nearly-circular disks with central cavities which from the side appear like an hour-glass. Calcium oxalate may occur as a sediment in an acid as well as in a neutral or alkaline urine. The quantity of calcium oxalate separated from the urine as sediment depends not only upon the amount of this salt present, but also upon the acidity of urine. The solvent for the oxalate in the urine seems to be the double-acid alkali phosplate, and the greater the quantity of this salt in the urine the greater the quantity of oxalate in solution. When, as above mentioned (page 427), the simple-acid phosphate is formed from the double-acid phosphate, on allowing the urine to stand, a corresponding part of the oxalate may be separated as sediment.

Calcium carbonate occurs in considerable quantities as sediment in the urine of herbivora. It only occurs in small quantities as a sediment in human urine, and indeed only in alkaline urines. It has either almost the same appearance as amorphous calcium oxalate, or it occurs as somewhat larger globules with concentric bands. It dissolves in acetic acid with the generation of gas, which differentiates it from calcium oxalate. It is not yellow or brown like ammonium urate and does not give the murexid test.

Calcium sulphate occurs very rarely as a sediment in strongly-acid urines. It appears as long, thin, colorless needles, or generally as plates grouped together.

Calcium phosphate. The CalCiUM tRIPHOSPhate, $\mathrm{Ca}_{3}\left(\mathrm{PO}_{4}\right)_{2}$, which only occurs in alkaline urines, is always amorphous and occurs partly as a colorless, very fine powder and partly as a membrane consisting of very fine granules. It differs from the amorphous urates in that it is colorless, dissolves in acetic acid, but remains undissolved on warming the urine. Calcium DipHosPHATE, CaHPO $4+2 \mathrm{H}_{2} \mathrm{O}$, occurs in neutral or only in very faintly, acid urine. It is found sometimes as a thin film covering the urine, and sometimes as a sediment. In crystallizing the crystals may be single, or they may cross one another, or they may be arranged in groups of colorless, wedge-shaped crystals whose wide end is sharply defined. These crystals differ from crystalline alkaline 
urates in that they dissolve without a residue in dilute acids and do not give the murexid test.

Ammonium-magnesium phosphate, TRIPLE PHOSPHATE, may separate indeed from an amphoteric urine in the presence of a sufficient amount of ammonium salts, but it is generally characteristic of an ammoniacal urine from alkaline fermentation. The crystals are so large that they may be seen with the unaided eye as colorless glistening particles in the sediment, on the walls of the vessel, and in the film on the surface of the urine. This salt forms large prismatic crystals of the rhombical system which are easily soluble in acetic acid. Amorphous magnesium triphosphate, $\mathrm{Mg}_{3}\left(\mathrm{PO}_{4}\right)_{2}$, occurs with calcium triphosphate in urines rendered alkaline by a fixed alkali. Crystalline magnesium phosphate, $\mathrm{Mg}_{3}\left(\mathrm{PO}_{4}\right)_{2}+$ $22 \mathrm{H}_{2} \mathrm{O}$, has been observed in a few cases in human urine (also in horse's urine) as strongly-refractive, long rhombical plates.

Kyestern is the film which appears after a little while on the surface of the urine. This coating, which was formerly considered as characteristic of urine in pregnancy, contains various elements, such as fungus, vibriones, epitheliumcells, etc. It often contains earthy phosphates and triple-phosphate crystals.

As more rare sediments we find cystin, tyrosin, hippuric acid, xanthin, homatoidin. In alkaline urines blue crystals of indigo may also occur, due to a decomposition of indoxyl-glycuronic acid.

\section{Urinary Calculi.}

Besides certain pathological constituents of the urine, all those urinary constituents which occur as sediments take part in the formation of the urinary calculi. EBsteIN considers the essential difference between an amorphous or crystalline sediment in the urine on one side and urinary sand or large calculi on the other to be the occurrence of an organic frame in the last. As the sediments which appear in normal acid urine, and in an alkaline urine due to fermentation are different, so also are the urinary calculi which appear under corresponding conditions.

If the formation of a calculus and its further development takes place in an undecomposed urine, it is called a PRIMARY formation. If, on the contrary, the urine has undergone alkaline fermentation and the ammonia formed thereby has given rise to a calculous formation by precipitating ammonium urate, triple phosphate, and earthy phosphates, then it is called a SECONDARY formation. Such 
a formation takes place, for instance, when a foreign body in the bladder produces catarrh accompanied by alkaline fermentation.

We discriminate between the nucleus or nuclei-if such can be seen-and the different layers of the calculus. The nucleus may be essentially different in different cases, for quite frequently it consists of a foreign body introduced into the bladder. The calculus may have more than one nucleus. In a tabulation made by ULTZMANN of 545 cases of urinary calculi, the nucleus consisted in $80.9 \%$ of the cases, of uric acid (and urates); in $5.6 \%$, of calcium oxalate; in $8.6 \%$, of earthy phosphates ; in $1.4 \%$, of cystin; and in $3.3 \%$, of some foreign body.

During the growth of a calculus it often happens that, for some reason or other, the original calculus-forming substance is covered with another layer of a different substance. A new layer of the original substance may deposit on the outside of this, and this process may be repeated. In this way a calculus consisting originally of a simple stone may be converted into a so-called compound stone with several layers of different substances. Such calculi are always formed when a primary formation is changed into a secondary. By the continued action of an alkaline urine containing pus, the primary constituents of an originally primary calculus may be partly dissolved and be replaced by phosphates. Metamorphosed urinary calculi are formed in this way.

Uric-acid calculi are very abundant. They are variable in size and form. The size of the bladder-stone varies from that of a pea or bean to that of a goose-egg. Uric-acid stones are always colored; generally they are grayish yellow, yellowish brown, or pale redbrown. The upper surface is sometimes entirely even or smooth, sometimes rough or uneven. Next to the oxalate calculus, the uricacid calculus is the hardest. The fractured surface shows regular concentric, unequally-colored layers which may often be removed as shells. These calculi are formed primarily. Layers of uric-acid sometimes alternate with other layers of primary formation, most frequently with layers of calcium oxalate. The simple uric-acid calculus leaves very little residue when burnt on a platinum foil. It gives the murexid test, but there is no mentionable development of ammonia when acted on by caustic soda.

Ammonium-urate calculi occur as primary calculi in new-born 
or nursing infants, rarely in grown persons. They often occur as a secondary formation. The primary stones are small, with a paleyellow or dark-yellowish surface. When moist they are almost like dough ; in the dry state they are earthy, easily crumbling into a pale powder. They give the murexid test, and develop much ammonia with caustic soda.

Calcium-oxalate calculi are, next to uric-acid calculi, the most abundant. They are either smooth and small (HEMP-SEED STONE) or larger, of the size of a hen's egg, with rough, uneven surface, or their surface is covered with prongs (MULBERRY CALCULI). These calculi produce bleeding easily, and therefore they often have a dark-brown surface due to decomposed blood-coloring matters. Among the calculi occurring in man these are the hardest. They dissolve in hydrochloric acid without developing gas, but are not soluble in acetic acid. After gently heating the powder it dissolves in acetic acid with frothing. After strongly heating the powder it is alkaline, due to the production of quick-lime.

Phosphate Calculi. These, which consist mainly of a mixture of the normal phosphate of the alkaline earths with triple phosphate, may be very large. They are as a rule of secondary formation, and contain besides these phosphates also some ammonium urate and calcium oxalate. These calculi ordinarily consist of a mixture of these three constituents, earthy phosphate, triple phosphate, and ammonium urate, surrounding a foreign body as a nucleus. Their color is variable-white, dingy white, pale yellow, sometimes violet or lilac-colored (from indigo-red). The surface is always rough. Caiculi consisting of triple phosphate alone are seldom found. They are ordinarily small, with granular or radiated crystalline fracture. Stones of simple-acid calcium phosphate are also seldom obtained. They are white and have a beautiful crystalline texture. The phosphatic calculi do not burn up, and the powder dissolves in acid without effervescence, and the solution gives the reactions for phosphoric acid and alkaline earths. The triplephosphate calculi generates ammonia on the addition of an alkali.

Calcium-carbonate calculi occur chiefly in herbivora. They are seldom found in man. They have mostly chalky properties, and are ordinarily white. They are completely or in great part dissolved by acids with effervescence.

Cystin calculi occur but seldom. They are of primary formation, of various sizes, sometimes attaining the size of a hen's egg. They have a smooth or 
rough surface, are white or pale yellow, and have a crystalline fracture. They are not very hard; they burn up almost entirely on a platinum foil, burning with a bluish flame. They give the above-mentioned reactions for cystin.

Xanthin calculi are very rarely found. They are also of primary formation. They vary from the size of a pea to that of a hen's egg. They are whitish, yellowish brown, or cinnamon-brown in color, medium hard, with amorphous fracture, and on rubbing appear like wax. They burn up completely when heated on a platinum foil. They give the xanthin reaction with nitric acid and alkali, but this must not be mistaken for the murexid test.

Urcstealith calculi have only been observed a few times. In the moist state they are soft and elastic at the temperature of the body, but in the dry state they are brittle, with an amorphous fracture and waxy appearance. They burn with an illuminating flame when heated on a platinum foil, and generate an odor similar to resin or shellac. Such a calculus, investigated by KRUKENBERG, consisted of paraffine derived from a paraftine bougie used as a sound on the patient. Perhaps the urostealith calculi observed in other cases had a similar origin, although the substances of which they consisted have not been closely studied.

Fibrin calculi sometimes occur. They consist of more or less changed fibrin coagulum. On curning they develop an odor of burnt horn.

The chemical investigation of urinary calculi is of great practical importance. To make such an examination actually instructive it is necessary to investigate separately the different layers which constitute the calculus. For this purpose saw the calculus, which has been wrapped in paper, with a fine saw so that the nucleus is sawed through and accessible. Then peel off the different layers, or, if the stone is to be kept, scrape off enough of the powder from each layer for examination. This powder is then tested by heating on a platinum foil. It must not be forgotten that a calculus is never entirely burnt up, and also that it is never so free from organic matter that on heating it does not carbonize. Do not, therefore, lay too great stress on a very insignificant unburnt residue or on a very small amount of organic matter, but consider the ealculus in the former case as completely burnt and in the latter as not burnt.

When the powder is in great part burnt up but a significant quantity of unburnt residue remains, then the powder in question contains as a rule urates mixed with inorganic bodies. In such cases remove the urate with boiling water, and then test the filtrate for uric acid and the expected bases. The residue is then tested according to the following schema of HELLER, which is well adapted to the investigation of urinary calculi. In regard to more detailed examination the reader is referred to special works on the subject. 


\begin{tabular}{|c|c|c|c|c|c|c|c|c|c|}
\hline \multicolumn{4}{|c|}{ Does not burn } & \multicolumn{6}{|c|}{ Does burn } \\
\hline \multicolumn{4}{|c|}{$\begin{array}{c}\text { The powder when treated } \\
\text { with } \mathrm{HCl}\end{array}$} & \multicolumn{3}{|c|}{ With flame } & \multicolumn{3}{|c|}{ Without flame } \\
\hline \multicolumn{3}{|c|}{ Does not effervesce } & \multirow{4}{*}{ 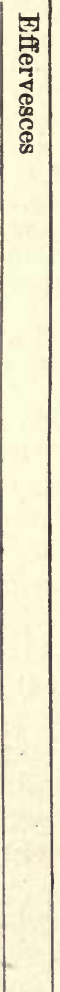 } & \multirow{4}{*}{ 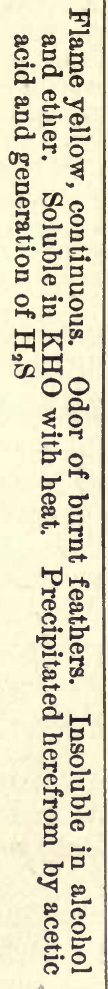 } & \multirow{4}{*}{ 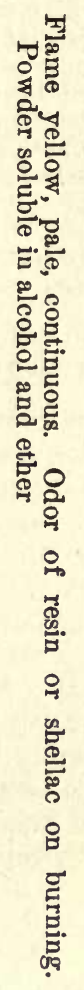 } & \multirow{4}{*}{ 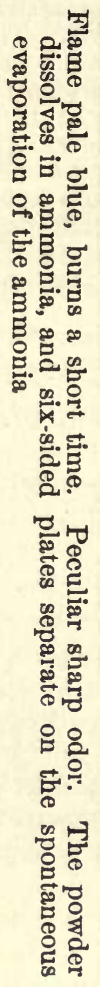 } & \multirow{4}{*}{ 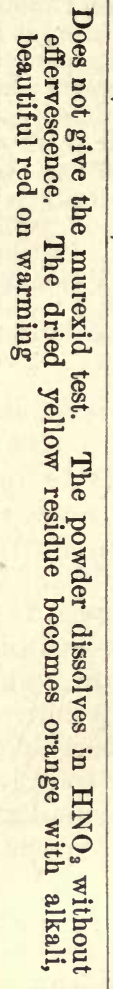 } & \multirow{3}{*}{\multicolumn{2}{|c|}{\begin{tabular}{|c}
$\begin{array}{c}\text { The powder } \\
\text { gives the } \\
\text { murexid test }\end{array}$ \\
$\begin{array}{c}\text { The powder } \\
\text { when treated } \\
\text { with KHO } \\
\text { gives }\end{array}$
\end{tabular}}} \\
\hline \multicolumn{3}{|c|}{$\begin{array}{l}\text { The gently heated } \\
\text { powder with } \mathrm{HCl}\end{array}$} & & & & & & & \\
\hline \multicolumn{2}{|c|}{$\begin{array}{l}\text { The powder when } \\
\text { moistened with a } \\
\text { little KHO }\end{array}$} & \multirow{2}{*}{ 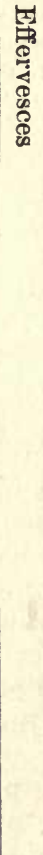 } & & & & & & & \\
\hline 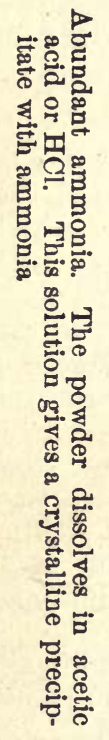 & 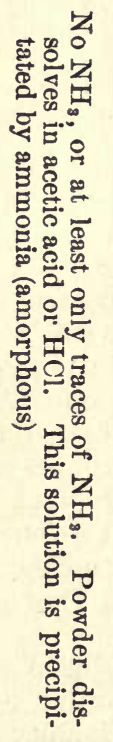 & & & & & & & 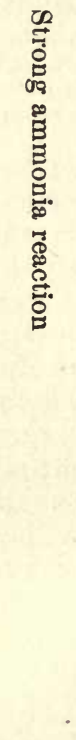 & 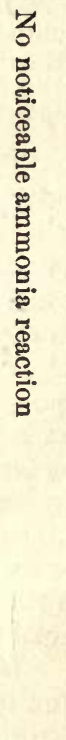 \\
\hline 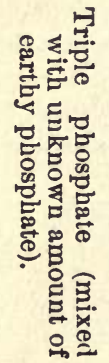 & 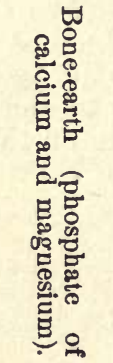 & 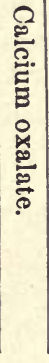 & 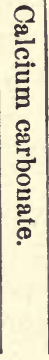 & 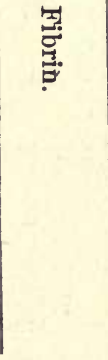 & 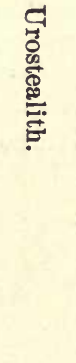 & 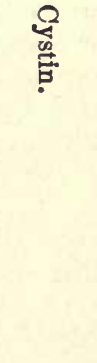 & 离 & 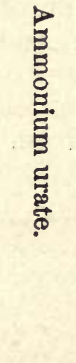 & 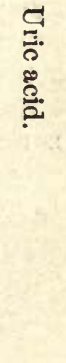 \\
\hline
\end{tabular}


CHAPTER XV.

\section{THE EXCHANGE OF MATERIAL ${ }^{1}$ WITH VARIOUS FOODS AND THE DEMAND FOR FOOD IN MAN.}

THE conversion of chemical tension into living energy, which characterizes animal life, leads, as previously stated in Chapter I, to the formation of the relatively simple compounds, carbon dioxide, urea, etc., which leave the organism and which moreover, being very poor in chemical tension, are for this reason of no or very little value for the body. It is therefore absolutely necessary for the continuance of life and the normal course of the functions of the body, that the organism and its different tissues should be supplied with new material to replace that which has been exhausted. This is accomplished by means of food. Those bodies are designated as food which have no injurious action upon the organism and which replace those constituents of the body that have been consumed in the exchange of material or that can prevent or diminish the consumption of such constituents.

Among the numerous dissimilar substances which man and animals take with the food all cannot be equally necessary or have the same value. Some perhaps are unnecessary, while others may be indispensable. We have learned by direct observation and a wide experience that besides the oxygen, which is necessary for oxidation, the essential foods for animals in general, and for man especially, are water, mineral bodies, proteid bodies, carbohydrates, and fats.

It is also apparent that the various groups of food-stuffs neces-

1 The translator will use in the following pages for the German word "Stoffwechsel" Dr. Burdon-Sanderson's (Syllabus of Lectures, 1879) translation, exchange of material, and at the same time the more general term "metabolism." 
sary for the tissues and organs must be of different importance; thus, for instance, water and the mineral bodies have another task than the organic foods, and these again must vary in importance among themselves. The knowledge of the action of various nutritive bodies on the exchange of material from a qualitative as well as a quantitative point of view must be of fundamental importance in determining the value of different nutritive substances relative to the demands of the body for food under various conditions and also in deciding many other questions, for instance the proper nutrition for an individual in health and in disease.

Such knowledge can only be attained by a series of systematic and thorough observations, in which the quantity of nutritive material, relative to the weight of the body, taken and absorbed in a given time is compared with the quantity of final products of the exchange of material which leave the organism at the same time. Researches of this kind have been made by several investigators, but above all should be mentioned those made by Bischoff and VorT, by Pettenkofer and Voit, and by Voit and his school.

It is absolutely necessary in researches on the exchange of material to be able to collect, analyze, and quantitatively estimate the excretions of the organism so that they may be compared with the quantity and composition of the nutritive bodies taken up. In the first place, one must know what the habitual excretions of the body are and in what way these bodies leave the organism. One must also have trustworthy methods for the quantitative estimation of the same.

The organism may, under physiological conditions, be exposed to accidental or periodic losses of valuable material-such losses as only occur in certain individuals, or in the same individual only at a certain period; for instance, the secretion of milk, the production of pus, the ejection of semen, or menstrul blood. It is therefore apparent that these losses can only be the subject of investigation and estimation in special cases.

The regular and constant excretions of the organism are of the very greatest importance in the study of the exchange of material. To these belong, in the first place, the true final products of the exchange-CARBON DIOXIDE, UREA (uric acid, hippuric acid, creatinin, and other urinary constituents), and a part of the WATER. 
The remainder of the water, the mineral bodies, and those secretions or tissue-constituents-MUCUS, DIGESTIVE FLUIDS, SEBUM, SWEAT, and EPIDERMIS FORMATIONS-which are either poured into the intestinal tract, or secreted from the surface of the body, or broken off and thereby lost for the body, also belong to the constant excreta.

The remains of food, sometimes indigestible, sometimes digestible but not acted upon, contained in the fæces, which vary in quantity and composition with the nature of the food, also belong to the excreta of the organism. Even though these remains, which are never absorbed and therefore are never constituents of the animal fluids or tissues, cannot be considered as excreta of the body in a strict sense; still their quantitative estimation is absolutely necessary in experiments on the exchange of material.

The determination of the constant loss is in some cases accompanied with the greatest difficulties. The loss from the detached epidermis, from the secretion of the sebaceous glands, etc., cannot be determined with exactness without difficulty, and therefore-as they do not occasion any mentionable loss because of their small quantity-they need not be considered in quantitative experiments on the exchange of material. This also applies to the constituents of the mucus, bile, pancreatic and intestinal juices, etc., occurring in the contents of the intestines and which, leaving the body with the fæces, cannot be separated from the other contents of the intestines and therefore cannot be quantitatively determined separately. The uncertainty which, because of the intimated difficulties, attaches itself to the results of the experiments is very small as compared to the variation which is caused by different individualities, different modes of living, different foods, etc. No general but only approximate value can therefore be given for the constant excretions of the human body.

The following figures represent the quantity of excreta for 24 hours, with a mixed diet, of a grown man weighing 60-r0 kilos. The numbers are compiled from the results of different investigators.

Grammes.

Water.............................. 2500-3500

Salts (with the urine)........................ 20-30

Carbon dioxide........................... 750-900

Urea.................................. 20-40

Other nitrogenized urinary constituents........... 2-5

Solids in the excrements ................ 30-50 
These total excreta are approximately divided among the various excretions in the following way-but still it must not be forgotten that this division may vary to a great extent under various external circumstances: by RESPIRATION about 32\%, by the EVAPORATION FROM THE SKIN 1\%\%, with the URINE 46-47\%, and with the EXCREMENTS 5-9\%. The elimination by the skin and lungs, which is sometimes differentiated by the name "PERSPIRATIO INSENSIBILIS" from the visible elimination by the kidneys and intestine, is on an average about $50 \%$ of the total elimination. This proportion, only quoted relatively, is subject to considerable variation, because of the great difference.in the loss of water through the skin and kidneys under different circumstances.

About $90 \%$ of the water in carnivora is excreted through the kidneys. In herbivora the excrements, which are $30-50 \%$ of the total excreta, may indeed eliminate $60 \%$ of the water. In man only a smaller fraction of the water (about 5\%) is eliminated with the fæces, and the great mass of the water is divided between the kidneys, lungs, and skin.

The nitrogenized constituents of the excretions consist chiefly of urea, or uric acid in certain animals, and the other nitrogenized urinary constituents. A disproportionally large part of the nitrogen leaves the body with the urine; and as the nitrogenized constituents of this excretion are final products of the transformation or metabolism of proteids in the organism, the quantity of proteids transformed in the body may be easily calculated by multiplying the quantity of nitrogen in the urine by the coefficient $6.25\left(\frac{100}{16}=6.25\right)$, if we admit that the proteids contain in round numbers $16 \%$ nitrogen.

Still another question is whether the nitrogen leaves the body only with the urine or by other channels. This last is habitually the case. The discharges from the intestine always contain some nitrogen which has a twofold origin. A part of this nitrogen depends upon undigested or non-absorbed remnants of food, and another part on the non-absorbed remains of digestive secretionsbile, pancreatic juice, intestinal mucus-and of epithelium-cells of the mucous membrane. It follows that a part of the nitrogen of fæces has this last-mentioned origin from the fact that discharges from the intestine occurs also in complete inanition. 
If the question to be decided is, how much of the nitrogenized bodies is absorbed in certain modes of nutrition or after taking a certain quantity of food, then naturally the quantity of nitrogen originating from the food and leaving the body with the excrements must be subtracted from the total quantity of nitrogen taken up with the food. To obtain the quantity of nitrogen leaving the body with the excrements it is necessary to subtract from the total quantity of nitrogen of the excrements the quantity of nitrogen coming from the digestive tract itself and its secretions, and the amount of this last must be known.

It is obvious that exact results which answer for all times cannot be given for that part of the nitrogen which has its origin in the digestive canal and fluids. It may not only vary in different individuals, but also in the same individual after more or less active secretion and absorption. In the attempts made to determine this part of the nitrogen of the excrements it has been found that in man, on non-nitrogenized or nearly nitrogen-free food, it amounts in round numbers to about $1 \mathrm{grm}$. per 24 hours (RIEDER; RUBNER). During starvation, in which a smaller quantity of digestive secretions is eliminated, it is less. MüLLER found in his observations on the faster CETTI that only $0.2 \mathrm{grm}$. nitrogen was derived from the intestinal canal.

Nitrogen also leaves the body through the horn formation. The quantity which is lost in this manner is, though it cannot be exactly determined, insignificant. Man loses only about $0.3 \mathrm{grm}$. nitrogen daily by means of the hair and nails (MOLESCHOTT) and about $0.3-0.5 \mathrm{grm}$. by the scaling-off of the skin. The quantity of nitrogen which leaves the body under ordinary circumstances by the perspiration must be so small that, like the loss by the horny structure, it need not be considered in experiments on the exchange of material. The elimination of nitrogen by the perspiration need only be considered in cases of profuse sweating.

The view was formerly held that in man and carnivora an elimination of gaseous nitrogen took place through the skin and lungs, and because of this, on comparing the nitrogen of the food with that of the urine and fæces, a nitrogen deficit occurred in the visible elimination.

This question has been the subject of much discussion and of 
numerous investigations. The conclusion has been drawn from the researches of REGNAULT and REISET on respiration that also an exhalation of nitrogen takes place. SEEGEN and NowaK especially have recently endeavored to prove the correctness of this conclusion. Such an experiment is, however, accompanied with so many difficulties, and there are so many sources of error, that it can scarcely be considered as conclusive. In fact, PETTENKofer and VoIT have demonstrated the existence of errors in the experiments of Seegen and Nowak. On the other hand, Pflüger and Leo have found no appreciable exhalation of nitrogen in rabbits. Also many investigators, especially Vort, PetTenkofer and Vort and RANKE, have shown by experiments on man that with the proper amount and quality of food we can bring the body into NITROGENOUS EQUILIBRIUM, in which the quantity of nitrogen voided with the urine and fæces is equal to the quantity contained in the food.

The experiments made by GRUBER in VorT's institute seem to be especially conclusive on this point. GRUBER fed a dog seventeen days on meat which in all contained 368.53 grms. nitrogen, and he found in the same time 368.28 grms. nitrogen in the urine and fæces. From this and other experiments we may conclude with Vort that a deficit of nitrogen does not exist; or if we consider the above-mentioned very small loss of nitrogen through the horny structure, etc., it is so insignificant that in experiments upon the exchange of material it need not be considered. In investigations on the metabolism of proteids in the body, ordinarily, it is only necessary to consider the nitrogen of the urine and fæces, but it must be remarked that the nitrogen of the urine is a measure of the extent of the metabolism of the proteids in the body, while the nitrogen of the fæces (after deducting somewhat less than $1 \mathrm{grm}$. on mixed diet) is a measure of the non-absorbed part of the nitrogen of the food.

In the oxidation of the proteids in the organism their sulphur is oxidized into sulphuric acid, and on this depends the fact that the elimination of sulphuric acid by the urine, which in man is only to a small extent derived from the sulphates of the food, makes nearly equal variations as the elimination of nitrogen by the urine. If we consider the amount of nitrogen and sulphur in the proteids as $16 \%$ and $1 \%$ respectively, then the proportion between the nitrogen 
of the proteids and the sulphuric acid, $\mathrm{H}_{2} \mathrm{SO}_{4}$, produced by their burning, is in the ratio $5.2: 1$, or about the same as in the urine (see page 382). The determination of the quantity of sulphuric acid eliminated with the urine gives us an important means of controlling the extent of the transformation of proteids, and such a control is especially important in cases in which we wish to study the action of certain nitrogenized non-albuminous bodies on the metabolism of proteids. A determination of the nitrogen alone is not in such cases sufficient.

If it is found, on comparing the nitrogen of the food with that of the urine and fæces, that there is an excess of the first, this means that the body has increased its stock of nitrogenized substancesproteids. If, on the contrary, the urine and fæces contain more nitrogen than the food taken at the same time, this denotes that the body is giving up part of its nitrogen-that is, a part of its own proteids. We can from the quantity of nitrogen, as above stated, calculate the corresponding quantity of proteids by multiplying by 6.25. Usually, according to VorT's proposition, the nitrogen of the urine is not calculated as decomposed proteids, but as decomposed muscle-substance. Flesh contains on an average about $3.4 \%$ nitrogen, and each gramme of nitrogen of the urine corresponds in round numbers to about 30 grms. flesh.

A disproportionally large part of the carbon leaves the body as carbon dioxide, which escapes chiefly through the lungs and skin. The remainder of the carbon is eliminated under the form of organic combinations by the urine and fæces, in which the quantity of carbon can be determined by elementary analysis. The amount of gaseous carbon dioxide eliminated is determined by means of PeTtenkofer's respiration apparatus, which is described in special works. By multiplying the quantity of carbon dioxide found by 0.273 we obtain the quantity of carbon eliminated as $\mathrm{CO}_{2}$. If we compare the total quantity of carbon eliminated in various ways with the carbon contained in the food, we obtain some idea as to the transformation of the carbon compounds. If the quantity of carbon in the food is greater than in the excretion, then the excess is deposited; while if the reverse be the case, it shows a corresponding loss of bodily substance.

The nature of the substances here deposited or lost, whether 
they consist of proteids, fats, or carbohydrates, is Iearned from the total quantity of nitrogen of the excretions. The corresponding quantity of proteids may be calculated from the quantity of nitrogen, and as the average quantity of carbon in the proteids is known, the quantity of carbon which corresponds to the decomposed proteid may be easily ascertained. If the quantity of carbon found is smaller than the quantity of the total carbon in the excretions, it is then obvious that some other nitrogen-free substance has been consumed besides the proteids. If the amount of carbon in the proteids is considered in round numbers as $54 \%$, then the relation between carbon (54) and nitrogen (16) is as 3.4:1. Multiply the total quantity of nitrogen eliminated by 3.4 , and the excess of carbon in the eliminations over the product found represents the carbon of the transformed non-nitrogenized compounds. For instance, in the case of a person experimented upon, $10 \mathrm{grms}$. nitrogen and $200 \mathrm{grms}$. carbon were eliminated in the course of 24 hours; then these 62.5 grms. proteid correspond to 34 grms. carbon, and the difference $200-(3.4 \times 10)=166$, which represents the quantity of carbon in the decomposed non-nitrogenized compounds. If we start from the simplest case, starvation, where the body lives at the expense of its own substance, then, since the quantity of carbohydrates as compared to the fats of the body is extremely small, in such cases in order to avoid mistakes the assumption must be made that the person experimented upon has only taken fat and proteids. As animal fat contains on an average $76.5 \%$ carbon, the quantity of transformed fat may be calculated by multiplying the carbon by $\frac{100}{76.5}=1.3$. In the case of the above example the person experimented upon would have used 62.5 grms. proteids and $166 \times 1.3=216$ grms. fat of his own bodily weight in the course of the 24 hours.

Starting from the balance of the nitrogen, we can calculate in the same way whether an excess of carbon in the food as compared with the quantity of carbon in the excretions is retained by the body as proteids or fat or as both. On the other hand, with an excess of carbon in the excretions we can calculate how much of the loss of the substance of the body is due to a consumption of the proteid or of fat or of both. 
The quantity of water and mineral bodies voided with the urine and fæces can easily be determined. The quantity of water eliminated by the skin and lungs may be directly determined by means of Pettenrofer's apparatus. The quantity of oxygen taken up is calculated as the difference between the weight of the individual before the experiment plus all the directly-determined substances taken in, and the final weight of the individual plus all his excreta.

\section{Exchange of Material in Starvation.}

In starvation the decomposition in the body continues uninterruptedly, though with decreased intensity; but as it takes place at the expense of the substance of the body, it can only continue for a limited time. When an animal has lost a certain fraction of the mass of the body death is the result. This fraction varies with the condition of the body at the beginning of the starvation period. Fat animals succumb when the weight of the body has sunk to $\frac{1}{2}$ of the original weight. Otherwise, according to CHOSSAT, animals die as a rule when the weight of the body has sunk to $\frac{2}{5}$ of the original weight. The period when death occurs from starvation not only varies with the different nutritive condition at the beginning of starvation, but also with the more or less active exchange of material. This is more active in small and young animals than in large and older ones, but different classes of animals show an unequal activity. Children succumb in starvation in three to five days after having lost $\frac{1}{4}$ of their bodily mass. Grown persons, according to ordinary statements, can live for three weeks if they bave water; we also have statements of much longer periods of starvation. For instance, a person suffering from melancholia, who drank water but took no food, died after 41 days, and the Italian MERLATtI withstood a starvation period of 50 days, during which it is stated he only took water. - Dogs can live without food from four to eight weeks, birds five to twenty days, snakes more than half a year, and frogs more than a year.

In starvation the weight of the body decreases. The loss of weight is greatest in the first fow days, and then decreases rather uniformly. In small animals the absolute loss of weight per day is naturally smaller than in larger animals. The relative loss of weight, 
that is, the loss of weight of the unit of the weight of the body, namely 1 kilo, is, on the contrary, greater in small animals than in larger ones. The reason for this is that the smaller animals have a greater surface of body in proportion to their mass than larger animals, and the greater loss of heat caused hereby must be replaced by a more active consumption of material (RUBNER).

Exact observations for a long time are necessary for a thorough study of the exchange of material in starvation. As these have seldom been made on man our knowledge of the exchange of material in starvation has been gained by observations on animals, especially on carnivora.

As the exchange of material in starvation takes place at the expense of the constituents of the body, it must take place in essentially the same way in both carnivora and herbivora. As the food of the herbivora is ordinarily richer in carbohydrates and nonnitrogenized nutritive bodies than that of the carnivora, so in starvation the body of the herbivora becomes relatively richer in proteids. On this account the elimination of urea is increased in herbivora in the first part of the period of starvation. In carnivora the elimination of urea decreases, as a rule, immediately at the beginning of the starvation, and in the later periods only small amounts of urea are voided by herbivora as well as by carnivora.

The extent of the metabolism of proteids and the elimination of urea, which is a measure for the same, does not show in carnivora any uniform decrease during the entire period of starvation. During the first few days the elimination of nitrogen is greatest and the quantity of the same depends essentially upon the amount of proteids in the organism and the nature of the food previously taken. The richer the body is in proteids from the food previously taken the greater is the metabolism of proteids, or, in other words, the elimination of nitrogen during the first days of starvation is greater. The rapidity with which the elimination of nitrogen decreases in the first days depends also, according to Vort, upon the proteid condition of the body. It decreases more quickly, that is, the curve of the decrease is more sudden, the first days of starvation, as a rule, the richer the food was in proteids which was taken before starvation. This condition is apparent from the following table. This table contains three different starvation experi- 
ments made by Vort on the same dog. This dog received 2500 grms. flesh daily before the first series of experiments, 1500 grms. flesh daily before the second series, and a mixed food relatively poor in nitrogen before the third series.

\section{TABLE I.}

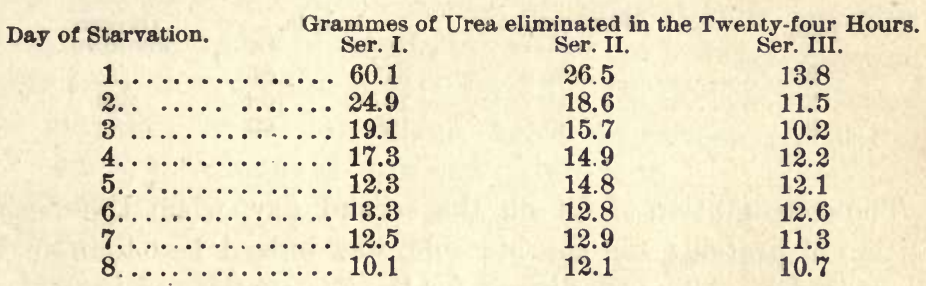

Other conditions, such as varying amounts of fat in the body, have an influence on the rapidity with which the nitrogen is eliminated during the first days of starvation. After the first few days the elimination of nitrogen, as is seen in the above table, is more uniform, and as the starvation proceeds it decreases as a rule rery slowly and uniformly. Cases also occur in which the elimination of nitrogen becomes constant in these stages, and in which indeed the elimination of nitrogen increases towards the end. 'This so-called premortal increase always occurs as soon as the adipose tissue in the body has sunk to a certain point, and it also depends on the fact that as soon as the fat is consumed a corresponding increase in the decomposition of proteids is necessary for the generation of heat as well as of other forces.

If fat occurs in the body besides proteids, it is also decomposed in starvation. Since fat has a diminishing influence on the destruction of proteids (see further on), the elimination of nitrogen in starvation is less in fat than in lean individuals. For instance, only 9 grms. of urea were voided in twenty-four hours during the later stages of starvation by a well-nourished and fat person suffering from disease of the brain, while J. MUNK found that 20-29 grms. urea were voided daily by CETTI, who had been poorly fed.

Like the destruction of proteids during starvation, the decomposition of fat proceeds uninterruptedly. The decomposition of fat does not show so great and rapid a decrease in the first days of 
starvation as the destruction of proteids. Pettenkofer and VolT found, for instance, in a starving dog the following losses of proteids and fat from the body on different days of starvation:

\section{TABLE II.}

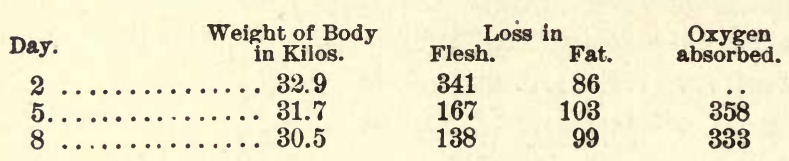

The consumption of fat on the second day, when the decomposition of proteids was considerable, was indeed less than in the following days. The conditions for the destruction of proteids in the animal body seem, as VoIT has suggested, to be different from those for the consumption of fat.

The constant decrease in the consumption of the adipose tissue and proteids during starvation must also cause a decrease in the extent of the exchange of gas; for instance, a diminished taking up of oxygen and a diminished elimination of carbon dioxide. This is found to be true. In starvation experiments made on a cat, ScHмidT found that the results for the carbon dioxide and oxygen fell in the course of eighteen days from $50.96 \mathrm{grms}$. carbon dioxide and 46.20 grms. oxygen on the first day of starvation to 22.26 grms. carbon dioxide and 22.12 grms. oxygen on the last day. Investigations on the extent of exchange of gas in human beings in the starving condition were made by LEHMANN and ZUNTz on Cetri, who only partook of water for ten days. These investigator's found that the absolute extent of exchange of gas during hunger decreases, but that when the oxygen consumed and the carbon dioxide eliminated were calculated on the unit of the weight of the body-1 kilo-its amount quickly sinks to a minimum, but then remains unchanged or may perhaps rise during the course of the fast. They found a consumption of 4.65 c. c. oxygen per minute for 1 kilo during the third to sixth day and 4.73 c. c. during the ninth to eleventh day. It is also a well-known fact that the temperature of the body of starving animals remains tolerably constant, without showing a mentionable decrease, during the greater 
part of the starvation period. The heat of the animal sinks only a few days before death, and at about $33^{\circ}$ to $30^{\circ} \mathrm{C}$. death results.

If the carbon is burnt with oxygen into carbon dioxide, the carbon dioxide produced occupies the same volume as the oxygen consumed, and the quotient $\frac{\mathrm{CO}_{2}}{\mathrm{O}}$ is therefore 1. The same is true of the burning of the carbohydrates, which contain in themselves the necessary quantity of oxygen to oxidize the hydrogen, and only the quantity of oxygen required to oxidize the carbon is necessary to be taken up for the burning of the carbohydrates into $\mathrm{CO}_{2}$ and $\mathrm{H}_{2} \mathrm{O}$. In the burning of fats and proteids this is different. In these cases an absorption of oxygen is necessary not only for the burning of the carbon but also for the hydrogen, and the volume of the carbon dioxide formed is therefore smaller than the oxygen consumed. The quotient $\frac{\mathrm{CO}_{2}}{\mathrm{O}}$ must therefore in these cases be smaller than 1. The conditions are still more complicated for the proteids, because these bodies contain sulphur which is oxidized into sulphuric acid, and also because they are not completely burnt in the organism, but yield nitrogenized decomposition products which contain hydrogen and oxygen as well as carbon.

From the above it follows that in man, on a mixed diet, the proportion between the inhaled oxygen and the expired carbon dioxide, or the so-called respiratory quotient, must be smaller than 1 . As a rule, it is $0.73-0.86$ on a mixed diet. On feeding with an exclusively vegetable food rich in carbohydrates it is closer to 1 ; with a strictly meat diet it is lowest, about $0 . \%$. In starvation, when the person or animal lives entirely upon his own body, it must be about the same as when fed entirely upon meat and fat. As the quotient for the burning of proteids is $0.81-0.75$, and for the burning of fats 0.7 , the respiratory quotient in starvation must be in the neighborhood of $0 . \%$. In the above-mentioned starvation experiments made by C. ScHмidT on a cat it was 0.765 , while in his observations on CETTI it was still lower, or 0.68-0.65.

Water passes uninterruptedly from the body in starvation even when none is given. If the amount of water in the tissues rich in proteids is considered as $70-80 \%$, and the amount of proteids in the same $20 \%$, then for each gramme of destroyed proteids about 
4 grammes of water are set free. A special increase in the demand for water does not seem to occur in starving animals.

The mineral substances leave the body uninterruptedly in starvation until death, and the influence of the destruction of tissues is plainly perceptible by their elimination. Because of the destruction of tissues rich in potassium, the proportion between potassium and sodium in the urine changes in starvation so that, contrary to the normal conditions, the potassium is eliminated in proportionally greater quantities. MUNK also observed in CETTI's case a relative increase in the phosphoric acid and calcium in the urine during starvation, which was due to an increased exchange of bonesubstance.

The question as to the participation of the different organs in the loss of weight of the body during starvation is of special interest. To illustrate this question we will give below the results of CHossat's experiments on pigeons and those of Vort on a male cat. The results are percentages of weight lost from the original weight of the organ.

TABLE III.

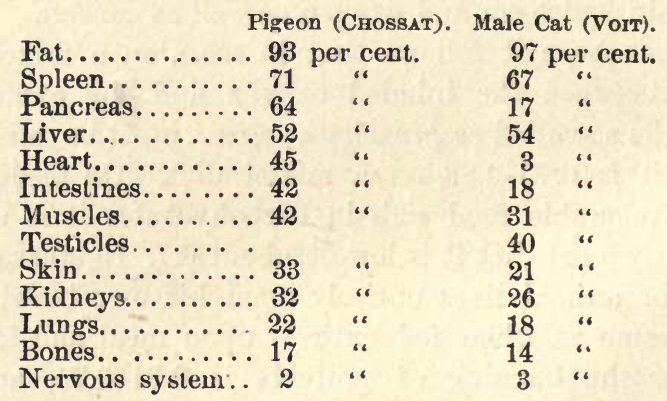

The total quantity of blood, as well as the amount of solids contained therein, decreases, as PANUM has shown, in the same proportion as the weight of the body. The statements in regard to the loss of water by different organs is somewhat contradictory; according to LUKJANOW, it seems that the various organs act somewhat differently in this respect.

The above-tabulated results cannot serve as a measure of the 
exchange of material in the various organs during starvation. For instance, the nervous system only shows a small loss of weight as compared to the other organs, but from this it must not be concluded that the exchange of material in this system of organs is not active. The condition may be quite different ; for one organ may derive its nutriment during starvation from some other organ and exist at its expense. A positive conclusion cannot be drawn in regard to the activity of the exchange of material in an organ from the loss of weight of that organ in starvation.

\section{Exchange of Material with Inadequate Nutrition.}

The food may be quantitatively insufficient and the final result is absolute inanition. The food may also be qualitatively insufficient, or as we say, inadequate. This occurs when any of the necessary nutritive bodies are absent in the food, while the others occur in sufficient or perhaps indeed in excessive amounts.

Lack of water in the food. The quantity of water in the organism is greatest during foetal life, and then decreases with increasing age ( $v$. BEZOLD). Naturally, the amount differs in various organs. Enamel, being almost free, contains only $2 \mathrm{p} . \mathrm{m}$. water, the teeth about $100 \mathrm{p}$. m., the fatty tissues and bones 60-120-150 p. m. The cartilage with $540-740$ p. m. is somewhat richer in water, while the muscles, blood, and glands with 750 to more than $800 \mathrm{p}$. m. are still richer. The quantity of water is even greater in the animal fluids (see preceding chapter), and the grown body contains in all about $600 \mathrm{p}$. m. water (BISCHOFF). If we bear in mind that two thirds of the animal organism consists of water; that water is of the very greatest importance in the normal, physical composition of the tissues ; moreover that all flow of juices, all exchange of substance, all supply of nutrition, all increase or destruction, and all discharge of the products of destruction are dependent upon the presence of water; besides this, that by its evaporation it is an important regulator of the temperature of the body, - we perceive that water must be necessary for life. If the loss of water be not replaced by fresh supplies sooner or later, the organism succumbs.

Lack of mineral substances in the food. We are chiefly in- 
debted to Liebig for showing that the mineral substances are just as necessary for the normal composition of the tissues and organs, and for the normal course of the processes of life, as the organic constituents of the body. The importance of the mineral constituents is evident from the fact that there is no animal tissue or animal fluid which does not contain mineral substance, and also from the fact that certain tissues or elements of tissues contain habitually certain mineral substances and not others, which explains the unequal division of the potassium and sodium compounds in the tissues and fluids. With the exception of the skeleton, which contains about $220 \mathrm{p}$. m. mineral bodies (VolKmaNN), the animal fluids or tissues are poor in inorganic constituents, and the quantity of such only amounts, as a rule, to about $10 \mathrm{p}$. m. Of the total quantity of mineral substances in the organism, the greatest part occurs in the skeleton, $830 \mathrm{p}$. m., and the next greatest in the muscles, about 100 p. m. (VolkmanN).

The mineral bodies seem to be partly dissolved in the fluids and partly combined with organic substances. In accordance with this the organism persistently retains, with food poor in salts, a part of the mineral substances, also such as are soluble, as the chlorides. On the burning of the organic substances the mineral bodies combined therewith are set free and are eliminated. It is also admitted that they in part combine with the new products of the burning, and also that they in part are attached to organic nutritive bodies absorbed from the intestinal canal which are poor in salts, or nearly salt-free, and are thus retained (VOIT, FonSTER).

If this statement be correct, it is possible that a constant supply of mineral substances with the food is not absolutely necessary, and that the amount of inorganic bodies which must be administered is insignificant. The question whether this be so or not has not, especially in man, been sufficiently investigated; but generally we consider the need of mineral substances by man as very small. It may, however, be assumed that man usually takes with his food a considerable excess of mineral substances.

Investigations on animals in regard to the action of an insufficient supply of mineral substances with the food have been made by several investigators, especially Forster. He observed, on experimenting on dogs and pigeons, with food as poor as possible in mineral sub. 
stances, a very suggestive disturbance of the functions of the organs, especially the muscles and the nerrous system, and death resulted after a time, indeed earlier than in complete starvation. In opposition to these observations BUNGE has suggested that the early death in these cases was not caused by the lack of mineral salts, but more likely by the lack of bases necessary to neutralize the sulphuric acid formed in the burning of the proteids in the organism, which must be then taken from the tissues. In accordance with this view, BUNGE and LUNIN also found on experimenting on mice that animals which received nearly ash-free food with the addition of sodium carbonate were kept alive twice as long as animals which had the same food without the addition of sodium carbonate. Special experiments also show that the carbonate cannot be replaced by an equivalent amount of sodium chloride, and that to all appearances it acts by combining with the acids formed in the body. The addition of alkali carbonate to the otherwise nearly ash-free food may indeed delay death, but cannot prevent it, and even in the presence of the necessary amount of bases death results for lack of mineral substances in the food.

In the above series of experiments made by BUNGE the food of the animal consisted of casein, milk-fat, and cane-sugar. While milk alone was an adequate and sufficient food for the animal, BUNGE found that the animal could not be kept alive longer by food consisting of the above constituents of milk and cane-sugar, with the addition of all the mineral substances of milk, than with the food mentioned in the above experiments with the addition of alkali carbonate. The question whether this result is to be explained by the fact that the mineral bodies of milk are chemically combined with the organic constituents of the same and can be assimilated only in such combinations, or whether it depends on other conditions, BUNGE leaves undecided. These observations, however, show how difficult it is to draw positive conclusions from experiments made thus far with food poor in salts. Further investigations on this subject seem to be necessary.

With an insufficient supply of chlorides with the food the elimination of chlorine by the urine decreases constantly, and at last it may stop entirely while the tissues still persistenly retain the chlorides. These last are, at least in part, combined in the body with

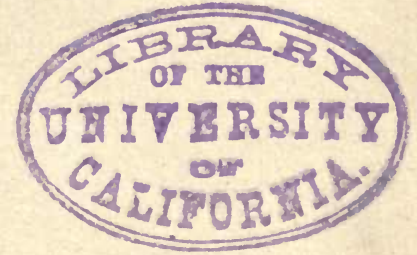


the organic substances which retain them. The great importance of such a retention of chlorides by the tissues is apparent if we bear in mind that the $\mathrm{NaCl}$ is not only a solvent for certain albuminous bodies, or a material for the elaboration of the gastric juice, but that it is also of the greatest importance as a so-called indifferent salt for the preservation of the normal consistency and the physiological imbibition relation of the tissues.

If there be a lack of sodium as compared to potassium, also if there be an excess of potassium compounds in any other form than $\mathrm{KCl}$, the potassium combinations are replaced in the organism by $\mathrm{NaCl}$, so that new potassium and sodium compounds are produced which are voided with the urine. The organism becomes poorer in $\mathrm{NaCl}$, which therefore must be taken in greater amounts from the outside (BUNGE). This occurs habitually in herbivora, and in man with vegetable food rich in potash. For human beings, and especially for the poorer classes of people who live chiefly on potatoes and foods rich in potash, common salt is, under these circumstances, not only a condiment, but a necessary addition to the food (BUNGE).

Lack of alkali carbonates or bases in the food. The chemical processes in the organism are dependent upon the presence of alkaline-reacting tissue-fluids, whose alkaline reaction is due to alkali carbonates. The alkali carbonates are also of great importance not only as a solvent for certain albuminous bodies and as constituents of certain secretions, as of the pancreatic and intestinal juices, but they are also a means of transportation of the carbon dioxide in the blood. It is therefore easy to understand that a decrease below a certain point in the quantity of alkali carbonate must endanger life. Such a decrease not only occurs with lack of bases in the food which accelerates death by a relatively too great production of acids by the burning of the proteids (see above: BUNGE and LUNIN), but it also occurs when an animal is given dilute mineral acids for a certain time. In herbivora the fixed alkalies of the tissues combine with the mineral acids, and the animal succumbs after a time. In carnivora the bases of the tissues are obstinately retained; the mineral acids unite with the ammonia produced by the decomposition of the proteids or their 
splitting products, and carnivora can therefore be kept alive for a longer time.

Lack of earthy phosphates. With the exception of the importance of the alkaline earths as carbonates and principally as phosphates in the physical composition of certain structures, such as the bones and teeth, their physiological importance is nearly unknown. The action which an insufficient supply of alkali-earths with the food causes is connected with the interesting question as to the effect of this lack upon the bony structure. This action, as well as the various results obtained by experiments on young and old animals, has already been spoken of in Chap. VIII, to which we refer the reader.

Lack of iron. As iron is an integral constituent of hæmoglobin, indispensable for the introduction of oxygen, so also it is an indispensable constituent of the food. In iron starvation iron is continually eliminated, even though in diminished amounts (HAMbUrGer, Dietl, v. Hösslin). From the observations of $\mathbf{v}$. HössLiN on dogs it also seems that an inadequate supply of iron with the food causes an insufficient formation of hæmoglobin. A special result of the lack of iron is chlorosis, which the physician has often to contend with and whose origin is not really a lack of iron in the food, but more likely an incomplete absorption of the foods containing iron (BUNGE). The iron-salts seem not to be absorbed at all in the intestinal canal, or only to a very small extent, so that it is questionable whether their absorption has any mentionable importance. It seems more probable that the absorption of iron from the food takes place in the form of protein bodies (nucleoalbumin) containing iron (BUNGE); and the importance of the iron-salts in preventing the lack of hæmoglobin consists chiefly, according to BUNGE, in that these salts counteract the decomposition in the intestines of the protein bodies containing iron, which split off iron as iron sulphide.

In the absence of proteid bodies in the food the organism must nourish itself by its own proteid substances, and on such nutrition it must earlier or later succumb. By the exclusive administration of fat and carbohydrates the consumption of proteids in these cases is reduced, for by an exclusive fat and carbohydrate diet the metabolism of proteids may indeed be smaller than in complete 
starvation (HIRSCHFELD). In conformity with this the animal may be kept alive longer by food containing only non-nitrogenized bodies than in complete starvation.

The absence of fats and carbohydrates in the food affect carnivora and herbivora somewhat differently. It is unknown whether carnivora can be kept alive for any length of time by food entirely free from fat and carbohydrates. But it has been positively demonstrated that they cannot only be kept alive by feeding entirely with meat freed as much as possible from visible fat, but that flesh, and perhaps also fat, is deposited. Men or herbivora, on the contrary, cannot live for any length of time on such food. On one side they lose the property of digesting and assimilating the necessarily large amounts of meat, and on the other a distaste for large quantities of meat or proteids soon appears.

\section{Exchange of Material with Various Foods.}

For the carnivora, as above stated, meat as poor as possible in fat may be a complete and sufficient food. As the proteids moreover take a special place among the organic nutritive bodies by the quantity of nitrogen they contain, it is proper that we first describe the exchange of material with an entirely meat diet.

Exchange of material with food rich in proteids, or feeding only with meat as poor in fat as possible.

By an increased supply of proteids the metabolization of proteids and the elimination of nitrogen is increased and indeed in proportion to the supply of proteids.

If a certain quantity of meat is given as food daily to carnivora and the ration of meat is suddenly increased, an increased metabolism of proteids or an increase in the quantity of nitrogen eliminated is the result. If we feed the animal daily for a certain time with larger quantities of the same meat, we find that a part of the proteids accumulates in the body, but this part decreases from day to day, while there is a corresponding daily increase in the elimination of nitrogen. In this way a nitrogenous equilibrium is established, that is, the total quantity of nitrogen eliminated is equal to the quantity of nitrogen in the absorbed proteids or meat. If, on the contrary, an animal which is in nitrogenous equilibrium having 
been fed on large quantities of meat is suddenly fed with a small quantity of meat per day, then the animal gives up its own bodily proteids, the amount decreasing from day to day. The elimination of nitrogen and the metabolism of proteids decrease constantly, and the animal may in this case also pass into nitrogenous equilibrium or nearly into this condition. These conditions are illustrated by the following table (VoIT):

TABLE IV.

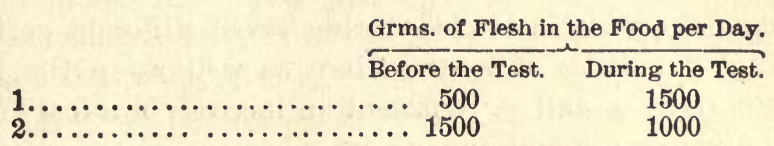

\begin{tabular}{ccccccc}
\multicolumn{8}{c}{ Grms. of Flesh metabolized in Body per Day. } & \multicolumn{3}{c}{ Bod } \\
& 2 & 3 & 4 & 5 & 6 & 7 \\
1222 & 1310 & 1390 & 1410 & 1440 & 1450 & 1500 \\
1153 & 1086 & 1088 & 1080 & 1027 & &
\end{tabular}

In the first case (1) the metabolism of flesh before the beginning of the actual experiment on feeding with 500 grms. meat was 447 grms., and it increased considerably on the first day of the experiment, after feeding on 1500 grms. meat. In the second case (2), in which the animal was previously in nitrogenous equilibrium with 1500 grms. meat, the metabolism of flesh on the first day of the experiment, with only 1000 grms. meat, decreased considerably, and on the fifth day a nearly nitrogenous equilibrium was obtained. During this time the animal gave up daily some of its own proteids. Between that point below which the animal loses from its own weight and the maximum, which seems to be dependent upon the digestive and assimilative capacity of the intestinal canal, carnivora may be kept in a nitrogenous equilibrium with a varying quantity of proteids in the food.

The supply of proteids, as well as the condition of the body, affects the extent of the proteid metabolism. A body which has become rich in proteids by a previous abundant meat diet must, to prevent a loss of proteids, take up more proteid with the food than a body poor in proteids. The quantity of fat in the body also has a great influence. As the fat of the body lowers the disintegration 
of proteids in starvation, so also it diminishes the disintegration of the proteids taken with the food.

PetTenkofer and Vort have made investigations on the metabolism of fat with an exclusively albuminous diet. These investigations have shown that by increasing the quantity of proteids in the food the daily metabolism of fat decreases, so that indeed a formation of fat from proteids, or more correctly an excess of carbon in the food as compared to the excretions, is produced, and this excess is considered as indicating a formation of fat from proteids. To illustrate this the following series of experiments were made on dogs, which were fed during seven different periods with increasing quantities of meat. Here as well as in the following pages the signs + and - represent an increase or a loss of the substances under consideration.

TABLE V.

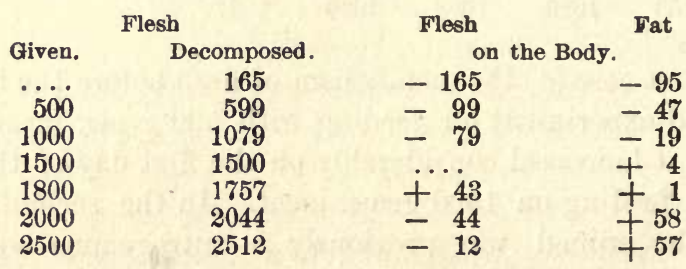

In these cases, according to the generally-received opinion, a formation of fat from proteids has taken place, even though it is small as compared to the quantity of decomposed proteid. In this formation of fat the proteids of the organism split into a nitrogenized part which ultimately yields urea, uric acid, etc., and a nonnitrogenized part which passes into fat or fat-forming substances. This non-nitrogenized part first diminishes the metabolism of fat and then, when it is formed in greater quantities, it is stored up as adipose tissue.

The accumulation of fat in the body is therefore only small with an exclusively meat diet. The same is true also of the storing up of proteids, which decreases from day to day and which only lasts for a short time, because nitrogenous equilibrium soon occurs. This is also the reason why with an exclusively meat diet a well- 
nourished body can be kept in its ordinary condition, while a poorly-nourished or diseased organism cannot be made fat.

The very considerable elimination of nitrogen and the peculiarities which it presents in an exclusively meat diet have, together with other circumstances, among which is the unequal behavior of the metabolism of proteids on the first and the following days of starvation, led to the view (VorT) that all proteids in the body are not decomposed with the same ease. Vort differentiates the proteids fixed in the tissue-elements, so-called organized proteids, tissueproteids, from those proteids which circulate with the fluids in the body and its tissues and which are taken up by the living cells of the tissues from the interstial fluids washing them. These circulating proteids are, according to VorT, more easily and quickly destroyed than the tissue-proteids. Although in a fasting animal which has been previously fed with meat an abundant and quicklydecreasing decomposition of proteids takes place, while in the further course of starvation this proteid metabolism becomes less and more uniform, still this depends upon the fact that the supply of circulating proteids is diminished chiefly in the first days of starvation and the tissue-proteids in the last days.

The tissue-elements constitute an apparatus of a relatively stable nature, which has the power of taking proteids from the fluids washing the tissues and digesting them, while a few proteids, the tissue-proteids, are ordinarily only disorganized to a small extent, about $1 \%$ daily (VoIT). By an increased supply of proteids the activity of the cells and their ability to decompose nutritive proteids is also increased to a certain degree. When nitrogenous equilibrium is obtained after increased supply of proteids, it denotes that the decomposing power of the cells for proteids has increased so that the same amount of proteids is metabolized as is supplied to the body. If the proteid metabolism is decreased by the simultaneous administration of other non-nitrogenized foods (see below), a part of the circulating proteids may have time to become fixed and organized by the tissues, and in this way the mass of the flesh of the body increases. During starvation or with lack of proteids in the food the reverse takes place, for a part of the tissue proteids is converted into circulating proteids which are metabolized, and in this case the flesh of the body decreases. 
VorT's doctrine of the circulating and tissue proteids is indeed a hypothesis, but it explains in a satisfactory manner a number of otherwise very intricate relations and it agrees best with the facts. This theory has received further confirmation from the investigations of several others, as PANUM, FALCK and FEDER, on the temporary secretion of urea after food rich in proteids. From the investigations on a dog it was found that the secretion of urea increases almost immediately after a meal rich in proteids, and that it reaches its maximum in about six hours, when about one half of the administered proteids is secreted as the corresponding quantity of urea. If we also recollect that, according to an observation of SCHMIDT-MÜLHEIM on a dog, about 33\% of the given proteids are absorbed in the first two hours after the meal and about $56 \%$ in the course of the first six hours, we may then infer that the increased elimination of nitrogen after a meal is due to a metabolization of the digested and absorbed proteids of the food. If we admit that the destroyed proteid must have been organized, then the greatly increased elimination of nitrogen after a meal rich in proteids supposes a far more rapid and comprehensive destruction and reconstruction of the tissues than has been generally admitted.

VorT's theory on the different behavior of the tissue proteids. and the circulating proteids in the animal body seems to have found support in the investigations of LUDWIG and TSCHIRJEW and of FORSTER. In TSCHIRJEW's experiments a dog was given at one time boiled dog's blood, and at another time the same quantity of defibrinated dog's blood was injected into a vein. In the latter case the secretion of urea was very little increased, while in the former case it increased proportionally to the food consumed. In FonsTER's experiments, on the contrary, a dog was transfused with defibrinated dog's blood and also with horse- or dog-blood serum. After the transfusion of the blood the secretion of nitrogen was somewhat greater than in starration, but the increase was only inconsiderable. In two experiments, in which 395 and 611 grms. of blood were transfused, the increase was only 3.6 and 3.4 grms. urea, while the proteids contained in the transfused blood corresponded to 32 and 42 grms. urea. After the tranfusion of $522 \mathrm{grms}$. of dog's-blood serum, in which the quantity of proteids corresponded to 10.6 grms. urea, the elimination of urea was only 6.4 
grins. greater on the day of the transfusion than on the following day. According to these experiments, the proteids of the bloodcorpuscles, which are considered as tissue-proteids, are less easily metabolized than digested blood-proteids or the proteids of the blood-serum, and therefore these experiments confirm Vorr's views.

It has been stated above that other foods may decrease the destruction of proteids. Gelatine is such a food. Gelatine and the gelatine-formers do not seem to be converted into proteid in the body, and this last cannot be entirely replaced by gelatine in the food. For example, if a dog is fed on gelatine and fat, its body sustains a loss of proteids even when the quantity of gelatine is so large that the animal, with an amount of fat and meat containing just the same quantity of nitrogen as the gelatine in question, may remain in nitrogenous equilibrium. On the contrary, gelatine, as Voit, Panum and Oerum have shown, has a great value as a means of sparing the proteids, and, according to VoIT, it may decrease the decomposition of proteids to a still greater extent than fats and carbohydrates. This is apparent from the following summary of Vorr's experiments on a dog:

TABLE VI.

$\begin{array}{cccc}\overbrace{\text { Meat. }} & \text { Gelatine. } & \text { Fat. } & \text { Sugar. } \\ 400 & 0 & 200 & 0 \\ 400 & 0 & 0 & 250 \\ 400 & 200 & 0 & 0\end{array}$

\begin{tabular}{|c|c|}
\hline \multicolumn{2}{|c|}{ Flesh. } \\
\hline $\begin{array}{c}\text { Decomposed } \\
450\end{array}$ & $\begin{array}{c}\text { On the Body } \\
-50\end{array}$ \\
\hline 439 & -39 \\
\hline 256 & +44 \\
\hline
\end{tabular}

This ability of gelatine to spare the proteids is explained by Vorr by the statement that the gelatine is decomposed instead of a part of the circulating proteids, whereby a part of this last may be organized.

Gelatine may also decrease somewhat the consumption of fat, although it is of less value in this respect than the carbohydrates.

The question of nutritive value of peptones stands in close relation to the nutritive value of the proteids and gelatine. The early investigations made by MaLY, PLOS'z and GYeRGYAY, and AdAMKIEWIC'z have led to the conclusion that an animal with food which 
contains no proteids besides peptones may not only preserve its nitrogenous equilibrium, but indeed its proteid condition may increase. In opposition to this Vort believes, basing his opinion upon some recent experiments conducted by FEDER, that the peptones are completely destroyed in the body; that indeed by their ability of sparing the proteids they entirely, or almost entirely, prevent the consumption of proteids, but cannot pass into proteid. The general view is still that (see page 231) peptones are reconverted into proteids in the body.

In the above-mentioned experiments with peptones a mixture of albumoses and peptones in the modern sense was used. Recently Zuntz and Pollitzer have made experiments on dogs partly with meat and partly with pure peptones and albumoses of various kinds. In these experiments a deposit of proteids (retention of a part of the nitrogen) seems to have taken place in the body, and if a correction be made for that nitrogen which is contained in the extractive bodies of the meat, then the investigated digestive products seem to have about the same nutritive value for the body as the corresponding quantity of proteids of the meat.

From experiments made by WEISKE on herbivora it appears that asparagin may spare albumin in such animals. In carnivora (J. MUNK) and in mice (VoIT and Politis) it was found that asparagin does not seem to have any sparing action on the proteids. It is not known how it acts in man.

Exchange of Material on a Diet consisting of Proteid and Fat. Fat cannot arrest or prevent the destruction of proteids; but it can decrease it, and so spare the proteids. This is apparent from the following table of Vort. A is the average for three days, and B for six days.

TABLE VII.

\begin{tabular}{|c|c|c|c|}
\hline \multicolumn{2}{|c|}{ Food. } & \multicolumn{2}{|c|}{ Flesh. } \\
\hline Meat. & Fat. & Metabolized. & On the Body. \\
\hline 1500 & 0 & 1512 & -12 \\
\hline 1500 & 150 & 1474 & +26 \\
\hline
\end{tabular}

The adipose tissue of the body acts like the food-fat, and the proteid-sparing effect of the former may be added to that of the latter, so that a body rich in fat may not only remain in nitrogenous 
equilibrium, but may even add to the store of bodily proteids, while in a lean body with food containing the same amount of proteids and fat there would be a loss of proteids. In a body rich in fat a greater amount of proteids is protected from metabolism by a certain quantity of fat than in a lean body.

Because of the sparing action of fats an animal by the addition of fat to its food may, as is apparent from the tables, increase its proteid condition with a quantity of meat which is insufficient to preserve nitrogenous equilibrium, The proportion of proteids and fats in the food is of the greatest importance in regard to increasing the proteids of the body. With an exclusively proteid diet the metabolism of proteids increases with the increased amount of proteids in the food, and this behavior is not prevented by the addition of fat to the food, even though the absolute extent of the metabolism of proteids is somewhat diminished. In the presence of a great deal of proteid in proportion to the fat of the food, very large quantities of proteid are necessary for the maintenance of nitrogenous equilibrium, as well as for an increase of proteid, while by the addition of a relatively large amount of fat to the proteids such a result is reached even with comparatively small amounts of proteids. The following table will elucidate this point:

\section{TABLE VIII.}

$\begin{array}{rr}\text { Meat. } & \text { Fat. } \\ 450 & 250 \\ 1000 & 250 \\ 1500 & 250\end{array}$

\begin{tabular}{|c|c|}
\hline Metabolized. & On the Body \\
\hline 344 & +106 \\
\hline 875 & +125 \\
\hline 1381 & +119 \\
\hline
\end{tabular}

The table shows that also in the presence of fat the proteid destruction decreases with increased amounts of proteids in the food, and that, because of this, in the given examples no essentially increased metabolism of proteid is obtained with a diet of $1500 \mathrm{grms}$. meat and $250 \mathrm{grms}$. fat than with $450 \mathrm{grms}$. meat and the same quantity of fat. The importance of this fact will be spoken of later.

If the quantity of meat in the food is kept constant by feeding with meat and fat, while the fat varies, then the destruction of pro- 
teids may gradually decrease with increased quantity of fat. 'This does not always take place, but on feeding with increased amounts of carbohydrates it does (VorT).

The same sparing action on proteids which the neutral fats have occurs, according to J. Munk, also with the fatty acids, while the second chief constituent of the neutral fats, glycerine, when it is taken in quantities equal to 1-2 grms. per kilo of the body does not seem to have any influence on proteid metabolism (J. MUNK).

In regard to the metabolism of fat, it has been found that with a constant quantity of proteid in the food the metabolism of fat increases with variable amounts of absorbed fats. The following table seems to show this:

\section{TABLE IX.}

$\begin{array}{cc}\text { Meat. } & \text { Fat. } \\ 500 & 0 \\ 500 & 100 \\ 500 & 200\end{array}$

$\overbrace{\text { Metabolized. }}^{\text {On the Body. }}$
$\begin{array}{cc}47 & -47 \\ 66 & +34 \\ 109 & +91\end{array}$

The fat of the body acts like the fat of the food. A body rich in fat decomposes a greater fraction of fat than a lean body, and the same quantity of absorbed fat of the food, which in a fat body is completely decomposed, may in a lean body cause a deposit of fat. If the body takes greater amounts of fat and proteids than it can decompose in the same time, then with increasing amounts of absorbed fats the fraction of the same which is deposited in the body also increases (see Table IX). The greatest deposit of proteids and fat occurs after taking medium quantities of both in proper proportion to each other (see below).

Exchange of Material with a Diet consisting of Proteids and Carbohydrates. That which has been said above in regard to the action of fats on the metabolism of proteids answers essentially also for the carbohydrates. The carbohydrates may also spare the proteids. By the addition of carbohydrates to the food the carnivora not only remains in nitrogenous equilibrium, but the same quantity of meat which in itself is insufficient and which without carbohydrates would cause a loss of weight in the body may with the addition of carbohydrates produce a deposit of proteids. The 
carbohydrates have a stronger sparing action on proteids than fats (VorT). This is apparent from the following table :

TABLE $\mathbf{X}$.

\begin{tabular}{rccccc}
\multicolumn{4}{c}{ Food. } & \multicolumn{3}{c}{ Flesh. } \\
Meat. & Fat. & Sugar. & Starch. & Metabolized. On the Body. \\
500 & 250 & $\ldots$ & $\ldots$ & 558 & -58 \\
500 & $\ldots$ & 300 & $\ldots$ & 466 & +34 \\
500 & $\ldots$ & 200 & $\ldots$ & 505 & -5 \\
800 & $\ldots$ & $\ldots$ & 250 & 745 & +55 \\
800 & 200 & $\ldots$ & $\ldots$ & 773 & +27 \\
2000 & $\dddot{50}$ & $\ldots$ & $200-300$ & 1792 & +208 \\
2000 & 250 & $\ldots$ & $\ldots$ & 1883 & +117
\end{tabular}

Because of the great sparing action of carbohydrates on the proteids, the herbivora, whose food generally contains large quantities of carbohydrates, easily increase in proteids (VorT).

While with the same amount of flesh increased amounts of fat in the food do not continuously decrease the destruction of proteids, according to VoIT the carbohydrates habitually decrease the metabolism of proteids. This is seen from the previous table, but is more apparent from the following :

TABLE XI.

$\begin{array}{cc} & \text { Food. } \\ \text { Meat. } & \text { Carbohydrates. } \\ 500 & 100 \\ 500 & 200 \\ 500 & 300 \\ 2000 & 100 \\ 2000 & 200 \\ 2000 & 200\end{array}$

\begin{tabular}{cc}
\multicolumn{2}{c}{ Flesh. } \\
Metabolized. & On the Body. \\
537 & -37 \\
505 & -5 \\
466 & +34 \\
1847 & +153 \\
1778 & +222 \\
1780 & +220
\end{tabular}

With the addition of carbohydrates to the food the destruction of proteids increases with an increased amount of proteids. In the presence of only small amounts of carbohydrates very large amounts of proteids are necessary in the food to produce an increase in the proteids of the body, while the result is more simply and advantageously attained by considerably less proteids and proportionally more carbohydrates.

The action of the carbohydrates on the accumulation of fat has been demonstrated by the investigations of Voit and PetrenKOFER showing that the carbohydrates not only decrease the metabolism of fat and prevent its loss, but also cause its accumu- 
lation. The various views in regard to the importance of the carbohydrates in the formation of fat which have been suggested from time to time have been given on page 249 , and it is there stated that, according to the present view, the carbohydrates not only spare the fat, but may also be converted into fat in the body.

The very important question as to the conditions for the deposition of fat and flesh in the body, stands in close relation to what has just been said in regard to food consisting of proteids and carbohydrates.

In the previous pages it was repeatedly stated that an exclusive diet rich in proteids causes an increased destruction of proteids, and that such a diet soon produces a nitrogenous equilibrium. By the exclusive increased administration of proteids the proteid condition of the body may be increased only for a short time and to a small extent, and this only when the body was previously proportionally well fed. This does not occur in bodies poor in fat from disease or from some other cause. If we wish to cause a deposition of proteids in the body, then we must administer in sufficient amounts with the food, besides proteids, also other bodies which will spare the proteids, such as gelatine, fat, or carbohydrates, and indeed, for several reasons, chiefly fat and carbohydrates.

It has also been previously stated that, because of the property of the proteids to raise the proteid metabolism, an accumulation of proteids may be caused more economically and better with a medium amount of proteids and proportionally more non-nitrogenized substances than with a greater amount of proteids and proportionally less non-nitrogenized bodies. Above all, such a proper relation between proteids and non-nitrogenized bodies is important when we aim at keeping the deposit of flesh for a longer time. The following extracts from VoIT's tables are instructive in this regard:

TABLE XII.

\begin{tabular}{ccccc} 
Number of Days & \multicolumn{2}{c}{ Food. } & Total Deposit of & Nitrogenous \\
of Experimentation. & Meat, grms. & Fat, grms. & $\begin{array}{c}\text { Notal } \\
\text { Flesh, grms. }\end{array}$ & Equilibrium. \\
32 & 500 & 250 & 1794 & not yet \\
3 & 750 & 250 & 271 & near \\
3 & 1000 & 250 & 375 & " \\
4 & 1500 & 250 & 476 & " \\
7 & 1800 & 250 & 854 & nitrogenous equilibrium \\
3 & 2000 & 250 & 352 & near
\end{tabular}


The greatest absolute deposition of flesh in the body was obtained in these cases with only 500 grms. flesh and 250 grms. fat, and even after 32 days the nitrogenous equilibrium had not occurred. On feeding with 1800 grms. meat and $250 \mathrm{grms}$. fat the nitrogenous equilibrium occurred after 7 days; and though the deposition of flesh per day was greater, still the absolute deposit was not one half as great as in the former case. Inasmuch as the quantity of proteids does not decrease below a certain amount, it seems that the most abundant and most lasting deposition of flesh is obtained with a food which does not contain too much proteids in proportion to the fat. The same is also true of a diet consisting of proteids and carbohydrates.

From the above conditions concerning a deposit of fat in the body it follows that such a deposit may indeed occur with an exclusively flesh diet, but that in this case it is only very small even when large amounts of proteids are taken. For the production of an abundant deposit of fat the body must take with the food, besides proteids, either fats or carbohydrates or, which is best for human beings, fat and carbohydrates simultaneously. The carbohydrates are of special importance because they are generally cheaper in comparison with the fats. As the non-nitrogenized bodies are, according to all appearances, the most important source of muscular activity, then diminished bodily work or rest must be a favorable condition for the deposition of fat in the body. Rest, with a proper combination of the three chief groups of organic food, is therefore of great importance in fattening, the object being to cause as great an increase as possible in the mass of the proteids and fats in the body in the cheapest way.

Action of certain other Bodies on the Exchange of Material. Water. If a quantity in excess of that which is necessary is introduced into the organism, the excess is quickly and principally eliminated with the urine.. This increased elimination of urine causes in fasting animals (VoIT, Forster), but not to any mentionable degree in animals taking food (SEEGEN, MUNK, MAYER), an increased elimination of urea. The reason for this increased elimination is sought for in the fact that the abundant drinking of water causes a complete washing out of the urea from the tissues. Another view, which is defended by VoIT, is that because of the more 
active current of fluids after taking large quantities of water an increased metabolism of proteids takes place. VoIT considers this explanation is the correct one, although he does not deny that by the abundant.administration of water a more complete washing out of the urea from the tissues takes place.

In regard to the action of water on the formation of fat and its metabolism, the view that abundant drinking of water is favorable for the deposition of fat seems to be generally admitted, while taking only very little water acts against its formation.

Salts. The excretion of urine, even when no great quantities of water are taken, is increased by common salt, and the elimination of urea is also increased at the same time. The same two possibilities may be considered for this last as in the action of water on the excretion of urea. The experiments continued for a long time by Vort, in which the absolute increase of the elimination of urea was considerable (106 grms. in 49 days), render the conclusion probable that common salt somewhat increases the metabolism of the proteids. Certain other salts, such as potassium chloride, sodium sulphate, sodium phosphate, acetate, saltpetre, and ammonium chloride, also seem to act like common salt. Sodium borate and the sodium salts of salicylic and benzoic acids also seem to have an increased action on the metabolism of proteids.

Alcohol. The question as to how far the alcohol absorbed in the intestinal canal is burnt in the body, or whether it leaves the body unchanged by various channels, has been the subject of much discussion. To all appearances the greatest part of the alcohol is burnt. According to BODLÄNDER, 1.18\% of the alcohol taken is eliminated with the urine, $0.14 \%$ by the evaporation from the skin, and $1.6 \%$ with the expired air. The remainder, or about $9 \% \%$, is burnt in the body. As the alcohol is in greatest part burnt in the body, then the question arises whether it acts sparingly on other bodies, and whether it is to be considered as a nutritive body. The investigations made to decide this question have led to no decisive result. In the experiments on the elimination of nitrogen in human beings sometimes a diminished (Hammond, E. SMith, Obernitr), sometimes an unchanged (PARKES and WoLLOWICZ), and in other cases an increased (FORSTER and RoMEYN) elimination of nitrogen was observed after the administration of small amounts of alcohol. 
FoKKER and J. MUNK after the administration of small amounts of alcohol to dogs found a diminished, and after large amounts an increased, metabolism of proteids.

Many observations have been made in regard to the extent of exchange of gas after taking alcohol. BOECK and BAUER observed in dogs after giving small amounts of alcohol that the consumption of oxygen as well as the elimination of carbon dioxide was increased. BODL ̈̈NDER found in rabbits and dogs a decrease in the consumption of oxygen and the elimination of carbon dioxide, while WoLFERs found an increased consumption of oxygen in rabbits. In an investigation on the human body ZUNTZ and BERDEZ, and also Geppert, observed no essential change in the respiratory exchange of gas after small, non-intoxicating doses of alcohol. As alcohol is in greatest part burnt up in the body and the exchange of gas is nevertheless not essentially raised, it seems as if the alcohol diminishes the combustion of other bodies and thereby has a sparing value. This value may still, if it really has such an action, be of essential importance ouly in certain cases, as large quantities of alcohol taken at once or the continued use of smaller quantities has injurious action on the organism. Alcohol may therefore be considered as a nutritive body in exceptional cases only, and it otherwise must be considered as an article of luxury.

Coffee and tea have no positively proved action on the exchange of material, and their importance lies chiefly in their action upon the nervous system.

\section{The Dependence of the Exchange of Material on Other Conditions.}

Rest and Work. According to LIEBIG, muscular activity is cennected with an increased metabolism of proteids, but, as has been mentioned on page $26 \%$, the investigations of others, especially VorT on dogs, and of Petrenkofer and Vort on man, show that the total elimination of nitrogen during activity or as a result of the same is not mentionably increased. It is indeed true that certain investigators have observed an increased elimination of nitrogen in special cases; but this increase has been explained in other ways.

Work may, for instance, when it is connected with violent 
movements of the body, easily cause dyspnœa, and this last may, as FRANKel has shown, since diminution of the oxygen supply increases the proteid metabolism, cause an increase in the elimination of nitrogen. In other series of experiments the quantity of carbohydrates and fats in the food was not sufficient; the supply of fat. in the body was decreased thereby, and the destruction of proteids was also correspondingly increased. Work may also increase the appetite, and an increase in the elimination of nitrogen may be caused by the greater quantity of proteids taken. According to the generally-accepted views, muscular activity has hardly any influence on the metabolism of proteids.

On the contrary, it has a very considerable influence on the metabolism of non-nitrogenized bodies and-as a measure of the extent of the metabolism-on the elimination of carbon dioxide and the consumption of oxygen. This action, which was first. observed by LavoIsier, has recently been confirmed by many investigators. PETTEnKoFer and Vort have made investigations as to the metabolism of the nitrogenized as well as of the nonnitrogenized bodies during rest and work, partly while fasting and partly on a mixed diet. The experiments were made on a full-grown man weighing 70 kilos. The results are contained in the following table:

\section{TABLE XIII.}

Consumption of

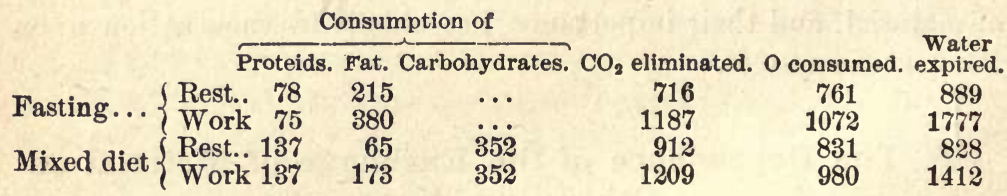

The work in this case had no influence on the destruction of proteids, while the consumption of non-nitrogenized bodies and the elimination of water by the skin and lungs was considerably increased.

The question of the exchange of material in sleep and waking stands in close relationship to the exchange of material in rest and activity. According to Pettenkofer and Vort, the metabolism of proteids is not constantly influenced by these two different conditions; on the other hand, the production of carbon dioxide is habitually greater during the day than in the night. If excessive 
work has been done during the day, the elimination of carbon dioxide may be decreased during the following night. In sleep the metabolism of non-nitrogenized substance is indeed smaller than in rest without sleep (LEviN), and it is less the more sound the sleep.

The reason for the less abundant elimination of carbon dioxide in sleep does not only depend upon muscular rest but also on several other conditions, among which are the absence of light and other excitants which act in the day and which, as it seems, cause a reflex of the chemical tonus of the muscles and thereby produce an exchange of material. Such a regulation of the exchange of material and the production of heat brought about by the nerves of the skin which is produced by the influence of the chemical tonus of the muscles shows that the external temperature is of the greatest importance in the exchange of material.

Action of the temperature of the surrounding air. In coldblooded animals the production of carbon dioxide increases and decreases with the rise and fall of the surrounding temperature. In warm-blooded animals this condition is the reverse. By the investigations of Ludwig and Sanders-Ezn, Pfü̈Ger, Duke Charles Theodore of Bavaria, and others, it has been demonstrated that in warm-blooded animals the change in the external temperature has different results, according as the animal's own heat remains the same or changes. If the temperature of the animal sinks, the elimination of carbon dioxide also sinks; if the temperature rises, the elimination of $\mathrm{CO}_{2}$ also rises. If, on the contrary, the temperature of the body remains unchanged, then the elimination of carbon dioxide increases with a lower and decreases with a higher external temperature. This condition may be explained, according to PFLÜGER and ZUNTZ, by the statement that the low temperature, by exciting a reflex action in the sensitive nerves of the skin, causes an increased metabolism of the muscles with an increased production of heat affecting the temperature of the body. The increased exchange of material produced at a low external temperature only applies, as far as is known, to the nonnitrogenized substances, but not to the proteids.

Weight of Body and Age. The greater the mass of the body the greater the absolute consumption of material; while, other things 
being equal, on the contrary, as above stated in speaking of the exchange of material in starvation, a small individual of the same species of animal, because of its relatively greater surface of body and therefore its relatively greater surface of heat, decomposes relatively more substance. With about the same bodily weight the destruction of proteids is smaller with a greater amount of fat. In women, who generally have less bodily weight and greater amount of fat than men, the consumption of proteids and the metabolism of material in general is therefore smaller, and the latter is ordinarily about $\frac{4}{5}$ of that of men. Otherwise the sex does not seem to hrve any special influence on the exchange of material.

Young animals have, for the reason above mentioned (page 444), a greater exchange of material than older ones, and they decompose a greater quantity of substance per kilo. In regard to the exchange of material in children the investigations of ScharLING and ForSTER on the elimination of carbon dioxide, and of CAMERER on the elimination of urea, are important.

Forster found in children at the ages given and at rest the following elimination of carbon dioxide per kilo in one hour:

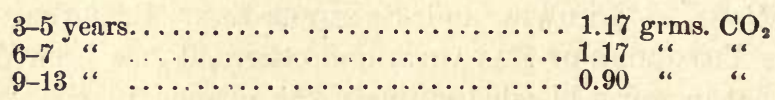

In a grown man at rest PETTENkofer and VoIt found the elimination of carbon dioxide with a mixed diet was $0.55 \mathrm{grm}$. per kilo in one hour. In children of 3 to 7 years the elimination of $\mathrm{CO}_{2}$ per kilo is more than double that of grown persons. At the age of 16 years the elimination of carbon dioxide per kilo is abont the same as in grown persons.

CAMERER has found the following results as to the elimination of urea in children:

TABLE XIV.

\begin{tabular}{|c|c|c|c|c|}
\hline \multicolumn{2}{|c|}{ Age. } & Weight of Body in Kilos. & \multicolumn{2}{|c|}{ Orea in grms. } \\
\hline & onth & 6.50 & 5.0 & 0.75 \\
\hline & years. & $\ldots \ldots \ldots \ldots, 8.95$ & 12.1 & 1.35 \\
\hline 3 & .6 . & ......... 1261 & 11.1 & 0.90 \\
\hline 4 & ." & ........ 17.43 & 14.6 & 0.84 \\
\hline 5 & “. & $\ldots \ldots \ldots \ldots 16.20$ & 12.3 & 0.76 \\
\hline 7 & “ & .......... 1880 & 13.9 & 0.74 \\
\hline 9 & “، & ….... 25.10 & 17.3 & 0.69 \\
\hline $12 \frac{1}{2}$ & “" & ......... 3260 & 17.6 & 0.54 \\
\hline 15 & “ & $\ldots \ldots \ldots \ldots 35.70$ & 17.9 & 0.50 \\
\hline
\end{tabular}


In adults weighing about 70 kilos about 30 to 35 grms. urea per day are eliminated, or 0.5 grm. per kilo. At about 15 years of age the destruction of proteids per kilo is about the same as in adults. The relatively greater metabolism of proteids in young individuals is explained partly by the fact that the metabolism of material in general is more active in young animals, and partly by the fact that the young animal is as a rule poorer in fat than the grown ones.

\section{Potential Energy and the Relative Nutritive Value of Various Organic Foods.}

With the organic foods the organism receives a supply of poten tial energy which is converted into living force in the body. This potential energy of the various foods may be represented by the amount of heat which is set free in their combustion. This quantity of heat, expressed in calories, if we consider the calorie as that quantity of heat which is necessary to raise $1 \mathrm{grm}$. of water from $0^{\circ} \mathrm{C}$. to $1^{\circ} \mathrm{C}$., is the following for $1 \mathrm{grm}$. of the substance.

\section{TABLE XV.}

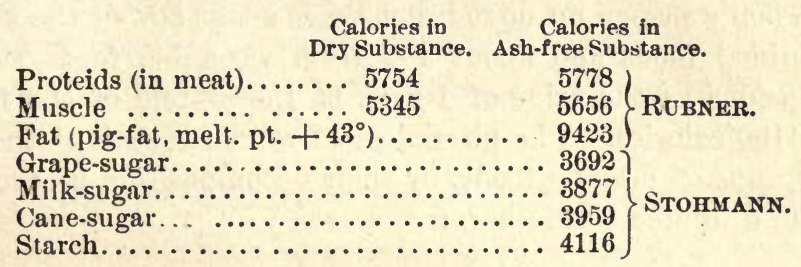

Fat and carbohydrates are completely burnt in the body, and we can therefore consider their combustion equivalent as a measure of the living force developed by them within the organism. The proteids act differently. They are only incompletely burnt, and they yield certain products of decomposition which, leaving the body with the excreta, still represent a certain amount of potential energy which is lost for the body. The heat of combustion of the proteids is smaller within the organism than outside of it, and they must therefore be specially determined. For this purpose RUnNer fed a dog on washed meat. He subtracted from the heat of com- 
bustion of the food the heat of combustion of the urine and fæces, which corresponded to the food taken plus the quantity of heat necessary for the swelling up of the albuminous bodies and the solution of the urea. RUBNER has also tried to determine the heat of combustion of the proteids (muscle-proteids) decomposed in the body of rabbits in starvation. According to these investigations, the physiological heat of combustion in calories for each gramme of substance is as follows:

\section{TABLE XVI.}

$1 \mathrm{grm}$. of the Dry Substance.

Calories.

Proteids from meat. . . . . . . . . . . . . . . . 4424

Muscle................................ 4000

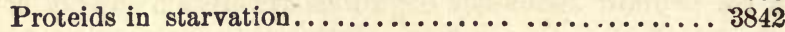

Fat (average for various fats)................ 9300

Carbohydrates (calculated average)............. 4100

The physiological combustion value of the various foods belonging to the same group is not quite the same. It is, for instance, 3969 calories for a vegetable albuminous body, conglutin, and 4424 calories for an animal albuminous body, syntonin. According to RUBNER, we may consider the normal heat value per $1 \mathrm{grm}$. of animal proteid as 4233 calories, and of regetable proteid as 3960 calories. When a person on mixed diet takes about $60 \%$ of the proteids from animal foods and about $40 \%$ from vegetable foods, then we may consider as the value of $1 \mathrm{grm}$. of the proteid of the food as about 4100 calories. The physiological value for each of the three chief groups of organic foods, by their decomposition in the body, is in round numbers as follows:

TABLE XVII.

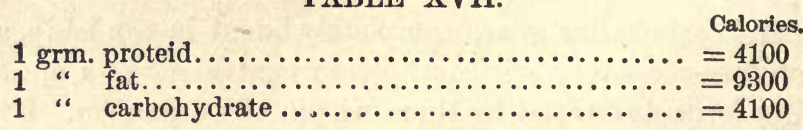

As above stated several times, the fats and carbohydrates may decrease the metabolism of proteids in the body, while, on the other hand, the quantity of proteids in the body or in the food acts on the metabolism of fat in the body. In the physiological combustion the various foods may replace one another to a certain extent, and it is therefore important to know in what proportion they replace one another. The investigations made by RUBNER have taught us 
that when we wish to diminish the loss of fat or to accumulate fat it takes place in proportions that correspond to the figures of the heat value of the same. 'This is apparent from the following table. In this we find the weight of the various foods equal to 100 grms. fat, a part determined from experiments on animals and a part calculated from figures of the heat values.

\section{TABLE XVIII.}

100 grms. fat are equal, to or isodynamic with :

\begin{tabular}{|c|c|c|}
\hline $\begin{array}{l}\text { From Experiments } \\
\text { on animals. }\end{array}$ & $\begin{array}{l}\text { From the } \\
\text { Heat Value. }\end{array}$ & $\begin{array}{l}\text { Difference, } \\
\text { per cent. }\end{array}$ \\
\hline Syntonin.........225 & & \\
\hline Muscle-flesh (dried).. 243 & 235 & +4.3 \\
\hline rch.......... 232 & 229 & +1.3 \\
\hline ae-sugar . . . . . . 234 & 235 & -0 \\
\hline pe-sugar........256 & 253 & \\
\hline
\end{tabular}

From the given isodynamic value of the various foods, it follows that these substances replace one another in the body almost in exact ratio to the potential energy contained in them. Thus in round numbers 240 grms. carbohydrate are equal to or isodynamic with $100 \mathrm{grms}$. fat, but only in regard to its ability to prevent the loss of fat. In regard to the sparing of proteids the carbohydrates accomplish more than the same quantity of fat (page 463). The knowledge of these isodynamic values, as well as of the potential energy in the various foods, is of fundamental importance in the calculation of the diet of human beings under various conditions.

\section{The Need of Man for Food under Various Conditions.}

Various attempts have been made to determine the daily amount of organic food needed by man. Certain investigators, such as Playfair, Moleschott, and others, have, from the total consumption of food by a large number of similarly-fed individuals, soldiers, sailors, laborers, etc., calculated the average quantity of food required per head. Others, such as Parkes, SMith, and VoIt, have calculated the daily demand of food from the quantity of carbon and nitrogen in the excreta. Others again, as PetrenKOFER and VoIT, have calculated the quantity of nutritive material in a diet by which an equilibrium was maintained in the individual for one or several days between the consumption and elimination of carbon and nitrogen. Lastly, others, especially Fonster, have 
quantitatively determined during a period of several days the organic nutritive substances consumed daily by persons choosing their own food, who were employed in various industries, and on which they felt well and fully capable of labor.

Among these methods a few are not quite free from reproach and others have not as yet been tried on a sufficiently large scale. Nevertheless the experiments collected thus far serve, partly because of their number and partly because of the methods, to correct and control one another, and also serve as a good starting-point in determining the diet of various classes and similar questions.

If we convert the quantity of nutritive substance daily taken into calories, produced during physiological combustion, we then obtain some idea of the sum of the chemical tension which under different conditions is introduced into the body. It must not be forgotten that the food is never completely absorbed and that undigested or unabsorbed residues are always expelled from the body with the fæces. 'The gross results of calories calculated from the food taken must therefore, according to RUBNER, be diminished at least $8 \%$.

The following summary contains certain examples of the quantity of food which is consumed by individuals of various classes under different conditions. In the last column we also find the amount of living force, calculated as calories, which corresponds to the quantity of food in question with the above-stated correction. The calories are therefore net results, while the figures for the nutritive bodies are gross results.

\section{TABLE XIX.}

\begin{tabular}{|c|c|c|c|c|c|}
\hline & Proteids. & Fat. & Carbohydrates. & Calories. & Authority. \\
\hline Soldier during peace... & 119 & 40 & 529 & 2784 & FlayfaIr. \\
\hline " light service... & 117 & 35 & 447 & 2424 & HILDESHEIM. \\
\hline " in field... .... & .146 & 44 & 504 & 2852 & " \\
\hline Laborer............. & 130 & 40 & 550 & 2903 & MOLESCHOTT. \\
\hline " at rest......... & 137 & 72 & 352 & $2458 \mathrm{P}$ & 'ETTENKOFER \& VC \\
\hline Cabinet-maker ( 40 years) & ) 131 & 68 & 494 & $28: 35$ & FORSTER. \\
\hline Young physician...... & 127 & 89 & 362 & 2602 & \\
\hline & 134 & 102 & 292 & 2476 & “. \\
\hline Laborer. . & 133 & 95 & 422 & 2902 & “ \\
\hline English smilh......... & 176 & 71 & 666 & 3780 & PlaAyfair. \\
\hline " pugilist........ & 288 & 88 & 93 & 2189 & \\
\hline avarian wood-chopper & $\mathrm{r} 135$ & 208 & 876 & 5589 & LIEBIG. \\
\hline rer in Silesia ....... & 80 & 16 & 552 & 2518 & Meinert. \\
\hline Seamstress in London.. & . 54 & 29 & 292 & 1688 & Pla yfair. \\
\hline
\end{tabular}


It is evident that persons of essentially different weight of body who live under unequal exterual conditions must need essentially different food. It is also to be expected (and this is confirmed by the table) that not only the absolute quantity of food consumed by various persons, but also the relative proportion of the various organic nutritive substances, shows considerable variation. Results for the daily need of human beings in general cannot be given. For certain classes of human beings, such as soldiers, laborers, etc., results may be given which are valuable for the calculation of the daily rations.

Based on extensive investigations and a very wide experience, VoIT has proposed the following average quantities for the daily liet of adults :

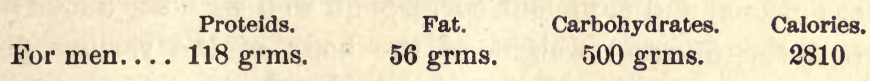

But we must here remark that these statements relate to a man weighing 70 to 75 kilos and who was engaged daily for ten hours with not too fatiguing labor.

The amount of food required by a woman engaged in moderate labor is about $\frac{4}{5}$ that of a laboring man, and we may consider the sollowing as a daily diet with moderate work.

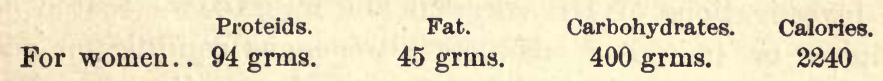

The proportion of fat to carbohydrates is here as 1:8-9. Such a proportion occurs often in the food of the poorer classes, while the ratio in the food of wealthier persons is 1:3-4. The maximum quantity of carbohydrates in the food must, according to VorT, be above 500 grms. ; and as the carbohydrates besides constitute the chief part of the often very bulky regetable foods, it has been suggested and is desirable on this and other grounds to increase the quantity of fat at the expense of the carbohydrates in such rations. But because of the high price of fat such a modification cannot always be made.

In judging the above numbers for the daily rations it must not be forgotten that the figures for the various nutritive bodies are gross results. They consequently represent the quantity of the 
nutritive vodies which must be taken in, and not those which are really absorbed. The figures for the calories, which here and in the following pages are so-called gross calories are, on the contrary, net results.

The various foods are, as is well known, not equally digested and absorbed, and in general the vegetable foods are less completely used up than animal foods. This is especially true of the proteids. When, therefore, VorT, as above stated, calculated the daily amount of proteids needed by a laborer as 118 grms., he starts with the supposition that the diet is a mixed animal and vegetable one, and also that of the above 118 grms. about 105 grms. are absorbed. The results obtained by PFLÜGER and his school, BLEIBTREU and BOHLAND, for the extent of the metabolism of proteids in man with a diet optional and sufficient correspond well with the above figures -when the unequal weight of the body of the various persons experimented upon is sufficiently considered.

As a rule, as a more exclusively vegetable food is employed, the amount of proteids in the same is also habitually smaller. The strictly vegetable diet of certain people-as of the Japanese-and that of the so-called vegetarians is therefore a proof that a person, if the quantity of food be sufficient, may exist on considerably smaller quantities of proteids than VoIT suggests. It follows from the investigations of HIRSCHFELD and KuMAGAwA that a nearly complete or indeed a complete nitrogenous equilibrium may be attained by the sufficient administration of non-nitrogenized nutritive bodies with relatively very small amounts of proteids. Hirschfeld, who weighed 73 kilos, could maintain nitrogenous equilibrium very nearly with a diet containing $43.5 \mathrm{grms}$. nitrogenized bodies, 165 grms. fat, 354 grms. carbohydrates, and 42.7 grms. alcohol. Kumagawa made experiments on himself with a purely vegetable diet, consisting mainly of boiled rice. On an average $50.5 \mathrm{grms}$. proteids and 569.83 grms. carbohydrates were daily introduced, and of this $3 \% .82$ grms. proteids and 566.7 grms. were daily used up By this food he was not able with a bodily weight of 48 kilos to attain nitrogenous equilibrium, but he found indeed that a part of the nitrogen was retained in the body. The weight of the body increased and the general condition was good. The total amount of calories in the absorbed food was in this case 2500 in round num- 
bers, or 52 per kilo. It follows from the experiments just mentinued that an adult may be sufficiently nourished with a considerably smaller quantity of proteids than VoIT considers necessary, if the total food corresponds to the demands of the body in calories, which may be accomplished by a corresponding increase in the administration of non-nitrogenized nutritive bodies.

If we keep in mind that the food of people of different countries varies greatly, and that the individual also takes essentially different nourishment according to the external conditions of living and the influence of climate, it is not remarkable that a person accustomed to a mixed diet cannot exist for a long time on a strictly vegetable diet deficient in proteids, even though not especially difficult to digest. No one doubts the ability of man to adapt himself to a heterogeneously-composed diet when this is not too difficult of digestion and is sufficient; but this ability does not seem sufficient reason for essentially altering the figures suggested by VorT. VoIT's figures are based on comprehensive experiments, also on experience and exact knowledge of the actual existing conditions, and they are, which is especially important, as above stated, only given for certain cases or certain classes of people. It is not denied by any one that these results are not applicable to all cases, as it is evident that the daily ration necessary for a laborer given by Vorr must be altered somewhat for other countries because of the existing conditions in middle Europe, where VoIT made his investigations. With regard to the conditions in Sweden we may propose as the daily diet of a laboring man about 120 grms. proteids, 100 grms. fat, and $400-450$ grms. carbohydrates. These figures are based on the experience obtained in Sweden and calculations made by the author.

If we compare the figures of Table XIX with the average figures proposed by Vort for the daily diet of a laborer, it would seem at the first glance as if the consumed food in certain cases was considerably in excess of the need, while in other cases, as for instance for the seamstress in London, it was entirely insufficient. A positive conclusion cannot, therefore, be drawn if we do not know the weight of the body as well as the labor performed by the person, and also the conditions of living. It is certainly true that the amount of nutriment required by the body is not directly propor- 
tional to the bodily weight, for a small body consumes relatively more substance than a larger one, and varying amounts of fat may also cause a difference; but a large body, which must maintain it greater quantity, consumes an absolutely greater quantity of substance than a small one, and in estimating the nutritive need one must also always consider the weight of the body. According to Vort, the diet for a laborer with 70 kilos bodily weight requires 40 calories for each kilo. In KUMAGAWA's series of experiments, in which the absolute quantity of food was smaller, the average was 52 calories for each kilo.

As above stated several times, the demands of the body for nourishment vary with its different conditions. Among these conditions two are practically important, namely, labor and rest.

In a previous chapter, in which muscular labor was spoken of, it was seen that the generally accepted view is that non-nitrogenized food is the most essential, if not the exclusive, source of muscular force. As a natural sequence it is to be expected that in activity the non-nitrogenized foods before all must be increased in the daily rations.

Still this does not seem to correspond to daily experience. It is a well-known fact that hard-working individuals-men and animals-require a greater amount of proteids in the food than less active ones. This contradiction is, however, only apparent, and it depends, as Vort has shown, upon the fact that individuals used to violent work are more muscular. For this reason a person performing severe muscular labor requires food containing a larger proportion of proteids than an individual whose occupation demands less violent exertion. Another question is, how should the relative and absolute amount of food be changed if increased exertion be demanded of one and the same individual ?

An answer based upon experience may be found in statistics concerning the maintenance of soldiers in peace and in war. Many such statements are obtainable. In a critical examination of the same it was found that in war-rations the quantity of non-nitrogenized bodies as compared to the proteids is only increased in exceptional cases, while usually the reverse is the case. Even in these cases the actual proportion does not correspond to the theoretical demand upon which, however, too great stress must not be placed, 
since in the case of soldiers in the field many other circumstances are to be considered, such as the volume and weight of the food, etc., etc., which cannot here be more closely discussed. The following table shows the average results of soldiers' rations in war and peace, calculated by ALMÉN from the detailed statement of several countries. ${ }^{1}$

These average results also include the figures for Sweden.

TABLE XX.

A. Peuce Ration.

\begin{tabular}{|c|c|c|c|}
\hline & Proteids. & Fat. & Carl \\
\hline 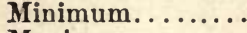 & .. 108 & 22 & \\
\hline$\ldots$ & .165 & 97 & $7: 31$ \\
\hline (5) & . 130 & 40 & 53 \\
\hline proposed) & . 179 & 102 & 59 \\
\hline
\end{tabular}

B. War Ration.

$\begin{array}{crc}\text { Proteids. } & \text { Fat. } & \text { Carb. } \\ 126 & 38 & 484 \\ 197 & 95 & 688 \\ 146 & 59 & 557 \\ 202 & 137 & 565\end{array}$

If we do not consider the very abundant rations proposed for the soldier in Sweden, and if we only adhere to the above mean figures, we obtain the following results for the daily rations:

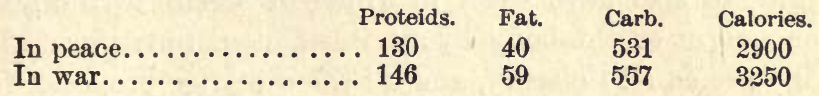

If we calculate the fat in its equivalent amount of starch, then the relation of the proteids to the non-nitrogenized foods is :

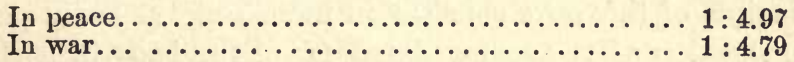

The proportion is nearly the same in both cases; the small difference which occurs shows a slight relative increase in the proteids in the war ration. On the contrary, what is especially apparent from the total of the calories, the total quantity of nutritive bodies is greater in the war than in the peace ration.

As more work requires an increase in the absolute quantity of food, so the quantity of food must be diminished when little work is performed. 'The question as to how far this can be done is of importance in regard to the diet in prisons and poor-houses. We give below the following as example of such diets.

1 Germany, Austria, Switzerland, France, Italy, Russia, and the United States. 
TABLE XXI.

\begin{tabular}{|c|c|c|c|c|c|}
\hline & Proteids. & Fat. & Carb. & Calories. & \\
\hline (not working & $\begin{array}{l}\ldots 87 \\
\ldots 85\end{array}$ & $\begin{array}{l}22 \\
30\end{array}$ & $\begin{array}{l}305 \\
300\end{array}$ & $\begin{array}{l}1667 \\
1709\end{array}$ & $\begin{array}{l}\text { Sch } \\
\text { Vol }\end{array}$ \\
\hline & 92 & 45 & 332 & 1985 & Forster. \\
\hline & 80 & 49 & 266 & 1725 & \\
\hline
\end{tabular}

The figures given by Vort are, according to him, the lowest figures for a non-working prisoner. He considers the following as the lowest diet for old non-working people:

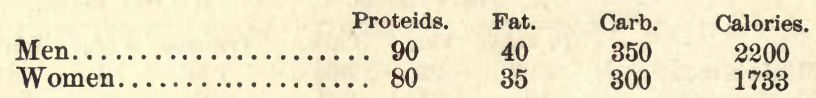

In calculating the daily diet it is in most cases sufficient to ascertain how much of the various nutritive bodies must be daily administered to the body to keep it in the proper condition to perform the work required of it. In other cases it may be required to improve the nutritive condition of the body by properly selected food; but we also have cases in which we desire to diminish the mass or weight of the body by an insufficient nutrition. This is especially the case in obesity, and all the dietaries proposed for this purpose are chiefly starvation cures.

The oldest and most generally-known diet cure for corpulency is that of HARVEY, which is ordinarily called the BANTING method. The principle of this cure consists in increasing, as far as possible, the consumption of the accumulated fat of the body by as limited a supply of fat and carbohydrates as possible and a simultaneous increased supply of proteids. A second cure, called EBstein's cure, is based on the assumption (not correct) that the fat of the food is not accumulated in a body rich in fat, but is completely burnt. In this cure large quantities of fat are therefore allowed in the food, while the quantity of carbohydrates is diminished very much. The third cure, called OER'TEL's cure, is based on the correct view that a certain quantity of carbohydrates has no greater influence in the accumulation of fat than the isodynamic quantities of fat. In this cure, therefore, carbohydrates as well as fat are allowed, provided the total quantity of the same is not so great as to hinder the decrease in the fatty condition. A greatly dimin- 
ished supply of water is also one of the features of OERTEL's cure, especially in certain cases. The average amount of the various nutritive substances supplied to the body in these three cures is as follows, and we give also for comparison in the same table VoIT's diet necessary for a laborer.

\section{TABLE XXII.}

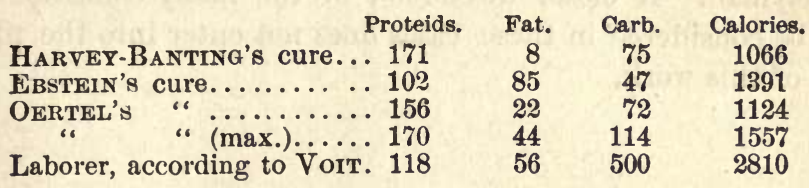

If the fat in all cases is recalculated in starch, then the proportion of the proteids to the carbohydrates is :

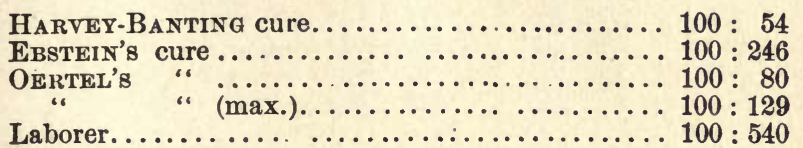

In all these cures for corpulence the quantity of non-nitrogenized bodies is diminished as compared to the proteids; but chiefly the total quantity of food, as is shown by the number of calories, is considerably diminished.

HARVEY-BANTING's cure differs from the others in a relatively very much greater amount of proteids, while the total number of calories in it is the smallest. On this account this cure acts very quickly; but it is therefore also more dangerous and more difficult to accomplish. In this regard EBstein's and Oertel's cures (especially OERTEL'S), having a greater variation in the selection of food, are better. As the adipose tissue has a proteid-sparing action, we have to consider in using these cures, especially BANTING's, that the destruction of proteids in the body is not increased with the decrease in the adipose tissue, and one must therefore carefully watch the elimination of nitrogen by the urine. All diet cures for obesity are moreover, as above stated, starvation cures; and if the daily amount of food required by an adult man, represented as calories, is in round numbers 2500 calories (according to the average 
figures found by Forstrk in the case of a physician), then one immediately sees what a considerable part of its own mass the body must daily give up in the aboveteures. This reminds us of the great care necessary in employing these cures; but each special case should be conducted with regard to the individuality, the weight of the body, the elimination of nitrogen in the urine, ete., ete, and always under strong control and only by physicians, never by a layman. A closer discussion of the many conditions which must be considered in these cases does not enter into the plan and scope of this work. 
TABLE I.-FOODS. ${ }^{1}$

\begin{tabular}{|c|c|c|c|c|c|c|c|c|c|}
\hline \multirow[b]{2}{*}{ 1. Animal Foods. } & \multicolumn{6}{|c|}{1000 Parts contain } & \multicolumn{3}{|c|}{ Relatlonshis of } \\
\hline & 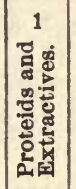 & 离 & 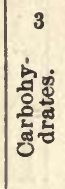 & 4 & 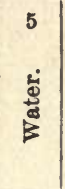 & $\begin{array}{c}6 \\
\text { sं } \\
\text { s. } \\
\sum^{\infty}\end{array}$ & 1 & $: 2$ & $: 3$ \\
\hline a. FLesh WIthout BoNes. & & & & & & & & & \\
\hline Fat beef ${ }^{2} \ldots \ldots \ldots \ldots \ldots \ldots$ & 183 & 166 & & 11 & 640 & & 100 & 90 & \\
\hline Beef (average fat ${ }^{3}$ ). & 196 & 98 & & 18 & 688 & & 100 & 50 & 0 \\
\hline Beef ${ }^{2}$................................ & 190 & 120 & & 18 & 672 & & 100 & 63 & \\
\hline Corned beef (average fat)..... . & 218 & 115 & & 117 & 550 & & 100 & 53 & \\
\hline 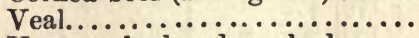 & 190 & 80 & & 13 & 717 & & 100 & 42 & 0 \\
\hline Horse, salted and smoked...... & 318 & 65 & & 125 & 492 & & 100 & 20 & \\
\hline Smoked ham.. & 255 & 365 & & 100 & 280 & & 100 & 143 & \\
\hline Pork, salted and smoked ${ }^{4} . . .$. . & 100 & 660 & & 40 & 130 & & 100 & 660 & \\
\hline lesh from hare............. & 233 & 11 & & 12 & 744 & & 100 & 5 & \\
\hline " " chicken............ & 195 & 93 & & 11 & 701 & & 100 & 48 & 0 \\
\hline " " partridge.......... & 253 & 14 & & 14 & 719 & & 100 & 6 & 0 \\
\hline " " wild duck......... & 246 & 31 & & 12 & 711 & & 100 & 13 & 0 \\
\hline b. Flesh With Bones. & & & & & & & & & \\
\hline Fat beef ${ }^{2} \ldots \ldots \ldots \ldots \ldots \ldots$ & 156 & 141 & & 9 & 544 & 150 & 100 & 90 & 0 \\
\hline Beef, average fat $^{3} \ldots$. & 167 & 83 & & 15 & 585 & 150 & 100 & 49 & 0 \\
\hline Beef, slightly corned.......... & 175 & 93 & & 85 & 480 & 167 & 100 & 53 & 0 \\
\hline Beef, thoroughly corned....... & 190 & 100 & & 100 & 430 & 180 & 100 & 53 & 0 \\
\hline Mutton, very fat $\ldots \ldots \ldots \ldots \ldots$ & 135 & 332 & & 8 & 437 & 88 & 100 & 246 & 0 \\
\hline$f_{\Omega}$ & 160 & 160 & & 10 & 520 & 150 & 100 & 100 & \\
\hline Pork, fresh, fat... & 100 & 460 & & 5 & 365 & 70 & 100 & 460 & 0 \\
\hline Pork, corned, fat.. & 120 & 540 & & 60 & 200 & 80 & 100 & 450 & 0 \\
\hline Smoked ham............... & 200 & 300 & & 70 & 340 & 90 & 100 & 150 & 0 \\
\hline c. Frshes. & & & & & & & & & \\
\hline River eel, fresh, entire. & 89 & 220 & & 6 & 352 & 333 & 100 & 246 & 0 \\
\hline Salmon, “ & 121 & 67 & & 10 & 469 & 333 & 100 & 56 & $\theta$ \\
\hline Anchovy, “ & 128 & 39 & & 11 & 489 & 333 & 100 & 31 & 0 \\
\hline Flounder, “ & 145 & 14 & & 11 & 580 & 250 & 100 & 9 & 0 \\
\hline River perch,“" & 100 & 2 & & 8 & 440 & 450 & 100 & 2 & 0 \\
\hline Torsk, “ & 86 & 1 & & 8 & 455 & 450 & 100 & 1 & 0 \\
\hline
\end{tabular}

1 The results in the following tables are chiefly compiled from the summary of ALMtiv and of Kösig. As "waste" we here designate that part of the foods which is lost in the preparation of the food or that which is not used by the body; for instance, the bones, skin, egg-shell, and the cellulose in the vegetable foods.

2 Meat such as is ordinarily sold in the markets in Sweden.

- Beef such as is delivered by large purveyors to public institutions in Sweden.

- Pork, chiefly from the breast and belly, such as occurs in the rations of Swedish soldiers. 
TABLE I.-FOODS.-(Continued.)

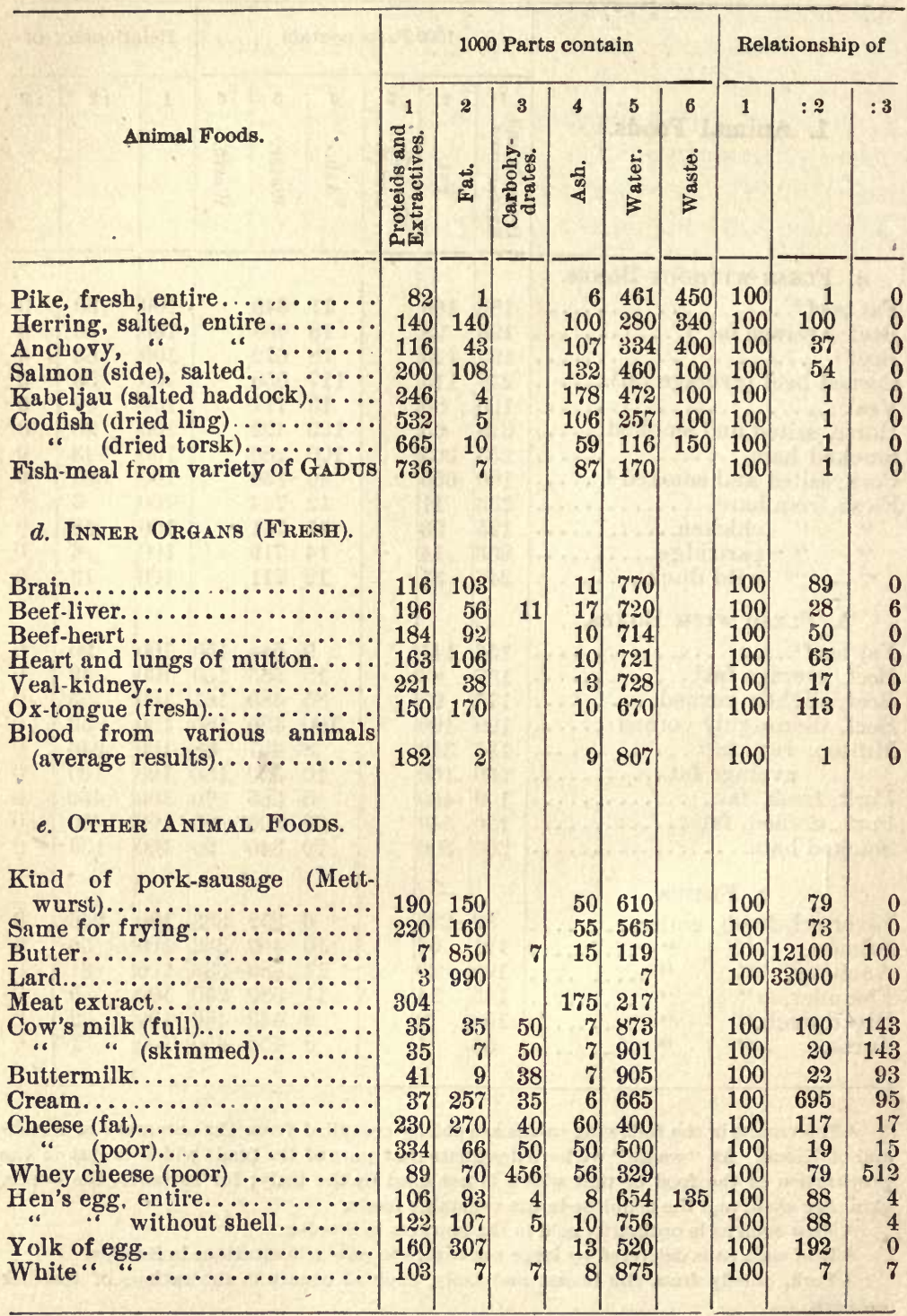


TABLE I.-FOODS.-(Continued.)

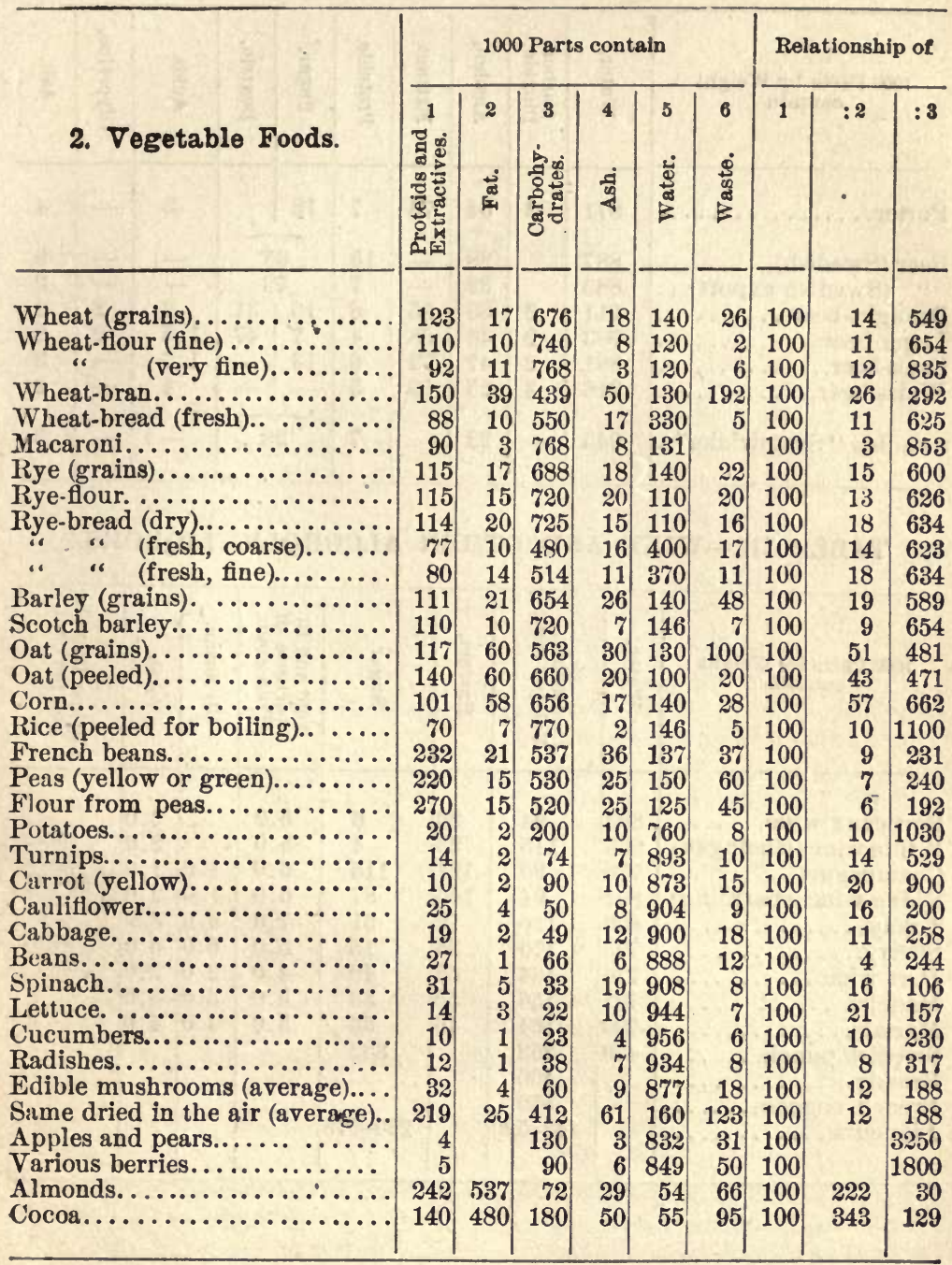


TABLE II.-MALT LIQUORS.

\begin{tabular}{|c|c|c|c|c|c|c|c|c|c|c|}
\hline $\begin{array}{l}1000 \text { Parts by Weight } \\
\text { contain }\end{array}$ & 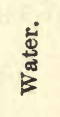 & 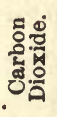 & वृं & 荵 & 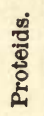 & 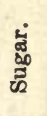 & 产 & : & 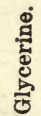 & क् \\
\hline Porter............... & 871 & 2 & 54 & 76 & 7 & 13 & & 3 & - & 4 \\
\hline 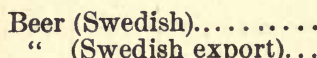 & $\begin{array}{l}887 \\
885\end{array}$ & & $\begin{array}{l}28 \\
32\end{array}$ & 二 & 15 & 6 & & $\overline{-}$ & - & 5 \\
\hline Draught-beer........... & 911 & 2 & 35 & 55 & 8 & $10^{\circ}$ & 31 & 2 & 2 & 2 \\
\hline Lager-beer... ........ & 903 & 2 & 40 & 58 & 4 & 7 & 47 & 1.5 & 2 & 2 \\
\hline Bock-beer............. & 881 & 2 & 47 & 72 & 6 & 13 & - & 1.7 & - & 3 \\
\hline 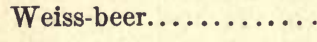 & 916 & 3 & 25 & 59 & 5 & & - & 4 & - & 2 \\
\hline Swedish "Svagdricka". . & 945 & - & 22 & & 7 & 2 & & - & - & 3 \\
\hline
\end{tabular}

TABLE III.-WINE AND OTHER ALCOHOLIC LIQUORS.

\begin{tabular}{|c|c|c|c|c|c|c|c|c|}
\hline $\begin{array}{l}1000 \text { Parts by Weight } \\
\text { contain }\end{array}$ & 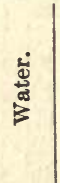 & 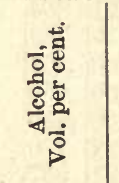 & 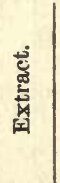 & 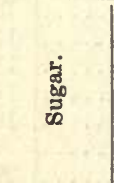 & 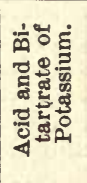 & 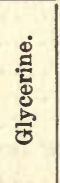 & 完 & 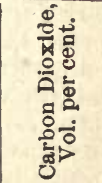 \\
\hline 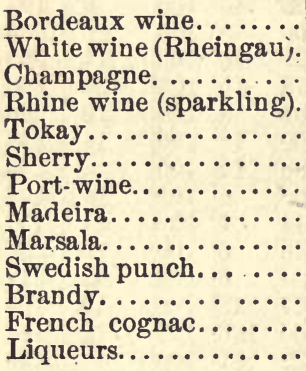 & $\begin{array}{l}883 \\
863 \\
776 \\
801 \\
808 \\
795 \\
774 \\
791 \\
790 \\
479\end{array}$ & $\begin{array}{r}94 \\
115 \\
90 \\
94 \\
120 \\
170 \\
164 \\
156 \\
164 \\
263 \\
460 \\
550 \\
442-590\end{array}$ & $\begin{array}{r}23 \\
23 \\
134 \\
105 \\
72 \\
35 \\
62 \\
53 \\
46\end{array}$ & $\begin{array}{r}6 \\
4 \\
115 \\
87 \\
51 \\
15 \\
40 \\
33 \\
35 \\
332\end{array}$ & $\begin{array}{l}5.9 \\
5.0 \\
6.0 \\
6.0 \\
7.0 \\
5.0 \\
4.0 \\
5.0 \\
5.0\end{array}$ & $\begin{array}{l}1.0 \\
1.0 \\
9.0 \\
6.0 \\
2.0 \\
3.0 \\
4.0\end{array}$ & $\begin{array}{l}2.0 \\
2.0 \\
1.0 \\
2.0 \\
3.0 \\
5.0 \\
3.0 \\
3.0 \\
4.0\end{array}$ & 60-70 \\
\hline
\end{tabular}




\section{INDEX.}

Absorption, 227-232 ; importance of the cells for the same, $225,231,232$; action on the putrefactive processes in the intestines, 220.

Absorption-ratio, 81; of the blood-coloring matters, 82.

Acetic acid, in the gastric juice, 177 ; in the contents of the stomach, 196 ; passage into the urine, 374,389 .

Aceto-acetic acid, 422; in urine, 420, 421.

Acetone, 421 ; in blood, 114 ; in urine, 420.

Acetonuria, 421.

Acetylen hænıglobin, 74.

Acholia, pigmentary, 158.

Achroo-dextrin, 171.

Acid albuminate, 14 ; properties and behavior, 23, 24; in pepsin digestion, $182,183$.

Acid amides, behavior in the body, 389 .

Acid rigor, 262.

Acids, organic, behavior in the body, 389.

Acidity, of the contents of the stomach, 195 ; of the urine, 334 .

Acrolein, 244 ; test, 244, 246.

Actinochrom, 325.

Acrylic acid, action on the elimination of uric acid, 351.
Acrylic acid di-ureid, 350.

ADAMKIEWICZ's reaction, 20.

Adenin, 48; properties and occurrence, 51 ; in urine, 359.

Adhesion, importance in the coagulation of blood, 89.

Adipocere, 248.

Agagropila, 226.

Albumin glands, 167 .

Albumins, 14 ; general properties, 22 (see also various albumins).

Albumin, detection in the urine, 397 ; quantitative estimation, 399 (see also Protein bodies).

Albuminous bodies, general behavior, reactions, splitting products and com. position, 15-21 ; summary, 14, 22-30 (see also various albuminous bodies of the tissues and fluids).

Albuminate, 14 ; properties and beh:Irior, 23,24 ; ferruginous albuminate in the spleen, 130 .

Albuminoids, 14,34 ; in cartilage, 234. 236 ; in the yolk membrane, 291.

Albumoses, 14 ; general properties, 2530 ; in the putrefaction of proteids, 216 ; in pepsin digestion, 182 ; in tryp. sin digestion, 206 ; in urine, 398 ; nutritive value, 460 .

Alcapton, 365. 
Alcohol, see Ethyl-alcohol.

Alcoholic fermentation, 7, 307, 409.

Alanin, 39.

Aleurone crystals, 292.

Alizarin, in the urine, 393.

Alizarin blue, behavior in the tissues, 5 .

Alkali albuminate, 14 ; properties, 23 , 24 ; in the eye, 280,283 ; in the yolk of the egg, 293 ; in the brain, 273 ; in the non-striated muscles, 272 ; Lieberkuhn's alkali albuminate, 23.

Alkali carbonate, physiological importance, 452 ; action on the secretion of gastric juice, 176 ; occurrence, see the various tissues and fluids.

Alkali earths, in the urine, 384 ; in the bones, 238, 239 ; insufficient supply, 241.

Alkali phosphates, in urine, 384 ; occurrence, see the various tissues.

Alkali urates, in sediments, 428 ; in calculi, 431.

Alkaline fermentation of the urine, 374, 427.

Alkaloids, action on the muscles, 262 ; passage into the urine, 393 .

Allantoic fluid, 358.

Allantoin, properties, occurrence, etc., 358 ; formation from uric acid, 350 ; in transudations, $122,124$.

Alloxan, 350.

Almín-Böttger's bismuth test, 409 , 411.

Almén's guaiacum test for blood, 402.

Amanitin, 44.

Ambergris, $22 \%$.

Ambrain, 227.

Amido-acids, relation to the formation of urea, 338 ; to the formation of uric acid, 352; production in putrefaction, 216 ; in pepsin digestion, 182 ; from protein bodies, $15,16.35,36,206$, 216 ; in trypsin digestion, 206.

Amido-acetic acid, see Glycocoll.

Amido-acrolein, 37.
Amido-caproic acid, see Leucin.

Amido-cinnamic acid, behavior in the organ ism, 391.

Amido-ethylsulphuric acid, see Taurin.

Amido-phenylacetic acid, behavior in the organism, 392.

Amido-phenylpropionic acid, formation in the putrefaction of proteids, 360 ; behavior in the organism, 391, 392.

Amido-succinic acid, see Aspartic acid.

Ammonia, formation in the putrefaction of proteids, 216 ; from proteid substances, $16,17,35,36,206,216$; in trypsin digestion, 206; in the blood, 114 ; in the urine, 383.

Ammonia, elimination in disease of the liver, 383 ; after the administration of mineral acids, 333 ; after extirpation of the liver, 352 .

Ammonia, estimation in the urine, 384 .

Ammonia salts, relation to the formation of urea, 338 ; to the formation of uric acid, 352.

Ammonio-magnesium phosphate, in urinary calculi, 432 ; in urinary sediments, 430.

Ammonium urate, in urinary calculi, 431 ; in urinary sediments, 428.

Amniotic fluid, 298.

Amphicreatin, 258.

Amphopeptone, 26.

Amyl-alcohol, 390.

Amyl nitrite, poisoning, 115, 407.

Amyloid, 14, 39.

Anæmia, 111, 113 ; malignant, 112.

Anhydride theory of the formation of glycogen, 140.

Aniline, behavior in the organism, 391.

Anisotropous substance, 251.

Antedonin, 325.

Anthrax spores, behavior with gastric juice, 192.

Antialbumose, 26.

Autipeptone, 26. 
Antimony, passage into milk, 320 ; action on the elimination of nitrogen, 337.

Antipyrin, action on the urine, 394 .

Apatite, 238.

Arachidic acid, in butter, 303.

Arachnoid membrane, fluid of, 122.

Arbutin, importance in the formation of glycogen, 139 ; behavior in the organism, 365.

Aromatic combinations, behavior in the organisin, 390-394.

Arsenic, passage into milk, 320 ; into sweat, 328 ; action on the elimination of nitrogen, 337 .

Arsenic acid, action on pepsin digestion, 181.

Arseniuretted hydrogen, poisoning, 160-163, 401.

Arterin, 68.

Ascitic fluids, 124.

Asparagin, importance in the synthesis of proteids, 17 ; nutritive value, 460.

Asparaginic acid, see Aspartic acid.

Asparagus, in the urine, 394.

Aspartic acid, relation to the formation of urea, 338; to the formation of uric acid, 352 ; formation from proteids, $17,206,210$; behavior in the organism, 389.

Ass's milk, 312.

Atmid-albumin, 27.

Atmid-albumose, 27.

Atropin, action on the secretion of saliva, 173.

Auto-intoxication, 12.

Bacterium ureæ, $42 \%$.

Banting's diet cure, 480, 481.

Bases, nitrogenized from albumin, 17.

Beeswax, 250.

Benzoar stone, 226.

Benzoic acid, formation from protein substances, $17,37,359$; passage into the sweat, 328 ; behavior in the or- ganism, 2, 360, 392; occurrence in the urine, 362 ; action on the exchange of material, 466 ; substituted benzoic acids, behavior on the body, 392 .

Benzol, behavior in the organism, 390 , 391.

Benzoyl amido-acetic acid, see Hippuric acid.

Benzoyl chloride, behavior with carbohydrates, 410 , to cystin ; 426 .

Benzoyl cystin, 426.

Benzyl alcohol, behavior in the organism, 3.

Bile, 143-163 ; general chemical behavior, 144 ; analyses of the same, 157 , 158 ; antiseptic action of, 220,221 ; constituents, 144-156; in disease, 158 ; diastatic action of, 212 ; action on the digestion of proteids, 214,215 ; on the emulsification of fats, 213,214 ; on the secretion of bile, 144 ; on the absorption of fat, 213, 220, 232 ; on the splitting of neutral fats, 214 ; on trypsin digestion, 206, 215 ; quantity, 143 ; passage of foreign substances into, 158; occurrence in the urine, 404,405 ; in the contents of the stomach, 193; in meconium, 224 ; composition, 157.

Bile-acids, 146-150 ; in blood, 114, 159 ; pus, 130 ; in urine, $162,228,404$; absorption, 228.

Bile-acids, Pettenkofen's test for,146. Bile-formation, 159-163.

Bile-pigments, $152-156$; origin, 160 , 161 ; reactions, $154,405,406$; passage into urine, $162,405,406$; occurrence in blood-serum, 63,114 ; in egg shells, 296.

Bile-salts, 145, 146.

Bile-secretion, 143.

Bilianic acid, 148.

Biliary calculi, 163.

Biliary fistula, 143 ; influence on putrefaction in the intestines, 220 . 
Bilicyanin, 152, 154, 156.

Bilifulvin, 153.

Bilifuscin, 152, 156, 163.

Bilihumin, 152, 156.

Biliphæin, 153.

Biliprasin, 152, 156.

Bilirubin, relation to the blood-coloring matters, 160,161 ; to hæmatoidin, 80 , 153,160 ; properties and occurrence, 153 ; in urine, 405 ; in the placenta, 298.

Bilirubin calcium, 153, 163.

Biliverdin, properties and occurrence, 155 ; in the egg-shell, 296 ; in excrements, 223 ; in urine, 405 ; in the placenta, 298.

Bitch's milk, 312.

Biuret, 339.

Biuret reaction, 20, 340.

Blister-fluid, 126.

Blood, 54-116; general behavior, 54, 86,87 ; analyses, quantitative, 105 107 ; arterial and venous, $68,87,94$, 95 ; defibrinated, 55 ; suffocationblood, $5,68,87,95$; quantity in body, 115 ; detection, medico-legal, 80 ; behavior in starvation, 110,448 ; composition under pathological conditions, 111-115 ; under physiological conditions, 108-111; in urine, 401 , 402 ; in contents of the stomach, 193. Blood-clot, 55.

Blood, coagulation of, 54, 59, 87-94.

Blood-coloring matters, $68-83$; in the urine, 401.

Blood-corpuscles, red, 66,67 ; in urine, 401 ; composition, $83,106,112$; colorless, 84 ; behavior in the coagulation of the blood, 84, 90, 91 .

Blood-cylinders, 402.

Blood, distribution of, in the body, 116 .

Blood, gases of, 94-105.

Blood-loss, 115.

Blood-plasma, 54-62 ; composition, 64, 107.
Blood-serum, 55, 62-66 ; composition, 64, 106.

Blood-spots, 80.

Blood-sweat, 328.

Blood-tablets, $54,84,85$; importance in coagulation, 91 .

Blood-transfusion, 111, 115.

Blonds, milk of, 316.

Blueberry, coloring matter of, in urine, 394.

Blue stentorin, 325.

BöTTCHER's spermine crystals, 286.

BöTtGer-Almén's bismuth test, 409, 411.

Bones and bone structure, 238-242.

Bone earths, 238, 239.

Bone, softening of, 340 .

Bonellin, 325,

Borneol, behavior in the organism, 393.

Borax, action on the exchange of material, 466 ; on trypsin digestion, 206.

Bowman's disks, 251.

Brain, 273-280.

Bread, behavior in the stomach, 187 : excrements after feeding on bread, 220.

Bromanil, 17.

Bromine combinations, passage into the saliva, 173.

Bromoform, 17.

Brunner's glands, 197.

Brunette, milk of, 316.

Brusæ mucosæ, contents of, 127.

Buccal mucus, 169.

Bufidin, 326.

Butter-fat, 303.

Buttermilk, 312.

Butyric-acid, in contents of the stomach, 193, 196; in the gastric juice, 177 ; in butter-fat, 303 .

Butyric-acid fermentation, 4, 409; in intestines, 218.

Butyl-alcohol, behavior in the body, 390. 
Butyl-chloral, behavior in the body, 390.

Byssus, 14, 39.

Cadaverin, 376, 425.

Caffein, 48; influence on the elimination of uric acid, 352 ; action on the muscles, 262.

Cairin, action on the urine, 394 .

Calcium carbonate, in urine, 332 ; in urinary calculi, 432 ; in urinary sediments, 429 ; in bones, 238,239 ; in tartar, 174.

Calcium, lack of, in the food, 241,453 ; occurrence, see various fluids and tissues.

Calcium oxalate, 357 ; in urinary sediments, 428 ; in urinary calculi, 432.

Calcium phosphate, relationship to the coagulation of casein, 305 ; to fibrin coagulation, 97 ; occurrence in intestinal calculi, 226 ; in urine, 332,384 ; in urinary sediment, 429 ; in urinary calculi, 432 ; in protein bodies, 13,97 ; in salivary calculi, 174 .

Calcium sulphate, in urinary sediments, 429.

Calories of the food, $471-473$; various diateries, 474-482.

Campherol, 393.

Camphor, 375, 393.

Camphor-glycuronic acid, 375, 393.

Cane-sugar, behavior with intestinal juice, 198 ; with gastric juice, 183.

Capric acid, 303.

Caproic acid, formation from phenol, 6 , 390 ; in fatty tissues, 243 ; in milkfat, 303.

Caprylic acid, 303.

Carbamic acid in the blood, 63.

Carbohydrates, importance for the formation of fat, 249,463 ; for the glycogen formation, 139 ; for muscular activity, $265,268,269$; exclusive feeding on carbohydrates, 249 ; action on the metabolism of proteids, 462 ; on putrefaction, 220 ; absorption, 227 , 229,407 ; inadequate supply of, 454 (see various carbohydrates).

Carbolic acid, action on pepsin digestion, 181 (see Phenol).

Carbolic urine, 365.

Carbon dioxide in the blood, 95, 96 ; in diabetes, 104 ; in poisoning with mineral acids, 176 ; in the intestines, 216 ; in the lymph, 118 ; in the contents of the stomach, 189 ; in the muscles in activity and at rest, 264, $265,268,269$; in rigor, 263 ; in secretions, $158,170,197,311,385$; in transudations, 122 ; binding of $\mathrm{CO}_{2}$ in the blood, 96-100; action on the elimination of gastric juice, 176; tension in blood, 102, 104; tissues, 104; lymph, 118; transudation, 121, 122.

Carbon-monoxide blood test, HoppeSEYLER's, 73.

Carbon dioxide, elimination, dependence of the external temperature, 469 ; in activity and rest, $264,269,467$; by the skin, 329 ; elimination in sleep and waking, 468 ; in various ages, 470 .

Carbon-dioxide hæmoglobin, 74, 97.

Carbon-monoxide hæmoglobin, 73, 76.

Carbon-monoxide methæmoglobin, 75.

Carbon-monoxide poisoning, 73, 114 ; action on the elimination of nitrogen, 337 ; on the elimination of sugar, 407.

Carminic acid, 325.

Carnin,48; properties,258; in urine, 359.

Carp, sperma of, 287.

Cartilage, 234-238 ; behavior with gastric juice, 183,187 ; with pancreatic juice, 211.

Cartilage glue, 39, 234, 238.

Casein, origin, $300,318,319$; from woman's milk, 313 ; from cow's milk, 304 ; quantitative estimation, 309 ; relationship to rennet, 304,305 ; to gastric juice, 313 . 
Caseoses, 27.

Castoreum, 326.

Castorin, 326.

Cataract, 283.

Cat's milk, 312.

Cell, animal, 41-53 ; nucleus, 46 ; membrane, $43,183$.

Cell-globulin, 58.

Cellulose, marsh-gas fermentation of, 213,219 ; behavior in the intestine, 213.

Cement, 241.

Cephaline, 274.

Cerebrin, 129, 274 ; properties, 275-278.

Cerebro-spinal fluid, 125.

Cerolein, 250.

Cerotinic acid, 250.

Cerumen, 326.

Cetin, 250.

Cetyl-alcohol, 250.

Cetylid, 276.

Chalaza, 294.

Charcot's crystals, 113, 286.

Cheese, 302, 305.

Chenotaurocholic acid, 148.

Chitin, 323 ; behavior in trypsin digestion, 211.

Chloral hydrate, behavior in the body, $375,390$.

Chlorate, poisoning with, 115, 401 .

Chlorazol, 17.

Chlorbenzol, behavior in the body, 393 .

Chlorides, elinination by the urine, 65 , 377 ; by the sweat, 328 ; insufficient supply, 451 ; action on the metabolism of proteids, 377, 466 (see the various fluids and tissues).

Chlorocruorin, 83.

Chloroform, action on the elimination of chlorine, 377 ; on the muscles, 262.

Chlorophan, 282.

Chlorophenyl-cystein, 393.

Chlorphenyl-mercapturic acid, 393.

Chlorophyll, 325.

Chlorosis, 112, 113.
Chlorrhodinic acid, 130.

Cholalic acid, 148 ; relation to cholesterin, 164.

Cholanic acid, 150.

Cholecyanin, 154.

Choleglobin, 162.

Choleic acid, 147, 149.

Cholera bacillus, behavior with gastric juice, 192.

Cholera, blood, $111,112,113,114$; contents of the stomach, 225; sweat, 328 .

Cholesterin, general chemical properties and occurrence, 164 ; in gallstones, 164 ; in the brain, 274,279 ; in the urine, 424 ; importance for lifeprocesses in the cell, 53 .

Cholesterin stones, 164.

Cholesterilin, 164.

Cholestrone, 164.

Choletelin, 152, 155; relation to urobilin, 370 .

Cholic acid, 146, 148.

Cholin, 44, 156.

Cholohæmatin, 156.

Choloidic acid, 150, 151.

Chondrigen, 37, 234.

Chondrin, 39, 234; in pus, 130.

Chondrin balls, 236.

Chondroitic acid, 235, 236.

Chondromucoid, 34, 234.

Chorda saliva, 168.

Chorioidea, 283 ; pigment, 324.

Christensen \& MygGe, approximative estimation of albumin in the urine, 400.

Chromidrosis, 328.

Chromogens, in the urine, 369 ; in the suprarenal body, 134.

Chrysophanic acid, action on the urine, 393.

Chyle, 117, 118.

Chylopericardium, 123.

Chyluria, 424.

Chyme, 186; investigation of, 193-196.

Chymosin, 184. 
Cinnamic acid, behavior in the body, | Corpora lutea, 288. 360.

Citric acid, in milk, 304, 311.

Coagulation of blood, 54, 59, 87-94; of milk, $187,301,302,305$; of muscleplasma, 252, 254, 255.

Coccygeal glunds, secretion of, 326 .

Cochineal, 325.

Cofficient, HASER's, 387.

Coffee, action on the exchange of material, 467.

Collagen, $14,37,38$; in connective tissues, 233; in the cornea, $23 \tilde{i}$; in cartilage, 234, 236.

Colloid, 34, 289.

Colluid corpuscles, 289.

Colloid cysts, 289.

Coloring matters, of the eye, 280 ; of the blood, 68-83; of blood-serum, 63; of the corpora lutea, 288; of the eggshell, 296; of the fat-cells, 243 ; of the bile, $145,152,156,160,161$; of the urine, 369-374; of the skin, 324, 325; of the lobster, 325 ; of bird-feathers, 325 ; medicinal coloring matters in the urine, 406.

Colostrum, of woman's milk, 315; of cow's milk, 311.

Colostrum corpuscles, 302, 311, 319.

Combustion, heat of, of foods, 471-473.

Comma bacillus, behavior in gastric juice, 192.

Conchiolin, 14, 39.

Concrements, see various calculi.

Cones of the retina, pigments of, 282.

Conglutin, 472.

Cornikrystalline. 39.

Connective tissue, 233.

Copaiva balsam, action on the urine, 394.

Copper, in blood, 107; in hæmocyanin, 83: in the liver, 137; in protein substances, 13.

Cornea, 237, 283.

Cornein, 14, 39.
Corpsewax, 248.

Corpulence, diet cures, 480-482.

Cow's milk, 301-312; general behavior, 301,302 ; analysis of, $308-310$; constituents, in organic, 310; organic, 304-307; coagulation with rennet. 302,304 ; in stomach, 187 ; composition, 310-312.

Cream, 312.

Creatin, relationship to the formation of urea, $25 \%, 339$; to muscular activity, 266, 268; properties and occurrence, 257 ; behavior in the organism, 389.

Creatinin, relationship to muscular activity, 266, 268, 347 ; properties and occurrence, $34 \%$; creatinin zinc chloride, 348 .

Cresol, 363.

Cresol-sulphuric acid, 363, 364.

Crotonic acid, 423.

Cruor, 55.

Cruso-creatinin, 258.

Crustaceorubin, 325.

Crusta inflammatoria or phlogistica, 87 , 113.

Cumic acid, 392.

Cuminuric acid, 393.

Curara poisoning, action on the muscles, 264 ; on the elimination of sugar, 407.

Crystalbumin, 283.

Crystalfibrin, 283.

Crystallin, 282.

Crystalline lens, 282.

Cyanocrystallin, 296, 325.

Cynogen, in the proteid molecule, 4.

Cyanuric acid, 350.

Cyanurin, 370.

Cymol, behavior in the body, 392 .

Cynurenic acid, 377.

Cystein, 425; grouping in the body, 393 , 425.

Cystin properties and occurrence, 425 ; in the urine, 376 ; in urinary sediment, 
430 ; in urinary calculi, 432 ; in the Dimethyl carbinol, behavior in the liver, 137 ; in the kidneys, 376 ; in sweat, 328.

Cysts, tapeworm, 126; thyroid, 133 ; ovarial, 288-291.

Cystinuria, 376.

Damaluric acid, 376.

Damolic acid, 376.

Delomorphic cell, 175.

Density method of determining albu$\min , 401$.

Dentin, 239, 241.

Dehydrocholalic acid, 148.

Dehydrocholeic acid, 150.

Desoxycholalic acid, 148.

Deutero-albumose, 27.

Dextrin, formation from starch, 171, 202 ; occurrence in the contents of the stomach, 187 , in the muscle, 260.

Dextrin, substance similar to, in urine, 374.

Dextrose, see Grape-sugar.

Diabetes mellitus, elimination of am. monia by the urine, 384 ; relationship of the liver, 142 , and the pancreas, 407 , to the elimination of sugar; blood, fat, 114; amount of sugar, 63 , 114 ; urine, general behavior, $332,38 \%$, 407; amount of sugar, 407 ; amount of $\mathrm{CO}_{2}$ of the blood, 104; oxybutyric acid in blood, 104 ; in the urine, 423 ; relation to the crystalline lens, 283.

Diabetic sugar, see Grape-sugar.

Diacetic acid, see Aceto-acetic acid.

Diamine, in the urine, 376,425 ; in the contents of the intestines, 425 .

Diastatic enzyme, see Enzymes.

Diazo-oxyacrylic acid ester, 37.

Diet for various classes of people, 474482.

Diet cures for obesity, 480-482.

Digestion, 167-232.

Digestibility of various foods, 189, 190 , $191,476$.

organism, 390.

Dimethyl-ketone, see Acetone.

Dioxybenzol, 391.

Dioxynaphthalin, 391.

Distearyl-lecithin, 43.

Doglingic acid, 246.

DonNé's pus test, 404 .

DoREMUs's urea determination, 346.

Dotterplättchen, 17, 292, 296.

Dropsy, 112, 113.

Dys-albumose, 27.

Dyslysin, 150.

Dys-peptone, 182.

Dyspnœa, action on the metabolism of proteids, 337, 468 .

Earthy phosphates, elimination by the urine, $379,384,448$; solubility in albuminous liquids, 241 ; occurrence in bone-earths, 238, 239 ; in concrements, $163,174,226,431,432$; in sediments, 429, 430 (see various earthy phosphates).

Ebstein's diet cure, 480.

Echinochrom, 83.

Eel, blood-serum, 64 ; flesh, 271.

Egg, 287; hen's egg, 291-298; hatching of, 297.

Egg-albumin, see Ovalbumin.

Egg-globulin, 295.

Egg, yellow, 291.

EHRLICH's bile-pigment test, 406

EIsELT's reaction, 403.

Elaic acid, 245.

Elaidic acid, 246.

Elaidin, 246.

Elastin, 14, 36 ; behavior with gastric juice, 183 ; with trypsin, 211

Elastinalbumose, 36.

Elastinpetone, 36.

Ellugic acid, $22 \%$.

Emydin, 296.

Enamel, of teeth, 242.

Encephalin, 276, 277. 
Endolymph, 284.

Energy, potential, of foods, 471 .

Enzymes, general, 8, 9, 10 ; pancreatic diastase, 201; in blood, 142; bile, 210; urine, 376 ; muscle, 256 ; in secretions of the mucous coat of intestines, 197; in saliva, 171; enzymes in the mucous coat of the intestines which dissolve proteids, 197; in urine, 376 ; in stomach, 178 ; in lower animals, 178; in pancreas, 201, 204; in the plant kingdom, 178 ; fat-splitting enzymes, 202,203 ; enzymes producing coagulation, see Fibrin, Ferment and Rennet; urea-splitting enzyme, 427.

Erythrodextrin, 171.

Erythropsin, see Visual purple.

EsBAch's albumin determination, 400 ; urea determination, 346.

Euxanthonic acid, 375.

Ethal, 250.

Ether, action on blood, 67 ; on the secretion of gastric juice, 176; on the muscles, 262; on the secretion of pancreatic juice, 201.

Ethereal oils, action on the muscles, 262.

Ethereal sulphuric acids, in the urine, $216,363,391,392$; in sweat, $32 \%$.

Ethidine lactic acid, 260.

Ethyl-alcohol, passage of, into milk, 320 ; behavior in the organism, 466 ; action on the secretion of gastric juice, $1 \% 6$; on muscles, 262 ; on digestion, 182, 189.

Ethylen lactic acid, 260.

Ethylenimin, 286.

Exchange of material, 435-471.

Excrements, 219, 222-224; in dogs with biliary fistula, 221 ; in starvation, 439 ; with various foods, 222 ; elimination of water with the excrements, 438.

Excretin, 223.

Excretolic acid, 223.
Exostose, 241.

Extinction coefticient, 81.

Exudations, 117, 121.

Eye, 280-283.

Fæces, see Excrements.

Fats, origin in the body, 247; general properties, detection, and occurrence, 242-247: emulsification of fats, 198, $202,203,213,214,227,244$; fat in blood-serum, $62,111,113$; chyle, 118 ; yolk of egg, 293 ; pus, 129; fatty tissues, 242 , 243 ; bile, 156 ; brain, 274 ; urine, 424 ; bones, 239 ; milk, $302,303,310,313,317,318$; nutritive value, $460,461,471-473$; absorption, 227, 232 ; heat of combustion, 471-473 ; behavior to the intestinal juice, 198 ; to gastric juice, 183 , 188 ; to pancreatic juice, 203 ; saponification of, $203,213,214$; action on the secretion of bile, 144 .

Fat, metabolism in activity and at rest, 268,269 ; in starvation, 445 , with various foods, $456,461-466,480-482$. Fat cells, 183, 211, 242.

Fat sweat, 326.

Fatty acids, general properties, detection, and occurrence, $243-247$; feeding with, 247 ; nutritive value, 461 ; syntheses to neutral fats, 248.

Fatty series, behavior in the organism. 388.

Fatty tissues, 242, 243; behavior with gastric juice, 183, 188.

Feathers, 34, 325.

Fehling's solution, 415.

Fellic acid, 150.

Ferments, general, 8 (see various ferments).

Fermentation, 4,8 ; of the urine, 374 , 427 ; of the contents of the stomach, $187,188,193$ (see also various fermentations, such as the alcoholic, butyric fermentations). 
Fermentation lactic acid, properties, oc- Furfuracrylic acid, 389.

currence, etc., 260, 261; in brain, 274 ; in the contents of the stomach, 188 ; gastric juice, 177 ; production in the souring of milk, 301 ; in the fermentation of urine, 427 ; detection in the contents of the stomach, 194.

Fermentation test, on the urine, 412.

Fevers, elimination of ammonia, 384 ; uric acid, 352 ; urea, 337 ; potassium salts, 383 ; blood in fevers, 113,114 ; metabolism of proteids in, $33 \%$.

Fibrin, 14, 55; properties, 57; occurrence in transudations, $117,121,123$; Henle's fibrin, 285.

Fibrin coagulation, 58, 89-94.

Fibrin concrements, 226.

Fibrin ferment, 55, 58, 85, 89-93, 117. Fibrin globulin, 59, 62 .

Fibrine, soluble, see Serum-globulin.

Fibrinogen, 14, 56, 62, 92, 117.

Fibrinoplastic substance, see Serum globulin.

Fibroin, 14, 39.

Fish, egg, 18, 296 ; bones, 240 ; scales, 50 ; air-bladder, 50.

Flesh, amount of nitrogen in, 272, 441; digestibility, 187, 190 ; composition, 270, 271 (see also Muscle).

Flesh, metabolism of, in starvation, 445 ; in various foods, 454-465.

Flesh accumulation of, with various foods, $454,455,462,463$.

Fluorine, in bones, 238, 239 ; in enamel, 242.

Fly-maggots, formation of fat in, 248 .

Formic acid, in butter, 303 ; in contents of the stomach, 196 ; passage into the urine, 374.

Frog's eggs, membrane of the same, 32.

Fumaric acid, 17.

Fundus glands, 175, 185.

Furfurol, relation to Petrenkofer's test for bile acids, 146 ; behavior in the body, 389 .

FürBRINGER's albumin reagent, 397.

Fuscin, 282.

Galactose, 306; from cerebrin, 377; from vegetable bodies, 320 .

GaLloIs's inosit test, 259.

Gases of the blood, 94-105; of the contents of the intestine, 218 ; of the bile, 158 ; of the urine, 385 ; of the hen's egg, 296 ; of the chyle, 118 ; of milk, 311 ; of muscles, 262,264 ; of transudations, 112.

Gas, exchange of, with various ages, 470 ; by the skin, 328 ; in starvation, 446 ; in various conditions of the body, 467, 468 ; in the muscles, 264 ; with various foods, 447 .

Gastric juice, 175 ; secretion of, 176 ; degree of acidity, 194 ; artificial gastric juice, 179 ; action, $23,26,38$, 179-184, 186-192.

Gelatine, 38 ; importance in the forma. tion of glycogen, 139 ; putrefaction, 37,216 ; nutritive value, 459 ; behavior with gastric juice, 183,211 ; to pancreatic juice, 211.

Gelatine, forming tissues, see Collogen.

Gelatine peptone, 38 .

Generative organs, 285-299.

Germ, of the egg, 291.

Globulins, 14; general character, 22 : in the urine, 397,400 ; in the protoplasm, 42 (see various globulins).

Globulin tablets, 86.

Globuloses, 27.

Glucosamine, 323.

Glucose, formation by the action of saliva, 171; of pancreatic juice, 202 (see Grape-sugar).

Glue sugar, see Glycocoll.

Glutamic acid, 16.

Glutin, in pus, 130 (see Glue).

Glycerin. importance for the formation of glycogen, 139, 140; relationship to 
the synthesis of fats, 248 ; action on the elimination of uric acid, 351 ; solvent for enzymes, 9; nutritive value, 462.

- Glycero-phosphoric acid, 43, 113, 127, $131,134,156$; in the urine, $374,376$.

Glycin, see Glycocoll.

Glycocholic acid, 145; properties, 146 ; quantity in excrements, 219 ; in various animal bile, 158 ; behavior in the putrefaction in the intestines, 219.

Glycocoll, properties, 150 ; production in the putrefaction of gelatine, 37 , 216 ; from protein substances, $37-39$; relation to the formation of uric acid, 350,352 ; to the formation of urea, 338,339 ; syntheses with glycocoll, $3,160,359,389,392$.

Glycogen, 138-143 ; origin, 139 ; general chemical behavior, 138, 139 ; relation to the formation of sugar, 141,142 ; to muscular activity, 265268 ; to rigor mortis, 263 ; occurrence in the muscles, 250 ; in the protoplasm $42,52,85$.

Glyconic acid, 374 .

Glycosuria, 63, 142, 407.

Glycuronic acid, properties, 374; paired glycuronic acids, 217, 367, 374; grouping in the body, 390, 393 ; origin, 390.

Glycuron, 374.

Glyoxyldiureid, 358.

Gmelin's test for bile pigments, 154; in the urine, 405 .

Goat milk, 312.

Gout, elimination of uric acid in, 351 , 352.

GraAfFian vesicles, 287.

Grape-sugar, properties and occurrence, 406-409; in urine, $63,142,406-419$; detection, 410-414; quantitative estimation, 414-419 ; reactions, 408-410. Guaiacum blood test, 402.

Guanin, properties and occurrence, 50;
In urine, 359 ; quantity in liver, 137 ; pancreas, 200 ; spleen, 287.

Guanin gout, 51 .

Guanin lime, 50.

Guano, 49, 50, 351.

Guano biliary acids, $14 \%$.

Guanovulit, 296.

Gum, animal, 32 ; in urine, 374.

Hæmin, 78, 79.

Hæmin crystals, 79, 403.

Hæmochromogen, 69 ; properties, 76 ; in muscle, 256.

Hæmocyanin, 83.

Hæmoglobin, properties and behavior, 72 ; quantity in blood, $69,108-111$; quantitative estimation, 82 ; behavior in the trypsin digestion, 211 (see Oxyhæmoglobin and its combinations with other gases).

Hæmoglobinuria, 401.

Hæmometer, 82.

Hæmatocrystallin, see Oxyhæmoglobin.

Hair, 34 ; ash, 322 ; pigments of, 324 , 325.

Hair-balls, 226.

HÄSER's coefficient, 387 .

Hæmatin, relation to bilirubin, 161 ; to urobilin, 161, 371 ; properties, 76 .

Hæmatinometer, 81.

Hæmatochlorin, 298.

Hæmatogen, 292, 297.

Hæmatoglobulin, see Oxyhæmoglobin

Hæmatoidin, relation to bilirubin, 80 , $153,160,162$; properties, 80 ; occurrence, corp. lutea, 288 ; in excrements, 223 ; sediments, 430.

Hæmatolin, 78.

Hæmatoporphyrin, relation to bilirubin, 78,161 ; to urobilin, 371 ; properties, 78 ; occurrence in lower animals, 325.

Hæmatosiderin, 162.

Hæmaturia, 401.

Hæmerythrin, 83. 
Heat, action on the exchange of material, 469; development of heat in plants, 2.

HELLER's albumin test, 19; in urine, 396.

Heller-Teichmann's blood test, 403.

Hemi-albumose, 26.

Hemi-collin, 38.

Hemi-elastin, 36.

Hemi-peptone, 26.

Hemp-seed stone, 432.

Heu's egg, 291-298; hatching, 297.

Heteroalbumose, 27.

Heteroxanthin, 48, 359.

Hippomelanin, 324.

Hippuric acid, 359; properties and reactions, 361; formation in the body, 3,360 , 361, 392; splitting, 360, 362; occurrence, 360; in sediments, 430 .

Histozyme, 363.

Hofmann's tyrosin test, 209.

Holothuria, Mucin, 34.

Homocerebin, 276, $27 \%$.

HoPPE-SEYLER's carbon monoxide test, 73; xanthin test, 49.

Horn, 35.

Horn substance, see Keratin.

Horse's milk, 312.

Humin substance, 369, 394.

Humor, aqueous, 126.

HUPPERT's bile-pigment reaction, 154, 405.

Hyaline, 34, 323.

Hyaline substance of Rovida, 67, 85, 129.

Hyalogen, 14, 34.

Hydracrylic, 260.

Hydraemia, 112.

Hydramnion, 299.

Hydrobilirubin, 152,156 ; relation to urobilin, $161,228,370$; formation in putrefaction, 219.

Hydrocele fluid, 125.

Hydrochinon, 365, 394.

Hydrochinon sulphuric acid, 363, 365.
Hydrocinnamic acid, behavior in the body, 360 .

Hydrochloric acid, secretion by the stomach, 176 ; anti-fermentive action, 192 ; action on opening the pylorus, 189 ; ou the secretion of pepsin, 177 ; quantity of, in the gastric juice, 177 ; reageuts for free $\mathrm{HCl}, 194,195$; action on albumin, 20, 23, 180.

Hydrocyanic acid, action on pepsin digestion,181; on trypsin digestion,206.

Hydrogen, in putrefactive and fermen. tive processes, 4, 216, 218.

Hydrogen peroxide, in urine, 385.

Hydrolytic splitting, 8.

Hydronephrosis fluid, 331.

Hydroparacumaric acid in putrefaction in the intestines, 216.

Hydrophenoketon, 6.

Hydrops folliculorum Graafii, 288.

Hyo-glycocholic acid, 147.

Hypalbuminose, 113.

Hyperalbuminose, 113.

Hyperinose, 113.

Hypinose, 113.

Hyposulphites, in the urine, 376.

Hypoxanthin, relation to the formation of uric acid, 352, 389 ; properties, etc., 50 ; quantity in the liver, 137 ; in muscles, 256 ; pancreas, 200 ; semen, 287.

Iceterus, 143, 162; blood, 114; in urine, 405.

Ichthidin, 296.

Ichthin, 296.

Ichthulin, 296.

Indican, 366-368.

Indican elimination in starvation, 219, 366 ; in disease, 366.

Indigo, 366; in sweat, 328.

Indigo blue, 367, 370.

Indol, properties, 218; formation from proteids, 16, 17; in putrefaction, $216,217,363,366$. 
Indophenol blue, behavior in the tissues, 5 .

Indoxyl, 216, 366.

Indoxyl glucoronic acid, 366, 367, 393.

Indoxyl red, 367.

Indoxyl sulphuric acid, 363, 366, 367.

Inosit, properties and occurrence, 256; in urine, 420 .

Inosinic acid, 256.

Intestive, contents, 212-225.

Intestine, putrefaction processes therein, 215-222; absorption, 215, 216, 225, 227-232; digestive processes, 212-216.

Intestine, glands of the mucous membrane, 197.

Intestinal calculi, 226.

Intestinal fistula, 197.

Intestinal gases, 218.

Intestinal juice, 197, 198.

Iodine combinations, passage of, into milk, 320; sweat, 328; saliva, 173.

Iodoform test, GunNing's, 421; LIEBEN's, 421.

Ischuria, 328.

Isodynamic value of foods, 473.

Isocholesterin, 165.

Isotropous substance, 251.

Iron in blood-coloring matters, 70,76 , $77,81,161$; in blood, $63,65,106$; in the bile, 157. 161; in urine, 385 ; in the liver, 136, 137; in milk, 310, 315, 320 ; in the spleen, $130,131,136$; in muscles 262; in protein substances, $15,23,130,136$; elimination of iron, 157, 161, 174, 385: amount of iron in dog's milk and new-born dogs, 317 ; absorption. 453.

Iron salts, elimination by the urine. 385 ; action on the blood, 111; on trypsin digestion, 206; absorption, 453.

JAFFÉ's indican test, 367 ; creatinin reaction, 349.

Janthinin, 325.
Jecorin, properties and occurrence, 137.

Kephir, 307.

Kerasene, 276.

Keratin, 14, 322; properties, 34; in egg-shells, 296; in bones, 238; be: havior with gastric juice, 183; to pancreatic juice, 211.

Keratinose, 35.

Kidneys, 331 ; relation to the formation of uric acid, 352; to urea, 339; to hippuric acid, 361.

KJELDAHL's method of determining nitrogen, 341, 345.

KNaPP's titration, method, 417.

KNOP-HÜFNER method for determining urea, 346.

Kumyss, 307.

Kyestein, 430.

Lactalbumin, 14; properties, 306; in human milk, 313.

Lactates, 261.

Lactic acid, 260 (see Paralactic acid and Fermentation lactic acid).

Lactic acid, fermentation, 307, 409; in urine, 427; in stomach, 187, 188; in milk, 301.

Lacto-caramel, $30 \%$.

Lacto-globulin, 306.

Lacto-protein, 306.

Lactose, 306.

Lævulinic acid, 32.

Latebra, 291.

Laxatives, action on the blood, 113; on the secretion of intestinal juice, 197 ; action of, 225.

Lead, in blood, 107; in the liver, 137; passage into the milk, 320 .

Lecithin, properties and occurrence, 43 ; action on the coagulation of the blood, 92; putrefaction of, 45,219 ; relation to muscular activity, 266.

Lens, see Crystalline lens. 
Leus capsule, 282.

Lethal, 250.

Leucæmia, 48, 113 ; elimination of urea, 352 ; xanthin bodies, $48,113,132$, 359 .

Leuceines, 16.

Leucin, 16, 17, 206 ; relationship to the formation of uric acid, 352 ; to the formation of urea, 338,389 , preparation, 209 ; properties, occurrence, etc., 206 ; passage into the urine, 424 ; behavior in the body, 338 , 389.

Leucinimid, 17.

Leucocytes, see Colorless blood-corpuscles.

Leucomaines, 12 ; in urine, 376.

Levulose in urine, 419.

LIEBERKüHN's glands, 197.

Lresig's titration method for estimating the quantity of urea, 341 .

Ligamentum nuchæ, 36.

Lime, lack of, in foods, $241,453$.

Linseed oil, feeding with, 247.

Lipacidæmia, 114.

Lipæmia, 113.

Lipochrome, 63, 393.

Lipuria, 424.

Lithium in blood, 107.

Lithobilic acid, 227.

Lithofellic acid, 227.

Lithuric acid, 377.

Liver, 135-137 ; relationship to the formation of uric acid, 352,353 ; to the formation of urea, 338, 339; blood of the liver, 108, 141; albuminous bodies, 136; fat of, 136 ; amount of sugar, 141.

Liver, atrophy, acute yellow, secretion of ammonia, 338,384 ; of urea, 338 , 384 ; leucin and tyrosin, 424 ; lactic acid, $260,374$.

Liver, cirrhosis, ascitical fluids, 124 : sction on the elimination of ammonia and urea, 338, 384.
Liver extirpation, elimination of ammonia, 352 ; of uric acid, 352 ; lactic acid, 352,373 ; action on the formation of bile, 159, 160.

Luteines, 293 ; in corpora lutea, 288; in yolk of the egg, 293 ; in serum, 63; relationship to hæmatoidin, 80,288 .

Lymph, 117-121.

Lymphatic gland, 130.

Lymph fibrinogen, 85.

Lymph-cells, proteids, 42 see (Colorless blood-corpuscles).

Mackerel flesh, 271.

Madder in urine, 393.

Magnesium in the urine, 384 ; in bones, 238,239 ; in muscles, 262,270 (see various tissues and fluids).

Magnesium phosphate, in intestinal calculi, 226 ; in urine, 393 ; in urinary calculi, 431,432 ; in urinary sediments, 430 ; in bones, $238,239$.

Malaria, blood in, 113.

Malt diastase, 171.

Maltose, formation from starch, 171, 202 ; behavior to intestinal juice, 198 , 213.

Mammary glands, 300, 319, 320.

Mandelic acid, 392.

Mare's milk, 312.

Margarin and margaric acid, 245.

Marsh-gas, in intestine, 218, 219 ; in intestinal putrefaction, 216 ; in the fermentation of cellulose, 213,218 ; by the decomposition of lecithin, 219 .

Material, exchange of, dependence on the external temperature, 469 ; in various ages, 470 ; in activity and rest, $264-270,467,468$; in various sexes, 470 ; in starvation, 443-449; with various foods, $454-465$; in sleep and waking, 468 ; calculation of the extent of exchange of material, 441, 442.

Meconium, 224. 
Melanin, relation to blood-coloring matters, 162,324 ; properties, occurrence, and composition, 324,325 ; in the eye, 282 ; in the urine, 403.

Melanæmia, 114.

Melauogen, 403.

Melanotic tumor, coloring matters of, 324.

Melissyl alcohol, 250.

Mellitæmia, 114.

Menstrual blood, 109.

Menthol, behavior in the body, 393 .

Mercapturic acid, 393.

Mesitylen, behavior in the body, 392.

Mesitylenic acid, 392.

Mesitylenuric acid, 393.

Metalbumin, 289.

Metallic salts, action on enzymotic processes, 172, 181, 206.

Metaphosphoric acid, constituents of the nucleins, 23,46 ; albumin reagent, 19 ; in urine, 396.

Methal, 250.

Methæmoglobin, 74 ; in blood in poisoning, 115 ; in urine, 401.

Methyl-guanidin, 348.

Methyl-guanidin acetic acid, see Creaiin.

Methyl-glycocoll, see Sarkosin.

Methyl-hydantoin, $\mathbf{3 5 0}$.

Methyl hydantoinic acid, 389.

Methyl-indol, see Skatol.

Methylpyridyl-ammonium hydroxyl, 393.

Methyl-uric acid, 350.

Methyl-uramin, 348.

Micrococcus ureæ, $42 \%$.

Micro-organisms in the intestinal canal, $10,215$.

Milk, 300-322; secretion, 318-322; blue or red milk, 321 ; milk in disease, 320 ; passage of foreign bodies into, 320 (see various varieties of milk).

Milk-fat, 303; analysis, 309; formation, 319.
Milk-globules of cow's milk, 302, 303; of human milk, 313.

Milk, human, 312-316; behavior in stomach, 187, 313; cumposition, 314. Milk-plasma, 304.

Milk-sugar, properties, 306, 307; fermentation, 187, 301, 307; quantitative estimation, 308, 310; passage into the urine, 307,419 ; origin, 320.

Mineral acids, 104, 333, 450, 452; antifermentive action, 192; action on the elimination of ammonia, 333, 452.

Mineral bodies, elimination in starvation, 448; inadequate supply of, 449 ; behavior in the organism, 450 (see various fluids, tissues, and juices).

Millon's reagent, 20.

MoHR's titration method for chlorine, 378.

Moore's sugar test, 408.

Morphine, passage into milk, 320 ; into urine, 393.

Mucic acid, 306.

Mucin, 14; properties and composition, 32 ; in connective tissue, 233; in urine, 376, 395; in salivary glands, 32,168 ; detection, 401.

Mucin-like bodies in bile, 145; urine, 376,395 ; kidneys, 331 ; thyroid gland, 133; synovial fluid, 126.

Mucinogen, 32.

Mucinoid, 14, 31, 34.

Mucoid, 14, 31, 34; in ascitical fluids, 124; cornea, 237; vitreous humor, 234, 282.

Mucous tissues, 234.

Mucus, of the bile, 144; of the urine, $331,376,401$; of the synovia, 126.

Mulberry calculi, 432.

Murexid test, 354.

Muscles, non-striated, 272; striated, 251-272; blood of the same, 109, 264, 265,268 ; chemical processes in activity and at rest, 264-270; in rigor mortis, 263; albuminous bodies, 252- 
256; extractives, 256-261; coloring Naphthol glycuronic ucid, 313, 393. matters, 256; fat, 261, 268, 270, 271; Navel-cord, mucin of, 32, 234. gases, 262, 264-268; mineral bodies, Nebecula, 331. 261, 262, 270; reaction, 251, 252, 266; Neossin, 34 . amount of water, 272 ; composition, 270.

Muscle-coloring matters, 256.

Muscle-fibre, 252.

Muscle-plasma, 252 ; coagulation of, $252,254,263,272$.

Muscle-serum, 252.

Muscle-sugar, 260.

Muscle-stroma, 255.

Muscle-syntonin, 256.

Muscular activity, chemical processes in the muscles, 264-270; action on the urine, 333, 347; on the exchange of material, 264-270, 467, 468, 478, 479.

Musculin, 14; properties, 255.

Mutton tallow, 247.

Myeline, 274.

Myeline forms, 274.

Mygge and Christensen's albumin determinate, 400 .

Myoalbumin, 255.

Myoalbumose, 255.

Myosin, 252 ; properties, 253, 254 ; in protoplasma, 42,84 .

Myosin-ferment, 254.

Myosinogen, 254.

Myosinoses, 27.

Myoglobulin, 255.

Myohæmatin, 256.

Myricyl alcohol, 250.

Myrisin, 250.

Myristinic acid in butter, 303.

Myxœderma, 133.

Myxoid cyst, 288.

Nails, 34, 35, 322.

Naphthalin, changing of the urine, 394 ; behavior in the body, 394 .

Napthol, reagent for sugar, 410, 414 ; behavior in the vody, 394 .

Nerves, 273.

Neuridin, 278, 291.

Neurin, 44 ; in supra-renal body, 134.

Neurokeratin, 35, 273, 280.

Neutral fats, see Fats.

Nitrates in urine, 383.

Nitric-oxide hæmoglobin, 74.

Nitrobenzoic acid, 17.

Nitrobenzyl-alcohol, 393.

Nitrogen, elinination in rest and activity, $267-270,467,468$; in starvation, 444,445 ; with various foods, $454-465$; elimination, by the excrement, 440 ; urine, 337 , $338,380,382,438,439,440$; horn-formation, 439,440 ; sweat, 439 ; relation to the elimination of phosphoric acid, 380 ; to sulphuric acid, 338.

Nitrogen, free, in blood, 94 ; in intestines, 218 ; in stomach, 189 ; in secretions, $158,170,311,385$; in transudations, 122; combined nitrogen, quantity of, in the discharges of the intestines, 440 ; in meat, 272,441 ; in urine, 337,338 ; determination, $341-347$; in protein substances, $15,32,35,39$.

Nitrogen deficit, 439.

Nitrogenous equilibrium, 440 ; with various foods, $454-465$.

Nitrophenyl-propiolic acid, reagent for sugar, 410, 414; behavior in the body, 366,367 .

Nitrosoindol-nitrate, 217.

Nitrotoluol, behavior in the body, 393 .

Nitrotyrosin-nitrate, 209.

Non-striated muscles, 272.

Nuclein, properties and occurrence, 46; in blood-corpuscles, 67 ; pus, 129 ; brain, 273 ; sperma, 287.

Nuclein protamin, $28 \%$.

Nucleoalbumin, 14 ; properties, 22 ; in 
bile, 145 ; in urine, 376, 396; kidneys, Ossein, 37, 238, 240.

331 ; mammary glands, 300,319 ; synovia, 126.

Osteomalacia, 241 ; lactic acid in urine, 374.

Nutritive bodies, necessary, 435 ; heat of combustion of, 471-473.

Nutrition, need of man for, 473-480.

Nutrition, influence on the secretion of intestiual juice, 197 ; bile, 143, 144 ; gastric juice, 176 ; pancreatic juice, 200: on the elimination of ammonia, 384; uric acid, 351 ; urea, 337,454 , 462,465 ; carbon dioxide, 447 ; mineral bodies, $337,379,382$; on the exchange of material, 449-465 ; various foods rich in proteids, $454-460$; proteid and fat, 460-462; proteid and carbohydrates, 462-465 ; insufficient, 449-454.

NYLANDER's test for sugar, 411.

Odoriferous bodies in urine, 394.

Edema, fluid of, 126.

OrTel's diet cure, 480, 481.

Oleic acid, 245.

Olein, 243-245.

Oligmia, 112.

Oligocythæmia, 112.

Oliguria, $38 \%$.

Olive-oil, action on the secretfon of bile, 144.

Onuphin, 34.

Ö̌cyan, 296.

Oörodein, 296.

O pium, passage into the milk, 320 .

Optogram, 282.

Ornithin, 389, 392.

Ornithuric acid, 392.

Organs, loss of weight in starvation, 448.

Organic acids, behavior in the body, 389.

Organized proteids or tissue-proteids, $45^{1 \%}, 458$.

Orthonitrophenyl-propiolic acid, see Nitrophenyl-propiolic acid.

Otholiths, 284.

Ovalbumin, 14 ; properties, 295 ; behavior in the body, 284.

Ovarial cysts, 288-291.

Ovovitellin, 14 ; properties, 292.

Ovum, $28 \%$.

Oxalic acid, in blood, 114 ; in urine, 357 ; behavior in the body, 389 .

Oxalic-acid diathesis, $35 \%$.

Oxalate lime in urine, 357 ; calculi, 432 ; sediments, 428.

Oxalate stone, 432.

Oxaluric acid, $251,35 \%$.

Oxaluria, $35 \%$.

Oxamid, 16.

Oxidation, 1-5, $71,94,95,96,155,216$, $217,350,363,389,391,392$.

Oxyacids, formation of, in putrefaction, 216; passage of, into the urine, 216,368 ; in sweat, 327.

Oxybenzoic acid, grouping in the body, 392.

Oxybenzol, 391.

Oxybutyric acid, in blood, 104; in urine, $384,423$.

Oxygen, activity of, in the animal body, $5,71,95$; in blood, 95, 96, 100, 101, 102 ; in intestines, 158, 170; lymph, 118; in stomach, 189; in transudations, 122; binding of the oxygen in the blood, 70, 95; tension of, in the blood, 100, 101; in the expired air, 101.

Oxygen, consumed in activity and rest, 265,468 ; in starvation, 446 ; by the skin, 328 .

Oxyhæmatin, 76.

Oxyhæmocyanin, 83.

Oxyhæmoglobin, 69; dissociation of, 70,100 ; properties and behavior, $69-$ 71; quantity in blood, $69,108-111$; in muscle, 256; passage into the urine, 
401; behavior with gastric juice, 183; | Parovarial cysts, 291.

to trypsin, 211.

Oxynaphthalin, 391.

Oxynitroalbumin, 17.

Oxyphenylamido-propionic acid, see Tyrosin.

Oxyproto-sulphonic acid, 17.

Ozone, 3, 96.

Ozone-exciter, 96.

Ozone-transmitter, 7 .

Palmitic-acid, 245.

Palmitic-acid cetyl-ether, 250.

Palmitic-acid melissyl-ether, 250.

Palmitin, 243, 245.

Pancreas, 199, 200; extirpation, action on the elimination of sugar, 407; charge, 132 ; change during secretion, 199, 211, 212.

Pancreatic juice, 200 ; secretion, 200, $201,211,212$; enzymes, 202-206; action on nutritive bodies, 202-206, 214, 215.

Para-amido-phenol, 391.

Parabamic acid, 351.

Paracasein, 305.

Paracresol, formation in putrefaction, 216, 363.

Paraglobulin, see Serum-globulin.

Parahæmoglobin, 71.

Paralactic acid, relation to uric acid, 350,351 ; properties and occurrence, 260 ; production in muscle during activity, 266, 268; in rigor mortis, 263 ; in osteomalacia, 241 ; passage of, into the urine, 352,374 .

Paralbumin, 289, 290.

Paramyosin, 253, 255.

Paraoxyphenyl-propionic acid, 216, 369.

Parapeptone, 182.

Paraphenyl-acetic acid, 216, 368.

Paraxanthin, 48, 359.

Parietal cells, 175.

Parotid, 167.

Parotid saliva, 169, 170.

Pentacumin, 325.

Pentamethylendiamin, 376.

Pepsin, 175, 178; properties, 178; in urine; 228,376 ; in muscles, 256 ; detection in the contents of the stomach; 193; quantitative estimation, 180 . 181 ; action on albumin, 179 ; on other bodies, 183.

Pepsin digestion, 179, 180, 181, 183; products of the same, $26-29,182,183$ 186.

Pepsin-glands, 175.

Pepsin test, 180.

Pepsin hydrochloric acid, 183.

Pepsinogen, 186.

Peptone, 25; in the putrefaction of proteids, 216; in pepsin digestion, 27, 182; in trypsin digestion, 27,206 ; assimilation, 231, 232; preparation, 29; nutritive value, 459; absorption, 227 232; passage into the urine, 228, 398.

Peptone-plasma, 54.

Peptonuria, 398.

Pericardial fluid, 122, 123.

Perilymph, 284.

Peritoneal fluid, 122, 124.

Perspiratio insensibilis, 438.

Pettenkofer's test for bile-acids, 146.

Phacozymas, 283.

Phaseomanuit, 258.

Phenaceturic acid, 362, 393.

Phenol, elimination by the urine, 216, 363 ; in starvation, 219 ; estimation in urine, 364; action on the urine, 394; electrolysis of, 6,390 ; formation in putrefaction, 216, 363; behavior in the body, 216, 263, 393, 394.

Phenol glycuronic acid, 364, 393.

Phenol-sulphuric acid in urine, 363, 364, 393; in sweat, 327.

Phenyl-acetic acid, formation in putrefaction, 216; in body, 391, 392.

Phenyl-rmido-acetic acid, 392.

Phenyl-amido-propionic acid, 391, 392. 
Phenyl-glucosazone, 410.

Phenyl-hydrazine test, 307; in urine, 410, 413.

Phenyl-lactosazone, 307.

Phenyl-propionic acid, formation in putrefaction, 216, 361; in body, 392.

Phlebin, 68.

Phloridzin diabetes, 143, 407.

Phosphorus-poisoning, action on the elimination of ammonia, 338,384 ; of urea, 337, 338, 384; lactic acid, 374; production of fatty degeneration, 248 ; changing the urine, $337,338,374$, 424.

Phosphorized compounds in urine, 376.

Phosphoric acid, elimination by the urive, 379-382, 448; formation in muscular activity, 266, 268.

Phosphates in urine, 379-382, 395 (see various phosphates).

Phosphate diabetes, 380 .

Phosphate stone, 432.

Phenosin, 276.

Phthalic acid, 391.

Phymatorusin, 324; in urine, 403.

Physetoleic acid, 250.

Picric acid, reagent for albumin, 20, 400 ; for creatinin, 349 ; for sugar, 410, 414.

Pig-milk, 312.

Pike, flesh of, 272.

Pilocarpin, action on the secretion of intestinal juice, 197; sweat, 327 ; saliva, 173.

Piria's tyrosin test, 208.

Placenta, 298.

Plants, chemical processes in the same, $1,2$.

Plasma, see Blood-plasma.

Plasmoschise, 92.

Pleural fluid, 123.

Plums, influence on the elimination of hippuric acid, 360, 361.

Polycythæmia, 111, 116.

Polyperythrin, 325.
Polyuria, 380, 387.

Portal vein, blood from, 108, 141, 230.

Potassium combinations, elimination in fevers, 383; in starvation, 383,448 ; by the urine, 383,448 ; by the saliva, 173; division in the form elements and fluids, 53.

Potassium chlorate, poisoning with, 114, 401.

Potassium phosphate, in yolk ef the egg, 294; muscles, 263; cells 53.

Preputial secretion, 326.

Principal cells, 175, 186.

Propepsin, 186.

Propyl-benzol, in body, 392.

Prostatic calculi, 287.

Prostatic secretion, 285.

Protagon, 273, 274, 275.

Protamin, 287.

Proteid, 31; of the mammary glands, $300,319,320$; in the protoplasm, 42 , 43.

Proteids, separation from liquids, 21; approximate estimation in the, 400 ; influence on the formation of gly. cogen, 140; living and dead, 4; detection, 20; in urine, 394-398; quantitative estimation, 21 ; in the urine, 399; absorption, 227, 229; passage into the urine, 394-399; heat of combustion, 471-473; digestibility of, in gastric juice, 182, 183, 190.

Protein bodies, 13-40 (see various bodies).

Protic acid, 256.

Protocatechinic acid, in body, 365 .

Protoplasm, 42, 52, 53.

Pseudocerebrin, 276, 277.

Pseudomucin, 34; properties, 289.

Pseudoxanthin, 258.

Purple, 325.

Purple cruorin, 72.

Pus, 127-130; blue pus, 130; pus in urine, 404.

Pus-cells, 127, 128.

Pus-serum, 127. 
Putrefaction processes, 4 ; in intestines, 216-222, 363-369; regulation of the putrefaction in the intestines, 220 , 221.

Putrescin, 376, 425.

Ptomaines, 11 ; in urine, 376.

Ptyalin, 171 ; behavior with $\mathrm{HCl}, 172$, 174, 212; action on starch, 171.

Pyæmia, 113.

Pyin, 14, 123, 128, 130.

Pyinic acid, 130.

Pyloric glands, 175, 185.

Pyloric secretion, 186.

Pyocyanin, 130 ; in sweat, 328.

Pyoxanthose, 130.

Pyridin, in body, 393.

Pyrocatechin, properties, 365 ; occurrence in the urine, 365 ; in the suprarenal body, 134; in transudations, 125.

Pyrocatechin-sulphuric acid, 363, 365.

Pyromucic-acid, 389.

Pyromucin-ornithuric acid, 389.

Pyromucuric acid, 389.

Quinic acid, behavior in the body, 360 . Quinin, passage into the urine, 393 ; in sweat, 328 ; action on the elimination of uric acid, 352,353 ; on the spleen, $132,353$.

Rachitis, 240, 241.

Reduction processes, $1,2,6,7,95,96$, $153,155,156,218,250,360,369,370$, 390.

Reducing substances, production in putrefaction and fermentation, 4, 218 ; occurrence in blood, $4,62,218$; in urine, 374 ; in transudations, 122.

Rennet, 175-177 ; properties, 184 ; detection in the contents of the stomach, 193 ; passage into the urine, 376.

Rennet-cells, 175.

Rennet-glands, 175.

Rennet-zymogen, 184.
Resinous acids, passage into the urine, $393,396$.

Respiration, of the hen's egg, 297; of plants, 2 (see Exchange of gases).

Respiratory quotient, 447.

Rest, exchange of material, 264-270, $467,468$.

Retina, 280.

Rhodophan, 282.

Rhodopsin, 280.

Rhubarb, action on the urine, 394.

Rigor mortis of the muscles, $262,272$.

RoBERT's method for determining the quantity of sugar, 418.

Rodents, biliary acids of, 147,158 .

Rods of the retina, coloring matter, 280.

RovidA's hyaline substance, 67,89 , 129.

Saccharogen, in the mammary glands, 320.

Saccharic acid, 374.

Sal ammonia, action on the exchange of material, 466 .

Salicylic acid, in the body, 392; action on the pepsin digestion, 181; on the exchange of muterial, 466; on trypsin digestion, 206.

Saliva, 168-174; secretion, 174; poisonous properties, 11; mixed saliva, 170, physiological importance, 174; behavior in the stomach, 174, 187; various kinds, 168-170; action, 170; composition, 173.

Salivary calculi, 174.

Salivary diastase, see Ptyalin.

Salivary glands, 165.

Salmon, flesh, 271; sperma of, 287.

Salts, see various salts.

Salt-plasma, 55.

Saltpetre, action on the exchange of material, 466.

Samandarin, 326.

Santonin, action on the urine, 394, 406. 
Saponification of fat, 203, 213, 244 . Sarcolactic acid, see Paralactic acid.

Sarcolemma, 251.

Sarcosin, 257; behavior in the body, 389.

Sarkin, see Hypoxanthin.

Schreiner's base, 286.

Sclerotica, 283.

Scyllit, 131.

Sebacic acid, 246.

Sebum, 326.

Sediments, see Urinary sediments.

Sedimentum lateritium, 332, 354.

Semen, 285.

Semen-fibres, 285, 286.

Semiglutin, 38.

Serum, see Blood-serum.

Serum-albumin, 14,56 ; properties, 60 ; detection in urine, 397 ; quantitative estimation, 61,399 ; behavior in the body, 60 .

Serum-casein, see Serum-globulin.

Serum-fibrinogen, 62.

Serum-globulin, 14, 56, 59; importance for the coagulation of the blood, 90 ; properties, 59 ; detection in the urine, 397 ; quantitative estimation, $60,400$.

Senna, action on the urine, 394,406 .

Sericin, 39.

Sericoin, 39.

Serin, 39.

Serous fluids, 121-127.

Shell-membrane of the egg, 35, 296.

Sheep's milk, 312.

Silicic acid, in feathers, 322; in urine, 385; in hen's egg, 294, 296.

Sinkalin, 44.

Skatol, 216; properties, 217; formation in putrefaction, $216,363,368$; behavior in the body, $217,363,368$, 393.

Skatol-carbonic acid, 368.

Skatol coloring matters, 368 .

Skatoxyl, 216, 368.

Skatoxyl-glycuronic acil, 368, 393.
Skatoxyl-sulphuric acid, 363, 368; in sweat, $32 \%$.

Skin, 322-329; secretion by the same, 326-3:9, 437, 440.

Sleep, exchange of material, 468 .

Smegma præputii, 326.

Soaps, in blood-serum, 62; chyle, 118; pus, 129; excrements, 222, 223 ; bile, 156 ; importance for the emulsification of fats, $214,244$.

Sodium combinations, elimination by the urine, 383; division among form elements and juices, 53 (see various tissues and fluids).

Sodium chloride, elimination by the urine, 65,377 ; by the sweat, 328 ; importance physiologically, 451; influeuce on the quantity of urine, 466 ; on the elimination of urea, 337 ; on the secretion of gastric juice, 176; quantitative estimation, $377-379$; lack of, 451.

Sodium phosphate in urine, 380 ; action on the exchange of material, 466.

Sodium salicylate, action on the secretion of bile, 144.

Sodium sulphate, absorption, 232; action on the metabolism, 466 .

Spectro-photometric method, 81, 82.

Spermaceti, 250.

Spermatin, 287.

Spermatocele-fluid, 125.

Spermatozoa, 286.

Spermine crystals, 286.

Spirographin, 34.

Spleen, 130; relation to the formation of blood, 132; to the formation of uric acid, 132, 353; to digestion, 132; blood of, 109.

Splitting processes, general, 1, 2, 7 (see various enzymes and ferments).

Spongin, 14, 39.

Staphylococcus, behavior in gastric juice, 192.

Starch, hydrolytic splitting, by intes- 
tinal juice, 197; pancreatic juice, 201; saliva, 171, 174; behavior in the stomach, 187.

Starvation, action on the blood, 110, 115; on the secretion of bile, 143; secretion ofiudican, 219, 366; pancreatic juice, 199; on the elimination of phenol, 219; on the exchange of material, 443-449.

Starvation cures, 480-482.

Starvation diabetes, 407.

Stearic acid, 244.

Stearin, 243, 244.

Stercobilin, 153, 223.

Stethal, 250.

Stomach, importance of, in digestion, 191; self-digestion, 192; digestion in the stomach, 186-191.

Stomach, catarrh, 193.

Stomach, contents of, see Chyme.

Stomach-fistula, 175.

Stomach, glands of, 175 .

Stomach, mucous membrane of, $\mathbf{1 7 5}$.

Stomach-saliva, see Pancreatic juice.

Streptococcus, behavior in gastric juice, 192.

Stroma of the blood-corpuscles, 66 ; of the muscles, 255

Stromafibrin, 67.

Strychnin, passage into the urine, 393. Sublingual saliva, 169.

Sublingual gland, 167.

Submaxillary gland, $\mathbf{1 6 7}$.

Submaxillary mucin, 32 .

Submaxillary saliva, 168.

Succinic acid, in transudations, 121-126; in spleen, 131; in thyroid gland, 133; passage into the urine, 374 ; in sweat, 328.

Sugar, formation, in the liver, 141, 142; after the extirpation of the pancreas, 407.

Sugar, in stomach, 188; absorption, 229, 231,232 ; relation to muscular activity, 265-268 (see various varieties of sugar).
Sugar test in the urine, 411.

Sulphur, in albuminous bodies, 15; in urine, neutral or acid, 376.

Sulphur-methæmoglobin, 75.

Sulphocyanide of potassium, in saliva, $169,170,173$; in urine, 376.

Sulphuric acid, ethereal and sulphate sulphuric acid; in urine, 363; estimation, 382 ; in sweat, 328 ; elimination by the urine, 363 ; relationship to the elimination of nitrogen, 382, 441 .

Sulphurous acid in the urine, 376, 389.

Sulphuretted hydrogen, in intestinal putrefaction, 216, 218; in urine, 376.

Supra-renal body, 134.

Sweat, 326; secretion, 327; action on the urine, $333,335,386,38 \%$.

Synovial fluid, 126.

Syntheses, 1, 2, 7, 135, 140, 217, 248, $249,336,350,360,363,366,390,392$. Syntonin, 24; from muscles, 256.

Sympathetic saliva, 168.

Tartaric acid, 328.

Tartar, 174.

Tatalbumin, 294.

Taurin, 151; behavior in the body, 389 . Taurocarbaminic acid, 389.

Taurocholic acid, 147, 148; quantity in various animal biles, 158 ; decomposition in the intestines, 219.

Tea, action on the exchange of material, 467.

Tears, 283.

Teeth, 241.

Teichmann's crystals, 78, 79.

Tension of the carbon dioxide in blood, $102-105$; in the lymph, 118; of the oxygen in the blood, 100-102.

Terpentine, action on the secretion of bile, 144 ; on the urine, 394 ; behavior in the body, 375 .

Terpenglycuronic acid, 375 .

Testicles, 285.

Tetanin. 11. 
Tetronerythrin, 83, 325.

Thallin, action on the urine, 394.

Theobromin, 48.

Theophyllin, 48.

Thyreoprotein, 133.

Thyroidea, 133.

Thyroid gland, 133.

Thymus, 132.

Tissue-fibrinogen, 88, 90.

Tissue-proteids, $457,458$.

Toluic acid, 392.

Toluric acid, 393.

Toluol, behavior in the body, 360 , 391.

Toluylendiamin-poisoning, 162.

Tonus, chemical, of the muscles, 264, 469.

Tortoise-shell, 35.

Toxine, 11.

Transudations, 117, 121-127.

Transudations in the intestine, 225 .

Tribrom-acetic acid, 17.

'Tribromamido-benzoic acid, 17.

Trichlorbutyl-alcohol, behavior in the body, 390.

Trichlorbutyl-glycuronic acid, 390.

Trichlorethyl-glycuronic acid, 390.

Trinitroalbumin, 17.

Triolein, 245.

Tripalmitin, 245.

Triple phosphate, in urinary sediments, 430 ; in urinary calculi, $430,432$.

Tristearin, 245.

Trommer's sugar test, 409, 411: behavior to glycuronic acid, 375; uric acid, 354; creatinin, 348.

Tuberculosis virus, behavior with gastric juice, 192.

Tubo-ovarial cysts, 291.

Tunicin, 323.

Turacoverdin, 325.

Turacin, 325.

Trypsin, 201, 204; action on proteids, 205 ; on other bodies, $38,210,211$.

Trypsin digestion, influence of various conditions on the same, 205, 206; products, 206.

Trypsin-zymogen, 200, 211, 212.

Typhotoxin, 11.

Tyrosin, properties and occurrence, 208 ; in urine, 424 ; in sediments, 424 , 430; detection, 209, 424; origin, 16, $35,206,216$; behavior in putrefaction, 360, 363; in the body, 391, 39:. Tyrosin-sulphuric acid, 232.

Uræmia, blood, 115; bile, 158; contents of the stomach, 193; sweat, 328 .

Uramido-acids, 328.

Urates, 354; in sediments, $332,428$.

Urea, 336; elimination in activity and in rest, $267,268,467,468$; in starvation, 444; in children, 470; in disease, $337,338,383$; after various foods, $337,454-467$; secretion of urea after meals, 458; properties and reactions, 339,340 ; formation in the organism, 338, 339; quantitative estimation, 341347; splitting by ferments, 340, 427; synthesis, 336, 338; occurrence, 337.

Ureids, 16.

Ureometer, of EsBACH, 346 ; of DoREMUs, 346.

Uric acid, 350; relation to urea, 350 , 351 ; properties and reactions, 353 , 354; formation in the body, 352, 353; relation of the spleen to the same. 132, 352; quantitative estimation. 355,356 ; synthesis, 350 ; hehavior in the body. 351 ; occurrence, 351 ; in sweat, 328 ; in sediments, 332,427 , 428.

Uric-acid calculi, 431.

Uric-acid sediments, $332,426-430$.

Urine, 330-433; secretion, 385, 386, constituents, inorganic, 377-385: poisonous, 11, 376; organic, pathological, 394-426; physiological, 336377; casual, 388-396; color, 332, 369, 388 , 394, 405, 406; solids, calcula- 
tions of the same, 387; fermentation, Vegetarians, nutrition, 476; excrement, alkaline, 374, 427; acid fermentation, 427; gases, 385; quantity, 386, 387, 388; physical properties, 331-336; reaction, 332,427 ; degree of acidity, 332 , 333; determination of acidity, 334; specific gravity, 335-387; determination of, 336; passage of foreign bodies, 388-394; composition, 388.

Urinary calculi, 430-433.

Urinary coloring matters, 369-374, 405, 406.

Urinary indican, 466, 467.

Urinary indigo, 466, 467 .

Urinary sand, 430.

Urinometer, 336.

Urobilin, 370, 371; relation to bilirubin, $153,161,370$; to choletelin, 371 ; to hæmatin, 161, 162, 371; to hæmatoporphyrin, 371 ; to hydrobilirubin, 371; properties, 370-373.

Urobilin icterus, 371.

Urobilinogen, 369, 370.

Urobilinoidin, 371.

Urocanic acid, 377.

Urochloralic acid, 390.

Urochrom, 373.

Urocyanin, 370.

Uroerythrin, 373, 403.

Urofuscohæmatin, 403.

Uroglaucin, 370.

Urohæmatin, 3i0, 404.

Urohodin, 370.

Uroleucic acid, 365.

Uromelanine, 370.

Urophæin, 370.

Urorosein, 370, 403.

Urorubin, 370.

Urorubrohæmatin, 403.

Urostealith stone, 433.

Uroxanthin, 366.

Uterine milk, 298.

Valerianic acid, 16, 243. 222.

Vernix caseosa, 326.

Visual red, 280.

Visual purple, 280.

Vitellin, 14; in the egg-yolk, 292; in the protoplasm, 42 .

Vitellolutein, 294.

Vitellorubin, 294.

Vitelloses, 27.

Vitreous humor, 234, 282.

Water, drinking, action on the elimination of chlorides, $37 \%$; of urea, 337 , 465 ; on the accumulation of fat, 465 ; on the secretion of bile, 144; on the secretion of urine, 385-387.

Water, elimination by the urine, $385-387,438$; by the skin, 327,438 ; in starvation, 447; importance for the body, 449; amount of, in the organs, 449 ; lack of water in the nutrition, 449.

Whey, 312.

Whey albumin, 305.

White of the hen's egg, 294.

Witch's milk, 316.

Woman's milk, see Human milk.

Wool-fat, 326.

Work, influence of, on the elimination of chlorine, 377; on the exchange of material, $267,269,467,468$.

Xanthin, properties and occurrence, 49 ; in urine, 359 ; in urinary sediments, 430; quantity in the liver, 137; in pancreas, 200 ; detection and quantitative estimation, 51, 52, 359.

Xanthin bodies, general on the importance and occurrence, 47, 48; in blood in leucæmia, 48 ; in urine, 359 ; relation to muscular activity, 269: steps to the formation of uric acid, 353.

Xanthin-stones, 433.

Vegetable acids,salts of, in the urine,334. Xanthocreatinin, 258, 350. 
Xanthophan, 282.

Xanthoproteic acid, 17, 20.

Xanthoproteic-acid reaction, 20.

Xylol, behavior in the body, 392 .

Yolk, of hen's egg, 291.

Yolk, membrane, 291.
Zinc, in the liver, 137; passage into the milk, 320.

Zoöerythrin, 325.

Zoöfulvin, 325.

Zoörubin, 325.

Zymogens, see various enzymes: Rennet, Pepsin, and Pancreatic enzyme.

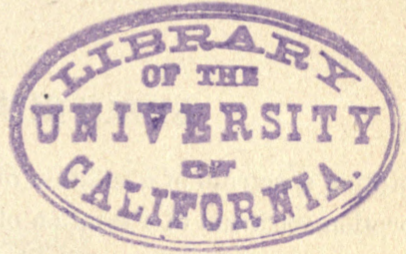




\section{SPECTRUM PLATE.}

1. Absorption spectrum of a solution of oxyhamoglobin.

2. Absorption spectrum of a solution of homoglobin, obtained by the action of an ammoniacal ferro-tartrate solution on an oxyhæmoglobin solution.

3. Absorption spectrum of a faintly-alkaline solution of methamoglobin.

4. Absorption spectrum of a solution of homatin in ether containing oxalic acid.

5. Absorption spectrum of an alkaline solution of hamatin.

6. Absorption spectrum of an alkaline solution of hamochromogen, obtained by the action of an ammoniacal ferro-tartrate solution on an alkalinehæmatin solution.

7. Absorption spectrum of an acid solution of urobilin.

8. Absorption spectrum of an alkaline solution of urobilin after the addition of a zinc-chloride solution.

9. Absorption spectrum of a solution of lutein (ethereal extract of the egg-yolk). 


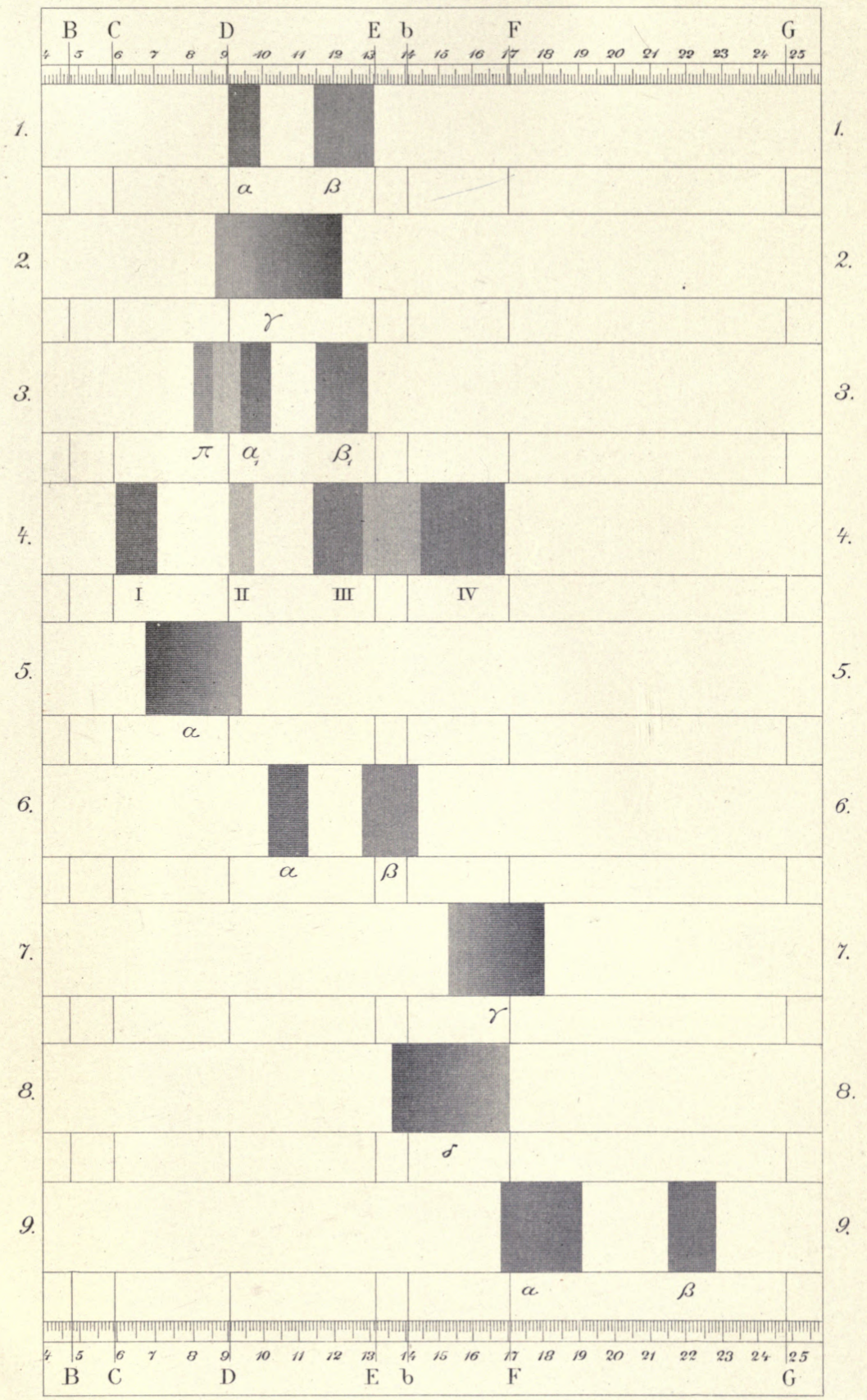




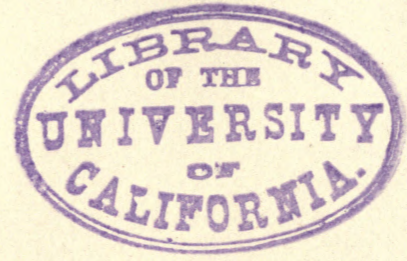




\section{CHEMISTRY.}

Qualitative-Quantitative-Organic-Inorganic, Eto.

\section{A MANUAL OF QUALITATIVE CHEMICAL ANAL- YSIS.}

By C. R. Fresenius. Translated into the New System, and newly edited by Samuel W. Johnson, M.A., Prof. of Theoretical and Agricultural Chemistry in the Sheffield scientific School of Yale College, New Haven. New edition, revised and enlarged with a beautifully colored frontispiece showing spectra. Eightl edition .................. 8 vo, cloth, $\$ 400$

A SHORT COURSE IN QUALITATIVE CHEMICAL ANALYSIS.

With the new notation, by Prof. I. M. Crafts. Revised with additions. By Prof. Chas. A. Schaeffer, of Cornell Institute. Sixth edition ......................... cloth,

A SYSTEM OF INSTRUCTION IN QUANTITATIVE CHEMICAL ANALYSIS

From the last English and German editions. Edited by Prof. O. D. Alleu, of the Sheffield Scientific School of Yale College, with the co-operation of Prof. Sam'l W. Johnson, of the same. This is an entirely new edition from new plates, the New Notation and Nomenclature being employed throughout. 900 page:. Tenth edition...................... 8 vo, cloth, 600

QUANTITATIVE CHEMICAL ANALYSIS.

By 'T. E. 'Thorpe, Prof. of Chemistry, Glasgow. Eighth

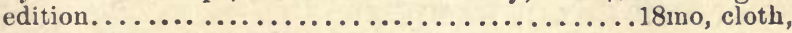

QUANTITATIVE ANALYSIS.

'The Student's Guide in Quantitative Analysis, designed as an aid to Fresenius' la ruer work. By H. Carrington Bolton, Ph.D. With many original methods by the author. Third edition. 8 vo, cloth, 150

QUANTITATIVE CHEMICAL ANALYSIS BY ELECTROLYSIS.

According to Original Methods. by Dr. Alexander Classen, of the Royal School of Technology at Aachen. Authorized translation from the second revised and enlarged German edition, by Prof. Wm. Hale Hurrick, of Penna. State College.

8 vo, cloth,

AN ELEMENTARY TEXT-BOOK OF CHEMISTRY. By William G. Mixter, Professor of Cliemistry, Slieffield Scientific School, Yale College, New Haven. Third revised

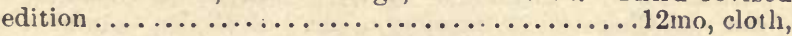

A HAND-BOOK FOR SUGAR MANUFACTURERS AND THEIR CHEMISTS.

Containing Practical Instruction in Sugar-House Control, the Diffusion Process, Belected Methods of Analysis, Reference Tables, etc., etc. Bv Guilford L. Spencer, A.C., of the U. S. Vepartment of Agriculture ...........12no, morocco flaps,

SUGAR ANALYSIS, ETC.

A Hand-Bonk in Schools of Chemical Technology, Etc. By F. C. Wiechmann, School of Mines........... 8vo cloth, 


\section{ROCKS AND SOILS.}

Their Origin, Composition, and Characteristics; Chemical, Geological, and Agricultural. By Horace Edward Stockbridge, Ph.D., Professor of Chemistry and Geology in the Imperial College of Agriculture, Sapporo, Japan. Chemist to the Hokkaido Cho. With 13 full page plates....... 8vo, cloth, \$2 50

A LABORATORY GUIDE IN CHEMICAL ANALYSIS. Third edition, entirely rewritten and revised by Prof. David O'Brine, of Colorado State Agricultural College....8vo, cloth,

INTRODUCTION TO ORGANIC CHEMISTRY.

Translated from the German of A. Pinner, Prof. of Chemistry at Berlin, with additions and revisions by Prof. P. T. Austen, of Rutgers College. A Text-book for the use of Colleges and High Schools. Second edition..............12mo, cloth,

TEXT-BOOK OF INORGANIC CHEMISTRY.

A short Text-book of Inorganic Chemistry, by Dr. Hermann Kolbe, Prof. in the University of Leipzig. Translated and edited by Prof. T. S. Humpidge, of the University of Wales. With a colored Spectra and numerous wood engravings. (Over 600 pages)...................... 12mo, cloth,

\section{CHEMICAL LECTURE NOTES.}

Containing Notes and Observations on Topies that Often Give the Student More or Less Trouble. Br Prof. P. 'T. Austen.

$12 \mathrm{mo}$, cloth, 100

\section{CHEMICAL REACTIONS,}

Being a thorough discussion of Chemical Reactions, which occur in the ordinary course of Qualitative Analysis and General Elementary Chemistry. By E. Drechsel. Translated, and specially adapted for the use of American students and teachers, by N. F. Merrill, Ph.D. Third edition, 12mo, cloth,

IABORATORY CALCULATIONS.

And Specific Gravity Tables. By John S. Adriance, A. B., Fellow of the Chemical Society. Second edition....12mo, cloth,

WATER SUPPIY.

Considered mainly from a Chemical and Sanitary standpoint. By Prof. Wm. Ripley Nichols. With numerous plates. Third

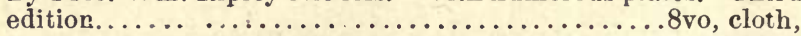

A TEXT-BOOK OF PHYSIOLOGICAL CHEMISTRY. $\mathrm{Sy}$ Olof Hammarsten, Professor of Medical and Physiological Chemistry in the University of Upsala. Authorized Translation from the second Swedish edition, and from the author's enlarged and revised German edition. By John A. Mandel, Asșistant to the Chair of Chemistry, etc., in the Bellevue Hospital Medical College and in the College of the City of New York. First edition................... cloth,

LECTURE-NOTES ON THEORETICAL CHEMISTRY. By Ferdinand G. Wiechmann, Ph.D., Instructor in Chemical Physics and Chemical Philosophy, School of Mines, Columbia College. First edition ................. 12mo, cloth,

PUBLISHED AND FOR SALE BY

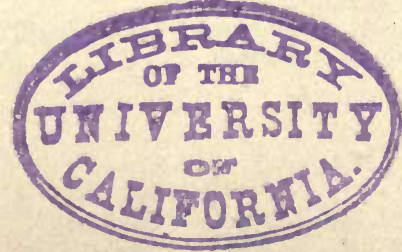




RETURN TO the circulation desk of any

- University of California Library or to the

NORTHERN REGIONAL LIBRARY FACILITY Bldg. 400, Richmond Field Station University of California Richmond, CA 94804-4698

ALL BOOKS MAY BE RECALLED AFTER 7 DAYS 2-month loans may be renewed by calling (510) 642-6753

1-year loans may be recharged by bringing books to NRLF

Renewals and recharges may be made 4 days prior to due date

DUE AS STAMPED BELOW

\section{MAY 141993}


65872

BIOLOGY

UBRARY

$G$

THE UNIVERSITY OF CALIFORNIA LIBRARY 
